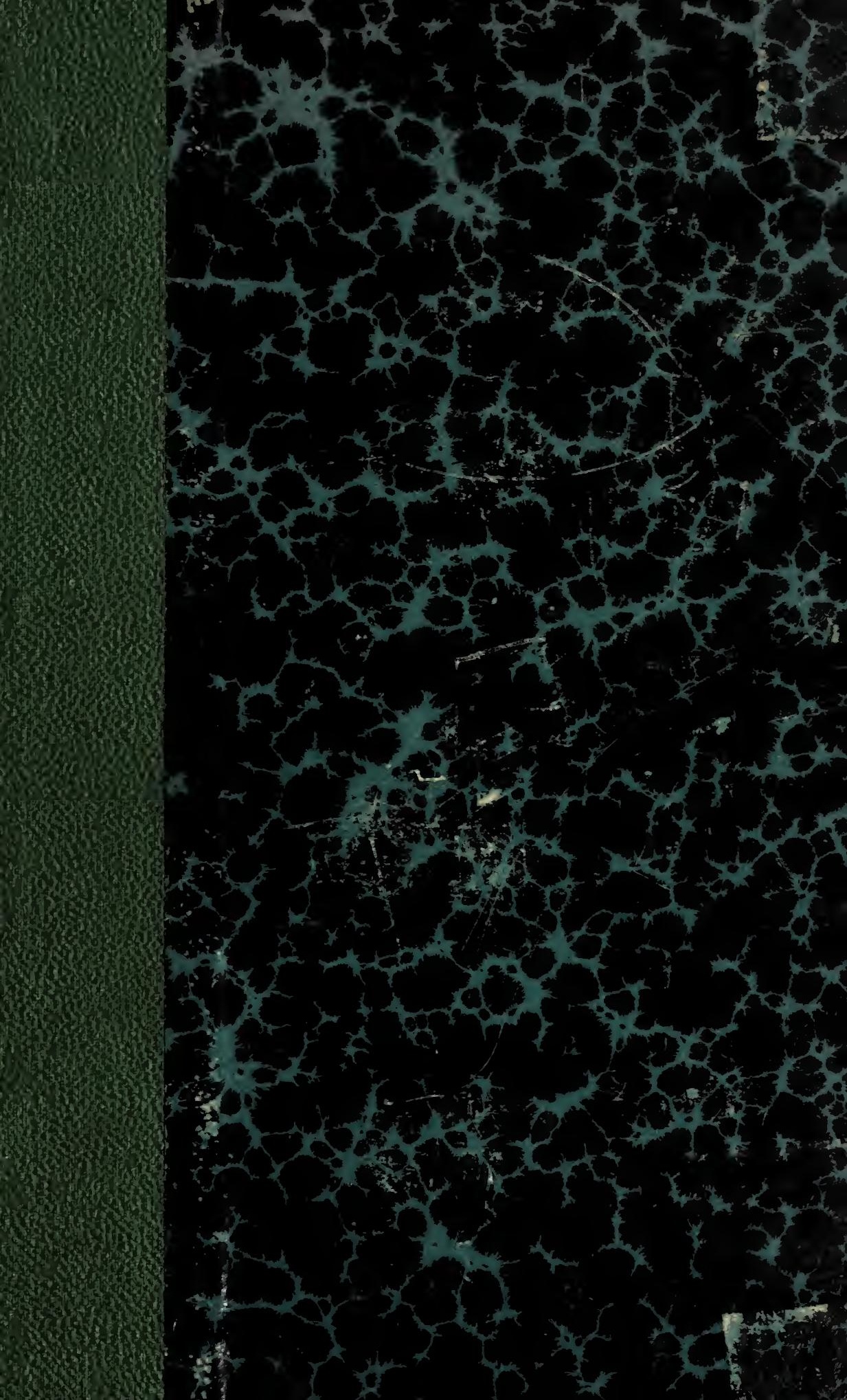




\section{OAK ST. HDSE}

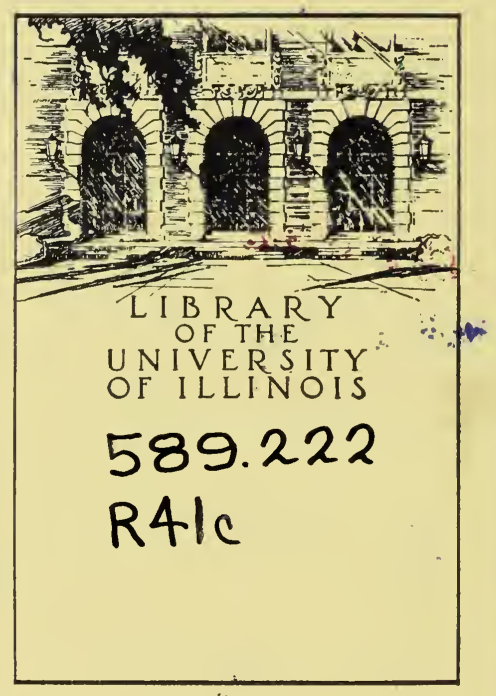


The person charging this material is responsible for its return on or before the Latest Date stamped below.

Theft, mutilation, and underlining of books are reasons for disciplinary action and may result in dismissal from the University.

University of Illinois Library

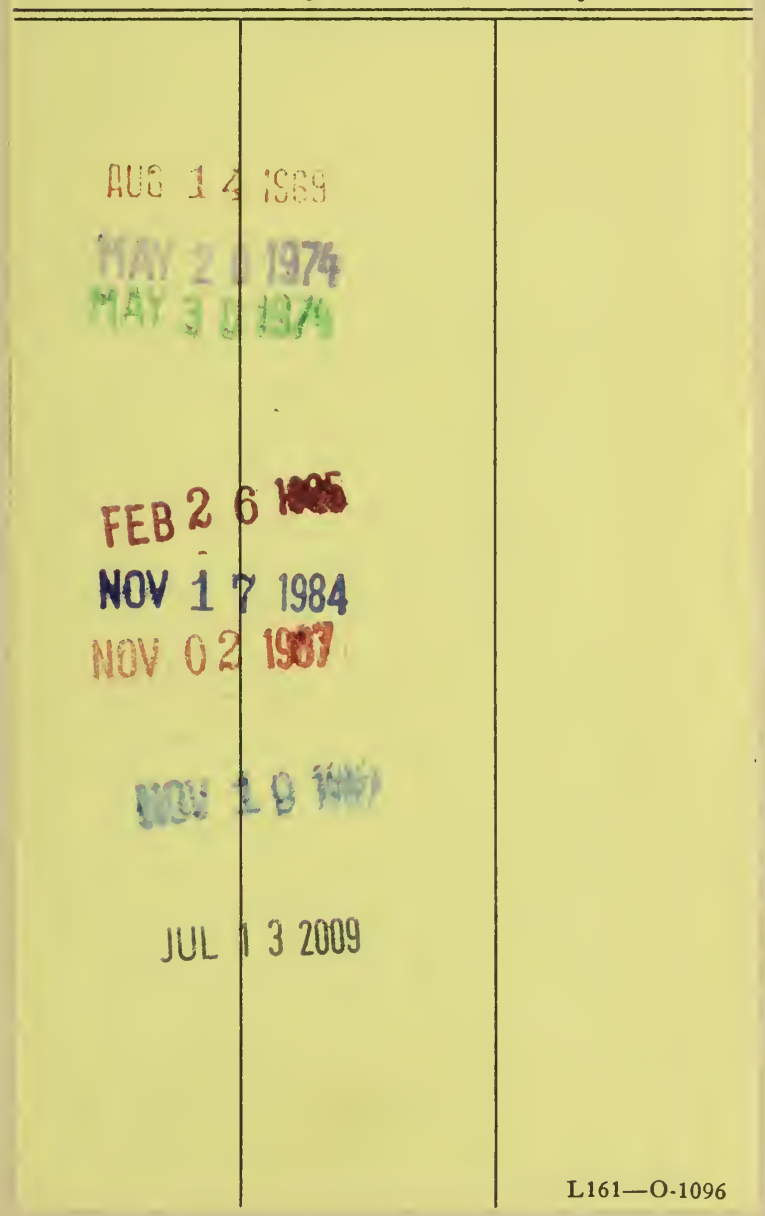



PLANTES CELLULAIRES.

\section{CATALOGUE}

RAISONNÉ

DES CHAMPIGNONS

QUI CROISSENT

DANS LE DÉPARTENENT DE LA MARNE. 
PUBLIGATION DE LA SOGIÉTÉ DES SGIENGES ET ARTS DE VITRY-LE-FRANGOIS. 


\section{CATALOGUE}

RAISUNNÉ

\section{DES CHAMPIGNONS}

QUI CROISSENT

\section{DANS LE DEPARTEMENT DE LA MARNE,}

Etabli d'après les Classifications des Auteurs modernes,

Fries, Quélet, Boudier, Saccardo.

ORNÉ DE DESSINS FAITS D'APRÈS NATURE, LITHOGRAPHIÉS,

représentant les Types des principales F'amilles,

ET DE DEUX TABLEAUX DE M. E. ROZE

sur les AGARICINÉEs,avec 4 planches à l'appui de sa Classification;

Suivi d'une Table Alphalétique d'environ 700 Genres

ascompagnés chacun de la figure et de la dimension des spores qui les caractérisent,

ET D'UNE EXPOSITION MÉTHOdIQUE DES CHAMPIGNONS

Vénéneux et Comestibles CITÉs DANS L'ouvrage,

$$
\text { Par CH. RICHON, }
$$

Membre titulaire de la Société Botanique de France, de la Société d'Agriculture,

Sciences et Arts de la Marne, et de la Société des Sciences et Arts

de Vitrụ-le-François.

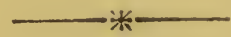

VITRY-LE-FRANGOIS

TYPOGRAPHIE VUE TAVERIIER ET FILS

1889. 
Digitized by the Internet Archive in 2016 with funding from

University of Illinois Urbana-Champaign Alternates

https://archive.org/details/catalogueraisonn00rich_0 
589.222

$R 4 / \mathrm{C}$

I MON PETIT-FILS BIEN AINÉ.

CHA RLES RICHON,

Encouragement A L'Étude de LA Mrcologie. 
Après trente ans de recherches sur la Mycologie, j'ai pensé qu'il convenait d'utiliser une partie de mes collections cryplogamiques en dressant un catalogue des champignons que j'ai récoltés moi-même dans le département de la Marne. Aujourd'hui, mon travail est terminé, je le prèsente à la Société des Sciences et Arts de Vitry-le-François.

Déjá en 1816, M. Léonce de Lambertye fit paraître un catalogue raisonné des plantes vasculaires du Département, publiẻ sous les auspices de la Société d'A griculture, Sciences et Arts de Châlons; il résumait les découvertes de MM. de Lambertye, de Mellet, Saubinet, Levant. Périn, Royer et Jules Remy; toutes les espèces litigieuses avaient été revues par M. Godron de Nancy ; une carte botanique de la Narne indiquant les principaux bassins et terrains géologiques avec les hauteurs et un aperçu géologigue et topographique surle Département, précédaient le texte.

Cet ouvrage remarquable conquit de nombreux 
adeptes dans tous les arrondissements, et en peu d'années le chiffre des espéces découvertes ne le cédait en rien à celui de la flore parisienne.

Un tel succès me donne le courage, peut-être téméraire, de suivre l'exemple de in. de Lambertye en ce qui concerne les champignons. Je ne me dissimule pas les difficultés qui peuvent surgir et s'opposer à la réussite immédiate de mon entreprise ; elles tiennerit à des causes multiples dont je signalerai les principales :

$1^{0}$ L'indifférence qui s'attache à cette classe de plantes humbles et cachées comparée à la faveur accordée sans réserve, aux Phanérogames qui ornent nos parcs et nos jardins.

$2^{\circ}$ L'opinion généralement répandue de l'inutilité ou des qualités dangereuses des Champignons.

3o L'ignorance absolue de leur degré d'importance dans le règne végétal et de la profusion avec laquelle ils sont disséminés.

$4^{0}$ La difficulté de détermination des espèces. réputée insurmontable; enfin, la nécessité de l'emploi du microscope.

Quelques réflexions modifieront, jel'espére, les idées faussement élablies sur ce sujet.

Les Phanérogames attirent instinctivement l'attention par leur taille élevée et par l'éclat de leurs fleurs visibles; les plantes Cryptogames passent ordinairement inaperçues, cependant examinées de prés, elles étonnent et intéressent au même degré le naturaliste et l'artiste, depuis le Lycoperdon giganteum qui mesure près d'un mètre de circonférence jusqu'aux monades et aux microbes qui atteignent á peine un millième de millimètre. 
Les mucédinées surtout affectent des formes variées et élégantes dont la seule vue captive et récompense le botaniste observateur.

L'opinion, très contestable de l'inutilitè et des qualités dangereuses des champignons en général, est le fait de la méfiance motivée par les accidents qui résultent de leur emploi culinaire. cela se comprend chez les gens du monde qui n’ont aucun moyen de contrôle. mais elle est sans valeur pour le botaniste destiné par ses études à en faire un choix judicieux. Du reste, cette méfiance ne vise que les champignons d'un certain volume et qui peuvent exciter la convoitise.

Une foule d'autres espéces, dont le nombre est incalculable intéressent les savants et occupent, suivant Fries, la plus grande place daris le règne végétal: elles envahissent tous les milieux. mème l'air qu'on respire et leur influence sur notre organisme est à cette heure l'objet de recherches fructueuses qui font honneur à la science. Envisagée sous ce point de vue, la Mycologie a droit au respect du botaniste et à sa préférence. Il peut sans hésiter en entreprendre l'étude certain d'arriver à la détermination des espéces avec le concours des nouvelles méthodes d'analyse, et l'emploi constant et indispensable du microscope. Il ne rencontrera plus les obstacles qui ont entravé la marche des anciens Mycologues privés d'ouvrages clairs et précis, de dessins fidèles et de guides expérimentés: ces éléments précieux de travail créés récemment par des naturalistes infatigables, sont désormais à sa disposition. Parmi ces derniers nous citerons : Léveillé. Boudier, Quélet, Bernard, Mougeot, Gillet dune part; de Seynes, Roze, Maxime Cornu, Vantiegliem, Pasteur 
de l'autre; ces courageux champions à force de travail et de persévérance ont aplani les difficultés et débrouıllé un véritable chaos. Les uns habitant la campagne, ont rectifié bien des erreurs en saisissant sur place les secrets de la nature en analysant consciencieusement les espéces; les autres, recherchant plus spécialement au moyen des cultures en cellules les phénoménes de la physiologie végétale chez les infiniment petits, ont enrichi la science et l'humanité de précieuses découvertes; leurs ouvrages destinés à l'étude sont écrits la plupart en français et accompagnés d'analyses dichotomiques.

Depuis 15 ans, les Sociétés savantes ont favorisé ces tendances vers la Mycologie. La Société botanique de France posséde sa section de Cryptogamie et. à l'instar de l'Angleterre, organise des Sessions et des Congrès Mycologiques où sont convièes les célébrités botaniques étrangères.

De plus, une Société mycologique nouvelle s'est organisée et rayonne par toute la France : elle ne compte pas moins de 200 membres.

Ces heureuses innovations contribuent puisamment aux progrès rapides de la Cryptogamie; l'étude des champignons est à l'ordre du jour, non seulement en France mais dans toute l'Europe et déjả (sans parler des œuvres magistrales des Tulasne, des Fries, des Berkeley, des Saccardo) des catalogues et des flores locales mycologiques existent dans les départements du Doubs, du Jura, des Vosges, de Vaucluse, des Charentes, de Seine-et-Oise, etc.

Notre catalogue du Département de la Marne vien! prendre une modeste place dans cette phalange de 
livres utiles ; il est patronné etpubliẻ par la Société des Sciences et Arts de Vitry-le-François à qui j’exprime ici toute ma reconnaissance.

Les Champignons sont des plantes cellulaires privées de chlorophylle: pourvues d'un système végétatif et d'un système de fructification.

Le systéme végétatif, Mycelium, vulgairement blanc de champignon, dınne naissance au système de fructification, Réceptacle, chargé de porter et de protéger les organes de la reproduction spores. Fries compare (abstraction faite du mode de développement) le Myc'elium à un arbre, le Réceptacle au fruit, et les spores aux semences.

Autrefois, le Réceptacle considéré comme le champignon tout entier. préoccupait seul les naturalistes et les classifications étaient basées sur sa forme, sa consistance, sa couleur, etc. Il en résultait, surtout pour les petites espéces, des erreurs et une confusion regrettables qui ont souvent causé le découragement et l'abandon de cette intéressante partie de la Cryptogamie. Aujourd'hui, sans négliger l'étude du Mycelium et du Réceptacle, on donne (de même que pour les Phanérogames) la plus haute importance aux 
organes de la reproduction, et prenant pour base les caractéres précieux fournis par les spores, portées sur des basides ou renfermées dans des thèques, ainsi que la couleur et la forme de ces spores, on a créé de nouvelles classifications qui suppriment les difficultés anciennes.

Tous les champignons connus, le microscope aidant, ont èté soumis à cette méthode et le succès le plus éclatant en a couronné l'épreuve.

C'est alors que des milliers d'espèces ignorées ou mal déterminées, appartenant aux Pyrénomycètes, aux Mucédinées, aux Urédinées etc., ont èté casées sans peine et scientifiquement dans les cadres préparés à l'avance.

J'ai suivi avec le plus grand intérêt ces innovations utiles à la Mycologie et, chaque fois qu'elles se sont produites, j’ai dû modifier les Catalogues que j’avais coutume de renouveler chaque année.

Notre Catalogue dela Marne profite de ces changements successifs et j’ai eu soin de revoir attentivement, avant sa publication, les espéces qu'il renferme. Mes remerciements les plus sincères à mes amis Boudier, Quélet. Andréle Breton et Roze qui ont maintes fois rendumon travail facile par leurs savants conseils et leurscommunications.

Plus de 2.200 espéces et de nombreuses variétés forment le contıngent des récoltes que j'ai faites pendant 30 ans dans le département; une centaine d'espéces sont de nouvelles découvertes pour la science; nous en donnons plus loin la liste alphabétique. Ce chiffre déjà respectable le serait beaucoup plus si j'avais eu dans la Marne des compagnons d'étude et de recherches: 
j'estime qu'il pourrait à l'avenir être augmenté d'une centaine d'espéces par an.

Une moisson abondante et précieuse est certainement réservée à ceux qui voudront poursuivre ma tâche dans les riches contrées du département que je n'ai pu explorer. Mes investigations en effet ont été dirigées principalement dans l'arrondissement de Vitry dont le sol est composé, au sud-est, de grès verts supérieurs et d'une bande de craie-tufau ; à l'est, de craie blanche, terrain crétacé supérieur.

J'ai parcouru entre-temps les bois de la montagne de Reims reposant sur le calcaire siliceux, et quelques localités dans les autres arrondissements. En somme, les bois de Vanault-les-Dames et de Charmont, de St-Remy-en-Bouzemont,la forêt de Sermaize à St-Dizier. les plantations de pins sylvestres qui couvrent le sol crayeux entre la Moivre et Vitry-le-François, les terrains d'alluvion avec les parties boisées qui bordent les cours d'eau, les plateaux élevés qui dominent la vallée de la Marne, de Dizy à Rilly-la-Montagne, telles sont les stations favorables que j'ai visitées si longtemps et toujours avec fruit.

On voit par cette énumération que les arrondissements d'Epernay et de Ste-Menehould sillonnés de forêts importantes font complètement défaut; nous signalerons particulièrement aux Mycologues les bois et les marais d'Anglure, la forêt de Traconne, les marais de St-Gond, les bois de St-Martin-d'Ablois, de Montmort et la forêt d'Epernay d'une part; de l'autre, la forêt de l'Argonne qui gagne Ste-Menehould et se prolonge jusqu'au Chatellier. Ces centres d'exploration complètementiguorés recélent, à n'en pas douter, des 


\section{VIII}

richesses qui surprendrontles naturalisles etles dédommageront de leurs peines. Ils devront avant d'entreprendre ces excursions mycologiques connaître suffisamment la flore phanérogamique de la contrée et posséder des données sommaires sur lasaison, l'habitat. la durée et la récolle des cryptogames. Quelques renseignements à ce sujet ne seront pas inutiles.

S'il est vrai de dire cu'il y a des champignons partout et qu'on peut en récolter toate l'année, (puisque certaines espéces, comme les Polypores ligneux, vivent de 10 à 15 ans et que les plantes mortes ou languissantes, voire même l'organisme animal n'échappent en aucun temps à leur envahissement), il faut reconnaître néanmoins, que l'apparition de chaque espéce se fait avec une régularité bien constatée, sous l'influence des milieux et de la température.

L'humidité et la chaleur modérée favorisent le développement des champignons charnus, de sorte que leur saison par excellence est la fin de l'été et l'automne. A cette époque, l'humus des bois formé par les détritus des feuilies: les vieux trones d'arbres. les matières azotées, les végétaux pourris en sont littéralement couverts. Chaque espèce a choisi pour croître le moment et le lieu qui conviennent à sa nature; le Pin, le Chêne, le Charme, etc. abritent des espéces différentes; c'est encore en automne que les plantes herbacées et les feuilles des arbres vivantes mais privées de leur vitalité première, deviennent la station spéciale des Urédinées et d'un grand nombre de Pyrénomycètes.

$\mathrm{Au}$ printemps, le nombre des grandes espèces est plus restreint, c'est la saison des Morilles, des Mousserons, des Pezizes. 
Les grandes chaleurs de l'été et la sécheresse ralentissent ou interdisent leur végétation.

En hiver, les espéces charnues et tendres ont disparu complètement et ont fait place aux champignons (improprement dits infẻrieurs) à enveloppe dure, charbonneuse, Pyrénomycètes, ou de structure celluleuse et filamenteuse telles que les Mucédinées.

La végétation active et prompte des champignons est proverbiale; on dit "pousser comme un champignon ". Un ou deux jours suffisent au développement total des A garicinées charnues et des Bolets; les Lycoperdons et les Bovista, dont le volume est parfois si considérable. poussent en une seule nuit; il ne faut que quelques heures pour les Coprins dont l'existence totale ne dépasse pas une journée. Unepluie abondantesuivie de chaleur peuple abondamment une forêt qui, la veille n'offrait aucune trace de cryptogames. C'est au botaniste á tenir compte de ces conditions atmosphériques et à visiter plusieurs fois dans une saison les mêmes localitès; son œil scrutateur doit sonder et fouiller les taillis, les replis des terrains humides, les hautes herbes, les amas de feuilles et les racines qui recèlent des espéces précieuses; autour des habitations, les vieilles haies, les palissades, les fumiers, les fagots mis en tas, les chantiers, les caves et toutes les substances putrescibles.

La plus humble plante, avant sa décomposition complète, intéresse; l'Ortie suivant Cooke donne asile à 24 espéces de champignons.

Lorsque la récolte des espéces a pour objet l'étude, on doit prendre la précaution de recueillir, sans les froisser, des échantillons de différents âges, même avec 
leurs supports, sans lesquels il serait difficile de constater et d'apprécier leur parasitisme.

Il s'agit ensuite de les transporter sans encombre au logis; pour cela faire, il est d'usage de placer les petites espéces avec un peu de mousse dans des boîtes qui tiennent dans la poche, et les grandes espéces dans la boîte traditionnelle des botanistes ou dans des paniers confectionnés exprès. Quant à moi, j'emploie un procédé rustique et primitif qui m'a rendu de bons services; un clayon en osier, rond, de 30 centimètres de diamétre et un mouchoir assez grand en font tous les frais. Le clayon posé sur le mouchoir et les extrémités decelui-ci nouées ensemble, constituent un panier improvisé dont les parois ne froissent pas les champignons.

Quand l'excursion est terminée, je profite d'un quart d'heure de repos pour organiser solidement mon précieux colis; j'installe une á une, sur le sol, mes trouvailles qui ont été placéessans ordre au fur et á mesure de la récolte; puis garnissant le clayon d'une mince épaisseur de mousse ou de graminées je fais une première couche avec les champignons les plus résistants; je les recouvre également de mousse et par dessus une seconde couche de champignons plus fragiles et ainsi de suite; de sorte que, dans le voyage qui me reste á faire, même en voiture et en chemin de fer les espèces souffrent peu du tassement et de la pesanteur.

Aussitôt rentré à domicile je débarrasse le clayon des champignons exposés à se déformer et á moisir dans la mousse humide et je me hâte d'étudier d'abord. les individus les plus tragiles, laissant les plus fermes et les plus coriaces pour la fin. 
J'ai soin de poser certaines espéces sur du papier blanc, la face fructifére tournée en bas pendant quelques heures, afin de recueillir les spores qui s'y déposent en masse ; je puis alors en apprécier la couleur et la forme. Je dessine dans leur état de fraìcheur les champignons charnus, putrescibles, déliquescents ou fugaces et je réserve pour mon herbier les plus résistants.

Je fais suivre ces considérations générales d'une exposition méthodique de la classification adoptée pour notre Catalogue.

Pour établir les groupes des familles, des genres et des espéces, j’ai eu recours aux classifications modernes et jai emprunté ce qui pouvait élever notre Catalogue á la hauteur des découvertes récentes de la Science. Ces éléments m'ont permis de dresser un cadre susceptible de donner asile á nos richesses mycologiques et á celles que les progrés de la botanique nous réservent.

La classification de Fries modifiée par le $D^{r}$ Quélet pour les Hyménomycètes, celle de $\mathrm{M}$. Boudier de Montmorency pour les Discomycètes et le travail gigantesque du Dr Saccardo de Padoue pour les Pyrénomycètes en général et pour les Hyphomycètes, ont èté mes meilleurs guides. 
Tenant compte en outre, des caractères essentiels et fondamentaux reconnus depuis peu dañs quelques groupes, voire même dans quelques espéces, j'ai admis sans hésiter avec rang d'ordre. les Plasmodiophores de Fuckel, les Phycomycètes de de Bary, Jes Asconémées de Kickx et les Onigénées de Tulasne. Ces ordres une fois établis, j'ai groupé sans difficultè une trentaine de familles,plus de six cents genres et des milliers d'espèces récoltées dans nos parages.

La classification des champignons de la Marne que nous avons adoptée pour le Catalogue comprend trois divisions principales et un appendice, subdivisés en neuf ordres et trente familles.

Tous les champignons arrivés au terme le plus complet de leur évolution font partie des deux premières divisions; les autres champignons sont rélégués dans la troisième.

La $1^{\text {re }}$ division se compose des Exospores, champignons dont les spores.(véritables semences) sont libres: elle est représentée par 4 orares "les Hyméniés, Quélet, les Péridiés, Quélet, les Plasmodiophores, Fuckel, et les Hypodermés , de Bary.

La $2^{\mathrm{e}}$ division se cormpose des Endospores, champignons dont les spores (sporidies) sont renfermées dans des thèques (petits sacs ou utricules cellulaires); elle est représentée par 5 ordres, les Phycomycètes, de Bary; les Asconémés. Kickx; les Onigénés. T'ulasne; les Cupulés, Quélet; les Nuclées, Quélet.

La $3^{\text {e }}$ division se compose des Fungi nondumperfecti ou champignons dont les organes de reproduction indiquent un état préformatif, très naturel d'ailleurs, dont le cycle de végétation n'est pas complet: elle 
forme un groupe de trois familles: conservées provisoirement, mais destinées à disparaître á mesure que de nouvelles découvertes permettront de les associer aux espéces parfaites dont elles dépendent; ce sont les Sphéropsidées, Léveillé ; Mélanconiées. Berkl; les Hyphomycètes. Martius. Les spores des Sphéropsidées sont dites sporules; celles des Mélanconiées et des Hyphomycètes, conidies.

Enfin, sous le titre d'Appendice est compris un nombre très restreint de productions fongines stériles ou de natıre douteuse et certaines altérations pathologiques considéréesjadıs comme des espéces autonomes.

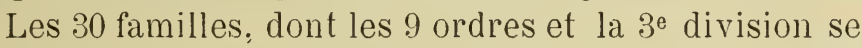
composent, sont séparées l'une de l'autre par des caractères très tranchés, signalés en partie par le Dr Quélet dans les Champignons du Jura et des Tosges et par le Dr Saccardo dans son Sylloge Fungorum. Dans notre tableau synoptique, nous les avons mis en regard du nom des familles; ces caractères différentiels méritent l'attention des botanistes commençants ; ils feront bien de les consulter souvent; en remontant ainsi à la source de la classification et en suivant scrupuleusement les tables analytiques et les descriptions des meilleurs auteurs appuyées par de bons dessins (comme l'a fort bien dit le $D^{r}$ Quélet à la première session mycologique française en 18\%6), ils arriveront á la détermination exacte des espéces.

Nous faisons précéder notre catalogue:

$1^{0}$ D'une énumération méthodique des principaux ouvrages que j'ai consultés de 1855 à 1887 ;

$2^{\circ}$ De l'indication des ouvrages français qui doivent diriger les premiers pas des mycologues : 
$3^{0}$ D'un tableau annoté de la classification toute entière :

$4^{\circ}$ De 6 planches de dessins d'après nature et lithographiés, accompagnés d'un texte explicatif assez étendu, représentant par ordre, les types de toutes les familles avec leurs organes de reproduction;

5' A titre de document intéressant,deux tableaux sur les Agaricinées, indiquant la répartition des genres par familles d'après la couleur des spores et d'un essai de classification des Agaricinées par M. E. Roze ; (*)

$6^{\circ}$ De 4 planches représentant les types des familles proposées par M. Roze pour la classification des Agaricinées.

La liste des genres dressée à la fin du catalogue sera suivie de plusieurs planches représentant les spores, sporidies et conidies des espéces considérées comıne types de chaque genre inscrit.

Le but que nous nous proposons est de donner aux botanistes, à l'aide de notre classification, une idée générale des organes essentiels qui caractérisent chaque famille. de les renseigner sur la répartition des genres et des espéces, enfin d'indiquer les stations naturelles des plantes et les localités oú elles ont été récoltées, leur utilité, leur degré de rareté et le moment de leur apparition.

Là. se borne la prétention de notre œuvre qui ne peut être assimilée à une Flore complète ou à un cours de Mycologie.

(*) Je dois à la bienveillance et à l'amitié de l'auteur la permission de publier ce tableau. 


\section{OUVRages CONSUltés DE 1855 A $188 \%$.}

\section{BOTANIQUE DESCRIPTIVE.}

\begin{tabular}{|c|c|}
\hline Ordre $1^{\mathrm{er}}$ & 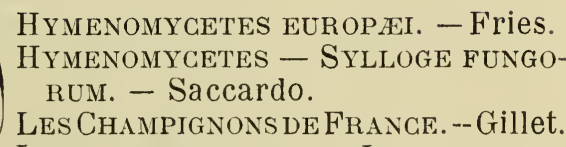 \\
\hline HYMÉNIÉES & $\begin{array}{l}\text { LES GHAMPIGNONS DU JURA ET DES } \\
\text { VOSGES. - Quélet. } \\
\text { GLA VIS HYMENOMYGETUM. - Gooke et } \\
\text { Quélet. }\end{array}$ \\
\hline
\end{tabular}

Ordre $2^{\mathrm{e}}$
PERIDIÉS $\quad\{$ CARPOLOGIA FUNGORUM. - Tulasne.

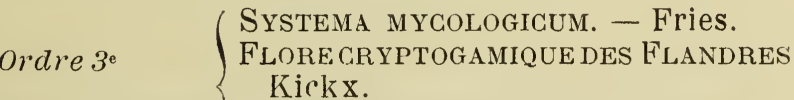

PLASMODIOPHORES NOTIGE SUR LES MYXOMYGÈTES. E. Roze.

\begin{tabular}{|c|c|}
\hline $\begin{array}{l}\text { Ordre } 4^{\circ} \\
\text { HYPODERMĖS }\end{array}$ & $\left\{\begin{array}{l}\text { FLOF } \\
\text { SYME } \\
\text { FLOH }\end{array}\right.$ \\
\hline $\begin{array}{l}\text { Ordre } 5^{\circ} \\
\text { HYCOMYCETES }\end{array}$ & $\left\{\begin{array}{l}\text { Flore des Flandres. - Kickx } \\
\text { MucorineEs. - Van Tieghem. } \\
\text { Peronosporées. - Max-Cornu. }\end{array}\right.$ \\
\hline & $\begin{array}{l}\text { ORE DES FLANDRES. Kick } \\
\text { MBOLA MYCOLOGICA. - Fuc } \\
\text { EILZE. - Otto Vünsche. }\end{array}$ \\
\hline
\end{tabular}




\begin{tabular}{|c|c|}
\hline $\begin{array}{l}\text { Ordre } 7 . \\
\text { ONIGENES }\end{array}$ & ANN.SCIENCES NATURELLES. -- Tulasne. \\
\hline $\begin{array}{l}\text { Ordre 8. } \\
\text { CUPULÉS }\end{array}$ & $\begin{array}{l}\text { FL.ORE DU JURA ET DES VosGeS. - } \\
\text { Quélet. } \\
\text { NouvELLE CLASSIFICATION DES DIS- } \\
\text { COMYCÉTES. - Boudier. } \\
\text { MÉMOIRE SUK LES Ascoboles. - } \\
\text { Boudier. } \\
\text { SYSTEMA MYGOLOGICUM. - Fries. } \\
\text { MYCOLOGIA FENNICA. - Karsten. }\end{array}$ \\
\hline $\begin{array}{l}\text { Ordre } 9^{\circ} \\
\text { NUCLEES }\end{array}$ & $\begin{array}{l}\text { SYLLOGE FUNGORUM. - Saccardo. } \\
\text { Les deux premiers volumes et sup- } \\
\text { pléments. }\end{array}$ \\
\hline $\begin{array}{l}\text { NGI NONDUM } \\
\text { PERFECTI }\end{array}$ & 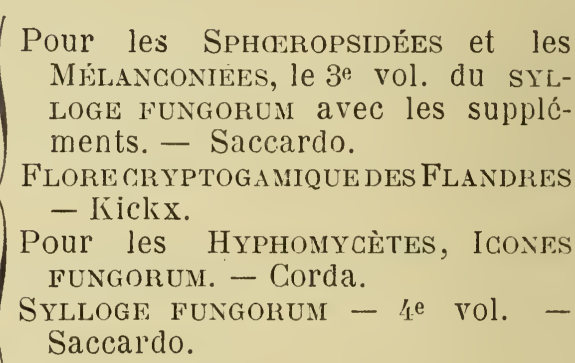 \\
\hline
\end{tabular}

\section{ICONOGRAPHIES.}

ICONES FUnGorum. - Schœffer - Ratisbonne 1761. Champignons de France. - Bulliard 1780-1798.

Traité des Champignons. - paulet - 1793-1835.

linglisch Fungi. - Sowerby - London - 1797-1815.

ICONES SELECTA HYMENOMYCETUM. - Fries - $18 \%$.

ABBILdUNGEN DER SCHWAMME. - Krombholtz - 1831-1819.

ICONES Fungorum. - Corda - Prague - 1887-1842.

Champignons de France. - Gillet.

Atlas des Champignons venéneux et comestibles. Richon et Roze 1886-188\%. 


\section{MYCOLOGIE GÉNERALE.}

Systema mycologium. - Fries.

SyNopsis FUngorum. - Persoon.

ICONES FUNGORUM. - Corda.

SYLLOGE FUNGOKUM. - Saccardo.

Ouvrages français particulièrement recommandés aux commençants.

Flore du JURA ET DES VOSGES avec les suppléments, par le Dr Quélet.

Les Ascoboles ét les Pezizes, par M. Boudier.

Les Chanpignons de France, par Gillet.

Les Ghampignons de France, par Bulliard. (Iconographie).

L'ATLAS DES Champignons véNÉNEux ET COMESTIBLES publié en commun par MM. Roze et Richun. - Aunée 1887. Texte et gravures.

Tables analytiques des Champignons, publication nouvelle de M. N. Patouillard. 


\section{CLASSIFICATION}

\section{DES \\ CHAMPIGNONS}

DISPOSITION GENEERALE DES FAMILLES.

$1^{\text {re }}$ DIVISION.

EXOSPOTES.

1 er ORdre. - Hyméniées. - Quélet.

1. AGARICINÉES Fries. Hymenium lamellé.

2. POLYPOREES. Mich. Hymenium tubuleux ou poreux.

3. HYDNEES. Fries. Hymenium aiguillé ou denté.

4. AURICUlarinées. Fries. Hymenium uni et horizontal.

5. CLAVARIÉES. Bull. Hymenium uni et vertical, simple et ramenx.

6. TRÉMELLINÉES. Fries. Hymenium indéfini et gélatineux. 
Ze ORDRE. - Péridiés. - Quelet.

1. NIDUlariées. Cord-Tul. Péridium (*) à hymenium charnu. ni pulvérulent ni déliquescent.

2. PHALLOIDÉEs. Fries. Péridium double, renfermant une couche de gélatine; hymenium déliquescent,

3. LYCOPERDINÉES. Fries. Péridium à hymenium charnu puis pulvérulent.

't. HYPOGÉES. Berk. Péridium membraneux, indéhiscent ; glèbe lacuneuse. déliquescente, privée de capillitium.

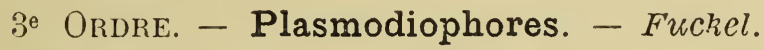

MYXOMYCETES. de Bary. Mycelium absent ; d'abord substance mucilagineuse, informe, fluxile, puis prenant une consistance et une forme determinée suivant les genres.

$4^{e}$ Ordre. - Hypodermès. - de Baiy.

USTILAGINÉES. Tul. Plantes parasites. sous épidermiques. pulvérulentes. détruisant les tissus.

URÉDINÉES. Tul. Plantes parasites, sous-épidermiques ne détruisant pas les tissus.

\section{ENTDSPORES.}

5e Ordre. - Phycomycètes. - Kunz. - de Bary.

1. PERONOSPORÉEs. de By. Petites plantes filamenteuses, parasites sur les feuilles virantes. á ramifications extérieures et intérieures terminèes par des Sporanges qui contiennent les spores.

('Péridium. Enveloppe close. tapissée intérieurement par l'hymenium. Hymenium. Membran fructifère.

Mycelium. Tissu cellulaire composant le système vẻgétatif des Champignons. 
2. MUCORINÉES. de By. Plantes filamenteuses, parasites sur les corps qui entrent en décomposition. Ramifications extérieures terminées par des sporanges.

$6^{\circ}$ Ordre. - Asconémées. - Kickx.

1. ASCOMYCÉES. Kickx. Petites plantes parasites sur les feuilles vivantes, réduites à une seule cellule ou théque contenant les spores.

$$
\text { ye OrdRe. - Onigénés. - Tulasne. }
$$

1. ONIGÉNÉES. Tul. Plantes parasites sur les substances animales; apparence de Lycoperdinées: filaments du capillitium terminés par des théques.

8eORDRE. - Cupulés. - Quélet. Discomycètes. - Fries.

1. HELVELLEES. Fries. Cupules extérieures.

2. TUBERACÉES. Tul. Cupules intérieures. plissées chiffonnées.

ye Ordre - Nuclées. - Quélet.Pyrenomycètes.-Fries.

1. PERISPORIACÉES. Fries. Périthèces (1) membraneux, coriaces ou charbonneux sans ouverture.

2. SPHARIACÉES. Fries. Périthèces charbonneux, coriaces ou membraneux, s'ouvrant par un ostiole arrondi.

3. HYPOCREACÉES. de Not. Périthèces presque charnus, céracés-membraneux, la plupart de couleur rouge. ostiole arrondi.

4. DOTHIDÉACÉES. Nitsk. Périthéces formés par la substance du strôme (1) dans laquelle ils sont plongès ; ostiole papilliforme.

(1). Peridium des Nuclées et des Sphcropsidées.

(1) Strôme, parenchyme des champignons supportant ou renfermant dans *? substance plusieurs Périthèces. 
5. MICROTHYRIACÉES. Sace. Périthèces membraneux ou charbonneux, dimidiés. scutiformes; tissu à fibres rayonnantes.

6. LophiostomaCÉES. Sacc. Périthéces charbonneux. adnès á la base ou incrustés; ostiole comprimé s'ouvrant en fente.

7. HYSTÉRIACÉES. Corda. Périthèces oblongs ou linéaires,coriaces,ou charbonneux. - Ouverture en fentequi parcourt toutela longueur du périthéce. PHACIDIACÉS. Tul. Périthèces en disques, s'ouvrant par fentes valviformes, simples ou multiples.

$3^{e}$ DIVISION.

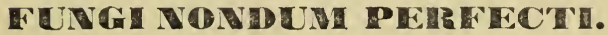

1. SPHÆROPSIDEES. Lév. Périthèces privès de thèques, contenant des sporules portées par des basides plus ou moins manifestes.

2. MÉLANCONIÉES. Berk. Champignons privés de perithéces et de theques; conidies portèes sur des basides variées, issues d'un substratum proligère.

HYPHOMYCETES. Marius. Champignons privés de perithèces el de théques consistant en filameuts plus ou moins développés et en conidies libres.

Nota. - Les Fungi imperfecti ou nondum perfecti des auteurs sont des champignons considérés comme des furnes primitives des Nuclées à endospores ; leurs organes delructificalion sont Exospoies, renfermés dans des conceptacles (sphæropsidées) ou libres (melanconiees, hyphomycètes). 


\section{APPENDICE.}

1. MYCELIA STERILIA. Fries. Tissus fongiques - privés de fructification.

2. EXANTHEMATA. Kickx. Altérations pathologiques dues aux piqures d'insectes.

3. NOSOPHLAEA. Fries. Renflements bulleux de l'épiderme des troncs et rameaux vivants, s'ouvrant par fentes et laissant échapper sous forme de corpuscules pulvérulents. les cellules désagrẻgées de l'ècorce. 

EYOOSPORES . Ier Ord. Hymẻnies. Quelet . I. Igaricinèes.
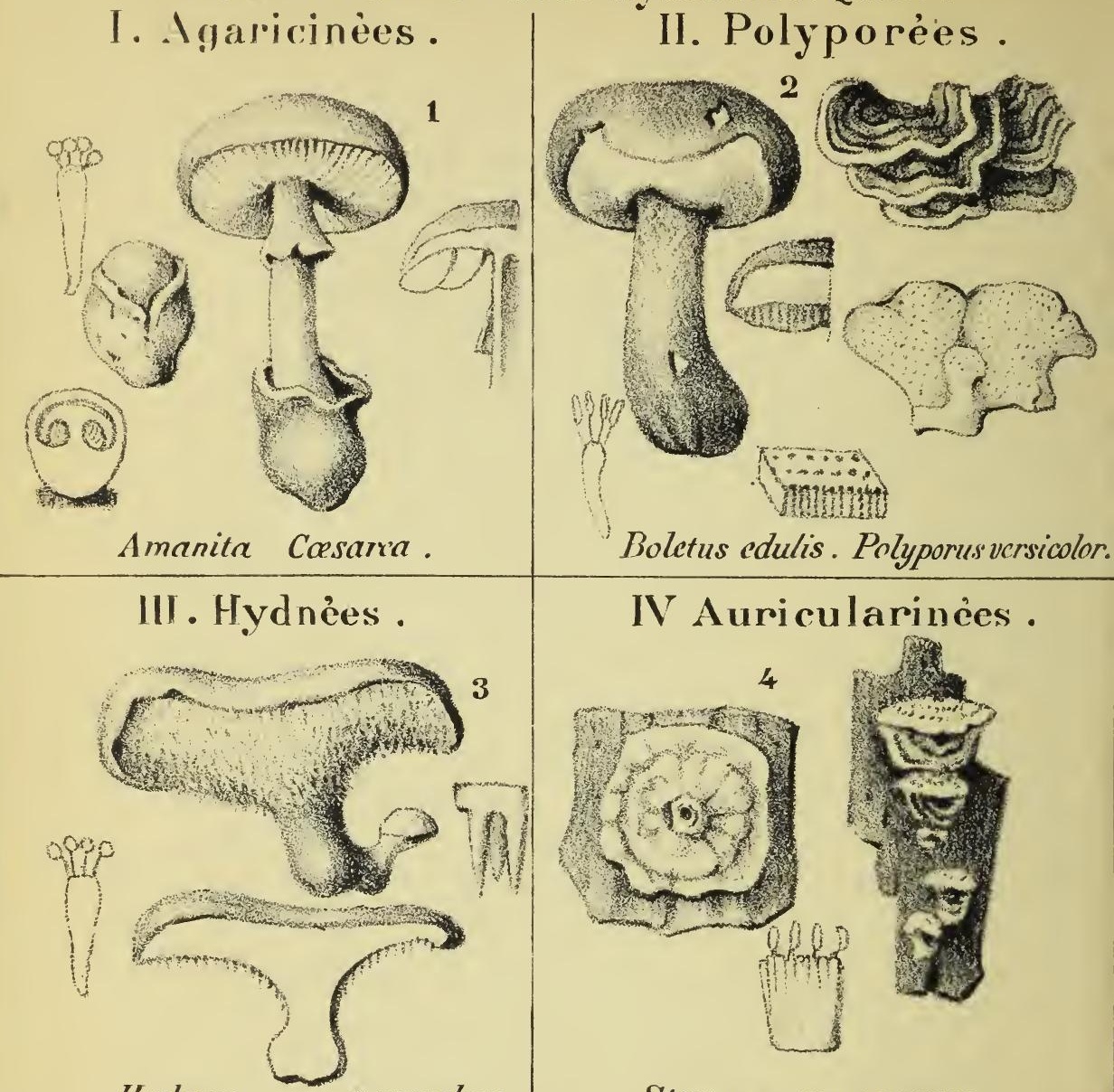

Hydnum

repandum.

V. Clavariées.

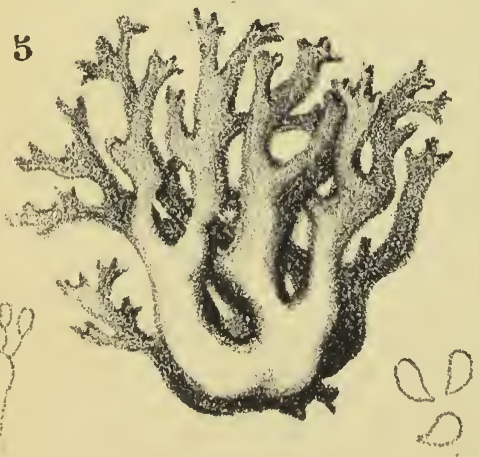

Boletus edulis. Polyporus versicolor.

IV Auricularinces.

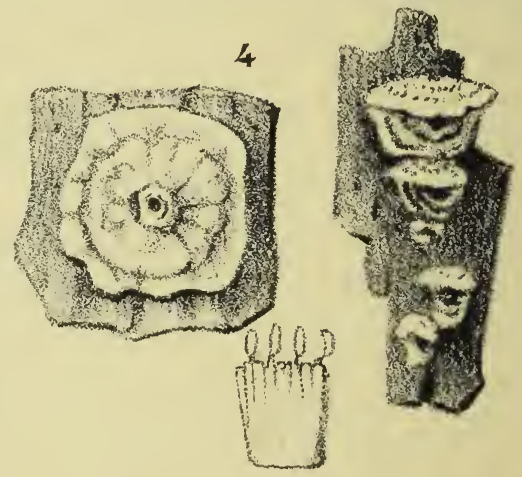

Stereum purpureum .

\section{Vl. Tremellinées.}

6

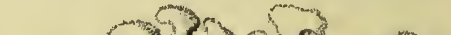




\section{EXPLICATION DES PLANCHES.}

\section{PLANCIE I.}

Fig. 1. AMANITA CCESAREA. Pers. Etat adulte: coupe de chapeau garnı en dessous de lamelles; état jeune. chapeau encore enveloppé par le volva; coupe du champignon avant la rupture du volva; basides et spores.

Fig. 2. BOLETUS EDULIS. Bull. Etatadulte; coupe du chapeau garni de tuhes; basides et spores. POLYPORUS VERSICOLOR. Fries. Chapeaux sessiles, superposés; chapeaux vus en dessous, sporeux; spores vus à la loupe.

Fig. 3. HYDNUM REPANDUM. Linn. Etat adulte; coupe du chapeau garni d'aiguillons; aiguillons vus à la loupe; baside et spores.

Fig. 4. STEREUM PURPUREUM. Fries. Champignon membraneux, á hymenium lisse.appliqué en rosace sur le bois ou réfléchi superieurement : basides et spores.

Fig. 5. CLAVARIA AMETHYSTEA. Bull. Champignon vertical, rameux; hymenium tapissant la surface des rameallx : basides et spores.

Fig. 6. TREMELLA MESENTERICA. Retz. Champignon gelatineux, sinueux. lobé, echantillon grandeur naturelle et échantillon plus petit sur. branche de peuplier; basides globuleuses puis émettant 4 apicules terminés par les spores: spores isolés. 


\section{$-10-$ \\ PL $\triangle$ NCHEII.}

Fig. 7. CYATHUS Striatus. Hoffm. Deux sujets grandeur naturelle, l'un fermé par un diaphragme, l'autre ouvert ; coupe d'un peridium; deux péridioles isolés; basides et spores.

Fig. 8. PHALLUS IMPUDICUS. Linn. Phallus adulte ; coupe du peridium et de la columelle ou stipe; cellules réticulées du capitule grossies, grandeur $1 / 3$.

PHALLUSCANINUS. Fuckel. Phallusadulte: presque de grandeur naturelle; hymenium capituliforme adhérant complètement au stipe : basides et spores.

Fig. 9. SCLERODERMA VERRUCOSUM. Pers. Peridium globuleux, verruqueux ; $1 / 3$ grand nat. spore bruse, entourée d'une bordure transparente; basides pirıformes et tetraspores.

\section{LYCOPERDON EXCIPULIFORME. SCOp.} Peridium muni d'un pédicule trés développé, couvert d'aspèrités côniques; basides renflées portant deux longs apicules sporifères; spore tuberculeuse avec apicule ; spore libre, bruneverdâtre.

Fig. 10. RHIZOPOGON LUTEOLUS. Fies. Peridium 1/2 grand. nat. lisse, fendillé : coupe. glébe jaune-verdâtre; hyınenium labyrinthifurme ; basides et spores ; spores isolees.

Hig. 11. DICTYDIUM UMBILICATUM. sichrad. Groupe sur bois; un pea plus grandque nature: Dictylium très développé : spores sphériques. oinbiliquèes.

SPUMARIA ALBA. Pers. Masse blanche à l'etat spumeux. puis organisée en membranes et en expansions rameuses. terminées par des 


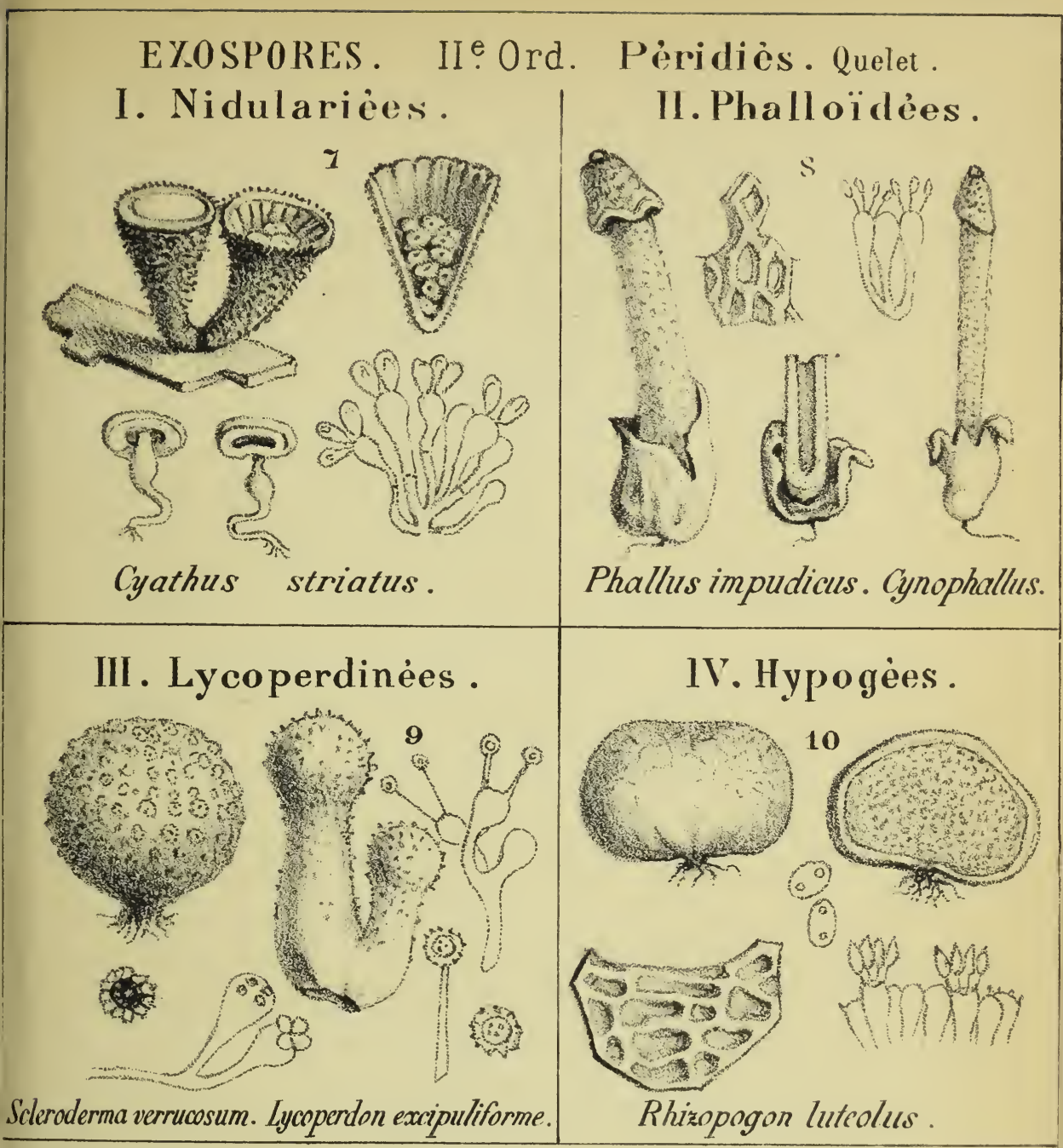

EXOSPORES . III Ord. Plasmodiophores. Fuckel. I. Myxomycètes.
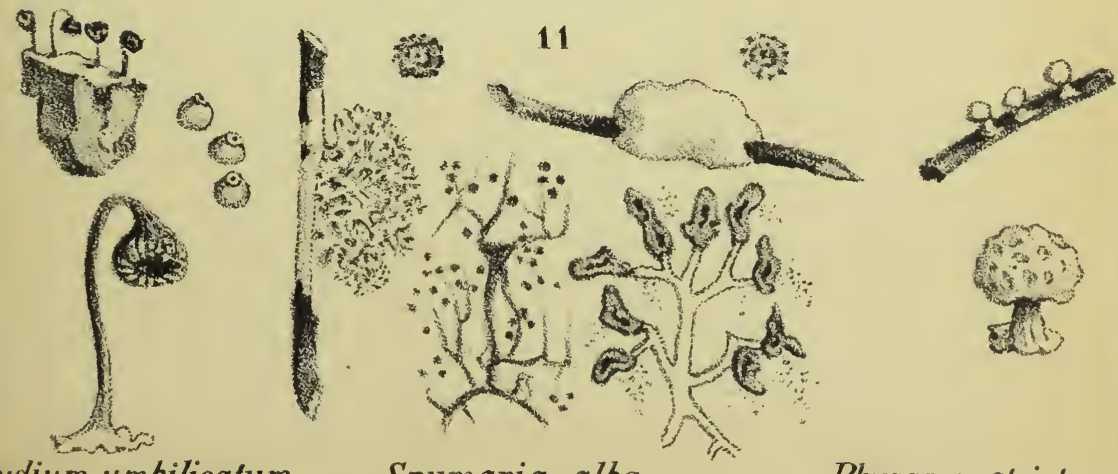

Dictydium umbilicatum. Spumaria alba.

Physarum striatum . 

EXOSPORES IVe Ord. Hypodermés . de Bary.

I. Ustilaginèes.

12

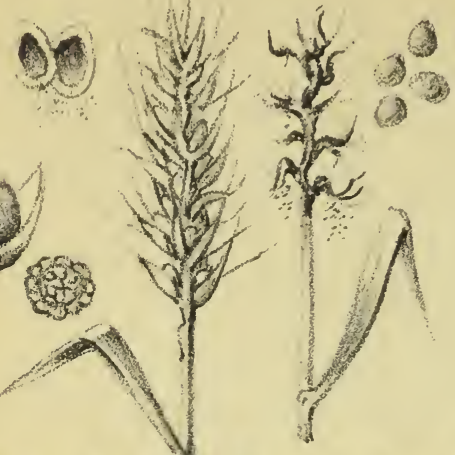

Tilletia caries. Ustilago segetum.
II . Urẻdinẻes.

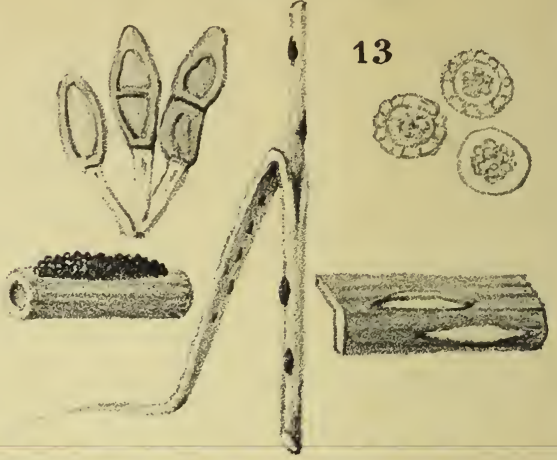

Puccinia graminis. Uredo linearis.

END OSP0RES. Ve Ord. Phycomycètes. deBary.

I. Peronosporées.

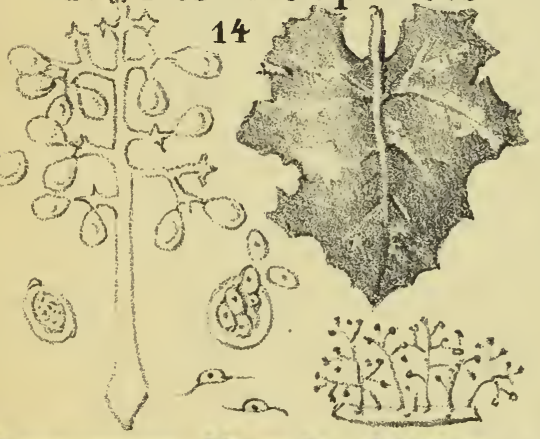

Peronospora Viticola. Mildew.
II. Mucorinées.

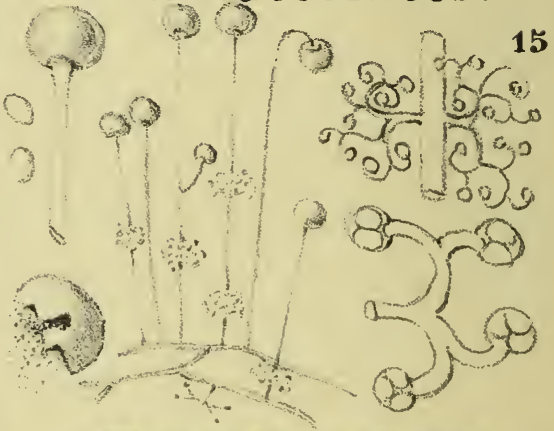

Thamnidium elegans.

END. VI ${ }^{e}$ Ord. Asconẻmẻs Kickı END.VIIe Ord.0nigènẻs . Tul.

I. Ascomycées.

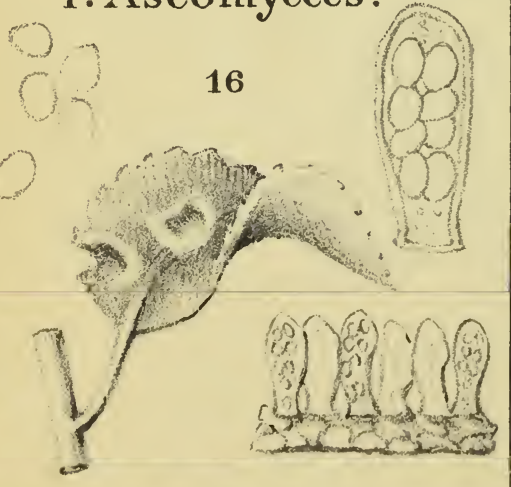

I. Onigẻnẻes.

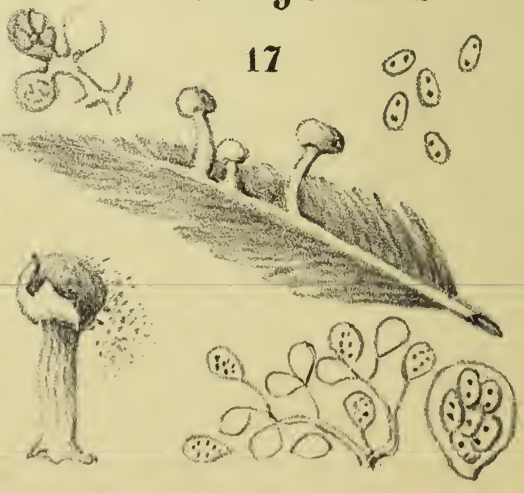

Onigena Corvina . 
logettes grises, réceptacles des spores, filaments bruns; spores libres, noires-bleuâtres. verruqueuses.

PHYSARUM STRIATUM. Fries. Groupe de Physarum un peu plus grands que nature: peridium blanc, globuleux, ombiliqué en dessous, pédicule strié, court. épais.

\section{P LA NCHE III.}

Fig. 12. TILlETIA CARIES. Tu. Epi de blé envahi: grain de ble maladesans changementue forme, brunâtre; coupe de grain de blé rempli de spores brunes áodeur fétide; spore globuleuse. réticulée.

USTILAGo SEGETUM. Corda. Epi d'avoine envahi : grains et gluınes détruits, remplacés par la masse des spores noires: spores lisses, noires, rondes-ovales.

liig. 13. PUCCINIA GRAMINIS. Pers. Groupe de puccinia sur feuille et gaîne de graminée; groupe grossi de forme elliptique, brun: spores brunes, pédicelles incolores.

UREDO LINEARIS. Pers. Stylospore de Puccinia graminis; groupe allongé. jaune : stylospores sessiles, globuleuses, jaune-d'or.

Fig. 1'í PERONOSPORA VITICOLA. De Bary. Feuille de vigne, envahie notamment le long des nervures, par les groupes blancs, pulvérulents de Peronospora (mildew.); groupe de filaments arborescents de peronospora filament arborescent isolé ; sporange isolé, émettant ses zoospores : zoospores ciliées.

Fig. 15. THAMNIDIUM ELEGANS. Link. Groupe de Thamnidium dont chaque filament est terminé 
par un sporange globuleux et souvent muni à son tiers inférieur d'une couronne de filaments dichotômes, deuxième sorte de fructification à petits sporanges contenant $2 m 3$ spores; sporange terminal isole et spores ; sporangebrisé; couronne de rameaux dichotômes inserée sur le filament principal; rameau dichotône et sporanges très grossies.

Fig. 16. EXOASCUS BULLATUS. Berk. Feuille de poirier boursoufflee par la présence de l'Exoascus; groupe de théques, constituant chacune un champignon tout entier ; théque isolèe renfermant 8 spores; spores libres.

Fig. 17. ONIGENA CORVINA. Alb. et Schu. Plume d'oiseau envahie par trois specimens d'onigena un peu plus grands que nature; onigena plus développé.peridium en partie détruit,laissant le capitule à nu, composédefilaments thécigères ; groupe de filaments munis de théques terminales; théque isolèe, trés grossie ovale. contenant de 6 à 8 spores; spores libres.

\section{PLANCHE IV.}

Fig. 18. MORCHELLA ESCULENTA. Vitt. Morille commune, stipe épais, creux, blanchâtre. mitre ou chapeau ovale, couvert d'aréoles et de fossettes profondes, ßlondes ou brunes; Théque et paraphyse; spore isolèe.

ACETABULA ACETABULUM. Boudier. Acetabula en forme de coupe. ornée extérieurement de côtes anastomosées, brune ; théque et paraphyse ; spore isolée.

Fig. 19. TUBER MESENTERICUM. Vill. Tubercule subglobuleux, brun, couvert de verrues pyra- 
ENDOSPORES. VIII e Ord. Cupulẻs . Quelet.

I. Tubéracẻes.

19

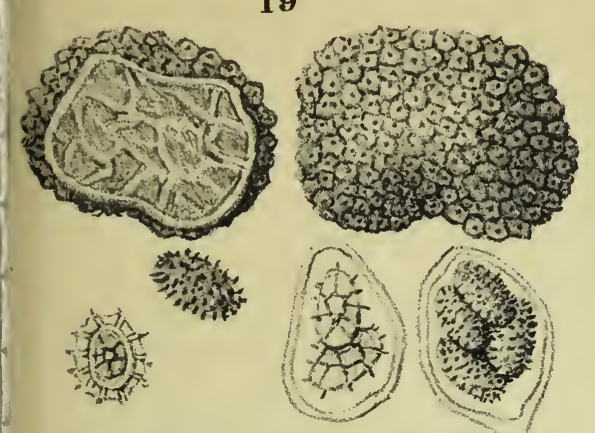

Tuber Mesentericum. T.Cibarium .
II. Helvellẻes.

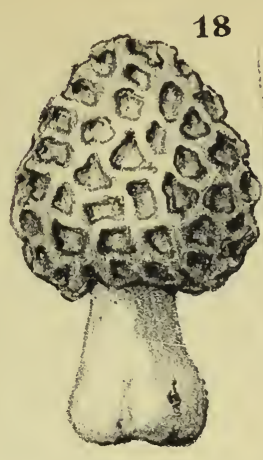

Morchella esculenta. Acetabula acetabulum.

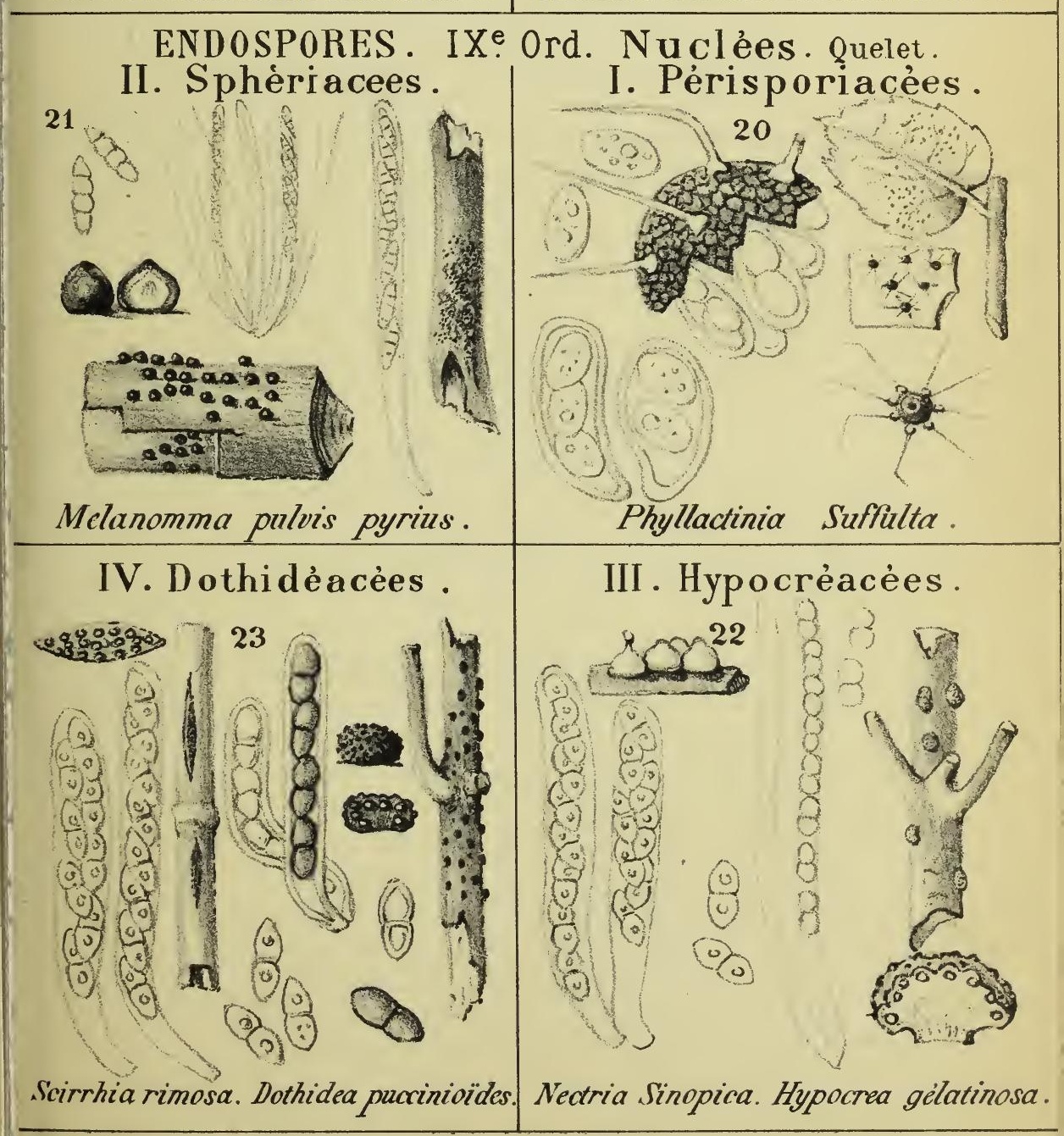

Richon, de?. 

midales; coupe mettant à nu la glèbe blanche puis brune et marbrée de blanc; thèque contenant 2 spores aréolées; spore libre.

TUBER CIBARIUM. Pers. Tubercule de même forme, mais plus gros que le précédent; théque renfermant quatre spores; spore noire, couverte d'aiguillons droits.

Fig. 20. PHYLLACTINIA SUFFULTA. Sacc. Feuille de Corylus avellana couverte de phyllactinia ; portion de feuille grossie; périthéce muni d'appendices bulbeux à la base ; pèrithèce brisé, théques à découvert; théques isolées renfermant 2-4 spores; spores isolees.

Fig. 21. MELANOMMA PULVIS-PYRIUS. Nke. Rameau de Corylus envahi par le Nelanomma; fragment du rameau grossi ; périthéces isolés, entier et avec coupe; groupe de théques et paraphyses ; thèque isolée; spores.

Fig. 22. NECTRIA SINOPICA. Fries. Groupe de Nectria grossis, sur sarment de lierre; théques et spores monostiques ou distiques; spores isolées.

HYPOCREA GELATINOSA. Fries. Sur branche de Pinus abies, tubercules épars d'Hypocrea, jaunes-roux, mous ; coupe de tubercule, périthéces à la périphérie; théques et spores uniseptées simulant 16 spores; spores isoléts.

Fig. 23. SCIRRHIA RIMOSA. Nke. Sur chaume de phragmites. strômes de Scirrhia elliptiquesaigüs, grisâtres; strôme coupé transversalement mettant á découvert les périthéces plongés dans la substance du strôme: théques : spores isolées. 
DOTHIDEA PUCCINIOIDES. Fries. Rameau de buis envahi par les tubercules de Dothidea; tubercules, l'un entier. l'autre coupé transversalement; théques tétraspores: spores isolées. incolores puis brunes.

\section{PL A NCHE V.}

Fig. '24. MICROTHYRIUM VISCI. Ch. R Rameau de Viscum album envahi par les périthèces de Nicrothyrium ; portion du rameau grossi. périthéces vus à la loupe ; périthéce isolé, brisé, laissant voir les théques disposées en rosette; thèques octospores: spores diverses. jeunes, adultes.

Fig. 25. LOPHIOSTOMA CRENATUM Nek. Rameau de prunier couvert de Lophiostoma: 2 périthéces grossis, dont l'un est incrusté à sa base dans le bois, libre supérieurement et l'autre coupé transversalement; groupe de therques et paraphyses; théque octospore et paraphyses; spores libres, incolores.

FIg. 26. HYSTEROGRAPHIUM FRAXINI. de Not. Rameau de Frêne envahi par l'Hysterographium ; périthèces incrustés dans l'écorce, vus á la loupe; thèques et paraphyses ; spore jaune-d'or, murale, adulte ; spore jeune, incolore entourée d'un sacculus.

Fig. 2\%. PHACIDIUM DENTATUM. Kae. Feuille de chêne, phacidium épars á la facesupérieure: portion de feuille vue à la loupe; groupe de théques ; théque isolée; spores filiformes. munies de gouttelettes, incolores.

Fig. 28. CYTOSPORA CHRYSOSPERMA. Sacc. Rameau de peuplier abritant sous leepiderme. les 
V. Microthyriacées.

24

$$
(\because \because \cdots)
$$

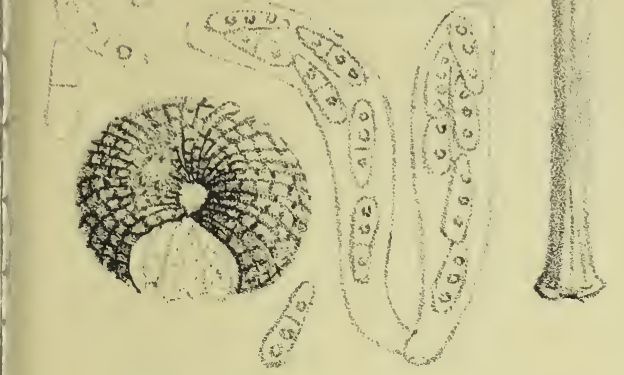

Microthyrium visci .

VII . Hystẻriacẻes .

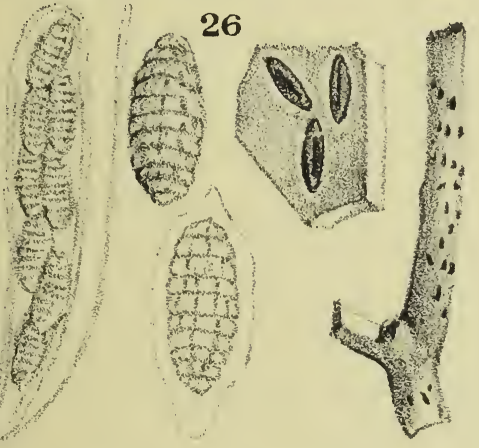

Hysterographium Fraxini.
VI . Lophiostomacèes.

25

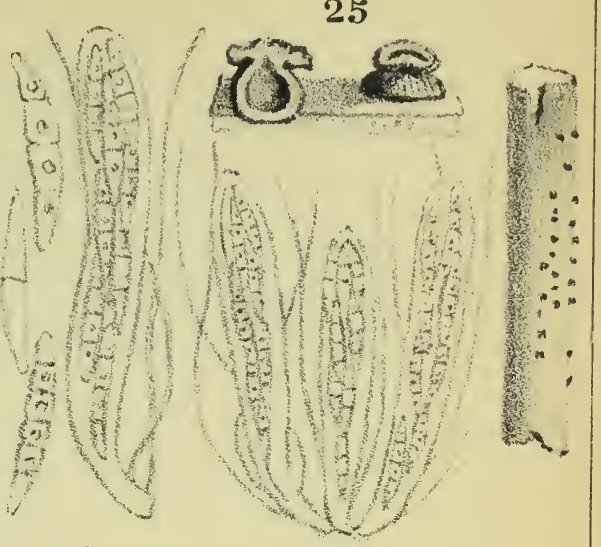

Lophiostoma crenatum.

VIII. Phacidiacẻes .
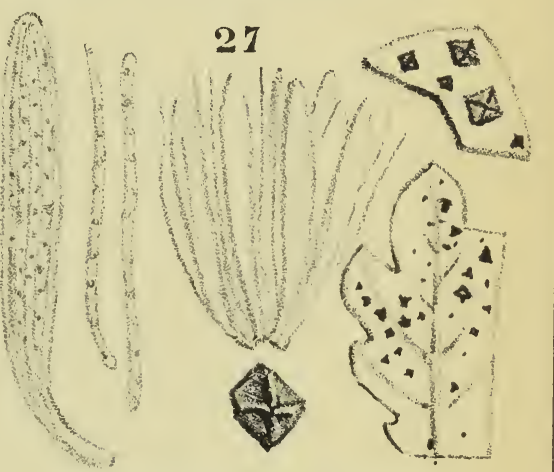

Phacidium dentatum .

\section{FUNGI NONDUM PERFECTI .}

I . Sphẻropsidẻes. Lev.

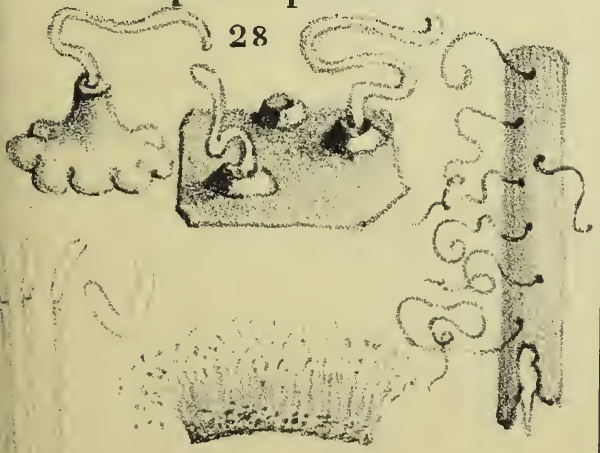

II. Mẻlanconiẻés . Berk .

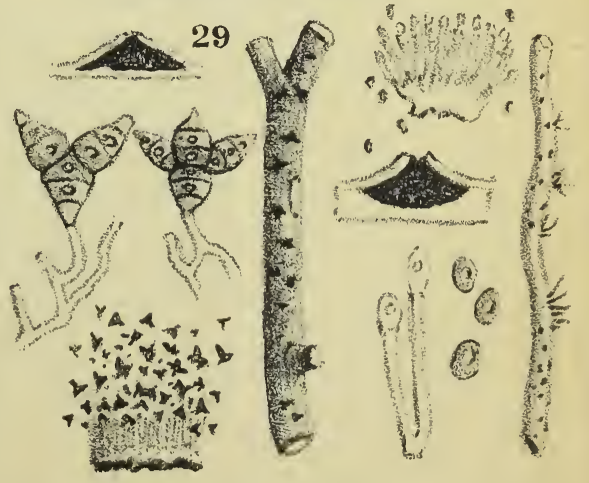

Asterosporium Hoffmanni. Melanconium Hedera 




\section{III . Hyphomycètes .}

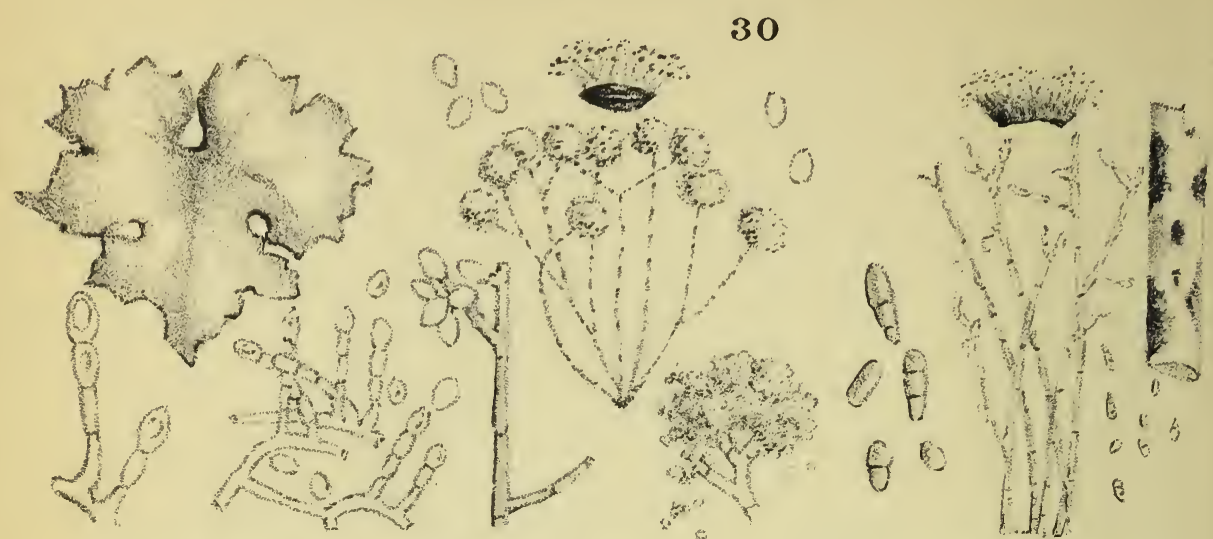

Oidium tuckeri. Botrytis cinerea. Cladosporium herbarum.

MYCELIA STERILIA . Fries.
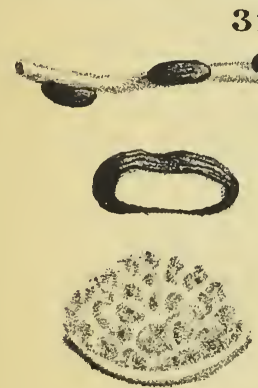

31

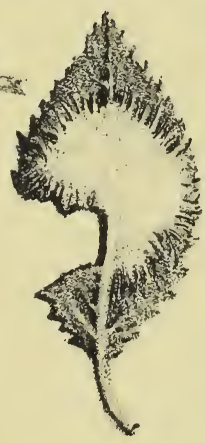

Sclerotium durum. Himantia candida.
EXAN THEMATA. Kickx.

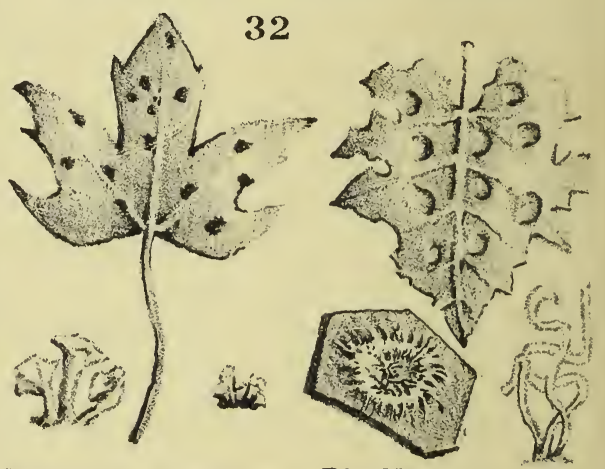

Erineum accrinum. Phyllerium vitis.

33

NOSOPHLFA . Fries .
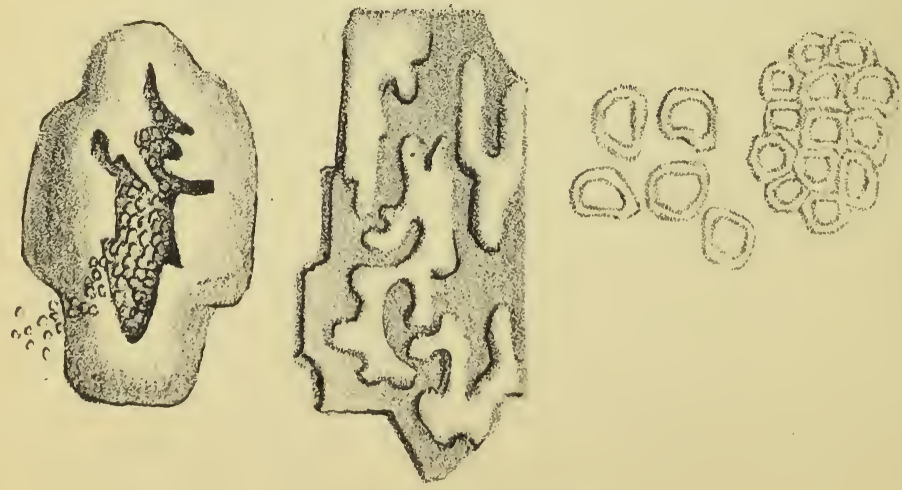

Nosophlaca Alnea. 
pustules de Cytospora ; de chaque pustule s'échappe un cirrhe de spores long; tortueux. d'un beau jaune-d'or; sommets des pustules vus á la loupe; pustule isolée; hymenium de cytospora; basides filiformes avec leur sporule terminale; sporules libres.

Fig. 29. MELANCONIUM HEDERAE.Pr.Sursarment de lierre, petitestaches noires de Melanconium; pustule sous-épidermique; groupe de basides et sporules; 2 basides etsporules très grossies; sporules libres.

ASTEROSPORIUM HOFFMANNI. Kze. Rameau de hêtre envahi ; pustule sous-épidermique d'Asterosporium; yroupe de basides et conidies ; conidies isolées, brunes, portées par des filaments rameux, composées de lobes cloisonnés disposés en étoile.

\section{PLANCHE VI.}

Fig. 30. CLADOSPORIUM HERBARUM. Link. Tige d'Althea officinalis couverte de taches velues. verdâtres de Cladosporium; groupe de cladosporium vu á la loupe; groupe de filaments fertiles plus grossis; spores diverses.

BOTRYTIS CINEREA. Pers. Sclerotium durum émettant ása surface un groupe compact de Botrytis cinerea var. sclerotiophila de Saccardo; plusieurs filaments grossis, terminés par des sporules disposées en capitule: capitule de spores plus développé; portion de filament très grossie ; sporules libres.

OIDIUM TUCKERI. Ber. Sur feuilles de vignetaches blanches arachnoïdes, confluentes. formées par l'Oïdium Tuckeri ; groupe de fila- 
ments et de sporules en chapelets; deux chapelets plus développés, à différents âges.

Fig. 31. Himantia CANDIDA. Pers. Sur feuille d'orme. expansion blanche. mince, fibrilleuse. stérile, précédant probablement l'apparition d'un corticium.

SCLEROTIUM DURUM. Per's. Sur tige herbacée trois tubercules sclérotiques, grandeur naturelle : coupe d'un sclérote noir, ridé extérieurement et de substance blanche, dure intérieurement; tissu sclérotique vu au microscope; les sclérotes sont des mycelium concentrés. destinés à donner naissance (aprés un laps de temps variable) à différentes espéces de champignons (agaries. pezizes, typhula, etc).

Fig. 32. PHYLLERIUM VITIS. Fries. Feuille de vigne dont la face infèrieure présente des groupes de filaments d'un blanc rosé ou jaunâtre qui dépriment la feuille; fragment de feuille avec dépression et filaments plus visibles ; filaments simples, tortueux, enchevêtrés.

ERINEUM ACERINUM. Pers. Feuille d'acer platanoides dont la face inférieure est envahie par les groupes rougeâtres de l'Erineum: filaments courts en forme de coupe ou de cône renversé. jaunes puis couleur de rouille.

Fig. 33. NOSOPHLEA ALNEA. Fries. Fragment d'écorce d'Alnus glutinosa dont l'épiderme est soulevé çá et lả en phlyctènes sinueuses; phlyctẻnes remplies de cellules désagrégées qui ressemblent á des sporules; cellules grossies. 
La Famille des garicinees de l'Ordre des Hyméniés est la première et en même temps la plus intéressante de la Classification.

Les espéces qui la composent ont des caractères communs et une physionomie uniforme qui permettent de les confondre ; aussi l'étude en est-elle difficile.

Persoon est le premier Mycologue à qui l'on doit la découverte des formes similaires, constantes dans certains groupes de champignons, qui ont servi de base à sa division des nombreuses espèces en Genres et en sous-Genres.

Le célèbre Fries, dans sa Classification plus récente, acceptant les Genres de Persoon, en fit des groupes distincts classés d'aprés la coloration des spores. Cette innovation eut le grand avantage de faciliter l'ètude des Agaricinées, mais sépara certains genres qui devaient ètre réunis. Sa méthode parait plusingénieuse que naturelle.

M. Roze, dans son essai de Classification publiẻ par la Société botanique de France, en Nars 1876, cherche 
á concilier les deux méthodes au moyen de tableaux que nous mettons sous les yeux du lecteur.

L'un représente la clef de son systeme qui se rapproche de la Classification de Persoon ; les deux autres (faits postérieurement) intitulés Répartition des Genres dans les Familles d'après la Coloration des spores, sont disposés de telle sorte que les lignes transversales indiquent les Familles et les Genres qu'il a réunis, en prenant pour base la similitude de forme et de consistance du Réceptacle; tandis que les lignes ou bandes longitudinales contiennent les Familles et les Genres differents qui possèdent des spores de même coloration et souvent de même forme.

Ces tableaux ainsi construits permettent de recueillir d'un seul coup d'œil, les renseignements de premier ordre nécessaires à l'analyse et à la détermination des Agaricinées. 


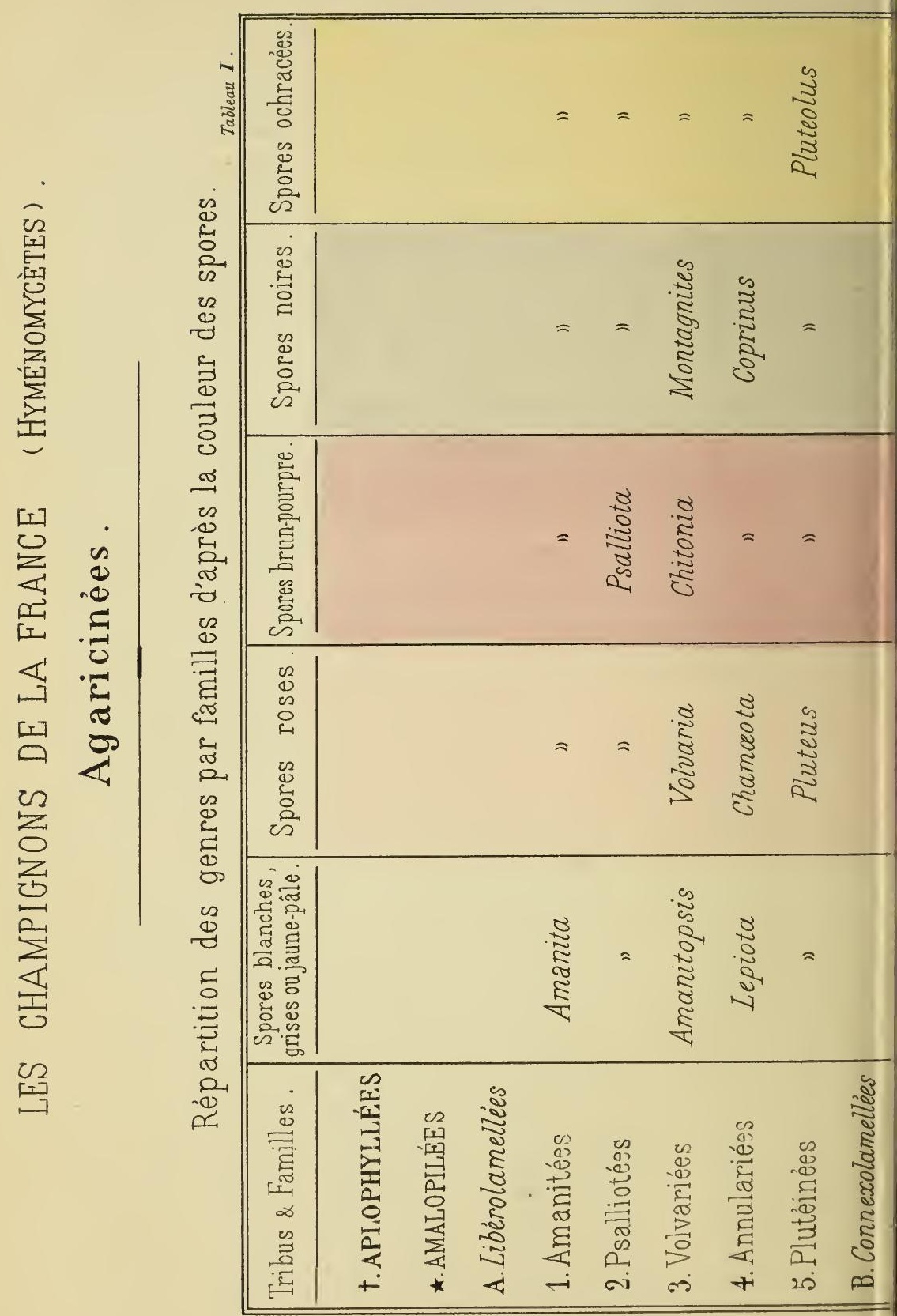




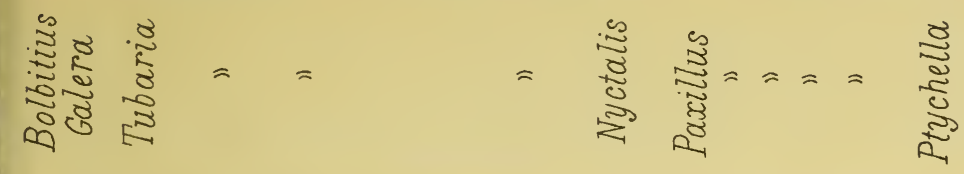
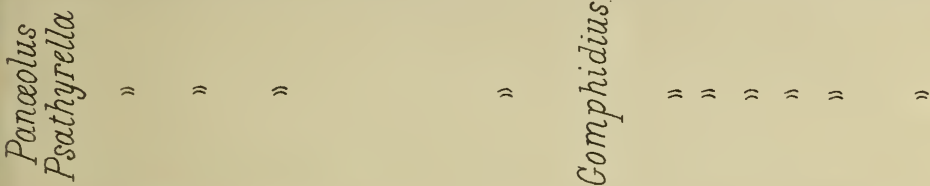

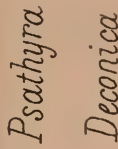

$\approx \quad=$

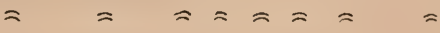

$\sum_{\pi}^{\infty} i_{i=1}^{\infty}=$

$\approx \approx \approx \approx=\approx \approx$

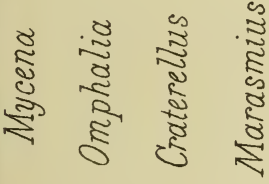

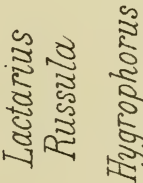

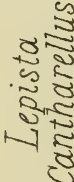

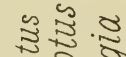

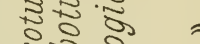

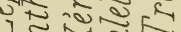

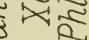

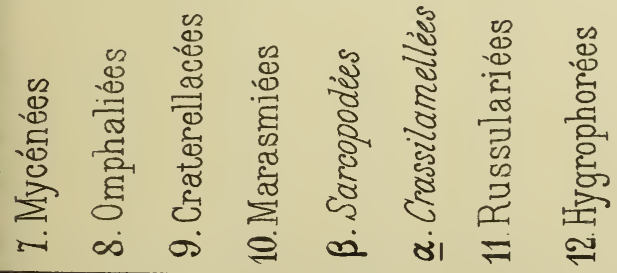

疍

क्ष

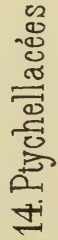




告

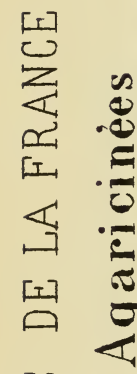

$\sum_{0}^{2}$

I 



\section{Agaricinèes .}

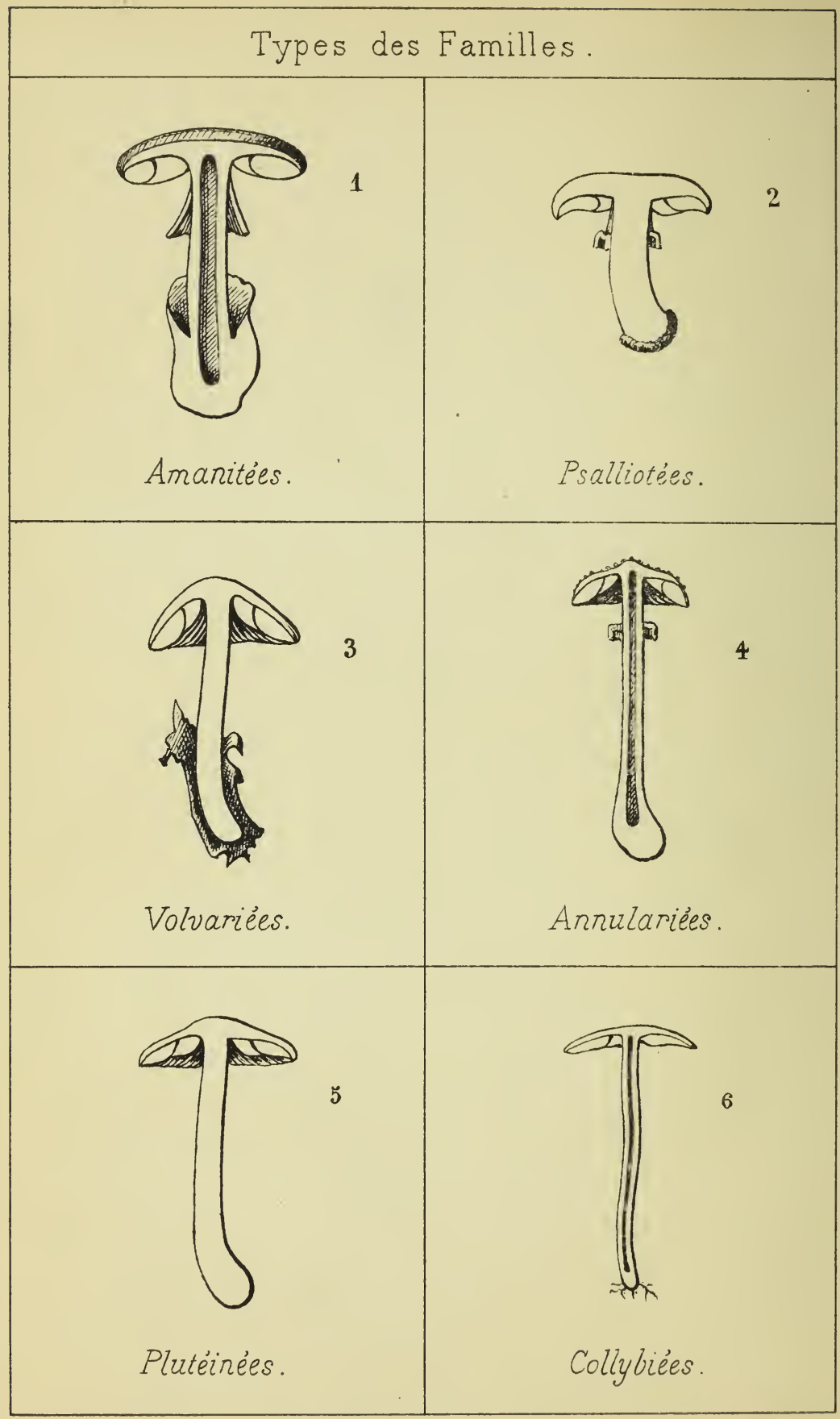





\section{Agaricinẻes .}

\section{Types des Familles.}

Mycérées

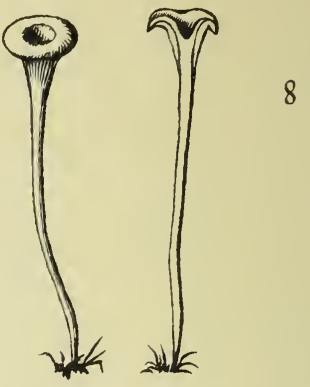

Omphaliées.

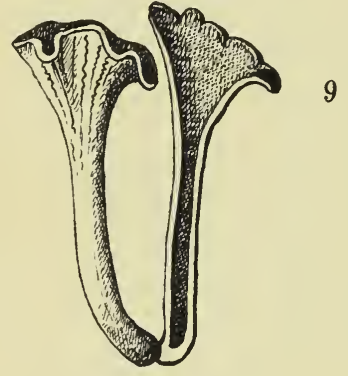

Cratérellacées.

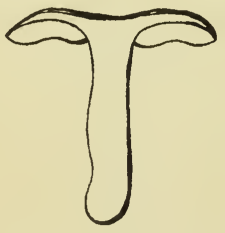

11

Russularièes.

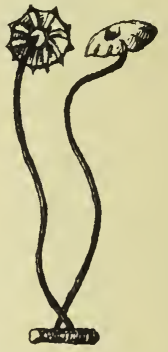

10

Marasmiées.

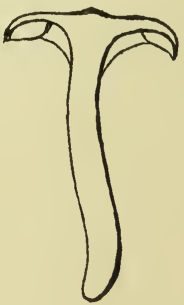

12

Hygrophorées. 


\section{Agaricinẻes .}

Types des Familles.

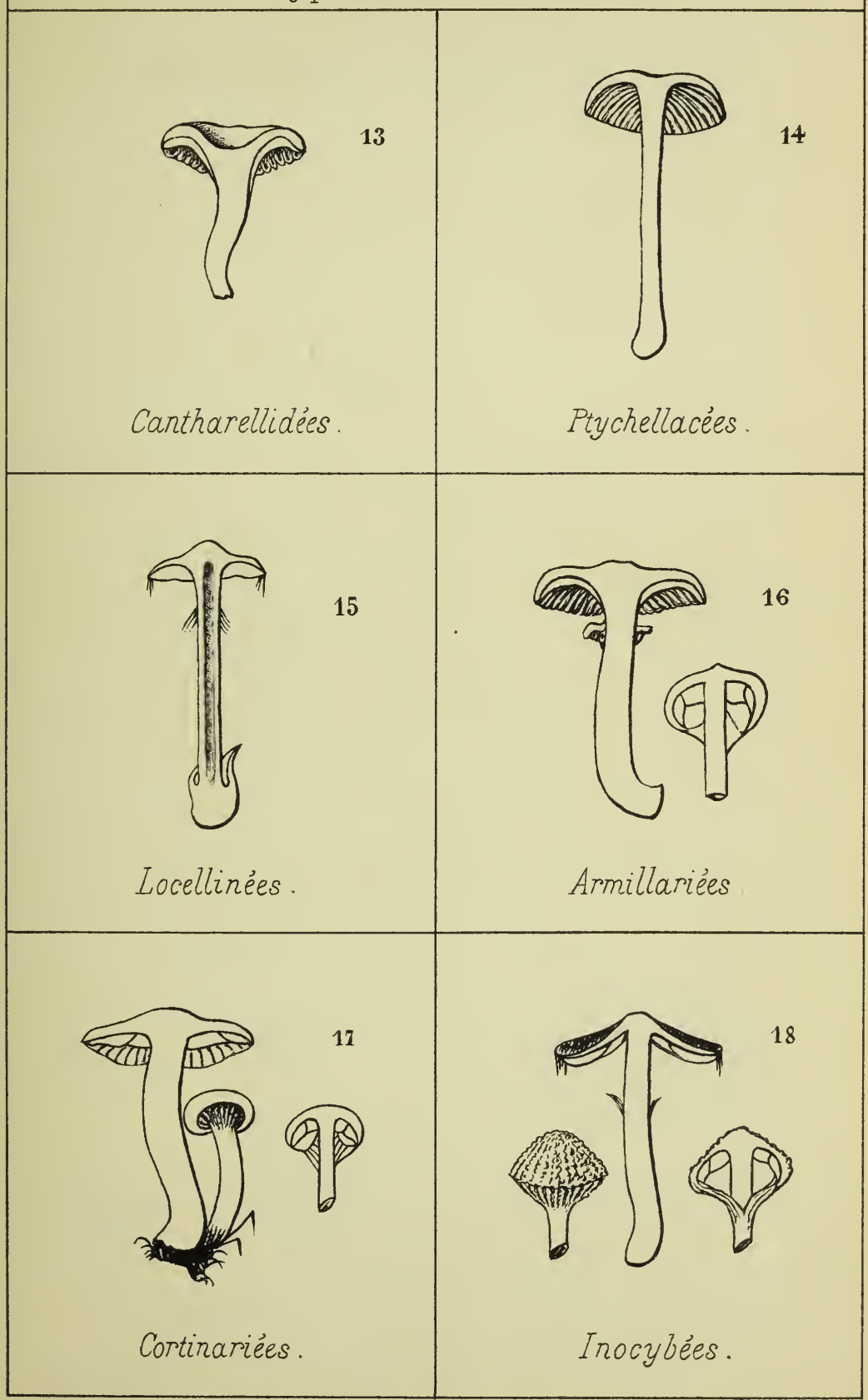

Ch. Richon, del. 



\section{Agaricinẻes .}

Types des Familles.
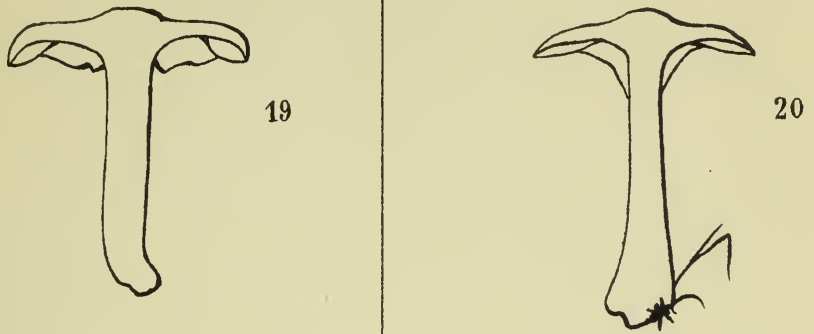

Tricholomées.

Clitopilées.
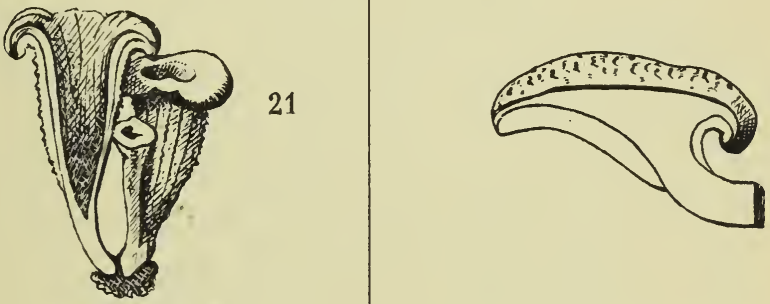

22

Pleuropodées.

Lentinées.

Hyptiotées.
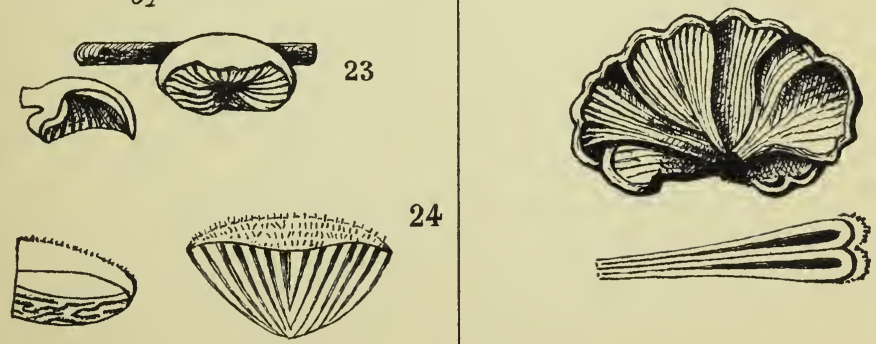

Lenzitéacées.

Schizophyllacées. 



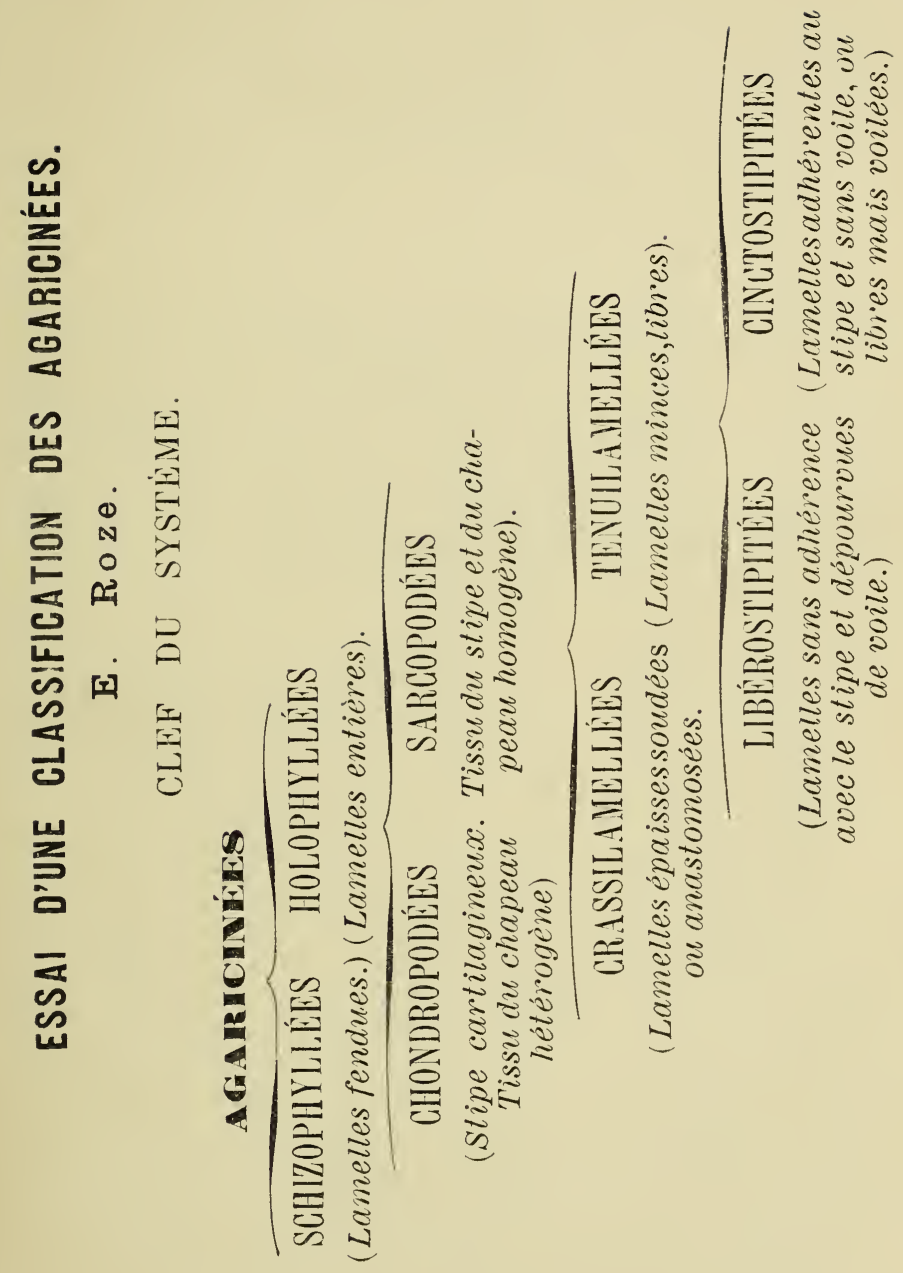



Suivant le tableau de la Classification proposée par M. Roze, les graricinées sont divisées en \$5 Familles distinctes l'une de l'autre par des caractères différentiels. Nous essayons de mettre ces caractères en évidence en reproduisant les dessins pris sur nature de la coupe des 25 Champignons-types dont voici la liste par numéro d’ordre :

1. AMANITA Cesarea. Schœff.

2. Psalliota campestris. Lin.

3. Volvaria BombYcina. Schof.

1. LEPIOTA PROCERA. Scop.

5. Pluteus cervinus. Hoff.

6. Collybia DRYOPHila. Bull.

7. MYCENA FLAVIPES. Quélet.

8. OMphalia fibula. Bull.

9. Craterellus tubeformis. Schceff.

10. Marasmius rotula. Scop.

11. Russula integra. Lin.

12 Hygrophorus ebURneus. Bull.

13. Cantharellus cibarius. Fries.

14. Ptychella OCRACEA. Ro $z$.

15. LOCELlina AlexAndri. Gillet.
Fam. Amanitées.

- Psalliotées.

- Volvariées.

- Annulariées.

- Plutéinées.

- Collybiées.

- Mycènées.

- Omphaliées.

- Craterellacèes.

- Marasmiées.

- Russulariées.

- Hygrophorées.

- Cantharellidées.

- Ptychellacées.

- Locellinées. 
16. Armillaria Mellea. Fl. $D$. - Armillariées.

17. Cortinarius cinereo-violaceus. Per's. - Cortinariées.

18. Inocybe amara. Alb. el Schw, - Inocybées.

19. Tricholoma terreum. Schceff. - Tricholomées.

20. Clitocybe clavipes. Pers. - Clitapilées.

21. Lentinus cochleatus. Pers. - Lentinées.

2:) Pleurotus conticatus. Fries. - Pleuropodées.

23. Claudopus variabilis. Pers. - Hyptiotées.

24. Lenzites abietina. Bull. - Lenziteacées.

25. Scinzophyllum commune, Bull. - Schizophyllacées. 


\title{
EXPLICATION DES ABRÉVIATIONS
}

\author{
QUI EXISTENT DANS LE GATALOGUE.
}

\section{AUTEURS}

Alb. et Schw. - Albertini et Schweinitz.

De By. - De Bary.

Bat. - Battara.

.... - Batsch.

Berk. - Berkeley.

Bolt. - Bolton.

Brond. - Brondeau.

Bull. - Bulliard.

Cast. - Castagne.

Chail. - Chaillet.

Ch. R. - Charles Richon.

Curt. - Curtis.

D. C. - De Candolle.

Desm. - Desmazières.

Ditm. - Dittmar.

Dur. - Durieu de Maisonneuve

Ehrh. - Ehrhenberg.

$F l$. D. - Flore Danoise.

Gil. - Gillet.

Gmel.-Gmelin.
Holms. - Holmskiold.

Hoff. - Hoffmann.

Huds. - Hudson.

Hedw. - Hedwig.

Jacq. - Jacquin.

Kalch. - Kalchbrenner.

Karst. - Karsten.

Kromb. - hrombholz.

...... - Kühn.

L. Lin. - Linné.

Lasch. - Lasch.

...... - Lenz.

Lév. - Léveillé.

Lib. - Libert.

L. K. - Link.

Mart. - Martius.

Mont. - Montagne

Müll. - Müller.

Nees. - Nees von Esenbeck

Noul. - Noulet.

Obs. - Obseck. 
Ott. - Otto Wünsche.

Pass. - Passerini.

Pers. - Persoon.

Poit. - Poiteau.

Purt. - Purton.

Quél. - Quélet.

Rbh. - Rabenhost.

Reb. - Rebentisch.

Relh. - Rellian.

Relz. - Retzius.

Roslk. - Rostkovius.

Saund. el Schm. - Saunders et Schmith.

Schoeff. - Schœfler.
Schrad. - Schrader.

Schulz. - Schulzer.

Schum. - Schumacher.

Scop. - Scopoli.

Somm. - Sommerfelt.

Sow. - Sowerby.

Str. - Strauss.

Trog. - Trog.

Tul. - Tulasne.

Wallr. - Wallrotht

Wern. - Weinmann.

Wild. - Willdenow.

Vill. - Vittadini.

Wesl. - Westendorp.

\section{ABRÉVIATIONS DIVERSES.}

Atl. Champ. - Atlas des Champignons vénéneux et comestibles.

$C-C C-C C C$ - Commun - très-commun - extrêmement commun.

$R-R R-R R R$ - Rare - trés-rare - extrêmement rare.

P. - Page.

$P l$. Planche.

Rec. - Recueil.

T. - Table.

Alb. de l'Esl. 


\section{CHAMPIGNONS}

$1^{\text {re }}$ D I VISI $0 \mathrm{~N}$.

\section{EXOSPORES.}

1er ORDRE. - HYMÉNIÉES. - Quélet.

$1^{\text {re }}$ FAM. - Agaricinées. Fries.

Hymenium lamellé; lamelles disposées en rayons.

A. Leucospori. - Spores blanches ou blanchâtres.

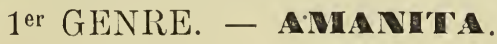

1. A. CAEAREA. Scop. - Atl. Champ. Ch. R. et Roze. p. 7. pl. 1. - ORONGE vRAIE. - JAZERAN. Comestible. - Forêts de Reims, Charmont. TroisFontaines. - Terrains sablonneux, calcaires-siliceux. - RR. -- Eté-automne.

2. A. OvoideA. Bull. t. 364. - Atl. Champ. Ch. R. et Roze. p. 9.pl. IV. Oronge Blanane.-Comestible. 
-- Forêts des plateaux de l'arrondissement d'Epernay et de la montagne de Reims. - Août-Septembre. - RR.

Les caractères qui distinguent cette belle espèce existent au moment de l'épanouissement du chapeau ; plus tard, le large collier moelleux et fragile disparaît. Si on la récoltait dans cet état on pourrait, comme certains auteurs, la nommer improprement A. Gloiocephala. A. Gigantea.

3. A. Virosa. Fries. - Atl. Champ. Ch. R. et Roze. p. 10, pl. III. - Vénéneux. - Forêt de Sermaize à Cheminon. - Fin d'Août. - RRR. Trouvée une seule fois.

4. A. PHALLOIDES. Fries. - Kromb. t. 28. - Atl. Champ. Ch. R. et Roze. p. 12, pl. XIII. A. bulbeux. - Bull. - Oronge gigue verte. Paulet. - Forêts de chênes, de charmes, etc. - rénéneux. - CCC. - Fité-Automne.

5. A. VERNA. Bull. t. 108. - Atl. Champ. Ch. R. et Roze. p. 11, pl. XIII. - Oronge Gigue BLANche. - Paulet. - Espéce printanière qu'on trouve isolément et rarement dans les terrains sablonneux de la forêt de Maurupt à Cheminon. - Mai-Juin. Ténéneux. - RRR.

6. A. MAPPA. Fries. - Schœff. t. 241. - $4 l 1$. Champ. Ch. R. et Roze. p. 17. pl. XI. - Bois couverls; Sermaize, Vavray-le-Petit, Cheminon, elc. - Etè-Automne. - CCC. - Ia Var. Alba doit son absence de couleur aux influences almosphériques et pour ce molifest très rare ou très commune. dans certaines années. 
7. A. RECUTITA. Fries, - Gon ct Rab. t. 2. Forêt de Maurupt. - Aoùt-Septembre. - AR. Vénéneux.

8. A. MUSCARIA. Lin. - Atl. Champ. Ch. R. et Roze. p. 14. pl. 1. - FAusse Oronge. - Chapeau d'un beau rouge-orangé, feuillets, collier, volva, et débris sur le chapeau tantôt blanc de neige, tantôt légérement teintés de jaune-citrin. -- ÉtéAutomne. - Lieux un peu humides dans toutes les forêts. - CC. - Vénéneux.

9. A. PANTHERINA. Fries. - Schcef. -- t. 90. Atl. Champ. Ch. R. et Roze. p. 20. pl. V. - Forèts de Charmont, Trois-Fontaines. Reims, etc. - ÉtéAutomne. - CC. - Vénéneux.

10. A. STRANGULATA. Fries, - Saund. et Schm. t. 40. - Bois de Vavray-le-Petit. - RR. Octobre. - Comestible.

11. A. STRobiliformis. Witt. t. 9. - Pomme de pin. - Forêt d'Eperuay. - A l'oree des bois, tranchées. - Automne. - R. - Comestible.

12. A. RUBESCENS. Fries. - kromb. t. 10. Atl. Champ. Ch. R. et Roze. P. 19, pl. VI. Golmet'te vraie. - Forêts de chênes, charmes. hêtres. - Étè-Automne. - CCC. - On en fait un grand usage dans les pays avoisinant les forêts de l'Est du département. - Comestible. - La chair rougit à la cassure.

13. A. SPISSA. Fries. - Kromb. t. 29. f. 1-5. - Atl。 Champ. Ch. R. et Roze. p. 22, pl. V. - Oronge PEPIÉE. - Forêl de Sermaize - Été-Automne. AR. - Suspect. 
1'́. A. VAginata. Bull. - Atl. Champ. Ch. R. et lioze.p. $25, p l$. Vili.-Grisette, Covlemelie grise. Bois et forèts humides. - CCC. - Comestible. Var. Jaune-fauve. - Courbmerde Jaune. - Atl. Champ. Ch. R. et Roze. p. 23. pl. XIX.

\section{4 (XENRE. - IEPIOTA. - Fries.}

15. L. PROCERA. Scop. - Atl. Champ. Ch. R. et Roze. p. 29. pl. XX.-Couleuvrée, (irande GouleMEI.LE. (KRISETTE.-Forêts de Sermaize. Cheminon, Epernay. - AR. - Automne. - Comestible.

16. L. RACHODES. Fries. - Atl. Champ. Ch. R. et Roze. p. 3', pl. XXII.-Très roisine de L. Procera. - Mèmes localitès.-RR.-Automne.-Comestible.

17. L. EXCORIATA. Scheff. - Atl. Champ. Ch. R. et Roze. p. 35. pl. XXII - Pàturage. limite de Seine-et-Marue. - AR. - Comestille.

18. L. MAstoIdeA. Fries. -- Atl. Champ. Ch. R. et Roze. p. 32, pl. XXII. - Bois de charmes et de hètres. - Charmont, Cheminon. - AR. - Automne. -- Comestible.

19. L. FRIESII. Lasch. - Atl. Champ. Ch. R. et Roze. p. 3:3, pl. XXIII. - Communiqué par MI. Boudier. - R. - Comestible.

2(1. L. BADHAMI. Berk et $B r$. - Album de l'Est. (h. R. - Récolté sur le tan à Sermaize. - RR. Suivant Fries odeur vireuse. - Suspect.

:1. L. CLYPEOLARIA. Bull.-Atl. Champ. ('h. R. et Roze. p. 31, pl. XXI. - Covlemelle D'EaU - Bois humides. - Possesse. Trois-Fontaines, St-Remyen-Bouzemont. - CC. - Automne. - Comestible. 
2\%. L. CRISTAtA. Bolt. - Atl. Champ. Ch. R. et Roze p. 32,pl. XXI.-Petite coulemelle.- Bois et taillis. - St-Amand, Soulanges, Bassu, etc. CC. - Automne. - Suspect.

23. L. HolosericEA. - Fries. - Atl. Champ. Ch. R. et Roze. p. 36, pl. X. - Champs des terrains calcaires à Soulanges. - Eté-Automne. - R.

2'. L. NAUCINA. Fries. - Atl. Champ. Ch. R. et Roze. p. 35,pl. VIII. - Pâturages - Clairières des bois. - Sermaize, St-Amand, Aulnay-l'Aître. AC. - Automne. - Comestible.

2i. L. CARNEIFOliA. Gill. - Alb. de l'Est, communiqué par le $\mathrm{D}^{\mathrm{r}}$ Reguis. - Départements $\mathrm{du}$ Midi, (inscrit pour mémoire).

26. L. CEPCESTIPES. Fries. - Alb. de l'Est. Croît dans les serres cháudes sur le tan. - R. - Sermaize. - Douteux.

Var. Flammula, de belle couleur citrine, puis tournant au gris-livide en vieillissant,même localité.

27. L. CARCHARIAS. Fries. - Album de l'Est. Ch. R. - Bois taillis, sapinières. - St-Amand. Soulanges. - AR. - Automne. - Comestible.

28. L. GRANULOSA. Batsch. - Album de l'Est. Ch. R. - Bois de pins. - St-Amand, Aulnayl'Aître. - RR. - Comestible.

29. L. AMianthina. Fries. - Album de l'Est. Ch. R. - Lieux herbus des bois, sapinières. CCC. - Etè-Automme. - Comestible.

30. L. HAMATites. All). de l'Est. Ch. R. - Belle espéce, pourpre-noirâtre, sur le tan des allées. dans un jardin á Sermaize. - Automne - RR. 
3e GENRE. - ARMLLA IRA. - Fries.

31. A. RobUsta. Fries. - Atl. Champ. Ch. R. et Roze. p. 60 .pl. XXIV. - Lieux découverts dans les bois de pins. - RRR. - Près de Reims, 1878. -.- Octobre.

32. A. Cingulata. Fries. - Atl. Champ. Ch. R. et Roze. - Croît en cercles sous lessaules Marceau plantés dans les bois de pins syl vestres. - St-A mand. - Automne.

3:3. A. Constricta. Fries. - Alb. de l'Est. Ch. R. - Un seul échantillon trouvé dans les bois de pins á St-Gibrien, prés de Châlons par M. Morize en 188'. - Automne.

31. A. MELLEA.Fl. D.-Atl.Champ. Ch. R. et Roze. p. 61,pl. XXIV.-TÊTE DE MÉDUSE.-Trés commun sur les troncs coupés à ras de terre, dans les bois, les jardins; son mycelium noir fibreux (rhizomorpha) - fruitiers, vignes. - CCC. - Automne. Comestible selon quelques auteurs.

35. A. MUCIDA. Schrcid. - Album de l'Est. Ch. R. - Croît en groupes sur les morceaux ou troncs de hêtres exposés à la pluie. - Automne. - AR. Charmont.

4e GENRE. - THICHOLOMA. - Fries.

36. T. EQUESTRE. Fries. - Atl. Champ. Cl. R. et Roze. P. 77, pl. XXXII. -- Bois de pins, terrains sablonneux, environs d'Epernay. - RR. - Automne 187\%. -- Comestible.

37. T. SEJunCtum. Sou. - Atl. Champ. Ch. R. et et Roze. P. 78, pl. XXXII. - Agaria Émarginé. 
Sous les chênes dans les forêts de Charmont, TroisFontaines.Vavray.-Automne. - CC. - Comestible. 38. T. Colossum. Fries. - Alb. de l'Est. - Récolté dans la Meuse sur la lisière de la Marne. Bois de pins. - RRR. - Comestible.

39. T. USTALE. Fries. - Atl. Champ. Ch. R. et Roze. P. 83, pl. XXVII. - Bois de Vavray-le-Petit. - R. - Automne. - Comestible.

40. T. PESSUNDATUM. Fries. - Atl. Champ. Ch. R. et Roze. p. 81 pl. XXVIII. - Forêts de Trois-Fontaines, St-Remy-en-Bouzemont. - AR. - Automne. - Comestible?

41. T. RUSSUlA. Fries. - Atl. Champ. Ch. R. et Roze. p. 80. pl. XXVIII. - Bois de charmes et de hêtres - Récolté à Brillon (Haute-Marne) avec le Dr Cordier, probablement dans la Marne. - R. Comestible.

42. T. RESPLENDENS. Fries. - Alb. de l'Est. Ch. R. - Espèce rare dans nos contrées; elle se rapproche de T. Columbetta. - Bois de Cheminon. Automne.

43. T. RUTILANS. Schœff. - Atl. Champ. Ch. R. et Roze. p. 81, pl. XXVII. - Bois de pins, environs d'Epernay, St-Martin d'Ablois. - AR. - Automne.

44. T. PSAMMOPUS. Kalch. -Alb. de l'Est. Ch. R. Bois de pins sylvestres, lieux arides, St-Amand. Soulanges, Aulnay-l'Aître. - AR. - Automne.

45. T. Columbetta. Fries. - Atl. Champ. Ch. R. et Roze p. 8\% pl. XL. - Forêt de: Cheminon á Sermaize - AR. - Automne. - Espéce blanche dont le pied bleuit par le froissement. - Comestible. 
46. T. IMMUNDUM. Berk. - Alb. de l'Est. Ch. R. - Bois de chênes à Vavray-le-Petit - Lieux ombragés. - R. - Automne. - Noircit en vieillissant et par le froissement.

47. T. TERREUM. Sou. - Alb. de l'Est. - Dans les bois de pins, croît en cercles très nombreux autour des arbres. - Automne et commencement de l'hiver. - CCC. - Comestible - peu savoureux.

Var. Album. Argyraceum de Bulliard. - AC. - mêmes localités.

Var. Chrysites. Feuillets prenant une teinte citrine en vieillissant, moins fréquent, dans les sapinières sur les terrains crétacés. - Automne.

18. T. SAPONACEUM. Fries. - Atl. Champ. Ch. R. et Roze. p. 84, pl. XXIX. - Dans les bois de chênes de haute futaie. Sermaize, Charmont, montagne de Reims. - C. - Automne. - Suspect.

49. T. MURINACEUM. Fries. - Alb. de l'Est. Ch. R. - Bois de Vavray-le-Petit. de Lisse, croît isolément - espèces AR. lamelles grises; chapeau et pédicule ornés de petites écailles noires, dressėes,ressemble un peu au $T$. Terreum.-Automne.

50. T. HoRDUM. Fries. - Alb. de l'Est. Ch. R. Trouvé a vec le précédent à Vavray-le-Petit. Sous les hêtres. - R. - Automne. - Récolté plusieurs années de suite dans l'allée du château d'Aulnayl'Aître. - suspect.

51. T. SUlfUREUM. Bull. - Atl. Champ. Ch. R. et Roze. p. \%\%,pl. XXXI,-Commun autour des chênes dans les lieux humidesdes forêts. Exhale une odeur vireuse trés prononcée. - Automne. - Vénóneux. 
52. T. BUFONIUM. Fries. - Atl. Champ. Ch. R. et Roze. p. 79, pl. XXXI. - Diffère du précédent par sa teinte vergetée-bistrée et par son odeur nulle ou à peine acccentuée. - Mêmes localités. - Automne.

\section{- Vénéneux.}

53. T. ALBELlUM. Fries. - Atl. Champ. Ch.R. et 54. T. GAMBOSUM. Fries. Roze. p. 85-8\% pl. 55. T. GEORGII. Fries. XXX, - Ces trois espéces de Fries connues sous le nom de MousseRON BLANG, nous paraissent être des variétés de la même espéce. - Récolte au printemps dans les prés qui avoisinent les forêts de Charmont, StEulien, etc. - Comestibles.

56. T. ACERBUM. Bull. $-\Lambda$ tl. Champ. Ch. R. et Roze: p. 79.pl.XXIX. - Bois de Possesse, Vanaultles-Dames, Vavray-le-Petit. - Chapeau à bords enroulés en dessous et fortement sillonnés. - AC. Automne. - Vénéneux selon le Dr Mougeot.

5\%. T. Personatum. Fries. - Atl. Champ. Ch. R. et Roze. p. 88, pl. XXXIV. - Récolté dans la Meuse à la fin d'octobre, peu commun,ressemble à T. Nudum mais est plus robuste et strié de violet et de blanc. - Comestible.

58. T. NUDUM. Bull. - Atl. Champ. Ch. R. et Roze. p. 89, pl. XXXIV. - Trés commun dans les bois de pins. - Automne. - Belle couleur violette changeant avec l'âge, persiste souvent dans les hivers doux. - Comestible.

59. T. Melaleucum. Pers. - Album de l'Est. Dans les bois de pins; le long des sentiers. Automne. - CCC. - Comestible. 
Var. Porphyroleucum. Moins commune. Bayarne. Ablancourt.

60. T. BREvipes. Bull. - Alb. de l'Est. - Sermaize. dans les allées d'un jardin oú l'on avait répandu du tan. - RR. - Automne. - Comestible.

61. T. HUMILE. Fries. - Alb. de l'Est. Ch. R. Trouvé une seule fois dans un chemin herbu à StAmand. - Le chapeau presqu'appliqué sur le sol est sinueux et les feuillets un peu décurrents. RR. - Automne.

62. T. Virgatum. Fries. - Album de l'Est. Ch. R. - Récolté dans les bois de Sermaize et de StLumier. - Chapeau remarquable par les lignes noires qui vergettent la surface du chapeau. Lamelles passant du blanc au gris sale. - R.

63. T. SORDIDUM. Fries. - Album de l'Est. Ch. R. - Cette espéce croît isolément dans les bois de pins à St-Amand, Bassu, Soulanges. - AC. Automne. - Comestible.

\section{5' GENRE. - CLITOCYHE.}

6'. CL. NEBULARIS. Batsch.-Atl. Champ. Ch. R. et Roze, p. 99. pl. XXXI. - Bois de Charmont, Trois-Fontaines. - AR.- Automne, - Suspect.

65. CL. Clavipes. Fries. - Atl. Champ. Ch. R. et Roze. p. 100, pl. XXXI. - Bois de pins de la Meuse et des Vosges. probablement dans la Marne. - Plus petit que $C l$. Neubularis. - Stipe renflé à la base. - Suspect.

65. CL. OdORA. Bull. - Alb. de l'Est. Ch. R. AgARIC ANISÉ - Vient solitaire dans les forêts; 
sa présence est dévoilée par une odeur d'anis trés prononcée. - CC. - Automne. - Comestible.

67. CL. PHyLlophila. Fries. - Alb. de l'Est. - Forêts de Charmont, Cheminon : St-Remyen-Bouzemont, etc. ; sur les feuilles de chênes pourries. - CC. - Eté-Automne. - Comestible.

68. CL. PITHYOPHILA. Fries. - Alb. de l'Est.Bois de pins sylves tres. - CC. - Automne. Comestible.

69. CL. DEALBATA. Sow. - Alb. de l'Est. Ch. R. - Commun en Eté et en Automne dans les sapinières; il forme des cercles sous les pins, en compagnie des Hebel. crustuliniforme. - Comestible.

70. CL. COFFEATA. Fries. - Alb. de l'Est. Ch. R. - Bois couverts de Vavray-le-Petit. AR. - Eté-Automne.

71. CL. DECASTES. Fries. - Alb. de l'Est. Ch. R. - Bois de Vanault-les-Dames, Sermaize ; dans les allées et les rives herbues des tranchées. - AR. - Automne.

Var. à chapeau rougeâtre. - Allée de jardin à St-Amand, 1881 .

72. CL. CATINA. Fries. - Alb. de l'Est. Ch. R. Dans les terrains calcaires plantés de pins, de saules Marceau. - AC. - A la fin de l'été. St-Amand, Soulanges, etc. - Comestible.

73. CL. INVERSA. Frics. - Atlas Champ. Ch. R. et Roze, p. 103, pl. XXXI - Dans les sapiniẻres et les taillis qui les avoisinent. AC. - Automne. 
14. CL. CYATHIFORMis. Bull. - Atl. Champ. Ch. R. et Roze. p. 105, pl. XXXII. - Dans bois-taillis, sapinières; présente plusieurs variétés différant seulement par la couleur qui passe du gris-fauve au bistre. - AC. - Automne. - Comestible.

75. CL. EXPAllens. Fries. - Alb. de l'Est. Même forme que le précédent, mais plus petit et de couleur blanc-sale ou grise. - AC. - Bois de Pins.

76. CL. OBBATA. Fries. - Alb. de l'Est. Dans les plantations de pins; hauteurs de Bassu, Lisse, Bayarne. - Lamelles grises, décurrentes. - AC. - Automne.

7\%. CL. SUAVEOLENS. Schum. - Atl. Champ. Ch. R. et Roze. P. 102 , pl. XXXII. - Boistaillis, sapirières; St-Amand, Ablancourt, Francheville, - AC. - Très odorant surtout étant sec. - Automne. - Coinestible.

78. CL. FRAGRANS. Sow. - Atl. Champ. Ch. R. et Roze, p. 103, pl. XXXII. - Dans la mousse, au pied des pins, broussailles ; stAmand, Soulanges, etc. - AC. - Odorant. Comestible.

79. CL. MetAChrous. Fires. - Alb. de l'Est. Ch. R. - En groupes dans la mousse des plantations de coniféres : lamelles grises en vieillissant, se détachant facilement du stipe. $\Lambda \mathrm{R}$. - Automne. - Comeslible.

86. CL. OBSOLETA. Fries. - Alb. de l'Est. Jans les sapinières. - $\mathrm{AC}$. Dans les Vosges... 
R. Dans la Marne. - Automne. - Comestible.

81. CL. LACCATA. Scop. - Atl. Champ. Ch. R. et Roze, p. 100, pl. XXXIV. - Espéce très commune, surtout au pied des saules, des peupliers. - Var. Violascens. - Dans les forêts - Automne. - Comestible. - peu délicat.

82. CL. PROXIMA. Boudier. - Atl. Champ. Ch. R. et Roze. p. 101, pl. XXXIV. - Espéce voisine de la précédente et confondue avec elle. Elles diffèrent entre elles par la forme des spores. - Comestible.

83. CL. TORTILIS. Fries. - Alb. de l'Est. Forêts de chênes et de charmes. Dans les chemins humides et les fossés. - Croît sans doute sur les radicelles mises à découvert. Vanault-lesDames, Possesse, Vavray-le-Petit, etc. - C.

- Etè-automne.

6e GENRE. - CoLLYana. - Fries.

8'. COL. RADICATA. - Relh. - Alb. de l'Est. Croît isolément dans tous les bois, près des troncs pourris. Son stipe est très long et renflé en fuseau á l'extrémité inférieure. - CC. Eté-automne. - Suspect.

85. COL. LONGIPES. Bull. - Alb. de l'Est. Ressemble au précédent, mais est velouté sur toute sa surface. Je ne l'ai jamais rencontré sur la craie. - Forêts de Cheminon, St-Remyen-Bouzemont. - AC. - Automne. - Comestible. 
86. COL. SEMITALIS. Fries, - Alb. de l'Est. Aspect d'un Tricholoma. - Dans les bois de Vavray-le-Petit. Sermaize ; sur les sciures de bois et la terre fraîchement remuée. - AC. Automne.

87. COL. PLATIPHYLlA. Pers. - Atl. Champ. Ch. R. et Roze, p. 159, pl. XXIX. - Đans les forêts de chênes à Cheminon, Charmont, TroisFontaines; stipe terminé par une Jongue racine träçante. - Été-Automne. - AC. - Indigeste.

88. COL. FUSIPES. Bull. - Atl. Champ. Ch. R. et Roze, p. 160, pl. XlVIII. - Ag.sRia EN fuseau. - Chenier ventru. - Croît en faisceaux à la base des vieux chênes. - ÉtéAutomne. - Le Col. cedematopus de Schœffer n'est qu'une variété à stipe ventru. - Comestible.

89. COL. MACULATA. Alb. et Schw. - Alb. de l'Est. - Récolté pour la première fois en octobre. à Sermaize près de la Colotte, en compagnie du Cortinarius armillatus et du Boletus felleus champignons également rares. - Suspect.

90. COL. BUTYRACEA. Bull. - Alb. de l'Est. - Forêt de Cheminon, sous les hêtres de haute futaie. - R. - Fin de l'Eté. Automne. Suspect.

91. COL. VELUTIPES. Cur . - Alb. de l'Est. Cette espèce, dont le stipe est brun velouté, croît en faisceaux sur les branches et troncs morts d'arbres de différentes essences, depuis le mois d'octobre jusqu'aux gelées. - Forêts. jardins. - CCC. 
92. COL. STIPITARIA. Bull. - Alb. de l'Est. Sur les rameaux, feuilles mortes, brindilles; dans les bois de toute nature. - CC. - Automne-Printemps.

93. Col. TUBERosA. Fries. - Alb. de l'Est. Commun dans les bois de pins sylvestres, sur la mousse et sur les vieux agarics. - Stipe terminé par un sclérote. - CC. - Automne.

9'. Col. CIRRHATA. Fries. - Alb. de l'Est. -Dans les sapinières ; ressemble au précédent. mais au lieu d'être terminée par un sclérote. la racine est tortueuse et fibrilleuse. - CC. Automne.

95. COL. ClAva. Fries. - Alb. de l'Est. - A la base des vieux troncs coupés à ras de terre et sur les racines exposées à l'air. - Forêts de Sermaize. - R. - Espéce très petite d'une belle couleur orangée.

96. COL. ESCULENTA. Wulf. - Atl. Champ. Ch. R. et Roze, p. 162, pl. XLVIII. - En automne et à la fin de l'été. dans les sapinières de Bayarne, St-Amand. - AR. - Malgré sa petite taille, - Comestible.

97. COL. DRYOPHILA. Bull. - Atl. Champ. Ch. R. et Roze, p. 161 , pl. XLIX. - Bois, forêts et sapinières. - Sur les feuilles tombèes. - Paraît toute l'année. - CCC. - Suivant le $\mathrm{D}^{\mathrm{r}}$ Quélet Comestible. - Le chapeau en vieillissant donne asile au Mucor fusiger.

98. COL. Ambusta. Fries. - Alb. de l'Est. Forêts de Trois-Fontaines et de Charmont. - 
Récolté autour des lieux abandonnés oủ l'on a fait du charbon. - RR.

99. COL. CONIGENA. Pers. - Alb. de l'Est. Dans tous les bois de pins, d'Epicea. - Croît sur les cônes couverts de terre ou humides. Pendant l'automne et même une partie de l'hiver et du printemps. - CCC.

\section{Fe GENRE. - MYCENA. -- Fries.}

100. MYC. PURA. Pers. - Atl. Champ. Ch. R. et Roze, p. 168, pl. XXXIII. - Fréquent en automne. dans les lieux herbus et dans la mousse des bois de toute essence. - suspect. 101. MYC. ADONIS. Bull. - Alb. de l'Est. - Croît sur les brindilles, feuilles mortes. - Forêts de Cheminon, Vanault-les-Dames. - Été-Automne. -- AĆ.

102. MYC. CHLORANTHA. Fries. - Alb. de l'Est. - Dans la mousse, au pied des pins sylvestres. La Chaussée, Bayarne. -- Été.

103. MYC. LACTEA. Pers. - Alb. de l'Est. - Bois couverts, sapinières, dans la mousse. - Automne. - CC.

10'. MYC. RUGOSA. Fries. - Alb. de l'Est. Sur les troncs pourris de vieux saules. - Partout. - Automne et hiver. - CCC.

105. MYG. GALERICUlata. Scop. - Alb. de l'Est. - Cespiteux sur les vieux troncs. - Sermaize. St-Remy-en-Bouzemonl, etc. - CC. Automne. 
Var. Calopus. Fries. - Souches. - Dans les sentiers des forêts. - Sermaize. Cheminon: dans les bois d'Aulnay-l'Aître. - Stipe variant du jaune al rouge-brun. - AR.

Var. Tenella. Fries. - Sur les troncs de pins coupés à ras de terre. - Taille grêle. - AR. 106. MYC. POLYGRAmma. Bull. - Alb. de l'Est.

- Parsemé ça et lá dans Jes forêts de chênes et de charmes. Sermaize, Cheminon, Vavray-lePetit. - AC. - Automne. - Stipe sillonné de stries longitudinales.

10\%. MYC. PARABolicA. Fries. - Alb. de l'Est. - Forêts de Cheminon á Trois-Fontaines, Vanaultles-Dames; sur les feuilles mortes, dans les lieux frais. - Automne. - AR.

108. MYC. AlCALINA. - Fries. - Alb. de l'Est.

- Trouvé une seule fois sur un tronc de saule à St-Amand. - Groupe cespiteux. - Odeur alliacée ou ammoniacale. - RRR. - Automne. 109. MYC. FILOPES. Bull. - Alb. de l'Est. Espèce trés commune dans les bois et dans les lieux moussus des sapins. - Automne et quelquefois au printemps. - CCC.

110. MYc. Amicta. Fries. - Alb. de l'Est. Dans les bois de St-Amand á St-Lumier. - Prés des souches. - RR. - Automne.

111. MYC. VITILIS. Fries. - Alb. de l'Est. Dans la mousse des sapinières de St-Amand à Francheville. - Automne. - AR.

112. MYC. ACICULA. Schceff.-Alb. de l'Est. $\mathrm{Au}$ pied des arbres mourants et des vieilles sou- 
ches. Couleur rouge-orangée. Lamelles jaunes. - CC. -- Automne. - St-Amand, Lisse. Soulanges, etc.

113. MYC. LUTEO-ALBA. - Fries. - Alb. de l'Est. - St-Amand. - Sur les aiguilles de pin et sur les feuilles mortes; dans les plantations de saules mêlés de pins sylvestres. - AR. Automne.

11'. MYC. GALOPA. Schrad. - Alb. de l'Est. Forêt de Cheminon, Trois-Fontaines, St-Remy; sur les feuilles pourries à terre. Le stipe brisé laisse échapper une goutte lactescente. - AC. Automne.

115. MYC. EPIPTERIGIA. Scop. - Alb. de l'Est. Fréquent dans les forêts après les pluies. Teinte du chapeau et du stipe jaune-citron lavé. - Visqueux. - AC. - Charmont, Vanault-lesDames. Cheminon, etc. - Automne.

116. MYC. RORIDA. Fries. - Alb. de l'Est. Plus petit que le précédent. blanc, stipe jaunâtre : très visqueux. lamelles décurrentes. Automne après les pluies. - R. - Sur les feuilles. - Sermaize.

117. MYC. VULGARIS. Pers. - Alb. de l'Est - Croît sur les aiguilles de pin, dans les sapinières. - Bassu, Francheville. - AR. - Automne. 118. MYC. DILATATA. Fries. - Alb. de l'Est. Sermaize, Vavray-le-Petit, etc. - Sur les feuilles de chêne inortes. - AR. - Automne.

119. MYC. CORTICOLA. Fies. - Alb. de l'Est. - Sur les mousses et lichens adhérents á l'écorce 
des arbres. - CCC. - En automne et pendant les hivers doux.

120. MYC. HYEMALiS. Obs. - Alb. de l'Est. Sur les troncs moussus des arbres fruitiers; jardins. - Automne-hiver. - AC.

121. MYC. CAPILlaRIS. Schum. - Alb. de l'Est.

- Très petite espéce à stipe capillaire. - Sur les feuilles mortes dans les forêts. - Vavray-lePetit, Cheminon, etc. - C. - Automne.

8 G'ENRE. - MMPHAMA.

122. OMPH. UMbilicata. Schoff. - Alb. de l'Est. - Dans les bois de pins, sur la mousse. CCC. - Vient en groupes. - Automne.

123. OMPH. SCYPHOIDES. Fries. - Alb. de l'Est.

- Sur la mousse et les touffes d'herbe au pied des pins des collines de Soulanges. d'Ablancourt. - AR. - Automne.

124. OMPH. TRICOLOR. Alb. el Schu. - Alb. de l'Est. - Récolté une seule fois à St-Amand, sur le bord d'un talus entourant un jardin. RRR. - Fin de l'été.

125. OMPH. UMBELLIFERA. L. - Alb. de l'Est. - Espéce rare trouvée dans les terrains marécageux de St-Amand à St-Lumier-en-Champagne. - RR. - Fin de l'été.

126. OMPH. PSEUDO-ANDROSACEA. Fries. Alb. de l'Est. - Bois du pont la Lisse entre St-Lumier et St-Amand. - RR. - Automne.

12\%. OMPh. STEllata. Fries. - Alb. de l'Est. - Prairies de Soulanges et d'Abiancourt, bois 
de Vavray-le-Petit. - Sur les racines et tiges languissantes de la grande Consoude. - Automne. - AC.

123. OMPH. INTEGRELlA. - Pers. -- Alb. de l'Est. - Bois de St-Amand à St-Lumier ; ressemble un peu à la précédente. Lamelles très décurrentes. - Automne. -. AR.

129. OMPH. FIBULA. Bull. - Alb. de l'Est. Très commun dans les bois et surtout sur la mousse qui tapisse les plantations de pins. Éte-Automne.

130. OMPH. GRISEA. Fries. - Alb. de l'Est. -

Dans l'herbe des bois de pins sylvestres. La Chausșée. - AR. - Automne

131. OMPh. Atropuncta. Pers. - Alb. de l'Est. - Collines plantées en pins sylvestres, entre St-Amand et Soulanges. - Cette espèce a une odeur tétide insupportable. AR. - Été.

9e GENRE. - PLEUTB'TUS. - Pers.

132. PL. Corticatus. Fries. - Alb. de l'Est.

- Sur les troncs de noyers languissants et sur les vieux saules creux. - St-Amand. Soulanges, Vavray-le-Petit. - On ne distingue le voile annulaire que chez les jeunes sujets. - AR. Été-Automne.

133. Pl. UlmariUs. Bull. - Atl. Champ. Ch. R. et Roze, p. 150 , pl. XLVI. - Cette belle et grande espèce croît sur les troncs languissants de l'orme. - Je ne l'ai trouvée 
qu'une seule fois dans le Département: prés du châleau d'Aulnay-l'Aître. - Automne. Comestible.

134. PL. Ostreatus. Jacq. - Atl. Champ. Ch. R. et Roze, p. 151. pl. XlVII. - Oreille DE NOURET. - Très fréquent à l'arrière-saison sur les vieux noyers dont les branches sont quelquefois garnies dans toute leur longueur. A la suite de l'hiver rigoureux de 1880 , le tiers des noyers centenaires en étaient couverts. On le rencontre aussi sur les troncs de peupliers coupés à ras de terre. - Dans ce cas, les feuillets des chapeaux situés près du sol sont attaqués par un insecte qui produit de petits tubercules glanduleux, d'oú vient le nom de la variété Pl. Glandulosus de Bulliard. - Automne et hivers doux. - St-Amand, St-Lumier, Soulanges, etc. - Comestible.

135. PL. SUbPalmatus. Fries. - sow. - Alb. de l'Est. - Sur les troncs d'ormes abattus. St-Amand, Merlaut, Soulanges. - AR. - J'ai récolté dans les bois d'Aulnay-l'Aître un échantillon á pied central de couleur rose, le chapeau était orné d'un réseau à mailles blanches, saillantes d'un très bel effet. J'ai donné alors á cet échantillon le nom D'AgARIG a RÉSEAU ; plustard jaai reconnu que c'était une variété remarquable de $\mathrm{Pl}$. Subpalmatus. - Été-Automne. 136. PL. ERYNGII. D. C. - Atl. Champ. Ch. R. et Roze, p. 154, pl. XlVi. - Ragoule, Oreille de Girardon. - Croil sur les racines de 
l'Eryngium campestre à Omey, sur les bords de la route, en face du château. - RR. - Comestible. 137. PL. GEOGENIUS. Fries. - Atl. Champ. Ch. R. et Roze, p. 149, pl. XLV. - Sur la terre avoisinant les bois de pins; Soulanges. RR. - Automne. - Suspect.

138. PL. PERPUSILluS. Fries. - Alb. de l'Est. - Petite espéce mesurant de 2 à 8 mill. sur rameau de Pinus sylvestris depuis longtemps dans l'herbe humide, insérée, sur le côté de la branche tournée vers le sol. - Aulnay-l'Aître. - R. - Automne.

139. PL. PORRIGENS. Faund et Schm. - Alb. de l'Est. - Sur les troncs de pins sylvestres en décomposition; palissades en sapin. - Aulnay-l'Aître. - RR. Automne.

140. PL. NIDUlans. Pers. - Alb. de l'Est. Forêts de Vanault-les-Dames, Trois-Fontaines. Sur les branches de chêne qui jonchent le sol. - AR. - Automne.

141. PL. APPLiCATUS. Batsch. - Alb. de l'Est. - Dans les jardins et les vignes, sur les échalas et les vieilles branches de chêne. - CCC.

Var. Cyphellœformis. Fries. - Varièté à teinte bleuâtre, stipe plus accentué. - Forêt de Maurupt. - R. - Automne.

142. PL. DICTYORHIZUS. Fries. - Alb. de l'Est. - Sur les detritus des végétaux, brindilles de chêne, d'érable ; dans les lieux qui ont été inondés. - St-Lumier-en-Champagne, fossés du château. - RR. -- Été. 
B. Hyporhodii. - Spores roses ou couleur de rouille claire.

10 GENRE. - VOLVARA - Fries.

143. VOL. BOMBYCINA. Schoff. - Alb. de l'Est.Amanite incarnate de Pers. - Récolté une seule fois dans l'excavation d'un vieux tronc de noyer à Bassu. - RRR. - Été. - Comestible.

144. VOL. SPECIOSA. Fries. - Atl. Champ. Ch. R. et Roze, p. 2\%, pl. IX. - Solitaire, sur les décombres et detritus des jardins. - Ponthion. Favresse, Ablancourt. - R. - Étè. - Automne. - Vénéneux.

145. Vol. GLOIOCEPHALA. Fries. - Atl. Champ. Ch. R. et Roze. p. 26, pl. VII. - Bords des routes avoisinant les bois ; sur les amas de sciures mélangées à l'humus. - Vanault-lesDames. - AR. - Été-automne. - Vénénerx.

146. VOL. PARVUlA. Fries. - Alb. de l'Est. - Dans les prairies, dans les jardins, allées herbues des bois ; St-Amand, La Chaussée, StLumier. - Été-automne. - AC.

\section{1e GENRE. - pLUTCOS. - Fries.}

14\%. PL. CERVINUS. Schœffer. - Atl. Champ. Ch. R. et Roze, p. 95, pl. XXXV. - Forêt de Trois-Fontaines, Charmont . Vanault-les-Dames, etc. - CC. - Automne. - Suspect. 
Var. Rigens. Fries. - Bois de St-Lumier à St-Amand. - Été-automne. - RR.

Var. Petasatus. Fries. - Dans les bois de Vanault-les-Darnes, sur les sciures de bois. - AC. 118. PL. EPhebeUs. Fries. - Alb. de l'Est. Récolté une seule fois sur un tronc d'Alnus glutinosus à St-Amand. - RR. - Automne.

149. Pl. Pellitus. Pers. - Alb. de l'Est. Bois de Sermaize et de St-Amand. - R. Automne.

150. PL. NANus. Pers. - Alb. de l'Est. - Sur la terre limoneuse des bords de la Marne, dans un bois planté d'ormes. - Soulanges. - Été. - R.

151. Pl. Semibulbosus. Lasch. - Alb. de l'Est. - Trouvé une seule fois à Ablancourt dans les bois et saussaies qui bordent la Marne. - R. - Automne.

152. PL. HisPIdUlus. Fries. - Alb. de l'Est. - Récolté en assez grand nombre sur le chemin herbu qui longe les sapinières de Bayarne à Soulanges, en 1885. - Automne. - R.

153. PL. CHRYSOPHEUS. Fries. - Alb. de l'Est. - Commun sur les écailles et sciures de peuplier. - Été-Automne. - CC.

15'. PL. PHLEBOPHORUS. Fries. - Alb. de l'Est. - Rameaux, brindilles et sciures de bois. - St-Amañd, Lisse, La Chaussée, etc. - CC. - Automne.

155. PL. LEONinus. Fries. - Alb. de l'Est. sur les écailles et morceaux de bois pourris. 
St-Lumier, La Chaussée. - Moins commun que les espéces précédentes. - Automne.

12 GENRE. - ENTOLOMA. - Fries.

156. ENT. Lividum. Bull. - Atl. Champ. Ch. R. et Roze, p. 90, pl. XXXV. - Forêts de Sermaize, de Charmont, de Reims. - CC. Été-Automne. - Vénéneux.

15\%. ENT. PRUNULOIDES. Fries. - Atl. Champ. Ch. R. et Roze, p. 91, pl. XXXV. - Croît isolément sur les chemins herbus prés des plantations de pins. - Soulanges, Francheville. AC. - Été-Automne. - Suspect.

15̊8. ENT. REPANDUM. Bull. - Alb. de l'Est Bois de Vanault-les-Dames. - Dans les sentiers herbus, apparence d'un Inocybe. - Été. - RR. 159. ENT. RHODOPOLIUM. Fries. - Alb. de l'Est. - Forêts de Cheminon, St-Remy-en-Bou: zemont, Charmont, etc. - Autour des chênes. CCC. - Automne.

160). ENT. SERICEUM. Bull. - Alb. de l'Est. Dans les sapinières de Bassu, Francheville, etc. - CC. - Automne.

161. ENT. JUbATUM. Fries. - Alb. de l'Est. Récolté en octobre 188.5 sur les chemins herbus qui longent les pièces de pins de Bayarne à Soulanges. - AR. - Suspect.

13 e GENRE. - CLITOPII S. - Fries.

162. Clit. PRUNUlus. Scop. - Atl. Champ. Ch. R. et Roze. p. 106. pl. XXXVl. - Mous- 
SERon-OraElle. - Prés autour des bois à Vavray-le-Petit. - Se distingue du suivant par la régularité de ses formes, sa consistance plus ferme et l'épaisseur plus égale de son stipe. AR. - Été-Automne. - Comestible.

163. Clit ORCElla. Bull. - Atl. Champ. Ch. R. et Roze, p. 107, pl. XXXVI. - Orcelle. - Espéce trés commune dans les forêts du Département. - Son chapeau est blanc-grisâtre, luisant, sinué ; ses feuillets roses, très décurrents, sur un stipe excentrique renflé à la base. - Fin de l'Été, Automne. - Elle exhale une odeur de farine prononcée.

1'te GENRE. - CEPTONIA - Fries.

164. LEPT. EUCHROA. Fries. - Atl. Champ. Ch. R. et Roze, p. 167, pl. XXXIII. - Sur les vieux troncs pourris de saule Marceau. Bois de St-Amand, Soulanges, Ablancourt. C. - Automne. - Teinte du chapeau grisbleuâtre, feuillets et stipe d'un bleu intense. AC. - Automne.

165. LEPT. EUCHLORA. Lasch, - Alb. de l'Est.

- Pelouses. collines herbues des bois de pins.

- Soulanges. Bassu. - Après les pluies d'Automne. - C.

$15^{\mathrm{e}}$ GENRE. - NOL NEA. - Fries.

166. NOL. MAmmosa. L. - Alb. de l'Est. Rècolté plusieurs fois sur la lisière d'un bois de pins, à Bassu. - RR. - Automne. 
16\%. NOL. ARANEOSA. Quélet. - Alb. de l'Est. - Récolté en 1873 à St-Amand, au bois du Rupt, et plusieurs fois depuis à St-Lumier, sous les feuilles mortes, à l'ombre des ormes et des érables. - RR. - Automne.

16 GENRE. - ECCHAA. - Fries.

168. ECC. Polita. Pers. - Alb. de l'Est. -

St-Amand; sur la terre des bois ombragés. RR. - Été.

169. ECC. ATROPUNCTA. Fies. - Aib. de l'Esl. - Bois de pins sylvestres. sur les collines de St-Amand. - R. Automne.

1\%̈e GENRE. - CLIUDOPUS. - Fries.

170. CL. VARIABILIS. Pers. - Alb. de l'Est. Très commun dans tous les bois et forêts, sur les rameaux et brindilles tombés à terre. Été-Automie, et hivers doux.

171. CL. DEPLUENS. Batsch. - Alb. de l'Est.

- Parasite sur les souches et troncs de pins sylvestres, près de terre. - St-Amand, Soulanges. - AC. - Automne.

C. - Dermini. - Spores ferrugineuses ou ocracées $18^{e}$ GENRE. - PHALIOTA. - Fries.

172. PH. CAPERATA. Pers. - Alb. de l'Est. Forêts de Charmont, Sermaize, Vanault-les-Dames. - C. - Automne. - Comestiule. 
173. PH. EREBIA. Fries. - Alb. de l'Est. Sermaize, Vanault-les-Dames; sur les tas de sciures exposées au soleil. Croît en groupes fasciculés. - Automne. - RR.

17\%. PH. OMBROPHILA. Fries. - Alb. de l'Est. - Dans les mêmes localités que le précédent. avec lequel il a quelqu'aralogie. - R. Automne.

175. PH. TOGULARIS. Bull. - Alł. de l'Est. Prairies près des bois à La Chaussée. - RR. Automne.

176. PH. BLATTARIA. Fries. - Alb. de l'Fist. St-Lumier, jardins, bords de la rivière. - AR. - Automne.

1\%. PH. DURA. Bolt. - Ats. Champ. Ch. R. et Roze, p. 63, pl. XXV. - Terrains cultivés pour jardins à la Cense des Prés. - Trouvé une seule fois en Été. - Suspect.

178. PH. PRECOX. Pers. - Atl. Champ. Ch. R. et Roze, p. 64, pl. XXX. - Croît le long des chemins herbus; pelouses : champs de luzerne ; prés. - C. - Été-Automne.

179. PH. GIBBEROSA. Fries. - Alb. de l'Est. St-Amand, St-Lumier; sur les souches de saules plantés près des habitations. - Fin de l'Été. - AR.

180. PH. RAdICOSA. Bu?l. - Alb. de l'Est. Bois et forêts; Possesse : Sermaize. Vavray-lePetit. etc. Au pied des chênes. - CCC. - ÉtéAutomne. - suspect.

181. PH. PUDICA. Fries. - Alb. de l'kst. - 
Forêt de Sermaize. simple ou cespiteux. - R. - Automne.

182. PH. CYLINDRICA. D. C. - Atl. Champ. Ch. R. et Roze, p. 65, pl. XXVI. - St-Amand. La Chaussée, Bassu. - Sur les vieux pieds de sureau et de saule. Stipe courbe, cylindrique. AC. - Automne. - Comestible.

183. PH. Attenuata. D. C. - Atl. Champ. Ch. R. et Roze, p. 66, pl. XXVI. - Pivoulade. - Bois de St-Amand à St-Lumier ; bords dı Fion à La Chaussée; Jalons-les-Vignes. Sur les troncs de peuplier coupés. - Automne. - AR. 18'. PH. DESTRUENS. Brond. - Alb. de l'Est. - Sur les bcis de peuplier travaillés pour la charpente; croît une année ou deux après l'abatage des arbres. Partout. - CCC. - ÉléAutomne.

185. PH. COMOSA. Frics.-Alb. de l'Est. - Récolté une seule fois sur des troncs de chêne mis en tas dans une cour à St-Amand. - Automne. Chapeau et stipe couverts de squames grises, stipe glabre au-dessus de l'anneau: lamelles blanches puis grises; stipe courbé, chair blanche. - RRR.

186. PH. AURIVELla. Batsch. - Mlb. de l'Est. - St-Amand, Lisse. Sur les saules et les noyers languissants. - RR. - Été-Automne.

18\%. PH. SQUARROSA. Mull. - Alb. de l'Est. Récolté sur de vieux troncs de noyer et de frêne, dans les bois de St-Lumier et à Bassuet. R. - Automne. - Comestible. - Peu délicat. 
188. PH. SPECTABILIS. Fries. - $\mathrm{llb}$. de l'Est. - Bois de Vanault-les-Dames, forêt de TroisFontaines. - Sur Jes souches de chêne, prẻs du sol. - RR. - Automne. - Comestible.

189. PH. AdIPOSA. Fries. - Alb. de l'Est. Bassuet, Lisse, sur les troncs de noyers. Le Baisil prés d'Epernay, communiqué par M. Royer, mycologue. - Suspect.

190. PH. MURICATA. Fries. - Alb. de l'Est. Vanault-les-Dames. - Sur les monceaux de sciures de bois exposés depuis plusieurs années au soleil et á l'humidité. - Croît en groupes nombreux, fasciculés. Récolté trois ans de suite dans la même localité. - Été-Automne. - RR. 191. PH. MUTABILIS. Schœff. - -4 lb. de l'Est. - Dans tous les bois; sur les souches d'arbres, prés de terre, en faisceaux nombreux. Automne. - CCC. - Comestible fade, selon Quélet.

192. PH. MARGinata. Batsch. - Alb. de l'Est. - Sapinières de St-Amand, La Chaussée, Bassu. etc. Dans la mousse qui entoure les pins. CCC. - Automne.

193. PH. ARRHenII. Fries. - Alb. de l'Est. St-Amand, St-Lumier. - Lieux herbus, champs en friche. - AC. - Automne.

19'. PH. MYCENOIDES. Fries. - Alb. de l'Est

- St-Lumier; Bois humides et pâturages aroinant la rivière; Jardins. - AC. - Étè-Automme. 
19e GENRE. - INOCYBE. - Fries.

195. IN. DULCAMARA. Alb. et Schu: - Alb. de l'Est. - Bois de pins et plantations de saules en ligne, dans les terrains calcaires. - StAmand, La Chaussée, Bassu, etc. Polymorphe. - CC. - Automne.

196. - IN. Plumosa. Balt. - Alb. de l'Est.

- Bois, saussaies, a St-Amand, La Chaussée. Automne. - AC.

19\%. IN. OBSCURA Pers. - Alb. de l'Est. Terrains calcaires plantés de pins. Soulanges, Bassu. - C. - Automne.

198. IN. PYRIOdORA. Pers. - Alb. de l'Est.

- Bois humides des bords du Fion ; Aulnayl'Aître, St-Amand. Notamment sous les peupliers. - AC. - Automne.

199. IN. CORYDALINA. Quélet. - Alb. de l'Est. Forêt de Cheminon. de Charmont; bois de Vavray-le-Petit. - Automne. - AC.

200. IN FLOCCULOSA. Berk. - Alb. de l'Est. Bois de pins, dans un parc réservé à Ablancourt. Automne. - RRR.

201. IN. FAstigiata. Schoeff. - Atl. Champ. Ch. R. et Roze, p. 9\%, pl. XXI. - Bois-taillis. St-Amand. Aulnay.l'Aître. - Plus rare que In. Rimosa. - Automne. - suspect.

202. IN. RIMOSA. Bull. - Atl. Champ. Ch. R. et Roze, p. 96, pl. XXI. - Forêts, bois, sapiniéres.jardins. - CCC. - Été-Automne. - Suspect. 
203. IN. GODEYI. Gillet. - Alb. de l'Est. - Sur la terre des bois de pins; collines crayeuses; chapeau et stipe lavés de rouge vermillon. Bassu, St-Amand. Soulanges. - AR. - Automne. 20'. IN. DESTRICTA. Fries. - Alb. de l'Est. Bois de St-Lumier-en-Champagne, autour des peupliers, lieux humides. - Automne. - R. 205. IN. GEOPHILA. Bull. - Alb. de l'Est. Sur la terre des bois humides. - Vanault-lesDames, Sermaize, St-Remy-en-Bouzemont, etc. CC. - Automne. - Chapeau blanc ou violet-lilas. 206. IN. PETIGINosA. Fries. - Alb. de l'Est.

- Lieux humides des bois couverts, taillis . forêts. - Possesse, Sermaize, St-Amand. - AC. - Automne.

20e GENRE. - HARHLCMA. - Fries.

207. HEB. FASTIBILE. Fies. - Atl. Champ. Ch, R. et Roze. p. 9' . pl. XXIII. - Bois de Vavray-le-Petit. L'échantillon que j’ai récolté avait l'aspect, le port et la couleur de Heb. crustuliniforme. de plus il était muni a'un voile (cortine) très apparent. - RR. - Fin de l'été. - suspect.

208. HEB. MESOPHAUM. Fries. - Alb. de l'Est.

- Bois de pins des terrains calcaires; St-Amand, Bassu, La Chaussée, etc. - CCC. - Automne. 209. HEB. FLOS-AURANTII. Comm. - Alb. de l'Est. - Bois de St-Amand, sous les peupliers. Forêt de Sermaize, dans les sentiers humides. - $\mathrm{C}$. - Automne. 
210. HEB. CRUSTULINIFORME. Bull. - Atl.

Champ. Ch. R. et Roze. p. 96, pl. XXVII. Bois couverts, forêts et notamment dans les sapinières des terrains calcaires dont le sol est jonché de champignons en cercles dits cercles magiques. - CCC. - Automne. - Comestible en Franche-Comté, suivant le $D^{r}$ Mougeot.

211. HeB. ElAtUM. Batsch. - Alb. de l'Est. - Forêts de Charmont, Vanault-les-Dames. StRemy-en-Bouzemont. - AC. - Automne.

212. HEB. NUDIPES. Fries. - Alb. de l'Est. Récolté dans une seule localité, dans les bois de Vanault-les-Dames; au rond-point oú aboutissent les grandes tranchées. - RR. - Automne.

$21^{\mathrm{e}}$ GENRE. - FumMuLA. - Fries.

213. FL. PARADOXA. Gillet. - Alb. de l'Est. Trouvé une seule fois dans les bois de Maurupt. Cet agaricinée a le port d'un bolet; ses feuillets sont d'un beau jaune et son chapeau d'un brun-pourpre. - RRR. - Été.

214. FL. GUMmoSA. Lasch. - Alb. de l'Est. Lieux humides des bois de Charmont. - AR. - Été. Automne.

215. FL. CARBonaria. Fries. - Alb. de l'list.

- Forêt de Sermaize à Cheminon, Charmont. Lieux oúl'on a fait ducharbon. - Automne.-AC. 216. FL. AlNicola. Bull. - Alb. de l'Est. Bois-taillis, sur les trones pourris d'Alnus glutinosa. - St-Amand, La Chaussée, etc. - CC. - Été-Automne. 
21\%. FL. FLAVIDA. Schœff. - Alb. de l'Est. Sur les écailles, racines et suuches de chênes : dans les forêts. - Sermaize, Vavray. Vanaultles-Dames. - AC. - Automne.

218. FL. CONISSANS. Fries. - Alb. de l'Est. Bois-taillis. forêts, jardins. - Croît en touffes considérables sur les vieux troncs à ras de terre. - CCC. - Été. Automne.

219. FL. TRICHOLOMA. Alb. et Schu. - Alb. de l'Est. - Récolte une seule fois dans les bois de St-Amand sur les feuilles pourries, probablement plus fréquent dans les forêts de chênes. - AR. - Automne.

20. FL. APICREA. Fries. - Alo. de l'Est. Sermaize , Trois-Fontaines ; cespiteux sur les souches. - C. - Été-Automne.

221. FL. GYMNOPODIA. Bull. - Alb. de l'Est.

- Les Mycologues paraissent incertains sur l'identité de cette espéce. - Je nomme ainsi le Flammula qui croit en groupes fasciculés sur les souches de chênes coupés à ras de terre. Il ressemble à l'Amillaria mellea, mais il est privé d'anneau. - CCC. - l)ans toutes les forêts. - Été-Automne.

\section{2e GENRE. - MACORIA. - Fries.}

222. N. CERODES. Fries. - Alb. de l'Est. Dans la mousse et dans l'herbe des chemins et tranchées des bois. - Sermaize. Vavray. Francheville. - AC. - Été-Automne. 
2223. N. MelinoIdes. Fries. - Alb. de l'Est. Lieux herbus, bords des routes, bois de pins : collines de Bassu. Soulanges, St-Lumier. - AC. - Automne.

2:2'. N. PEdiades. Fries. - Alb. de l'Est. Plantations de pins sylvestres de La Chaussée. Bassu, Meigneux, etc. - CC. - Automne.

225. N. SEMIORBICULARIS. Bull. - Alb. de l'Est. - Champs en friche. - Bords des chemins, prairies. - St-Amand, Aulnay-l'Aître . Ablancourt, etc. - AC. - Été-Automne. - Ressemble à un bouton d'or au milieu de la verdure.

226. N. Myosotis. Var. Tenax. Fries. - Alb. de l'Est. - Lieux humides des torêts. Vanault-les-Dames, Sermaize, Vavray-le-Petit. AR. - Chapeau campanulé, fauve. verdâtre à la marge ; stipe concolore ; lamelles olive. Automne.

227. N. ERINICEA. - Fries. - Alb. de l'Est. Sur les tiges mortes de Rosa canina. - Dans un bois de pins bordé de broussailles à Soulanges. - Automne et hivers doux. - RR.

2:28. N. SIPARIA. Fries. - Alb. de l'Est. Très voisin du précédent; s'en distingue par son stipe velu-hispide dans toute sa longueur. - Le stipe de $N$. Erinacea est nu à son tiers supérieur. - RR. - Automne.

229. N. ESChAROIDES. Fries. - Alb. de l'Est. - Bois humides. bords des ruisseaux. - StAmand, Sermaize, etc. - CCC. - Aulomne. 
230. N. ARVALis. Fries. - Alb. de l'Est. Commun en automne dans les vieux champs de luzerne, sur les bords des chemins ; jardins.

- Stipe terminé par une racine filiforme. St-Amand, St-Lumier, etc.

$23^{\mathrm{e}}$ GENRE. - GALERA. - Fries.

231. GAL. TENER. Schoff. - Alb. de l'Est. Jardins, prés, lieux herbus des bois. - CCC. Été. Automne. - Espèce extrêmement fragile. Var. Minor. - Mêmes localités.

232. GAL. HYPNORUM. Batsch. -- Alb. de l'Est.

- Forêts, bois de pins, dans la mousse. - CC.

- Été. Automne.

Var. Sphagnorum. - Beaucoup plus grand.

- Sermaize, tranchées des bois près du finage d'Andernay.

233. GAL. MYCENOPSIS. Fries. - Alb. de l'Est.

- Sermaize, Vavray. - Prés avoisinant les bois.

- AR. - Automne.

24 GENRE. - TCHBAIA. - Fries.

234. TUB. FURfurACEA. Pers. - Alb. de l'Est. - Croît en groupes sur les brindilles et sur la terre humide des bois - Partout. - Automne. - CCC.

235. Tub. Crobula. Fries. - Alb. de l'Est. Sur la terre des plantations de pins. vis-á-vis du château d'Aulnay-l'Aître. - RR. - Automne. 2:36. TUB. INQUILINA. Var. Ecbola. Fries. Alb. de l'Est. - Collines de La Chaussée à 
Erancheville, sapinières. - Sur les racines des graminées et sur la mousse. - Feuillets presque décurrents. fuligineux, brunâtres. - R. - Automne.

25 GENRE. - CREPIDG'RUS. - Fries.

23\%. CREP. MOLLIS. Schœeff. - Alb. de l'Est. - Très commun sur les souches de peupliers déterrées et sur les troncs languissants. - Partout. - Été. Automne et quelquefois en hiver.

D. - Pratelli. - Spores pourpres-brunes.

26e GENRE. - PSALLIOTA. - Fries.

238. PS. VAILlantiI. Fries. - Atl. Champ. Ch. R. et Roze. p. 46, pl. XII. - Bois de Charmont, Vanault-les-Dames, Scrupt. - A. Rare. - Été. Automne. - Comestible.

239. PS. ARVEnsis. Schoeff. - Atl. Champ. Ch. R. et Roze, p. 45, pl. X. - Cultivé sur couches, champs, bois, jardins. - CC. - Anneau doublé, chair blanche. immuable.

240. PS. VillaticA. Fries. - Atl. Champ. Ch. R. et Roze, p. 49, pl. XV. - Espéce très grosse, à chapeau couvert de squames brunes. concentriques; anneau simple, trés épais, distant: odeur fade, peu agréable. Récolté á Pargny-sur-Saulx par le Dr Mougin. - RRR. 
2'1. PS. HÉMORRHOIDALIS. Kalch. - Atl. Champ. Ch. R. et Roze, p. 49, pl. XVI. - A chair rougissant à la cassure. - Lieux découverts des forêts. - RR.

2i2. PS. PRATENSIS. Schueff. - Atl. Champ. Ch. R. et Roze, p. 54, pl. VIII. - Charmont, StEulien, etc. - Prairies avoisinant les bois. Printemps. Été. - CC. - Comestible.

243. PS. RICHONI. Roze. - Atl. Champ. Ch. R. et Roze, p. 48, pl. XVI. - Cette espéce dont je fais ample récolte tous les ans; est exquise et possède un parfum d'anis très prononcé. Elle croît notamment dans les caves construites depuis peu d'années et autour des granges et écuries. C'est dans les jointures des pierres qu'apparaissent les groupes de chapeaux d'une belle couleur blanche, jaunissant en vieillissant; leur développement est sans doute dû à la composition du mortier qui unit les pierres fait de terre, de chaux et de crottin de cheval. - StAmand. St-Lumier. - Été. Automne. - Rare ailleurs.

244. PS. Comtula. Fries. - Atl. Champ. Ch. R. et Roze, p. 5\%, pl. XVIII. - Espéce beaucoup plus petite que les précédentes. Récolté une seule fois dans la forêt de Charmont. Automne. - R.

2\% GENRE. - STTROPHARHA. - Fries.

215. STR. ERUGINOSA. Curt. - All. Champ. Ch. R. et Roze, p. 8\%. pl. XXXIII. - Commun 
dans la mousse des bois et sapinières, après les pluies d'été et d'automne. - Partout.

Var. Albocyanea, avec le type, p. 68, pl. XXXIII. - suspect.

2'6. STR. OBTURATA. Fries. - Atl. Champ. Ch. R. et Roze, p. 69, pl. XV. - Champs en friche , bords des chemins, prés. - La Chaussée, St-Amand. - C. - Automne. - Douteux. 217. STR. CORONILlA. Bull. - Atl. Champ. Ch. R. et Roze, p. 6i9, pl. XV. - Champs, lisières des bois, collines herbues. - St-Amand. Ablancourt. - C. - Été. Automne. - Douteux. 2'8. STR. MERDARIA. Fries. - Alb. de l'Est.

- Sur les excréments humains desséchés á l'ombre. - St-Amand. - Printemps. - RR. 249. STR. SEMIGLOBATA. Batsch. - Alb. de

l'Est. - Sur le crottin de cheval. - StAmand, Francheville, La Chaussée. - CC. Prés, collines herbues. - Automne. - Vénéneux. 250. STR. ACULEATA. Quélet. - Alb. de l'Est. - Sur les tas de sciures de peupliers, dans les bois exploités depuis un an ou deux. -St-Arnand, St-Lumier-en-Champagne. - Été. Après les pluies, en compagnie d'Hypholoma Candolleanum. - AR. - Automne.

28. GENRE - IHYHoloma. - Fries.

251. HYPH. SUBLATERITIUM. Schoff. - Atl. Champ. Ch. R. el Roze, p. 73, pl. XXV. Allées herbues et sentiers des forêts, prés des 
vieilles souches de chêne. - Vanault-les-Dames. Charmont, Trois-Fontaines. - C. - Automne. - Vénéneux.

252. HYPH. EPIXANTHUM. Fries. - Alb. de l'Est. - Sur les troncs de pins sylvestres abattus. - Sapinières de Soulanges. - Automne. - R.

253. HYPH. ELEODES. Fries. - Atl. Champ. Ch. R. el Roze: p. 74, pl. XXV. - En faisceaux sur les écailles et rameaux pourris de chêne. - Vanault-les-Dames. - AR. - Automne. I'́néneux.

254. HYPH. FASCICULARE. IIuds. - Atl. Champ. Ch. R. et Roze. p. 75, pl. XXV. - Souches de chênes, dans les forêts. CC. - Automne. Vénéneux.

255. HYPH. LACRYMABUNDUM. Fries. - Alb. de l'Est. - Bois humides . prés, jardins. Automne. - CCC. - Douter $x$.

256. HYPH. PYROTRICHUM. Holms. - Alb. de l'Est. - Bois couverts, chemins herbus, après d'abondantes pluies. - St-Amand. - C. - Automne. 25\%. HYPH. CANDOLLEANUM. Fries. - Alb. de l'Est. - Troncs de peupliers, frênes ; tas d'écailles; vergers, bois, prairies. - Partout. - CC. - Été. Automne.

258. HYPH. ANNULATUM. Ch. $R$. - Espéce nouvelle. - Alb. de l'Est. - Se rapproche d'Iyph. appendiculatum. - Stipe muni d'un collier distant, trés entier.

25!). HYPH. APPENDICULATUM. Bull. - Alh. 
de l'Est. - Groupes fasciculés au pied des frênes. - St-Amand, St-Lumier. - CC. - Été. Automne.

260. HYPH. PILULAFORME. Bull. - Alb. de l'Est. - Sur les souches d'ormes et de frênes à ras de terre. prés du château diulnayl'Aitre. - RR. - Automue.

261. HYPH. HYDROPHILUM. Bull. - Alb. de l'Est. - Bois et forêts; croît en touffes trés considerables sur les vieilles souches et sur les racines superficielles après les pluies abondantes.

- CC. - Automne.

29. GENRE. - PSILACYHE. - Firies.

262. PS. COPROPHILA. Bull. - Alb. de l'Est.

- Sur le crottin de cheral ; dans les lieux herbus des collines de Francheville, St-Amand; bords des bois. - AC. - Automne.

263. PS. CERNUA. $\mathrm{Fl}$. D. - Alb. de l'Est. Jardins, vergers à St-Amand. - R. - Automne. 26'. PS. PH INISECII. Pers.-Alb. de l'Est.-Prés. Jardins. - St-Amand. La Chaussée. - AC. - Étè. 265. PS. SPADICEA. Fries. - Alb. de l'Est. Groupé au pied des peupliers et des frênes St-Amand, La Chaussée; dans les bois qui bordent la rivière. - AC. - Été. Automne.

30 (iENRE. - PSATHYRA. - Fries.

266. PSATH. CONOPIleUS. Fries. - Alb. de l'Est. - Au pied des palissades et des haies des jardins ; partout. - CCC. - Automne. 
26\% PSATH. CORRUGIS. Pers, -- Alb. de l'Est.

- Terres fumées. jardins, bois. - rCC. Eté. Automne.

68. PSATH. GIROFLEXA. Fries. - Alb. de l'Est. - Le long des haies et des murs humides. - St-Amand. - R. - Été. Automne. 269. PSATH. OBtUSATA Fries. - tlb. de

l'Est. - Lieux herbus des collines couvertes de pins sylvestres: entre La Chaussée et Francherille. - AR. - Automne.

27). PSATH. FATUA. Fries. - Alb de l'Est. Trouvé une seule fois dans un jardin avoisinant la rivière. à St-Amand. - R. - Automne. 2i1. PSATH. GORDONI. Berk. et $B \%$ - Alb. de l'Est. - Rare - Dans les bois de St-Lumieren-Champagne. au pied des frênes. - Automne.

\section{E. Coprinarii. - Spores nuires.}

31. GENRE. - PACOAS. - Fies.

27. PAN. FIMIPUTRIS. Bull - Alb. de l'Est. - Bords des chemms herbus : jardins. allées des bois ; sur le crottin. - AC. - Automne. 27:. PAN. PHALENARUM. Fries. - Alb. de l'Est. - Isolé. - Bois de Vanault-les- Dames : sur les sciures de bois anciemnes et sur le crottin de cheval - Eté. Automne. - AC. 
2r4. PAN. Papillonaceus. Fries. - Alb. de l'Est. - Bois de Vanault-les-Dames. - Sentiers. - AC. - Été-Automne.

32e GENRE. - PSATHYRELA. - Fries.

2\%5. PSAT Tla GRACILIS. Fries. - Alb. de l'Est.

- Jardins, prairies, allées des bois. - CCC.

- Automne.

276. PSAT ${ }^{\text {lla }}$. DISSEMINATA. Pers. - Alb. de lEst. - En groupes très nombreux sur les troncs humides; bois, jardins. - CCC. - Été. Automne.

\section{3e GENRE. COPRINCS. - Pers.}

2\%i. COP. COMATUS. Batt. - Atl. Champ. Ch. R. et Roze, p. 38, pl. LI. - Jardins, chemins herbus. detritus des vègétaux en tas. - Après les pluies. - CCC. - Été. Automne. - Comestible étant jeune.

278. CoP. OVATUS. Schceff. - Atl. Champ. Ch. R. et Roze, p. 3\%, pl. LI. - Champs fumés. Sur les terrains crayeux. - St-Amand. - RR. Automne. - Comestible.

279. COP. STERQUILINUS. Fries. - Alb. de l'Est. - Jardins, autour des couches, pâturages. - St-Amand. Soulanges, La Chaussée. - AC. Automne. - Comestible.

280. COP. ATRAMENTARIUS. Bull. - Alb. de l'Est. - Bois humides, fossés herbus, après les pluies d'automne. - CC - Partoul. - Comestible. peu dèlicat. 
281. COP. PICACEUS. Fries. -- Alb. de l'Est.

Lieux lıumides et découverts des bois. - Forêts de Sermaize. - Automne. - RR.

28\%. COP. EXTINCTORIUS. Fries. - Alb. de l'Est.

- Prés d'un bois de pins, dans la vallée de Soulanges. Trouvé une seule fois. - RR. - Automne. '8:3. COP. FIMETARIUS. Lin. - Alb. de l'Est.

- Fumiers et couches de jardins, champs. CCC. - Partout.

Var. Macrorhizus. Pers. - Mêmes localités. surtout sur les couches. - AR. - Automne. 284. COP. CINEREUS. Bull. - Alb. de l'Est.

- Considéré par certains auteurs comme variété du précédent. Chapeau gris-cendré. - AC. Été-Automne.

285. COP. TOMENTOSUS. Bull. - Alb. de l'Est.

- Bords des bois, prairies; sur les brindilles recouvertes de terre. - Aulnay-:'Aître, St-Amand.

- R. - Autornne.

286. COP. MICACEUS. Bull. - Alb. de l'Est. -

Cespiteux, sur les troncs, pieux, palıssades. CCC. - Automne. Printemps.

:87. COP. TRUNCORUM. Schoeff. - Alb. de l'Est.

- Troncs de saules, de chênes. - Forêts. Ie paraît être une simple variété du précédent. - CC. - Automne.

¿\&8. COP. ERYTHROCEPHALUS. Lév. - Alb. de l'Est. - Récolté à Châlons : communiqué par lí. Barbat père, lithographe. - RRR.

289. COP. HENDERZONII. Berk. - Alb. de l'Est.

- Sur le fumier de cheral: detritus en tas. 
lieux azotés. -- CC. - Été aprés les pluies. Espèce délicate, trés éphémère, pourvue d'un petit anneau en godet: au milieu du stipe. St-Amand, La Chaussée, etc. - AR.

290. COP. LAGOPUS. Fries. - Alb. de l'Est. -

Sermaize ; dans les tranchées et les sentiers herbus de la forêt. - AC. - Automne.

291 COP. DOMEsticus. Pers. - Alb. de l'Est. - Intérieur des bâtiments. - Ecuries, sur les pompes et seaux de bois. - AC. - St-Amand. - Automne.

292. COP. EPHEMERUS. Bull. - Alb. de l'Est. Fumiers, lieux azotés. - CCC. - Automne. 293. COP. FRIESII. Quélet. - Alb. de l'Est. St-Lumier-en-Champagne, dans les fossés inondés du village; sur les feuilles de Phragmites communis. - Juillet. - RR.

29'. COP. Plicatilis. Fries. - Alb. de l'Est.

- Jardins, prairies. - CCC. - Partout el presque toute l'année.

Dans le Département, je n'ai pas encore vu de représentant du GENRE BORBATICS.

3'e GENRE. - Comtimarids. - Fries.

295. CORT. ANFrACtUS. - Fries. - Alb. de l'Est. - Sermaize, dans les bois de haute futaie. entre la Colotte et Trois-Fontaines. - RR. Automne. 
296. CORT. MULTIFORMIS. - Fries. - Alb. de l'Est, - Forêt de Sermaize ; sous les chênes. - AR. - Été. Automne.

297. CORT. CALOCHROUS. Fries. - Alb. de

l'Est. - Bois de Vanault-les-Dames. - AR. Automne.

298. CORT. TURBINATUS. Bull. - Alb. de l'Est. - Forêts de Charmont, Cheminon.AC. - Automne. - Comestible.

299. CORT. ORICHALCEUS. Batsch. - Alb. de l'Est. - Bois de Vavray-le-Petit. - AC. Automne.

300. CORT. PRASINUS. Schoff. - Alb. de l'Est.

- Forêts de Sermaize à Cheminon, Possesse.

- AC. - Automne.

301. CoRT. COLlinitus. Sou. - Alb. de l'Est.

- Trés commun en Automne et à la fin de l'Été dans toutes les forêts. - Reims. Maurupt, Charmont, etc. Sous les chènes et les charmes. - Automne surtout, aprés les pluies.

Var. Mucosus à stipe exempt d'anneaux squameux, concentriques.

302. CORT. MUCIFLUUS. Fries, - Alb. de l'Est. - Mêmes localités, mais beaucoup plus rare. - Se distingue du précédent par sa marge striée et l'absence des écailles sur le stipe. Automne.

303. CORT. ELATIOR. Sow. - Alb. de l'Est. Bois de Possesse et de Charmont. - AR. Automne. - Stipe élancé, chapeau plissé . rugueux. 
30'. CORT. NITIDUs. Fries. - Alb. de l'Est. Bois de Vanault-les-Dames, de Vavray, etc., dans les taillis, sous les coudriers. - AC. Automne.

30i. CORT. VIOLACEUS. Lin. - Atl. Champ. Ch. R. et Roze, p. 71, pl. XXXIV. - Forêt de Cheminon; sous les rameaux du houx. Lieux sablonneux. - RR. - Automne. - Comestible. 306. CORT. CINEREO-VIOLACEUS. Pers - Atl. Champ. Ch. R. et Roze. p. pl. XXXIV. Très commun dans les forêts de Charmont, de Trois-Fontaines, de St-Remy-en-Bouzemont. Automne. - Comestible.

30\%. CORT. Albo-VIolACEUS. Fries. - Atl. Champ. Ch. R. et Roze. p. 72, pl. XXXIV. - Bois de Vanault-les-Dames, Possesse. - C. - Automne. 308. CORT. BULliardi. Pers. - Alb. de l'Est. Forêt de Sermaize à Trois-Fontaines, au lieudit la Colotte. - RR. - Automne.

309. CORT. BOLARIS. Fries. - Alb. de l'Est.

- Bois de Cheminon, Vanault-les-Dames ; sous les charmes. - AR. - Automne.

310. CORT. SUBLANATUS. Fries. - Alb. de l'Est.

- Forêt de Sermaize; sous les charmes et les chênes : entre la Colotte et Trois-Fontaines. Automne.

311. CORT. OCHROLEUCUS. Fries. - Alb. de l'Est. - Trouvé une seule fois dans les sapinières de Soulanges: son stipe fusiforme me laisse quelques doutus sur son identité. - RR. - Automne. 
312. CORT. CAMURUS. Fries. - Alb. de l'Est. - Bois-taillis, à St-Amand. - R. - Fin de l'Été. 313. CORT. ANOMAlUS. Frics. - Alb. de l'Est. - Bois de Vavray-le-Petit. - Automne. - AR. 314. CORT. CinNAMOMEUS. Lin. - Alb. de l'Est. - Forêt de Sermaizeả Cheminon; dans les tranchées, et sous bois. - AR. - Automne. - Comestible. 315. CORT. RAPHANOIDES. Pers. - Alb. de l'Est. - Forêt de Sermaize à Cheminon ; bois de pins à St-Amand. Possesse. - AC. - Automne. 316. CORT. BIVEllus. Fries. - Alb. de l'Est. - Sermaize, Trois-Fontaines ; lieux humides ; fin de l'Été. - AR.

31\%. CORT. ARMillatus. Fries. - Alb. de l'Est. - Récolté dans un taillis de charmes à Sermaize, prés de la Colotte. - Automne. - RR. 318. CORT. HiNNUleus. - Sow. - Alb. de l'Est. - Trés commun dans les bois de Vavray. Vanault-les-Dames. Cheminon, etc. - Automne. 319. CORT. BIFORMIS. Fries. - Alb. de l'Est.

- Plantations de pins sylvestres, entre St-Amand et la Moivre. - RR. - Été.

320. CORT. ILIOPODIUS. Bull. - Alb. de l'Est.

- Sur les feuilles et sur les fruits de hêtre. St-Amand, Aulnay-l'Aître, etc. - C. - Automne. 321. CORT. PALEACEUS. Fries. - Alb. de l'Est. - Bois de Possesse à Vernancourt; le long des sentiers. - $\Lambda$ R, - Été. Automne. 322. CORT. SUBFERRUGINEUS. - $\Lambda \mathrm{lb}$. de l'Est - Bois découverts; dans une coupe à Vanaultles-Dames. - AR. - Été. Automne. 
323. CORT. LEUCOPUS. Bull. - $A \mathrm{lb}$. de l'Est.

- Bois de Possesse, et bois de pins sylvestres à Aulnay l'Aître. - R. - Automne.

32'. CORT. ACUTUS. Fries. - Alb. de l'Est. Possesse, Charmont; en compagnie du Cort. Paleaceus, mais plus commun. - Été. Automne.

\section{5* GENRE. - GOMPHIDIUS. - Fries.}

325. G. GLUTINosus. Schœff. - Atl. Champ. Ch. R. et Roze, p. 138, pl. XXIII. - Bois de pins à Ablancourt, Aulnay l'Aître. - AR. Comestible.

326. G. VIscidus. Lin. - Atl. Champ. Ch. R. et Roze, p. 189, pl. XXIII. - Très commun dans les sapinières de Bassu, Soulanges, St-Amand. etc. - Automne. - Comestible.

\section{$36^{\mathrm{e}}$ GENRE. - PAMLUS.}

327. PAX. SORDARIUS. Fries. - Alb. de J'Est. - Les échantillons que j'ai récoltés dans les bois de Bayarne et de Bassu diffèrent peu de ceux que j’ai reçus de Paris, sous le nom de Pax. Sordarius. Je les avais nommés Tricholoma Sordidum. espèce dont les caractères peuvent être confondus avec ceux du Paxilus sordarius. - AC. - Automne. - Comestible.

328. PAX. Involutus. Batsch. - Atl. Cinamp. Ch. R. et Roze. p. 140. pl. XLVII. - Bords des routes et des champs, allées des pares. St-Amand. Aulnay, etc. - CC. - Automne. - Comestible. 
Var. Leptopus. Fries. - Plus commun dans les bois que dans la plaine; de dimensions deux fois plus petites. - Charmont, Sermaize. AR. - Automne.

229. PAX. Atrotomentosus. Batsch. - Atl. Champ. Ch. R. et Roze. p. 141, pl. XLV. Commun au pied des troncs de pin sylvestre. - Soulanges, La Chaussée, Bassu, etc. Automne. - Suspect.

330. PAX. PANuOIdes. Fries. - Alb. de l'Est.

- Trouvé une seule fois sur une paroi de sapin vermoulue, à St-Amand. - Automne. - Récolté depuis en grande quantité à Fontainebleau, au pied des troncs de pins, dans les roches.

3\% GENRE. - HYGRorhoress. - Fries.

331. HYG. EBURNEUS. Bull. - Atl. Champ. Ch. R. et Roze, p. 135, pl. XXXIV. - Commun dans les forêts autour des chênes, après les pluies. - Étè. Automne. - Comestible.

332 HYG. MELizeUS. Fries. - Alb. de l'Est. Forêt de Sermaize; sous les chênes et les hêtres, au lieudit la Colotte. - AR. - Automne. - Comestible.

333. HYG. GLUTINIFER. Fries. - Alb. de l'Est. - Très commun autour des chênes, notamment sur les racines, dans les forêts. - Été. Automne. - Comestible.

33'. HYG. LimaCinUS. Fries. - Alb. de l'Est. - Dans les plantations de pins sylvestres, ter- 
rains calcaires. - St-Amand, Soulanges, etc. CCC. - Automne, jusqu'aux gelées.

335. HYG. OLIVACEO-ALBUS. Fries. - Alb. de

l'Est. - Récolté une seule fois dans les bois de Vavray-le-Petit, sous un vieux chêne. RR. - Autemne.

336. HYG. Discoideus. Fries. - Alb. de l'Est.

- Forêt de Charmont et bois de Vavray-le-

Petit; en compagnie de l'Inocybe corydalina. AC. - Automne.

33\%. HYG. VIRGINEUS. Wulf. - Atl. Champ.

Ch. R. et Roze, p. 13\%, pl. XL. - Collines herbues, bords des bois, prairies. - AC. Automne, - Comestible.

338. HYG. NIVEUS. Fries. - Alb. de l'Est. Bois de pins, Bayarne, Bassu, La Chaussée. etc. - AC. - Été, Automne. - Comestible.

339. GYG. SUBRADIATUS. Fries. - Alb. de l'Est. - Bords des bois de pins et collines herbues. - Soulanges, Francheville, Bassu, etc. - AC. - Automne. - Comestible.

310. HYG. Latus. Fries. - Alb. de l'Est. Bois de Vanault-les-Dames; lieux découverts. - RR. - Automne.

341. HYG. COCCINEUS. Schœff. - Alb. de l'Est.

- Bois de Charmont, Cheminon. - AR. Automne. - Comestible.

342. HYG. Miniatus. Fries. - Alb. de l'Est. - Collines crayeuses, presquarides. - Francheville, Soulanges, Gravelines, etc. - AC Automne. 
343. HYG. INTERMEDIUS. Pass. - Alb. de l'Est. - Bois de pins à St-Gibrien près de Châlons. Récolté par M. Morise. - RR. Automne.

344. HYG. CONICUS. Fries. - Alb. de l'Est. Collines herbues, sapinières, vergers, etc. CCC. - Automne, après les grandes pluies. Suspect.

38e GENRE. - LACTARIUS. -- Fries.

345. LACT. TORMINosUS. Schœff. - Atl. Champ. Ch. R. et Roze, p. 113, pl. XXXVII. - MorTON. - Bois-taillis. broussailles, sapinières ; StAmand, Ablancourt, Bassu, etc. - CC. Automne. - Suspect.

346. LACT. CONTROVERSUS. Pers. - Atl. Champ. Ch. R. et Roze, p. 113, pl. XXXIX. - Forêt de Trois-Fontaines à Sermaize, Vanault-les-Dames.

- Lattron-Roussette. - CC. - Automne. Comestible.

3ł\%. LACT. PUBESCENS. Kromb. - Alb. de l'Est. - Collines des terrains crayeux plantés de pins et de saules. St-Amand. - Localité unique. - RR. - Été-Automne.

318. LACT. INSUlsUS. Bull. - Atl. Champ. Ch. R. et Ruze, p. 115, pl. XXXVII. - Bois de Charmont, Vanault-les-Dames ; dans l'herbe des tranchées. - AC. - Automne. - Vénéneux. 319. LACT. BLENNIUS. Kromb. - Alb. de l'Est. - Forêts de la montagne de Reims, de Trois- 
Fontaines, de St-Remy-en-Bouzemont, etc. AC. - Automne. - suspect.

:350. LACT. UVIDUS. Fries. - Alb. de l'Est. - Allées des bois de Sermaize à Cheminon . Vanault-les-Dames. - AC. - Été, Automne. Suspect.

:301. LACT. PYROGAlUS. Bull. - Atl. Champ. Ch. R. et Roze, p. 116, pl. XXVII. - Dans les taillis de chênes. Sermaize, Charmont. - AC. - Automne. - Vénéneux.

352. LACT. PIPERAtUS. Bull. - Atl. Champ. Ch. R. et Roze, p. 112, pl. Xl. - Auburon vaGhe Blanche. - Très commun en été et automne dans les bois de chênes. - Comestible mais peu délicat.

353. LACT. PARGAMENUS. Kromb. - Alb. de l'Est. - Sous les charmes dans les forêts de Sermaize, Charmont. - AR. - Automne.

35'. LACT. VellerUS. Fries. - Atl. Champ. Ch. R. et Roze, p. 111, pl. XXXIX. - Bois de Cheminon, Maurupt. - AC. - Récolté plusieurs fois à St-Amand sous les hêtres. - Été. - Suspect. 355. LACT. Deliciosus. Lin. - Atl. Champ. Ch. R. et Roze, p. 109, pl. XXXVIII. - AGARIG DÉliaieux. - Dans les lieux herbus des bois de pins sylvestres: St-Amand, Soulanges, Vanault-le-Châtel : forêt de Vertus. - AC. Été, Automne. - Comestible.

356. LACT. Pallidus. Pers. - Alb. de l'Est.

- Bois de Vavray-le-Petit. Sermaize, etc. Été-Automne. - Comestible peu délicat. 
35\%. LACT. THEIOGAlUS. Bull. - Atl. Champ. Ch. R. et Roze, p. 109, pl. XXXVII. - Forêt de Cheminon, Possesse, Vanault-les-Dames. C. - Automne. - suspect. - Lait doré.

358. LACT. VIETUS. Fries. - Alb. de l'Est. Forêt de Trois-Fontaines. Tranchée de la Colotte à Cheminon. - Été-Automne. - AC. - Après les pluies.

359. LACT. PICINUS. Fries. - Alb. de l'Est. Récolté une seule fois dans les bois de Vanaultles-Dames, en été. - RRR. - Chapeau violacéfuligineux, tomenteux-velouté.

360. LACT. FULIGINOSUS. Fries. - L. Azonites. Bull. - Alb. de l'Est. - Lieux humides des forêts de St-Remy-en-Bouzemont et de Sermaize.

- AC. - Ėté-Automne. - suspect.

361. LACT. Volemus. Pers. - Atl. Champ. Ch. R. et Roze, p. 118, pl. XXXVIII. - Forêts de l'Est du Département. - On en fait un grand usage à Sermaize, Maurupt, Charmont et dans la Meuse sous le nom de Veau-Vaghe. - CCC - Été.

362. LACT. SUbdulcis. Fries. - Alb. de l'Est.

- Croît sur la mousse, au pied des chênes. Forêt de Cheminon, Vanault-les-I)ames, etc. CCC. - Été, Automne. - Comestible.

363. LACT. CAMphoratus. Fries. - Alb. de l'Est. - Ressemble beaucoup au précédent. Odeur légèrement musquée. - AR. - Automne.

- Mêmes localités. 
39e GENRE. - RUSsula. - Fries

36'. RUS. NIGRICANS. Fries. - Atl. Champ. Ch. R. et Roze, p. 120, pl. XLI. - Forêts de Charmont, Sermaize, St-Remy-en-Bouzemont, etc. au pied des chênes. - Été, Automne. - CC. - Suspect. - Cette espéce en vieillissant donne asile au Nyctalis asterophora et all Nyctalis parasitica.

365. RUS. AdUSTA. Kromb. - Atl. Champ. Ch. R. et Roze. p. 121, pl. XLI. - Plus petit que le précédent et noircissant à peine en vieillissant. - Sermaize. - AC. - Automne.-- Suspect. 366. RUS. DELICA. Fries. $-\Lambda$ tl. Champ. Ch. R. et Roze, p. 121, pl. XL. - Considéré jadis comme suspect. - On le mange sans hésiter dans la Meuse, sous le nom de Blavet. - Sermaize, Vanault-les-Dames. - AC. - Automne.

36\%. RUS. FURCATA. Fries. - Atl. Champ. Ch. R. et Roze, p. 129, pl. XLIII. - Lieux humides des bois. - Cheminon, Possesse. AR. - Été-Automne. - Vénéneux.

368. RUS. RUBRA. Fries. - Atl. Champ. Ch. R. et Roze. p. 129, pl. XliII. - Lieux humides des bois. - Cheminon. Possesse. - C. Automne. - Vénéneux.

369 RUS. ROSACEA. Fries. - Alb. de l'Est. Bois de pins à Aulnay-l'Aître. - AR. - Automne. - Suspert. 
3\%0. RUS. DEPALlens. Fries. - Atl. Champ. Ch. R. et Roze, p. 130, pl. XLIV. - Parties découvertes des bois nouvellement exploités. - AR. - Vavray-le-Petit. - Automne. - Comestible.

3\%1. RUS. SEROTINA. Quélet. - Alb. de l'Est. - Récolté plusieurs fois à St-Amand et à StLumier: sur les vieux troncs à ras de terre. nourri par les detritus du bois. - AR. Automne. - Vénéneux.

372. RUS. VIRESCENS. Fries. - Atl. Champ. Ch. R. et Roze, p. 125, pl. XLII. - Cul-VErT - Forêts de Charmont, de Sermaize. - Parties découvertes des bois. - AC. - Aliment très estimé. - Été-Automne.

373. RUS. CYANOXANTHA. Schweff. - Atl. Champ. Ch. R. et koze, p. 12\%, pl. Xlll. Charbonnier. - Trés commun en été dans les bois. Recherché par les habitants des campagnes à Maurupt, Sermaize, Charmont, etc.

$3 \%$. RUS. HETEROPHYLLA. Fries. - Atl. Charnp. Ch. R. et Roze, p. 12', pl. XLII. Se rapproche du précédent. - Bois de pins entre La Chaussée et Francheville. - - C. Été. - Comestible.

3\%. RUS. FEETENS. Fries. - Alb. de l'Est. Vanault-les-Dames. Cheminon, Vavray : etc. Prés des troncs de chênes après les pluies. Très visqueux. - Aulomne. - AC. - Suspect. :376. RUS. PECTINAtA. Fries. - Atl. Champ. Ch. R. et Roze, p. 126, pl. XLI. - Parties 
découvertes des bois nouvellement exploités. Bords des chemins et sentiers des forêts. Vavray-le-Petit, Sermaize. - AR. - Automne. - Vénéneux.

3\%7. RUS OCHROLEUCA. Fries. - Alb. de l'Est. - Bois et forêts. - Vavray : Sermaize, Charmont. - Automne. - AC. - Vénéneux. 378. RUS. FRAGILIS. - Fries. -- Atl. Champ. Ch. R. et Roze, p. 132, pl. XLIII. - Commun dans les sentiers herbus des forêts. - Très fragile et de saveur âcre. - AC. - Charmont. Cheminon, Vanault-les-Dames, etc. - Automne. - Vénéneux.

379. RUS. AURATA. Fries. - Alb. de l'Est. Sermaize. - Bois de la montagne de Reims : etc. - Croît isolément, en Été. - AR. feuillets dorés sur la tranche. - Comestible. 380. RUS. AlUtACEA. Fries. - Atl. Champ. Ch. R. et Roze, p. 113, pl. XliIV. - Cul-rouge. - Très commun en été dans la forêt de Sermaize à Trois-Fontaines, etc. - Très recherché des habitants de la campagne. - Comestible. 381. RUS. OCHRACEA. Fries. - Atl. Champ. Ch. R. et Roze, p. 126, pl. XLIII. - Bois de Vanault-les-Dames, Vavray-le-Petit. - AC. Automne. - Suspect.

382. RUS. LUTEA. Fries. - Atl. Champ. Ch. R. et Roze. Alb. de l'Est. - Croît ça et lá isolément dans les lieux découverts des coupes ou dans les sentiers. - Vanault-les-Daines, Sermaize, etc. - Été-Automne. - AC. - Suspect. 
40 GENRE. - CANTHA RELUS. - Fries.

383. CANTH. CIBARIUS. Fries. - Atl. Champ. Ch. R. et Roze, p. 144, pl. L. - Chanterelle, Girole, Jaunelet. - Très commun pendant l'été dans toutes les forêts. Aliment très recherché dans les campagnes. Odeur agréable. Chapeau glabre.

384. CANTH. AURANTIACUS . Wulf. - Atl. Champ. Ch. R. et Roze, p. 142, pl. XLIX. Plus rare que l'espéce précédente avec laquelle on pourrait la confondre. Chapeau tomenteux. pédicu?e mince. régulier, noircissant. Croît dans les sapinières. - Bassu, Francheville, Soulanges. - Été-Aulomne. - Suspect.

385. CANTH. CARBONARIUS. Alb. et Schu. Alb. de l'Est. - Dans les forêts ; sur la terre oú l'on a fait du charbon. - Vanault-les-Dames, Sermaize. - C. - Automne.

886. CANTH. MAMmosus. Quélet. - Alb. de l'Est. - D'après les indications du Dr Quélet j'ai placé cette espéce dans le genre Cantharellus. - Ses feuillets grisâtres et èpais, assez. nettement accusés lui donnent l'apparence d'un Clitocybe. - Collines arides de Soulanges plantées en pins sylvestres. - Été.

38\%. CANTH. TUBAFORMIS. Var. Lutescens. Bull. - Alb. de l'Est. - Bois de Vavray-le. Petit, Sermaize, Cheminon. - CC. - Automne. - Suspect. 
388. CANTh. Cinereus. Fies. - . Mllb. de l'list.

- Forêt de Charmont. - AR. - Automne. -

A peine Comestible.

389. CANTH. MUSCIGENUS. Fries. - Alb. de l'Est. - Sur les mousses humides, dans les bois de pins. - St-Amand. - AC. - Automne 390. CANTH. CRISPUS. Fries. - Trogia. Alb. de l'Est. - Trouvé sur les rameaux secs de saule formant une haie de jardin à St-Amand. $-\mathrm{RR}$.

41 $1^{\mathrm{e}}$ GENRE. - VYC'TALIS - Fries.

391. NYCT. ASTEROPHORA. Files. - Alb. de l'Est. - Dans les forêts. sur les chapeaux en décomposition du Russula nigricans. -- Sermaize, Vanault-les-Dames. - CC. - Automne. - Conidies en forme d'étoiles, sur la partie supérieure du chapeau.

392. NYCT. PARASITICA. Fries. - Alb. de l'Est. - Nême station et mêmes localités que le précédent. mais plus rare. - Sans conidies. Automne.

42e GENRE. - MARASMICS. - Fries.

393. M. URENS. Fries. - Atl. Champ. Ch. R. et Roze. p. 16', pl. XliX. - Agaria brulant. - Commun sur les feuilles pourries dans les forêts, en automne. - Vénéncux.

Var. Peronatus. Fries. - Atl. Champ. p. 168, pl. XLIX. - Stipe garni à la base de poils rairles. dressés; marge du chapeau striée. 
39'. M. PORREUS. Fries. - Alb. de l'Est. Bois de Vavray-le-Petit: sur les feuilles de chêne. AR. - Aulomne. - Odeur d'ail.

395. M. PRAsiosimus. Fries. - Alb. de l'Est. Forêt de Sermaize à Cheminon. Sur les feuilles CC. - Automne. - Odeur d'ail. Condiment.

396. M. ERYTHROPUS. Fries. - Alb. de l'Est.

- Forêt de Trois-Fontaines. Vavray. - Sur la terre et sur les brindilles de bois pourri. Été, Automne. - C.

397. M. DISPAR. Fries. - Alb. de l'Est. - Cette espéce diffère peu de la précédente ; on la reconuaît à la marge striée de son chapeau et à son stipe pruineux. Toutes deux passent pour être le Collybia hariolorum de Bull. - Bois de Charmont. Vavray-le-Petit. - AR. - Automne. 398. M. CALopus. Fries. - Alb. de l'Est. - Sur les feuilles de chênes dans les bois de Charmont. -- AR. - Automne.

399. M. Foetidus. Fries. - Alb. de l'Est. Forêt de St-Remy-en-Bouzemont. Lieux humides, sur les brindilles et feuilles pourries. Odeur infecte. - RR. - Automne.

400. M RAMEALIS. Bull. - Alb. de l'Est. Sur les rameaux de bois mort, sur les tiges de ronces desséchées: vient en groupes disposés par étages. - CCC. - Automne. - Dans les bois de charmes, de chênes, etc.

401. M. ROTULA. Scop. - Alb. de l'Est. - Croîl sur les vieilles souches et les petites racines de bois cachées sous les feuilles. - CC. - Été- $\Lambda$ utomne. 
402. M GRAMINUM. Fries. - Alb. de l'Est. -

Sur les tiges de Poa encore vertes. - Le long d'un terme. - Rues de St-Amand. - R. - Été. 403. M. Festudea. Ch. R. - Alb. de l'Est. J'ai récolté cette nouvelle espéce sur des chaumes de Festuca ovina, dans un bois de pins sylvestres à Bayarne, près de St-Amand. Été. - RR.

40'. M. ANDROSACEUS. Fries. - Alb. de l'Est. - Croît sur les aiguilles de pin á La Chaussée. Francheville. - AR. - Été. - Par les pluies. 405. M. HUdsoni. Pers. - Alb. de l'Est. Forêt de Trois-Fontaines à Cheminon. - Sur les feuilles de houx tombées à terre. - R. Automne.

406. M. EPIPHYLlUS. Fries. - Alb. de l'Est.

- Fréquent sur les feuilles de lierre et autres.

- Dans tous les bois. - Printemps-Automne. 4(i). M. SACCHARINUS. Fries. - Alb. de l'Est.

- Lieux humides des bois ; sur les feuilles de peuplier à St-Amand. St-Lumier. - AC. Automne.

$43^{\mathrm{e}}$ GENRE. - MENTINS. - Fies.

408. LENT. TIGRINUS. Bull. - Atl. Champ. Ch. R. et Roze, p. 170, pl. XLVIII. - Troncs et souches de saule; dans les prairies humides. - La Chaussée. Vavray-le-Petit, etc. - CC. Été-Automne. - Comestible.

109. LENT. COCHLEATUS. Sow. - Alb. de l'Est.

- Croit en groupes fasciculés sur les souches 
de hêtres à Sermaize, Vanault-les-Dames. AC. - Automne.

4'e GENRE. - PANus. - Fries.

410. Pan. Conchatus. Fries. - Alb. de l'Est.

- Sur les troncs de saules et de frênes. Plus grand que Pan. Torulosus; surface du chapeau couverte de légères squames. - StAmand, St-Lumier. - Été. - Comestible étant jeune.

411. PAN. RUDIS. Fries. - Alb. de l'Est. Cespiteux sur les troncs coupés à ras de terre, - St-Amand, Aulnay. - CC. - Été-Automne.

- Comestible.

412. PAN. VIOLACEO-FUlvUS. Fries. - Alb. de l'Est. - Récolté une seule fois sur les branches de saules Marceau bordant un bois de pins sur la route de Lisse à Bassu. - RRR. - Automne.

413. PAN. STYPtiCUS. Bull. - Atl. Champ. Ch. R. et Roze, p. 15\%, pl. XLV. - Croît en groupes étagés, sur les vieux troncs d'Alnus glutinosa et de chêne. - CC. - Vénéneux.

45" GENRE. - TRRgia. - Fries.

414. TROG. CRISPA. Fries. - Alb. de l'Est. Trouvé une seule fois sur une branche de saule morte clôturant une haie à St-Amand. Été. -- RRR. 
46. GENRE. - SCHIZORHYLCUM. - Fries, 415. SCH. COMMUNE. Fries. - Alb. de l'Est. -- Sur les troncs abattus de noyer. de pin sylvestre, de peuplier. - St-Amand, La Chaussée, etc. - CC. - Automne-Hiver.

$47^{\circ}$ GENRE. - I.ENGITES. - Fries.

416. LENZ. VARIEGATA. Fries. - Alb. de l'Est. - Palissades, souches de hêtres; StAmand, Vanault-les-Dames : etc. - CC. Automne-Hiver.

41\%. LENZ. ABIETINA. Bull. - Alb. de l'Est.

- Sur les poutres de sapin. - Bois de charpente vieillis dans les chantiers. - St-Arnand, Aulnay-l'Aître, etc. - AC. - Automne. 
$2^{e}$ Fam. - Polyporees. - Fries.

Hymenium tubuleux ou poreux.

1er GENRE. - moldetes. - Fries.

1. - Ochrospori. - Tubesjaunes; spores subocracées.

A. - Viscipelles.

418. B. LUteUS. Lin. - Atl. Champ. Ch. R. et Roze, p. 1\%, pl. LVI. - Dans tous les bois de pins sylvestres du Département. - CCC. Été-Automne. - Espèce munie d'un anneau brun, entourant le stipe. - Comestible peu délicat.

119. B. GRANULAtus. Lin. - Atl. Champ. Ch. R. et Roze, p. 179, pl. LVI. - Très commun dans les bois de pins. - Mêmes localités que l'espéce précédente. Forme de grands cercles sur la terre autour des arbres. Automne. - Comestible.

420. B. Bovinus Lin. - Alb de l'Est. - Très rare dans les bois des terrains crétacés: StAmand. St-Lumier: mais en abondance dans les taillis de Vanault-les-Dames. - Terrains tertiaires. - Comestible. 
421. B. Mitis. Kromb. - Alb. de l'Est. - Bois de Cheminon. - RR. - Automne.

422. B. SANGUINEUS. Fries. - Atl. Champ. Ch. R. et Roze, p. 181, pl. LIX. - Bois-taillis á Vavray-le-Petit. - RR. - Automne.-Vénéneux. 423. B. PIPERAtus. Bull. - All. Champ. Ch. R. et Roze, p. 181. pl. XV. - Forêt de la montagne de Reims et de Trois-Fontaines. AC. - Automne. - suspect.

B. - Subtomentosi.

42'. B. VARIEGAtUS. Sow. - Atl. Champ. Ch. R. et Roze, p. 178, pl. LV. - Bois de pins sylvestres; Soulanges, Francheville. - AC. Automne. - Comestible, peu estimé.

425. B. CHRYSENTERON. Bull. - Atl. Champ. Ch. R. et Roze. p. 192, pl. LV. - Bois de Cheminon, Varray-le-Petit, etc. - Sur les vieilles souches de chênes moussues. - AC. Automne. - Comestible.

426. B. Subtomentosus. Lin. - Atl. Champ. Ch. R. et Roze, p. 19\%, pl. LIX. - Forêts de Charmont, Sermaize, Vanault-les-Dames, etc. Été-Automne. - Douterux.

Var. Sistotremoides. Kickx. - Tanault-lesDames. - AR.

Var. Radicatus. Kromb. - Possesse, Vavrayle-Petit. - AC. - Près des trones pourris.

427. B. VERSICOLOR. Rost. - Alb. de l'Est. Bois de Charmont; dans l'herbe des grandes tranchées. - Automne. - AR. 
428. B. PRUinatus. Fries. - Alb. de l'Est. -

Bois-taillis près de Reims, Sermaize. - ÉtéAutomne. $-\Lambda \mathrm{C}$.

429. B. BARLaE. Fries. - $\Lambda \mathrm{lb}$. de l'Est. - Dans les bois de Charmont. - R. - Automne.

\section{C. - Calopodes.}

430. B. CALOpUs. Fries. - Atl. Champ. Ch. R. et Roze, p. 19', pl. LX. - Bois de Charmont, Vanault-les-Dames. - AR. - Automne. Suspect.

431. B. PAChypus. Fries. - Atl. Champ. Ch. R. et Roze, p. 193, pl. LIX. - Bois humides de St-Rerny-en-Bouzemont. - R. - Automne. - Vénéncux.

\section{D. - Edules.}

432. B. EDUlis. Bull. - Atl. Champ. Ch. R. et Roze, p. 196, pl. LXI. - Polonais - Grosse Queue. - CCC. - Dans les forêts, en été et en automne. - Il acquiert un volume considérable. - Comestible.

Var. Bulbosa. - Mêmes localités.

Var. Alba. - Anomalie dépendant des influences atmosphériques. - Après les pluies prolongées.

433. B. Reticulatus. Boud. - Atl. Champ. Ch. R. et Roze, p. 195, pl. LVIII. - Forêts de Charmont, Cheminon, etc. - Moins fréquent que B. Edulis. - Stipe entièrement réticulé, à côtes saillantes. - Automne. - Comestible. 
'33'. B. EREUS. Bull. Atl. - Champ. Ch. R. et Roze, p. 19', pl. LXI. -- Bois de la montagne de Reims; région des blocs erratiques. Été-Automne. - AR. - Comestible.

\section{E. - Luridi.}

135. B. SATANAS. Leñ. - Atl. Champ. Ch. R. et Roze, p. 18\%, LVIII. - Bois de la montagne de Reims, au dessus d'Ay ; bois de Vavray-lePetit. - AR. - Automne. - Vénéneux.

4:6. B. LURIdUS. Schatf. - Atl. Champ. Ch. R. et Roze, p. 169, LVII. - Lieux humides des forêts. - Vanault-les-Dames. St-Remy-enBouzemont, etc. - AC. - Été, Automne. Vénéneux.

Var. Erythropus. Pers. - Avec le type.

437. B. PURPUREUS. Fries. - Atl. Champ. Ch. R. et Roze, p. 18\%. pl. LIV. - Bois de Vavrayle-Petit. Vanault-les-Dames, etc. - RR. Automne. - Suspect.

138. B. OBSONIUM. ? Fries.-Alb. del'Est. - Cette espéce, récoltée en automne dans un bois découvert à V'avray-le-Petit, ne présentait que des échantillons jeunes, avec les caractères qui la distinguent ordinairement; mais les sujets adultes faisant défaut j'ai mis un point d'interrogation en attendant d'autres récoltes.

\section{F. - Versipelles.}

439. B. Versipellis. Fries. - Atl. Champ. Ch. R. et Roze, p. 183; pl. LIV. - Bois de 
Vanault-les-Dames, Sermaize, etc. - AC. ÉtéAutomne. - Comestible.

440. B. SCABER. Fries. - Atl. Champ. Ch. R. et Roze, p. 182, pl. LIV. - Très commun en été et automne dans les forêts. Le type à chapeau gris-cendré et la variété orangée dans les mêmes localités.

G. - Hyporhodii, à spores roses.

4'11. B. Felleus. Bull. - Atl. Champ. Ch. R. et Roze, p. 186, pl. LVII. - Forêt de TroisFontaines, Vavray-le-Petit. - Croît isolément.

- R. - Été-Automne. - Vénéneux.

442. B. Albus. Ch. $R$. - Alb. de l'Est. Récolté plusieurs échantillons dans un boistaillis mêlé de pins sylvestres, entre La Chaussée et Francheville. - Chapeau blanc, glabre; tubes très décurrents à orifice à peine teinté.

- Spores roses. - suspect.

\section{H. - Cariosi.}

443. B. Cyanescens. Bull. - Atl. Champ. Ch. R. et Roze, p. 18', pl. LIX. - Forêt de la montagne de Reims près d'Epernay. - Région des blocs erratiques. - R. - Étè-Automne. Vénénerx.

\section{I. - Gyrodon.}

4'4. B. RUBESCENS. Trog. - Alb. de l'Est. Récolté une seule fois dans les taillis d'aulnes à Vanault-le-Châtel. - RR. - Automne. 
'ł́. B. Sistotrema. Fries. - Alb. de l'est. - Bois de Vanault-les-Dames bordant les prairies près de la route d'Heillz-le-Maurupt. - R. Automne.

2e GENRE. - STROHLOMYCES. - Berk.

446. STR. STROBILACEUS. Scop. - Atl. Champ. Ch. R. et Roze, p. 17\%, pl. LIII. - Bois de Maurupt, Cheminon. - Lieux hurnides. Croît isolément. - R. - Été. - Spores brunes, échinulées, sphériques. - Suspect.

3e GENRE. -- FISTULINA. - Bull.

44\%. Fist. HEPATICA. Fries. - Atl. Champ. Ch. R. et Roze: p. 172, pl. LII. - Forêt de la montagne de Reims, Trois-Fontaines. Cheminon; dans les fissures des vieux troncs de chênes. - AR. - Automne. - Comestible. Se mange cru, en salade.

4e GENRE. - POLYPORCS. - Fries.

\section{A. - Mesopus.}

418. POLYP. BRUMALIS. Fries, - Alb. de l'Est. - Sur les branches et les racines d'aulne et de pommier. - St-Lumier-en-Champagne, Soulanges, etc. - AC. - Printemps.

149. POLYP. ARCULARIUS. Fries. - Alb. de l'Est. - Bois de hêtres et de chênes. - Cheminon, Aulnay-l'Aître, etc. - AC. - ÉtéAutomne. 
150. POLYP. VERNALIS. Var. Cyathoides. Quélet. - Alb. de l'Est. - Sur les racines et rameaux d'arbres morts recouverts de terre. - Sermaize, St-Amand. - AR. - Été.

451. POLYP. BIENNIS. Fries. - Alb. de l'Est. Dans les bois de chênes. - Sur les souches et troncs coupés à ras de terre. - Vanault-lesDames, Cheminon, etc. - Automne. - CC.

452. POLYP. PERENNIS. Lin. - Alb. de l'Est. - Lieux oú l'on a fait du charbon ; dans les forêts de Sermaize, Vavray-le-Petit. - AC. Été.

\section{B. - Pleuropus.}

453. POLYP. SQUAMOSUS. Fries. - Atl. Champ. Ch. R. et Roze, p. 201, pl. LXII. - Croît sur les noyers, les pommiers. - St-Amand, Soulanges, etc. - CC. - Été-Automne. - Comestible dur.

454. POlyP. Melanopus. Fries. - Alb. de l'Est. - Bois de pins de la montagne de Reims; sur la terre et sur les racines. - R. - Automne.

455. POLYP. PICIPES. Schultz. - Alb. de l'Est. Sur les troncs et racines de saule. - St-Amand. La Chaussée. - Été-Automne. - AC.

456. POLYP. VARIUS. Fries. - Alb. de l'Est. - Sur les troncs pourris de saule. - Soulanges, Lisse. - Polymorphe. - CC. - ÉtéAutomne.

45\% POLyP. ELEGANS. Fries. - Alb. de l'Est. 
Var. Nummularius. - Sur les brindilles de bois mort et les rameaux secs de chênes, dans les forêts. - CC. - Automne-Été.

458. POLYP. LUCIDUS. Fries. - Alb. de l'Est.

- Sur les troncs et souches de chènes ; dans les lieux humides des forêts. - Sermaize. Vanault-les-Dames. - C. - Automne.

\section{C. - Merisma.}

459. Polyp. uMBellatus. Fries. - At1. Champ. Ch. R. et koze, p. 203, pl. LXIII. - Eichase. - Quélet. - Forêt de Sermaize, au lieudit la Colotte; à la base des chênes. aprés les pluies. - R. - Automne. - Comestible.

460. POL YP. INTYBACEUS. Fries. - Atl. Champ. Ch. R. et Roze, p. 20', pl. LXIII. - Bois de St-Remy-en Bouzemont; sous les vieux chênes languissants. - Croît en bouquets énormes. Automne. - AR. - Comestible,

461. POLYP. SUlfuREUS. Fries. - Alb. de l'Est. - Vient sur les troncs de saules, de pommiers, etc. - Aulnay-l'Aître, Vitry-le-François, etc. - C. - Été-Automne. - Comestible.

\section{D. - Apus.}

462. POLYP. LACTEUS. - Fries. - Alb. de l'Est. - Récolté sur le tronc vermoulu d'un vieux poirier et sur le tronc d'un pommier à St-Amand. - Automne. - R. 
463. POLYP. FRAGILIS. Fries. - Alb. de l'Est. - Sur les troncs pourris de pins sylvestres abattus, tapissant les surfaces tournées vers le sol. - Soulanges. St-Amand. - AC. - Automne. - J'ai toul lieu de croire que le Ptychogaster. albus est l'état conidien de cette espéce.

46'. POLYP. NIDULANS. Fries. - Alb. de l'Est. - Sur les branches de chênes et de pins pourris. - Vanault-les-Dames, Trois-Fontaines, etc. - CC. - Été-Automne.

465. Polyp. Fumosus. Pers. - Alb. de l'Est.

- Imbriqué sur les troncs mourants de saules.

- St-Amand. Lisse, etc. - CC. - Été, Automne.

466. POLYP. ADUSTUS. Fries. - Alb. de l'Est.

- Sur les souches et troncs d'arbres coupés à fleur de terre. - Toute l'année. - CCC.

16\%. POLYP. HISPIDUS. Fries. - Alb. de l'Est.

- Troncs de noyers, pommiers et quelquefois de frênes. - Soulanges, St-Amand, etc. - CC. - Automne. - Il noircit en vieillissant et dans cet état, il est utilisé (sous le nom de SELle DE coucou) par les habitants des campagnes pour la teinture des parquets et des meubles. 468. POLYP. DICHROUS. Fries. - Alb. de l'Est. - Sur les branches de chênes et de charmes tombées à terre. - Forêt de Sermaize. - CC. - Automne.

469. POLYP. CUTICULARIS. Bull. - Alb. de l'Est. - Sur les souches d'A nus glutinosa coupées á la surface du sol. - Aulnay-l'Aitre. RR. - Automne. 
470. POlyp. Applanatus. Frics. - Alb. de l'Est. - Récolté une seule fois à la base d'un tronc de pommier à St-Amand. - RR. -Été. 471. POLYP. FOMENTARIUS. Fries. - Alb. de l'Est. - Récolté une seule fois sur un tronc de noyer près de l'Eglise de St-Lumier-en-Champagne. - Automne. - RRR.

472. POLYP. IGNIARIUS. Fries. - Alb. de l'Est. - Très rare. - Rencontré une seule fois sur un énorme peuplier. - Automne $188 \%$. - Confondu ordinairement avec Polyp. salicinus. - persistant.

473. POLYP. FUlvus. Fries. - Alb. de l'Est.

- Sur les troncs et branches d'arbres fruitiers.

- CCC. - Toute l'année. - Persistant.

4\%1 POLYP. RIBIS. Fries. - Alb. de l'Est. A la base des tiges et sur les racines du groseillier noir. - Soulanges, St-Amand. - AC. - Automne.

475. POLYP. CONChatus. Pers. - Alb. de l'Est. - Sur les troncs de vieux saules coupés en tête; disposé par étages. - AC. - St-Amand. Soulanges, etc.

476. POLYP. PINICOLA. Fries. - Alb. de l'Est. - Sur le pied et les racines de Pinus picea, dans un bois du parc d'Ablancourt. RR. - Automne.

4\%. POLYP. SAlicinUS. Fries. - Alb. de l'Est. - Très commun sur les trones de saules coupés en tête. - La surface fructifère se renouvelle chaque année pendant 10 ou 15 ans. 
478. POLYP. POPUlinUs. Fries. - Alb. de l'Est. - Sur les troncs abattus et sur les souches de peuplier; palissades; bois de charpente. - CCC. - Été-Automne.

179. POLYP. CRYPTARUM. Fries. - Alb. de l'Est. - Sur les parois et poutres de chêne dans les caves et dans les carrières. - AC. 480. POLYP. FIBULA. Fries. - Alb. de l'Est. - Barrières, palissades, pieux autour des jardins. - Aulnay-l'Aître. - AC. - Automne.

481. POLYP. ZONATUS. Fries. - Alb. de l'Est. - Commun dans les bois et dans les jardins; sur les vieux troncs languissants, tombés à terre ou coupés près du sol. - Base des chapeaux imbriqués tuberculeuse. - Été-Automne. - CCC.

182. POLYP. VERSICOLOR. Lin. - Alb. de l'Est. - Sur les rameaux et les troncs languissants. - Partout et toute l'année.

\section{E. - Resupinati.}

183. POLYP. FERRUGINOSUS. Fries. - Alb. de l'Est. - Sur les troncs et grosses branches d'Alnus glutinosa coupés et mis en tas pendant plusieurs années, à l'air libre. - St-Lumier-enChampagne. - R. - Automne.

48'. POLYP. CERASI. Ch. $R$. - Alb. de l'Est. - Cette espèce résupinée croît sur les branches de cerisier sous forme de coussinets elliptiques d'un à 3 centimétres, bruns-rougeâtres . saillants à marge glabre, stérile; tubes assez 
grands, à orifice canelle-rougeâtres, puis plus foncés; spores incolores. - Récolté près de St-Amand, dans les bois du Rut. - R. Automne.

485. POLYP. PURPUREUS. Fries. - Alb. de l'Est. - Plaques minces, étalées, interrompues. pourpre-violacées, á contour étroit, byssoïde. Sur planches de sapin pourries. - St-Amand. $-\mathrm{R}$.

486. POLYP. INCARNATUS. - Fries. - Alb. de l'Est. - Sur troncs de cytise. - Dans le parc d'ablancourt. - Automne.

48\%. POLYP. CRUSTACEUS. Lér. - Alb. de l'Est. - Sur bois de chêne travaillé. - A St-Amand. - AR. - Automne.

488. POLYP. RHODELlUS. Fries. - Alb. de l'Est. - Ressemble au précédent, mêmes localités. - Pores plus courts ; marge non byssoïde. R. - Automne.

489. POLYP. MEDUllapanis. Fries. - Alb. de l'Est. - Sur vieux troncs pourris et branches mortes dans les lieux humides des bois. - Surtout sur le pin sylvestre. - AC. Aatomne.

490. POLYP. VUlgaris. Fries. - Alb. de l'Est. - Souches pourries et vieilles planches de palissades. - Etalé, blanc, puis jaunâtre. Automne, IIiver. - CC.

491. POLYP. VAIllantir. Fries. - Alb. de l'Est. - Mycelium filamenteux. entrecroisé. interrompu çà et lá par des plaques de tissu 
blanc, mince, muni de pores très courts. Dans les celliers, sur les poutres vermoulues. - St-Amand.

5e GENRE. - TRAMETES. - Fries.

492. Tram. GibBosa. Pers. - Alb. de l'Ést. Sur les souches et troncs de peuplier, de foêne, de saule. - St-Amand, Ablancourt, etc. Automne-Hiver. - CC.

493. TRAM. RUFESCENS. Fries. - Alb. de l'Est. - Sur les troncs vivants de saules coupés en tête : prairie de Vavray-le-Petit. - R. - Automne.

494. TRAM. BULliARDI. Fries. - Alb. de l'Est. - Vieux troncs de saule Marceau. Chapeau glabre. - St-Amand, La Chaussée. Lisse. - CC. - Automne.

495. TRAM. SUAVEOLENS. Fries. - Alb. de l'Est. - Très commun sur les saules dans les prairies. - Soulanges, La Chaussée, etc. Automne. - Odeur d'anis.

6e GENRE. - DEDALEA. - Fries.

496. DEED. QUERCINA. Lin. - Alb. de l'Est. Commun sur les troncs de chênes coupés, dans les forêts et dans les chantiers. - Été-Automne. - Persistant.

497. DGED. UNICOLOR. Fries. - Alb. de l'Est. - Imbriqué sur les troncs d'arbres languissants: dans les bois et dans les jardins. CCC. - Automne. 
je GENRE. - Magroudus. - Fries.

498. MER. TREMELlosus. Schrad. - Alb. de l'Est. - Sur les vieux troncs d'arbres pourris, dans les lieux marécageux et sur les pins sylvestres vermoulus. - CC. - Automne.

199. MER. CORIUM. Fries. - Alb. de l'Est. Sur les bois de clôture, branches mortes. St-Amand, Aulnay-l'Aître, etc. - AC. Automne.

500. MER. RUFUS. Pers. - Alb. de l'Est. -

Récolté une seule fois sur un tronc de lilas, à St-Lumier-en-Champagne. - Hymenium rouge incarnat, brunissant.

501. MER. SERPENS. Tode. - Alb. de l'Est. Sur une palissade de sapin pourrie; à Aulnayl'Aître. - Automne. - AR.

502. MER. CRISPATUS. Fl. Dan. - Alb. de l'Est. - Sur racines d'Alnus glutinosa. Dans les bois de St-Amand à St-Lumier. - Automne, hiver. - AR.

503. MER. LACRYMANS. Fries. - Alb. de l'Est.

- Larges plaques orangées à contour cotonneux, blanc; sur les murs et les poutres des caves, celliers. - CC. - Printemps-Automne.

Var. Pulverulentus. - Dans les caves avec le précédent.

כ0\%. MER. UMBrinUS. Fries. - Alb. de l'Est.

- Sur les troncs de sapins cariés: surface 


\section{$-102-$}

pulvérulente, brune, plissée, contournée. St-Amand.

8e GENRE. - SOLENIA. - Fries. -

505. SOL. ANOMALA. Pers. - Sol. Ochracea.

- Hoffm. - Alb. de l'Est. - Pezisa anomala des auteurs. - Sur les rameaux de saule, de hêtre. de cerisier. - CC. - Toute l'année. Partout. 


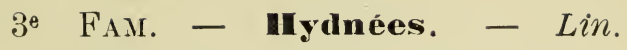

Hymenium aiguillé ou denté.

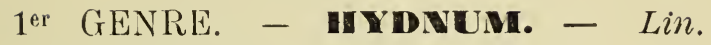

506. HYD. SUBSQUAMOSUM. Batsch. - Alb. de l'Est. - Bois humides, forêt de Trois-Fontaines. - AC. - Automne.

507. HYD. REPANDUM. Lin. - Atl. Champ. Ch. R. et Roze, p. 206. pl. LXV. - Au pied des chênes, des charmes et des hêtres, dans la plupart des forêts. - Charmont, Vanault-lesDames, etc. - CCC. - Automne. - Comestible.

Var. Rufescens. Pers. - Mêmes localités. Plus rare. - Atl. p. 208, pl. LXV.

508. HYD. ZONATUM. Batsch, - Alb. de l'Est.

- Commun dans les forêts de Cheminon, Possesse, etc. - Sous les chênes. - Automne. 509. HYD. NIGRUM. Fries. - Alb. de l'Est. Mêmes localités que le précédent, ainsi que la Var. Olivaceo-cinereum. - Aiguillons blancs. - Automne.

510. HYD. AURISCALPIUM. Lin. - Alb. de l'Est. - Très commun dans les bois de pins ; sur les cônes enfouis sous la mousse. Automne, hivers doux. - Bassu. St-Amand, etc.

- Comestible. 
511. HYD. CORALLOIDES. Scop. - Atl. Champ. Ch. R. et Roze, p. 211, pl. LXIV. - Récolté une seule fois, en abondance sur un tronc de noyer pourri; dans un fossé près de St-Quentin-les-Warais. - Automne. - Comestible.

512. HYD. ERINACEUM. Bull. - Atl. Champ. Ch. R. et Roze, p. 210, pl. LXIV. - Récolté plusieurs fois sur de vieux troncs de noyer encore vivants. - Bassu . Bassuet, St-Amand. - Comestible. - Cette espéce, outre les basides et les spores qui tapissent la surface des aiguillons, possède dans l'épaisseur du tissu du chapeau, un autre mode de reproduction qui consiste en conidies intracellulaires, (voir la notice et le dessin que j'ai communiqués à la Société botanique de France. Bullet. T. XXVIII; 10 juin 1881).

513. HYD. MEMBRANACEUM. Bull. - Alb. de l'Est. - Cet hydnum commence la série des espèces résupinées; elle croît sur les branches de chêne tombées à terre; forêt de Trois-Fontaines. Charmont, etc. - CC. - Automne.

514. HYD. FERRUGINOSUM. Fries. - Alb. de l'Est. - Assez commun sur les souches déracinées de peuplier et d'Alnus glutinosa, dans les lieux humides des bois. - St-Amand, StLumier. - Automne.

515. HYD. AlUtACEUM. Fries. - Alb. de l'Est. - Sur vieilles tiges de Rosa canina couvertes de mousse, dans les bois de pins. - Soulanges. - AC. - Automne. 
516. HYD. SUlfurEUM. Ch. $R$. - Alb. de l'Est - J'ai récolté plusieurs fois cet hydnum, au mois de septembre, sur des brindilles de peuplier, d'èrable et de chêne tombées dans un fossé humide près de St-Lumier-en-Champagne. La partie exposée á l'air libre est d'un beau jaune-soufre ; celle qui est tournée vers la terre ou enfouie sous les débris végétaux est de couleur gris-brun avec des aiguillons blancs. - l'Hydnum limonicolor de Berk, me semble voisin de notre espéce.

51\%. HYD. DENTICULATUM. Fries. - Alb. de l'Est. - Sur les souches d'Alnus glutinosa coupées près du sol. - St-Amand, St-Lumier. AC. - Automne.

518. HYD. NIVEUM. Pers. - Alb. de l'Est. Sur les vieilles souches et racines à demi enterrées dans les lieux humides des bois et des jardins. - CC. - Automne, hiver. - StAmand, Lisse, etc.

$2^{e}$ GENRE. - SISTO'TREMA. - Fries.

519. Sist. CONFLUENs. Pers. - Alb. de l'Est. - Dans les bois de Vavray-le-Petit, Cheminon, Sermaize; dans la mousse. - AC. - Automne. 520. SIST. PACHYODOM. Fries. - Alb. de l'Est. - J'ai récolté pendant plusieurs années cette espéce sur un vieux tronc de noyer languissant, entre St-Amand et Aulnay-l'Aître, - Elle est sessile, à chapeaux imbriqués, épais, gla- 
bres, de couleur ocracée; les lamelles planes sont mélangées de quelques aiguillons. Automne. - RR.

3e GENRE. - IH PEX. - Fries.

521. IRP. FUSCO-VIOLACEUS. Fries. - Alb. de l'Est. - Sur les troncs de pins sylvestres abattus. - St-Amand, Lisse, etc. - AC. Automne.

522. IRP. OBLIQUUS. Fries. - Alb. de l'Est. Sur les branches de chêne pourries, tombées à terre, pendant les temps pluvieux; dans les forêts. - Sermaize, etc. - Automne.

523. IRP. CANDITUS. Weinm. - Alb. de l'Est. - Récolté à Aulnay-l'Aître, sur une branche de saule Marceau. - Automne. - RR.

$4^{e}$ GENRE. - PHILEBala. - Fries.

52'. PHL. RADIATA. Fries. - Alb. de l'Est. Sur les trones de noyers, sur le bois dénudé et entre les fentes de l'écorce. - Lisse. Bassu, Ablancourt, etc. - AC. - Hiver, automne.

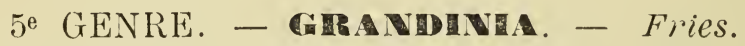

525. GRAND. CRUSTOSA. Pers. - Alb. de l'Est. - Sur les troncs et rameaux desséchès gisant dans la mousse, en automne. - Bois de pins à Soulanges, Lisse. - AC. 


\section{$4^{\mathrm{e}}$ FaM. - Auricularinees.}

Hymenium uni et horizontal.

$1^{\text {er }}$ GEnRE. - CRATERELLUS. - Fries.

526. CRAT. CORNUCOPIOIDES. Lin. - Atl. Champ. Ch. R. et Roze, p. 147, pl. LII. Sur la terre dans les taillis de charmes et de chênes, dans les forêts de Cheminon, Charmont, Reims. - CC. - Automne. - TromPETTE DES MORTS. - Comestible étant jeune. 52\%. CRAT. SINUOSUs. Fries. - Alb. de l'Est. - Broussailles et taillis des bois de Possesse, Sermaize, Vanault-les-Dames, etc. - CC. Automne-Été. - Comestible.

2 GENRE. - THELEPHORA. - Fries.

528. Thel. PAllida. Pers. - Alb. de l'Est.

- Sous les chênes et dans les sentiers de la forêt de Trois-Fontaines à St-Dizier. - R. Automne.

529. THEL. CARYOPHYLlEA. Fries. - Alb. de l'Est. - Terrains humides, dans les forêts de Sermaize, St-Remy-en-Bouzemont, Vanault-lesDames, etc. - CC. - Automne.

530. THEL. TERRESTRIS. Ehrh. - Alb. de

l'Est. - Bois découverts; terrains sablonneux.

- Sermaize. - AR. - Automne. 
531. THEL. BIENNIS. Fries. - Alb. de l'Est. - Assez commun dans les bois-taillis et broussailles, au pied des jeunes chênes. - St-Amand, Aulnay-l'Aître, etc. - CC. - Automne.

532. THEL. CRISTAta. Fries. - Alb. de l'Est. - Cette plante blanche, sébacée incruste les graminées et les mousses, dans les lieux humides des bois et des forêts. - CC. -

3 GENRE. - STEREUM. - Eries.

533. STER. PURPureum, Pers. - Alb. de l'Est.

- Sur les troncs et branches de peuplier, de bouleau, etc. - CCC. - Été-Automne.

534. STER. HIRSUTUm. Wild. - Alb. de l'Est.

- Très commun en automne et en hiver sur les troncs de chênes abattus et sur les palissades ou planches de clôture. Dans les bois et autour des habitations. - Été-Automne.

535. STER. FERRUGINOSUM. Fries. - Alb. de l'Est. - Coriace, brun-foncé. Imbriqué sur les vieilles palissades en chêne. - St-Lumier, St-Amand, etc. - Persistant. - CC.

536. STER. AVEllanUm. Fries. - Alb. de l'Est. - A la base des troncs et sur les racines déterrées des noisetiers. - St-Amand, La Chaussée. - CC. - Automne.

536 bis. STER. SANGUINOLENTUM. Fries. Alb. de l'Est. - Sur Pinus sylvestris : branche à demi pourrie. - Aulnay-l'Aître. - Printemps. - R. - Devient rouge-incarnat par le frottement. 
$4^{\mathrm{e}}$ GENRE. - AURICULARIA. - Fries.

537. AUR. MESEMTERICA. Bolt. - Alb. de l'Est. - Imbriqué en amphithéâtre sur les vieux troncs de noyer. - Trés commun surtout dans l'arrondissement de Vitry-le-François; Soulanges : St-Lumier : etc. - Automne et hivers doux. - Se dessèche par les temps secs comme une membrane papyracée, se gonfle et reprend sa forme première par les temps pluvieux.

$5^{\text {e }}$ GENRE. - CORTICHUM. - Fries.

538. CORT. GIGANTEUM. Fries. - Alb. de l'Est. - Commun sur les troncs de pins abattus. - Blanc, trés étendu. - St-Amand, Soulanges, etc. - Automne-Hiver.

539. CORT. LEVE. Pers. - Alb. de l'Est. Sur les trones et branches de peuplier, d'érable, etc. - CC. - Automne, hivers doux.

כ'10. CORT. FUSCUM. Fries. - Alb. de l'Est. Récolté sur une tige desséchée de Rosa canina. - RR. - Bois de St-Amand. - Été.

5'11. CORT. QUERCINUM. Fries. - Alb. de l'Est. - Dans les bois; sur les branches de chêne et de hêtre tombées à terre. - CCC. Automne. - Brun-pourpre puis noirâtre et retourné.

5'2. CORT. CALCEUM. Fries. - Alb. de l'Est. - Troncs et branches de peuplier et d'érable. 
couverts de mousse; dans les lieux humides des bois. - Automne, hiver.

543. CORT. CCERULeUm. Fries - Alb. de l'Est. - Commun sur les bâtons de vigne en chêne, mis en tas et reposant sur la terre humide. Automne, hiver. - Après les pluies. - Partout. 5't. CORT. OCHRACEUM. Fries. - Alb. de l'Est. - Tiges moites et couvertes de mousse de Rosa cunina. - Branches de pin sylvestre. - Soulanges, Bayarne, etc. - Automne. - AC. 545. CORT. CINEREUM. Fries. - Alb. de l'Est. - Sur les rameaux des hêtres, des cerisiers, etc., jonchant la terre. - St-Amand, Bassu. CCC. - Automne.

546. CORT. COMEdENS. Nées. - Alb. de l'Est. - Croît sous l'épiderme soulevé du coudrier, du noyer, etc. - Aulnay-l'Aître, St-Amand, etc. - Printemps, automne. - CC.

51\%. CORT. SAMBUCI. Fries. - Alb. de l'Est.

- Sur les rameaux et tiges de sambacus nigra; dans les vieilles haies clôturant les jardins. - CC. - St-Amand. - Automne.

548. CORT. TYPHE. Fuckl. - Alb. de l'Est.Enveloppe la base des faisceaux de feuilles de Carex paludosa et des Typha. - Bois marécageux de St-Arnand. - Automne, hivers doux. - CCC.

Var. Foliicolum. Ch. R. - Alb. de l'Est. Croît sur les feuilles tombées á terre, dans le voisinage des Carex déjá envahis. - AR. St-Amand. 
549. CORT. PUTANEUM. Var. areolatum. Fries. - Alb. de l'Est. - Sur les vieilles poutres des bâtiments abandonnés et humides. - CC. - Automne.

6 e GENRE. - HYPOCHNUS. Fries.

550. HYP. FERRUGINEUS. Pers. - Alb. de l'Est. - Lacis filamentelix de couleur ferrugineuse, trés dense. adhérent aux racines de pins sylvestres et aux plantes herbacées qui les avoisinent. - $\mathrm{AC}$. - Automne. - StAmand, Francheville, etc. - Les filaments se terminent chacun par un faible renflement qui émet quatre spores rondes, échinulées, rousses.

ye GENRE. - CYPHELLA. Fries.

5ว๊1. CYPH. AMPLA. Lév. - Alb. de l'Est. Cette espéce peu connue est extrêmement frèquente en automne et en hiver sur les branches et rameaux morts de peuplier d'Italie. La cupule varie entre la largeur d'un pois et celle d'une noix. - Son hymenium se rapproche de celui des Hirneola. - St-Amand, La Chaussèe, etc.

552. CYPH. ALBO-VIOLASCENS. Alb. et Schu. - Alb. de l'Est. - Je pense que cette espéce est la même que Peziza albo-violascens d'Albertini, Cyphella curreyi de Berk. et Cyphella dubia de Quélet. - Elle est commune sur les rameaux secs de la vigne. les brindilles de prunier de Pyrus oxyacantha, etc. - Automne. - CCC. 
553. CYPH. MUSCIGENA. Fries. - Alb. de l'Est. - Croît en groupes sur les mousses au pied des pins sylvestres. - St-Amand, Bassu. - C. - Automne.

554. CYPH. MUSCICOLA. Fries. - Alb. de l'Est. - Dans les lieux humides, sur les troncs coupés près du sol, couverts de mousse et de lichens. - St-Lumier, Ablancourt. - C. Automne.

555. CYPH. NECKERA. Fries. - Alb. de l'Est.

- Dans les bois de pins sylvestres à St-Amand, sur Neckera curlipendula. - CC. - Automne. 556. CYPH. LACERA. Fries. - Alb: de l'Est.

- Récolté sur une tige de Dipsacus à Aulnayl'Aître. - Extérieur villeux-noirâtre. - AR. Autornie.

55\%. CYPH. LETA. Fries. - Alb. de l'Est.

Cupule glabre. - Croît sur les tiges herbacées, Geranium robertianum, Galeopsis, etc. - StLumier. St-Amand. - CC. - Automne.

555. CYPH. RUBI. Fuckel. - Alb. de l'Est. Sur les sarments de Rubis fruticosus. - Espéce blanche, velue, extrêmement petite; se rapproche de Cyph. Villosa; elle est munie de poils plus courts et d'un disque plus aplati par l'humide. - Automne. St-Amand, le long du ruisseau. - AC.

559. CYPH. VIllosA. Pers. - Alb. de l'Est.

- Très commun sur les tiges herbacées; $B a r^{-}$ dane, Circium Arvense, Achilloea millefolia, elc. - Toute l'année. 
560. CYPH. CULMICOLA. Fuckel. - Alb. de l'Est. - Croît sur les chaumes de seigle humides et pourrissants. Récolté sur un tas de paille exposé depuis plusieurs années à l'air libre. - St-Amand. - AR.

561. CYPH. TUBA. Wein. - Alb. de l'Est. En forme d'entonnoir ou de conque, blanc-villeux, marge incisée. - Sur les tiges d'ortie dioique. - St-Amand. - Été-Automne. - C.

562. CYPH. CAPULA. Fries. - Alb. de l'Est. - Croît sur les souches de phragmites sorties du limon ou de la surface de l'eau. Fossés du château à Aulnay-l'Aître. - AR. 
5e Fam. - Clavariées. - Fries.

hymenium uni et vertical, simple ou rameux.

1er GENRE. - CLAVARIA. - Fries.

563. CLAV. FLAVA. Fries. - All. Champ. Ch. R. et Roze, p. 218, pl. LXVII. - Forêt de Trois-Fontaines à Sermaize. - RR. - Espèce á spores blanches souvent confondue avec Clax. aurca, à spores ochracées. - Automne. Comestible.

56'. CLAV. Botrytis. Pers. - Atl. Champ. Ch. R. et Roze, p. 217, pl. LXVII. - Forêts de Charmont, Sermaize, St-Remy-en-Bouzemont, etc. - CC. - Automne. - Comestible.

565. CLAV. AMETHYSTEA Bull. - Alb. de l'Est. - Lieux humides des forêts de Charmont, Sermaize. - RR. - Automne. Comestible.

566. CLAV. CORALLOIDES. Lin. - Alb. de l'Est. - Bois et forêts; Lisse. St-Amand, Sermaize; sous les chênes et les hêtres. - CC. Automne. - Comestible.

$56 \%$ CLAV. MUSCOIDES. Lin. - Alb. de l'Est. - Sur la terre, dans les bois de pins sylvestres. - St-Amand, Bassu. Soulanges. - AC. - Automne. 
5̋68. ClAV. CINEREA. Bull. - Alb. de l'Est. - Forêts de Sermaize, Vanault-les-Dames, elc. CC. - Automne. - Comestible.

569. ClAv. CRIStata. Pers. - Alb. de l'est. - Lieux humides des bois et forêts ombragés. - CC. - Automne. - St-Amand, Vavray-le-Petit, etc. - Clavaire ordinairement envahie et noircie par Helminthosphoria clavariarum.

5\% CLAV. RUGOSA. Bull. - Alb. de l'Est. Lieux humides des bois. - St-Amand, Possesse, La Chaussèe, etc. - CC. - Automne. - Comestible. 571. CLAV. EPICHNOA. Fries. - Alb. de l'Est. - Récolté à Sermaize sur le tan répandu dans les allées d'un jardin. - RR. - Automne.

572. CLAV. AUREA. Schoeff. - Atl. Champ. Ch. R. et Roze, p. 21\%, pl. LXVI. - Croît sur terre en touffes considérables dans les forêts; sous les chênes, les coudriers. - Sermaize, Charmont, Epernay, etc. - CC. Automne. - Comestible? - Spores ochracées. 573. CLAV. FORMOSA. Pers. - Atl. Champ. Ch. R. et Roze, p. 216, pl. LXVI. - Mêmes localités que le précédent. - Rameaux plus épais et de couleur orangée-rose. - AR. Automne. - suspert.

5\%. CLAV. FLACCIDA. Fries. - Alb. de l'Est. - Bois de pins sylvestres. - St-Lumier. Bassu. - AR. - Automne.

5\%. CLAV. FUSIFORMIS. Sow. - Alb. de l'Est. - Bois de Cheminon, Trois-Fontaines ; lieux humides, sur terre. -- CC. - Automne. 
5\%6. CLAV. INGEQUALIS. Fries. - Alb. de l'Est.

- I)ans les bois, Charmont, montagne de Reims, Sermaize. - Lieux découverts, ornières des sentiers. - CC. - Automne.

5\%. CLAV. VERMICULARIS. Scop. - Alb. de l'Est. - Dans les bois de St-Amand à St-Lumier.-Endroits humides et herbus. - CC. - Automne. 578. CLAV. FRAGILIS. Holmsk. - Alb. de l'Est. mêmes localités. - CC. Automne.

579. CLAV. PISTILlARIS. Lin. - Alb. de J'Est.

- Forêts de Cheminon. Possesse, Kieims. Dans les taillis des collines boisées. C. - Eté. Comestible.

580. CLAV. JUNCEA. Fries. - Alb. de l'Est. sur les feuilles de peuplier, d'érable, pourries sur terre. - Eté, Automne. - St-Amand. - CC. 581. CLAV, CANALICULATA. Fries. - Alb. de l'Est. - Bois de St-Lumier. - Echantillon isolé. - AR. - Été-Automne.

58\%. CLAV. INCARNATA. Wein. - Alb. de l'Est. - Récolté une seule fois dans les bois de St-Lumier-en-Champagne, près d'un ruisseau.

- RR. - Automne.

583. CLAV. TRICHOMORPHA. Fries. - Alb. de l'Est. - Sur tiges des Zéa maydis mises en tas et entrant en décomposition. - Aulnayl'Aître, près du château. - RR. - Automne. 58'. CLAV. MuCidA. Pers. - Alb. de l'Est. J'ai trouvé cette rare espéce près de Bassu, sur peuplier. - Automne. - Voir la notice sur Calocera corticalis. 
2e GENRE. - CALOCERA. - Fries.

58't bis. CAL. VISCOSA. Fries. - Alb. de l'Est. Terrains siliceux de la montagne de Reims et de Cheminon ; sur les vieux troncs et racines de pin. - Automne. - AR.

585. CAL. CORNEA. Batsch. - Alb. de l'Est. - Sur le bois de chène pourri, branches. pieux, palissade. - Automine. hiver. - CCC. Partout.

586. CAL. CORTICALIS. Fires. - Alb. de l'Est - Sur les rameaux tombés et pourris de pin sylvestre, de Rosa canina. sur tiges sarmenteuses de houblon, etc. - C. - Lté, automne; après les pluies - Partout. - Cette espéce me parait être plutôt un Cératium qu'une Claraire - J'ai comparé les échantillons qui m’ont èté adressés par M. Boudier de Montmorency et par le Dr Quélet, sous le nom de $\mathrm{Cl}$. mucida avec ceux que j'ai rẻcoltés à St-Amand, Lisse. Soulanges. - Ils présentent tous les mêmes caractères; substance malacoïde á l'origine et filamenteuse dans l'âge adulte. Les filaments se terminent à la surface du champignon par des basides munies de 1 à 3 spicules inégales, courtes, surmontées chacune d'une spore ovale, uniseptée, semblable à celle du Trichothecium roseum. - Il résulte, selon moi, de 
l'observation ci-dessus : que les espéces nommées par les auteurs Cl. mucida, Calocera corticalis, Fries , appartiennent probablement au Cératium pyxidatum d'Alb. et Schw. Le véritable $\mathrm{Cl}$. mucida est différent, à spore sans cloison. La note que je viens de donner sur Cal. Corticalis a été faite en 1885; en. Février 1886, j’ai récolté près ce Bassu, sur des écailles de peuplier le véritable Clavaria mucida, pourvu de basides tétraspores, de spores ovales, non septées - l'espéce était une fois plus grande que Calocera corticalis, les clavules ètaient simples et non bi ou trifunquées. de plus en forme de massue - caractéres qui n'existaient pas dans les différents échantillons qui m’avaient étè communiqués précédemment.

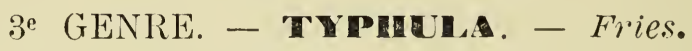

587. TYPH. ERYTHROPUS. Bolt. - Alb. de l'Est. - Sur les pétioles des feuilles de peuplier, d'érable, etc. inséré sur un sclérote rougeâtre. - Automne et hivers doux. - C.C.

598. TYPH. PHACORRHIZA. Fries.-Alb. del'Est.

- Filiforme. trés allongé, inséré sur sclerotium scutellatum, parasite des pétioles des feuilles de frêne; dans les bois de St-Amand à StLumier. - RR. - Automne.

589. TYPH. VARIABILIS. Ries. - Alb. de l'Est.

- Clavules simples, munies d'un pédicule court. velu, inséré sur un sclérote multilobé. - Cha- 
que mamelon porte une clavule; sur écailles de peuplier. - RR. - St-Amand. - Automne. 590. TYPH. GRAMINUM Karlt. - Alb. de l'Est. - Espéce blanche, presque filiforme, à pédicelle distinct, court, prenant naissance sous l'épiderme des chaumes de blè. Tas de paille depuis longtemps exposè à l'air libre. - St-Amand Printemps - AR.

591. THYPH. GREVILLEI. Fries. - Alb. de l'Est. - Commun sur les feuilles de peuplier amoncelées en tas: - à la fin de l'automne St-Amand. - CC.

$4^{\mathrm{e}}$ GENRE. - PISTILIARA. - Fries.

592. PIST. SCLEROTIOIDES? Fries. - Alb. de l'Est - Espèce haute d'un à 2 cent. insérèe sur un sclérote sphérique, caché sous l'épiderme des tiges de pommes de terre desséchées en plein air. La clavule est seule apparente au dehors. - Champs de Lisse - RR. - Automne.

593. PIST. MICANS. Firies. - Alb. de l'Est. Sur les tiges herbacées, notamment sur Eryngium campestre. - CC. - en Automne. - StAmand, Soulanges, etc. - D'une belle couleur vermillon ou rose.

594. PIST. CULmigenA. Fries. - Alb. de l'Est. - Sur les chaumes des graminées pourris sur terre, - pendant l'automme. après les pluies. AC. - St-Amand. 
5 e GENRE. - TREMELIA. - Fries.

595. TREM. Fimbriata. Pers -- Alb. de l'Est.

- Expansions membraneuses, brunes, hautes de 3 á 6 centimètres, sur le bois de chêne dénudé exposé à la pluie. - St-Amand. - Automne.

- R. - dans les chantiers de bois.

596. TREM. MESENTERICA. Retz. - Alb. de l'Est. - Très commun sur les branches des arbres languissants, couverts de lichens ; Peupliers. Erables. etc. - St-Amand. - Eté-Automne.

597. TREM. AlBidA. Huds. - Alb. de l'Est. Sur les branches de saule destinées à servir de rames et placées dans les lieux humides des jardins. - CC. - Printemps-Automne St-Amand, Lisse, etc.

593. TREM. VIOLACEA. Relh. - Alb. de l'Est. - Sur les troncs de cerisiers languissants, à Lisse. - Printemps. - RR. - En février j'ai observè sur les mêmes sujets les spores et les spermaties.

599. TREM. INDECORATA. Somm. - Alb. de l'Est. - Commun sur les tiges et branches de saules enlacés pour faire des haies de clôture. - St-Amand, Bayarne, etc. - CC. - Printemps, Automne. 
599 bis. TREM. INTUMESCENS. Fries. - Alb. de l'Est. - Sur branche d'orme à demi pourrie ; plaques brunes ressemblant á un Collema. assez larges, gaufrées comme les morilles -. Bois d'Aulnay - R. - Janvier.

6e GENRE. -- ERHDOCHAUH. - Fries.

600. EPID. NIGRICANS. Fries. - Alb. de l'Est.

- Petits tubercules trémelloïdes, noirâtres, se gonflant par l'humidité, possédant des basides globuleuses et des spores pyriformes - sortant de dessous l'épiderme de diffèrents arbusles, lilas, Colutea arborescens, coudrier, etc. - Dans les jardins. - Automne-Hiver. - AC. - StAmand.

601. EPID. MELANOCHLORUM. Desm. - Alb. de l'Est. - Espéce encore plus petite que la précédente, à peine d'un millimètre, noirâtre étant sèche, et verte par l'humide. - Spores en chapelet. - Sur les pédoncules des gousses de Cytisus laburnum. - AR. - Automne. Jardins. - St-Amand.

602. EPID. ATROVIRENS. West. - Alb. de l'Est. - Récolté au mois d'avril dans les bois de Lisse sur un rameau de Frêne. - R. Très pelits tubercules vert-foncé, passant au noir. Spores ou plutôt conidies assez grandes, légérement courbées, portées par des basides cylindriques. C'est probablement l'appareil conidien de Calloria-atrovirens. Fries. 


\section{Fe GENRE. - DACRYMYCES. - Fries.}

603. DACR. FRAGIFORMis. Nées. - Alb. de l'Est. - Sur branches et rameaux de chêne et de pins sylvestres. - AC. - Soulanges, Bassu, etc. - Automne.

60'. DACR. DELiquescens. Duby. - Alb. de l'Est. - Trés commun, partout au printemps et à l'automne, sur les branches pourries de sapin, de chêne, les vieilles palissades.

605. DACR. STillatus. Nées. - Alb. de l'Est.

- Cette espéce est plus ferme, arrondie et convexe que l'espéce précédente ; sa couleur orangée presque vermillon est aussi différente. Elle croît communément sur les bois de sapin qui servent de clôture aux jardins. - CCC. Automne, printemps.

606. DACR. CHRYSOCOMUS. Tul. - Alb. de l'Est. - (Calloria Fries). Sphérique: puis en forme de pézize ou d'Helotium, sessile, orangépâle ; sur les branches de pins sylvestres. AR. - Automne. - St-Amand, Soulanges.

60\%. DACR. Phragmitidis. Kick $x$. - Alb. de l'Est. - Cette espéce, décrite dans la Flore des Flandres, ressemble à un Tubercularia jaune-orangé dans sa jeunesse; en vieillissant elle prend la forme d'une cupule pézizoide. Elle croît sur les chaumes de Phalaris arundinacea et sur les chaumes et rhizòmes de 
Phragmites communis. - Bords du Fion à St-L.umier, et à Aulnay-l'Aître dans les fossés du château. - Automne - AR.

608. DACR. URTICE. Pers. - Alb. de l'Est. Etat conidien de Calloria fusaroides très commun sur les tiges mortes d'ortie. - Automne. - Partout.

8e GENRE. - EXIDIA. - Fries.

609. EXID. RECISA. Dithm. - Alb. de l'Est. Sur les branches mortes du saule Marceau. Automne, Printemps. - St-Amand, Aulnay. - CC.

610. EXID. GLANDULOSA. Fries. - Alb. de l'Est. - Sur les branches mortes de charme, de chêne, de hêtre, dans les forêts et dans un bois sur les bords du Fion à St-Amand Pargny: Sermaize. - AC. -- Automne.

9e GENRE. - HITRNEOLA. - Fries.

611. HIRN. AURICULA JUDGE. Lin. - Alb. de l'Est. - Ofeilide de Judas. -- Commun sur les troncs et rameaux languissants de sambucus nigra, dans les haies des clôtures de jardins; elle croît aussi. mais plus rarement sur Robinia pseudo-accacia et sur le noyer. Automne et hivers doux. - St-Amand, StLumier. - Suivant Quélet, purgatif. 
2e ORdRE. - PÉRIDIÉES. - Quélet.

$1^{\text {re }}$ FAM. - Vidulariées. - Tul.

Hymenium charnu, ni pulvérulent ni déliquescent.

1er GENRE. - CYATHUS. - Hall.

612. CYATh. STRIATUS. Hoff. - Alb. de l'Est.

- Sur les rameaux et racines de bois pourri.

- Bois découverts de Sermaize, Vanault-les-

Dames, Soulanges. - AC. - Automne. Intérieur du peridium strié.

613. CYATH. VERnicosus. Bull. - Alb. de

l'Est. - Sur les écailles et rameaux de chêne pourrissant sur terre. - Cheminon, Sermaize. - Automne. - AR. - Intérieur du péridium lisse.

614. CyATh. CRUCibulum. Hoff. - Alb. de l'Est. - Dans les lieux humides des forêts de Cheminnn, Maurupt. - AR. - Automne. - Sur les brindilles de chêne. - Couleur ochracée.

$2^{\mathrm{e}}$ GENRE. - SPHAEROHOLUS. - Tode.

615. SPH. STEllatus. Tode. - Alb. de l'Est.

- Sur les sciures et petites écailles de chêne.

- Sermaize, près de la fontaine. - AR. -Automne. 
2e FAM. - Phalloidées. - Fries. Péridium double renfermant une couche de gélatine. Hymenium déliquescent.

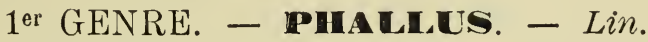

616. PH. IMPUdicus. Lin. - Alb. de l'Est. - Sous les chênes, pendant les pluies d'été et d'automne. - Forêts de Charmont, Sermaize. etc. - AC. - Vénéneux. - Odeur fétide.

$2^{e}$ GENRE. - CYNDPIIALLUS. - Fries.

617. CYN. CANINUS. Huds. - Alb. de l'Est. Bois de Cheminon; sous les chênes. les charmes et les buissons de houx. - AR. Automne. - Vénéneux.

3e FAM. - Lycoperdinées. - Fries.

Hymenium charnu puis pulvérulent.

1er GENRE. - PTyCHOGASTER. - Corda.

618. - PTYCH. ALBUS. Corda. - Alb. de l'Est. - Cette fongosité est considèrée par certains auteurs, comme une Lycoperdinée à cause de son péridium et de sa consistance charnue, pulvérulente. - Selon moi, elle n'est 
pas autonome, mais seulement un état conidien d'un Hydne ou d'un Polypore. (Fragilis ou boréalis). Elle croît sur les troncs de pins et de Thuya coupés prés du sol et sur leurs racines.

- Soulanges, St-Amand. - AR. - Automne.

\section{2e GENRE. - ECCHYMa. - Fries.}

619. ECCH. FAGINEA. Fries - Alb. de l'Est. Ce petit champignon nommé jadis Pilacre ou Onigena et en dernier lieu Ecchyna par Fries et Boudier est, cornme l'espéce précédente, un état conidien d'un type parfait non encore déterminé. - Il croît sur les troncs et les branches de hêtres abattus. - Charmont, TroisFontaines, etc. - $\mathrm{AC}$. - Automne.

620. ECCH. PORICOLA. Ch. $R$. - Alb. de l'Est. - J'ai récolté cette espéce une seule fois, mais en assez grande quantité sur les tubes d'un Polyporus salicinus parasite sur saule Marceau; elle diffère d'Ecchyna faginea par sa station, par la coloration plus foncée de son peridium et par l'épaisseur de son stipe. Elle est à n'en pas douter, comme la précédente un état conidien particulier. - St-Amand, dans les bois du Rupt. - Automne.

3e GENRE. - SCLERODERMA. - Pers.

651. SCLER. VULGARE. Fries. - Atl. Champ. Ch. R. et Roze, p. 223, pl. LXVI. - CC. En automne dans les allées et sous les chênes des forêts de Sermaize, Vavray-le-Petit. - rénéneux. 
622. SCLER. VERRUCOSUM. Bull. - Atl. Champ. Ch. R. et Roze, p. 223 , pl. LXVI. - Bois de St-Amand á Aulıay ; forêts de Reims, Trois-Fontaınes, etc. - CC. - Automne. Vénéneux.

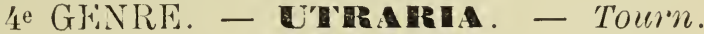

623. UTR. HIRTA. Batsch. - Alb. de l'Est. Dans les forêts de Charmont, Trois-Fontaines, etc. Sous les charmes et sous les chênes. AC. - Automne.

62'. UTR. ECHINATA. Pers. - Alb. de l'Est.

- Récoltè une seule fois en automne entre Sermaize et Trois-Fontaines, au lieu dit la Colotte. Peridium pourvu de longsaiguillons. - RRR. 625. UTR. PRATENSIS. Bull. - Alb. de l'Est. Prairies près des bois de Charmont. St-Eulien, etc. - AC. - Printemps, Automne.

626. UTR. EXCIPULIFORMIS. Pers. - Atl. Champ. Ch. R. et Roze, p. 220, pl. LXVII. - Commun en été dans les forêts de Cheminon. Vanault-les-Dames, etc.

62\% UTR. PYRIFORMIS. Linn - Alb. de l'Est. - Sur la terre des bois nouvellement défrichés. Croît en groupes compacts sous les chênes. St-Remy-en-Bouzemont, Vavray, etc. - Automne.

5e GENRE. - GLOHA IBAA. - Pers.

628. GLOB. GIGANTEA. Rotsk. - Alb. de l'Est. AR. - Jardins, champs par les temps humides - Péridium globuleux, couleur de gant de 
peau blanche; muni à la base d'un pédicule radiciforme mince et court, de grosseur variable. Un remarquable spécimen trouvé à Soulanges au mois de Juillet, sur la craie, mesurait un métre 15 centimétres de circonférence; tout en attribuant à la glébe les deux tiers de la substance du champignon: on peut affirmer qu'il ne renfermait pas moins de vingt millions de sporules ou graines. J'ai reçu aussi de St-Remy-en-Bouzemont la Var. Glob. Craniolare de Paulet, de 65 contimètres de circonférence, affectant la forme d'un crâne humain. - Comestible ètant jeune.

6e GFNRE. - GEATERe. - Mich.

629. GEAST. HYGROMETRICUS. Fries. - Alb. de l'Est. - Terrains silicieux de la montagne de Reims. - AR. - Eté.

ye GENRE. - TULASWODEA. - Fries.

630. TUL. MAMmosA. Tul. - Alb. de l'Est. Sur les collines herbues des terrains calcaires à St-Amand, Soulanges. etc. - dans le voisinage des sapinières. - Automne. - AC. 


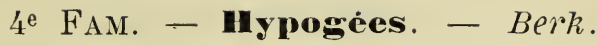

Peridium membraneux, indéhiscent ; glèbe lacuneuse, déliquescente, privée de capillitium.

1er GENRE. - RHIzOPOGON. - Fries.

631. RHIz. LUteolus. Fries. - Alb. de l'Est. - A demi enfoui ou superficiel sur la terre dénudée et crayeuse des bois de pins sylvestres. - Soulanges, Lisse, Bassu, etc. - CC. Automne, Été.

$2^{e}$ GENRE. - HYMEMOGASTER. - Witt.

632. HYM. LEPTONIASPORUS. Ch. $R$. - Alb. de l'Est. - Nov. spec. - Cette espéce a été récoltée par M. Jannet Sophrone de St-Lumieren-Champagne, en Septembre, dans les champs de la plaine située entre Bassuet et St-Lumier. Elle croît dans la terre comme les truffes, el c'est en culbutant le sol crayeux avec la charrue que les èchantillons ont été découverts en abondance dans plusieurs champs de luzerne. C'est la seule espéce de la famille qui possède des spores roses, anguleuses, semblables à celles de Leptonia. - Se retrouve tous les ans dans la même contrée. - RR. 
3e ORdRE. - PLASMODIOPHORES. - Fuckel.

$1^{\text {re }}$ FaM. - Myxomycètes. - De By. Mycelium absent, d'abord substance mucilagineuse, informe, fluxile. puis prenant une consistance déterminée suivant les Genres.

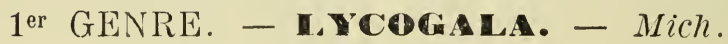

633. LYC. EPIDENDRON. Lin. - Alb de l'Est. Péridium rouge-minium, enfin brun-grisâtre. ponctué. Spores jaunâtres, tuberculeuses. - Sur les vieux troncs et souches d'arbres déracinés. - CC. - Automne, printemps.

634. LYC. LENTICULARE. Dur. - Alb. de l'Est. - Sur les trones et branches de frênes abattus depuis deux ou trois ans - Péridium lenticulaire, rouge - clair puis brun, inséré sur une membrane blanche; filaments du capillitium moins rugueux que dans l'espèce précédente - Bois et chantiers - AC. - St-Amand, Ablancourt. - Automme.

$2^{e}$ GENRE. - IRETHCCLARI. - Bull.

635. RET. UMBrina. Fries. - $\Lambda \mathrm{lb}$. de l'Est. Sur les trones et pieux de pins sylvestres ; d'abord argenté puis brunâtre - quelquefois 
de la grosseur d'un cuf $-\Lambda \mathrm{C}$. - Automne.

- Soulanges, St-Amand, etc.

636. RET. CARNEA. Schum. - Alb. de l'Est. Mêmes localités et station - Péridium rosé, marqué de veines anasthomosées, blanches puis brunes. - AR. - St-Amand. - Automne.

63\%. RET. ATrA. Alb. et Schw. - Alb. de l'Est.

- Sur le bois dénudé de pin sylvestre. Automne, printemps. - CC. - Soulanges, Li Chaussée. - Dabord blanc de lait puis noir ; capillitium noir-brun.

$3^{3}$ GENRE. - RETIMUM. - Link.

638. ETH. SEPTICUM. Lin. - Alb. de l'Est. Sur le tan dans les serres et les jardins. CC. - Eté, automne. - Sermaize, Soulanges, etc.

639. ETH. FLAVUM. Link. - Alb. de l'Est. Sur les troncs de pins et de peupliers coupés prés du sol; sur les tiges et les feuilles de graminées rans les bois. - AC. - Eté, automne.

$4^{e}$ GENRE. - THIIIIIA. - H Hr.

6'10. TRICH. FALlax Pers. - Alb. de l'Est. Sur les rameaux de chène pourris; bois de Sermaize. - Passant, selon l'âge, du blanc au rouge-vermillon puis au jaune, au brun et au noir. - Eté. - AC.

611. TRICH. RUBIFORMIS. Pers. - Alb. de l'Est. - J'ai récolté cette espéce sur une souche de peuplier, dans les bois de St-Amand, au 
mois d'Août. Elle se compose de faisceaux épars, chacun de 6 à 8 peridium noir-bleu, arec des opercules brillants comme du jais. Les filaments et les spores sont rouges. - RR.

642. TRICH. PYRIFORMIS. IIoff. - Alb. de l'Est. - Sur le bois pourri au niveau du sol. - Peridium en forme de poire jaune-orangé, à pédicule brun. - CC. - Printemps, Automne.

643. TRICH. BOtRYTIS. Pers. -Alb. de l'Est. - Sur troncs cariès, dans les lieux humides. - Automne. - St-Amand. Lisse, etc. - Le même pédicule porte deux ou trois péridium rouges. - CC. 64'. TRICH. CHRYSOSPERMA. Bull. - Alb. de l'Est. - Sur les souches, écailles, rameaux pourris. - CC. - Dans les bois, les jardins. - Automne, printemps.

6'5. TRICH. SERPULA. Fries. - Alb. de l'Est.

- Peridium flexueux, réticulés, jaunes. Naissant sur le bois de peuplier, d'aulne, etc. - CC. - Automne.

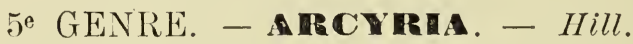

646. ARC. CINEREA. Fries. - Alb. de l'Est. Sur rameaux dépouillés d'écorce et branches pourries. - Peridium blanc-grisître ainsi que les spores. - Bois de Sermaize, Vanault-lesDames, etc. - Autornne. - C.

61\%. ARC. PUnicea. Pers. - Alb. de l'Est. Sur vieilles branches de chêne humides, dans les forêts - Cheminon, Charmont. etc. - Couleur de chair ou rose. - AC. - Automne. 
6'18. ARC. NUTANS. Bull. - Alb. de l'Est. -

Peridium très longs, groupés, fasciculés, jaunepâle : - AR. - Sur troncs cariés et humides.

- Sermaize. - Automne.

6e GENRE. - DIC'TYDLM. - Schrad.

6'9. DiCT. UMBiLiCATUM. Schrad. - Alb. de l'Est. - Sur troncs pourris de pins sylvestres. - CC. - Automne. - Soulanges, Bassu. etc.

ye GENRE. - CRIBARIA. - schrad.

6500. CRIB. VULGARIS. Schrad. - Alb. de l'Est. - Sur écailles de bois pourri à St-Amand. RR. - Peridium enveloppé supérieurement d'un réseau et muni inférieurement d'une membrane cupulaire crénelée. striée; pédicelle noir.

8e GFNRE. - FERICHIONA. - Fries.

651. PER. POPULINA. Fries. - Alb. de l'Est. - Sur l'écorce et les écailles humides du peuplier abattu. Dans les bois de Soulanges, StAmand, etc. - Automne. - CC. -- Capillitium rare ou nul.

652. PER. STROBILINA. Fries. - Alb. de l'Est. - Sur et entre les bractées des cônes de Pinus abies à Ablancourt. - Automne. - RR. - Spores rondes, ovales, granuleuses, incolores. - Selon Oersted, ce champignon appartient aux Urédinées et sa place serait près du Peridermium pini. 


\section{9e GENRE. - TuduldaA. - Pers.}

653. TUB. FRAGIFORMis. Bull. - Alb. de l'Est. - Cefte espéce peu commune croît sur le bois pourri et sur les mousses qui le recouvrent. Je l'ai trouvée plusienrs fois dans les bois humides à St-Amand et à St-Lumier ; elle ressemble à une fraise mûre.

\section{GENRE. - SPUMaRIA. - Pers.}

65̆'. SPUM. ALBA. Bull. - Alb. de l'Est. Croît en Automne, par les temps humides, sur les rameaux tombés, sur les plantes et les feuilles de graminées; d'abord sous forme d'une goutte de crême blanche, puis d'une substance grise, sèche, friable, remplie de spores brunes ou noirâtres. - CCC. - Surtout dans les sapinières.

11 GENRE, - STEMONITIS. - Fries.

65๊. STEM. TYPHOIDES. Bull. - Alb, de l'Est. - Peridium á l'ètat de maturité rassemblés en faisceaux sur les vieux troncs cariés. Lieux humides des bois. - CC. - Automne. Ressemble en miniature à des groupes de têtes de Typha. - St-Amand.

656. STEM. OVATA. Pers. - Alb. de l'Est. Sur le bois pourri de pin sylvestre. - groupé mais chaque individu distinct. - Peridium ovale, brun. - AC. - Aulomne. 
6.\%. STEM. PHYSAROIDES. Alb. et Schu. Alb. de l'Est. - Récolté une seule fois à Ablancourt. - Automne. - RR. - Péridium sphériques.

\section{GENRE. - CARCERA. - Fries.}

658. CARC. SPUMAROIDES. Fries. - Alb. de l'Est. - Cette espéce, dans sa jeunesse ressemble à Spumaria alba; en vieillissant. la membrane extérieure blanche devient rose et les peridium sessiles. serrés l'un contre l'autre s'accusent nettement: leur sommet est alors couvert de la première enveloppe blanchâtre. fugace, et dessous se trouve une seconde enveloppe brune plus résistante. Quand les peridium sont vides de leurs spores brunes, ils ressemblent à des alvéoles d'abeilles. - AutomneHiver. - Dans les sapinières. - St-Amand. - AC.

$13^{\text {e }}$ GENRE. - HADAMAR. - Berk.

659. BADH. UTRICULARE. Berk. - Alb. de l'Est. - Trés belle espéce sur l'écorce du bois de tremble et de bouleau; dans les chantiers à St-Amand. - Peridium en grappes. couleur d'or, puis d'un beau bleu-violacé. - AR. - Automne.

1'e GENRE. - NGIDIRIDICMI. - Grev.

660. ANGIOR. SiNuOsUm. Bull. - Alb. de l'Est.

- Commun sur les feuilles mortes et les détritus de végétaux dans les bois, les jar.. dins. - Automne, Printemps. - CCC. 


\section{5e (iENRE. - mIDERMA. - Pers.}

661. DID. DIFFORME. Fries. - Alb. de l'Est. On rencontre cette espéce toute l'année pendanl les lemps humides sur les feuilles pourries et sur les végétaux mis en tas dans les jardins, les bois. - Partout.

(66\%. DID. GLobosum. Fries. - Alb. de l'Est. Cette espéce enveloppe les feuilles et les brindilles tombées à terre. dans les lieux herbus des plantations de pins et dans les bois ombragès. Elle ressemble à une masse d'œufs d'insectes placés sur une membrane blanche. CCC.

668. DID. CONTEXTUM. Fries - Alb. de l'Est. - Récolté une seule fois sur un tronc de Sabine à Ablancourt. - Peridium irréguliers, sinueux. jaune-soufre pâle: spores granuleuses brunes. - R. - Automne.

66' 1 . DID. TREVELYANI. Fries. - Alb. de l'Est. - Bois de St-Lumier. - Sur rameaux de peuplier. - Peridium jaune-nankin, sessiles, divisés en 4 ou 5 segments rabattus en rosette. - Automne. - R.

665. DID. FLORIFORME. Fries. - Alb. de l'Est. - Bois d'Aulnay, St-Amand. - Automne. - Sur les troncs coupés de peuplier. - Le péridium est disposé comme celui de Did. Trerelyani. mais porté par un pédicule tlexueux, élégant. inséré sur une membrane blanche très visible.- $R$. 


\section{$16^{\mathrm{e}}$ GENRE. - DIDYMUM.}

666. DIDYM. HEMISPHARICUM. Bull. - Alb. de l'Est. - Sur les feuil!es et sur les tiges pourries mises en tas dans les jardins, etc. Printemps, Automne. - Peridium blanc, aplati, pédiculé. - Partout.

667. DIDYM. MELANOPUS. Schrad. - Alb. de l'Est. - Bois couverts de St-Lumier. St-Amand, La Chaussée, etc. - En Automne. - Sur écailles de peuplier. - Peridium blanc, squameux-farineux; pédicelle noir. - AR.

668. DIDYM. MARGINATUM. Fries. - Alb. de l'Est. - J'ai récoltè cette espèce sur les chaumes de blé mis en tas. - Elle est remarquable par son peridium sphérique, blanc-verdâtre : porté par un pédicule couleur de chair ou jaune, strié longitudinalement, terminé au sommet par un bourrelet ou marge crénelée. Spores rondes, chagrinées, brunes. - Automne. - Bois de St-Amand. - RR.

17e GENRE. - LEOCA RPOS. - Pers.

669. LEOC. VERNICOSUS. Pers. - Alb. de l'Est. - Commun en automne dans les sapinières. sur les brins d'herbe et sur la mousse. - Soulanges, Lisse, etc. - Peridium jaune d'or. brunissant, à surface lisse et luisante. Automne. 
18 GENRE. - CRITERIUM. - Fries.

670. CRAter. Pedunculatum. Fries. - Alb, de l'Est. - AC. - Sur brindilles de bois. racines et tiges rampantes encore vertes. Lieux humides des bois et jardins. - St-Amand, St-Lumier.

671. CRATER. Minutum. Fries. - Alb. de l'Est. - Plus petit que le précédent; pédicule plus court, lacuneux. - $\Lambda \mathrm{R}$. - Mêmes localités. - Automne.

6i2. CRATER. LEUCOCEPHALUM. Hoff. - Alb. de l'Est. - Sur feuilles et tiges encore vertes. Bois de St-Lumier. -- Automne. - AR. Pédicule violacé-strié.

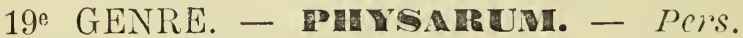

673. PHYS. ALBIPES. Fries. - Alb. de l'Est. Fréquent sur le bois et les feuilles pourries. Peridium et pédicule blancs, rugueux. striés. - AC. - Dans les bois humides, les jardins. - Automne.

67\%. PHYs. COLUmininum. Pers. - Alb. de l'Est. - Sur écailles de reuplier. inséré sur une membrane blanche. - Espéce élégante et très petite. Pédicelle noir. filiforme ; peridium sphérique, violacé-noir ou blenâtre. - AR. Automne. - Soulanges. 
6\%5. PHYS. STRIATUM. Var. Cœsium. Schum. - Alb. de l'Est. - Sur souches et troncs cariés, à fleur de terre. - AC. - St-Amand, Soulanges, etc. - Aulomne.

Var. Aurantium. Fries. - Sur le bois de chêne :pourri ; Forêts de Sermaize, Vanault-lesDames. - AR. - Capillitium blanc, spores brunes.

\section{4e ORdRE. - HYPODERMES. - De By.}

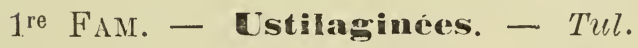

Petites planles parasites, sous-épiderimiques, pulvérulentes, délruisant les tissus.

1er GENRE. - USTILAGO. -- Tul.

6\%6. UST. ANTHERARUM. Tul. - Alb. de l'Est.

- Sur les pelouses et bords des bois de Verzy; les capsules fructifères de silene otites sont envahies par celte espéce à spores brun-pourpre, échinulées - RR. - Automne.

$6 \%$ UST. URCEOLORUM. Tul, - Alb. de l'Est. - Envahit les épillets du Carex glauca. Ces épillets sont couverts de tubercules formés par le développement anormal des ovaires noircis et agglomérés. d'oú s'échappent des amas sphériques de spores noires; les épillets sains 
restent verts et fertiles. - En Eté, sur les pelouses séches des sapinières de Soulanges. AC. - Automne.

(i78. UST. OLIVACEA. Tul. - Alb. de l'Est. Croît sur les épillets de Carex maxima. - Il détruit les ovaires sans déformer sensiblement l'épillet el produit des masses de spores ovoïdes, brun-verdâtre, très petites $\left(0^{\mathrm{m}} 006\right)$ - Bords des fossés et des ruisseaux - St-Lumier. AR. - Automne.

679. UST. RECEPTACULORUM. D. C. - Alb. de l'Est. - Cette espéce altaque les réceptacles du Tragopogon pratense, dont il arrête le développement et détruit la substance. Les spores sont violettes-noirâtres, tuberculeuses et semblent munies de cloisons comme dans celles des Thecaphora. - CC. - Automne. - Dans les prairies : Soulanges, La Chaussée, etc.

680. UST. VAILLANTII. Tul. - Alb. de l'Est.

- Cet Ustilago envahit l'intérieur de la corolle du Muscari comosum. et bien que cette dernière reste intacte en apparence ainsi que l'ovaire, la plante devient stérile. Les spores d'un brun-noirâtre sont elliptiques ou rondes. - AR. - Côte de Gravelines. - Automne.

681. UST. SEGETUM. Pers. - Alb. de l'Est. Croît sur les glumes, les ovaires et les épillets de l'orge et de l'avoine. - Il détruit complètement ces organes, de sorte qu'il ne reste plus que le rachis couvert de spores noires, rondes ou ovales. - CCC dans les moissons. 
682. USt. LONGISSIMA. Sou. - Alb. de l'Est. Croît sur les feuilles de Poa aquatica en formant de longues lignes brunes ; l'épiderme blanc d'abord, brunit, se rompt et laisse échapper des spores rondes trés petites $\left(0^{\mathrm{m}} 003\right)$ Automne. - Le long des ruisseaux. - CCC. 683. UST. DESTRUENS. Duby. - Alb. de l'Est. - Envahit les épillets de Millet qu'il convertit en une poussière noire, masse de spores brunes, rondes ou ovales. - AC. dans les jardins. - Temps humides d'Automne. - St-Amand. prẻs du moulin Husson.

68'. UST. MAYDIS. Tul. - Alb. de l'Est. Cet Ustilago attaque les tiges et les épis de Maïs. - Il forme de gros tubercules blancsrosés qui égalent la grosseur d'une noix ou d'un œuf de pigeon. L'enveloppe se crève et laisse échapper des myriades de spores brunes échinulees, entremêlées de lambeaux filamenteux - Champs de maïs. - Automne. - StAmand. - RR.

\section{‘e GENRE。 - THLE'TIA. - Tul.}

685. TILL. CARIES. Tul. - Alb. de l'Est. Le Tilletia, carie du blé, attaque les ovaires du blé sans en changer la forme. - Le grain noircit et en le triturant on fait sortir des spores rondes; brunes, tuberculeuses mêlèes à quelques filaments, répandant une odeur de marée. - CC. dans certaines années, moissons.

- Partout. 
3e GENRE. - CHOCYSTIS. - Lév.

686. UROCYST. POMPHOLIGODES. Lev. - Alb. de l'Est. - Cette espéce croît sur les feuilles des Renonculacées, notamment de la Ficaire Elle forme des bulles bleuâtres recouvertes par l'épiderme qui se déchire et met à nu des groupes distincts de spores agglutinèes (15 à 20) : mêlés à quelques filaments fugaces. Spores ovales, brunes. - AR. - Printemps. Bords des ruisseaux - St- $\lambda$ mand.

68\%. UROCYST. COLCHICI. Str. - Alb. de l'Est. - Croît sur les feuilles languissantes d'Ornithogalum umbellatum et forme des phyctènes bleuâtres, elliptiques qui renferment des spores semblables á celles de l'espéce précédente et affectant la même disposition en glomérules. - Bois de St-Amand à St-Lumier. Au mois de mai. - AR. 688. UROCYST. VIOLAE. Berk. - Alb. de l'Est. - J'ai récolté cette espéce sur Viola odorata ; elle croît sur les tiges, les stolons et les pédoncules fructiféres; le renflement des phlycténes est relativement plus considérable que dans les autres espèces, mais les spores et les globules sont absolument semblables.

$4^{\mathrm{e}}$ GENRE. - THICCA PHOMEA. - Tul.

689. THEC. DELASTRINA. Tul. $-\Lambda \mathrm{lb}$. de l'Est. - Celte espéce envahit au printemps les capsules de Véronica agrestis. La capsule 
prend une teinte bleuâtre due à la présence des spores bleues. verruqueuses du Thecaphora. - Champs de blé ; St-Amand, Lisse. $-\mathrm{AC}$.

690. THEC. VERONICA HEDERAFOLIA. Tul. Alb. de l'Est. - Ce Thecaphora attaque les capsules de Ver. hederafolia. - Il se comporte comme le précédent, seulement ses spores sont lisses et non verruqueuses. - Printemps. mêmes localités. - AC.

\section{2e FAM, - Urédinées. - Tul.}

Plantes parasites, sous-épidermiques, ne détruisant pas les tissus.

1er GENRE. - PRERTERTMUM. - Tul.

691 PER. PINI. Pers. - Alb. de l'Est. - Renflements vésiculeux d'un beau jaune-d'or: disséminés sur les troncs et les branches de jeunes Pins sylvestres, remplis de spores rondes de couleur jaune-orangée, superposées en manière de chapelet. On appelle vulgairement cette espéce Balai des Sorcières. Sa présence est un fléau qui détruit des plantations entiéres. Automne. - Soulanges. Bassu, etc. - $\mathrm{AC}$. 
2e GENRE, -- ENDOPUYMLUM. - Lév.

692. ENDOPH. PERSONII. Lév. - Aib. de l'Est. - Cette espéce croît sur les feuilles de Serum sempervivens. Le tissu du Sedum forme un pseudo-péridium qui contient les stylospores placées en chapelet comme celles des Ecidium. L'épiderme qui recouvre la pustule se rompt rarement. Sur la Joubarbe des toits à Soulanges. Mars. - RR.

$3^{e}$ GENRE. - Coledsporerum. - Lév.

693. Coleosp. Miniatum. Pers. - Alb. de l'Est. - Croît sur les tiges, pédoncules et feuilles encore verts de Rosa canina et forme des pustules d'un beau rouge vif, pulvérulentes , oblongues. Les spores en massues allongées , bi-triloculaires, jaune-d'or, sont entremêlèes de stylospores rondes, échinulées. - AC. - Dans les haies et les jardins. - Été-Automne.

69'. COLEOSP. SONCHI. Tul. - Alb. de l'Est. Sur les feuilles de Sonchus arvensis. Spores jaunes. Stylospores rondes, échinulées, de même couleur. - CC. - Champs. - Été-Automne. Partout.

695. COlEOSP. TUSSilaginis. Lév. - Alb. de l'Est. - Sur les feuilles de Trussilago farfara. - Eté, Automne. - Champs, bords des routes. -.. CCC. 
696. COLEOSP. CAMPANULACEARUM. Fries. - Alb. de l'Est. - Envahit entièrement la face inférieure de diverses campanules, notamment de Campanula rapunculö̈des. - ÉtéAutomne. - CCC.

69\%. COLEOSP. SENECIONIS. Schlecht. - Alb. de l'Est. - Sur les feuilles de Senecio vulgaris. CCC. - Jardins, champs. - Automne. - Partont.

698. COLEOSP. RHINANTHACEARUM $\quad$ D. C. - Alb. de l'Est. - Commun sur les feuilles et les tiges de Melampyrum. Euphrasia. Rhinanthus. - Autumne. - Champs, bords des routes. - Partout.

699. COLEOSP. OCHRACEUM. Bon. - Alb. de l'Est. - Sur les feuilles disposées en rosette à la base des tiges d'Agrimonia eupatoria. Automne. - Broussailles, bords des chemins. - CCC.

$4^{\mathrm{e}}$ GENRE. - MELAMPSOMA. - Tul.

700. MEL. EUPHORBIÆ. Tul. - Alb. de l'Est.

- Petites taches irrégulières, d'un noir luisant, sous les feuilles et sur les tiges des Euphortia Esula, Cyparissia. Helioscopia, composées de Téleutospores brunes en forme de graines de Tournesol. l'Uredo Euphorbice est son état stylosporifère.

701. MEL. LINI. Tul. - Alb. de l'Est. - Fréquent sur les tiges et les feuilles de Limum. 
calharlicum. - Sur les pelouses séches, sur le bord des routes en automne. - AC. - Stylospores, Uredo Lini.

702. MEL. SALICINA. Tul. - Alb. de l'Est. Recouvre souvent les feuilles languissantes de Salix caprea en automne, sous forme de petites taches rousses puis noires, proéminentes. Styl. Uredo Caproarum.

703. MEL. POPULINA. Tul. - Alb. de l'Est.

- Commun sur les feuilles de Populus nigra et Pyramialis tombées. - Automne. - Styl. Uredo longicapsula.

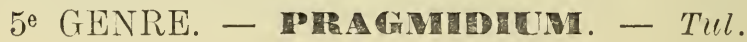

70'. PHRAGIM. BREVIPES. Fuckel. - Alb. de l'Est. - Cette espèce croît sous les feuilles de Polerium Sanguisorba,dans le voisinage de l'Uredo potentillarum ou sur le coussinet qui lui donne asile. Les téleutospores sont courtes, obtuses au sommet. munies seulement de 2 à 3 cloisons et d'un court pédicelle. - Styl. Uredo potentillarum. - AC. - En Automne. - Champs, côteaux herbus.

705. PHRAGM. INCRASSATUM. Tul. - Alb. de l'Est. - Croît sous les feuilles des Rubus en compagnie de l'Uredo ruborum. - Styl. Téleulospores cylindriques 5.6 cloisons, brunes. - CCC.

706. PHRAGM. ASPERUM. Wallr. - Alb. de l'Est. - Envahit pendant l'hiver la face inférieure des fevilles de Rubus fruticosus. Elle sc 
distingue des autres Phragmidium par sa surface verruqueuse, par ses trois cloisons et son pédicelle assez long, renflé brusquement à la base. - Dans les bois et le long des haies. $\mathrm{AC}$.

707. PHRAG. EFFUSUM. Fuckel. - Alb. de l'Est. Croît à la face inférieure des feuilles de Rubus coesius, dans les bois de Sermaize. - AR. Styl. Uredo gyrosum plus fréquent.

708. PHRAGM. ROSARUM. Fuckel. - Alb. de l'Est. - Trés fréquent après l'été sous les feuilles des rosiers dans les jardins. Sa Téleutospore est allongée. brune, à 6 cloisons, verruqueuse et munie d'un mucron conique au sommet; le pédicelle est de même longueur et renflé à la base. - Styl. Uredo Rosa. Partout.

709. PHRAG. SPARSUM. Ch. R. - Alb. de l'Est.

- Cette espéce croît en groupes peu nombreux sur les tiges de Spiroei Ulmaria et sur les chaumes d'Agrostis vulgaris; les individus franchement séparés l'un de l'autre ne sont pas issus d'un strôme commun comme chez Ja plupart des autres Phragmidium. Les sporidies sont cylindriques, apiculées, brunes, munies de 5 à 6 cloisons et de très fines aspérités; les pédicelles sont allongés, incolores et cylindriques. - Récolté dans les bois entre St-Amand et Aulnay-l'Aître. - AR. 
6e GENRE. - NENDDOCHES. - Fuckel.

710. XEN. CARBONARIUS. Fuckel. - Alb. de l'Est. - Le Xenodochus possède des Téleutospores et des Stylospores comme toutes ies Puccinées. La disposition remarquable des Téleustopores a motivé la création d'un Genre à part ; elle consiste dans la présence d'une vingtaine de cloisons ou segments ressemblant à des grains de Maïs, superposés et maintenus par l'épispore unique servant de capsule générale. Ces segments ne se désagrégent pas comme dans les Torula. - RRR. - Sous les feuilles de Sanguisorba déjá envahies par un Uredo qui en est la Stylospore. - Sapinières de Bayarne près de Soulanges. - Automne.

ye GENRE. - TRIPHRAGMIDIUM. - Tul.

711. TRIPH. ULMARIE. Tul. - Alb. de l'Est. - Cette espéce croît á la fin de l'été sur les feuilles de Spirca Ulmaria, à la face inférieure. Les Téleutospores sont orunes, rondes ou ovales, tuberculeuses, divisées en trois et plus rarement quatre loges. - Styl. Uredo Ulmaria. C. - Bords des ruisseaux.

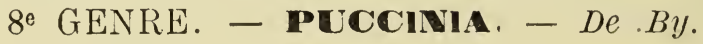

712. PUCC. ANEMONes. Pers. - Alb. de l'Est.

- Hypophylle sur Anemone nemorosa. Pustules brun-pâle, éparses. Téleutospores à 
stipes courts, tuberculeuses, brunes. - R. Etė. - Forêt de Charmont, Vanault-les-Dames. 113. PUCC. CALThe. Duby. - Alb. de l'Est.Iypophylle sur Caltha palustris. Pustules petites. éparses, rousses. Téleutospores brunes ovales; loges resserrées al milieu. - CC. - Automne. - Bords des ruisseaux ; St-Amand. - Styl. Uiedo Calthce.

71'. PUCC. PRUNORUM. Link. - Alb. de l'Est, - IIypophylle sur Prunus domestica. Groupes bruns, épars. Téleustopore courte, échinulée, étranglée au milieu. - CC. - Jardins. - Styl. Uredo Prunorum.

715. PUCC. LYCHNIDEARUM. Link. - Alb. de l'Est. - Hypophylle sur Dianthus sinensis. Dans les jardins. Pustules brunes sur une tache jaune-pâle, disposées en cercles concentriques. Téleutospores brun-clair, stipe court. - CC. Rencontré aussi sur Agrostema Githago.

Var. Behenis. Link. - IIypophylle sur Silene inftrte sans décoloration de la feuille. - CC. - Automne. - Champs crayeux. - Styl. Uredo Lychnidearum.

716. PUCC. FALCARIE. Fuckel. - Alb. de l'Est. - Hypophylle sur Buplevrum falcarice. Pustules éparses, brunes. Téleutospores courtes. á court pédicelle. - Styl. Uredo falcarice, habitant les mêmes pustules. -- AR. - StAmand. - Automne.

717. PUCC. AEGOPODII. Link. - Alb. de l'Est.

- Hypophylle sur Egopodium podagraria. Teleu- 
tospore ovale, brune: stipe trés court. - R - liais commune à st- 1 mand. -Bois humides.-Automne.

ils. PUCC. TANACETI. D. C. - Alb. de l'Est. IIypophylle sur Trnacetum rulyare. Teleutospore elliptique, rouge brun. stipe trés long.-Styl. Tiedo Tanareti. - Bords de la Marne. - AC. - Automne.

719. PUCC. CHONDRILLA. Fucke?. - Alb. de l'Est. - (Jroît sur les feuilles et les tiges de Lartuca perennis. Pustules noires. arrondies. Téleutospores brunes à court pédicelle. - Styl. Uredo aichoracearum - Champs de terre légère. - AC. - Juillet.

\%). PUCC. OBTEGENS. Tul. - Alb. de l'Est. - Hypophylle sur Cirsium arrense mélangé avec la Styl. Uredo Suareolens. Téleutospore ovale. granuleuse. Stipe court. - CC. - Partout. - Été-Automne.

i21. PUCC. BARDANAE. Corda. - Alb. de l'Est. - Iypophylle sur Lappa tomentosa. Téleutospore brune, tuberculeuse, à stipe court. - Styl. Uredo Tunaceti. - C. - Été-Automne. - StAmand, Francheville.

7:2. PUCC. CONII. Fuckel. - Alb. de l'Est. Commune sur les tiges de Conium maculatum. Eile forme des boursouflures de l'épiderme gris-noir allongèes, se déchirant rarement. Téleutospores brunes, à court pédicelle. - Automne. - Partout.

72:3. PUCC. CIRSII. Fuckel. - Mlb. de l'Est. Cette Puccinie rare est hypophylle sur Cir- 
sium oleraceum; elle forme des tubercules bruns-rougeâtres, épars, aplatis en disques. Tẻleutospores rougeâtres, vues en masse et d'un jaune doré sous la lentille. Pédicelle égal aux deux loges. - RR. - Bords du Fion.

72'. PUCC. TRAGOPOGONIS. Fuckel. - Alb. de I'Est. - Croît sous les feuilles et sur les tiges de Salsifis. en Juillet, accompagné de l'Ecidium Tragopogonis. Téleutospores brunes. presque rondes, à stipe très court. - Bois herbus et prairies. - CC. A la fin de l'Été. 725. PUCC. THESII. Chaill. - Alb. de l'Est. Hypophylle et sur les tiges de Thesium humifusum. Pustules noires, allongées. Téleutospores brunes, ovales ou pyriformes; stipe long. Collines crayeuses de Soulanges; Aulnay. AR. - Automne.

726. PUCC. BUXI. D. C. - Alb. de l'Est. Hypophylle sur Buxus sempervivens. Pustules discoïdes brunes. Téleutospores allongées, jaunemiel foncé. Pédicelle trés long. - RR. Jardins. - Printemps.

727. PUCC. POLYGONORUM. Link. - Alb. de l'Est. - Hypophylle sur Polygonum convolvulus et hydropiper. Pustules brunes, longtemps recouverts par l'épiderme. Téleustopores brunes, long pédicelle. - Styl. Uredo polygonorum. CC. - Dans les champs aprés moissons, et sur le bord des eaux oú croît Poly. hydropiper.

728. PUCC. RUMICIS. Tul. - Alb. de l'Est. Hypo. et épiphylle sur Rumex acetosa en 
Automne. Téleutospores brunes, courtes, elliptiques, pédicelle presque rudimentaire. - Slyl. Uredo Rumicum. - CC.

7\%9. PUCC. CIRCEA. Pers. - Alb. de l'Est. IIypophylle sur Circea lutetiana. Tubercules rouge-pourpre, disséminés. T'éleutospores allongées, brun-rougeâtre pâle; stipe d'égale grandeur. - CC. - Été. - Bois de St-Amand, Sermaize.

750. PUCC. EPILOBII. D. C. - Alb. de l'Est. Hypophylle sur Epilobium hirsutum; pustules en cercles concentriques; Tèleutospores brunes, oblongues, stipe plus ou moins long. - Styl. Uredo epilobii. - AC. - Bords des eaux. St-Amand, Lisse, etc. - Automne.

731. PUCC. ASParagi. D. C. - Alb. de l'Est. - Sur tiges et pétioles d'Asparagus officinalis; pustules oblongues, confluentes, brunes; Téleutospores allongées, brun-clair ; très long pédicelle. Styl. Uredo Asparagi. - Hiver, Automne. - CC. - Dans les jardins.

732. PUCC. VAILlANTIE. Link. - Alb. de l'Est. - Hypophylle sur raillantia cruciata. Pustules brun-clair; Téleutospores claviformes, de même couleur; stipe très long. - Été , Automne. - CC. - Partout.

733. PUCC. STELlaria. Duby. - Alb. de l'Est. - Hypophylle sur Alsine media; groupe de pustules en cercles brun-roux. Teleustopores oblongues, rousses. - AR. - Bois de StLumier. - Été. 
734. PUCC. TEUCRII. Fuckel. - Alb. de l'Est. - Hypophylle sur Teucrium chamcedrys. Pustules tuberculeuses, brun-rougeâtre ; Tèleutospores brun-rougeâtre, stipe long. - CC. Automne. - Lieux arides, bords des routes.

735. PUCC. GLECHOM 2 . Pers. - Alb. de l'Est. Hypophylle sur Glechoma hederacea; pustules en cercles brun-rougeâtre. Téleutospores de même couleur; stipe long. - CCC. - Automne. - Bois, jardins.

736. PUCC. RIBIS. D. C. - Alb. de l'Est. - Epiphylle sur Ribes rubrum; pustules réunies, formant des taches rondes, brunes,éparses. Teleutospores brun-rougeâtre;stipe plus court. - RR. Récolté une seule fois. - Jardins. - St-Amand. 73\%. PUCC. MENThAe. Pers. - Alb. de l'Est. - Hypophylle sur Mentha sylvestris; Téleutospores brunes, courtes, tuberculeuses. - Pédicelle court. - Styl. Uredo Menthce. - CC. Champs et jardins. - Automne.

738. PUCC. CALAMiNTHeE. Fuckel. - Alb. de l'Est. - Hypophylle sur Clinopodium vulgare. - Tèleutospore elliptique, verruqueuse, jaune d'or foncé. - Styl. Uredo Clinopodii. - Cette espéce donne asile aux périthèces de Darluca filum. - CC.

739. PUCC. Gentian e. Link. - Alb. de l'Est. - Hypophylle sur Gentiana cruciata. - Collines boisées de pins sylvestres. - St-Amand . Soulanges. - AC. - Téleutospore rondebrune, stipe court. 
740. PUCC. DiANTHI, PUCC. AGRostemma, PUCC. LYCHNIDIS DIOICE, que j'ai fréquemment récoltés dans nos localités, appartiennent à Pucc. Lychnidearum, cité plus haut.

i't. PUCC. Malvacearum. Mont. - Alb. de l'Est. - Hypophylle sur toutes les Mauves. Téleutospores allongées, brun-clair ; pédicelle très long. Cette espèce est aujourd'hui très commune; quand je l'ai récoltée pour la première fois à St-Amand en 18\%2, elle n'avait pas encore été signalée en Europe; la priorité de ma découverte a été confirmée par la Société botanique de France. - Elle cause de grands ravages dans les jardins.

712 . PUCC. LiliACEARUM. Duby. - Alb. de l'Est. - Hypo. et Epiphylle sur Ornithogalum pyreneaicum. Téleutospores ovoïdes, oblongues, brun-pâle; stipe très court. - Automne. Bois de St-Amand. - R.

743. PUCC. VINCAE. Cast. - Alb. de l'Est. Hypophylle sur Vinca major encore en pleine vigueur; les Téleutospores sont brunes, courtes et à peine pédicellées. - Styl. Uredo Vinca. St-Amand. - Automne. - AR.

i'́t. PUCC. CALCITRAPA. D. C. - Alb. de l'Est. - Hypophylle sur Centaurea calcitrapa. Téleutospores elliptiques, à peine pédicellées, brunes. - Styl. Uredo Calcitrapes. - Champs après les moissons. - Automne. - AR. - La même espèce sous le nom de Pucc. Centaurea; croît aussi sur Centaurea Jacea. - St-Amand. 
“'5. PUCC. CHAROPHYLli. Purt. - Alb. de l'Est. - Hypophylle sur Chcerophyllum temulum. - CC. - Automne. - Dans les bois et les jardins. - Téleutospores brunes, elliptiques; stipe court.

746. PUCC. ARUNDINACEA. Hedw. - Alb. de l'Est. - Envahit les feuilles d'Arundo phragmites encore vertes; elle forme des stries brunes, longitudinales, visibles des deux côtés de la feuille. Téleutospores oblongues: brunes, stipe de même dimension. - CC. - Bords des ruisseaux. - Automne. - Partout.

i'i. PUCC. GRAminis. Pers. -- Alb. de l'Est. - Epiphylle sur les graminées en général, principalement sur le blé et le seigle; les pustules longıtudinales contiennent des Téleutospores oblongues, amincies aux deux bouts, brunes, munies d'un pédicelle d'égale longueur. - Styl. Uredo Linearis; état hymenifère Ecidium berberidis. - CC. - Étè.

718. PUCC. STRIAFFormis. West. - Alb. de l'Est. - Sur les gaînes de Phalaris arundinacea. Sries brunes ; Téleutospores claviformes, brunes; stipe très court. - AC. - Bords des ruisseaux. - Automne.

749. PUCC. CARICIS. D. C. - Alb. de l'Est. Hypo. et épiphylle sur Carex acuta. Téleutospores brunes, claviformes; pédicelle égal. - Bois marécageux. - St-Amand, St-Lumier. - CCC. 750. PUCC. LUZULAE. Lob. - Alb. de l'Est. Sur feuilles de Luzula pilosa. Téleutospores en 
massue : brun-clair; pédicelle égal. - Styl. ronde, Uredo Luzula.

751. PUCC. Coronata. Corda. - Alb. de l'Est.

- Cette espéce dont certains auteurs ont fait le Genre Solenodonta, croît sur les feuilles et sur les chaumes de l'avoine; Téleutospores d'un brun-fauve , claviformes ; la loge supérieure est ornée, à son sommet de dents radiées en forme de couronne. - CC. - Automne. Sur les rives des champs qui ont été moissonnés et dans leur voisinage.

9c GENRE. - CROMYCES. - $D e B y$.

752. UROM. FICARIA. Fuckel. - Alb. de l'Est. - Hypophylle et sur les pédoncules de Ranun culus ficuria. Groupes brunâtres, tuberculeax. décolorant les tissus voisins. Téleutospores brunclair, sub-ovoïdes, anguleuses; pédicelle court. - CC. - A la fin du Printemps, dans les bois humides. - St-Amand, La Chaussée, etc. Etat hymenifère Ecidium ranunculacearum.

753. UROM. PHASEOLORUM. Euckel. - Alb. de l'Est. - Hypophylle sur Phaseolus vulgaris. Téleutospore brun-clair. - AC. - En Automne - Jardins. - St-Lumier, St-Amand, etc. - Etat. hym. Ecidium Phaseolorum; plus rare.

754. UROM. FABæ. De By. - Alb. del'Est. - Iypophylle et sur les gousses et tiges de Vicia faba. Pustules couleur de roujlle; Téleutospores rousses. long pédicelle. - Styl. Uredo fabœ. - CC. Jardins, champs, prés des villages. - Automne. 
155. UROM. PISI. De By. - Alb. de l'Est. Hypophylle sur Pisum sativum. Téleutospores brun-roussâtre, ovoïdes cunéiformes. Pédicelle court. - CC. - Jardins. - Été, Automne. Styl. Uredo leguminosarum.

Var. Orobi. Fuckel. - Hypophylle sur Orobus tuberosus. Mêmes caractères que le type. AR. - Forêt d'Epernay.

756. UROM. LABURNI. Fuckel. - Alb. de l'Est. - Hypophylle sur Cytisus laburnum. Téleutospores brun-rougeâtre, tuberculeuses, à pédicelle très court, en compagnie de l'Uredo laburni dans les mèmes pustules. - Commencement de l'hiver. - Jardins.

75\%. UROM. BET E. Kü̈n. - Alb. de l'Est. Epi. et hypophylle sur la Betterave. Champs. - Automne. - Téleutospores ovoïdes, rousses ; stipe court. - Styl. Uredo Betce.

758. UROM. POLYGONI. Fuckel. - Alb. de l'Est. Hypophylle et sur tiges de Polygonum aviculare: Téleutospores rondes ou anguleuses, brunes, stipe long. - Styl. Uredo polygoni avicularioe. - AC. - Champs, aprés les moissons.

759. UROM. SCUTEllatA. Pers. - Alb. de l'Est. - Hypophylle sur Euphorbia Cyparissias. Les feuilles attaquées sépaississent et se déforment. Téleutospores rondes, tuberculeuses ; pédicelle court. - CC. - Au printemps. - Sur les collines séches de la Champagne. - Etat hym. Ecidium Euphorbice. 
ilo. URom. excAvata. Lév. - Alb. de l'Est. - Hypophylle et sur les tiges d'Euphorbia dulcis. - Champs des terrains crétacés. - StAmand. - AR. - Téleutospores brunes, ovoïdes: stipe court. - Cette espèce ne déforme pas l'Eupborbe comme la précèdente.

761. UROM. GERANII. Otth. -- Alb. de l'Est. Hypophylle sur Geranium dissectum et molle. Téleutospores ovoïdes cunéiformes, trunes. Styl. Uredo Gerani. - Coteaux d'Epernay. AR. - Automne. - Champs voisins de la forèt.

762. UROM. AMbiguA. Tul. - Alb. de l'Est.

- Hypophylle et sur la tige d'Allium sphorrocephalum. - AR. - Bois de St-Amand. Tèleutospores coniques, anguleuses, pédicelle de moyenne grandeur. - Styl. Uredo limbata. 763. UROM . FLOSCULOSORUM Kickx. Alb. de l'Est. - Groupes hypo. et épiphylles sur Taraxacum dens leonis, brun-foncé. tachant la feuille d'une auréole jaune. Téleutospores brunes, ovoïdes-anguleuses. - Styl. Uriedo flosculosorum.

10 GENRE. - UIREDO. - Pers.

76'. URED. HYPERICORUM. D.C. - Alb. de l'Est. - Hypophylle et sur les tiges d'hypericum pulchrum. Pustules éparses ; la face supérieure de la feuille correspondante présente des taches rougeâtres-violacées. Stylospores orangées. 
i65. URED. ARI. Desm. - Alb. de l'Est. -

Hypophylle sur Arum maculatum. Stylospores rondes, tuberculeuses, jaunes. - AC. au Printemps mais plus rare que l'Cecidium. - Dans les bois de St-Amand. St-Lumier; etc.

766. URED. LINI. D.C. - Alb. de l'Est. -

Epiphylle et sur les tiges de Linum catharticum Stylospores rondes, jaunes, tuberculeuses avec stipe court, entourées de paraphyses. Téleutospores Melampsora lini. - CC. - Automne. Collines sèches.

\%6\% URED. EPILOBII. D.C. - Alb. de l'Est. Hypophylle sur Epilobium hirsutum. Pustules brunes, disposées en cercle. Stylospores brun-clair. Teleut.Puccinia epilobii. - AR. - bords du Fion, 768. URED. LONGICAPSULA. D.C. - Alb. de

l'Est. - Hypophylle sur Populus alba. Stylospores jaune-d'or, de forme oblongue. Téleut. Melampsora populina. - CCC. - A la fin de l'Eté. - Partout.

769. URED. CAPREARUM. D.C. - Alb. de l'Est. - Hypophylle sur Salix caprace. Stylospores rondes, tuberculeuses, entourées de cys $^{-}$ tides. - Téleut. Melampsora salicina. - Les pustules de cet Uredo donnent quelquefois asile au Darluca vagans. - CC. - Automne.

7\%0. URED. EUPHORBIEE. Pers. - Alb. de l'Est. - Hypophylle sur Euphorbia helioscopia. Stylospores rondes jaune-d'or. - Téleut. Melampsora Euphorbice. CC. - Automne. Jardins, vergers. - Partout. 
771. URED. PRUNORUM. Fuckel. - Alb. de l'Est. - Hypophylle sur Prunus domestica ; stylospores brunes, rondes. irréguliéres, tuberculeuses, munies d'un très court pédicelle (an Uromyces?) - Téleutospores Puccinia prunoruin. - CC. - Automne. - Jardins.

772. URED. POTERII. Rbh. - Alb. de l'Est. Hypophylle sur Poterium sanguisorba. Stylospores rondes, jaunes, entourées de cystides.

- Tèleut. Phragmidium mucronatum. - CCC. - Champs. - Partout.

7\%3. URED. ROSA. Pers. - Alb. de l'Est. Hypophylle sur Rosa canina et autres. Stylospores rondes, jaune-pâle, tuberculeuses entourées de cystides. - Téleut. Phragmidium rosarum. - CCC. - Automne.

794. URED. GYROSA. Reb. - Alb. de l'Est. Hypophylle, disposé en cercle et décolorant la feuille. Stylospores arrondies, jaunâtres. portées par un stipe plus ou moins long. - Téleutospores Phragmidium effusum. - AR. - forêts de Sermaize, Reims.

795. URED. RUBORUM. D.C. - Alb. de l'Est. Hypophylle sur Rubus fruticosus. Stylospores arrondies, ponctuées, jaune-pâle, entourées de cystides. - Téleut. Phragmidium incrassatum. - C. - Automne, hivers doux. - Partout.

\%\%. URED. VINCETOXICI. D.C. - Alb. de l'Est. - Hypophylle sur Cynanchum vincetoxicum. stylospores rondes, jaune-pâle, habitant les mêmes pustules que les téleutospores, Cronar- 
tium asclepiadeum. - Automne. - AC. Collines herbues de Soulanges.

77\%. URED. PAONIÆE. Tul. - Alb. de l'Est. Hypophylle sur Poenia officinalis ; stylospores rondes, jaunes, à épispore ponctué-épineux, mélangées avec les téleutospores, Cronartium Pconice. - Fin de l'Eté. - AR. - Jardins.

778. URED. CALTHA. Rabh. - Alb. de l'Est. Hypophylle sur Caltha palustris. Pustules rousses, èparses, peu nombreuses. Stylospores rondes, chagrinées, brun-pâle. - Téleut. Puccinic caltho. - Bords des ruisseaux ou dans les hois marécageux. - AC. - Eté. - StAmand, St-Lumier.

799. URED. FICARIE. Alb. et Schw. - Alb. de l'Est. - Voir Uromyces Ficarice.

780. URED. VALERIANAE. D.C. - Alb. de l'Est.

- Hypophylle sur Valeriana officinalis ; pustules rousses, souvent disposées en cercles ; stylospores de même couleur, rondes-ovales. Téleut. Puccinia Valeriance. - CC. en Eté.- Bois humides, prés des ruisseaux.

781. URED. GERANII. Lév. - Alb. de l'Est. Hypophylle sur Geranium dissectum et molle. Stylospore brune, ronde, tuberculeuse. - AR. - Epernay. - Téleut. Uromyces Geranii.

782. URED. POLYGONORUM. D.C. - Alb. de l'Est. - Hypophylle sur Polygonum convolvulus ; Pustules brunes, saillantes et allongées sur les tiges; stylospores jaune foncé ou 
café au lait ; rondes. - Téleut. Uromyces polygonorum. - CC. dans les champs après les moissons.

783. URED. RUMICUM. D.C. - Alb. de l'Est. - Hypo. et epiphylle sur Rumex acetosa, crispus, etc ; stylospores rondes, tuberculeuses, brun-clair, habitant les mêmes pustules que les Téleutospores Puccinia Rumicum. - CC. Automne. - Bois et jardins.

78'. URED. PISI. D.C. - Alb. de l'Est. - Hypophylle sur Pisum sativum. Pustules éparses brunes, contenant les stylospores brun-pâle, rondes, finement échinulées, mélangées avec les Téleut. Uromyces Pisi. - CC. - Automne. - Jardins.

785. URED. LABURNI. D.C. - Alb. de l'Est.Hypophylle sur Cytisus laburnum. Pustules réunies formant de larges taches rousses contenant chacune des stylospores rondes, rouxclair et les Téleut. Uromyces laburni. - CC. avant la chûte des feuilles. - Jardins. - Partout.

786. URED. FABÆ. D.C. - Alb. de l'Est. Hypophylle et sur tiges de Vicia faba ; pustules rouillées; stylospores rondes, tuberculeuses. - Téleut. Uromyces fabo. - CC. - en Automne.

787. URED. FALCARIE. Fuckel. - Alb. de l'Est. - Hypophylle et sur tiges de Buplevrum falcarioe. Stylospores rondes, brunes mélangées avec les Téleut. Pucrinio falcaria. 
AR. - Automne. - L'Ecidium falcarice. état hym. est au contraire trés commun. Lieux herbus des coteaux secs. - Partout. 788. URED. VINCAE. D. C. - Alb. de l'kist. Hypophylle sur Vinca maior; pustules brunes. contenant les stylospores brun-clair. rondes, ètat Téleut. Puccinia vincer. - R. - St-Amand, soulanges. - Automne. - Les tiges des feuilles attaquées meurent dalls l'annèe.

789 URED. ULMARIAE. Mart. - Alb. de l'Est.

- Hypophylle sur Spircea ulmarice; stylospores rondes, jaunâtres, ou rousses mélangées aux Tèleut. Thriphragmidium Umarice. - RR. Automne. - St-Amand.

790. URED. BET E. Per's. - Alb. de l'Est. Epi. et hypophylle sur la betterare; stylospores rondes, granuleuses, rousses, dans les mêmes pustules que celles de la Téleut. Uromyces Betre. - AC. - Été et Automne, aprés les pluies. - Jardins et champs cultivés. - Partout. 791. UREd. SCUTEllata. Pers. - Aib. de l'Est. - IIypophylle sur Euphorbia cyparissires. Stylospores rondes, brunes, tuberculeuses ; sa présence déforme les teuilles. - CC. - Au printemps. - Le long des chemins herbus.

792. URED. CALAMINTHA. Fuckel. - Alb. de l'Est. - Hypophyl!e sur Clinopodirm vulgare. Stylospores rondes, ponctuées, roux très pâle, mélangées avec les Styl. Puccinia Calamintha. - Automne. - AR. - Bords des haies, voisinage des jardins. - St-Amanr, etc. 
79:3. URED. MENTHAE. Pers.- Alt. de l'list. - Iypophylle sur Mentha sylrestris; pustules disposees en cercles. Stylospores brun-clair mélangèes aux Tèleut. Purciniu Menthce. C(. - Le long des haies, autour des habitations. - Partout.

i!'. URED. LIMBATA. Khb. - Alb. de l'Est. Sur tiges d'Allium sphcerocephahure, pustules d'un beau jaune, Jordées par l’épiderme. Stylospores blanches, globuleuses, granulèes . jaunâtres au centre. - Téleut. Puccinia Alliorum. - AR. Automne et Étè. - St-Amand. - Champs, bois i95. URED. VIOLARUM. D. C. - Alb. de l'Est. Hypophylle sur Tioia canina et sylrestris. Stylospores brun-clair, rondes, granuleuses. Téleut. Puccinia violarum. - AC. - Bois de St-Amand à st-Lumier. - Été.

796. URED. CYNAPII. D. C. - Alb. de l'Est. - Iypcphylle sur Ethusa cynapium. Stylospores rousses, rondes, brun-clair. - Téleut. Puccinia athusa. - AR. - Jardins, champs fumés. - Automne.

797. URED. CONDRILLEE. Corda, - Alb. de l'Est. - Hypophylle sur Lactuca perennis. Stylospores rondes, brun-clair: verruqueuses. - Téleut. Puccinia chondrille. - AR. - Champs d'aroine. dans les terrains crayeux. - St-Amand. Solllanges. - Été.

798. URED. FLOSCULOSORUM. All. et Schuc. - Alb. de l'Est. - Sur Taraxacum. - Voir Uromules flosculosorum. 
799. URED. CENTAUREA. Fuckel. - Alb. de l'Est. - Epi. et hypophylle sur Centaurea calcitrapa et Centaurea jacea. Stylospores brunes, rondes, granuleuses ou lisses. - Téleut. Puccinia calcitrapce. - AC. - Champs, prairies. bords des chemins après les moissons.

800. URED. BARDANÆ. Fuckel. - Alb. de l'Est. - Hypophylle sur Lappa communis. Stylospores rondes, brun-clair, ponctuées, mélangées aux Téleut. Puccinia bardance. - AC. Automne. - Bords des chemins, sur les décombres autour des jardins, $\in$ tc.

801. URED. TANACETI. Str. - Alb. de l'Est.

- Hypophylle sur Tanacetum vulgare. Stylospores brunes, globuleuses. - Téleut. Puccinia tanaceti. - AR. - Bords de la Marne et sur les graviers destinés à l'entretien des routes.

802. URED. LAPSANAE. Fuckel. - Alb. de l'Est.

- Hypophylle sur Lapsana communis. - CC.

- Étè. Automne. - Téleut. Puccinia Lapsana. 803. URED. PUSTUlatA. Pers. - Alb. de l'Est.

- Hypophylle sur Epilobium roseum. Pustules jaunes se rompant rarement; stylospores rondes. jaune-pâle, échinulèes. - AR. - St-Amand. Bois humides. - Automne.

804. URED. SUAVEOLENS. Pers. - Alb. de l'Est. - Hypophylle sur Cirsium arvense; stylospores brun-olive, rondes, très petites. AC. - Dans les jardins. - Automne. - Champs.

- Téleut. Puccinia obtegens.

805. URED. CONCENTRICA. Desm. - Alb. de 
l'Est. - Hypophylle sur Iucca gloriosa; Pustules en cercles concentriques. Stylospores brun. olive, rondes, très petites. - AC. - Dans les jardins. - Automne.

806. URED. LINEARIS. Pers. - Alb. de l'Est. - Hypo. et épiphylle sur Triticum vulgare; secale cereale; pustules longitudinales; stylospores rondes, granuleuses, jaunes. - Téleut. Puccinia graminis. - CCC. - Dans les moissons. - ROUILIE DU BLÉ.

807. URED. ARUNDINACEA. Nouel. - Alb. de l'Est. - Hypo. et épiphylle, sur Phragmites communis. Stylospores rondes ou ovales, brunclair, ponctuées. - Téleut. Puccinia arundinacea. - CCC. - A la fin de l'Automne et en Hiver.

11' GENRE. - GECIDIUM. -- Pers.

808. ACID. RANUNCULACEARUM. D. C. Alb. de l'Est. - Hypophylle sur Ranunculus ficarice et R. Bulbosus, cupules multifides : renfermant des spores rondes, jaunes. Téleut. Uromyces ficarice. - AC. - Sur la Ficaire; plus rare sur R. Bulbosus. - Printemps. - Lieux humides des bois. - Partout.

809. ACID. PHASEOLORUM. Wllr. - Alb. de l'Est. - Hypophylle sur Phaseolus vulgaris ; cupules à l'imbe blanc; spores blanches. Téleut. Uromyces phaseolorum. - RR. Trouvé une seule fois à St-Amand à la fin de l'Été, dans un jardin près de la rivière. 
810. ACID. QUADRIFIDUM. D. C. - Alb. de l'Est. - Hypophylle sur Anemone coronaria: cupules blanches à l'imbe à 4 segments; spores blanches. - AR. - Jardins. - Téleut. Puccinia anemones.

811. AECID. LeUCOSPERMUM. D. C. - Alb.de l'Est. - Hypophylle sur Anemone sylvatica; cupules blanches; spores blanches. - AC. Printemps. - Bois de St-Amand, de Charmont, etc.

812. AECID. URTICA. Schum. - Alb. de l'Est. - Hypophylle sur Urtica divica. Cupules blanches ; spores blanches. - RR. - Bois de St-Lumier.

813. AECID. GENTIAN ÆE. Ch. $R$. - Alb. de l'Est.

- Hypophylle sur Gentiana cruciata. Cupules et spores jaunes. - Téleut. Puccinia gentiance. - J'ai découvert cette espèce en 1872, dans les sapinières de Soulanges, longtemps avant qu'elle ne soit signalée par Fuckel. - RR.

814. AECID. CLEMATIDIS. Schwz. - Alb. de

l'Est. - Hypophylle sur Clematis vitalba. Cupules et spores juunes. - RR. - Dans les bois et haies de St-Remy-en-Bouzemont. - Été. 815. AECID. TARAXACI. Kze. - Alb. de l'Est. - Hypophylle sur Taraxacum dens leonis ; cupules et spores jaunes. - Téleut. Puccinia chondrillce. - AC. - En Automne. - champs. bois découverts, partout.

816. ACID. CONVALLARIARUM. Schum. Alb. de l'Est. - Hypophylle sur Convallaria 
polygonatum; cupules en cercles sur une tache jaune; spores orangées. - AR. - Été. Bois de St-Lumier, etc.

81\%. ACID. TRIFOLII. Cast. - Alb. de l'Est.

- Hypophylle sur Trifolium repens; cupules jaunè-pâle; spores orangées. - Téleut. Uromyces trifolii. - AC. - Le long des chemins, dans les prairies. - Été.

818. \#CID. EUPHORBIÆ. Pers. - Alb. de l'Est. - Hypophylle sur Euphorbia cyparissias Cupules jaunes dont la présence déforme considérablement les feuilles ; spores jaunes. Téleut. Uromyces Tuberculatus. - CC. - Soulanges, St-Amand, etc, - Au printemps.

819. ÆCID. RUBELlATUM. Eries. - Alb. de l'Est. - Hypophylle sur Rumex crispus ; cupules blanches, disposées en cercles sur une tache rouge-pourpre, - Téleut. Uromyces rumicum. - AR. - Dans les bois; au bord des ruisseaux. - St-Amand, Aulnay, etc. - Eté, automne. 820. FCID. GROSSULARIA. D.C. -- Alb. de l'Est. - Hypophylle et sur les fruits de Ribes Grossularice. Cupules à limbe blanc, à spores jaune d'or, sur des taches orangé-pourpre. R. - Printemps. - Dans les jardins à StAmand. - Téleut. Puccinia Ribis.

821. ACID. VIOLARUM. Schrin. - Alb. de l'Est. - Hypophylle et sur les pétioles de Viola canina et sylvestris. - Tèleut. Puccinia Violarum. AC. - Bois, bords des champs. - Eté. Spores jaunes. 
822. ACID. TUSSILAGINIS. Pers. - Alb. de l'Est. - Hypophylle sur Tussilago farfara. Spores jaunes. Le côté correspondant de la feuille, supérieurement, présente des taches rondes, roussâtres, d'un bel effet. - CC. Champs, bords des routes, partout.

823. ECID. TRAGOPOGONIS. D.C.-Alb . de l'Est. - Hypophylle et sur les tiges de Tragopogon pratense ; spores rondes, jaune-pâle. Téleut. Puccinia Tragopogonis. - CCC.-Champs, prairies ; partout. - Eté: Automme.

824. ACID. FALCARIAE. D.C. - Alb. de l'Est. - Hypophylle sur Buplevrum falcarice ; pustules éparses, à limbe blanc. Spores jaunes, rondes. - Téleut. Puccinia buplevri. - AC. Lieux herbus des collines crayeuses. - StAmand. - Automne.

825. AECID. ARI. Desm. - Alb . de l'Est. Hypophylle sur Arum maculatum. Cupules et spores jaunes sur une tache de même couleur. - AC. - Printemps. - Dans les bois ; partout. - L'Uredo ari est plus rare.

826. ACID. BERBERIDIS. Gmel. (Tubularium). - Alb. de l'Est. - Hypophylle sur Berberis vulgaris. Spores jaunes. - Téleut. Puccinia graminis. - D’après les auteurs la stylospore est la rouille du blé, Uredo linearis. - CC. - Jardins, haies. - A la fin de l'été. - Partout.

82\%. ACID. Elongatum. Pers. - Alb. de l'Est. - Cet æcidium (Tubularium) est hypophylle sur Rhamnus Cathartica. Cupules tubu- 
lées, jaunes. sur une tache brune. Spores jaunepâle. - Téleut. Puccinia coronata. - AC. Dans les jardins, les haies. - Automne. La Chaussée, St-Amand, etc.

\section{2e GENRE. - PODISOMA. - Oest.}

828. POD. JUNIPERI. Fries. - Alb. de l'Est. Tubercules jaune-orangé, coniques ; sur les rameaux du Genévrier commun qu'il déforme et détruit avec le temps. Ces tubercules sont composés d'une masse de Téleutospores biloculaires, réunies, agglutinées en faisceaux, émettant des spores ovales difficiles à observer. AC. - En Automne. - Soulanges, Aulnay, etc. - L'état hyménifére est Rastelia cancellata.

Var. Clavariæforme. Duby . - Tubercules allongés, aplatis en lanières, surtout pendant les pluies. - Mêmes localités.

13 GENRE. - R IRSTCLIA. - Rebent.

829. RAST. CANCELlatA. Rebent. - Alb. de l'Est. - Hyponhylle sur Pirus communis et sur les pétioles. - CC. - Dans les jardins, en Automme. - La partie correspondante du côté supérieur de la feuille présente une tache d'un beau rouge, couverte de Spermogonies ponctiformes. Stylospores Podisoma Juniperi. Spores brunes, rondes.

830. RAST. LACERATA. Var. Mali. Sow. Alb. de l'Est. - RR. - Hypophylle sur Pyrus 
malus. v. Sylrestris. - Découvert une seule fois dans une vallée boisée de Soulanges, sur un pommier sauvage dont toutes les feuilles envahies par les taches rouge-pourpre étaient d'un très bel effet. - Fin de l'Eté.

Var. Oxyacantha. Alb. de l'Est. - Cette variété est beaucoup plus commune ; elle attaque les feuilles, les pétioles et les fruits de l'aubépine en Automne. - Lisse, St-Amand, etc. 831. RAET. CORNUTA. Fries. - Alb. de l'Est. - Hypophylle sur Sorbus acuparia; Péridium aliongés, tubuleux, jaunâtres, placés sur une tache brune. Les feuilles attaquées restent longtemps vertes; spores rondes-irrégulières, brunrougeâtre. - R. dans la Marne. - Récolté à Bassuet.

14" GENRE. - GRAPHIOLA. - Poit.

832. GRAPH. PHÆNICIS. Poit - Alb. de l'Est. - Hypophylle sur les dattiers dans les serres. - Apparences de petites pezizes noires á centre jaune, remplies de filaments blancs; longs, réunis en faisceau cylindrique, couvert de spores blanches, rondes, très petites. - Communiqué par M. Marcilly, récolté chez lui à Châlons-sur-Marne.

15 GENRE. - CROMARTIMI. - Tul.

863. CRON. A.SCLEPIADEUM. Tul. - Alb. de l'Est. - Hypophylle sur Cynanchum Vincetoxicum. Téleutuspores rondes, échinulées, jaune 
d'ambro. Styl. Uredo Vincetoxici. - Collines de Soulanges, Possesse. - AR. - Automne. 834. CRON. PAONIAE. Fuckel. - Alb. de l'Est. - Espéce voisine de la précédente, hypophylle sur Pceonia officinalis. Columelles couvertes de basides 2-4 spores, jaune-d'or, lisses. Stylosp. Uredo Pconice. - AR. - Dans mon jardin à St-Amand. - Automne.

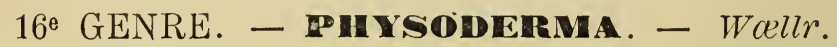

835. PHYS. ERYNGII. Corda. - Alb. de l'Est.

- Dans les cellules du parenchyme des feuilles d'Eryngium campestre. Sporidies jaunâtres ovales-irrégulières. CC. - Automne-hiver.

836. PHYS. MACULARE. Wllr. - Alb. de l'Est.

- Petites taches brunes, rondes; sur feuilles d'Alisma plantago. contenant des sporidies assez grosses, rondes. jaune-pâle entourées d'une bordure rougeâtre. - AR. - Automne ; Bords des eaux, lieux inondés des prairies, à Soulanges. La Chaussée, etc. 


\section{ENDOSPORES.}

5 e ORDRE. - PHYCOMYCÈTES - De Bary.

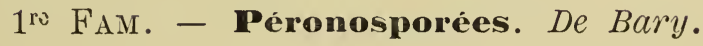

Petites plantes filamenteuses, parasites sur les feuilles vivantes, à ramifications extérieures et intérieures terminées par des sporanges qui contiennent les spores.

1er GENRE. - PERONOSPOBA. - De $B y$.

837. PERON. AFFINIS. De By. - Album de l'Est. - Cette espèce croît à la face inférieure et au sommet des lobes des feuilles de Fumaria officinalis, sous forme de taches grises. velues. -.. Eté, automne. - Jardins et champs nouvellement fumés. - AC. - Terrains crayeux.

838. PERON. DEVASTATRIX. Casp. - Album de l'Est. - Hypophylle sur les Pommes de terre. - Maladie de la Pomme de Terre. - 
Taches d'abord blanches, puis grises, enfin brunes, envahissant graduellement tout le végétal et occasionnant sa mort. - Acrospores et Zoosporanges (organes de la fructification) ovales. - CCC. - Dans les jardins et dans les champs pendant les saisons d'été pluvieuses. Partout.

839. PERON. CHENOPODII. Schlecht. - Album de l'Est. - Hypophylle sur Chenopodium viride. Taches jaune-pâle, de teinte violacée au centre dûe à la couleur des acrospores. - Etéautomne. - Jardins, champs. - CC. - Partout. 840. PERON. Viticola. De By. - Album de l'Est. - Hypo. et épiphylle sur les différentes espèces de Vigne. - Ce champignon a fait son apparition dans le midi de la France en 1879. C'est en automne 1883 que j'ai signalé sa présence dans nos vignobles de la Marne, pour la première fois. Les feuilles de vigne envahies brunissent, se dessèchent et tombent avant la maturité du raisin. Les dommages que peut occasionner le Peronospora viticola. surnommé le Mildew des Aliéricains, le font considérer comme un fléau. - CC - Sur les treilles des Jardins et dans les vignobles du Département dont les plants sont de nature délicate. - (Voir : Rapport sur les maladies de la Vigne au Comité de Vigilance contre le Phylloxera de la Marne par Ch. Righon, 1883). 811 PERON. GANGLIFORMIS. Berk. - Alb. de l'Est. - Hypophylle sur Sonchus oleraceus. 
Senecio vulgaris, Lactuca sativa. Taches blanchâtres, éparses, arrondies, acrospores presque rondes, portées sur des rameaux, terminaux. trifurqués, en petits plateaux triangulaires. CC. - Été, Automne. - Jardins, vergers; partout. 8'2. PERON NIVEA. De By. - Alb. de l'Est.

- Hypophylle sur les Ombelliféres. - Cherophyllum temulum , Egopodium podagnaria. Angelica sylvestris, etc. Acrospores ovoïdes : blanches. - Été, Automne. - CCC. - Bois : bords des eaux, jardins. - Partout.

813. PERON. PARASITICA. De By. - Alb. de l'Est. - Cette espèce envahit les feuilles, les fruits et les tiges des Crucifères, notamment $\mathrm{du}$ Capsella bursa pastoris; Les organes attaqués se déforment et donnent souvent asile au Cystopus candidus. - CC. - Sur le bord des routes, dans les rues des villages, etc. Été-Automne.

\section{¿ GENRE. - CYSTOPUS. - $D e \quad B y$.}

844. CYST. CANDIDUS. De By. - Alb. de l'Est. - Commun sur les feuilles, les tiges et les siliques des Crucifères, Capsella bursa pastoris: Cochlearia amoriaca, Alyssum, etc. Spores et zoosporanges incolores, contenus longtemps sous l'épiderme bulleux de la plante. - AC. - ÉtéAutomne. - Partout. 
Les Genres de cette curieuse Famille, classés autrefois parmi les Urédinées, possèdent une fructification Endospore et doivent prendre place daus cette 2 e Division. Les sporanges internes et externes qui contiennent des Anthéridies font l'office des thèques qui caractérisent les Endospores en général.

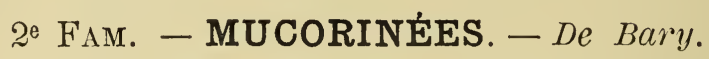

Plantes filamenteuses. parasites sur les corps en décomposition; Ramifications extérieures terminées par des sporanges.

$1^{\text {er }}$ GENRE. - PILOBboLUS. - Coemans.

8'5. PIlob. CRYSTAllinUs. Tod. - Alb. de l'Est. - Filament court, dressé, renflè-vésiculeux vers le milieu, incolore, transparent; sommet de la vésicule surmonté d'un sporange brun. lenticulaire, contenant des spores ovoïdes. Croît en automne sur le crottin de cheval et de brebis. - Sur la lisière des bois. - St-Amand. Soulanges. - AR.

2 GENRE. - MUCOrs. - De Bary.

816. MUC. MUCEDo. Lin. - Alb. de l'Est. Touffes de filaments blancs, longs, surmontés chacun d'un sporange brun, relativement petit. - CCC. - Sur la plapart des substances qui entrent en décomposition. - Printemps, Automne.

- Partout. 
817. MUC. CANINUs. Pers. - Alb. de l'Est. Duvet blanc très dense de filaments ; les uns fertiles surmontés d'un sporange jaune, puis brun; les autres rampants à la surface des crottes de chien. - Automne. - Partoul.

818. MUC. STERCOREUS. Tod. - Alb. de l'Est. - Touffes de filaments blancs porteurs de sporanges sphériques, jaunes, puis verdâtres. AC. - Sur les excréments humains - Partout. - Automne.

8'9. MUC. FUNGICOLA. Corda. - Alb. de l'Est.

- Filaments fertiles simples ou bifurqués; sporanges incolores; spores elliptiques. - Sur les agarics en décomposition. - Fréquent en Automne. - Partout.

850. MUC. RAMosus. Bull. - Alb. de l'Est. Croît sur les chapeaux d'agarics en décomposition. Sporanges d'abord blancs, puis gris-hruns . portés par des filaments trés rameux, en buisson. - Automne. - CC. - Bois de pins. et forêts.

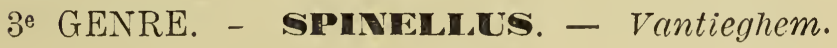

851. SPINEL. FUSIGER. Vantieghem. -. Alb. de l'Est. - Parasite sur chapeaux de diffèrends agarics, notamment de Collybia dryophila. Filaments fertiles très longs, bruns, sporange noir, spores fusiformes, noires. - Bois de pins. St-Amand. - AR. 


\section{$4^{e}$ GENRE. - RHWLPPUS. - Ehrenb.}

852. RHIZ. NIGRICANS. Ehrenb. - Alb. de l'Est. - Filaments et sporanges bruns, parasites sur le pain moisi. - En Automne. - Mycelium radiciforme, spore elliptique, chagrinée. - AR.

\section{5e GENRE. - THAMNIDIUM. - Link.}

853. THAMN. ELEGANS. Link. - Alb. de l'Est.

- Cette espéce croît sur le crottin de cheval, dans les écuries. en Automne et au Printemps. - CC. - Elle possède deux sortes de sporanges renfermant des spores de même forme et d'égale grosseur. $1^{\circ}$ Un sporange très gros , terminant le filament principal, contenant une grande quantité de spores incolores, ovoïdes ; $2^{0}$ vers le milieu du filament une couronne élégante de ramifications dichotômes, munies chacune d'un petit sporange contenant de 2 à 4 spores.

6e GENRE. - SPDRODINIA - Link.

85'. SPOROD. GRANDIS. Link. - Alb. de l'Est. - - Parasite en Automne sur les chapeaux des Agarics en décomposition. - Cette espéce présente d'une façon très distincte ses Sporanges et ses Zygospores, organes qui caractérisent complètement la fructification des $M$ Mucor. Les sporanges sphériques, à spores rondes- 
ovales, gris-bleuâtre sont portés par des filaments arborescents, dichotômes. Les Zygospores, résultant de la conjugaison de deux rameaux renflés et du mélange de leur protoplasma, sont brunes, échinulées, visibles à l'œil nu. AC. - St-Amand, Sermaize, etc.

7e GENRE. - MORTIER ELLA. - Coemans.

855. MORT. POLYCEPHALA. Coemans. - Album de l'Est. - J'ai récolté cette espèce sur le crottin de cheval, en mars 1886, à St-Amand. On dirait à première vue un Botrytis. mais au microscope on distingue au sommet de chaque rameau latéral ou terminal un sporange contenant une vingtaine de spores incolores. - Les filaments ne sont pas cloisonnés et à leur base on découvre des chlamydospores échinulées, à court pédicelle. - AC.

8e GENRE. - CHAETOCLADIUM. - Fresenius.

8.j6. CHAT. JONESII. Fresenius. - Album de l'Est. - Cette espéce croît au printemps et en automne, sur le crottin de cheval, dans le voisinage de Mucor mucedo et de Thamnidium elegans. Ses filaments grêles et rameux rampent comme des lianes; les branches fructifères, arborescentes se terminent par une pointe allongée; les ramuscules en verticiles sont terminées par des sporanges granuleux : monospermes, gris ardoisés. - A.C. 
6e ORdRE. - ASCONÉMÉS. - Kickx.

$1^{\text {ra }}$ FAM. - Ascomycées. - Kickx.

Petites plantes parasites sur les feuilles vivantes, réduites à une Cellule ou Thèque contenant les spores.

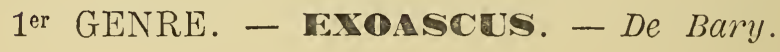

85\%. EXOASC. ALNI. Fuckel. - Alb. de l'Est.

- Sur feuilles d'Alnus glutinosa. Taches grises puis brunes, gauffrant et crispant les feuilles formées par la masse des théques superficielles, contenant chacune 8 spores ovales, incolores, bisériées. - A.C. - St-Amand, dans les bois avoisinant la riviére.

858. EXOASC. BULlatus. Berk. - Alb. de l'Est. - Sur les feuilles de poirier, boursouflées par sa présence; thèques cylindriques ou en massue, contenant 8 à 12 spores incolores. - C. Dans certaines années. - Jardins. - St-Amand.

859. EXOASC. PRUNI. Fulkl. - Alb. de l'Est. - Cette espéce envahit les jeunes fruits des pruniers et occasionne le développement mons. trueux que les horticulteurs nomment la LÈPRE 
DU Prunier. Les thèques prolongées en un court mycelium contiennent 8 spores rondes ou ovales. - AC. - Printemps.

860. EXOASC. DEFORMANS. Fuckel. - Alb. de l'Est. - AC. sur les pêchers ; vulgairement nommé Cloque du PÈcher. - Automne. Jardins. - Cette espéce attaque les feuilles et les déforme, d'oú leur vient le nom de Cloque.

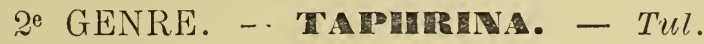

861. TAPH. POPUliNA. Tul. - Alb. de l'Est. - Croît à la face inférieure des feuilles de peuplier d'Italie, dès la fin du printemps, sous forme de tache jaune-d'or, situéc au fond d'une dépression de la feuille. Théques cylindriques, renflées au sommet; sporules très nombreuses, jaune-pâle. - CC. - St-Amand.

\section{7\% ORDRE. - ONIGÉNES. - Tul.}

$$
1^{\text {re }} \text { FAM. - Onigénées. - Tul. }
$$

Plantes parasites sur les substances animales, ayant l'apparence de Lycoperdinées ; filaments du capillitium terminés par des thèques.

1or GENRE. - ONGGN. - Pers.

862. ONIG. CORVINA. Alb. et Schw. - Alb. de l'Est. - J'ai trouvé cette espéce une seule fois, à St-Lumier-en-Champagne, sur un tas de 
détritus oủ sejournaient depuis longtemps des plumes de pigeon. - RR. - L'Onigena equina qui croît sur les sabots de cheval doit être plus commun, bien que je ne l'aie pas encore rencontré dans la Marne. 
8. OrdRe. - CUPULÉS. - Quélet. - (Discomycètes).

$1^{\text {re }}$ Fam. - Helvellées. - Fries. (*)

Cupules extérieures.

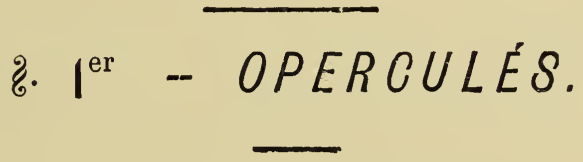

1re Tribu. - MITRÉS.

M OR C HELLÉS.

1er GENRE. - Most CHELLA. - Dill.

863. MORCH. ESCULENTA. Dill. - (Morch.) Atlas Champ. Ch. k. et Roze, p. $226 \mathrm{pl}$. LXVIII. - Morille. - Espèce comestible assez

(") Nota. - Cette nombreuse Famille vient d'être l'objet d'un important travail de mon savant ami E. Boudier de Montmorency ; nous empruntons sa classification qui résume, pour les Helvellées, depuis la tribu des Mitrés jusqu'aux Patellariacés, les progrès les plus récents de la science.

Dans cette Classification, le Genre Peziza de Linn. jusqu'alors adopté a presque complètenent disparu ; il renfermait toutes les espèces qui font partie des Operculés (à part le Rhizina et les Ascobolés) et les deux tiers des Inoperculés. Comme renseignement, nous avons cru devoir rappeier en abrégé et entre parenthèse, le Genre ancien auquel appartenait chaque espèce. 
commune dans les bois des terrains calcaires et dans les plantations de pins sylvestres. Mi-avril. - Soulanges. St-Amand, etc.

86'. Var. Villatica. - Cette variẻté est plus rare et croît spécialement dans les bois tourbeux qui avoisinent la rivière à St-Amand, StLumier-en-Champagne ; elle atteint de grandes diménsions, sa couleur est blonde, ses aréoles très larges et son pédicule renflé outre mesure. - Avril, Mai. - AC.

865. MORCH. COMICA. D.C. - Atl. Champ. Ch. R. et Roze, p. $228 \mathrm{pl}$. LXIX. - Morille DES Sapins. - Cette espéce croît au mois de Mai dans les Sapiniéres de Soulanges, Bassu. $-\mathrm{AC}$.

2 GENRE. - MHTHOFHOHR. - Lév.

866. MitR. SEMILIBERA. D.C. - Atl. Champ. Ch. R. et Roze, p. 230 pl. LXIX. - Comestible. - Commune dans les bois de St-Amand, Aulnay, La Chaussée ; sous les Erables et sous les Ormes ; croît environ quinze jours après $M$. Esculenta; réceptacle conique.

86\%. Var. Rimosipes. D. C. - Diffère de l'espéce précédente par son stipe sillonné dans son tiers supérieur de stries longitudinales. - AR.

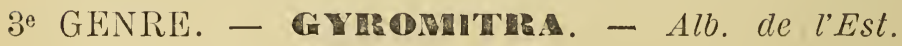

Gyr. Esculenta. - Commun dans les Vosges; manque au Département. 


\section{H E L VEL L ÉS . - Q Quél.}

1er GENRE. - VERPA. - Schwartz.

868. VERP. DIGITALIFORMIS. Pers. - Alb. de l'Est. - Comestible. - Espèce en forme de doigt terminé par un dé à coudre. - Taillis, broussailles. - Soulanges, St-Amand. - AR.Printemps.

869. Var. Morchelloides. - Réceptacle creusé de petites aréoles. - RR. - Mêmes localités.

2 GENRE. - LEPTOPOHA. - Boudier. 870. LEPT ELASTICA. Bull. - Alb. de l'Est. - Comestible. - Bois ombragés, dans les lieux humides. - St-Amand, Sermaize, etc. - Eté, Automne. - AC.

3e GENRE. - HeLUderLA. - Linn.

871. HELV. CRISPA et Var. Fries. - Atl. Champ. Ch. R. et Roze. p. 232, pl. LXIX. - Comestible. - Commun dans les bois de pins sylvestres à Soulanges, Francheville. Dans les taillis à StAmand, Aulnay. - Printemps et quelquefois en Automne. - AC.

8\%. HeLV. LACUNOSA. Afz. - Atl. Champ. Ch. R. et Roze, p. 233 pl, LXIX. - Comestible. - Plantations de pins sylvestres sur les collines crayeuses de Bayarne, Francheville. Eté-Printemps. - AC. 
873. HELV . MONACHELLA Fies - Atl . Champ. Ch. R. et Roze, p. 234 pl. LXIX. Comestible. - Trouvé une seule fois dans les bois de la montagne de Reims au-dessus de Dizy. - RRR. - Mai.

8\%. HELV. ATRA. Kon. - Album de l'Est. Comestible. - Espèce plus petite que les précédentes. - Récoltée une seule fois dans les lieux sablonneux de la Forêt de Sermaize. Eté. - RR.

$2^{e}$ Tribu. - CUPUlés.

\section{R H I ZI N É S .}

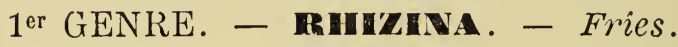

8\%5. RHIz. UNDUlata. Fries. - Alb. de l'Est. - Terrains siliceux de la montagne de Reims; dans la forêt. - Septembre-Octobre. - RR. Cette espéce est adhérente au sol par des appendices radiciformes.

\section{PÉZIZÉS.}

GENRE. - DISCIOTLS. - Boud.

876. DISC. VENOSA. Pers. - (Peziza). - Atl. Champ. Ch. R. et Roze, p. 239, pl. LXX. Comestible. - Très grande espèce étalée en 
rosace sur la terre des bois et des vergers, autour de la rivière à St-Amand, Aulnay, St-Lumier. - Mois de Mai. - CC.

877. Disc. RETICUlATA. Grev. - Alb. de l'Est. - $(P e z i z a)$. - Se rapproche de la précédente et croît dans les mêmes localités. - RR. Mai-Juin.

2e GENRE. - ACETAaULA. - Fuckel.

878. ACET. ACetabulum. Linn. - (Peziza). Atl. Champ. Ch. R. et Roze, p. 236, pl. LXX. - Comestible. - Sur la terre, dans les taillis épais de St-Amand à St-Lumier ; à Aulnayl'Aître. - Printemps. - AC. — Forme de ciboire au pied ciselé.

879. ACET. LeUCOMElAs. Pers. - (Peziza). Alb. de l'Est. - Sur la terre dénudée des plantations de pins sylvestres, sur les hauteurs de Soulanges, La Chaussée, Bassu. - CC. - Commencement du printemps.

3e GENRE. - MACROPODIA. - Fuckel.

880. MACR. MACROPUS. Pers. - (Peziza). Alb. de l'Est. - Sur l'humus des bois ombragés. - St-Amand, Trois-Fontaines, Possesse. AC. - Été.

$4^{\mathrm{e}}$ GENRE. - ALEURIA. - Fries.

881. Aleur. Vesiculosa. Bull. - (Peziza). Alb. de l'Est. - Espèce ayant l'apparence d'un gros grelot, croissant sur les fumiers et les 
lerreaux autour des habitations et dans les jardins. - CC. - Été, Automne. - Partout. 832. AlEUR. CEREA. Sow. - (Peziza). - Alb. de l'Est. - Sur la terre des bois ombragés. Sermaize, St-Amand, etc. - Printemps-Été. CC. - J'ai trouvé dans les bois de Vavray-leGrand une variété Purpurcescens qui se rapproche de Pez. Nigrella. - RR.

883. Aleur. EPIXYLA. Ch. R. - (Peziza). Alb. de l'Est. - Toutes les espèces du Genre Aleuria sont terrestres; j'ai récolté celle-ci sur les branches de Juniperus communis mises en tas sur la terre des bois d'Aulnay-l'Aître; c'est donc la seule qui soit epixyle; les échantillons étaient assez nombreux pour affirmer cette nouvelle espèce que j'ai publiée dans les Bulletins de la Société des Sciences et Arts de Vitryle-François. - RRR.

881. Aleur. AMPliata. Cooke. - (Peziza). Alb. de l'Est. - Cette espèce est très voisine d'Al. Vesiculosa; elle croît sur les écailles de bois de peuplier humides et mises en tas l'hiver; plus petite que Vesiculosa, sa cupule extérieure est moins furfuracée et sa station est différente. - AC. - Printemps. - St-Amand.

5e GENRE. - GALACThNia. - Cooke.

885. GALACT. SUCCOSA. Berk. - (Peziza). Alb. de l'Est. - Coupe fragile d'un à 2 centimètres de largeur, exsudant un suc blanc puis 
jaunâtre à la cassure. - Bois de Sermaize à Trois-Fontaines. - AR. - Été. - Terrestre. 886. GALACT. COChleAtA. Pers. - (Peziza). Atl. Champ. Ch. R. et Roze: p, 238, pl. LXX. - Coupe fragile, contournée en limaçon; extérieur brun-clair, hymenium brun-pourpre. Dans les allées des forêts de Cheminon. Sermaize, etc. - AC. - Automne. - On la dit Comestible.

6e GENRE. - SARCOSPHAERA. - Aw.

88\%. SARC. CORONARIA. Jacq. - (Peziza). Alb. de l'Est. - Cette bella espèce presqu'hypogée, croît dans les plantations de Pins sylvestres de St-Amand, de Francheville dés les premiers jours de Mai; sa déhiscence a lieu par déchirure au sommet qui se divise en 4 ou 6 fragments triangulaires. Ces fragments se déjettent en dehors et donnent à la cupule la forme d'une tulipe dont l'intérieur est d'une couleur violette-tendre puis purpurine. - AR. - Cette espèce a été décrite sous différents noms " Peziza Corona. Jacq. - P. Eximia. Lév. - Schizostoma.Ch. R. - P. Macrocalyx. Riess, etc.

\% GENRE. - OTHDEA. - Fuckel.

888. OTID. ONOTICA. Pers. - (Peziza). - Alb. de l'Est. - Forêt de Trois-Fontaines, Cheminon, dans les tranchées. - RR. - Octobre. Espèce couleur 'orangée, en forme d'oreille de lièvre. 
889. S. G. Pseudotis-Abietina. Pers. -- (Peziza). - Alb. de l'Est. - Plantations de Conifères dans les Parcs. - Ablancourt, Aulnay. - RR. - Cupule concave, flexueuse, couleur de rouille. - Automne.

8 GENRE. - PUSTULARIA. - Fuckel.

890. PUST. CUPULARIS. (Linn. - (Peziza). Alb. de l'Est. - Bois-taillis à St-Amand, Charmont, elc. - Printemps. - AC.

$9^{\circ}$ GENRE. - PEZIZA. - Dill.

891. Pez. Aurantia. Pers. - (Peziza). - Atl. Champ. Ch. R. et Roze, p. 239, pl. LXX. Cupules d'une belle couleur orangée-rouge, groupées sur les souches et troncs de chênes coupés à ras de terre. - CC. - Forêts de Sermaize, Charmont, etc. - Automne.

892. PEZ. SPLENDENS ? Quélet. - (Peziza). Alb. de l'Est. - Récoltée une seule fois près de Cheminon, dans les lieux sablonneux de la forêt ? ? 893. PEZ. POLYTRIChINA. Pers. - (Peziza). - Alb. de l'Est. - Espéce rare, récoltée sur les mousses (polytrichs) qui l'enveloppent et ne laissent voir que la belle cupule orangée qui tranche sur le vert sombre de son entourage. - Sermaize. - Automne.

10 GENRE. - Caloscypha. - Boud.

894. Calosc. Fulgens. Pers. - (Peziza). Alb. de l'Est. - Lieux herbus. pâturages près 
des bois de Charmont. - RRK. - Cupules orangées; spores rondes.

11 GENRE. - SARCOSCYPHA. - Fries.

895. SARCOSC. COCCINEA. Jacq. - (Peziza). Alb. de l'Est. - Cupule pédiculée, blanche extérieurement et d'une brillante couleur rougevermillon, pourprée à l'intérieur; croissant sur les brindilles de bois. - St-Lumier-en-Champagne, Méry-sur-Marne, Soulanges. - AR. Automne.

12e GENRE. - RHIZOPODELLA. - Cooke.

896. RHIZ. MELASTOMA. Sow. - (Peziza). Alb. de l'Est. - Cupules plus petites que les espéces précédentes brunes, à hymenium noir; terminées inférieurement par des houppes filamenteuses, radicantes. - Croît sur le bois pourri. - Plantations de pins à Soulanges, - AR.

13 GENRE. - PSEUDOPLECTANA. - Fuckel.

89\%. PSEUd. NIGELlA. Pers. - (Peziza). Alb. de l'Est. - Croît sur la terre des bois à Sermaize. - AR. - Son disque a la couleur et la taille de Bulgaria inquinans. - Printemps

1'e GENRE. - LEUCOSCYPIA. - Boudier.

898. LEUCOSC. LEUCOTRICHA. Alb. el Schw. - (Peziza). - Alb. de l'Est. - Cupules blanches, velues, hemisphériques. - Sur la terre des lieux ombragés. - Forêt de Charmont. - RR. 
15 GENRE. - LACHNEA. - Fries.

899. LACHN. HEMISPHARICA. Weber.-(Peziza).

- Alb. de l'Est. - Commun sur la terre humide des forêts. - Sous les chênes, les charmes, etc. - Automne.

900. LACHN. BRUNNEA. Alb. et Schw. (Peziza). - Alb. de l'Est. - Croît dans les forêts, sur la terre oú l'on a brûlé du charbon. - Cupule brune; disque chamois. - AC. - Sermaize, Charmont, etc. - Automne.

901. LACHN. GREGARIA. Cooke. - (Peziza). Alb. de l'Est. - Cupules petites, en groupes serrés, sur le sol de mon jardin, le long des pins qui bordent les allées. - Extérieur velu, brun; disque blanchâtre. - AR. -- Automne.

16 GENRE. - STPULTARHA. - Cooke.

902. SEPULT. ARENOSA. Boud. - (Peziza). Alb. de l'Est. - Croît enfouie dans le sable. Lieux découverts des forêts de Cheminon à Trois-Fontaines. - Cette espèce en vieillissant est envahie par une sphériacée remarquable. le Melanospora Zobelii qui m'a été gracieusement communiqué par M. Boudier. - Automne. 


\section{$3^{\mathrm{e}}$ TRIBU. - LENTICULÉS.}

\section{G I L I A R IÉS.}

1er GENRE. - TRICHOPHAEA. - Boud.

903. TRICHOPH. HISPIDULA. Sch. - (Peziza).

- Alb. de l'Est. - Cette petite espéce jaunâtre extérieurement et parsemée de poils brunsrougeâtres, rigides; à disque plan. blanchâtre. croît. tantôt sur le sol, tantôt sur les rameaux de peuplier mis en tas. sur la terre humide. - Pendant l'hiver. Je l'ai récoltée à St-Quentinles-Marais. le 15 Novembre 1885.

2e GENRE. - CILIARIA. - Quélet.

90'. Cillaria. SCUTELlATA. Linn. - (Peziza). - Alb. de l'Est. - Espèce très commune dans les bois, sur la terre près des souches d'arbres abattus. - Été. Automne. - Partout.

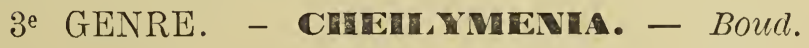

905. CHEIL. STERCOREA. Pers. - (Peziza). Alb. de l'Est. - Sur les houses de vaches. Bords des chemins; prairies. - AC. - Automne. 906. CHEIL. PULChERRIMA? Crcuan. - (Peziza). - Alb. de l'Est. - Sur les bouses de vaches. - Cette espèce considérée jadis comme un Ascobolus, ayant un disque non papillé et des 
thèques cylindriques est reléguée avec raison dans le Genre Cheilymenia.

$4^{\mathrm{e}}$ GENRE. - ANTHiracomin. - Boud.

90\%. ANTHR. MELALOMA. Alb. et Schw. $(P e z i z a)$. - Alb. de l'Est. - Croît dans les forêts, sur la place oú l'on a fait du charbon. - Récolté prés de Reims, en Automne. - AR.

- Cupule rouge-brique; extérieur squameux.

5e GENRE. - MELAsTIza. - Boud.

908. MELAST. MiNIATA. Fuckel. - (Pеziza). - Alb. de l'Est. - Sur la terre calcaire des fosses où l'on met en réserve les pulpes de betteraves. - AC. à St-Amand, Bassu, etc.

\section{H U M A R I É S .}

10r GENRE. - huMaria. - Fries.

909. HUM. CONVEXULA. Pers. - (Peziza). - Alb. de l'Est. - Sur la terre des sentiers humides; dans les forêts. - Charmont, Sermaize. - AR. - Automne.

910. HUM. LEUCOLOMA. Hedw. - (Pe $\approx i z a)$. Alb. de l'Est. - Sur la terre; dans les lieux oú séjournent des détritus azotés et putrescibles; dans le voisinage des jardins et des maisons de culture.

911. Var. Uvicola. Ch. $R$. - Commun dans les vignes sur les dépôts anciens des marcs 
de raisin. - Le Type et la Var. C. en Automne. - St-Amand, Bassuet, etc.

2 GENRE. - LAMPRASPORA. - De Not.

912. LAMPR. CRECQ'HERAULTII. Crouan. $(P e z i z a)$. - Alb. de l'Est. - Cette petite espèce, jaune-indien, si remarquable par sos spores rondes, ciliées, m'a été communiquée par M. Boudier, et a été retrouvée depuis sur la terre sablonneuse des bois de Reims. RR.

913. LAMPR. CROUANI. Boud. - (Peziza). Alb. de l'Est. - Récolté une seule fois sur la mousse qui tapissait le sol d'un endroit découvert de la forêt, prés de Trois-Fontaines. Automne.

Je posséde deux espéces voisines intéressantes: le Lamprospora Wrigtii et le Boudiera areolata. - Berk. trouvées par M. Boudier dans les localités identiques du Département de Seine-etOise. Des recherches ultérieures permettront sans doute de les récolter dans la Marne.

914. LAMPR. HUMOSA. Berk. - Alb. de l'Est.

- Sur la terre humide, forêt de Sermaize. - AC.

3e GENRE. - COPROABIA. - Boud.

915. COPROB. GRANUlAtA. Bull. - (Peziza).

- Alb. de l'Est. - Cette espéce croît sur le fumier de vaches. -- On la rencontre en Automne dans les prairies oú l'on fait pâturer les bestiaux. - CC. 
4e GENRE. - PYRONEMA. - Carus.

916. PYRON. OMPHALODES. Bull. - (Peziza).

- Alb. de l'Est. - Cette espéce d'un beau rouge vermillon, entourée d'une bordure de mycelium blanc, crô̂t en groupes très nombreux sur la terre oú l'on a fait du charbon. - Automne. - Sermaize, Vanault-les-Dames, etc. - CC.

\section{A S COBOLÉS.

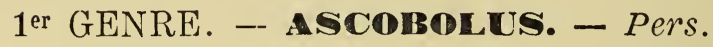

917. ASCOB. FurfuraceUs. Pers. - Alb. de l'Est. - Cette espèce la plus développée du Genre croît sur la bouse de vache et sur le crottin de cheval. - Dans les prairies et dans les enclos servant à la pâture. - Partout. Automne. - CC.

918. ASCOB. IMMERSUS. Pers. - Alb. de l'Est.

- Croît sur les bouses de vaches. plorigé dans la croûte grise qui en tapisse la surface. Bords des chemins, prairies. - Automne. - CC. 919. ASCOB. GLABER. Pers. - Alb. de l'Est. Commun sur le crottin de cheval. - Dans les champs et les écuries, presque toute l'année. Partout.

920. ASCOB. DenUdatus. Fries. - Alb. de l'Est. - Récolté dans mon jardin sur tiges de Dahlia et de Zimia en décomposition. - AR. - Été-Automne. 
2e GENRE. - SACCOBOLUS. - Boud.

921. SACC. NEGLECTUS. Boud. - Alb. de l'Est. - Récolté sur les crottes de brebis. - Hauteurs de Bayarne, Francheville. - AC. - Automne.

3e GENRE. - HASIOBOLUS. - Boud.

922. LASIOB. PILOSUS. Boud. - Alb. de l'Est. - Très commun sur le crottin de cheval et de brebis dans les pâturages; sur les collines herbues. - Automne.

$4^{\mathrm{e}}$ GENRE. - SCOPHANS. - Boud.

923. ASCOPH. CARNEUS. Boud. - Alb. de l'Est. - Commun sur les crottes de brebis et les bouses de vaches; dans les champs et sur les collines herbues. - Soulanges, St-Amand, etc. - Automne.

92't. ASCOPH. SUBFUSCUS. Boud. - Alb. de l'Est. - Très petite espèce souvent confondue avec Riparobius Cookei, qui l'accompagne sur les excréments de chien. - AR. - St-Amand. - Automne.

925. ASCOPH. SPADICEO-NIGER. Ch. $R .-$ Alb. de l'Est. - Cette espéce granuliforme, sphérique. brun-foncé, croit sur les crottes de lapin et les brindilles qui cntourent les fumiers. - Elle est voisine d'Ascoph. Ochraceus de Boud. Ses paraphyses ne sont pas renflées au sommet et 
ses spores prennent en vieillissant une légère teinte orune. - St-Amand. - Automne. - Dans les jardins.

926. ASCOPH. SACCHARINUS. Bord. - Alb. de l'Est. - Croît sur les substances mélangées aux fumiers, débris végétaux, vieux papiers, toiles pourries, etc. - St-Amand.

5e GENRE. - IRHPAROHEUS. - Boud.

927. RIPAR. COOKEI. Crouan. - Alb. de l'Est. - Récolté sur les crottes de chien recouvertes d'une cron̂te grise, en compagnie de stilbum Erythrocephalum. Cupule très petite : brun-clair. d'abord sphérique puis aplatie. Thèques larges. contenant 6't spores ovales, incolores. - AC. Automne-Printemps. - St-Amand, Aulnay.

928. RIPAR. BRUNNEUS. Boud. - Alb. de l'Est. - Ressemble à l'espéce précédente, mais plus brune et ne contenant que 32 spores. - Mêmes localités. - AR.

929. RIPAR. MYRIOSPORUS. Boud. - Alb. de l'Est. - Très petite espéce, croissant sur le crottin de cheval. - Inappréciable à l'œil nu; remarquable par ses thèques larges, courtes. ovales, contenant une quantité innombrable de spores. - Printemps. - Dans les écuries. St-Amand. - AR.

6e GENRE. - SCOZONUS. - Boud.

930. ASCOZ. CUNICULARIA. Boud. - Alb. de l'Est. - Croît sur les crottes de lapin. - Espèce 
presqu'imperceptible à l'œil nu . Réceptacles sphériques, blanchâtres, couverts de poils courts, blancs, hispides. Thèques larges, à déhiscence en fente bilabiée; spores elliptiques, allongées , incolores, très nombreuses. - RR.

\section{2'. -- INOPERCULÉS.}

$4^{e}$ Tribu. CLAVULÉS.

GÉOGLOS SÉS.

1'r GENRE. - TRECHOGLOSSUM. - Boud.

931. TRICH. HIRSUTUM. Boud. - (Geoglossum).

- Alb. de l'Est. - C'ette espéce plus petite que ses congénéres atteint à peine 2 centimétres de hauteur. Elle est d'un brun-foncé, couverte de poils courts, noirs, dressés ; ses spores sont baculiformes, d'un blanc-grisâtre, septées. - Elle croît très rarement dans les plantations de pins. - Bassu. - Automne.

2e GENRE. - GEOGROSSUMI. - Pers.

932. Geogl. Glabrum. Pers. - Alb. de l'Est. - Cette espéce croît sur la terre des plantations de pins sylvestres, dans tout le Département. - Elle est noire, haute de 4 ì 6 centi- 
metres; sa forme est celle d'une langue ou d'une spatule. - CC. - Automne. - Spore brune, cylindrique, cloisonnée.

\section{3e GENRE. - MICHAGLOSSUM. - Sacc.}

933. MICROGL. VIRIDE. Pers. - Alb. de l'Est. Cette espèce presque de même forme que la précédente est de couleur verte. Ses spores sont incolores, cylindriques mais courtes, un peu courbes. - Elle croît dans les taillis des bois de Possesse, Charmont. - Été. - RR.

\section{$4^{\mathrm{e}}$ GENRE. - LERTGLOSSUM. - Cooke.}

931. LEPTOGL. OLIVACEUM. Quél. - Aib. de l'Est. - Cette espéce m’a été communiquée par le $\mathrm{D}^{\mathrm{r}}$ Quélet, et a été récoltée depuis dans la Meuse sur les confins de la Marne. - Nous la citons ici parce qu'elle représente un Genre particulier (existant sans doute dans le Département) remarquable par ses spores incolores : triseptées, en fuseau.

\section{LÉOTIÉS.}

$1^{\mathrm{er}}$ GENRE. - IREIDEIBIA. - Fries.

935. HEYD. CUCULLATA. Batsch. - Alb. de l'Est. - J'ai récolté cette petite et élégante espéce dans les bois de pins sylvestres, à Aulnayl'Aître et à Ablancourt. - En Automne, après les pluies. - Elle est remarquable par son 
capitule d'un beau jaune-d'or et son pédicelle brun. - Elle croît en groupes nombreux, sur la mousse - RR.

$2^{e}$ GENRE. - LEOTA. - Hill.

936. LEOT. LUBRICA. Scop. - Alb. de l'Est. - Croît dans les lieux humides des forêts de Sermaize, Vanault-les-Dames, etc. - AC. Automne.

\section{3e GENRE. - PILACRE. - Fries.}

937. PIL. FRIESII. Wein. - Alb. de l'Est. Cette espéce dont les organes caractéristiques ont été imparfaitement signalés par les auteurs, est le type du Genre Pilacre, jadis décrit par Weinmann et définitivement fixé par M. Boudier, dans sa classification des Discomycètes, 1885. C'est le fameux champignon des racines de la vigne nommé improprement Rosleria hypogea, par Von-Thümen, et attribué par nous au Genre Vibrissea. J'en ai fait la première découverte en France en 1872, et M. Boudier en 187\%-1878, sur les racines et souches de l'orme, de l'érable, de la vigne et du pêcher. Depuis cette époque, le Pilacre a envahi les vignes du Midi et de la Champagne. - CC. sur la vigne; RR. sur les racines des autres régétaux. - StLumier, St-Amand. 


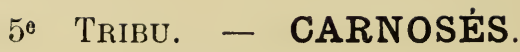

\section{O M B R O P H I LÉS .}

1er GENRE. - OMHROPHALA. - Fries.

938. OMBR. CLAVUS. Alb. et Schw. - Alb. de l'Est. - La varièté Purpurascens et la variété Lilacina se rencontrent sur les brindilles de bois, notamment sur les écailles de peuplier qui ont séjourné dans les fossés humides. - $R$. dans les bois de St-Lumier à St-Amand. Automne.

2e GENRE. - CACYCHLA. - Fries.

939. CAL YC. CITRINA. Batsch. - Alb. de l'Est. - (Peziza). - J'ai récolté une seule fois cette espèce sur un fragment de cerisier, à demi enfoui dans la mousse, dans une garenne de sapins à St-Amand. - Automne.

3e GENRE. - CORYNE. - Tul.

940. CORYNE SARCOIDES - Jacq. - Alb. de l'Est. - (Peziza). - Espèce couleur vineuse, très fréquente sur les troncs abattus des chênes, peupliers, Érables, etc. - Son état conidiophore ressemblant à une trémelle frisée, violacée. l'accompagne souvent. - Automne. - Partout. 


\section{GENRE. - BULGARIA. - Fries.}

941. BULG. INQUINANS. Pers. - Alb. de l'Est.

- Peziza nigra de Bull. - Espéce très commune sur les troncs de chênes abattus, dont elle envahit l'écorce en automne et en hiver. - Forêts et chantiers. - Partout.

9'2. BULG. PULlA. Karst. - Alb. de l'Est. (Patellaria). - Sur les souches de peuplier à demi cariées, au bord des cours d'eau à Lisse et á Soulanges. - RR. - Automne.

\section{G A L L OR IÉ S.}

1er GENRE. - CaLLorian. - Fries.

913. CALl. FusAroides. Berk. - Alb. de l'Est. - (Peziza). - Croît en Automne et en Hiver sur les tiges desséchées de l'Ortie, sous forme de petits boutons rouge-minium, en compagnie de l'état conidien (Tremella urticce des auteurs. CC. - Les long des haies, des jardins. Partout.

2 GENRE. - CORYMELLA. - Boudier.

9'1'. CORYN. Atrovirens. Pers. - Alb. de l'Est. (Peziza). - J'ai récolté cette espéce sur une écaille de bois de frêne cariée, sur la terre, dans les bois de Sermaize. La forme conidiophore, Epidochium virens est plus commune. 
9'5. CORYN. AUREA. Fuckel. - Alb. de l'Est. - (Peziza). - Cette espèce croît sur les chaumes pourrissantes de l'Arundo phragmites et sur les rhizomes. - Je l'ai récoltée en Automne dans les fossés du château d'Aulnayl'Aître et le long d'un ruisseau à Lisse. - Etat conidien CC. - Type RR.

3e GENRE. - ORBHLIA. - Fries.

946. ORB. ROZEI. Quélet. - Alb. de l'Est. (Peziza). - J'ai rencontré maintes fois cette très petite espèce transparente et rouge. à laaisselle des feuilles mortes de l'Helleborus viridis. Ses théques larges contiennent huit spores ovales, incolores. - Soulanges, sur les collines crayeuses. - Automne.

9'\%. ORB. FERRUGINEA. Pers. - Alb. de l'Est. - (Peziza). - Cette espéce récoltée une seule tois à Sermaize sur un morceau de bois pourri, est petite, d'un jaune orangé foncé-transparent, ses spores sont fusiformes, incolores. - RRR. 9'8. ORB. RUBELLA. Kartlen. - Alb. de l'Est. - Espèce trémelloïde, glabre, rouge-incarnat , à bords laciniés, crénélés; thèques cylindriques; tronquées au sommet. - Paraphyses terminées en fer de lance. - Spores fusiformes. - Croît en groupes sur l'écorce des rameaux et branches d'orme tombées à terre. - St-Annand. Automne. - AR 
4e GENRE. - IYYALIIA. - Boud.

919. HYAlin. CRYSTALliNA. Quél. - Alb. de l'Est. - Petite espèce transparente, sessile, blanchâtre, à bords dentés. - Spores vermiformes. - Croît sur les écailles de bois pourries et jonchant la terre humide. - St-Amand, St-Lumier. - Automne. - AĆ.

6e Tribu. - CYATHULÉS.

HÉ L OT I ÉS.

1er GENRE. - CIBdoria. - Fuckel.

Sous-Genre. - Stromatinia. - Boud.

950. STrom. RApulum. Bull. - Alb. de l'Est.

- $(P e z i z a)$ - Lieux humides et tourbeux des bois de St-Lumier, d'Aulnay-l'Aître oú croît ordinairement le Convalliara polygonatum. - R. - Eté-automme.

Sous-Genre. - Ciboria. - Fuckel.

951. CIB. AMFinTi. Batsch. - Alb. de l'Est. (Peziza). - Sur les chatons du Salix caprcea, en Avril, dans les lieux oú ils ont séjourné l'hiver sous des tas de feuilles. - Aulnay-l'Aître. St-Lumier. - C. 
952. CIB. AmentACEA. Balb. - Alb. de l'Est. - (Peziza). - Sur les écailles des chatons femelles de l'Alnus glutinosa. - En automne et en hiver. dans les lieux humides des bois de St-Amand à St-Lumier. - CC.

$2^{e}$ GENRE. - PHLLEA. - Fries.

953. Phial. Phialea. Pers. - Alb. de l'Est. - (Peziza). - Sur les branches mortes d'Alnus glutinosa planté dans les lieux marécageux des bois entre St-Amand et St-Lumier. - AC. - Automne-Printemps.

95'. PHIAL. BOLARIS. Batsch. - Alb. de l'Est. - (Peziza). - Sur les racines et sur les rameaux du Coudrier tombés à terre. - Printemps-automne. - AC.

955. PHIAL. FIRMA. Bolt. - Alb. de l'Est. $($ Peziza). - Sur les troncs coupés à ras de terre et sur les branches tombées d'Alnus glutinosa. - Automne, hiver. - A.C.

33 GENRE. - ChLomosplenium. - Fries.

956. CHLOR. AERUGINOSUM. $F l$. D. - Alb. de l'Est. - (Peziza). - AR. sur les branches de chêne tombées à terre et pourries. - Cette espéce est de couleur verte, son mycelium envahit le support et lui donne la même teinte. - Bois de Charmont, Sermaize, etc. - Automne.

95\%. CHLOR. VERSIFORME. Pers. - Alb. de l'Est. - (Peziza). - Espèce rare, récoltée pen- 
dant plusieurs années dans l'intérieur d'un saule creux. Dans les bois entre St-Amand et Lisse. - Eté-automne.

$4^{\mathrm{e}}$ GENRE. - MELOTHUM. - Fries.

958. HELOT. VIRGULTORUM. Walr. - Alb. de l'Est. - (Peziza). - Commun sur les rameaux et sur les brindilles pourrissant sur la terre humide des bois. - Automne, Printemps. - Partout.

959. Helot. EPIPHyllum. Pers. - Alb. de l'Est. - (Peziza). - Commun dans les forêts de chêne, sur les feuilles tombées. - Sermaize. Charmont, etc. - Automne.

960. Helot. Herbarum. Pers. - Alb. de l'Est. - (Peziza). - Sur les grandes plantes herbacées, notamment sur Urtica divica. Dans les haies, jardins, etc. - CCC. Automne, hiver.

961. HELOT. ACICULARE. Bull. - Troncs pourris. - CC. - Dans les forêts.

962. Helot. AlNiellum. Karsten. - Alb. de l'Est. - (Peziza). - Commun à la fin de l'automne sur les chatons femelles de l'Alnus glutinosa tombés sur le sol humide. - StAmand, Lisse, etc.

963. Helot. Clavatum. Pers. - Alb. de l'Est - (Peziza). - Commun en Automne sur les liges sèches d'ombellifẻres; sur les collines crayeuses, St-Amand, Soulanges. 
96'. HELOT. CAUliCOLUM. Fries. - Alb. de l'Est. - (Peziza). - Sur les tiges d'asperges. - Automne et Printemps. - Jardins, parmi les detritus des végétaux mis en tas. - StAmand. - AC.

965. HELOT. CYATHOIDEUM. Bull. - Alb. de l'Est. - (Peziza). - Dans les bois sur les tiges herbacées et sur les pétioles des feuilles. - CC. - St-Amand, Aulnay, etc. - Automne. Printemps.

966. HELOT. PHYLLOPHILUM. Desm. - Alb. de l'Est. - (Peziza). - Croît sur les feuilles de Fagus sylvatica, tombées ; à peine visible à l'œil nu. - Bois du château d'Aulnay-l'Aître. AR. - Automne.

967. HELOT. SALICELLUM. Fries. - Alb. de l'Est. - (Peziza). - Sur les branches et petits rameaux de Saule morts, encore adherents au tronc. - Hiver, printemps. - Dans les saussaies marécageuses à Soulanges. - AR.

968. HELOT. FRUCTIGENUM . Fries. - Alb. de l'Est. - (Рeziza). - Sur les glands et sur les pétioles des feuilles de Chêne. - Dans les bois. - Partout. - Automne.

969. HELOT. PINETI. Batsch. - Alb. de l'Est. (Peziza). - Sur les rameaux et aiguilles de pin après les piuies. - CC. au printemps. - St-Amand. 970. HELOT . FAGINEUM. Pers . - Alb . de l'Est. - (Peziza). - Sur les péricarpes de Fagus sylvatica tombés à terre ; - Forêt de Trois-Fontaines. - Automne. - AC. 
971. HELOT. IMBERBE. Fuckel. - Alb. de l'Est.

- Sur bois pourri d'Alnus glutinosa. - AC. Bois de St-Amand, etc.

5e GENRE. - CYaThICuLA. - De Not.

972. CYATH. CORONATA. Buli. - Alb. de l'Est. - (Peziza). - Commun sur les tiges des plantes herbacées, surtout sur Urtica divica. Dans les haies. - Automme. - Partout.

\section{A S Y S C Y PHÉS.}

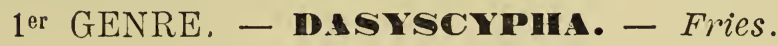

973. DASYSC . VIRGINEA. Fries. - Alb. de l'Est. - (Peziza). - Commun sur les bois cariés et sur les écailles de bois pourries ; dans les bois. - Partout. - Aatomne.

9\%. DASYSC. BICOLOR. Bull. - Alb. de l'Est. - (Peziza). - Très commun sur les petites branches de chênes tombées ; dans les bois et les forêts. - Sermaize, St-Amand ; etc. Automne, hiver.

975. DASYSC. BRUNNEOla. Desm. - Alb. de l'Est. - (Peziza). - Croît sur les petites branches mortes de comus sanguinea, - AR. Eté. - St-Amand.

976. DASYSC. CERINA. Pers. - Alb. de l'Est. $($ Peziza). - Commun sur la tranche des troncs de peuplier et de frêne coupés á ras de terre ; 
se présente sous deux formes, sur le même tronc ; pédécellée et sessible. - Eté, automne. - Partout.

977. DASYSC. ACUUM. Fries. - Alb. de l'Fist. - (Peziza). - Sur les aiguilles de pin sylvestre encore adhérentes aux rameaux pourris, sur la mousse. - CC. en automne et au printemps. - Partout.

978. DASYSC. CILIARIS. Schrad. - Alb. de l'Est. - (Peziza). - Espèce extrêmement petite. éparse sur les feuilles de chêne. -- Lisse, StAmand. - Automne. - AR.

979. DASYSC. FUSCESCENS. Pers. - Alb. de l'Est. - $($ Peziza) . - Visible seulement à ì loupe ; croît sur les feuilles de hêtre. - Bois entre St-Amand et Aulnay-l'Aître. - AR . Automne.

980. DASYSC. NIVEA. Fries. - Alb. de l'Est. (Peziza). - Espéce très petite, difficile à déterminer à cause de sa ressemblance avec Cyphella villosa de la famille des Auricularinées, et de son hymenium souvent stérile.

981. DASYSC. PALEARUM. Desm. - Album de l'Est. - (Peziza). - Assez commun sur la paille de blé pourrie et mise en tas. - Printemps, hivers doux. - AC. - St-Amand, StLurnier.

982. DASYSC. MOLLISSIMA. Lasch. - Alb. de l'Est - (Peziza). - Croît sur tiges mortes d'Eupatorium cannabinum, dans les jois de StLumier. - En automne et au printemps. - AC. 
933. DASYSC. SERICEA. Alb. et Schu. - Alb. de l'Est. - (Peziza). - Récolté deux fois sur les souches de chène, dans la forêt de TroisFontaines: au lieu dit la Colotte. - RR. Automne.

98'. DASYSC. RUFO OLIVACEA. - $A l b$. et Schw. - Alb. de l'Est. - (Peziza). - Espéce très curieuse qui se rapproche beaucoup des Cenangium. - Elle croît isolément ou en petit nombre sur les rameaux secs de Rubus fruticosus. - Dansles broussailles et le long des termes à St-Amand, Sermaize. - AR. - Automne.

985. DASYSC. HYPOCASTANI. $C h . R .-A l b$. de l'Est. - (Peziza). - J'ai trouvé cette nouvelle espéce, plusieurs années de suite à la face interne des involucres du Marron d'Inde pourrissant sur la terre des bois de St-Lumieren-Champagne. - Elle est voisine de Pez. Godroniana ou Episcopalis de Montagne. Automne. - RR.

2e GENRE. - LACHYELLA. - Fries.

986. LACHN. SULFUREA. Pers. - Alb. de l'Est. - (Peziza). - Espéce commune sur les grandes herbes, au pied des haies, notamment sur Urtica dioica. Sambucus ebulus. - Automne et hivers doux.

98\%. LACHN. CORTICALIS. Pers. - Alb. de l'Est. - (Peziza). - Croît sur l'écorce de peuplier qui entoure les troncs coupés à ras de terre.CC. en Automne. - Partout. 
988. LACHN. JUNIPERI. $C h$. $R$. - Alb. de l'Est.

- (Peziza). - Espéce nouvelle récoltée sur les branches de Juniperus communis, mises en tas de fagots dans les bois du château à Aulnayl'Aître. - L'hyménium de plusieurs sujets était blanc-pruineux; cette pruine ètait due à la présence d'un champignon parasite, nouveau, que j'ai nommé Didymaria pezizce. - RRR. en Automne.

989. LACHN. PRASINA. Quel. - St-Lumier. Mai 1886, sur chaumes mortes de Phragmites et de sparganium. - R.

\section{3e GENRE. - TRICROSCYPHA. - Boud.}

990. TRICH. CALYCINA. Fries. - Alb. de l'Est. - (Peziza). - Espéce jaune d'or à l'intérieur et blanche velue extérieurement, croissant en groupes de 5 à 6 sur les branches mortes de Pinus sylvestris. - St-Amand, Bassu, etc. Automne. - AC.

991. TRICH. PYGMEA. Fries. - Alb. de l'Est. - (Peziza). - Cette espéce plus grande que la précédente est munie d'un pédicelle allongé; elle lui ressemble par la couleur de son hyménium et par son extérieur villeux - beaucoup plus rare que la première, je ne l'ai récoltée qu'une seule fois sur les branches de pin sylvestre à Bayarne. - Automne.

992. TRICH. NIDULUS. Kunze. - Alb. de l'Est.(Peziza). - Commune en hiver sur les tiges du Sceau de Salomon. - St-Amand, Aulnay. - Bois. 
4eGENRE. - HYMLOSCYPHA. - Boud.

993. HYAl. Dentata. Pers. - Alb. de l'Est.

- (Peziza). - J'ai récolté cette espéce sur un morceau de bois de peuplier carié ; elle vient en groupes très denses; la cupule sessile, blanche, est urcéolée, dentée sur les bords, très petite. - Bois de St-Amand. - R. en automne.

\section{UR CÉ OLÉS.}

1er GENRE. - URe CEldeLA. - Boud.

99'. URCeol. ASterostoma. Phill. - Alb. de l'Est. - (Peziza). - Petite pezize blanche. urcéolée, dont les poils extérieurs disposés en faisceaux séparés simulent une étoile - sur les écailles de peuplier tombées à terre, dans les bois humides. - A.C. - Printemps.

995. URCEOL. FILICINA. Cooke. - Alb. de l'Est. - (Peziza). - Petites cupules brunes, parsemées de papilles blanches, brillantes Croît sur tiges de Pteris aquilina. - RR. Sermaize.

996. URCEOL. ARENUlA. Alb. et Schw. Alb. de l'Est. - (Peziza). - Commun en Automne, hiver; sur les tiges desséchées de Spirea Ulmaria. - Le long du ruisseau de St-Amand à St-Lumier. 
997. URCEOL. HYALINA. P. Quel. p. 398.

- Se rapproche de la précédente. Elle est sphérique, transparente, blanche. tuberculeuse à côtes ciliées, très petite. - Sur le bois humide. notarnment sur le peuplier, le noyer. - StAmand et ailleurs . - Commun - Automne. Printemps.

998. URCEOL. POPULICOLA. Ch. R. - Alb. de l'Est. - (Peziza). - J'ai trouvé cette espéce sur un morceau de peuplier carié, déjá envahi par Bulgaria pulla; en automne 1885. à Lisse. Ses petites cupules urcéolées, sessiles rouge-incarnat, parsemées de poils blanchâtres sont d'un bel effet; elles reposent sur les fibres Jigneuses humides, colorées en rose. - RR. Nouv. Esp.

2e GENRE. - TAPESA. - Fries.

999. TAPES. ROS E. Pers. - Alb. de l'Est. (Pe$z i z a)$. - Commun sur les rameaux secs de Rosa canina. - Bois et bords des chemins. - ÉtéAutomne. - Partout.

1000. TAPES. PRUNICOLA. Fuckel. - Alb. de l'Est. - (Peziza). - Croît sur les branches mortes de Prumus spinosa tombées à terre et recouvertes de mousse. - AC. - Soulanges, St-A mand, etc.

1001. TAPES. FUSCA. Pers. - Alb. de l'Est. (Peziza). - Sur les rameaux et sur les branches d'Alnus glutinosa, tombés sur le sol humide. - CCC. - Partout. - Automne. Printemps. 
1002. TAPES, ERRATICA. Pers. - Alb. de l'Est. - (Peziza). - Commun sur les lattes et planches de chêne pourrisant dans les caves ou sur des troncs cariés. - AC. -. St-Amand.

1003. TAPES. STOBILINA. Fries. - Alb. de l'Est. - (Peziza). - Sur les cônes de Pinus abies tombés à terre. Subiculum brun. - AC. Ablancourt, Aulnay-l'Aître.-A utomne, hivers doux.

3e GENRE. - MOLLISHA. - Fries.

100'. MOLL. CINEREA. Batsch. - Alb. de l'Est.

- (Peziza). - Espèce très commune sur le bois pourri des vieux troncs et charpentes séjournant à l'ombre dans les lieux humides.

- Consistance trémelloüde. - Toute l'année. Partout.

1005. MOLL. ATRATA. Pers. - Alb. de l'Est. - (Peziza). - Sur tiges d'Angelica sylvestris, et de Sambucus ebulus. -- AC. - AutomnePrintemps. - St-Amand. Ablancourt, etc.

1006. MOLL. POA. MICROPEZIZA POA. Ch. $R$.

- Vavray. - Bois. - Sur chaumes de Poa. AR. - Automne.

1007. MOLL. IMBERBE. Bull. - Alb. de l'Est. - (Peziza). - Commun sur les troncs cariés, à demi enfouis dans le sol. - Bois de St-Amand.

- Printemps, Automne.

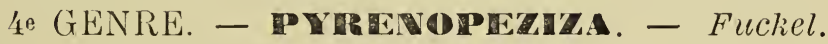

1008. PYREN. GRAMINIS. Desm, - Alb. de l'Est. - (Peziza). - Cette espèce croît au printemps 
sur les chaumes et sur les feuilles de carex sylvatica et de Poa. - Dans les bois de StAmand à St-Lumier. - AC.

1009. PYREN. ChaIlletil. Pers. - Alb. de l'Est. - (Peziza). - Sur tiges d'Angelica sylvestris desséchées. - AC. Dans les bois, sur les bords du Fion de St-Amand à La Chaussée. - Automne. hiver.

1010. PYREN. ERYNGII. an. LABIATA. Desm? - Alb. de l'Est. - (Peziza). - Petites cupules noires, à marge décolorée, denticulée. - AC. Sur les feuilles d'Eryngium campestre desséchées. - St-Amand, Soulanges. - Printemps.

1011. PYREN. JACE $A$ et PYREN. GALII. Alb. de l'Est. - (Peziza). - Ces deux espèces dont la première croît sur ies tiges de Centaurea Jacea et la seconde sur les tiges de Galium Mollugo me semblent être l'ancien Peziza phacidiö̈des de Fries. - On les rencontre après l'hiver le long des termes et des chemins, à Soulanges, Bassu, etc. - AC.

\section{5e GENRE. - PSECDOPLZIZA. - Fuckel.}

1012. PSEUdO - PEZ. TRIFOliI. Desm. - Alb. de l'Est. - (Ascobolus). - Croît à la face inférieure des feuilles de Trifolium pratense, sur de petites taches rondes, brunes. Le disque grisâtre est ponctué granulé comme celui des Ascobolus. - Fin de Juin ; dans les champs . sur le bord des chemins. - Parlout. - CC. 
1013. PSEUDO - PEZ. MEDICAGINIS. Fuckel. Alb. de l'Est. - (Phacidium). - Cette espéce envahit la face supérieure des feuilles de Medicago sativa, en Été et en Automne, dans les prairies artificielles. - St-Amand. - CCC.

1014. PSEUDO - PEZ. RANUNCULI . Kickx. Alb. de l'Est. - (Phacidium). - Croît à la face supérieure des feuilles de Ranunculus repens et $R$. bulbosus. - Prairies, bords des chemins. - St-Amand, La Chaussée, etc. - C. - Automne.

\section{PA TEL LAR I A CÉS.}

1er GENRE. - HETERDSPII AERA. - Grév.

1015. Heter. PATELla. Fries. - Alb. de l'Est. - Très commun sur les tiges desséchées de Daucus carota et de Linaria vulgaris. - La forme conidiophore se présente en automne et ce n'est qu'après l'hiver qu'apparaît la forme ascophore. - Chemins herbus; le long des champs; autour des habitations.

2e GENRE. - HECANHION. - Rabenh.

1016. LECAN. ATRUM. Rab. - Alb. de l'Est. (Pritellaria). - Espéce commune sur les rameaux des rosiers et des ronces, sur le bois de chêne et de sapin. - Partout. - Toute l'année. 
3e GENRE. - DERMATE. - Fries.

101\%. DERM. CERASI. Fries. - Alb. de l'Est. (Cenangium). - Envahit les branches et rameaux de cerisier, coupés et mis en tas à l'humidité. - C. - Automne-hiver. - Dans les bois. Les cupules sont précédées ou accompagnées par leurs pycnides. (Micropera).

1018. DERM. FURFURACEA. Fr. - Alb. de l'Est. - (Cenangium). - Récoltè sur les branches d'Alnus glutinosa tombées à terre, dans le parc du château d'Aulnay-l'Aître. - AR. - Été. 1019. DERM. VERNICOSUIM ? Fuckel. - Alb. de l'Est. - (Cenangium). - AR. sur les branches mortes du Prunus spinosa. Se rapproche de Dermatea cerasi par la forme générale de ses cupules ell cônes renversés et entourées de spermogonies. - Elle s'en distingue néanınoins par son disque noir à marge rousse, striée; par ses spermogonies noirâtres et non rosées. (Microper( $)$ enfin par ses spermaties plus courtes et non septées. - Récoltè à Ablancourt dans le mois de fèvrier.

1020. DERM. FASCICULARIS. Fries. - Alb. de l'Est. - (Cenangium). - Récolté sur rameaux et branche de salix capraca une seule fois. A Aulnay-l'Aître au lieudit la liouge-Terre. Automne. 
4e GENRE. - PEZLCULA. - Fuckel.

1021. PEZIC. CRATAEI. Awd. - Alb. de l'Est. J'ai récolté cette rare espèce sur les branches mortes de Cratcegus oxyacantha, encore adhérentes au tronc; dans la plantation de pins réservée du château d'Ablancourt. - Printemps. 1022. PEZIC. RESINA. Fuckel. - Alb. de l'Est.

- Cette espéce d'un beau jaune d'or croît sur la résine du Pinus sylvestris. - Fuckel et Nylander la considéraient comme l'état ascophore du Nectria Resince, dont on ne connaissait que la spermogonie. La découverte que j'ai faite du Nectria muni de théques, lève toute espéce de doute et permet de supprimer la parenté de ces deux espéces, si différentes l'une de l'autre! - AC. - Automne-hiver. St-Amand.

1023. PEZIC. EUCRITA. Kasten. - Alb. de l'Est. - Espéce commune en Automne sur les racines déterrées et sur les branches tombées de Pinus sylvestris. Sessile, obconique, immarginée, glabre jaune d'ocre. Thèques assez larges contenant de 4 à 6 spores elliptiques, allongées, un peu courbes, incolores, munies de plusieurs gouttelettes. - St-Amand, Soulanges, etc.

5e GENRE. - CEMAMGIUN. - Fuckel.

102'. Cenang. Ferruginosum. Tul. - Alb. de l'Est. - Cupules brunes sortant de dessous 
l'épiderme des branches et rameaux du Pinus sylvestris, en groupes de 2 à 6 . - Soulanges, Bassu, etc. - AC. - Automne.

Var. Strobilini. - Plus petit que le type; croît sur les écailles des cônes. - Mêmes localités.

1025. CENANG. PRUNASTRI. Fuckel. - Sur Prunus Spinosa. - Ablancourt. - AC. - Automne. 1026. CENANG. Viticolum. Fuckel. - Alb. de l'Est. - (Tympanis). - J'ai rencontré une seule fois cette espèce rare sur de vieux sarments de vignes mis en tas et exposés toute l'année à l'humidité. - Près du village de Lisse , en Octobre 1882.

1027. CENANG. SAlignUM. Fuckel. - Alb. de l'Est. - (Tympanis). Cette espéce croît sur les branches mortes du Salix caprcea; elle est trés rare. je l'ai récoltée dans une plantation de saules et de pins, sur les côtes de Bassu. Les cupules noir-verdâtre groupées étaient, les unes sphériques (spermugonies) les autres pézizoïdes (ètat parfait.) - Printemps.

10\%8. CENANG. ERICGE Fuckel. - Alb. de l'Est. - Je n'ai récolté que deux fois ce Cenangium sur les tiges mortes de Calluna vulgaris; la première, dans les bois de Sermaize, en Automne, et la deuxième au mois d'Août, dans les $A r$ dennes, à la dernière session de la Société botanique de France. - Fuckel ne cite que la Pycnide de l'espéce, j'ai été assez heureux pour trouver l'état ascophore. - RR. 
1029. CENANG. CONSPERSUM. Fuckel. - Alb. de l'Est. (Tympanis). - Sur branches mortes de pommier et de peuplier. Jardins. Automne. - AR. - Groupes composés de cupules ascophores et de pycnides pyriformes entourant la base des cupules. Thèques grandes; spores rondes, très nombreuses ; spermaties ovales portées par des basides arborescentes.

Var. Oxyacanthæ. - Sur les branches de l'aubépine. - St-Amand. - RR.

6e GENRE. - TrochmLA. - Fries.

1030. TROCh. CRATERIUM. Fries. - Alb. de l'Est. - Croît sur les feuilles de lierre dont elle envahit les parties mortes et décolorées d'une foule de petits points noirs, pézizoïdes. - CC. - Automne. - Partout.

\section{P H A CI D I A CÉS.}

1er GENRE. - RHITHMMA. - Fries.

1031. RHYT. ACERINUM. Tul. - Alb. de l'Est.

- Croît sur les feuilles d'Acer pseudo-platanus.

- CC. en Automne. - Partout. - Fructification complète au printemps. - RR. - Spore plus petite que celle de Rhy. Salicinum.

1032. RHYT. PUNCTATUM. Rbh. - Alb. de l'Est. - Ce Rhytisma me semble n'être qu'une variété de l'espéce précédente; cependant Fuckel 
l'adopte comme une espéce bien distincte. Il croît sur le même support et à la même époque. - AC. - St-Amand.

1033. RHYT. SAliCiNUM. Fries. - Alb. de l'Est. - Sur les feuilles de saule Marceau, dans les bois en Automne. - Partout. - CC. - Ce n'est qu'au mois d'Avril suivant qu'apparaît l'état thécasporé sur les feuilles tombées à terre. Spores aculéiformes.

103'. RHYT. MAXIMUM. Tul. - Alb. de l'Est. - J'ai récolté plusieurs fois l'espéce désignée sous ce nom par Tul. Sur les branches des saules qui bordent la Marne à Soulanges. J'ai pu constater par l'examen microscopique, que les formes secondaires. Discella carbonacea. Br. et Pilidium fuliginosum. Sacc. envahissaient seuls les rameaux à l'exclusion du type ascophore. Suivant Fuckel ce type parfait serait le Cryptospora salicella inscrit dans notre catalogue dans l'ordre des Nuclées: (Pyrénomycètes). Été, Automne. - AR.

1035. RHYT. ONOBRYCHIDIS. $D C$ - Alb. de l'Est. - De même que pour l'espéce précédente, nous avons récolté très fréquemment sur les feuilles de sainfoin la spermogonie nommée par Saccardo Placospharia Onobrychidis - Je doute que l'état ascophore existe dans nos parages. - Fin de l'Eté. - Partout.

2: GENRE. - S'TEGRA. - Frics.

1036. STEG. Illicis. Rok. - Alb. de l'Est. - 
Sur les feuilles mortes d'Ilex. aquifolium. - Bois de Sermaize à Cheminon. - AC.

- Automne. - Théques souvent remplies de granulations, mais normalement octospores.

$3^{\mathrm{e}}$ GENRE. - PHA CHDHM. - Fries.

103\%. PHAC. CORONATUM. Fries. - Alb. de l'Est. - Sur les feuilles de chêne desséchées. - Petits groupes au centre d'une tache blanche. Forme arrondie. - CC. - Dans les forêts - Automne, hiver.

1038. PHAC. DENTATUM. Schmit. - Alb. de l'Est. - Sur les feuilles de chêne, au centre d'une tache blanche; forme anguleuse. - CC. Automne, hiver.

1039. PHAC. PINI. Fries. - AC. - Sur Pinus Sylvestris.

1010. PHAC. REPANDUM . Fries. - Alb. de l'Est. Phac. Vernale de Fuckel. - Commun sur les tiges des grandes herbes. notamment sur les Rubircées : Galium mollugo, Sherardia. arvensis, etc. - Automne. Eté. - Moissons. bords des chemins. - CC. - Fuckel a divisé cette espéce en $P h$. vernale et $P h$. autumnale. 10'1. PHAC.? VIRIDE. Ch $R$. - Alb. de l'Est. - J'ai trouvé cette nouvelle espéce sur un rameau de peuplier dénudé - Je l'ai placée parmi les Phacidiacés en raison de la déhiscence de sa cupule en plusieurs segments et à cause de son disque pézizoïde - Elle croît incrustée dans l'écorce. - Les cupules d'abord 
fermées, extérieurement brun-clair et granuleuses, se déchirent en 4 ou 5 segments et laissent voir un disque vert-foncé. - Les cupules ont à peine 1 à 2 mill. de largeur. Les thèques renflées en massue contiennent huit spores aculéiformes (0:04), remplies de gouttelettes. Paraphyses simples, incolores, un peu plus longues, recouvertes au sommet par un epithecium brunâtre analogue à ceux des Lichen. - RR. - St-Amand. - Printemps.

$4^{\mathrm{e}}$ GENRE. - PROROLIS. - Fries.

1042. PROP. VERSICOLOR. Fries. - Alb. de l'Est. - Croît communément sur les branches mortes de saule, de prunier, de hêtre etc. Toute l'annèe - Hymenium blanchâtre, puis gris de fer, ponctué. - Bois, Jardins. Partout.

10'13. Var. Strobilina. - Cette variété un peu plus rare et plus petite, croît sur les cônes de Pinus abies et Pinus sylvestris.

\section{S T R I C T É S.}

1er GENRE. -MABROSTICTIS. - Fuckel.

10'依. HABR. CHRYSOPHAA. Fuckel. - Alb. de l'Est. - Sur les branches et sur les rameaux des jeunes peupliers, encore adhérents au tronc. - St-Amand, Lisse, Bassu. - Automne - AC. Disque d'un rouge-cuivre entouré par l'épiderme. 
1015. HABR. RUBRA. Fuckel. - Alb. de l'Est.

- Ressemble á la précédente espéce, mais elle est plus petite, le disque moins foncé. - sa station a lieu sur les branches d'Orme tombées à terre. - AC. - au printemps. - St-Amand, Soulanges, etc.

2 GENRE. - STICTIS. - Pers.

10'6. Stict. RAdiAta. Nées. - Alb. de l'Est. - Très commun sur les tiges herbacées et sur les brindilles de bois tombées à terre, dans les bois. - St-Amand, Vanault-les-Dames, etc. Toute l'année.

3e GENRE. - ScHIzozYLCM. - Pers.

10'ii. SCHIZ. ERUGINOSUM. Fuckel. - Alb. de l'Est. - Sur les tiges mortes de Rubus ccesius à l'automne et au printemps. - Dans les bois de St-Amand à St-Lumier. - AC. - Cette petite espéce a la forme d'un Ophiobolus.

10'18. SCHIZ. PUPULA. Fries. - Alb. de l'Est. Sur les rameaux de Cornus Sanguinea, de Prunus Spinosa etc. - Dans les bois taillis à StAmand, Soulanges, etc. - Spermogonie, Melanostroma, Corda.

10'19. SCHIZ. GRAMINUM. Karst. - Alb. de l'Est.

- Récolté plusieurs fois sur les feuilles mortes de Festuca Sylvatica, au Printemps. - Bois de St-Amand à St-Lumier. - Cette espéce ressemble extérieurement à Oomyces carneo-albus de Cooke. 
4e GENRE. - XYLOGRAPHA. - Fries.

1050. XYLOG. ? PARAllela. Fries. - Alb. de l'Est. - Sur tiges desséchées de Solidago virga aurea, et sur Genista tinctoria. - En automne, à Aulnay-l'Aître au lieu dit la Rouge-terre, et dans les bois de Charmont. - Le disque est rouge-incarnat, entouré par un limbre noirâtre qui se ferme par le sec. - AR.

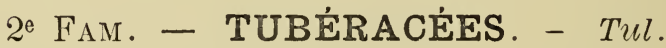

Cupules (Plissées-chiffonnées) intérieures.

19r GENRE. - ELAPMOMYCES. - Tul.

1051. ElAPH . GRANUlatus. Fries. - Atl . Champ. Ch. R. et Roze: p, $249 \mathrm{pl}$. LXXI . Récolté au pied des chênes ou croît ordinairement la trufte à Verzy, près de Reims. - Eté. - AR.

1052. ElAPH. MACUlatus. Lév. - Atl. Champ. Ch. R. et Roze, p. 2'18, pl. LXXI . - Récolté dans les mêmes terrains que le précédent et á la même époque. Une exploration sérieuse pourrait y faire découvrir les espéces voisines. Elaph aculeatus, Leveillei. etc. que j'ai trouvées près de Montmorency dans des conditions semblables. 
$2^{e}$ GENRE. - TUBERE. - Mich.

1053. TUB. MESENTERICUM. Vitt. - Atl. Champ. Ch. R. et Roze, p. 2't6:pl. LXXII . - Cette espéce croît dans les bois de la Montagne de Reims. - Elle m'a été communiquée par le $\mathrm{D}^{\mathrm{r}}$ Maillart. - Sa surface est verruqueuse. brune ; elle possède un parfum assez prononcé. Les spores sont brunes, entourées d'une aréole. - RR. - Février, mars. - Verzy.

105'. TUB. ABUMr. Bull. - Atl. Champ. Ch. R. et Roze. p. 2ł6, pl. LXXII. - Cette espéce a été récoltée en Eté sous des peupliers qui servaient de limite à un pré, dans les environs de Vitry-le-François. - AR . - La suriace esi lisse et d'une couleur blanc-jaunâtre. Les spores sont jaunâtres, entourées d'une aréole, sans ởeur spéciale.

1055. TUB. UNCINATUM. Chatin. - Atl. C'iamp. Ch. R. et Roze, p. 2'16, pl. LXXII.

$3^{3}$ GENRE. DOG S'ARE. - Corda.

1056. OOG. CIBARIUM. Cord. - Atl. Champ. Ch. R. et Roze. p. '2'3. pl. LXXII. - Cette espéce ainsi que l'oog. brumalis (distinctes l'une de l'autre par des caractères peu appréciables) m'a été envoyée par le $D^{r}$ Maillart médecin ‘ Verzy. - Elle a un parfum qui rappelle les truffes récoltées dans le Périgord. Aussi la 
récolte, souvent abondante, ètait-elle entreprise, il y a quelques années, par des commerçants des environs de Périgueux pour en tirer parti. - Les spores sont noires et couvertes d'aiguillons. - Signalée seulement (dans le Département) sous les chênes de la Montagne de Reims, entre Ay et Verzenay. 
9e Ordre. - NUCLÉÉS. - Quélet. (Pyrénomycètes). - Fries.

$1^{\text {re }}$ FAM. - Périsporiacées. - Fries.

Périthèces membraneux, coriaces ou charbonneux. sans ouverture.

Sub-Famil. 1. - Erysipheæ. - Lév.

19R GENRE. - PODOSPHACRA. - Kunze.

105\%. PODOSPH. TRIDACTYLA. Wallr. - Alb. de l'Est. - Croît sur les feuilles de Prunus spinosa. Les pèrithèces ne contiennent qu'une thèque octospore ; les filaments mycéliens sont allongés, terminés par des découpures dichotômes élégantes. - CC. - Automne.

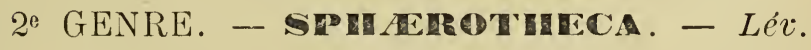

1058. SPHAR . CASTAGNEI . Var humuli . Kickx. Sacc. - Alb. de l'Est. - Cette espéce croît à la face supérieure des feuilles de houblon. Les périthèces brunâtres, enfoncés dans le mycelium, ont des appendices flexueux qui se confondent avec ceux du mycelium; ils renferment une seule théque octospore. AC. Juillet. - St-Amand. 
30 GENRE. - PIIYHACTIMHA. - Lév.

1059. PHYLL. SUFFULTA. Sacc. - (Ph. gutiata. Walir.) - Alb. de l'Est. - Espéce très commune à la face inférieure des feuilles du Corylus avellana. - Les périthèces garnis de poils raides, renflés-bulbeux à la base, renferment piusieurs théques $2-4$ sporidies. Automne.

\section{4e GENRE. - UNCINULA. - Lév.}

1060. UNCIN. ADUNCA. Lév. - Alb. de l'Est. Cette espéce croît à la face supérieure des feuilles de Salix caprcea. - Les filaments blancs äressés en colerette autour des périthèces leur donne l'aspect de petites marguerites en miniature. - CCC. - Automne.

1061. UNCIN. ACERIS. Sacc. - Alb. de l'Est. Cette espéce croît abondamment à la face supérieure des feuilles d'Acer campestre - Les Périthéces un peu dẻprimés sont garnis d'appenåices bifides-oncinulés au sommet. - Eté. Automne.

5e GENRE. - MEHCHOSPRAERA. - Lév.

1062. MICROSPH. GROSSULARIAE. Lév. - Alb. de l'Est. - Ceite espèce envahit les feuilles de Ribes grossularia. - Elle croît de préférence à la face supérieure dont l'aspect est poudreux ; c'esî le résultat des débris de 
l'Oidium erysiphoïdes qui a précédé l'apparition des périthéces. - CC. en Automne ou á la fin de l'été - dans les haies - St-Amand. 1063. MiCROSPH. PENICjLlata. Lév. - Alb. de l'Est: - Commun sur les feuilles d'Alnus glutinosa. - Périthèces munis de 8 à 12 appendices terminés par des divisions plusieurs fois dichotômes. - Fin de l'Eté, automne.

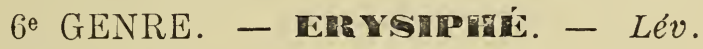

106'. ERYS. LAMPROCARPA. Lév. - Alb. do l'Est. - Croît sur les feuilles de Plantago et de Galeopsis ladanum. ordinairement sur les deux faces. - Périthèces à filaments courts, inégaux, bruns; théques de 10 à 20 munies de 2 sporidies. - AC. en Automne. - StAmand.

1065. ERYS. TORTILIS. Lév. - Alb. de l'Est. Hypophylle sur Cornus sanguinea. - Mycelium fugace; appendices très longs, fasciculés. thèques 3-4 renfermant 4 sporidies. - CC. Automne.

1066. ERYS. HORRIDULA. Lév. - Alb. de l'Est.

- Hypophylle sur Symphytum et sur Borrago. Périthèces à appendices courts, flexueux, contenant 10 à 20 Théques à $2-4$ sporidies. - AC. - Prés, bois, bords de la Marne.

1067. ERYS. UMBELLIFERARUM. De Bary. Alb. de l'Est. - Cette espéce croît communèment sur les feuilles et tiges d'Heracleum spondylum et sur Angelica sylvestris, à la fin de 
l'Eté. - Elle occupe la face supérieure qui prend une teinte grisâtre, ponctuée, appendices peu nombreux confondus avec le mycelium ; plusieurs thèques contenant de 2 à 4 sporidies. - Automne. - Prés, bois.

1068. ERYS. COMMUNIS. Fries. - Var. Nitida Wallr. - Alb. de l'Est. - Cette variété de l'Erys. Communis envahit les feuilles et les tiges d'Aconit napel. - Les périthèces sont plus superficiels et d'un noir plus luisant que dans le type, ils contiennent de 2 à 5 théques munies de 4 à 8 sporidies. - AR. - Jardins. - St-Amand, St-Lumier.

1069. ERYS. MARTII. Lév. - Alb. de l'Est. Cette espéce, commune sur les crucifères et sur les ombellifères, ne diffère pas, selon de Bary, de l'espéce précédente. - St-Amand. Soulanges, etc.

10\%. ERYS. ULMARIÆ. Pers. - Alb. de l'Est.

- Espèce commune à la fin de l'Eté sur les feuilles de la Spirée Ulmaire. - Ses périthéces sont très petits, luisants ; ils contiennent de 6 à 8 thèques octospores. - Bords du Fion à St-Amand et ailleurs.

1071. ERYS. GRAMINIS ? D. C. - Recueil 10. p. 31. - L'Erysiphe graminis, marqué ici d'un point de doute, envahit les feuilles et les tiges de Poa nemoralis et de Festuca sylvatica. Les pèrithèces sont ornés de 6 ou 8 appendices simples et renflés à la base. Il constitue probablement une varièté de Phyllactinia suffulta 
non signalée par les auteurs et différente de l'Erysiphe graminis de Saccardo.

1072. ERYS. TUCKERI. Berk. Kickx. - Alb. de l'Est. - Cette espéce si redoutée des viticulteurs ne se présente dans nos contrées que sous la forme conidienne, Oidium Tuckeri, et sous la forme spermogonienne, Cicinnobolus Cesati ; ces états præformatifs sont indiqués dans notre catalogue, le $1^{\mathrm{er}}$ parmi les Hyphomycétes, le $2^{\mathrm{e}}$ parmi les Sphéropsidées.

\section{Sub-Famil. 2. - Perisporieæ. - Sacc. \\ 1 er GENRE. - EUROTICMT. - Link.}

1073. EUROT. HERBARIORUM. Link. - Alb. de l'Est. - Très commun sur les plantes mal séchées des herbiers et sur les fruits. - Ses périthèces sphéroïdes, jaune-soutre, renferment des théques sphériques, octospores; l'état conidien est l'Aspergilius glaucus.

10\%. EUROT. CORIORUM. Wallr. - Alb. de l'Est. - J'ai trouvé cette espéce sur les harnais de cuir, graissés et sejournant depuis longtemps dans une écurie. - Sa couleur est d'un beau rouge-carminé ; son appareil conidien est le Torula Corii, (Ch. R.) habitant le même support.

1075. EUROT. EPIXYLON. Kunze. - Alb. de l'Est. - Commun sur les claies d'osier anciennes, humides placées dans les caves. 


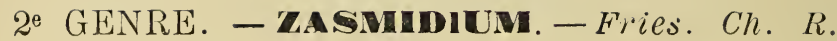

1076. ZASM. CELLARE. Kickx. Ch. R. - Alb. de l'Est. - J'ai récolté plusieurs fois cette espéce sur les lattes de chêne qui supportent les bouteilles mises en tas dans les caves; les filaments mycéliens sont le Racodium cellare et son appareil thécasporè est un véritable Eurotium pour la fructification. Les Périthèces sont petits, sphériques, astomes, noirs, hérissés de filaments en massue, cloisonnés, jaunes . Les thèques sont rondes, octospores ; sporidies ovales hyalines puis brunes. - St-Amand. -Eté, automne.

3e GENRE. - CETHAMOTHECA. - Fuckel.

107\%. CEPH. SULFUREA. Sacc. Fuckel. - Alb. de l'Est. - On rencontre cette espéce sur les chaumes de blé humides et pourrissants au milieu des gerbes mises en tas; elle tire son nom de la forme exceptionnelle de son hyménium composé de filaments rameux, courts, terminés par des théques rondes, renfermant 8 sporidies, rondes-ovales, brunes; les perithéces qui les contiennent sont glabres, jaunàtres, munis seulement à la base de longs filaments rampants ; ils succèdent à la forme conidienne beaucoup plus commune l'Actinospira chartareum de Corda. - St-Amand, St-Lumier. - 
Automne, printemps. - Dans les meules de paille exposées à l'injure du temps.

1078. CEPH. HISPIDA. Ch. $R$. - Alb. de l'Est.

- Cette périsporiacée que nous plaçons dans le Genre Cephalotheca. à cause de ses périthèces astômes, hispides et de ses théques rondes, a été trouvée sur écaille de vieux pommier mort, encore debout, dans un jardin de StAmand. - Les théques ne contiennent que 4 sporidies ovales, hyalines et non colorées.

4e GENRE. - AsCOTRICHA. - Berk.

10\%. ASC. Chartarum. Berk. - Alb. de l'Est.

- Assez commun sur le papier de tenture et sur les fragments de journaux délaissés dans les lieux sombres et humides. Les périthèces sphériques sont hérissés de poils rigides, noirs, ils reposent sur les filaments dichotômes et conidiféres de Myxotrichum chartarum. Théques cylindriques. - Sporidies brunes 6-8. - StAmand. - Automne.

5 e GENRE. - ASTERINA.

1080. ASTER. SCABIOS.AE. Ch. R. - Alb. de l'Est. - J'ai trouvé cette espèce sur tige desséchée de Scrbiosa Columbaria, à St-Amand, en Automne. - Elle appartienl à la section Asteridium de Saccardo, à sporidies pluriseptées et à théques ovoïdes; elle est très voisine d'Asterina Eugenice de Montagne, et est munie, comme cette dernière d'un ostiole central. 
6e GENRE. - ANMXIA. - Fries.

1081. ANIX. WALLROTHII. Fuckel. - Alb. de l'Est. - J'ai récolté cette espèce sur les èxcréments de chien, le 7 mars 1880. - Ses périthèces sont d'un noir mat, sphériques, de la grosseur d'un grain de navette; les théques sont cylindriques à 8 sporidies rondes, incolores, lisses, monostiques, occupant le sommet de la thèque. Sa forme conidienne me parait être le Mycogala parietinum de Saccardo. - RR. - St-Amand.

\%e GENRE. - IRICIIONIA. - Boud.

1082. RICH. VARIOSPORA. Boud. - Rev. Myc. 1885. - Rec. 10, p. 42. - Cette espèce m'a été dédiée par mon savant ami Boudier de Montmorency; elle croît sur les racines de l'asperge. - Les périthèces sont sphériques , astômes, noirs, superficiels; les théques larges, entourées de paraphyses rameuses, contiennent une à 3 sporidies elliptiques, comprimées au milieu, incolores, munies de 2 grosses sporules; ces spores, en vieillissant, grossissent considérablement, deviennent uni-cloisonnées, d'un brun-noir opaque et sont hérissées de filaments tortueux remarquables, identiques pour la forme et la structure aux paraphyses. - On peut voir par le dessin de notre Album, les théques jeunes avec leurs sporidies incolores de dimension 
normale, puis une sporidie brune: enveloppée de fragments de paraphyses et quadruplée de volume après sa sortie de la thèque. - RR. 12 Mai 1885.

8e GENRE. - PERISPORIUM. - Fries.

1083. PERISP. FIMETI. Sacc. - PREUSSIA. FIMETI. Fuckel. - Alb. de l'Est. - Cette espéce croît sur les crottes de lapin; elle est précédée par des taches blanches de Torula nivea, sa forme conidienne. - AC. au printemps. - St-Amand.

108'. PERISP. SECALE. Ch. R. Sacc. - Alb. de l'Est. - J'ai trouvé cette espéce plusieurs années de suite sur les chaumes du seigle, à St-Amand. - La description en a été publiée dans le Bulletin de la Socièté de Vitry-le-François et par Saccardo; elle se rapproche de l'espéce précédente par ses caractéres généraux, et s'en distingue par sa station et par les filaments blancs et rayonnants situés à la base des périthèces. - RR.

1085. PERISP. MATRICARIÆ. Ch. R. - Alb. de l'Est. - Cette espéce croît sur les tiges pourrissantes de la Matricaire des jardins mélangées aux detritus mis en tas. - Elle paraît au printemps; à son aspect extérieur, on la prendrait pour Mycogala parietinum; elle en différe par ses sporidies lisses et non tuberculeuses, obovales et non sphériques, brun-clair et non jaune-soufre. L'absence des théques classe cet 
état præformatif parmi les Species dubice Perisporiacearum de Saccardo prés de Perisp. Tragopogi.

\section{9e GENRE. - DHMEROSPARIRM. - Sacc.}

1086. DIMER. PUlChrum. Sacc. - Alb. de l'Est. - Jusqu'á présent, je n’ai trouvé sur les feuilles languissantes de Cornus sanguinea, que l'ètat conidien Coniothecium Questieri. Sacc.

10 GENRE. - ZOPHIA. -Rabh. Sacc. Alb. de l'Est.

108\%. ZOPF. RHIZOPHILA. Rabh. Ch. R. - Rec. 11. p. 41. - Cette Périsporiacée croît ainsi que le Richonia Variospora, sur les racines d'asperges. - Elle a le même aspect extérieur, mais elle en diffère par la forme des sporidies et des thèques. Les théques sont larges, ovales ou obovales, elles contiennent de 4 à 8 sporidies apiculées; celles de Richonia sont allongées, claviformes et ne renferment que deux ou 3 spores sans apicules. - RR. - Jardins.

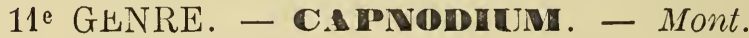

1088. CAPN. SAlicinUm. Mont. - Alb. de l'Est. - Les feuilles et les rameaux de saule envahis par cette espéce semblent noircis par la fumée; la forme conidienne composée de filaments toruleux, bruns, donne naissance aux périthèces thécasporés, pyriformes, en massue, renflés et percés au sommet par un ostiole. Les théques 
obovales renferment 6 à 8 sporidies brunes, elliptiques, muriformes. - AR. - Kécolté entre St-Amand et Lisse.

1089. CAPN. TILIE. CAPN. CITRI. CAPN. FUMAGO. (Vitis). - Ces Périsporiacées sont fréquentes, à l'état conidien seulement, sur les feuilles de Tilleul, d'Oranger et de la Vigne, sous le nom de Cladosporium fumagi.

2e FAM. - Sphériacées. - Fries.

Périthèces charbonneux, membraneux ou coriaces, sans ouverture.

Segt. I. - Allantosporées

1er GENRE. - ENCHMOA. - Fries.

1090. ENCHN. FRIESII. Fuckel. - Alb. de l'Est.

- Cette espéce croît sous l'épiderme des tiges ou troncs de sambucus nigra, entre les fentes de l'écorce et sur le bois dénudé. - Les périthèces sont réunis comme ceux des Valsa ou épars, munis de longs cols glabres et noirs, plongés à la base dans un substratum tomenteux, brun, envahissant parfois les sphéries; les théques sont en massue allongée et les sporidies botuliformes avec 4 gouttelettes. - AC. Automne, hiver. - St-Amand. 
1091. ENCHN. CLEMATIDIS. Ch. $R$. - Alb. de l'Est. - Cette espèce croît sous l'écorce des vieilles tiges mortes de Clematis vitalba. - Elle ressemble beaucoup à Ench. Friesii, parasite sur Sambucus, par la disposition de ses périthèces agglomérés en cercle et par ses longs cols flexueux; elle s'en distingue cependant par deux caractères importants ; l'existence de longues paraphyses et la cloison médiane des sporidies. Je l'ai truuvée, de même que l'Ench. Friesii, accompagnée d'un Cephalotrichum (Stysanus) qui semble en être l'état conidien. CC. -- St-Amand. - Bois, haies des jardins. Automne-hiver.

GENRE. - CAELSPHART - Sacc.

1092. CAELOSPH. CUPULARIS. Karsten et Sacc.

2e GENRE. - CALASPHAERA. - Tul.

1093. CAL. PRINCEPS. Tul. - Alb. de l'Est. Cette belle sphérıcée croît sur les troncs et sur les grosses branches de Cerisier coupés et exposés à l'air depuis longtemps. - Ses périthèces placés sous l'écorce sont sphériques. disposés en cercles et leurs longs cols tortueux se joignent en un disque à la surface de l'épiderme; les pycnides sont mêlées aux périthèces, mais en petit nombre. - CC. en automne et au printemps. - Jardins.

109'. CAL. MiNima. Tul. - Alb. de l'Est. Celte espéce, moins développée que la précé- 
dente croît aussi sur le Cerasus, elle ressemble un peu au Cal. Vibratilis; ses thèques sont extrêmement petites et pourraient être prises pour des sporidies, elles sont portées par des pédicules arborescents compacts et nombreux. - AR. - Avril-mai 1883. - St-Amand.

1095. CAL. VIBRATILIS. Fuckel. - Alb. de l'Est.

- Cette espèce croît sur les branches de Prunus spinosa; elle posséde des périthèces disposés en rosette. moins nombreux que ceux de Cal. princeps, renfermant des théques plus développées et remarquables par la disposition de leurs pédicules en faisceaux arborescents. AC. - Février 1882, - St-Amand.

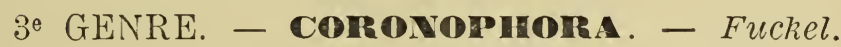

1096. CORON. GREGARIA. Fuckel. - Alb. de l'Est. - J'ai trouvé cette espèce sur le bouleau en compagnie du Melanconis lanciformis; les

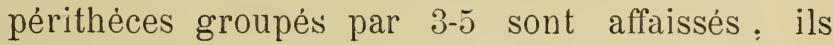
contiennent des théques ovales, amples, terminées brusquement par un prolongement filiforme; les sporidies en nombre indéterminé sont courbes. - AC. - Printemps. - St-Amand, 1879.

109\%. CORON. ANNEXA. Fuckel. - Alb. de l'Est.

- Croît en automne sous l'épiderme des rameaux d'Alnus glutinosa. - Les périthèces au nombre de 5-8 sont munis. á la base, de fibrilles brunes; les théques plus resserrées que dans 
l'espèce précédente renferment des sporidies plus grosses. - AR. - St-Amand. - Décembre 188 '.

4 GENRE. - QUATERNARIA. - Tul.

1098. QUAT. PERSOONII. Tul. - Alb. de l'Est. - Croît sur l'écorce des branches et des troncs du hêtre. - Les périthèces groupés régulièrement par 5-8 sous l'épiderme en forme de rosettes, contiennent des théques très longuement pédicellées et des sporidies un peu courtes, faiblement teintées. - Récoltée aussi sur le noyer, en février 1882. - AC. - St-Amand. - La spermogonie Libertella Faginea est plus commune.

5e GENRE. - VALsA. - Fries.

1099. VAls. CERATOPHORA. Tul. - Alb. de l'Est. - Cette espéce croît sur les petits rameaux morts de Rosa canina. - Elle forme des tubercules saillants, noirâtres, recouverts par l'épiderme et surmontés de 5 à 10 cols, divergents, du sommet desquels s'échappent de longs filaments sporigères. - AR. - Bois, haies. St-Amand.

1100. VALS. RUBI. Fuckel. - Alb. de l'Est. Ce Valsa croît sur les tiges des Rubus; il se rapproche du V. Ceratophora, Var. Rosarum; ses périthèces sont plus petits et munis de cols plus courts avec un ostiole béant, très visible. - Je n'ai pas observé de paraphyses. - 28 décembre 1885. - St-Amand. - $\Lambda \mathrm{R}$. 
1101. VALS. PINI. Alb. et sche. - Alb. de l'Fst. - Cette espéce est commune en hiver sur l'écorce des troncs de Pinus sylreatris abattus. - Elle forme des petits tubercules arronds. noir's au centre et entourés par une matière purérulente. jaune, mêlée aux ostioles réunis en disque. Theques sans paraphyses. - Sapinières: - Partout.

11(1). VALS. VITIS. Fuckel. - Alb. de l'Wst. Cette espéce croit sur les vieux sarments de vigne en compagnie de sa spermogonie, le Cytismor ritis. - Ses tubercules contiemnent 10 à 1.j périthéces qui émergent de l'écorce en lignes longitudinales. - AC. - St-Amand, etc. Hiver. printemps.

111:3. VALS. JUNIPERINA. Sacr. - Alb. de l'Est. - rroit en hiver sur les rameaux morts tombès à terre du Juniperus communis, sous forme de petits tubercules blancs, farineux, enfoncès dans l'écorce. - Cette espéce est plus petite dans toutes ses dimensions que le Vals. rini. - AC. - St-Amand. - Printemps.

110 '. VAls. GLANDULOsa. Cooke. - Alb. de l'Est. - Ce Valsa envahit les rameaux secs de l'illunthus glcudulosa; ses pustules coniques. á disque aplati au sommet. naissent sur un stròme noir. etalé sous l'épiderme ; ils contiennent de 15 a 20 périthèces: les théques sont petites, renflées en massue - AC. - Dans les jardins à soulanges. St-Amand. - Février $188 '$ '. 
110.). VALS. SYNGENESIA. Sace - Sur Sambucus nigin. - CC.

1106. VALs. Ambiens. Pers. - Alb. de l'Est. Frequent sur les branches et rameaux recouverts d'écorce de l'orme, du pommier, du prunier. etc. - Les pustules de cette espece, aplaties au sommet, sont traversées par les ostioles des périthéces qui apparaissent au dehors, disposés en cercle ou couronne. - Les thèques sont très fugaces. - CCC. - Hiver et printemps. - Partout.

110\%. VALS. SALICINA. Firies. - Alb. de l'Est. Cette espéce vient communément sur les branches séches de Salix alba; elle a l'aspect du Valsa niver dont elle diffère, par l'absence du conceptacle. par le nombre moindre des périthéces; ses théques sont fugaces et contiennent 8 sporidies moyennes ou 4 grandes spores. - CC. - Hiver, printemps.

1168 . VALS. NIVEA. Fries. - Alb. de l'Fist. -- Sur l'écorce des rameaux de peupliers morts et mis en fagots. - Remarquable par le disque rond. blanc-farineux des pustules pointillè de noir. par les ostioles des périthéces. - CCC. - Hiver. Partout. - Spermogonie. Cytispora nivea.

1109. VAls. Leucostoma. Pers. - Alb. de l'Est.

- Fréquent sur les rameaux des cerisiers. pruniers, et sur l'épine blanche. - Disque elliptique, blanc-farineux; pustules contenant de 5 à 10 périthéces. - Spermogonie. Cytispora leucostoma. -- CCC. - Partout. 
6e GENRE. - EETTEILA. - Nits.

1110. EUTYP . PRUNASTRI . Sacc . - Alb . de l'Est. - Espéce commune au printemps sur les rameaux morts du Prunus spinosa. - Tubercules munis diun conceptacle, renfermant de 15 á 20 périthéces dont les ostioles épais, 3-5 angulaires réunis à la base et divergents au sommet paraissent au dehors aprés avoir romıpu l'épiderme du rameau transversalement. - CCC. Jardins, haies. - Spermogonie. Cytispora rubescens.

1111. EUTYP. STELLUTATA. Sacc. - Alb. de l'Est. - Cette espéce est commune sur les rameaux secs de l'Orme. - Elle rompt l'épiderme en étoile et laisse apparaître une dizaine d'ostioles 4 angulaires. sortant d'un conceptacle noir: arrondi, logé dans l'écorce. - Hiver, printemps.

1112. EUTYP. BRUNAUDIANA. Sac. - Alb. de l'Est. - J'ai récolté cette espéce plus rare que les deux précédentes sur les rameaux morts et secs de Ribes rubrum, dans mon jardin. . Automne 1886. - Les ostioles sont cylindriques.

\% GENRE. - EUTYPA. - Tul.

1113. EUT. ASPERA. Fuckel. - Alb. de l'Est. Les tiges mortes de Lonicera envahies par l'Eutypa aspera sont recouvertes d'un strôme mince, cendré-rougeâtre: parsemé çá et lá de 
petites aspérités dues à la sortie des ostioles arrondis des périthèces incrustés dans le bois. - AC. dans les jardins en hiver et au printemps. - St-Amand.

1114. EUT. SPINOSA. Tul. -- Alb. de l'Est. Cette espèce est remarquable par ses ostioles coniques. tétragones et sa large cruste noire étalée sur les branches dénudées de peuplier; elle est trés commune en hiver. - St-Amand.

1115. EUT. FLAVOVIRESCENS. Tul. - Alb. de l'Est. - Fréquent sur les rameaux secs de Rosa canina et de Prunus spinosa. - Le strôme aplati, noir, de forme irrégulièrement arrondie est d'une belle couleur jaune-verdissant à l'intérieur. - Printemps, hiver. - St-Amand 1882. 1116. EUT. Ligustri. Ch. $R$. - Alb. de l'Est. - Cette nouvelle espéce croît sur les vieux rameaux de Ligustrum vulgare. - L'épiderme qui la recouvre est percé par les ostioles noirs. arrondis et sans changement de couleur, quand on le soulève on voit l'écorce convertie en une croûte noire et çá et lá des strômes en disques irréguliers. de substance noirâtre, contenant les périthéces à nucleus blanc. Les thèques sont longuement pédicellées et atténuées en fuseau au sommet. - Cet Eutypa se rapproche du Flavo virescens, à part le strôme vert-jaunâtre. - AR. - St-Amand. - Mars. IIaies, jardins.

111\%. EUT. HETERACANTHA. Sacc. - Alb. de l'Est. - Croît fréquemment sur les jeunes 
branches et sur les rameaux de Sambucus nigra morts depuis longtemps; le rameau envahi est entouré d'un strôme noir, parsemé de pustules valsiformes qui contiennent 6 ou 8 périthèces dont la base est piongée dans la substance du bois. - Jardins. - St-Amand. Soulanges. - Mars.

1118. EUT. LATA. Sacc. - Alb. de l'Est. Commun sur le bois et les branches dénudés de Noyer, d'Acer pseudo-platanus, etc. Strôme mince, noirâtre, très étendu, superficiel; sphérules incrustées dans le bois; sporidies petites; thèques accompagnées de rares paraphyses à peine visibles. - CC. - St-Amand. Hiver, printemps.

8 GENRE. - CiRYPTOSPIIARIA. - Grev.

1119. CRYPT. POPULINA. Sacc. - Alb. de l'Est. - Sur rameaux morts de peuplier noir et sur tremble. - Strôme étendu, irrégulier sous l'épiderme un peu soulevé et d'une teinte grise causée par les ostioles rugueux qui sont disséminés à la surface. - CG. dans tous les bois. - Hiver.

1120. CRYPT. MillepUNCTATA. Sacc. - Alb. de l'Est. - Sur branches mortes et troncs de Fraxinus excelsior; Spharia eunomia de Fries. Se rapproche du précédent; strôme moins ètendu et ne colorant pas ou trés peu l'épiderme ; ostioles punctiformes. - AC. dans les bois . Hiver, printemps. - St-Amand, St-Lumier, etc. 
9' GENRE. - CRYPTOVALSA. - Ces.

1121. CRYPT. PRUNI. Sacc. - Alb. de l'Est. Sur les rameaux secs de Prunus spinosa. Plaques disciformes sous l'épiderme, plus oll moins larges, traversées çà et là par les ostioles noirs, obtus, anguleux ; périthèces logés dans la couche corticale ; théques polyspores. - CCC. - Jardins, vergers. - St-Amand. hiver, printemps.

11 2. CRYPT. EFFUSA. - Sacc. - Alb. de l'Est.

- Sur sarments de Rubus fruticosus et sur Rosa canina. - Thèques polyspores; disposition des Périthèces comme dans Cryp. millepunctata. - CC. - Hiver, printemps. - StAmand. - Bords des routes et talus boisés.

1123. CRYPT. PROTRACTA. Sacc. - Alb. de l'Est. - Sur le bois dénudé d'Acer pseudo-platanus. - Strômes allongés, étroits, rugueux par la présence des ostioles coniques; périthèces incrustés dans le bois. Théques polyspores. -. AC. - Hiver, printemps. - St-Amand, etc.

10 GENRE. - DIATERTE. - Fries.

1124. DiATR. BULlatA. Sacc. - Alb. de l'Est.

- Sur rameaux secs de Salix caprcea et Populus alba. - CCC. -- Partout ; dans les haies de clôture et bois des villages. - Hiver, printemps. - Strômes en disques entourés par l'épiderme. 
1125. DIATR. STIGMA. Sacc. - Alb. de l'Est.Sur branches sèches du hêtre, du charme, de Cratcegus, etc. - Larges plaques entourant les rameaux, parsemés de petits points saillants et tétragones, formés par les ostioles des périthéces sous-jacents. - CCC . - Partout . Toute l'année.

1126. DIATR. ACERICOLA. Sacc. - Alb. de l'Est.

- Sur branches d'Erable sèches. - J'ai rencontré cette espéce une seule fois à St-Amand. - Elle n'a été signalée par Saccardo qu'en Italie. - Elle ressemble à Diatrypella quercina par ses pustules et ses disques rugueux ; mais sa station sur Acer et le nombre de ses sporidies limité à 8, le placent dans le Genre Diatrype. - RR. - Printemps. - St-Amand.

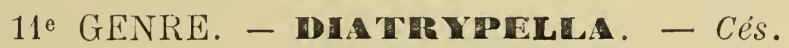

1127. DIATRplla. VERRUCIFORMIS. Sacc. - Alb. de l'Est. - Commun sur les branches mortes d'Alnus glutinosa. - Strôme verruqueux entouré par l'épiderme fendu en étoile; théques polyspores. - Ressemble beaucoup à Diatrype quercina. - Bois, bords des eaux. - St-Amand, etc. - CC. - Printemps.

1128. DIATRplla. FAVACEA. Sacc. - Alb. de l'Est.

- Sur rameaux et troncs de Bouleau servant de barrières et clôtures des jardins, à St-Amand. - AC. - Hiver, printemps. - Strômes elliptiques sortant de dessous l'épiderme fendu transversalement. 
1129. DIATRplla. NIGRO ANNULATA. Sacc. - Alb. de l'Est. - Croît sur les branches de Bouleau. - Elle se distingue du Diatr. verrucceformis par ses pustules sphéroïdes et par la ligne noire qui circonscrit les pustules á la base. - Hiver, printemps. - St-Amand. - AR.

1130. DIATRplla. MINUTA. Sacc. - Alb. de l'Est. - Sur les branches mortes du châtaignier déjà envahies par Clypeospharia castanea. - Pustules saillantes: rondes, aplaties au sommet, entourées à la base par une ligne noire, renfermant 10 à 12 périthéces. - RR. - Bois de la Montagne de Reims. - Printemps.

1131. DIATRplla. QUERCINA. Sacc. - Alb. de l'Est. - Espéce très commune sur les branches et les rameaux morts du chêne. - Pustules éparses. orbiculaires, rugueuses, noires, entourées par l'épiderme lacinié. - Dans tous les bois et forêts. - Automne, Printemps.

1132. DIATRplla. RIBIS. Ch. $R$. - Alb. de l'Est. - Cette espéce croît sur les branches mortes de Ribes nigrum. - Les pustules elliptiques sortent par une fente transversale de l'épiderme. - On pourrait la confondre avec Diatrype strumella sans ses théques polyspores, allongées. - AR. - Jardins de St-Amand. - Hiver, Printemps. 


\section{SEGT. II. - PHROSPORÉES.}

12e GENRE. - CERA'TOSTOMA. - Ces.

1133. CERAT. ROSE. Ch. R. - Alb. de l'Est. - J'ai trouvé cette nouvelle espéce prés de Bassu, sur les bords de la route de St-Amand; sur les tiges mortes de Rosa spinosissima. Il ne faut pas la confondre avec Valsa ceratophora dont les sporidies sont incolores et botuliformes. Dans Cerat. Rosce, les ostioles sont filiformes; les thèques rondes comme celles des Eurotium et les sporidies brunes, ovoïdes. - Je n'ai constaté que 4 spores dans chaque théque. - RR. - Automne.

Le Ceratostoma Piliferum, de Sacc. doit être reporté au Genre Melanospora. - Croît sur les écailles de chêne. - CCC.

Il en est de même du Cerat. hæmatorrhynchum, qui est sans doute le Melanospora Caprece.

13 GENRE. - CHATOMHUM. - Kunze.

1131. CHAT. CRISPATUM. Sacc. - Alb. de l'Est. - Sur les murs des caves et sur le bois pourri; formant des taches noires, velues. - Les périthéces ovoïdes sont couverts de plusieurs sortes de filaments, les uns bruns, raides, septés. disséminés à la surface; les autres très longs, tortueux, septés, placés au sommet du périthèce 
et formant une houppe assez semblable à une grenade; sporidies incolores dans les thèques, brunes et limoniformes étant mûres. - AC. Hivers doux, Printemps. - St-Amand.

1135. CHet. Comatum. Sacc. - Alb. de l'Est. - Très commun sur les pailles de seigle et de blé pourrisantes. - Automne, Printemps. Partout.

1136. Chat. Elatum. Sacc. - Alb. de l'Est. - Sur les poutres et bois pourris, formant de larges taches velues; dans les lieux humides et sans lumière. - Voisin du précédent. - Caves, celliers, décombres. - Partout. - Hiver et Printemps.

113\%. CHAT. GRISEUM. Sacc. - Alb. de l'Est. - Sur bois pourri et sur tiges mortes des plantes herbacées : Brassica, Solanun tuberosum. etc. - Périthèces ovoïdes; couverts de très longs poils grisâtres, mous; courbés au sommet; sporidies peu colorées; St-Amand. - Automne. - AR.

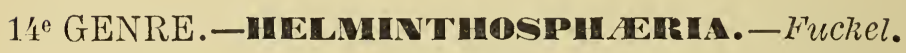

1138. Helm. Clavariarum. Euckel. - Alb. de l'Est. - Cette espéce intéressante croît à la base des rameaux de Clavaria cristata et lui donne un aspect noirâtre et velu. - Les périthèces globuleux sont couverts de poils courts, raides, noirs. Les thèques cylindriques et les sporidies brunes limoniformes. Ils accompagnent l'état conidien qui est Helminthosporium clava- 
rice, beaucoup plus commun que l'état thécasporé. - AC. - Dans les forêts de Cheminon, Ser:naize. - Été-Automne.

\section{GENRE. - SORdARIA. - Ces.}

1139. SORD. FIMISEDA. Sacc. - Alb. de l'Est. - Sur crottes de brebis et de vache. - Les périthèces sont ovoïdes, à col allongé ; ils renferment des théques, 8 sporidies cylindriques avec des spores elliptiques, brunes, munies d'un appendice caudal incolore, de même longueur, - AC. - Automne. - Champs, lieux oú pâturent les troupeaux.

1140. SORD. CARBONARIA. Sacc. - Alb. de l'Est. - Croît sur le sol oú l'on a fait du charbon. - Les périthèces sont rugueux, ovales, noirs; les théques cylindriques, longuement stipitées; les sporidies naviculaires, présentant un appendice hyalin trés court et difficile à observer. - RR. - Sermaize. Automne. - Forêts.

1141. SORD. SQUAMULOSA. Sacc. - Alb. de l'Est. - Sur le crottin de cheval, en Été par l'humide. - Périthéces mous, verdâtres, recouverts de papilles plus foncées, éparses, terminés en un ostiole noir conique. longues thèques; sporidies diverses suivant l'âge; d'abord vermiformes, puis renflees au sommet; le renflement brunit et se sépare du prolongement hyalin. C'est la sporidie définitive, limoniforme, 
tronquée inférieurement. Cette évolution de la spore est la même que celle de Bombardia. AR. - Automne.

1142. SORD. CAUdATA. Sacc. - Alb. de l'Est. - Sur le bois pourri. - Elle différe du Squamulosa par ses périthèces noirs, charbonneux. sans papilles. Les thèques et les sporidies sont semblables, cependant les jeunes sporidies munies de leur appendice sont munies de 4 à 5 cloisons. - AR. - Lieux humides, azotés. St-A mand. - Automne.

1143. SORD. ANSERINA. Sacc. - Alb. de l'Est. - Podospora Fimeti de Ces. - AC. sur les crottes de brebis dans les pâturages. - Très voisin de Sord. fimiseda. - S'en distingue par la prèsence de 4 sporidies dans chaque thèque au lieu de 8. - Automne, printemps. - Partout.

16' GENRE. - IIYPOCORPA. - Fuckel.

1144. HYP. DISCOPORA. Sacc. - Alb. de l'Est. - Epars sur les crottes de lièvre. - Périthèces munis de poils noirs, courts ; thèques cylindriques et sporidies en forme de disques. Soulanges, Bayarne, dans les bois de pins sylvestres. - AR. - Automne.

1145. HYP. MACROSPORA. Sacc. - Alb. de l'Est. - Croît aussi sur les crottes de lièvre. - Périthèces noirs, glabres; sporidies atteignant $0^{\mathrm{mm}, 02}$ - Environs de Reims. - Communiqué par le $\mathrm{D}^{\mathrm{r}}$ Jolicœur. 
1146. HYP. MAXIMA. Sacc. - Alb. de l'Est. Sur crottes de lapin de garenne. - Périthèces en forme d'obusier ; thẹques ne contenant que 4 sporidies encore plus grandes que celles d'Hyp. macrospora $0^{\mathrm{mm}}, 03$. - Récolté en décembre dans les sapinières de Lisse à Bassu. - RR. 114\%. HYP. CAPILLIFERA. Sacc. - Alb. de l'Est.

- Récolté sur tige pourrie de porreau près d'un tas de fumier. - Périthèces en forme de bouteille blanc's-jaunâtres avec un col noir, couverts çá et lá de poils blancs, courts, plus longs à la base. - RR. - St-Amand. - Automne.

1\% GENRE. - COFRMLEPA. - Fries.

1148. COPR. MERDARIA. Fuckel. - Alb. de l'Est. - Sur crottes de lapin noircies par les sphærules et le tapis tomenteux-brun dans lequel elles sont plongées; sporidies grandes, elliptiques, entourées d'un limbe très visible. RR. - Lieux incultes des plantations de Pins. - Soulanges. - Automne.

1149. COPR. FIMETI. Sacc. - Alb. de l'Est. Sur crottin de cheval. - Périthèces plongés dans un pseudo-strôme crustacé, gris ; ostioles coniques superficiels. - CC. - Après l'hiver dans les champs fumés. - St-Amand, etc.

1150. COPR. EQUORUM. Fuckel. - Alb. de l'Est. - Sur crottin de cheval. - Périthèces globuleux, glabres à ostioles courts, plongés dans un strôme brun. - CCC. - Fumiers anciens, jardins, etc. - Hiver, printemps. 
18 GENRE. - PHILOCOPRA. - Speg.

1151. PHIL. SETOSA. Sacc. - Alb. de l'Est. Sur crottin de cheval. - Rare espéce récoltée à St-Amand, hameau de la Cense des Près. Périthéces pyriformes, munis de poils bruns ; thèques amples, fusiformes, renfermant une très grande quantité de sporidies brunes, elliptiques, munies d'appendices hyalins.

\section{9e GENRE. - RosELLRMA. - De Not.}

1152. ROS. AQUILA. De Not. - AJb. de l'Est. Très commun sur les branches et rameaux pourris, fichés en terre, dans les haies. - Périthèces globuleux, plongés dans un subiculum tomenteux, brun. - Partout. - Conidie, Sporotrichum fuscum.

115̃3. ROS. THELENA. Sacc. - Alb. de l'Est. Sur planches et bois pourris. - Périthèces placés sur un subiculum presque glabre, brunpourpre. - Moins commun que Ros. aquila. St-Amand, etc.

115't. ROS. MAMMAEFORMIS. Ces. - Alb. de l'Est. - Sur les troncs et les rameaux pourrissants. - Périthéces sans subiculum, mammiformes, glabres, plus ou moins agglomérés ; sporidies brunes pourvues d'appendices hyalins. CCC. - Haies, tas de bois. - St-Amand, etc. 
1155. ROS. PULVERACEA. Fuckel. - Alb. de l'Est. - Sur bois dénudé et troncs de hêtre et de peuplier. - Larges plaques formées par les périthéces nombreux, noirs, chagrinés, ressemblant à des grains de poudre; sporidies brunes elliptiques. - CC. Dans les chantiers et dans les bois. - St-Amand, St-Lumier, etc.

1156. ROS. LIGNIARIA. Fuckel. - Alb. de l'Est.

- Sur bois et planches de chêne humides. Périthèces disposés en ligne, trés petits, sphériques, couverts de poils noirs, raides. - AC. dans les celliers et les vieilles charpentes abandonnées dans lieux frais. - St-Amand, etc.

115\%. ROS. MALACHOTRICHA. Niessl. - Alb. de l'Est. - Sur branches mortes de Pinus sylvestris. - : Petits amas de périthéces logés dans les fentes de bois pourri, placès sur un tapis de poils courts, noirs : recouverts euxmêmes entièrement de poils raides. Thèques cylindriques, sporidies brunes, presque sphériques. CCC. dans les sapinières. - Soulanges, Bassu, etc.

20 GENRE. - BOMEA IPIA. - Fries.

1158. BOMB. FASCICULATA. Fries. - Alb. de l'Est.

- Sur vieux troncs d'Erable et d'Aulne coupés près du sol. - Périthéces réunis en faisceaux, allongés en massue ; bruns, glabres. Théques très longues et tronquées au sommet; sporidies semblables à celles de Sordaria squamulosa décrites plus haut. - AC. - Automne, hiver. - St-Amand, etc. 
21. GENRE. - NTMOSTOMELLA. - Sacc.

1159. ANTH ${ }^{\text {lla }}$. TOMICUM. Sacc. - Alb. de l'Est. Sur tiges de Juncus Glaucus et $J$. Communis. - Petites taches brunes, rondes: éparses, entourant les ostioles des périthèces nichés dans le parenchyme. Théques elliptiques; sporidies brunes, bi-ocellées. - AC. - Lieux marécageux. - Hiver, Printemps. - St-Amand, etc.

1160. ANTH Hla CONORUM. Sacc. - Alb. de l'Est. - Sur les écailles des cônes du Pin sylvestre. - Périthèces à demi immergés dans l'écorce. épars. Théques cylindriques, sporidies branes, elliptiques, sans cloison. - CC. - Plantations de pins. - Sou!anges. Bassu, etc.

1161. ANTH ${ }^{\text {lla }}$. LimitAtA. Sacc. - Alb. de l'Est. - Sur rameaux de Cornus sanguinea. Sous-épidermiques. formant des plaques tuméfiées, noicies ; sporidies brunes, elliptiques, munies de 2 gouttelettes. - AC. - St-Amand, St-Lumier, etc.

1162. ANTH ${ }^{l l a}$. INTERMEDIA. Sacc. - Alb. de l'Est. - Sur branches mortes de Salix caprcea - Groupes de Périthèces très petits, épars. Théques cylindriques ; sporidies elliptiques : brunes. avec 2 gouttelettes. - $\Lambda$ R. - Aulnayl'Aître, Lisse, etc. - Automne.

1163. ANTHilla. VISCI. Sacc. - Alb. de l'Est. Sur rameaux morts de Viscum album. - Péri- 
thèces épars, nombreux. placés sous l'épiderme. assez semblables à ceux du Diplodia visci. Thèques amples, cylindriques, entourées de paraphyses confuses; sporidies grandes $0^{\mathrm{mm}} .03$. - RR - Ablancourt. - Automne, printemps. 1164. ANTH Hla, BERBERIDIS. Ch. $R$. - Alb. de l'Est et Bull. de la Soc. bot. de France. Sur petits rameaux encore verts de Berberis vulgaris. - Récolté dans mon jardin en automne. - Périthéces sous-épidermiques . isolés ou réunis 2-3. - Thèques cylindriques ; sporidies elliptiques, brunes. aiguës aux extrémités. - Je crcis cette espéce assez commune, bien qu'ignorée jusqu'alors.

1165. ANTH ${ }^{11 a}$. BROMI. Ch. $R$. - Alb. de l'Est. - J'ai trouvé cette espéce nouvelle sur chaumes de Bromus asper. - Elle diffère d'Anthlla. Tomicum par ses périthèces plus saillants, elliptiques, et par ses sporidies cymbiformes, courbes, munies d'une gouttelette comme celle des Rosellinia. - RR. - Bois entre St-Amand et St-Lumier. - Automne.

\section{2e GENRE. - ANTHOSTOMA. - Nits.}

1166. ANTh. XYLOSTEI. Sacc. - Alb. de l'Est.

- Sur rameaux de Lonicera Xylosteum et $L$. Caprifolium. - Périthéces en groupes, plongés dans un strôme mince et dans le bois ; l'épiderme noirci, en plaques allongées, elliptiques est couvert d'aspéritès dues aux ostioles érum- 
pents. Sporidies elliptiques, sans cloison ni gouttelettes. - AC. - Jardins. - St-A rnand, Aulnay. - Automne.

116\%. ANTH. GASTRINUM. Sacc. - Alb. de l'Est.

- Sur branches mortes d'Ulmus campestris. Se présente sous forme de pustules grosses. saillantes, noir-bleuâtre. - Thèques cylindriques et sporidies elliptiques, brunes avec quelques gouttelettes. - CC. dans les bois. - Automne. - Partout.

$23^{\mathrm{e}}$ GENRE. - XYLA IBA. - Hill.

1168. XYL. POLYMORPHA . Grév. - Alb. de l'Est. - Sur vieilles souches d'Orme, de Peuplier, etc. coupées au niveau du sol. - Très varié de forme; le strôme est vertical, noir. extérieurement, blanc à l'intérieur, strié en rayonnant vers la circonférence qui est couverte de sphérules contenant des théques cylindriques très longues et des sporidies brunes, elliptiques, courbes. - CC. - Bois de St-Amand. La Chaussée.

1169. XYL. HYPOXYLON. Grér. - Alb. de l'Est.

- Sur le bois pourri, les écailles de bois gisant sur le sol humide. - Strômes minces, claviformes couverts d'abord de poils noirs à la base. puis en spatule aplatie, blanche, conidifère (fusidium parasiticum) au sommet; plus tard munis de périthéces sphériques presque superficiels. - CCC. dans les bois, haies, jardins. Partout. 
1170. XYL. TULAsNei. Nits. - Alb. de l'Est. - Sur les crottes de lapin de garenne. - Très rare espèce ayant des strômes de formes variées. portant au sommet un capitule garni de périthèces linguiformes ; théques cylindriques : sporidies noires, elliptiques, entourées d'un limbe hyalin. - Garenne de Meigneux entre St-Amand et Vanault-le-Châtel. - Automne.

1171. XYL. CARPOPHILA. Fries. - Alb. de l'Est. - Espèce filiforme, sur les fruits du hêtre tombés sur la terre humide des bois. Aulnay-l'Aître, St-Amand. - CC. - AutomneHiver.

117\%. XYL. DigitATA. Grév. - Alb. de l'Est. - Sur le bois de chêne pourri, pieux et palissades. - Vient en touffes épaisses ; strômes égaux, plus minces et pius réguliers que ceux de Xyl. polymorpha. - AC.

1173. XYL. HIPPOTRICHOIDES. Sacc. - Alb. de l'Est. - J'ai récolté ce Xylaria sur écailles de bois pourri de pin sylvestre. - Ses strômes sont filiformes, rameux, comme ceux des Rhizomorpha; les sphérules ovoïdes ou lagéniformes sont disposées en petit nombre le long des filaments tenus; elles renferment à l'intérieur un mucilage rempli de glomérules de sporidies petites, ovales, hrunes qui résultent sans doute. des théques fugaces disparues. - RR. - Bois de pins à Soulanges. - Automne. 


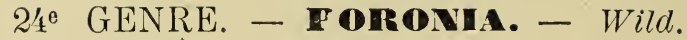

11\%'. POR. PUNCTATA. Wild. - Alb. de l'Est.

- Sur le crottin de cheval depuis longtemps á l'air libre. - Strômes dressés en clavules ou cônes renversés; disque blanc: ponctué-noir . conidifère ; sphérules sous-jacentes ; thèques cylindriques; sporidies ovales brunes. - RR. - St-Amand, au lieu dit Chemin des bois. Automne.

25e GENRE. - CSTUMINA. - Tul.

1175. USTUl. VUlgaris. Tul. - Alb. de l'Est. - Sur les troncs moris d'Erable, de Peuplier. etc. - Larges plaques crustacées, noirâtres, friables, couvertes de conidies dans le jeune âge (sporotrichum agaricinum); sporidies brunes, fusiformes. - CC. - Automne, printemps.

26e GENRE. - HYPOXYLON. - Bull.

11\%. HyPOX. COCCINEUM. Bull. - Alb. de l'Est. - Sur troncs et branches de Frêne et de Prunier. - Strômes de la grosseur d'un pois, minium puis brun-rouille, précédé quelquefois par l'état conidien (anthina flavovirens). - CC. - Bois. - St-Amand, St-Lumier, etc. - Hiver, printemps.

117\%. HYPOX. ARGILlACEUM. Berk: - Alb. de l'Est. - Sur troncs et branches d'Almus glutinosa, de Corylus, etc. - Strômes bruns, recou- 
verts d'une couche tomenteuse couleur d'argile (état conidien) ; - AC. dans les bois . - StAmand, Aulnay-l'Aître, etc. - Hiver, printemps. 11\%. HYPOX. FUSCUM. Fries. - Alb. de l'Est.

- Sur troncs et branches d'Alnus glutinosa. de Coryluis, etc. - Strômes bruns puis noirs, hémisphèriques, nombreux. - CCC. - Partout.

1179. HYPOX. MULTIFORME. Fries. - Alb. de l'Est. - Sur tronc de Bouleau. - Strômes arrondis ou aplatis, irréguliers-confluents. AC. dans les bois. - Printemps, hiver. - StAmand, St-Lumier, eic.

1180. HyPoX. RUBIGINOSUM. Fries. - Alb. de l'Est. - Sur troncs de Pommier et de Hêtre. Plaques minces, passant du brun-rouge au noir ; Péritièces peu saillants, elliptiques, logés dans l'épaisseur du strôme. - CC. - Bois, chantiers. - Automne, printemps.

1181. HYPOX. SERPENS. Fries. - Alb. de l'Est.

- Croît sur le buis des vieux saules pourrissants. - Plaques irrègulières, étroites, paralléles ; sporidies brunes, un peu courbes, à 2 gouttelettes, - CC. - Bords des ruisseaux, saussaies.

1182. HYPOX. UDUM. Fries. - Alb. de l'Est. Sur bois pourri de chêne. - Plaques irrégulières, moins ètendues que celles d'Hypox. serpens ; sporidies elliptiques-allongées, droites. - CC. - Bois. - Hiver, printemps.

1183. HYPOX. MINUTUM. Nits. -- Alb. de l'Est. - Sur bois de chêne. - Ressemble á l'espèce 
précédente, mais plus petite avec des périthéces confluents et distincts, roussâtres. á ostiole noir ; sporidies presque fusiformes . - CC . - Bois. hiver.

2\% GENRE. - DALDINIA. - De Not.

118'. DALD. CONCENTRICA. Cés. - Alb. de l'Est. - Sur les troncs et souches de Frêne et de Bouleau. - Strômes de la grosseur d'une noix, globuleux, noirs extérieurement et couverts de Périthèces à la périphérie ; chair grisàtre munie de lignes concentriques plus foncées. - AR . - St-Amand, St-Quentin-lesMarais. - Printemps.

SeGt. III. - hyalosporÉEs. - Sacc.

28 GENRE. - CER a'TOSTMMELLA. - Sacc.

1185. CERAT Tla . ROSTRATA. Sacc. - Alb . de l'Est. - Sur le bois et les écailles de peuplier pourries sur la terre humide des bois. Pèrithèces presque superficiels, distincts, à col très long ; sporidies incolores, courtes, botuliformes. - AC. - Bois. - Printemps.

29e GENRE. - GNOMHNHELLA. - Sacc.

1186. GNOM ${ }^{\text {lla }}$. TUBIFORMIS. Sace. - Alb. de l'Est. - Croît à la face inférieure des feuilles 
d'Alnus glulinosa. - Périthèces nichès dans le parenchyme ; très long col ; sporidies fusiformes. - CC. - Bois, Haies, Jardins. - Hiver, Printemps.

118\%. GNOM ${ }^{\text {lla }}$. VULGARIS. Sacc. - Alb. de l'Est. - A la face inférieure des feuilles du Corylus avellana. - Périthèces s'aplatissant en cupules, cols en tubes trés minces; sporidies en fuseaux presqu'aculéiformes. - CC. - Hiver, printemps.

30 GENRE. - LAESTADA. - Auersw.

1188. LAEST. COOKEANA. Sacc. - Alb. de l'Est. - Sur feuilles de chêne encore adhérentes au rameau ; à la face inférieure. - Périthèces discoïdes, épars ; sporidies elliptiques, assez grandes, un peu resserrées aux extrémités, granuleuses. - AR. - St-Lumier, dans les bois. - Printemps, hiver.

1189. LAEST. VENETA. Sacc. - Alb. de l'Est. - Sur feuilles de Platane. - Périthèces nichés dans le parenchyme; ostiole apparent au dehors ; sporidies elliptiques-fusiformes, munies d'une petite cloison à l'une des extrémités, - AR. - Ablancourt. - Eté, automne.

1190. LEST. EXCENTRICA. Sacc. - Alb. de l'Est. - Sur branches terminales de Buis mort. - Périthéces formant au dehors une tache elliptique blanche, à pourtour et point central noirs, nichés sous l'épiderme; spores elliptiques. - CC. - Haies, Jardins. - Printemps. - Partout. 
1191. LAEST. CARPINEA. Sacc. - Alb. de l'Est. - Sur feuilles de carpinus betulus mortes, en hiver. - Périthèces épars, nombreux ; ouverture de l'ostiole comme un point blanc central. Sporidies elliptiques-oblongues, granuleuses. - CC. - Haies, bois.

1192. LAST. PYROL $\mathbb{E}$. Ch. R. - Alb. de l'Est.

- Sur feuilles vertes de Pyrola roiundifolia. - Apparence d'un phyllosticta; taches rouges puis brunes; Périthèces presques superficiels ; sporidies elliptiques-oblongues, granuleuses. (An Last. malbrancheana de Sacc. ?) - AR. - Automne. - St-Amand.

31 GENRE. - mHOMATOSPORA. - sacc.

1193. PHOMspra. MOLLUGINIS. Sacc. - Alb. de l'Est. - Sur tiges de Galium mollugo. - Périthèces peu nombreux prés des nœuds; sporidies elliptıques, munies de 2 gouttelettes semblables aux sporules des Phoma. Théques cylindriques, monostiques. - AC. - St-Amand - Talus et broussailles; le long des routes.

119'. PHOM ${ }^{\text {spra }}$. BERBERIDIS. Ch. $R$. - Alb. de l'Est. - Sur jeunes rameaux morts de Berberis vulgaris. - Théques presque filiformes ; sporidies elliptiques, 2 gouttelettes. AR. - Dans mon jardin. - Hiver.

32e GENIRE. - PHYSALOSPORA. - Niessl.

1195. PHYS. GREgARIA. Sacc. - Alb. de l'Est.

- Sur tigelles et feuilles de Taxus baccata. - 
Pustules noires, rangées en ligne comme celles du Diplodia Taxi. - Thèques larges: sporidies granuleuses, ovoïdes-oblongues. - AR. Automne. - Jardins. - St-Amand.

1196. PHys. MiNutula. Sacc. - Alb. de l'Est.

- Sur tiges mortes d'Euphorbia sylvatica. Groupes de Périthéces sous-épidermiques formant des taches grises; oblongues; théques courtes, renfermant de 6 à 8 sporidies ovoïdesoblongues. - Forêl de la Montagne de Reims. - Automne. - RR.

1197. PHYS. ONONIDIS. Ch. $R$. - Alb. de l'Est.

- Sur tiges mortes d'Ononis natrix. - Périthéces sous-épidermiques; ostioles noirs, érumpents. Théques fusiformes; larges; sporidies elliptiques; oblongues; quelques paraphyses fugaces. - AR. - Bords du chemin de Soulanges à St-Amand. - Automne. - Voisin de Phys. minutella. Sacc.

\section{3" GENRE. - Ditopella. - De Not.}

1198. DITOP. FUSISPORA. De Not. - Nlb. de l'Est. - Envahit les branches et rameaux d'Alnus glutinosa. - Périthéces nichés sous l'épiderme qu'il est difficile de soulever sans les arracher. Théques larges renfermant une grande quantitè de sporidies fusiformes. - CC. dans les bois. - Automne, hiver. - Partout.

34 GENRE. - WALLROTHIELLA. - Sace. 1199. WALlR. Minima. Sacc. - Alb. de l'Est. 
- Croît sur les lattes de chêne qui soutiennent les bouteilles dans les caves. - Périthèces à peine visibles, sphériques, superficiels, luisants; théques cylindriques; sporidies ovales fusiformes. - AR. - St-Amand. - Automne, hiver.

\section{$35^{\mathrm{e}}$ GENRE. - HO'TRYOSPII AERIA. - Ces.}

1200. BOTRYOS. BERENGERIANA. Sacc. - Alb. de l'Est. - Sur les branches mortes de Peuplier. - Forme des groupes de 5-8 périthéces réunis dans un même strôme irrégulier, elliptique et non en pustule comme celui de Botryos. advena; sporidies ovoïdes-fusiformes granuleuses. - AC. - Automne. - St-Amand. - Est accompagné quelquefois du Botryıdiplodia, sa forme pycnidienne.

1201. Botryos. AdVEnA. Ces. - Alb. de l'Est. - Dothidea Melanops de Tul. - Sur les branches et rameaux de chêne, accompagné de sa forme conidienne Dothiorella advena. - Sporidies ovoïdes, fusiformes, granuleuses. - AC. - Automne. - Bois de St-Lumier à St-Amand, etc.

1202. Botryos. DOTHIDEA. Ces. - Alb. de l'Est. - Sur tiges encore vertes de Rosa canina. - Tubercules dartreux, fendillés, renfermant les périthèces à nucleus blancs; théques larges : sporidies ovales; paraphyses toruleuses. CCC. dans les bois et les jardins. - Partout. 
36e GENRE. - CRYP'TOSPORELLA. - Ces.

1203. CRYPT ${ }^{11 a}$. HYPODERMIA. Sacc. - Alb. de l'Est. - Croît sur les rameaux d'Ulmus campestris. - Périthèces sous-èpidermiques; thèques amples; sporidies fusiformes trés grandes; la conidie Phoma. ulmicola. Sacc. l'accompagne souvent. - AC. - St-Amand. - Automne.

120'. CRYPT ${ }^{\text {lla }}$. AUREA. Sacc. - Alb. de l'Est. - Sur branches et rameaux de Carpinus betulus - Forme des pustules composées de 6-8 périthèces réunis par un pseudo-strôme jauneor ngé ; larges théques; sporidies ovoïdes, grandes. - AR. - Automne. - Bois. Aulnay-l'Aître.

1205. CRYPT ${ }^{\text {lla }}$. POPULINA. Sacc. - Alb. de l'Est. - Commun sur les branches de Populus pyramidalis. - Périthèces 6-8 à cols réunis en faisceau sous l'épiderme des rameaux : sporidies elliptiques un peu courbes. -- CCC. Bois, jardins. - Partout.

1206. CRYPT ${ }^{1 / a}$. CHONDROSPORA. Rehm. - Alb. de l'Est. - Sur les petits rameaux encore adhérents au trone du tilleul. - Sporidies obovales, teinte jaunâtre-claire, granuleuses, partie inférieure exempte de granulations et paraissant munie d'une cloison. - AC. - Soulanges, Aulnay. - Automne. 
SEGT. IV. - HYALODIDYMÉES. - Sacc. 3re GENRE. - SPHAERELLA. - Ces.

1207. SPH ${ }^{\text {lla }}$ MACULIFORMIS. Auersw. - Alb. de l'Est. - Hypophylle sur Quercus robur. Périthèces très petits. réunis en groupes de teinte grisâtre; théques nombreuses; sporidies oroïdes, septées, peu visibles. - CCC. dans les bois. - Automne, printemps. - Partout.

1208. SPH ${ }^{\text {lla }}$. HEDERICOLA. Cooke. - Alb. de l'Est. - Sur feuilles de Lierre. - Taches blanches orbiculaires, entourées d'un limbe rouge ; périthèces peu nombreux, assez semblables à ceux de la spermogonie, Septoria hederce; sporidies elliptiques, uniseptées. - AR. - Ablancourt, St-Amand. - IIiver.

1209. SPH ${ }^{11 a}$. GENUFLEXA. Auersw. - Alb. de l'Est. - Sur feuilles mortes de Castanea vulgaris . - Petits groupes à peine visibles; thèques amples ; sporidies allongées, courbes, munies d'une cloison; lobe supérieur renflé ; lobe inférieur étroit. - AC. - Bois de la Montagne de Reims. - Printemps.

1210. SPH ${ }^{l l a}$. POPULI. Auersw. - Alb. de l'Est. - Sur feuilles de Populus nigra ayant séjourné l'hiver sur le sol humide. - Périthèces hémisphèriques, thèques renflées; sporıdies cylindriques, septées, amincies aux extrémités. AC. - St-Amand, St-Quentin, etc. - Printemps. 
1211. SPH ${ }^{\mathrm{lla}}$. CRASSA. Aucrsuc. - Alb . de l'Est.

- Sur feuilles de Populus cordifolia, à la face inférieure ; - Petits groupes épars de pèrithéces hémisphériques ; thèques en massue renflée ; sporidies presqu'ovales, uniseptèes. AR. - St-Quentin-les-Marais. - Printemps.

1212. SPH ${ }^{\text {lla }}$. RUMICIS. Gon. - Alb. de l'Est. Sur feuilles de Rumex crispus. - Périthèces 3-6. très petits, insérés sur une tache brune ; théques en massue; sporidies ovales, uniseptées, ressemblant aux sporules de Trichcthecium roseum. - AC. - Printemps. - Ablancourt. etc. - Bois.

1213. SPH ${ }^{\text {lla }}$. DEPAZEAFORMIS. Gon. - Alb. de l'Est. - Petits groupes de Périthèces épars à la face inférieure des feuilles mortes de Poterium sanguisorba; thèques en massue ; 4-8 sporidies elliptiques, allongées, courbes, uniseptées, 4 gouttelettes. - AC. - Champs crayeux, herbus, bois de Pins. - St-Amand, Soulanges. - Automne.

1214. SPH ${ }^{1 l a}$. PLANTAGINIS. Sacc. - Alb. de l'Est. - Sur les scapes de Plantago major et media. - Groupes de périthèces très petits. sphériques ; thèques claviformes , renfermant 6 sporidies obovales-allongées. - AR. - Bords du chemin de Lisse à Bassu. - Printemps.

1215. SPH ${ }^{l l a}$. DAHLIE. C. et Ell. - Alb. de l'Est. - Sur tiges de Dahlia mortes, encore adhérentes au tubercule. - Groupe de périthèces très nombreux; thèques en massue; 
sporidies grandes, ressemblant à celles des Diaporthe, munies de 4 sporules. - AC. Hiver, Prinlemps.

1216. SPH ${ }^{11 a}$. VINCETOXICI. Sacc. - Alb. de l'Est. - Sur les tiges de Cynanchum Vincetoxicum, formant des taches grisâtres semblables à celles de Sphceropsis nebulosa. Les spermogonies viennent de préférence sur les siliques de la plante. Théques cylindriques: sporidies assez grandes, ovales-allongées. AR. - Collines de Soulanges. - Automne, hiver.

121\%. SPH ${ }^{l l a}$. RUBELLA. Niessl. - Alb. de l'Est. - Sur tiges d'Angelica sylvestris. - Groupes allongés de petits périthèces sur une tache rouge-pourpre. Théques en massue et sporidies elliptiques-allongées ; rarement observées. - CC. - Automne, printemps. - Bords des eaux.

1218. SPH ${ }^{\text {lla }}$. CAULINCOLA. Var. Succisæ. Karst. - Alb. de l'Est. - Sur tiges mortes de Scabieuse succise. - Très petits périthèces disséminés sur des taches brunes qui entourent les tiges et les feuilles desséchees ; thèques en massue; sporidies incolores d'abord. puis un peu jaunâtres. - Bois de Sermaize. RR.

I219. SPH ${ }^{l l a}$. EPILOBII. Sacc. - Alb. de l'Est. Envahit les deux côtés des feuilles d'Épilobium roseum et angustifolium. - Sur les bords du Fion à St-Amand. - Théques la plupart cylin- 
driques; sporidies ovoïdes-allongées, teintèes de jaune en vieillissant. - AC. - Automne.

1220. SPH ${ }^{\text {lla }}$. VULNERARIE. Sacc. - Alb. de l'Est. - Sur feuilles d'Anthyllis vulneraria. Petits groupes maculiformes ; théques assez larges; sporidies allongèes: étroites, un peu courbes. - AR. - Bois de pins, bords des chemins herbus. - Automne, Printemps. St-Amand, Lisse.

1221. SPH ${ }^{l l a}$. EBULI. Ch. $R$. - Alb. de l'Est. Sur tiges mortes de Sambucus ebulus. - Périthéces disséminés donnant à l'épiderme une teinte grise; théques en massue; sporidies uniseptées, un peu étranglèes à la cloison. AC. - Aulnay-l'Aître, Lisse, etc. - Automne. 1222. SPH ${ }^{l l a}$. SUPERFLUA. Sacc. - Alb. de l'Est. - Sarments de houblon. - Théques courtes, sans paraphyses, renfermant 6 sporidies ovalesallongèes. - AC. dans les jardins et les bois à St-Amand, Aulnay, etc. - Hiver.

38e GENRE. - STHGMATEA. - Fries.

1223. STIGM. ROBERTIANi. Fries. - Alb. de l'Est. - Sur la face supérieure des feuilles vivantes de Geranium Robertianum. - Groupes de périthèces petits, hémisphériques, noirs, luisants; théques distiques ou monostiques; sporidies ovoïdes, uniseptées, incolores puis jaunâtres. - CC. - Automne. - Partout. 
39e GENRE. - DIDYMELLA. -- Sacc.

1224. DIDYMlla. VEXA'TA. Sacc. - Alb. de l'Est.

- Sur les rameaux de Cornus sanguinea. Périthéces souscutanés, groupés ; ostioles petits, érumpents, formant une tache grisâtre ; thèques amples : sporidies grandes, incolores puis jaunâtres, uniseptées ; paraphyses fugaces, septées. - AC. - St-Lumier, St-Amand, etc. - Printemps.

1225. DIDYMlla. MEDIA. Sacc. - Alb. de l'Est. - Sur tiges sèches de Verbena officinalis. -Périthèces épars. sous-cutanés ; théques amples ; sporidies didymes, munies de quelques gouttelettes ; paraphyses très visibles. - AC. Ablancourt, La Chaussée, etc. - Printemps.

1226. DIDYM ${ }^{\text {lla }}$. HELLEBORI. Sacc. - Alb. de l'Est. - Sur les vieilles tiges d'Helleborus viridis dont la surface noire, rugueuse est couverte de périthéces disposés en lignes; thèques monostiques; sporidies ovoïdes, uniseptées. AR. - Soulanges, Ablancourt. - Hiver, Printemps.

1227. DIDYMlla. BRYONIÆ. Rehm. - Alb. de l'Est. - Sur les tiges mortes de Bryonia dioica. - Périthèces sous-cutanés puis érumpents; thòques grandes; 8 sporidies didymes, lobe supérieur plus large que l'inférieur; paraphyses nébuleuses. - AC. - Haies, jardins. - Hiver. 
1228. DIDYM $M^{\text {lla }}$. CULMIGENA. Sacc. - Alb. de l'Est. - Sur les chaumes de Bromus asper. prés des nœuds. - Périthéces aplatis à la base ; thèques renfermant 6 sporidies elliptiquesfusoïdes, contenant deux grosses gouttelettes; paraphyses remplies de granulations. - AR. Printemps. - St-Amand.

1229. DIDYM $\mathbf{M}^{\text {lla }}$. EPILOBII, Sacc. - Alb. de l'Est. - Sur tiges d'Epilcbium spicatum. - Périthèces sous-cutanés, érumpents ; s'affaissant en cupules; ostioles coniques; apparence de Pleospora. Thèques monostiques ; sporidies assez grandes. - AR. - St-Amand. - Jardins.

40 GENRE. - GNOMOMIA. - Ces.

1230. GNOM. DEPRESSULA. Karst. - Alb. de l'Est. - Sur tiges séches de Rubus cassius dont la surface est hérissée de petits aiguillons qui sont les ostioles des périthèces sous-jacents. Théques renfermant 4 sporidies elliptiques-allongées, munies de 4 gouttelettes. - AR. - StAmand. - Automne.

12:1. GNOM. RHOIS. Ch. R. - Alb. de l'Est. Sur les pétioles de feuilles de Rhus Typhinum. - Périthéces sous-cutanès; ostioles en forme d'épines, érumpents; théques sans paraphyses , amincies à la base; sporidies fusiformes, étroites. - AK. - Dans mon jardin. - St-Amand. Printemps.

1232. GNOM. SETACEA. Ces. - Alb. de l'Est. Sur les écailles et les involucres des glands 
du chêne, presqu'invisibles à l'œil nu. - Périthèces globuleux à cols filiformes; thèques en massue renflée, contenant de 4 à 6 sporidies baculiformes, un peu courbes, \& gouttelettes. AR. en fructification. - St-Amand. - Printemps.

1233. GNOM. ISCHNOSTYLA. Fuckel. - Alb. de l'Est. - Sur les nervures et sur les pétioles d'Acer platanoides. - Périthèces sous-cutanés, cols érumpents, entourés à la base d'une tache noire; théques renfermant 8 sporidies fusoïdes, étroites. - CCC. dans les bois. - Automne, hiver. - St-Amand.

1234. GNOM. ERYTHROSTOMA. Auersw. - Alb. de l'Est. - Sur les feuilles de Prunus avium encore adhérentes à l'arbre. - Périthèces disséminés à la face inférieure en forme de petits mamelons rougeâtres et de cupules déprimées à la face supérieure; théques renfermant 8 sporidies distiques, oblongues, granuleuses. - RR. - St-Amand. - Printemps.

1235. GNOM. LePtostyla. Ces. - Alb. de l'Est. - Sur feuilles de noyer desséchées; espéce voisine de Gnom. Isthnostyla, mais plus développée, thèques contenant 8 sporidies fusiformes. - AC. - Hiver, Printemps. - Partout.

41 GENRE. - MELA NOPSAMMA. - Niessl.

1236. MEL. POMIFORMIS. Sacc. - Alb. de l'Est.

- Sur troncs d'arbres, Noyer, Aulne, etc. Agglomération considérable de périthéces superficiels, petits, rugueux, s'affaissant en cupule : 
formant de larges plaques irrégulières. - Thèques octospores; sporidies uniseptées; 4 gouttelettes. - AC. - Chantiers, bois. - St-Lumier, St-Amand. - Printemps.

1237. MEL. CRYPTOSTOMA. Sacc. - Alb. de l'Est. - Sur racines d'Erica, cachées sous terre. - Périthéces presqu'astomes, couverts de poils divergents-étoilés et placés sur un subiculum velu, brun; théques renfermant de 2 à 4 sporidies fusoïdes. allongées, uniseptées; 4 gouttelettes. - R. - Forêt de Sermaize. - Automne.

42 GENRE. - BERTIA. - De Not.

1238. BERT. MORIFORMIS. De Not. - Alb. de l'Est. - Sur rameaux de chêne et sur cônes de Pinus sylvestris. - Périthéces réunis en petits groupes. noirs, tuberculeux, superficiels, allongés ou sphéroïdes; thèques avec paraphyses filiformes; sporidies allongées-étroites, munies d'une cloison. - AR. - Bois, sapinières. Soulanges. - Forêt de Sermaize. - Automne.

43 GENRE. - LENTOMH'TA. - Niessl.

1239. LENT. AUERSWALDII. Sacc. - Alb. de l'Est. - Sur tiges mortes de Genista tinctoria, notamment à la base. - Les périthéces sous-cutanés sont réunis 3-5 ou épars; les cols plus ou moins allongés, épais, percent l'épiderme. - Le Genre Lentomita diffère du G. Rhinchospora par ses sporidies incolores. - AR. - Bois de Charmont. - Automne. 
4'4 GENRE. - VENTURIA. - De Not.

12'10. VENT. KUNZEI. Sacc. - Alb. de l'Est. Sur feuilles de Rubus casius. - Petits tubercules ou périthèces noirs, velus, épars, souvent stériles, renfermant quand ils sont fertiles des thèques claviformes, octospores; sporidies ovales. uniseptées, incolores puis teintées jaunâtres. CC. dans les bois en Automne.

1241. VENT. CIRCINANS. - Sacc. - Alb. de l'Est. - A la face inférieure des Geranium molle et Rotundifolium. - Groupes de Périthèces couverts de poils courts, renfermant des thèques amples, octospores; sporidies à deux loges, la supérieure plus grande. - AC. - Bois, jardins. - Partout.

1242. VENT. EXOSPORIOIDES. Sacc. - Alb. de l'Est. - Sur chaumes de Poa et de Festuca. - Petits groupes de périthéces noirs, à poils courts, divergents, s'affaissant en cupules. Thèques octospores: sporidies fusoïdes, uniseptées. - AR. - Bois de St-Amand à St-Lumier. Automne.

45e GENRE. - ERIOSPHARIA. - Sacc.

1243. ERIos. Alligata. Sacc. - Alb. de l'Est.

- Sur lattes de chêne exposées à l'air humide.

- Très petits périthéces à peine visibles, couverts de poils noirs, placés sur un subiculum 
velu, brun. - Thèques cylindriques: sporidies elliptiques, uni-cloisonnées , 2 gouttelettes. AR. - St-Lumier. - Automne.

\section{6e GHNRE. - MELANCONIS. - Tul.}

1214. MEL. STilbostoma. Tul. - Alb. de l'Est.

- Sur branches de Belulus alba. - Pustules corticales composées de 6 à 8 périthèces réunis en cercle; cols en faisceau dont le sommet apparaît comme des points noirs sur un disque blanc. Thèques octospores; sporidies elliptiques uni-cloisonnées, granuleuses. - AC. - Bois, chantiers.

1245. MEL. MODONIA. 'Tul. - Alb. de l'Est. Sur branches de châtaignier. - Pustules formées par la réunion de 6-8 pèrithèces; ostioles courts, disposés en disque; thèques longues ; sporidies grandes, elliptiques, un peu jaunâtres; la Conidie Steganosporium Castance l'accompagne. - RR. - Bois de la Montagne do Reims.

1246. MEL. ALNI. Tul. - Alb. de l'Est. - Sur branches d'Alnus glutinosa. - Périthèces en cercle, munis de théques amples, renfermant 8 sporidies elliptiques, resserrées au milieu et terminées aux extrémités par des appendices minces, allongées. - CC. dans les bois. St-Amand. - Automne, Printemps.

1247. MEL. THELEBOLA. Sacc. - Alb. de l'Est.

- Sur branches d'Alnus glutinosa. - Pustules corticales renfermant 5-7 périlhèces; thèques 
fusiformes, contenant 8 sporidies allongées, botuliformes ou droites, granuleuses, terminées par deux appendices filiformes. - AC. - Bois de St-Lumier à St-Amand. - Hiver.

\section{$4 \%$ GENRE. - HER COSPORA. - Tul.}

12'18. HerCOSP. TILIAE. Tul. - Alb. de l'Est.

- Très commun sur les branches mortes de Tilleul qu'il entoure complètement ; $\check{\jmath}-6$ périthèces en cercle dont les cols réunis en un seul tube percent l'épiderme; théques octospores; sporidies elliptiques, uni-cloisonnées; la Pycnide Hercospora Tilice l'accompagne souvent - Bois, jardins. - Automne, Printemps.

\section{GENRE. - DIAPOR'THE. - Nits.}

1249. DIAP. CARPINI. Fuckel. - Alb. de l'Est.

- A la base des rameaux secs de Carpinus beiulus. - Périthèces réunis en pustules disposées en lignes et perçant l'épiderme en forme d'ètoile. Théques fusiformes; sporidies elliptiques allongées, uniseptées: munies de 4 gouttelettes. caractére commun à presque tous les Diaporthe. - AC. - Haies des jardins el dans les forêts. - Hiver, printemps.

12500. DIAP. ACERIS. Fuckel. - Alb. de l'Est.

- Sur branches d'Acer pseudo-platanus. Périthéces réunis en pustules circonscrites par une ligne noire; thèques oblongues-fusiformes, 4 gouttelettes. - CC. - Bois. - Partout. Printemps. 
1251. DIAP. ONCOSTOMA. Fucke!. - Alb. de l'Est. - Sur rameaux de Robinia pseudo-acacia. - Pustules sous-cutanées, disposées en lignes. circonscrites par une ligne noire; périthèces réunis, à cols allongés. un peu divergents; thèques oblongues; sporidies fusoïdes. - CC. - Automne, Printemps. - Jardins. - Partout.

1252. DIAP. STRUMELLA. Fuckel. - Alb. de l'Est. - Sur branches desséchées de Ribes nigrum. - Pustules contenant de 10 à 15 Périthèces, disposées en lignes ; ostioles plus ou moins longs; théques fusoïdes; sporidies uni ou triseptées, fusoïdes un peu courbes. CCC. - Jardins. - Automne, Printemps.

1253. DIAP. LEIPHAMA. Sacc. - Alb. de l'Est. - Sur branches de chêne. - Pustules à disques pâles; périthèces peu nombreux; théques presque cylindriques; sporidies distiques, ovales lancéolées. - CCC. - Bois, forêts.

125'. DIAP. QUERCINA. Fuckel. - Alb. de l'Est. - Sur rameaux de chêne. - Pustules éparses, corticales, limitées par une gne noire, théques allongées. contenant 8 sporidies distiques, oblongues-lancẻolées. - 4 gouttelettes. - CC. -- Bois.

1255. DIAP. FIBROSA. Fuckel. - Alb. de l'Est. - Sur branches desséchées de Rhamnus frangula. - Pustules éparses, entourées à la base par les fibres de l'écorce; théques renfermant des sporidies monostiques, elliptiques : courtes; 
2 goulteletles seulement; paraphyses fugaces, très longues. - AR. dans les haies à La Chaussée. - Printemps.

1256. DIAP. DETRUSA. Fuckel. - Alb. de l'Est.

- Sur Berberis vulgaris. - Pustules corticales, reposant sur un strôme noir qui entoure le rameau; théques fusoïdes; sporidies elliptiques, 4 gouttelettes; paraphyses longues, fugaces. CC. - Hiver, Printemps. - Jardins.

125\%. DIAP. CRASSIUSCULA. Sacc. - Alb. de l'Est. - Sur rameaux desséchés de Mahonia. - Pustules corticales entourées par une ligne noire; périthéces à ostioles courts, érumpents, larges, pézizoïdes ; théques en massue; sporidies ovales-oblongues, resserrées au milieu; 4 gouttelettes. AC. - Jardins. - Hiver. - St-Amand.

1258. DiAP. SAlicElla. Sacc. - Alb. de l'Est.

- Sur rameaux de Salix alba. - Périthèces épars, sous-cutanés, adhérents à l'épiderme ; ostioles courts; thèques en massue; sporidies distiques, ellipsoïdes-courbes, sans gouttelettes. - CCC. - Bords des eaux. - St-Amand. Automne.

1259. DIAP. SYNGENESIA (?). Fuckel. - Alb. de l'Est. - Sur rameaux de Rhamnus frangula. - Périthèces sous-cutanès, peu apparents au dehors, se rapprochant de l'espéce voisine; thèques fusoïdes; sporidies longues, étroites : resserrées au milieu. un peu courbes; 4 gouttelettes écartées. - AR. - La Chaussée. Hiver. 
1260. DiAP. TALEOLA. Sacc. - Alb. de l'Est. - Sur les jeunes rameaux du chêne. - Pustules corticales, peu apparentes extérieurement; périthèces 5-8 contenant des théques presque cylindriques ; sporidies monostiques, munies de 4 appendices filiformes dont deux aux extrémités et deux au centre en forme de croix. - AR. - St-Amand, St-Lumier. - Automne.

1261. DiAP. GALERICULATA. Sacc. - Alb. de l'Est. - Sur rameaux de Fagus sylvatica. Pustules corticales, blanchâtres; périthèces agrẻgés en cercles; théques fusoïdes, tronquées supérieurement, contenant 6 sporidies eliiptiques fusoïdes, munies de 2 appendices ciliẻes. - AR. - St-Amand, Aulnay. - Printemps.

1262. DIAP. PULlA. Nits. - Alb. de l'Est. - Sur tiges de Solanum dulcamara et sur Hedera helix. - Périthéces enfoncés dans le ligneux; ostioles èrumpents , spiniformes, traversant un strôme noir, superficiel ; thèques entourées de paraphyses, renfermant 8 sporidies elliptiques-oblongues, 4 gouttelettes. $-\mathrm{AC}$. - Printemps.

1263. DIAP. CRYTICA. Nits. - Alb. de l'Est. Sur tiges sarmenteuses dénudées de Lonicera periclymenum. - Strôme grisâtre, parsemé de petits points noirs, ostioles des périthèces incrustés dans le bois; théques claviformes contenant 6-8 sporidies elliptiques; 4 gouttelettes. AC. - Haies des jardins, parcs, etc. - Automne. - St-Amand, Soulanges. 
1261. DIAP. CONORUM. Niessl. - Alb. de l'Est. - Sur cônes de Pinus sylvestris. - Périthèces épars, sphériques, peu nombreux; théques claviformes; sporidies distiques, oblongues, resserrées au milieu, 4 gouttelettes. - CC. Bois de pins. - Bassu, Lisse, etc. - Hiver.

1265. DIAP. OCCULTA. Nits. - Alb. de l'Est. Sur cônes de Pinus sylvestris. - Diffère du précédent par l'ostiole trés allongé des périthéces et par ses sporidies munies aux extrémités d'appendices très courts. - AC.

1266. DiAP. NIGRELlA. Niessl. - Alb. de l'Est.

- Sur vieilles tiges d'Eryngium campestre. Périthéces incrustés dans un strôme noirâtre, superficiel; sporidies fusoïdes, issez larges ; 4 gouttelettes. - AR. - Bords des chemins. St-Amand.

126\%. DIAP. ORTHOCERAS. Nits. - Alb. de l'Est. - A la base des tiges d'Achillea millefolium. - Aspérités superficielles, dues à la présence des ostioles coniques des périthéces incrustés dans le ligneux; thèques claviformes; sporidies distiques, fusiformes ; 4 gouttelettes. AC. le long des chemins herbus. - St-Lumier. - Printemps.

1268. DIAP. LINEARIS. Nits. - Alb. de l'Est.

- Sur tiges sèches de Solidago virga aurea.

- Périthéces disposés en ligne; ostioles courts. saillants; thèques claviformes; sporidies fusiformes, resserrées au milieu; 4 gouttelettes. AC. - St-Amand, Lisse. - Automne. 
1269. DIAP. EUPHORBIA. Cooke. - Alb. de l'Est.

- Sur tiges d'Euphorbia cyparissias. - Périthèces épars, 6-8 sous l'épiderme noirci et formant tache sur la tige; théques claviformes; sporidies fusoïdes. - AR. - St-Amand. - Le long des chemins herbus. - Printemps.

12\%. DIAP. VINCA. Cooke. - Alb. de l'Est. Sur tiges desséchées de Vinca major. -- Strômes noirâtres formant des taches irrégulières; périthèces dont les ustioles sont saillants, coniques; thèques claviformes; sporidies fusoïdes. resserrées au milieu; 4 gouttelettes. - R. dans les Jardins. - Printemps.

12\%1. DIAP. IN EQUALIS. Nits. - Alb. de l'Est.

- Sur tiges et racines mortes de Genista prostrata; groupes épars de périthèces disposés en cercle, déchirant l'épiderme; théques octospores et paraphyses fugaces, très longues; sporidies monostiques semblables à celles de Diap. fibrosa. - AR. - Lisse, Bassu. - Sapinières. Printemps.

1272. DIAP. VEPRIS. Fuckel. - Alb. de l'Est. Sur tiges de Rubus fruticosus. - Périthèces en lignes avec ostioles coniques dirigés en tous sens; thèques claviformes; sporidies un peu courbes, resserrées au milieu. - AC. - StAmand. - Automne.

1273. DIAP. ROSTELlATA. Nits. - Alb. de l'Est - Sur tiges de Rubus fruticosus. - Diffère de l'espéce précédente par ses périthèces superficiels. munis de cols allongés el par ses spores 
oblongues terminées à chaque extrémité par un appendice court, spiniforme. - AR. - StLumier. - Printemps.

12\%. DiAP. LIRELLA. Fuckel. - Alb. de l'Est.

- Sur tiges de spircea ulmiria. - Taches elliptiques ayant l'apparence des Leptostroma. recouvrant 4 à 6 périthèces, sphèriques, un peu aplatis, contenant des thèques claviformes, octospores; sporidies baculiformes, droites; 4 gouttelettes. - AR. - Bords du Fion. - St-Amand.

12\%. DIAP. CERASI. Fuckel. - Alb. de l'Est. Sur les rameaux de Cerasus avium. - Pustules corticales contenant 5-8 périthèces; thèques claviformes; sporidies distiques-fusiformes. acuminèes ; 4 gouttelettes. - AR. - Printemps. St-Amand.

12\%6. DIAP. RESECANS. Nits. - Alb. de l'Est. - Sur rameaux de Syringa vulgaris. - Strôme noir, cortical, logeant les périthèces épars . incrustés dans le bois; ostioles courts; théques claviformes ; sporidies acuminées: oblongues ; paraphyses fugaces; 4 gouttelettes. - AC. dans les jardins. - Printemps. - St-A mand.

12\%. DIAP. ALNEA. Fuckel. - Alb. de l'Est. Sur rameaux 'secs d'Alnus glutinosa. - Périthèces sous-cutanés, à peine visibles, épars, noirs, à nucleus blanc ; théques octospores; sporidies didymes, resserrées à la cloison. AC. - St-Lumier. - Bois. - Été, Aulomne.

1278. DIAP. RYCKHOLTII. Nits. - Alb. de l'Est. - A la base des rameaux secs de symphori 
carpon. - Pustules sous-cutanées composées de 5-6 périthèces; théques octospores; sporidies elliptiques-oblongues, un peu acumirées, distiques; 4 gouttelettes. -- Paraphyses fugaces . longues et larges. - AC. - Jardins. Hiver.

1279. DIAP. CORNI. Fuckel. - Alb. de l'Est. Sur rameaux morts de Cornus sanguinea. Strômes noirs sous-cutanés; périthéces en groupes ellipsoïdes; sporidies distiques : fusoïdes ; 4 gouttelettes. - AC. - Soulanges, Ablancourt. - Printemps.

1280. DIAP. LASCHII. Nits. - Alb. de l'Est. Sur rameaux d'Evonymus Europceus. - Strôme noir, étendu, sous-cutané : traversé par les ostioles des périthèces épars, logés dans le bois : sporidies ovales-oblongues, non resserrées à la cloison ; 4 gouttelettes. - La Spermogonie Phoma foveolaris croît à la surface de l'épiderme qui recouvre le strôme. - AR. Ablancourt. - Printemps.

1281. DIAP. PUTATOR. Nits. - Alb. de l'Est.

- Sous l'écorce des rameaux de Populus nigra.

- Périthèces confluents, elliptiques, incrustés dans le bois ; théques claviformes; sporidies elliptiques, allongées; 4 gouttelettes. - StAmand. - Bois. - C. - Hiver.

1282. DIAP. EPILOBII. Cooke. - Alb. de l'Est. -- Sur tiges mortes d'Epilobium hirsutum. Périthèces sphériques, épars, traversant un strôme à peine coloré; théques avec paraphyses ; 
sporidies fusoïdes, resserrées au milieu ; 4 gouttelettes. - AC. - Printemps. - Bords du Fion. 1283. DIAP. STRIAFORMIS. Nits. - Alb. de

l'Est. - Sur tiges d'Epilobium spicatum. Groupes de périthèces distincts, disposés en ligne sous l'épiderme légèrement teinté ; theques cylindriques; sporidies monostiques, ovoïdesoblongues. - AR. - Jardins. - St-Amand. - Été.

1284. DIAP. PARDALOTA. Fuckel. - Alb. de l'Est. - Sur tiges de Convallaria multiflora. - Petites taches grises. irrégulières, formées par des strômes minces, contenant de 6 à 8 périthèces ronds, aplatis ; thèques octospores ; sporidies elliptiques-oblongues; 4 gouttelettes. - AC.

1285. DIAP. BECKAUSII. Nits. - Alb. de l'Est. - Sur rameaux de Viburnum opulus. - Strôme noirâtre, ètendu, sous-cutané, traversé par les ostioles des périthèces nombreux, plongés dans le bois ; thèques octospores; très longues paraphyses, fugaces ; sporidies étroites, non resserrées ; 4 gouttelettes. - AR. - Automne. Bois de St-Amand.

SEct. V. - PHAEODIDYMÉES. - Sacc. 49e GENRE. - DIDYMOSPHACRIA. - Sacc.

1286. DIDYM. GENISTA (Spartii ?) Fuckel. Alb. de l'Est. - Sur tiges et racines de Genista 
prostrata. - Strôme noirâtre, mince; périthèces épars, sphériques; théques cylindriques avec paraphyses ; sporidies elliptiques, brunes, uniseptées, monostiques, - AC. - Bassu, Soulanges. - Automne.

1287. DIDYM. DIPLOSPORA. Rehm. - Alb. de l'Est. - Sur les sarments de Rubus fruticosus. - Périthéces sous-cutanés, hémisphériques, noirs, luisants ; l'épiderme qui les recouvre est noirci ; théques cylindriques ; sporidies monostiques, brunes-olivâtres. - AC. - St-Lumier, Soulanges ; - Printemps.

1288. DIDYM. ACERINA. Rehm. - Alb. de l'Est.

- Sur les rameaux secs d'Acer platanoides. Périthèces sous-cutanés, hémisphériques, aplatis ; thèques cylindriques; sporidies monostiques. elliptiques-allongées, brunes. - AC. - Bois de St-Amand. - Printemps, automne.

50 GENRE. - AMPHISPIHARIA - - Cés.

1289. AMPH. UMBRINA. De Not. - Alb. de l'Est. - Sur rameaux de chêne ; Périthèces souscutanés ; thèques cylindriques avec paraphyses filiformes, libres ; sporidies fuligineuses, brunpâle. - AC. - St-Amand. - Automne, hiver.

1290. AMPH. PSEUDO-UMBRINA. Sacc. - Alb. de l'Est. - Sur rameaux d'Ulmus campestris. - Périthèces sous-cutanés, épars, sphériques ; théques cylindriques ; paraphyses indécises, muqueuses ; sporidies monostiques. brunes, elliptiques. - AC. - Soulanges, St-Amand. - Prinlemps. 
1291. AMPH. PUSIOLA. Karsten. - Alb. de l'Est. - Sur hois de Chêne dénudé. - Groupes de périthéces sphériques, trés petits, luisants, entourés de poils noirs qui leur semblent étrangers; nucleus blanc ; théques cylindriques ; sporidies elliptiques, allongées; brunes. - RR. - Bois de Sermaize. - Eté.

1292. AMPH. POSIDONIAe. Cés. - Alb. de l'Est.

- Inscrit dans notre catalogue pour mémoire.

- Celte plante m'a été communiquée par M. Marcilly, Inspecteur des forêts à Châlons-surMarne. Je copie ici textuellement la notice dont j'ai accompagné le dessin de mon album, après examen. Cette espèce croît sur les tiges radicantes du Posidonia oceanica végétant encore au fond de la mer ; l'échantillon a été récoltè en 18\%, dans la mer d'Eza prés de Monaco. Les souches brunes, luisantes, écailleuses du Posidonia sont couvertes çá et lá de périthèces dimidiés, hémisphériques, noirs, à ostiole papilliforme; les thèques et les sporidies sont trés développées ; ces dernières ont la forme des Diplodia; les périthèces croissent au milieu de concrétions maritimes diverses; coraux, etc.

51e GENRE. - DELITSCHMA. - Auersw.

1293. DELITS. AUERSWALdII. Fuckel. - Alb. de l'Est. - Sur les crottes de lièvre. - Périthèces à demi émergés, noirs, sphériques avec un col èpais plus ou moins proéminent; théques amples ; sporidies brunes, grandes, biloculaires, 
entourèes d'un limbe muqueux. - Est accompagné par Delitschia minuta. - RR. - Sapinières de Soulanges. - Printemps.

129'. DELITS. MiNUTA. Fuckel. - Alb. de l'Est.

- Sur la même station que la précédente espéce et possédant les mêmes caractères. -- Se distingue par ses dimensions trois fois plus petites; sporidies des Diplodia entourées d'un limbe fugace.

52e GENRE. - OTTHIIA. - Nits.

1295. отTH. POPULiNA. Fuckel. - Alb. de l'Est. - Sur rameaux de Populus nigra et pyramidalis. - Pustules contenant de 15 à 20 périthéces globuleux, agglomérés ; thèques cylindriques; paraphyses fugaces; sporidies mono. stiques. didymes, brunes. - AC. - St-Amand, St-Lumier. - Printemps.

1296. OTTH. BRUNAUdIANA. Sacc. - Alb. de l'Est. - Sur rameaux de Ribes rubrum. Pustules de substance vert-noirâtre, renfermant de nombreux pèrithèces globuleux, noirs, érumpents par une fente transversale de l'épiderme; thèques cylindriques; sporidies ovoïdes-oblongues, septèes. - Espece accompagnée souvent par Diplodia ribis. - AC. - Jardins. - StAmand. - Printemps.

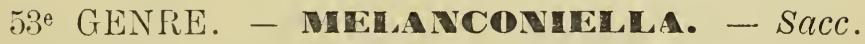

1297. MELla . SPODIAEA. Sacc. - Alb. de l'Est.

- Sur écorce de Carpinus betulus. - Groupes 
en cercles de périthèces sphériques, aplatis, thèques claviformes, octospores ; sporidies brunclair, biloculaires, elliptiques, munies de deux grosses sporules et, à chaque extrémité, d'un court appendice incolore, hémisphérique. - RR. - Bois de Sermaize. - Automne.

\section{5'te GENRE. VALSARIA. - De Not.}

1298. VALS. INSITIVA. Ces. - Alb. de l'Est. Sur rameaux de Rhamnus frangula et de Cytisus laburnum. - Périthèces sous-cutanés, agglomérés sur un strôme noir, plus ou moins èten$\mathrm{du}$; théques presque cylindriques. entourées de paraphyses ; sporidies monostiques, incolores puis brunes, biloculaires : 2 gouttelettes; l'ètat conidien Coniothyrium insitivum, croît sur le même strôme. - AC. - St-Amand, La Chaussée, etc. - Printemps.

1299. VALS. CINCTA. Sacc. - Alb. de l'Est. Sur écorce de Hêtre et de Frêne. - Pustules en disques, rangées en lignes, entr'ouvrant l'épiderme, renfermant des groupes de périthèces ovoïdes; thèques cylindriques avec paraphyses ; sporidies brunes, elliptiques, biloculaires. - AC. - Bois. - St-Lumier, Aulnay. - Printemps. - Melogramma rubricosa de Plowright.

1300. VALS. NIESSLII. Sacc. - Alb. de l'Est. sur écorce de Betulus alba . - Périthèces nombreux dans un strôme ovale, sous-cutané. 
munis d'ostioles allongés, émergents ; thèques claviformes ou cylindriques; sporidies biloculaires, incolores puis brunes. - AR. - StAmand ; Cense des Prés. - Printemps.

\section{SEGT. VI. - PHÆOPHRAGMIÉES. - Sacc.}

55e GENRE. - Massaria. - De Not.

1301. MASS. FAEDANS. Fuckel. - Alb. de l'Est.

- Sur les branches et rameaux d'Uimus campestris. - Groupes de 6-8 périthèces sous-cutanés, sphériques, réunis en cercle, émettant au dehors des sporidies brunes qui tachent l'épiderme; théques trés amples avec paraphyses continues ; sporidies pyriformes à 2 cloisons, brunes, entourèes d'un limbe muqueux. - AC. - Bois. - Automne, printemps.

1302. MASS. PUPUlA. Tul. - Alb. de l'Est. Sur rameaux secs d'Acer pseudo-platanus. Périthėces épars, sous-cutanés, arrondis, aplatis. Théques larges surtout au sommet; sporidies brunes, oblongues-claviformes, à 3 cloisons ; limbe muqueux. - La conidie Steganosporium pyriforme l'accompagne. - AC. - Bois. - St-Amand. - Printemps.

1303. MASS. INQUINANS. Fries. - Alb. de l'Est. - Sur rameaux d'Acer campestris. - Périthèces sous-cutanés, rejetant une masse de sporidies noires qui tachent l'épiderme comme les stylospores, thècyues. grandes avec paraphyses 
filiformes; sporidies à 3-6 cloisons, fusiformes. brunes. - CC. - Bois. - Hiver, printemps. - Très voisin de Mass. gigaspora.

130'. MASS. ULMI. Fuckel. - Alb. de l'Est. Sur rameaux d'Ulmus campestris. - Périthéces sphériques, sous-cutanés; théques larges avec paraphyses; sporidies distiques. fusoïdes, brunes, à 4 cloisons, plus petites que celles de Mass. inquinans. - CC. - St-Amand. - Bois . - Hiver.

1305. MASS. FAGI. Fuckel. - Alb. de l'Est. Sur rameaux de Fagus sylvatica. - Périthèces faisant irruption au dehors par de petites fentes; théques allongées; paraphyses muqueuses; sporidies fusoïdes à 3 cloisons, brunes, entourées d'un limbe. - La conidie Steganosporium fagi habite le même support. - AC. - St-Amand, Aulnay. - Bois. - Printemps.

1306. MASS. RUBI. Fuckel. - Alb. de l'Est. Sur sarments de Rubus fruticosus. - Périthèces sous-cutanés, épars; thèques accornpagnées de longues paraphyses; sporidies brunes, fusiformes, à 5-6 cloisons et entourées d'un limbe - RR. - St-Lumier. - Printemps.

130\%. MAsS. Vomitaria. $B$. et $C$. - Alb. de l'Est. - Sur écorce d'Acer. -- Périthèces sphériques, sous-cutanés; théques amples avec paraphyses muqueuses; sporidies brunes, pyriformes, 2 cloisons; limbe apparent dans les jeunes sporidies. - Reims. - Communiqué par le Dr Jolicœur. - Hiver. 
1308. MASS. MACROSPORA. Sacc. - Alb. de l'Est. - Sur rameaux de Fagus sylvatica. - Périthèces en groupes peu nombreux, sphériques . entourés de l'état conidien Sporidesmium Vermiforme; théques et paraphyses d'égale grandeur; sporidies fusoïdes à 3 cloisons, incolores puis fuligineuses; limbe mucilagineux. - CC. surtout la Conidie. - Printemps. - Aulnay . St-Amand.

\section{6e GENRE. - LEPTOSTPH AERAA. - Cés.}

1309. LEPT. DOLIOLUM. De Not. - Alb. de l'Est. - Sur tiges d'Angelica sylvestris. Périthèces épars, superficiels, noirs, entourés de la base au sommet de plis concentriques ; théques allongées; sporidies submonostiques, fusiformes, droites, à 3 cloisons ; teinte jaunâtre. - CCC. - Bords des eaux. - Printemps.

1310. LEPT. SUFFulta. Niessl. - Alb. de l'Est. - Sur tiges desséchés de Melampyrum pratense. - Groupes de périthéces conoïdes, à base aplatie avec plis concentriques; se rapproche de l'espéce précédente ; théques oblongues; paraphyses filiformes, continues; sporidies fusiformes à 3 cloisons, jaune-olive. AR. - Bois de Sermaize. - Eté.

1311. LEPT. OLERICOLA. Sacc. - Alb. de l'Est.

- Sur tiges mortes de Brassica oleracea. Périthéces irréguliers, difformes, noirs-luisants ; théques cylindriques ; sporidies submonosti - 
ques, fusiformes à 3 cloisons, jaunâtres ou cafẻ au lait étant mûres. - AC. -- Jardins . - Accompagné par Phoma lingam.

1312. LEPT. APARINES. Fuckel. - Alb. de l'Est.

- Sur tige de Galium mollugo et aparines. Périthèces épars, sphériques, s'affaissant en cupules ; thèques cylindriques; sporidies elliptiques allongées, à 3 cloisons un peu resserrées, jaunâtres. - AC. - Bords des bois. Automne.

1313. LEPT. ACONITI. Sacc. - Alb. de i'Est. Sur tiges mortes d'Aconitum napel. - Périthèces épars, sphériques ou conoïdes; thèques en massue allongée inférieurement; sporidies distiques, fusiformes, arquées, à 3 cloisons. olives ou brunes. - AR. - Jardins. - St. Amand. - Printemps.

1314. LEPT. VAGABUNDA. Sacc. - Alb. de l'Est.

- Sur tiges de Seseli montanum et sur Cornus sanguinea ; - Périthèces épars, sous-cutanés : thèques allongées ; paraphyses noduleuses ; sporıdies brunes, fusoïdes-aiguës, à 3 cloisons.

- AR. - St-Lumier, St-Amand. - Printemps.

1315. LEPT. OGILVIENSIS. Ces. - Alb. de l'Est.

- Sur tiges mortes de Picris et de Senecio.

- Périthéces épars ; thèques en massue, allongées ; paraphyses simples, septées ; sporidies lancéolées, à 5 cloisons ; vert-d'eau clair. AC. - St-Amand, Lisse. - Automne.

1316. LEPT. MACULANS. Ces. - Alb. de l'Est. A la base des tiges desséchées d'Erysinum 
alliarice. - Périthèces sphériques, épars sur une tache noire qui entoure le support ; thèques en massue, allongées ; sporidies fusiformes-lancéolées à 5 cloisons, jaune-miel. AC. - Le long des haies à St-Amand. - Printemps.

131\%. LEPT. MODESTA. Karsten. - Alb. de l'Est. - Sur les tiges desséchées d'Eupatorium et d'Achillaca millefolium. - Périthèces èpars, sous-custanés, conoïdes ; thèques en massue ; sporidies distiques; fusiformes-allongées, aiguës, à 5 cloisons; cellule médiane noduleuse renflée. - CC. - St-Lumier, Soulanges. - Printemps. 1318. LEPT. AGNITA. De Not. - Alb. de l'Est.

- Sur tiges d'Eupatorium cannabinum . Périthèces épars, sphériques, sous-cutanés; thèques en massue; sporidies fusiformes allongées , à 6 cloisons. - CC. - Bois. - St-Lumier, St-Amand. - Printemps.

1319. LEPT. ACUTA. Karsten. - Alb. de l'Est.

- Sur tiges mortes d'Urtica divica. - Périthèces sous-cutanés puis libres, en forme de cônes aigus; théques en massue; sporidies jaunâtres, fusiformes-allongées, à 6-10 cloisons. - CCC. - Jardins, haies. - Partout.

1320. LEPT. EUSTOMA. Sacc. - Alb. de l'Est.

- Sur feuilles de Phragmites communis. Périthèces sous-cutanés, très petits, aplatis ; théques amples, entourées de paraphyses; sporidies fusoïdes, courbes. olivâtre-clair, à 3 cloisons. - C. - Printemps. - Aulnay, St-Amand. 
1321. LEPT. ARUNDINACEA. Sacc. ..- Alb. de l'Est. - Sur chaumes de Pliragmiles communis; - Groupes de 6 à 10 périthèces disposés à la base du chaume et prés des nœuds, elliptiques; thèques en massue; sporidies droites, étroites. allongées, à 3 cloisons, jaune-miel. - CC. Bords des eaux. - Partout. - Hiver-printemps. 1322. LEPT. TYPHARUIM. Karsten. - Alb. de l'Est. - Sur les chaumes de Typha latifolia. - Périthéces épars, cachés dans le parenchyme; ostiole à peine visible au dehors; thèques cylindriques ou en massue; sporidies un peu jaunàtres, fusoïdes à 3 cloisons. - CC. - StLumier, Soulanges, etc. - Printemps.

132'. LEPT. JUNCINA. Sacc. - Alb. de l'Est. Sur chaumes de Juncus conglomeratus. - Périthèces sous-cutanés, ronds, aplatis, disposés en lignes; thèques courtes, renflées; sporidies fusoïdes à 3 cloisons; loge médiane plus large. - AC. - Lieux marécageux. - St-Amand. Lisse. - Été.

1324. LEPT. NIGRANS. Cés. - Alb. de l'Est. Sur feuilles et gaînes de Dactylis glomerata - Périthéces sous-cutanés, distincts, hémisphériques, entourés de filaments bruns rampants: théques renfermant 8 sporidies distiques, fusoïdes, allongèes, jaunâtres, á 5 cloisons, l'avantdernière loge renflée. - CC. - Bois. - StAmand. - Printemps.

1325. LEPT. RUSCI. Sace. - Alb. de l'Fist. Sur feulles et rameaux de Ruscus aculcatus. 
- Périthèces épars ; thèques claviformes; paraphyses de même grandeur; sporidies distiques, elliptiques-oblongues, à 3 cloisons ; jaune-miel. - AR. - Bois de Sermaize. - Automne.

1326. Lept. CULmifragA. Cés. - Alb. de l'Est. - Sur chalmes de Bromus asper. - Périthèces disposés en lignes, à demi émergés; théques allongées; sporidies distiques, fusoïdes, longues, à 6-8 cloisons, jaune-citron. - AC. Printemps. - Bois de St-Amand à St-Lumier. 132\%. LEPT. RIPARIA. Sacc. - Alb. de l'Est. Sur chaumes de Juncus communis. - Périthèces sous-cutanés, en groupes épars; thèques ovoïdes entourées de paraphyses pleines do gouttelettes; sporidies fusoïdes oblongues, un peu courbes. à 5 cloisons; jaune-verdâtre. AR. - Entre St-Amand et Lisse. - Automne. 1328. LePt. Planiuscula. Cés. - Alb. de l'Est. - Sur tiges de Solidago-virga aurea. Périthèces disposés en lignes, ronds, aplatis; théques en massue allongée ; sporidies brunpâle, fusoïdes, courbes, à 5 cloisons. - $-A R$. - Entre St-Amand et Aulnay. - Printemps.

132:9. LEPT. STELLARIE. Ch. $R$. - Alb. de l'Est. - Sur les tiges anciennes de Stellaria media mélangées aux tiges vivantes. - Périthéces sphériques, noirs, un peu aplatis, épars; thèques en massue; paraphyses filiformes, très longues; sporidies fusiformes, allongées, aiguës à 3 cloisons, faiblement jaunâtres. - AR. St-Amand. - Printemps. 
1330. LEPT. BERBERIDIS. Ch. $R$. - Alb. de l'Est. - Sur jeunes rameaux de Berberis vulgaris vivants. - Périthèces situés près des fascicules d'épines, épars, sous-cutanés, aplatis lenticulaires; théques claviformes enveloppées par les paraphyses; sporidies fusoïdes-oblongues, à 3 cloisons, olive-clair. - AR. - Récolté dans mon jardin. - St-Amand. - Printemps.

1331. LEPT. LEMOINII. Ch. R. - Leptosphcerites. Bull. Soc. bot. de Fr. 1880. - Cette sphériacée. l'un des plus anciens représentants de la mycologie, a été trouvée dans les envirorıs de Reims par le $D^{r}$ Lemoine qui me l'a communiquée pour en faire l'analyse. J'ai constaté son habitat sur une Monocotyledonnée! de l'époque tertiaire, incrustée dans un fragment d'argile à lignite dont j'ai soulevé avec soin les lamelles pour le mettre à jour; je l'ai nommé alors Leptosphcerites à cause de sa station spéciale ; l'examen microscopique m'a donné la preuve la plus évidente de la conservation intacte de ses organes de reproduction. - Périthèces noirs, hémisphériques, à ostiole court, renfermant des sporidies fusoïdes, brunes, un peu courbes, à 3 cloisons; Spermogonies avec spermaties (bâtonnets) disposées en chapelet.

Quand je fis hommage de ce précieux specimen au Museum, lors de la Session mycologique de 188\%, M. le professeur Bureau reconnut que notre espéce, dont les organes de reproduction s'étaient si bien conservés, était un 
témoignage frappant de la grande vitalité des sporidies capables de résister à la destruction après tant de siècles écoulés et que, malgré son ancienneté, une place devait lui être réservée dans le Genre d'espèces vivantes Leptosphceria que nous adoptons aujourd'hui.

5\% GENRE. - CLYPEOSPIH ARIA. - Fuckel.

1332. CLYP. NOTARISII. Fuckel. - Alb. de l'Est.

- Sur sarments de Rubus fruticosus. - Pèrithèces sous-cutanés; ostiole peu saillant entouré d'une tache noire en forme de disque ou de bouclier; thèques cylindriques; paraphyses filiformes; sporidies brunes, lancéolées-oblongues; courbes, à 3 cloisons.

Var. Robiniæ. Ch. R. - Disque moins apparent; sporidies plus petites. - AR. - St-Lumier, St-Amand. -- Automne.

\section{GENRE. - CH AETOSPH AERIA. - Tul.}

1333. CHAT. PHAOSTROMA. Fuckel. - Alb. de l'Est. - Récolté sur une planche depuis longtemps placée dans une cave. - Groupe peu nombreux de Périthèces petits, noirs, astomes, entourés de poils à la base ; théques en massue renflée, fugaces; paraphyses simples; sporidies elliptiques-oblongues à 3 cloisons, les deux loges médianes brunes, les deux extrêmes hyalines. - RR. - St-Amand. - Hiver.

183'. CHAT. CALlimorPhA. Sacc. - Alb. de l'Est. - Sur sarments de Rubus. - Périthèces 
très petits, sphèriques, au milieu d'un coussinet noirâtre de poils rigides; théques claviformes; sporidies fusoïdes, munies de 3 cloisons et de 4 gouttelettes, à peine teintées. - RR. St-Amand. - Hiver.

59" GENRE. - MRELANOMM. - Nits.

1335. MELAN. PULVISPYRIUS. Fuckel. - Alb. de l'Est. - Sur rameaux de Corylus avellana. - Groupes trés nombreux de périthéces petits, sphériques, noirs, ressemblant á des grains de poudre; théques cylindriques; sporidies légèrement teintées, oblongues, monostiques à 3 cloisons. - AC. - St-Amand, Soulanges, etc. - Printemps.

1336. MEL. VILE. Fuckel. - Alb, de l'Est. Sur écailles de Noyer. - Périthéces plus petits que dans l'espéce précédente, épars; théques cylindriques; paraphyses filiformes, bifurquées au sommet; sporidies brun-clair, oblongues à 3 cloisons. - R. - St-Amand. - Printenups.

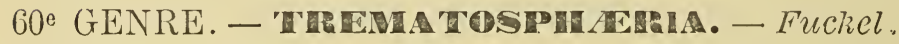

1337. TREM. FRAXINI. Ch. R. - Alb. de l'Est - Sur rameaux morts de Fraxinus excelsior. - Périthéces incrustés dans le bois, noirs. sphériques; ostioles corirts, conoïdes ; théques en massue terminées par un long pédicelle; paraphyses simples; sporidies oblongues-cylindriques, courbes, brun-clair, á $5-6$ cloisons, dis- 
tiques, noircissant l'épiderme autour de l'ostiole. - Bois de St-Amand. - RR. - Printemps.

61e GENRE. - STPIRORMIIA. - De Not.

1338. SPOR. INTERMEDIA. Auersw. - Alb. de l'Est. - Récolté sur tige d'Allium cœpa, dans le voisinage du fumier de cheval. - Pèrithèces sphériques, superficiels, noirs; thèques subcylindriques; sporidies distiques. paralléles, composées de 4 articles brun-verdâtre qui se désagrègent facilement. - AR. - St-Amand. Été.

1339. SPOR. OVINA. Sacc. - Alb. de l'Est. Sur crottes de brebis. - Périthèces petits, à demi émergés: ostiole conique; thèques subcylindriques entourées de paraphyses ténues ; sporidies distiques, à 4 segments, brun-verdâtre. - CC. - Champs, pâturages. - St-Amand. - Automne.

629 GENRE. - ATILOSPORA. - De Not.

13’0. Agl. PROFusA. De Not. - Alb. de l'Est. - Sur rameaux de Robinia. - Pustules elliptiques, contenant 2 à 5 périthéces circonscrits à la base par une ligne noire; thèques cyindriques á 4 sporidies brunes, elliptiques oblongues, droites, à 3 cloisons et 4 grosses sporules. - CC. - Jardins. - Printemps.

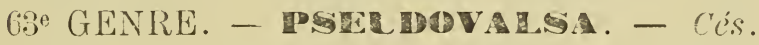

1311. PS. VALS. LANCIFORMIS. Cés. - Alb. de 
l'Est. - Sur branches et rameaux de Betulus alba. - Pustules verdâtres, tronquées au sommet, contenant 3-6 périthèces; thèques cylindriques; sporidies brunes, obtuses, à 5 cloisons; paraphyses fugaces, longues. - CC. Bois, haies. - St-Amand. - Printemps.

1342. PS. VALS. BERKELEYI. Sacc. - Alb. de l'Est. - Sur branches et rameaux d'Ulmus campestris. - Périthèces disposés en cercles sous l'épiderme; thèques oblongues; sporidies brunes, distiques. elliptiques-oblongues, à 3 cloisons, munies à chaque extrémité d'un appendice hyalin assez long; recourbé. - AR. Soulanges, St-Amand. - Printemps.

1313. PS. VALS. AUCTA. Sacc. - Alb. de l'Est. - Sur branches d'Alnus glutinosa. - Périthèces 5-6 en cercles, à cols convergents ; théques oblongues - ovales, sessiles; paraphyses fugaces; sporidies elliptiques - oblongues, brunclair, á cloison et plusieurs gouttelettes ; appendices très courts aux extrémités. - AR. - St-Amand. - Printemps.

1344. PS. VALS. HAPALOCYSTIS. Sacc. Alb. de l'Est. - Sur rameaux de Platane. Pustules hémisphériques, renfermant 5-6 périthéces à ostioles courts; thèques ovales; sporidies agglomérées, brunes, elliptiques à 2 cloisons . terminées par deux appendices hyalins en forme de coin. - RR. - Ablancourt. Jardins. - Hiver.

1345. PS. VALS. KICKXII. Sacc, - Alb. de l'Est. 
- Sur rameaux de Platane oriental. - Périthéces disposés en cercles comme ceux des Valsa; thèques amples, courtes, contenant 4 sporidies fuligineuses, elliptiques-allongées, à 5 cloisons. munies à chaque extrémité d'un assez long appendice, cylindrique, hyalin. AR. - Ablancourt. - Jardin du château. Hiver.

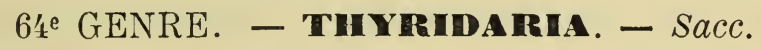

1346. THYR. INCRUSTANS. Sacc. - Alb. de l'Est. - Sur rameaux de Sumac amaranthe. - Périthèces nombreux, agglomérés en pustules ; ostioles larges, noirs, saillants . entourés d'un cercle ruage foncé tomenteux ; thèques cylindriques, paraphyses nombreuses; sporidies monostiques, brunes, oblongues, à 3 cloisons un peu resserrées. - AR. - Jardins. - St-Amand. - Printemps.

13ł\%. ThYR. AILANTHI. Rehm. - Alb. de l'Est.

- Sur rameaux d'Ailanthus glandulosa. Périthèces 8-10 rouge-brun, plongés dans un strôme valsiforme, brunâtre, tomenteux ou brunrouge. Théques cylindriques; sporidies brunes, oblongues, à 3 cloisons. - La Pycnide Coniothyrium ailanthi l'accompagne. - RR. - Jardin du château d'Ablancourt. - Printemps.

65 GENRE: - MELOGRAMMA. - Tul.

1318. MELOG. VAGANS. De Not. - Alb. de l'Est. - Sur branches et troncs de Carpinus betulus. 
- Périthéces 10-15, réunis dans des pustules rondes, aplaties, disposées en lignes ; ostioles courts, saillants ; théques en massue ; sporidies distiques, fusiformes, étroites, courbes, à 3 cloisons et à 4 sporules. - CC. Dans les forêts et les haies de charmille. - Hiver.

SEGT. VII. - HYALOPHRAGMIEES. - Sacc.

66 GENRE. - MASSARHA. - Sacc.

1319. MASS. EBURNEA. Sacc. - Alb. de l'Est. Sur rameaux de Platane. - Périthèces souscutanés, épars, hémisphériques ; théques claviformes ; sporidies incolores, oblongues-fusoïdes, uniseptées, resserrées à la cloison, 4 sporules, entourées d'un limbe muqueux ; paraphyses sinueuses. - AR. - Ablancourt, Vitry-la-Ville. - Automne, printemps.

1350. MASS. CORNI. Sacc. - Alb. de l'Est. Sur rameaux de Cornus sanguinea. - Périthèces épars, solls-cutanés; thèques amples entourées de paraphysẹs sinueuses. indécises; sporidies distinctes, à 3 cloisons, 4 gouttelettes oblonguesfusoïdes ; limbe peu apparent. - AC. - St-Amand. - Printemps.

1351. MEtAs. OCELIATA. Sacc. - Alb. de l'Est. - Sur tiges mortes d'IIypericum perforatum. Périthèces sous-cutanés, brunissant l'épiderme ; théques claviformes entourées de paraphyses 
simples, courtes ; sporidies sub-monostiques fusoïdes, 2 septées, resserrées aux cloisons, à 3 gouttelettes. - $\Lambda$ R. - Printemps. - St-Lumier. St-4mand.

1352. METAS. SEPINCOLA. Fuckel. - Alb. de l'Est. - Sur rameaux de Rosa canina. - Périthèces épars, sous-cutanès; théques cylindriques ; sporidies elliptiques-oblongues, monostiques, 3-4 septées. - CC. - Dans les haies et broussailles. - St-Amand, etc. - Automne.

1353. METAS. CORTICOLA. Sacc. - Alb. de l'Est. -- Sur rameaux de Prunus domesticus. Périthèces sous-cutanés; théques cylindriques. 3-4 septées, elliptiques-oblongues, présentant quelquefois une cloison longitudinale. - AR. St-Amand, Aulnay. - Automne.

135'. METAS. CINEREA. Sacc. - Alb. de l'Est. - Sur rameaux de Salix caprcea, près des bourgeons. - Thèques cylindriques ; sporidies monostiques. 4 septées, déprimées vers le centre. AR. - St-Amand. - Printemps.

1355. METAS. VINCÆ. Sacc. - Alb. de l'Est.

- Sur tiges de Vinca major. - Périthéces èpars, entourant la tige, sous-cutanés; théques claviformes ; sporidies fusoïdes, un peu courbes, étroites, aiguës, à 3 cloisons. - AC. Jardins. - St-Amand. - Printemps.

1356. METAS. COCCODES. Sacc. - Alb. de l'Est.

- Sur chaumes de Phragmites communis. Pustules grisâtres, allongées, disposées en ligne, renfermant de 5 à 15 périthèces à nucleus 
blanc ; théques claviformes ; paraphyses simples ; sporidies distiques, fusiformes, à 3 cloisons. - AC. - St-Amand. - Printemps.

135\%. Metas. GeI. Ch. $R$. - Alb. de l'Est. Sur tiges de Geum urbanum desséchées. Périthèces sous-cutanés, épars ; thèques claviformes ou cylindriques; paraphyses septées ; sporidies mono-distiques, fusoïdes, chloro-hyalines, à 3 cloisons. - AR. - St-Amand, StLumier. - Printemps.

68: GENRE. - CERMOSPORA. - Niesst.

1358. CERIOSP. XANTHA. Sacc. - Alb. de l'Est.

- Sur sarments de Clematis vitcelba. - Périthèces nichés dans l'écorce, à demi erumpents, groupés pyriformes, à cols allongés ; théques cylindriques ; sporidies distiques, fusoïdes, un peu teintées jaunâtres en vieillissant, courbes, uniseptées, resserrées à la cloison, munies à chaque extrémité d'un appendice hyalin, filiforme. - AR. - Soulanges, St-Amand. Printemps.

69" GENRE. - SPHAERULINA. - Sacc.

1359. SPH ${ }^{\text {na }}$ INTERMIXTA. Sacc. - Alb. de l'Est. - Sur tiges de Rosa canina. - Périthéces épars, sous-cutanés, noircissant l'èpiderme, sous forme de petits disques luisants; théques sans paraphyses, ovales-oblongues, disposées en roselte ; sporidies pyriformes, à 3-4 cloisons. hyalines. 
Var. Corni. Sur rameaux de Cormus sanguinea. - Thèques et sporidies plus développées que celles du type. - AC. - St-Amand, Aulnay. - Printemps.

\section{0e GENRE. - HYPOSPHA. - Fries.}

1360. HYP. PUSTULA. Karsten. - Alb. de l'Est.

- Sur feuilles mortes de Chêne. - Périthéces brun-clair, amphigènes, formant des pustules coniques sous - épidermiques; Théques claviformes ; sporidies distiques, fusoïdes, incolores, à 3 cloisons et à $2-4$ sporules . AC. - Automne, printemps. - Bois de St-Amand.

71e GENRE. - LASIOSPIIARIA. - Cés.

1361. LAsiosph. RHACOdiUm. Cés. - Alb. de l'Est. - Sur écailles de bois pourri. - Périthèces superficiels, ovoïdes, noirs, couverts de poils conculores dressés, placés sur un subiculum de poils bruns, longs, rampants, partant de la base du périthéce ; théques cylindriques, sporidies vermiculaires, munies de 6 - 8 cloisons et gouttelettes. - CC. - Lieux humides des bois et jardins. - Printemps.

1362. LASIOSPH. SPERMOIDES. Ces. - Alb. de l'Est. - Sur troncs et bois pourrissants. Périthèces simples, ovoïdes, glabrescents , groupés, noirs ; ostiole papilliforme ; théques cylindriques : sporidies monostiques : botuliformes. - CCC. - Partout. - Automne. printemps. 
1363. LASIOSPH. CAUdATA. Sacc. - Alb. de l'Est. - Sur écailles de Pin sylvestre. - Périthèces ovales ou pyriformes, noirs, couverts de poils très courts. - La conidie Fuckelina microspora les accompagne et forme un subiculum noir autour d'eux; thèques cylindriques; sporidies sub-fusiformes, un peu courbes. - AR. - St-Amand. - Printemps.

1364. LASIOSPH. CRINITA. Sacc. - Alb. de l'Est. - Sur écailles de hois humides tournées vers le sol. - Les Périthèces sont sphériques, couverts de poils noirs, courts, raides ; ils croissent aussi dans le voisinage du support et constituent la Var. Terrestris; théques cylindriques; sporidies vermiculaires à 5-6 gouttelettes. CC. - Partout.

1365. LASIOSPH. SULFURELLA. Sacc. - Alb. de l'Est. - Sur écailles pourrissantes de Peuplier. - Périthèces ovoïdes ou coniques, recouverts d'un duvet de filaments jaune-soufre ; ostiole noir oblique; thèques cylindriques; paraphyses simples: sporidies vermiculaires, munies d'appendices filiformes aux deux extrémités. $-R R$. - Bois entre St-Lumier et St-Amand. - Été.

1366. LASIOSPH. OVINA. Cés. - Alb. de l'Est. - Sur les bois pourris, humides. - Périthèces groupés, ovoïdes, couverts d'un duvet blanc d'oú émerge un ostiole noir, court. Théques cylindriques; sporidies distiques, vermiculaires. - CC. - Automne, printemps. - Partout. 
72e GENRE. - ENCHNOSPHARIA. - Fuckel.

136\%. ENCHN. PINETORUM. Fuck. - Alb. de l'Est. - Sur écailles de Pin sylvestre. - Périthèces coniques ou pyriformes, noirs, superficiels, couverts de poils courts, rigides; théques cylindriques; sporidies fusiformes, étroites, jaunâtres, à 3 cloisons. - Diffẻre beaucoup de $L a$ siosph. caudata qu'elle accompagne souvent. - RR. - St-Amand. - Bois. - Automne.

\section{3e GENRE. MeLOMAS'TIA. - Nits.}

1368. Melom. FRIESII. Nits. - Alb. de l'Est. - Sur rameaux de Fraxinus et de Hedera helix. - Périthèces épars, base incrustée dans le bois, sommet conique, noir, luisant; thèques cylindriques; paraphyses filiformes ; sporidies menostiques, oblongues, à 2 cloisons. - CC. - Automne, printemps. - Partout.

\section{"4te GENRE. - ZIGNOELLA. - Sacc.}

1369. ZIGN. OVOIDEA. Sacc. - Alb. de l'Est.

- Sur bois dénudé des branches de Corylus avellana. - Périthèces ovoïdes ou coniques, épars; théques claviformes entourées de paraphyses; sporidies fusoïdes, distiques, à 3 cloisons et 4 sporules incolores . - AR . Automne. - St-Amand. 
75e GENRE. CEIRATUSPHAREA. - Niessl.

13\%0. CERAT. LAMPADOPHORA. Niessl. - Alb. de l'Est. - Sur racines et troncs pourris d'Alnus glutinosa. - Périthèces groupés, à demi incrustés dans le bois, la partie supérieure amincie en un long col flexueux; théques allongées; sporidies fusoïdes, étroites, longues, aiguës aux deux extrémités, à 8-10 cloisons, incolores. - CC. - Bois de St-Amand. Automne, Printemps.

SEGT. VIII. - DICTYOSPOREES. - Sacc.

76" GENRE. - PLEOMASSA IRA. - speg.

1371. PleOM. SIPARIAE. Tul. - Alb. de l'Est. - Sur branches de Betula alba. - Périthéces sous-cutanés, épars, arrondis-déprimés, tomenteux; théques simples, paraphyses sinueuses; sporidies brunes, distiques, oblongues, à 5-7 cloisons-murales. entourées d'un cercle mucilagineux. - La pycnide est le Prosthemium betulinum. - CC. - Bois de St-Lumier, St-Amand. - Automne.

7\%e GENRE. - PLEOSTPIRA. - Rab.

13\%. Pleosp. VUlgaris. Niessl. - Alb. de l'Est. - Sur tiges d'Euphorbia Gerardiana. Périthéces épars, sous-cutanés, hémisphériques 
puis aflaissés; théques claviformes; paraphyses simples; sporidies murales, distiques, à 5 cloisons transversales et une longitudinale jaunecitron. - AR. - Chemin de St-Amand à Dampierre-sur-Moivre. - Automne.

13\%3. PleOSP. MEdiA. Niessl. - Alb. de l'Est.

- Sur tiges de Scabieuse des jardins. - Périthèces épars, déprimés, bruns, noirâtres ; thèques amples; sporidies jaune-d'or, ellipsoïdesovales, murales, à $3-5$ cloisons transversales et une longitudinale; paraphyses septées. -- AC.

- Printemps.

13\%. PLEOSP. HERBARUM. Rabh. - Alb. de l'Est. - Sur les plantes herbacées, notamment Asparagus officinalis. - Pẻrithèces épars, souscutanés, aplatis ; thèques amples ; sporidies monostiques ou distiques, jaune-d'or, murales. 7 cloisons transversales et 2 longitudinales. La Spermogonie est le Phoma herbarum. CCC. - Partout.

13\%5. PLEOSP. LEGUMINUM. Raben. -- Alb. de l'Est. - Sur les gousses de Vicia sativa. Phaseolus, etc. - Périthèces épars, hémisphériques; théques larges, contenant de 6 à 8 sporidies jaune-rniel, elliptiques-ovales, murales. 8 à 12 cloisons transversales et $3-4$ longitudinales. - CC. - Jardins. - Partout.

13\%. PLEOSP. SYRINGÆ. Fuckel. - Alb. de l'Est. - Sur la face inférieure des feuilles de Syringa vulgaris. -- Périthéces épars, hẻmisphériques; théques amples; sporidies ovales- 
oblongues. obtuses : murales , sub-monostiques. jaunes. - AC. - Jardins. -- St-Amand. Printemps.

137\%. PLEOSP. SAMARA. Fuckel. - Alb. de l'Est. - Sur les samares d'Acer plalanoides. Périthèces peu nombreux, épars, hémisphériques. Thèques amples; sporidies ovales. grandes, murales. jaune-d'or. - CC. - Bois de StAmand. - Printemps.

1378. PLEOSP. SOCIALIS. Niessl. - Alb. de l'Est. - Sur scape d'Allium vineale. - Périthéces sous-cutanés, épars ; théques cylindriques; sporidies sub-monostiques à 5 cloisons transversales et une longitudinale jaunes, brunissantes: ellipsoïdes, oblongues; paraphyses très grandes. - AC. - Soulanges, St-Amand. - Été.

13\%9. PLEOSP. INFECTIORA. Fuckel. - Alb. de l'Est. - Sur les chaumes des graminées. du Seigle principalement. - Périthéces disposés en lignes parallèles, 5-6, globuleux; thèques cylindriques; sporidies murales. 5 cloisons transversales, ovoïdes-oblongues, jauned'or puis brunes. - CC. - Printemps, Été. Champs. - Partout.

1380. PLEOSP. VAGANS. Niessl. - Alb. de l'Est. - Sur chaumes de Blé. - Périthéces hémisphériques, fibrilleux à la base; théques cylindriques; sporidies fusoïdes, à 5 cloisons transversales et une longitudinale jaune-d'or.

- AC. - Fité. 
1381. Pleosp. AlliI. Ces. - Alb. de l'Est. Sur les tiges de l'Allium cepa et porrum. Périthèces épars, sphériques à ostiole conjque; thèques amples ; paraphyses courtes, septées ; sporidies murales, ovales, jaune-d'or ; 7 cloisons transversales et 4-5 longitudinales. CC. - Jardins. - Printemps.

1382. PLEOSP. JUNCI. Pass. - Alb. de l'Est. Sur chaumes de Juncus communis. - Périthéces épars: sphériques ; théques amples; paraphyses noduleuses, septées; sporidies elliptiques, amincies aux extrémités, murales, resserrées au milieu; 7-8 cloisons; brunes puis opaques. - AR. - Bords des ruisseaux de Lisse à Saint-Amand. - Hiver.

78 GENRE. - FTEREMPHORA. - Fries.

1383. PYREN. RELICINA. Sacc. - Alb. de l'Est. -- Sur les chaumes du Seigle après l'hiver. Périthèces èrumpents : hémisphériques, surmontés de quelques poils noirs, septés, dressés; théques amples; sporidies elliptiques-oblongues, jaune-miel, à 3 cloisons entourées d'un cercle muqueux. La conidie Vermicularia relicina l'accompagne souvent. - CC. - Printemps.

1384. PYREN. PELLITA. Sacc. - Alb. de l'Est.

- Sur les tiges mortes de Papaver somniferum envahi déjà par l'état conidien Brachycladium penicillatum. - Périthèces globuleux. munis de quelques poils, placés au milieu d'un coussinet formé par la conidie. Thèques cylin- 
driques; paraphyses bifurquées au sommet; sporidies monostiques, ovoïdes ou fusoïdes, à 3 cloisons transversales et une longitudinale. Vertd'eau clair, puis brunes. - AC. - Jardins. St-Amand. - Printemps.

79 GENRE. - CA PRomia. - Sacc.

1385. CAPR. JUNIPERI. Ch. $R$. - Alb. de l'Est. - Bull. Soc. Bot. de Fr. 188. spec. nov.

- Récolté sur branches et racines dénudées de Juniperus communis jonchant la terre, dans les bois de Pins entre Aulnay et Soulanges. - Printemps. - Périthéces épars, ovales ou hémisphériques, noirs, hispides, très petits. munis à la base de filaments bruns, septés; thèques larges, ovales, contenant 16 spores murales, brun-clair, à 3 cloisons transversales et 2 longitudinales ovoïdes. - Diffère de Cap. sexdecemspora par le nombre des cloisons des spores et par la station.

80" GENRE. - TEICHOSPORA. - Fuckel.

1386. TEICH. TAPHRINA. Fuckel. - Alb. de l'Est. - Sur rameau dénudé de Peuplier. Périthèces épars, glabres, base incrustée dans le bois, sommet hémisphérique, théques cylindriques ; sporidies oblongues-ovales, jaune-miel puis brunes. - AR. - Bois de St-Amand. Printemps.

1387. TEICH. ORDUCENS. Fuckel. - Alb. de l'Est. - Sur Fraxinus, dans les cicatrices des 
rameaux tombés à terre. - Périthéces groupés, superficiels ovoïdes noirs, glabres ; thèques cylindriques ; paraphyses filiformes ; sporidies ellipsoïdes, á 5-7 cloisons transversales et une longitudinale. Jaune-foncé puis brunes CC. - Bois de Lisse, St-Amand, etc. - Automne, printeinps.

81 GENRE. - PLEOSPIIARAA. - speg.

1388. PlEOSPH. PILOSELlA. Sacc. - Alb. de l'Est. - Sur rameaux de Pinus sylvestris, dans les sinus formés par les insectes. - Périthéces ovoïdes, noirs, épars, couverts de poils hispisdes; théques très amples ; sporidies ovoïdes-oblongues, murales, 8-10 cloisons trans. versales vert-glauque puis brunes. - AR. - Soulanges. - Printemps.

\section{GENRE. - RMAMPHORIA - Niessl.}

1389. RAMPH. BUXI. Ch. R. - Alb. de l'Est. - Bull. Soc. bot. de Fr. 188\% - Sur tige morte de Buxus, dans les fentes de l'écorce. - Périthéces groupés distincts, noirs, glabres, sphériques: surmontés d'un long col flexueux ou dressé ; sporidies ellipticques, murales à 3 cloisons transversales brun-clair. - RR. St-Amand. - Jardins. - Automne.

83" GENRE. - CUCURARARA. - Gray's.

1390. CUCURB. BERBERIDIS. Gray's. - Alb. de l'ist. - Sur rameaux morts de Berberis 
vulgaris. Périthéces groupés dans les lentes de l'écorce, sphériques ridés, s'ouvrant en étoile au sommet; théques cylindriques ; sporidies sub-monostiques, ellipsoïdes, resserrées au milieu, à 8-9 cloisons murales, jaunes puis brunes. - CC. - Jardinss. - Printemps. Partout.

1391. CUCURB. LABURNi. De Not. - Alb. de l'Est. - Sur rameaux de Cytisus laburnum. Périthéces globuleux, rugueux, noirs, réunis en un strôme compact ; thèques cylindriques; sporidies elliptiques, fusoïdes, à 5-7 cloisons murales, monostiques, jaunes puis brunes. CCC. - Jardins. - Automne, printemps.

1392. CUCURB. Elongata. Grev. - Alb. de l'Est. - Sur rameaux de Robinia psendacacia. - Périthéces noirs, globuleux, cespiteux, sur un strôme noir, étendu ; théques cylindriques; sporidies monostiques, ovales-oblongues, brunes murales à 5-7 cloisons. - CCC. - Jardins. Printemps.

1393. CUBURB. UlmicolA. Fuckel. - Alb. de l'Est. - Sur rameaux d'Ulmus campestris. -Groupes de 5-6 périthèces globuleux, noirs, rugueux entourés par l'épiderme; thèques cylindriques ; paraphyses muqueuses ; sporidies sub-monostiques, elliptiques, resserrées au milieu à 8-10 cloisons murales, jaune-miel puis brunes. - La conidie Sporodesmium ulmicolum l'accornpagne. - AR. - St-Amand, Aulnay, etc. Printemps. 
139'. CUCURB. COLUTEA. Auersw. - Alb. de l'kst. - Sur rameaux de Colutea arborescens. - Périthéces globuleux, noirs, cespiteux ; thèques cylindriques; sporidies jaunes puis brunes. à 5 cloisons transversales et une longitudinale. CC. - Jardins, printemps.

1395. CUCURB. CORONILLA. Sacc. - Alb. de l'Est. - Sur rameaux de Coronilla Emerus. Considéré par Fuckel comme une variété de Cucurb. Elongata. - AC. - Jardins.

1396. CUCURB. RHAMNI. Fuckel. - Alb. de l'Est.

- Sur rameaux morts de Rhamnus frangula.

- Périthèces agrégés 6-8 sur un strôme noir; théques cylindriques entourées de paraphyses sporidies ovoïdes-oblongues : à 5-6 cloisons murales, brun - clair ; la partie infèrieure n'a qu'une seule cloison souvent incolore. - R. La Chaussée, dans les haies. - Printemps.

1397. CUCURB. NAUCOSA. Fuckel. - Alb. de l'Est. - Sur rameaux d'Ulmus campestris. Périthèces sous-cutanés, astômes, peu nombreux; thèques cylindriques; sporidies ovoïdes-oblongues, à 4-5 cloisons murales, jaunes puis brunes. - AR. - St-Amand. - Printemps. - Sur le même support j'ai récolté la pycnide Diplodia Ulmi, le Camarosporium cruciatum macrostylospore et la conidie Sporidesmium Ülmicolum.

1398. CUCURB. ROSÆ. Wint. - Alb. de l'Est. Sur tiges de Rosa canina. - Périthéces globuleux ; noirs, rugueux, placés sur un strôme étendu, noirâtre; théques cylindriques; spori- 
dies oblongues-fusoïdes, monostiques à 3-4 cloisons transversales et 1 longitudinale olive clair. - AR. - Lisse. - St-Amand. - Hiver. 1399. CUCURB. MAHONIAE. Ch. $k$. - Alb. de l'Est. - Sur rameaux de Mahonia aquilifolium déjá envahis par Diaporthe crassiuscula. - Périthèces groupés sous l'épiderme déchiré et placés sur un strôme noir, ètendu, sphèriques, tuberculeux. Thèques cylindriques; sporidies jaunes puis brunes, ovales-ohlongues, resserrées au milieu, à 6-8 cloisons murales. - AR. Jardins. - St-Amand. - Hiver.

1400. CUCURB. STAPHYLEAE. Ch. $R$. - Alb. de l'Est. - Sur rameaux de staphylea primata. - Tubercules sous-cutanés, arrondis ou allongès, renfermant de $5^{\circ}$ à 10 périthèces disposés en cercles ou en lignes; théques cylindriques entourées de paraphyses simples, très longues; sporidies monostiques, ellipsoïdes, incolores puis brunes. à 5 cloisons transversales et 1 longitudinale. Les mêmes périthèces contiennent aussi une forme spermogonienne à basides arborescentes portant des petites spermaties (Dendrophoma). - RR. - St-Amand. - Jardins. - Hiver.

1101. CUCURB. RIBIS. Niessl. - Alb. de l'Est.

- Sur rameaux de Ribes rubrum. - Périthèces noirs, sub-sphériques, puis déprimés, réunis en groupes sous l'épiderme déchiré; thèques cylindriques; sporidies monostiques : obovales, brunes;olivâtres, murales à 5 cloisons 
transversales et 1-2 longitudinales. - St-Amand - Jardins. - Printemps.

1402. CUCURB. SPARTII. Cés. - Alb. de l'Est.

- Sur tiges et rameaux de Sarothamnus sco. parius. - Périthèces sphériques, épars ou disposés en lignes et placés sur un strôme noir: mince; théques cylindriques entourées de para. physes sinueuses; sporidies monostiques. brunes, murales, à 3-5 cloisons transversales, resserrées au milieu. - AR. - Prés d'Epernay; sur la lisière de la forêt. - Automne.

\section{8'e GENRE. - THYRHIUMI. - Sacc.}

1'03. THYR. PHILAdElPhi. Ch. $R$. - Alb. de l'Est. - Sur rameaux de Philadelphus coronarius. - L’épiderme soulevé laisse voir les périthèces agglomérés. sub-astomes, s'affaissant en cupule, réunis dans un strôme brun, mince, ellipsoïde, byssoïde; thèques cylindriques ; sporidies monostiques, elliptiques, murales, à 5-7 cloisons transversales et une longitudinale. Le Camarosporium macrosporum de Berk. l'accompagne. - R. - Jardins. - St-Amand. - Printemps.

85 GENRE. - FENESTELLA. - Tul.

1'10'. FENEST. PRINCEPS. Tul. - Alb. de l'Est.

- Sur branches de Betula alba, Alnus glutinosa. Carpinus. - Périthèces réunis en pustules valsiformes; théques amples. claviformes ; sporidies distiques, jaunes puis brunes, elliptiques, amin- 
cies aux deux extrémités enfin munies de deux apicules hyalins, à 3 cloisons principales et de nombreuses cloisons intermédiaires transversales et longitudinales. - AC. - St-Amand, St-Lumier . - Printemps.

1405. FENEST. MACROSPORA. Fuckel. - Alb. de l'Est. - Sur rameaux secs de Corylus et de Fagus. - Périthèces réunis en un strôme valsiforme, logé dans l'écorce; thèques cylindriques; sporidies sub-monostiques, jaunes puis brunes. elliptiques-oblongues, murales, didymes par une cloison médiane très accentuée, terminées par un mucron trés court. - R. - Communiqué par le $\mathrm{D}^{\mathrm{r}}$ Jolicœur de Reims. Automne.

1406. Fenest. Vestita. Sacc. - Alb. de l'Est. - Sur rameaux de Ribes aureum et de Juglans regia. - Périthèces 10-15 disposés en cercles; ostioles réunis en disque tronqué ; thèques cylindriques; sporidies monostiques, elliptiques, murales, à 5 cloisons transversales brunes,verdâtres. - AR. - St-Amand. - Printemps.

\section{SeGt. IX. - SCOLECOSPOREES. - Sacc.}

86e GENRE. - DPHMOBOLCS. - Riess.

140\%. OPHIOB. PORPHYROGONUS. Sacc. - Alb. de l'Est. - Sur tiges d'Angelica sylvestris. Périthèces sphériques puis déprimés, à ostiole conique, placés sur une tache de l'épiderme 
rouge-pourpre ; thèques sub-cylindriques ; sporidies filiformes, incolores puis jaunâtres, remplies de gouttelettes. - CCC. - Bords des eaux. - St-Amand. - Printemps.

1408. OPHIOB. ACUMINATUS. Duby. - Alb. de l'Est. - Sur les tiges mortes des Carduus et Cirsium. - Périthèces épars. ovoïdes, à ostiole conique, allongé, érumpent ; théques cylindriques; sporidies jaunâtres, filiformes. - CC. Partout. - Printemps.

1409. OPHIOB. HYPERICI. Sacc. - Alb. de l'Est.

- Sur tiges d'Hypericum perforatum. - Périthèces épars, peu nombreux, petits; ostiole conique; thèques claviformes, amincies en pointe à la base; sporidies hyalines, remplies de gouttelettes, filiformes. - AC. - St-Amand, St-Lumier. - Bois. - Printemps.

1410. OPHIOB. ACONITI. Sacc. - Alb. de l'Est. - Sur tiges d'Aconit napel. - Périthèces rugueux, velus, roussâtres ; ostiole noir ; thèques cylindriques : sporidies filiformes, jaunâtres. - AC. - Jardins. - St-Amand. Printemps.

1411. OPHIOB. CAMPTOSPORUS. Sacc. - Alb. de l'Est. - Sur tiges de Teucrium chamcedrys desséchée. - Périthèces épars, à demiémergés; ostiole conique; thèques claviformes; sporidies baccillaires, très recourbées, septéesnoduleuses avec renflement d'une cellule vers le milieu, jaunâtres. - AC. - St-Amand, Sou langes. - Printemps. 
1412. OPHIOB. EUSPORUS . Sacc. - Alb. de l'Est. - Sur tiges d'Origanum vulgare. - Périthèces épars, très petits ; ostiole court ; thèques claviformes; sporidies baccillaires munies de 14 à 16 cloisons et sporules; les deux cellules médianes sont renflées. - AC. - Le long des chemins. - Eté, automne. - StAmand, etc.

1413. OPHIOB. AFFinIs. Sacc. - Alb. de l'Est. - Sur tige de Mentha rotundifolia. - Périthèces épars, aplatis ; ostiole conique ; thèques claviformes ; sporidies baccillaires, à 5-6 cloisons ; la cloison médiane resserrée. - AC. Aulnay-l'Aître, Lisse. - Printemps.

1414. OPHIOB. FRUTICUM. Sacc. - Alb. de l'Est. - Sur tiges d'Ononis spinosa. - Périthèces épars, sous-cutanés ; ostioles courts, érumpents ; thèques sub-cylindriques; sporidies baccillaires: jaunâtres, septées. - CC. - Le long des chemins herbus. - Soulanges. - Printemps.

1415. OPHIOB. GRAMINIS. Sacc. - Alb. de l'Est. Sur chaumes d'Agrostis stolonifera. - Périthèces presque superficiels, glabres, coniques, épars ; thèques cylindriques; sporidies filiformes, remplies de gouttelettes. - AC. - Bords des champs humides. - Printemps.

1116. OPHIOB. CULMORUM. Sacc.-Alb. de l'Est.Sur chaumes de Phragmites communis - Périthèces ovoïdes, fuligineux, mous, incrustés dans une tache noire près du Rhizome; théques claviformes; paraphyses rameuses au sommet; spo- 
ridies baccillaires, remplies de gouttelettes. AR. - St-Lumier. - Fossés humides. - Automne. 1417. OPHIOB. ENCRYPTUS. Sacc. - Alb. de l'Est. - Sur feuilles et gaînes de Carex glauca. - Périthéces ovoïdes. nichés sous l'épiderme au milieu d'un feutrage de filaments bruns; ostiole très petit; théques fusoïdes-allongées; sporidies linéaires-courbes, munies de gouttelettes; paraphyses très longues. - AR. entre St-Amand et Bassu. - Automne.

1418. орніов. GALII. Ch. R. - Alb. de l'Est. - Sur tiges de Galium mollugo. - Périthèces sphériques, petits, épars, peu nombreux ; thèques cylindriques renfermant des sporidies incolores, disposées en spirales, entre-croisées en forme de treillis. - Se rapproche d'Ophiob. helicosporus. - R. - St-Amand. - Printemps. 1419. OPHIOB. MELIOLAEOIDES. Ch. R. - Alb. de l'Est. -- Bull. Soc. Bot. de Fr. (pour mémoire). - Périthèces pyriformes, velus. grisâtres, à ostiole noir, entourés de faisceaux de filaments noirs, septés, dressés comme ceux des Meliola. Thèques cylindriques; sporidies filiformes, continues. - Cette espéce m'a été communiquée par le $\mathrm{D}^{\mathrm{r}}$ Roussel qui l'avait reçue de M. Veillard botaniste à la Nouvelle-Calédonie.

1420. OPHIOB. HERPOTRICHUS. Sacc. - Alb. de l'Est. - Sur chaumes de Seigle. - Périthéces ovales, couverts de longs poils bruns, septés, apprimés en descendant et rampants 
ensuite sur le support; thèques fusoïdes; sporidies filiformes, remplies de gouttelettes. CC. - En Hiver et au Printemps. - Champs. 1421. OPHIOB. CHATOPHORUS. Sacc. - Alb. de l'Est. - Sur feuilies de Glyceria aquatica et de Carex. - Périthéces ovoïdes, à large ostiole; gris-noirâtres, mous, couverts de longs poils raides, septés, divergents; théques cylindriques: sporidies filiformes, munies de gouttelettes. - AC. - Bords des eaux. - St-Amand. Lisse, etc. - Printemps, Automne.

8\% GENRE. - HIIOSPORA. - Fuckel.

1422. LINOSP. CAPREA. - Fuckel. - Alb. de l'Est. - Sur feuilles de Salix caprcea. - Périthèces noirs, disposés en lignes le long des nervures, recouverts par de petites taches brunes de l'épiderme, rondes ou anguleuses, sorte de pseudo-strômes; ostioles proéminents, allongés. recourbés : thèques cylindriques, sporidies filiformes. - AC. - St-Amand. - Hiver-Printemps.

1423. LINOSP. POPULINA. Schrot. - Alb. de l'Est. - Sur feuilles de Populus tremula. Périthèces nichés dans le parenchyme, recouverts par des taches noires ou pseudo-strômes anguleux, aplatis, sub-astomes ; théques cylindriques terminées en un pédicelle aminci; sporidies filiformes. - AR. - Bois de St-Amand. - Printemps. 
88e GENRE. - DILopHIA. - Sacc.

1424. DILOPH. GRAMINIS. Fuckel. - Alb. de l'Est. - Sur chaumes de graminées. - Nous ne possédons que la pycnide Dilophospora graminis sur les épis de blé. - Bull. de la Soc. des Sc. de Vitry-le-François. - RR. - Mais a été très commun et nuisible aux céréales pendant trois années consécutives à St-Amand . St-Lumier, Lisse, etc. - Fin de l'Été. - Avant les moissons.

89e GENRE. - OPHHOCERAS. - Sacc.

1425. OPHIOC. CORNI. Ch. R. - Alb. de l'Est. - Sur rameau dénudé de Cornus sanguinea. - Périthèces groupés, peu nombreux , sphériques, noirs, surmontés d'un ostiole trés long. flexueux; thèques cylindriques; sporidies filiformes, remplies de gouttelettes. - RR. Trouvé une seule fois à Soulanges. - Bords du canal.

90e GENRE. - SHLLIA. - Karsten.

1426. Sillia. FERRUGiNeA. Karsten. - Alb. de l'Est. - Sur branches de Corylus avellana. - Périthèces 15-20 réunis dans un strôme disciforme, entourè par l'épiderme de substance jaune-d'ocre. ferrugineuse; ces pèrithèces sont ovoïdes, à ostioles allongés, érumpents ; thè- 
ques fusiformes; sporidies bacillaires, amincies aux extrémités, munies de 6-7 gouttelettes. RR. - Ablancourt. - Printemps.

$91^{\circ}$ GENRE. - CRYPTOSPORA. - Tul.

142\%. CRYPT. SUFFUSA. Tul. - Alb. de l'Est.

- Sur rameaux d'Alnus glutinosa. - Périthèces 8-10 groupés en pustule sous l'épiderme, noirs, aplatis. Théques en massue renflée, contenant 8 sporidies cylindriques, courbes, hyalines. La conidie est le Cryptosporium Neesii. - CC. Dans les bois, au printemps. - St-Amand.

1428. CRYPT. CORYLINA. Fuckel. - Alb. de l'Est. - A la base des rameaux de Corylus avellana - Périthèces 10-15 sphériques : surmontés de longs cols égaux; réunis en pustule. Thèques fusoïdes, aiguës aux extrémités ; sporidies linéaires, septèes. - CC. Bois, Jardins. - Hiver, printemps.

1429. CRYPT. BETULA. Tul. - Alb. de l'Est.

- Sur branches de Betula alba. - Périthèces réunis en cercle, 5-8 dans une pustule en strôme elliptique, cortical ; théques en massue: amincies en pointe, tronquées au sommet; sporidies baculiformes, continues, hyalines. AC. - Bois de St-Amand. - Printemps.

1130. CRYPT. QUERCUS. Ch. $R$. - Alb. de l'Est. - Sur rameaux de Qucrcus. - Périthèces 4-6 réunis en cercle dans une pustule valsiforme, entourée par l'épiderme; ostioles courts, érumpents sur le disque ; thèques 
cylindriques, obtuses, à 3-5 cloisons, incolores, granuleuses. - Des conidies grandes, brunes, pédicellées, à 5-6 cloisons (Coryneum), naissent sur le même support. - R. - Hiver. - StAmand.

\section{A P P EN D I CE. - Sacc.}

Sphériacées qui n'ont pu être introduites avec certitude dans la classification.

1431. SPHARIA. RENIFORMIS. Ch. $R$. - Alb. de l'Est. - Sur branches mortes de Betula alba. - Périthéces sous-cutanés, groupés mais distincts, hémisphériques puis aplatis, noirs, rugueux, à ostiole papilliforme ; théques $(0,03)$ très petites, en massue tronquées au sommet, simulant les spores d'une hyménomycète: disposées en rosette, renfermant chacune .4-6 sporidies allantoïdes ou réniformes, continues, incolores ; sans paraphyses. - Les caractères de cette espéce lui assignent une place dansle Genre Calosphceria, près de Cal. minima.

1432. SPHARIA. DICHANOIDES. Ch. $R$. Alb. de l'Est. - Sur tige morte et moussue de Rosa canina. - Périthèces agglomérés en groupes formant des taches rugueuses, noires. Ces périthèces sont elliptiques, aplatis supérieurement et munis d'une fissure longitudinale comme celle des Hysteriacées, placés sur un coussinet tomenteux, brun (Alitosporium) ; 
théques claviformes; paraphyses simples ou peu rameuses ; sporidies elliptiques-oblongues; uni-cloisonnées, resserrées au milieu, incolores, renfermant 4 sporules, semblables à celles des Diaporthe. - RR. - Récolté en Automne, à St-Amand, dans les broussailles des bois de pins.

\section{$3^{e}$ Fam. Hypocréacées. - De Not.}

Périthèces presque charnus, céracés, membraneux, la plupart de couleur rouge; ostiole arrondi.

SEGT. I. - HYALOSPORÉES. - Sacc. 19r GENRE. - NECTRIELLA. - Sacc.

1'133. NECTR ${ }^{\text {lia. }}$ RESINA. Ch. R. - Alb. de l'Est. - Buil. Soc. Bot. de Fr. 1882. - Sur la résine de Pinus sylvestris. - Périthèces ovoïdes, glabres, orangés. groupés 4-6, très petits; Théques en massue $\left(0^{\mathrm{mm}}, 066\right)$; sporidies distiques, elliptiques-oblongues, continues, incolores $\left(0^{\mathrm{mm}}, 011\right)$. - RR. - Bois de pins à St-Amand, Printemps. - Cette forme thécasporée n'avait jamais été observée ; la spermogonie (Nectria Resince. Fr.) Phomapsis cucurbitula? Sacc. est extrêmement commune.

1'43'. NECTR ${ }^{\text {lla }}$. ROUSSELLIANA. Sacc. - Alb. de l'Est. - Sur feuilles de Buxus sempervirens mortes, exposées à l'humide. - Péri- 
thèces globuleux, jaunes-orangés ou verdâtres, couverts au sommet et à la base de poils blancs; thèques claviformes ou fusoïdes; sporidies elliptiques, oblongues, continues, incolores. - L'état conidien Volutella buxi l'accompagne. - AR. - St-Amand, Bassuet. - Printemps.

2 GENRE. - ELEUTHÉ RoMYĆ́s. - Euckel.

1435. ELEUTHER. SUBULATUS. Fuckel.-Jusqu'à présent nous ne possédons de cette espéce que la Spermogonie Sphceronema subulatum et l'ètat conidien Isaria brachiata.

3e GENRE. - POLYSTIGMA. - Fuckel.

1436. POLYST. RUBRUM. D. C. - Alb. de l'Est. - Sur les feuilles mortes de Prunus spinosa après l'hiver. - Strômes orbiculaires ou taches bruries extérieurement, rougeâtres à l'intérieur, renfermant 8-10 périthèces ; thèques claviformes ; sporidies ovales, incolores, continues. R. - St-Amand, Gravelines . - Printemps. -En Automne les strômes sont rouges-pourpres et contiennent les spermogonies, Polystigmina rubra.

SEGT. II. - PHÆOSPORÉES. - Sacc.

ie GENRE, - Melanospora. - Corda.

1437. Melan. VerVecina. Fuckel. - Alb. de l'Est. - Sur morceau de bois pourri. - Péri- 
thèces globuleux, bruns, mous. diaphanes, recouverts de filaments blancs issus d'un subiculum brun; ostioles bruns, très longs: glabres, terminés par une houppe de filaments blancs qui retiennent les sporidies à leur sortie $\mathrm{du} \mathrm{col}$; théques larges-obovales ; sporidies brunes, distiques, ellipsoïdes-aiguës. - RR. Printemps.

1438. MELAN. CAPRINA. Sacc. - Alb. de l'Est.

- Sur rameaux de Salix caprcea. - Périthéces réunis en petit groupe à la surface dụ bois dénudé, teinté rouge-pourpre, petits, globuleux, couverts de poils blanchâtres et munis d'un très long ostiole brun, dressé, laissant échapper les sporidies ; thèques indécises ; sporidies ellipsoïdes, brunes. - RR. - Bords de la Marne à La Chaussée. - Eté.

1439. MELAN. ZOBELII. Fuckel. - Alb. de l'Est.

- Sur le disque de Peziza (Sepultaria) arenaria. - Communiquée par mon ami Boudier. - Périthéces pyriformes: mous, noirâtres ; thèques obovales; sporidies ellipsoïdes ou en amande, incoiores puis brunes, distiques. RRR. - Automne.

1440. MELAN. VITRAA. Sacc. - Alb. de l'Est. Sur le chapeau d'un Bolet en décomposition. Périthéces très nombreux, blancs-jaunâtres, globuleux, à ostiole allongé, formé de fibres longitudinales qui s'écartent en éventail au sommet : les Périthèces sont remplis d'une masse de théques cylindriques, sans paraphyses, con- 
tenant chacune 8 sporidies monostiques, incolores, elliptiques. - RR. - Rècolté à Sermaize, en Automne.

14'1. MELAN. PARASitica. Tul. - Alb. de l'Est. - Sur la carapace d'un hanneton (Melalontha vulgaris). - Périthéces globuleux, noirs, munis d'un long col flexueux brun, à demi enfouis dans un subiculum blanc-filamenteux, couvert de conidies incolores; théques pyriformes ou ovales; sporidies agglomérées ; brunes, cylindriques très courtes. - Espéce remarquable communiquée par M. Boudier. - Printemps.

\section{Sect. III. - HyALOdIDYMÉES. - Sacc.}

\section{5 e GENRE. - HYPOMYCES. - Tul.}

1442. HYPOM. LATÉRITIUS. Tul. - Alb. de l'Est.

- Sur les lamelles de Lactarius deliciosus. Périthèces rouges puis bruns, ovoïdes, issus d'un mycelium blanchâtre qui recouvre les lamelles, thèques cylindriques; sporidies fusiformes-acuminées, incolores ou jaunâtres, uniseptées. - AC. - Bois de pins. - St-Amand. - Automne.

1443. HYPOM. AURANTIUS. Fuckel. - Alb. de l'Est. - Sur les tubes de Polyporus versicolor. et adultus. - Périthẻces nombreux, ovoïdes, rouges-orangés, à demi plongés dans un subiculum rose ou rouge-clair tapissant la face inférieure du Polypore. Théques cylindriques; spo- 
ridies fusoïdes, acuminées, incolores, uniseptées. - L'appareil conidien Diplocladium majus. Sacc. l'accompagne.

Les formes secondaires des autres Hypomyces sont inscrites dans le Catalogue, à la place qui leur est assignée par leurs caractères particuliers.

6e GENRE. - NECTRIA. - Fries.

1444. NeCtr. CinNabarina. Fries. - Alb. de l'Est. - Sur troncs d'Acer, de Fraxinus, etc. - Périthèces 10-15 sphériques, rugueux , rougecinabre, cespiteux sur un strôme hémisphérique rosé; thèques claviformes; sporidies oblongueselliptiques, incolores, uniseptées. distiques. CC. - Partout. - Le Tubercularia vulgaris est son appareil conidien.

1445. NECTR. SINOPICA. Fries. - Alb. de l'Est. - Sur sarments d'Hedera helix. - Périthèces sphéroïdes, glabres, rouges, réunis 10-15 sans strôme; thèques cylindriques, sub-monostiques ; munies de gouttelettes. - CC. - Automne. Conidie Tubercularia sarmentorum.

1446. NeCTR. DESMAZIERI. De Not. - Alb. de l'Est. - Sur rameaux de Buxus sempervirens. - Périthèces 10-15, roses ou rougeâtres, ovales, cespiteux sur un strôme jaunâtre; thèques cylindriques; sporidies elliptiques, monostiques, uniseptées, incolores. - Conidie Tubercularia versicolor. - CC. - St-Amand. - Jardins. Printemps. 
141\%. NECTR. DISCOPHORA. Mont. - Alb. de l'Esst. - Sur rameaux d'Alnus glutinosa. Envoi de M. Boudier. - Au Printemps. Périthéces globuleux, glabres, rouges-bruns, tronqués au sommet en disque, à large ostiole noirâtre; théques cylindriques; sporidies fusoïdes, incolores, uniseptees, monostiques. - RR.

1448. NECTR. STILBOSPORA. Tul. - Alb. de l'Est. - Sur écorce de Fagus sylvatica. - Périthèces ovoïdes : rouge-carmin, groupés et cespiteux sur les tubercules de Fusisporium carneum; thèques cylindriques, sporidies elliptiques; incolores, uniseptees à 2 gouttelettes. - R. - Bois de St-Amand et à Vitry-le-François, sur les chantiers du Canal. - Printemps. 1449. NECTR. BRASSICE. Ell. - Alb. de l'Est. - Sur tiges de Brassica oleracea pourrissantes. - Périthèces réunis en groupes, mais distincts, rouges-bruns, rugueux, sphèriques ; thèques claviformes ; sporidies distiques, elliptiques - allongées, uniseptées , resserrées au milieu, incolores. - AR. - St-Amand. Jardins. - Automne.

1450. NECTR. PALUdOSA. Sacc. - Alb. de l'Est. Sur feuilles d'Iris pseudoacorus pourries sur l'humus des marais, à St-Lumier. - Périthèces globuleux, glabres, couleur de chair. épars ; théques claviformes; sporidies allongées-fusoïdes, un peu courbes, uniseptées, incolores. - AR. Automne.

1451. NECTR. SANGUINEA. Fries. - Alb. de 
l'Est. - Sur troncs cariés de Juglans, d'Acer etc. - Périthèces groupés, ovoïdes, mous, sanguins ou incarnats ; thèques claviformes ou cylindriques, paraphyses simples ; sporidies distiques et monostiques, elliptiques, uniseptées à 2 sporules. - CC. - St-Amand. - Automne, printemps.

1452. NECTR. EPISPH ÆRIA. Fries. - Alb. de l'Est. - Parasite sur Diatrype stigma, Hypoxylon, etc. - Périthèces ovoïdes puis affaissés, glabres, rouge-sanguin , épars ; théques cylindriques ; sporidies incolores, elliptiques, uniseptées. - CC. - St-Amand, Aulnay, etc. Hiver, printemps.

1453. NECTR. FUCKELII. Sacc. - Alb. de l'Est. - Sur scutelles du Parmelia croissant sur les troncs de Noyer. - Périthèces ovoïdes, rouges, à demi immergés dans le tissu lichénoïde ; thèques claviformes; sporidies incolores, fusoïdes uniseptees, distiques. - AC. - Automne, printemps. - St-Amand.

1454. NECTR. PUNCTUM . Boudier. -- Alb. de l'Est. - Communiqué par M. Boudier et inscrit pour mémoire. - Croît sur les feuilles de Jungermannia. - Remarquable par ses périthèces très petits, ovoïdes, noirs-velus ; thèques cylindriques; sporidies elliptiques oblongues, uniseptées. - RRR. - Printemps.

1455. NECTR. COCCINEA. Fries. - Alb. de l'Est. - Sur écorce d'Ulmus campestris, placé dans les fissures. - Périthèces ovoïdes, d'un rouge- 
vif: réunis sur un strôme un peu convexe, rosé; théques cylindriques ; sporidies monostiques, incolores, elliptiques, uniseptèes, 2 gouttelettes. - CC. - St-Amand. Printemps.

1456. NECTR. DELICATULA. Sacc. - Alb. de l'Est. - Sur tiges mortes d'Helleborus icetidus. - Périthèces épars, très petits, orangés-pâles, mous; thèques claviformes; sporidies fusoïdes . un peu courbes, allongées, munies de 4 gouttelettes : uniseptées. - R. - Jardins. - Été. - St-Amand.

145\%. NECTR. SOLANI. Reinke. - Alb. de l'Est.

- Sur tiges mortes de Solanum tuberosum. Périthèces rouges-rosés, ovoïdes, plongés dans un subiculum blanc-villeux, glabres mais entourés près de l'ostiole, par une couronne de poils blancs; théques claviformes; sporidies incolores, elliptiques-allongées. uni-septées, à 4 gouttelettes, distiques. - RR. - St-Amand. Printemps.

1458. NECTR. DAHLiÆ. Ch. R. - Alb. de l'Est. - Sur tiges pourrissantes de Dahlia. - Périthèces globuleux, bruns-rougeâtres puis noirs, réunis en groupe mais distincts; thèques claviformes entourées de paraphyses muqueuses; sporidies incolores, remplies de gouttelettes, elliptiques-allongées, un peu courbes. - Récolté dans mon jardin en hiver. - Quelques périthèces étaient recouverts par des conidies blanches portées sur des basides à 1-2 cloisons, que j'ai nommées Oospora nectricecola. Ch. R. - RR. 


\section{7e GENRE. - HYPOCREA. - Fries.}

1459. HYPOCR. OLIVACEA. C. et Ell. - Alb. de l'Est. - Sur l'écorce de Pinus sylvestris. - Strôme sub-globuleux, de couleur olive, ponctué par les ostioles des périthèces sphériques logès à la périphérie du tubercule; thèques cylindriques; sporidies elliptiques, uniseptées composées de deux articles qui, en se dèsagrégeant, simulent 16 sporidies dans la théque. - RR. - St-Amand. - Automne, par les temps humides.

1460. HYPOCR. RIGENS. Sacc. - Alb. de l'Est. Sur les troncs abattus de Pinus sylvestris. Strômes noirs-verdâtres, hémisphériques ou aplatis-sinueux ponctués à la surface; périthèces blanchâtres, sous-jacents; thèques cylindriques filiformes, incolores, simulant 16 cellules séparées. - AC. - St-Amand. - Hiver, Printemps.

1461. HYPOCR. GELATINOSA. Fries. - Alb. de l'Est. - Sur branches de Pinus sylvestris et Abies. - Strômes tuberculeux, irrégulièrement arrondis, jaune-nankin, ponctués, substance blanche; périthèces périphériques, mous; théques cylindriques, très longues; sporidies bi-loculaires, caractères des espèces précédentes. - AR. Automne, Printemps.

8e GENRE. - LETENDRAEA. - Sacc.

1462. LETENDR. EUROTIOIDES. Sacc. - Alb. de l'Est. - Bull. Soc. bot. de Fr. 1881, sous 
le nom de Nectria helminthicola. Ch. R. - Sur les tiges mortes de Ribes rubrum, et Symphoricarpos. -- Périthèces glabres, jaunes-pâles , ovoïdes, groupés à la base des filaments d'Helminthosporium appendiculatum son appareil conidien; théques claviformes, entourées de paraphyses simples; sporidies elliptiques, brunes, uniseptées, resserrées à la cloison, 2 gouttelettes. - AR. - St-Quentın-les-Marais, St-Amand. - Automne, Printemps.

\section{SEGT. IV. - PHRAGMOSPORÉES. - Sacc.}

ge GEnRE. - CaLonectirha. - De Not.

1463. CALONECT. RICHONI. Sacc. - Alb. de l'Est. - Bull. Soc. bot. de Fr. sous le nom de Nectria mellince. Mont. Sur tiges mortes de Salix caprcea et de Symphoricarpos. - Périthèces sphériques puis déprimés, roses ou rougeâtres puis jaune-miel, mous, épars, renfermant des thèques fusoïdes, sans paraphyses; sporidies tristiques, cylindracées un peu courbes, incolores, munies de 3-5 cloisons. - R. - Soulanges, St-Amand. Printemps.

10e GENRE. - GIBbERELLA. - Sacc.

1461. GIBB ${ }^{\text {la }}$ PULICARIS. Sacc. - Alb. de l'Est. - Sur lécorce des rameaux de Sambucus nigrce et sur Solanum dulcamara. - Périthèces 15-20, 
sphériques puis aplatis en cupule, noirs, rugueux, insérés sur un strôme tuberculaire brun; théques oblongues-fusiformes: sporidies incolores, elliptiques-fusoïles, triseptées, resserrées aux cloisons. - CC. - St-Amand. Ablancourt. - Hivers doux, printemps. - Conidie Fusarium sambucinum.

1465. GIBB ${ }^{l a}$. SAUBINETII. Sacc. - Alb. de l'Est. - Sur chaumes d'Arundo phragmites, près des nœuds. - Périthéces groupés 15-200, ovoïdes, noirs-bleus, rugueux, sphériques puis affaissés; thèques oblongues-lanceolées; sporidies distiques, fusiformes, un peu courbes, tri-septées. - AC. Bords des eaux. - St-Amand. - Printemps.

1'166. GIBB ${ }^{l a}$. ACERVALIS. Sacc. - Alb. de l'Est. - Sur branches mortes de Salix caprcea. - Périthèces ovoïdes, bruns, rugueux, groupès ou épars sur l'écorce; thèques claviformes ; sporidies elliptiques-oblongues, 2-3 septées. - La forme thécas. porée AR. - La spermogonie Phoma acervalis plus commune. - St-Amand. - Automne.

1467. GIBB ${ }^{l a}$. AgGlomerata. Sacc. - Alb. de l'Est. - Sur les tiges mortes et humides de Vinca maior. - Périthėces sub-sphériques, glabres. noirs-bleus, épars; théques claviformes ; sporidies distiques, elliptiques-oblongues, munies de 2 cloisons. - AR. - Jardins - Printemps. - StAmand.

11e GENRE. - PLEONECTRIA. - Sacc.

1468. PleON. LAMYI. Sacc. - Alb. de l'Est. - Sur rameaux de Berberis vulgaris. - Périthèces 
rouges-orangés; sporidies muriformes hyalines. - RR. - Reims. - Communiqué par le Dr Jolicœur.

12 GENRE. - THYRONECTRIA. - Sacc.

1469. THYR. PATAVINA. Sacc. - Alb. de l'Est.

- Sur branches de Noyer. - Périthèces globuleux, verdâtres, épars sur un strôme discoïde ; sporidies hyalines, muriformes à 10 cloisons transversales. - St-Amand. - Printemps. - RR.

14\%0. THYR. PYRROCHLORA. Sacc. - Alb. de

l'Est. - Sur branches d'Acer campestris. - Périthèces globuleux, 10-2í pulvérulents. verdâtres au sommet,substance pourpre; ostiole noir; sporidies hyalines, murales. - St-Amand. - Automne. RR.

SECT. V. - SCOLECOSPORÉES. - Sacc. 13e GENRE. - DOMYCES. - B. et $B r$.

1471. OOMYC. CARNEO-ALBUS. $B$, et $B r$. - Alb. de l'Est. - Sur chaumes de Festuca nemoralis. AR. - Bois de St-Amand. - Périthéces ovoïdes, surface à papilles blanchâtres, ostioles noirs; théques cylindriques; sporidies filiformes, cloisonnées, articles se désagrégeant. - Automne. Printemps. 
14 $4^{\mathrm{e}}$ GENRE. - CLAVICLPS. - Tul.

1472. ClAV. PURPUREA. Tul. - Alb. de l'Est.-Sur grains de Secalis, Avena, Triticum, etc. - StAmand, Ablancourt, etc. - CC. - Fin de l'Eté. - Conidie Sphacelia purpurea.

15e GENRE. - CORDICERS. - Link.

1'173. CORD. MILITARIS. Link. - Alb. de l'Est.

- Sur larves d'Insectes. - Bois de St-Amand.

- Automne. - Périthèces périphériques, rougesorangés, sur un strôme claviforme. - AC. Conidie Isaria farinosa.

11\%4. CORD. OPHIOGLOSSOIDES. Link. - Alb. de l'Est. - Sur Elaphomyces granulatus. Strômes allongés terminés par un capitule cylindrique, obtus, verdâtres, puis bruns, enfin noirâtres. - AR. - Bois de la Montagne de Reims. - Automne.

1475. CORD. CAPITATA. Link. - Alb. de l'Est. - Sur Elaphomyces granulatus. - Strômes élevés, capitule globuleux, jaunâtres-noircissants ; sporidies filiformes se désagrégeant en articles cylindriques. - Bois de Verzy, Verzenay. RR. - Printemps.

16 GENRE. EPICHLOE. - Tul.

1176. EPICHL. TYPHINA. Tul, - Alb. de l'Est.

- Sur chaumes de Phleum pratense et Dactylis. 
qu'il entoure près des nœuds dans une longueur de 4 à 6 centimètres. - CC. - Dans les bois et les prairies. - Fin de l'Eté. - Partout. Conidie Sphacelia typhina.

$4^{\mathrm{e}}$ FAM. - Dothidéacées. - Nits.

Périthèces formés par la substance du strôme dans lequel ils sont plongés; ostiole papillijorme.

1er GENRE. - MAzzantia. - Mont.

147\%. MAzz. GALII. Mont. - Alb. de l'Est. Sur tiges de Galium aparine. - RR. - environs de Reims. - Communiqué par le Dr Jolicœur.

- Hiver.

2 GENRE, - PHYLLACHORA. - Nits.

1478. PHYLL. ULMI. Fuckel. - Alb. de l'Est. Sur feuilles d'Ulmus campestris. - Bois de StAmand, etc. - Hiver, automne. - Conidie Septoria ulmi.

1'179. PHYLL. GRAMINIS. Fuckel. - Alb. de l'Est. - Sur feuilles de Fétuque. - Dans les bois de St-Amand. - CC. - Automne, hiver. 1480. Phyll. POPUli. Ch. $R$. - Alb. de l'Est.

- Sur feuilles de Populus cordifolia. - AR.

- St-Lumier. - Espèce ambiguë, consulter le Rec. 9, page 12. 
1181. PHYLL. JUNCI. Fuckel. - Sur chaumes de Juncus communis. - CC. - Dans les fossés humides. - St-Amand, Lisse. - Automne.

3e GENRE. - SCHRIRHA. - Nits.

1482. SCIRRH. RIMOSA. Fuckel. - Alb. de l'Est.

- Sur chaumes d'Arundo phragmites. - CCC.

- Bords des eaux. - St-Amand, Soulanges, Vanault-les-Dames, etc. - Hiver, printemps.

$4^{\mathrm{e}}$ GENRE. - PLOWRHGHTA. - sacc.

1483. PLOWR. RIBESIA. Sacc. - Alb. de l'Est.

- Sur rameaux arides de Ribes rubrum. CCC. - Jardins. - Partout. - Conidie Podosporium ribis. - Hiver. printemps.

1484. PLOWR. INSCULPTA. Sacc. - Alb. de l'Est. - Sur sarments de Clemaits vitalba. AR. - Solulanges. - Automne.

1485. PLOWR. MORBOSA. Sacc. - Alb. de l'Est.

- Sur rameaux pourrissants de Prunus domesticus. - R. - Entre Lisse et St-Amand. Automne.

5e GENRE. - DOTHIDEA. - sacc.

1486. Doth. SMILACICOLA. In. Grév. - Alb. de l'Est. - Sur feuilles de Smilax laurifolia. Serres. - RR. - Printemps.

1487. DотH. PUCCINIOIDES. Tul. - Alb. de l'Est.

- Sur tiges mortes de Buxus sempervirens. Jardins à St-Amand. - Hiver-Printemps. 
6e GENRE. - TROPOGRA PHUS. - Fuckel.

1488. ROPOGR. FILICINUS. Fuckel. - Alb. de l'Est. - Sur tiges de Pteris aquilina. - Bois de la Montagne de Reims. - AR. - Hiver.

5" FAM. - Microthyriacées. - Sacc.

Périthèces membraneux ou charbonneux, dimidiés, scutiformes; tissu à fibres rayonnantes.

1er GENRE. - MHCROTHYRIUM. - Desm.

1489. MICR. MICROSCOPICUM. Desm. - Alb. de l'Est. - Sur feuilles mortes de Buxus sempervirens. - Hiver-Printemps. - St-Amand. - AK. - Haies, jardins.

1490. MICR. VISCI. Ch. $R$. - Alb. de l'Est. - Sur rameaux secs de Viscum album, après l'hiver. en compagnie de Camurosporiuni visci et d'Anthostomella visci. - RR. - Printemps. - Ablancourt. - Espéce nouvelle.

1491. MiCR. PLAtANI. Ch. $R$. - Alb. de l'Est. -- Sur feuilles de Platanus orientalis. - AR. - Soulanges, dans le parc du château. - Voir Recueil 10, p. 29. - Espéce nouvelle. 
6e FAM. - Lophiostomacées. - Sacc.

Périthèces charbonneux, adnés à la base, incrustés; ostiole comprimé, s'ouvrant en fente.

$1^{\mathrm{er}}$ GENRE. - LOPHIOTHICHA. - Richon.

1492. LOPH. VIBURNI. Ch. $R$. - Alb. de J'Est. - Bull. soc. Bot. de Fr. 1885. - Sur ramilles de Viburnum Lantana. - RR. - Soulanges. - Printemps. - Sacc. add. au Vol. IV. p. 25'

2e GENRE. - LOPIIOSPIIARA - Trév.

1493. LOPH ${ }^{\text {ra }}$. FUCKELII. Sacc. - Alb. de l'Est.

- Sur tiges mortes de Rubus fruticosus. - AC.

- St-Lumier, St-Amand. - Ostiole comprimé ; sporidies hyalines, fusoïdes, uniseptées.-Hiver. 1494. LOPH ${ }^{\mathrm{ra}}$. DONACIS. Ch. R. - Alb. de l'Est.

- Sur chaumes d'Arundo donaci desséchés, cultivé dans les jardins. - Sermalze. - Printemps.

3e GENRE. - LOPHHGTR EMA. - sacc.

1495. LOPH ${ }^{\mathrm{ma}}$. CRENATUM. Sacc. - Alb. de l'Est. - Sur rameaux de Pmonus domestica et Viburnum opulus.- CC.-Ostioles comprimés, en forme de crête. - Hiver, printemps. - Partout. 1496. LOPH ${ }^{\text {ma }}$ EPILOBII. Ch. R. - Alb. de l'Est. - d'Epilobium molle ct Roseum. - Printemps. - St-Amand, bords du Fion. - Rec. 12, p. 15. 
ie GENRE. - LOPHIOSTOMA. - Ces.

1497. LOPH ${ }^{\text {ma }}$. QUADRINUCLEATUM. Kar'st. Alb. de l'Est. - Sur branches dénudées de Peuplier, en Automne. - Bois de St-Amand, etc. - CC.

1498. LOPH ${ }^{\text {toma }}$. CESPITOSUM. Sacc. - Alb. de l'Est. - Sur rameaux de Cratcegus oxyacantha. - Printemps. - St-Amand, St-Lumier. - C.

1499. LOPH ${ }^{\text {toma }}$. ARUNDINIS. Ces. - Alb. de l'Est.

- Sur chaumes d'Arundo phragmites. - CC. Bords des eaux. - Partout. - Hiver, Printemps. 1500. LOPH toma EXCIPULIFORME. Ces. - Alb. de l'Est. - Sur écorces de Peuplier d'Italie. Printemps-Été. - CC. - Partout. - Périthèces gros, émergés, à larges ostioles. - Sur Genista prostrata. Var. -. Collines de Bassu.

1501. LOPH toma. INSIDIOSUM. Ces. - Alb. de l'Est. - Sur tiges d'Ononis spinosa et sur Erysimum alliaria. - AC. - Soulanges, Aulnay. Sporidies olivâtres, à 5 cloisons et terminées par 2 apicules. - Automne, Printemps.

5e GENRE. - LORHIDIUM. - Sacc.

1502. LOPHID. COMPRESSUM. Sacc. - Alb. de l'Est. - Sur rameaux de Salix caprcea, de Cornus sanguinea, etc. - CCC. - Bois, haies. - Partout. - Printemps, Hiver. 
1503. LOPHID. POPULI. Sacc. - Alb. de l'Est.

- Commun sur branches dénudées de Peuplier.

- Toute l'année, notamment au printemps. Bois de St-Amand, Soulanges, etc.

Yo FAM. - Hystériacées. - Corda.

Périthèces oblongs ou linéaires, coriaces ou charbonneux. Ouverture en fente qui parcourt toute la longueur du périthèce.

¿er GENRE. - SCHIZOTHYRIUM. - Desm.

1504. SCHIz. PTARMICE. Desm. - Alb. de l'Est.

- Sur les tiges et feuilles encore vertes d'Achillcea ptarmica. - En Automne. - AC. dans les prairies humides de Soulanges.

\section{2e GENRE. - AULdGRAPIUM. - Lib.}

1505. AULOGR. JUNCINUM. Lib. - Alb. de l'Fist.

- Sur chaumes de Juncus communis desséché.

- AC. - Entre Lisse et St-Amand dans les fossés humides. - Automne. - Thèques larges renfermant 4 sporidies uniseptées. -- Automne, hiver.

3e GENRE. - GLovium. - Miehl.

1506. GLON. PYGMAEUM. Karsten. -- Alb. de l'Est. - Sur branches dénudées de Juniperus 
communis. - AC. dans les plantations de Pins sylvestres, entre St-Amand et Soulanges. Printemps.

$4^{e}$ GENRE. - HYSTERIUM. - Tod.

150\%. HYSTER. ANGUSTATUM. Alb. et Schw. Alb. de l'Est. - Sur sarments de Rubus fruticosus. - AC. - St-Lumier, Soulanges. Printemps.

$5^{\mathrm{e}}$ GENRE. - DICHAENA. - Fries.

1508. DICHÆN. STROBILINA. Fries. - Alb. de l'Est. - Sur cônes de Pinus sylvestris. - AC. - Bois de pins. - St-Amand, Soulanges. Hiver-Printemps.

6e GENRE. - GLONIOPSIS. - De Not.

1509. GLONpsis. BIFORMIS. Sacc. - Alb. de l'Est.

- Sur lattes de Chêne exposées à l'air libre. Palissades, vignes. - CCC. - Jardins. - Toute l'année. - Partout.

Fe GENRE. - HYSTEIROGRAPHIUM. - Corda.

1510. HYSTER. FRAXINI. De Not. - Alb. de l'Est. - Sur branches et rameaux de Fraxinus excelsior. - CCC. - Bois. - Printemps, hiver. Partout.

1511. HYSTER. SYRINGAE. Sacc. - Alb. de l'Est. - Sur rameaux de Syringa vulgaris. - AR. Jardins. - St-Amand. - Printemps. - Très voisin du précédent. 
8e GENRE. - HYPODERMA. - $D . C$.

1512. HYPOD. VIRGULTORUM. D. C. - Alb. de l'Est. - Sur sarments de Rubus fruticosus. AC. - Dans les haies, les broussailles des forêts. - Sermaize, St-Amand. - Automne.

9e GENRE. - LOPHODERMUUM. - Chev.

1513. LOPH ${ }^{\text {mium }}$ HYSTERIOIDES. Sacc. - Alb. de l'Est. - Sur feuilles de Cratcegus oxyacantha tombées à terre, aprés l'hiver. - Dans les bois de pins, entre St-Amand et Bassu. - AR.

1514. LOPH ${ }^{\text {mum }}$. PINASTRI. Chev. - Alb. de l'Est. - Sur écailles de cônes de Pinus sylvestris. - CC. dans les plantations de Pins, après l'hiver. St-Amand, Soulanges.

1515. LOPH ${ }^{\text {mium }}$. CULMIGENUM. Chev. - Alb. de l'Est. - Sur gaîne des chaumes des Graminées, Poa, Festuca. etc. - AC. - Bois humides de St-Lumier, Lisse, etc. - Automne, Été.

1516. LOPH ${ }^{\text {mium }}$. ARUNDINACEUM. Chev. - Alb. de l'Est. - Sur chaumes de Calamagrostris, d'Arundo phragmites. - AC. - Bords des ruisseaux. - St-Amand, Soulanges, etc. - Automne.

1517. LOPH ${ }^{\text {mum }}$. CARICINUM. Duby. - Alb. de l'Est. - Sur feuilles de Carex glauca. - CC. dans les bois humides et dans les bois de Pins. - Dans cette dernière station il est assez souvent accompagné de Myrothecium caricis. Aulomne. - St-Amand. 
10 GENRE. - LOPIHUM. - Fries.

1518. LOPHIUM. MYTILINUM. Fries. - Alb. de l'Est. - Sur le bois mort et les cônes de Pin sylvestre. - Espéce ressemblant à une moule en miniature. - CC. - Soulanges, etc. Printemps, Auiomne.

11 GENRE. - CoLPOMA. - Vallroth.

1519. COLP. QUERCINUM. Wallr. - Alb. de l'Est.

- Très commun sur les rameaux et petites branches de Chêne vertes que sa présence fait périr. - Dans tous les bois. - Hiver. - Partout.

12. GENRE - OSTROPA. - Fries.

1520. OSTR. CINEREA. Fries. - A!b. de l'Est.

- Sur rameaux de Prunus spinosa et domestica. -AR. - Dans les bois et broussailles de Soulanges, St-Amand, Lisse, etc. - Hiver-Printemps.

$13^{e}$ GENRE. - ROBETGEA. - Desm.

1521. ROBERG. UNICA. Desm. - Alb. de l'Est.

- Sur Fraxinus Excelsior, sur Lonicera xylosteum. et Viburnum opuli. - AR. - Bois de St-Amand. - Printemps.

14' GENRE. - A CROSPERMUM. - Tode.

1522. ACROSP. COMPRESSUM. Tode. - Alb. de l'Est. - Sur les tiges mourantes des grandes herbes, Urtica. Cirsium arrense, etc. - CC. - 
Automne et Été, par les temps humides. - StAmand, etc.

1523. ACROSP. GRAMINUm. Lib. - Alb. de l'Est.

- Sur les chaumes et sur les feuilles des Graminées, Poa, Festuca, etc. - CC. - AutomnePrintemps. - Partout.

\section{5e GENRE. - GODRoNIA. - Ch. Rïchon.}

152'. GODR. MUHLENBECKII. Ch. $R$. - Alb. de l'Est. - Sur chaumes d'Arundo phragmites. RR. - Se présente sous deux aspects. $-10 \mathrm{La}$ forme thécasporée, avec théques claviformes; sporidies filiformes, remplies de gouttelettes; paraphyses simples; $2^{\circ}$ la for:ne spermogonienne. renfermant des spermaties aculéiformes, septées, en faisceaux. - Bul. soc. Bot. de France. 


\section{FUNGI NONDUM PERFECTI.}

$1^{\text {re }}$ FAm. - Sphéropsidées. - Lév. Périthèces privés de thèques, contenant des sporules portées par des basides plus ou moins manifestes.

\section{SEGT. I. - HYALOSPORÉES. - Sacc.}

sporules continues, hyalines.

1er GENRE. PIIYLLOSTIC'TA. - Pers.

1525. PHYLL ta ACUPARIÆE. Sacc. - Alb. de l'Est. - Sur feuilles mourantes de Sorbus acuparia. - Automne-Hiver. CC. - Jardins. St-Amand.

1526. PHYLLta. RUBICOLA. Rabb. - Alb. de l'Est. - Sur feuilles de Rubus ccesius. Depazea areolata de Fuckel ? - L'état conidien Calloria ruborum l'accompagne. - AC. - Bois de StAmand à St-Lumier. - Automne. 
1527. PHYLL ${ }^{\text {ta }}$ ROSAE. Kickx. - Alb. de l'Est. Sur feuilles de Rosa Bengalis et Rosa maialis encore vertes. - Jardins. - St-Amand. - AR. 1528. PHYLL ${ }^{\text {ta }}$. CYTISI. Desm. - Alb. de l'Est.

- Sur la face supérieure des feuilles de Cytisus laburnum. - CC. - Jardins et parcs. - Soulanges, Ablancourt. - Automne.

1529. PHYLL ta. ACERIS. Sacc. - Alb. de l'Est.

- Sur feuilles d'Acer campestre. - CC. dans ies bois, à la fin de l'Été. - Partout.

1530. PHYLL ${ }^{\text {ta }}$. VULGARIS. Desm. - Alb. de l'Est. - Sur les feuilles encore vertes de Lonicera Xylostcum. - CC. - Automne. - St-Amand. Ablancourt.

1531. PHYLL ta SYMPHORICARPI. West. - Alb. de l'Est. - Sur feuilles de Symphoricarpos. CC. - Jardins. - St-Amand. - Automne.

1532. PHYLL ${ }^{\text {ta }}$. SAMBUCICOLA. lialch. - Alb. de l'Est. - Sur feuilles de Sambucus cbulus encore vertes. - Accompagne souvent le Septoria ebuli.

- CC. - Automne. - Lisse, St-Amand.

1533. PHYLL ta. SYRINGA. West. - Alb. de l'Est.

- Sur feuilles encore vertes de Syringa vulgaris.

- CC. en Été. - Jardins. - St-Amand, etc.

153'. PHYLT ta. Limbalis. Pers. - Alb. de l'Est.

- Sur feuilles de Buxus sempervirens. - CC.

- Automne. - Jardins. - St-Amand.

1535. PHYLL ${ }^{\text {ta }}$. BUXINA. Sacc. - Alb. de l'Est. - Sur feuilles de Buxus sempervirens. - Jardins. - Automne. - Plus commun que le précédent. - Partout. 
1536. PHYLLta. MAHONIÆ. Sacc. - Alb. de i'Est.

- Sur feuilles vertes de Mahonia aquifolius.

- Jardins. - St-Amand. - Printemps. - Me paraît être un Macrophoma par la forme.

1537. PHYLLta. BERBERIDIS. Rabb. - Alb. de l'Est. - Sur feuilles vertes de Berberis vulgaris.

- AR. - Aulnay-l'Aître. - Automne. - En compagnie du Leptothyrium ribis.

1538. PHYLLta. ALNIGENA. Thüm. - Alb. de l'Est. - Sur feuilles d'Alnus glutinosa. - AR. - Entre Lisse et St-Amand. - Bois. - Automne, fin de l'Été.

1539. PHYLLta ${ }^{\text {ta }}$ CORYLI. West. - Alb. de l'Est.

- Sur feuilles vertes du Corylus avellana. AC. à la fin de l'Été. - Jardins, parcs. - StAmand. - Sporules se rapprochant de celles des Macrophoma.

1540. PHYLL ${ }^{\text {ta }}$. CARPINI. Schultz. - Alb. de l'Est.

- Sur feuilles mortes de Carpinus betula. - AC. dans les charmilles, jardins. - Printemps. Sporules grosses $\left(0^{\mathrm{mm}}, 015\right)$. - St-Amand.

15'1. PHYLLta. QUERCUS. Sacc. - Alb. de l'Est. - Sur feuilles de Quercus desséchées. - AC. dans les bois. - Printemps. - Partout. - Sporules cylindriques.

15吩. PHYLL. BRASSICA. West. - Alb. de l'Est.

- Sur feuilles languissantes de Brassica oberacea.

- CCC. - Jardins. - Automne. - Partout.

15'3. PHYLLta DIANTHi. West. - Alb. de l'Est. - Sur feuilles de Dianthus barbatus. - CC. 
dans les jardins. - St-Amand. - Automne. Sporules cylindriques. courbes.

15'1'. PHYLL ta ANGELIC $A$ E. Sacc. - Alb. de l'Est.

- Sur feuilles d'Angelica sylvestris. - CCC.

en Automne, au bord des rivières. - St-Amand, Ablancourt.

15'5. PHYLL ${ }^{\text {ta }}$. ATRIPLICIS. Desm. - Alb. de l'Est. - Sur feuilles d'Atriplex hastata. - CCC. - Automne. - Champs, jardins. - Partout.

15'16. PHYLL ${ }^{\text {ta }}$. ALISMATIS. Sacc. - Alb. de l'Est.

- Sur feuilles de Sagittaria et d'Alisma plantago. - CC. - Lieux marécageux. - Automne. - La Chaussée, Togny.

15'17. PHYLL ${ }^{\text {ta }}$. PERSICAE. Sacc. - Alb. de l'Est.

- Sur feuilles de Persica vulgaris. - CU. Jardins. - Autornne. - St-Amand, etc.

15'18. PHYLL ${ }^{\text {ta }}$. LIGUSTRINA. Sacc. - Alb. de l'Est. - Sur feuilles de Liguslrum vulgare. CC. - Automne. - Haies des jardins, bois. St-Amand.

1549. PHYLL ${ }^{\text {ta }}$. TRIFOLII. Ch. R. - Alb. de l'Est.

- Sur les feuilles de Trifolium repens. - Dans le voisinage du Polythrincium Trifolii, dont il est sans doute la spermogonie. - Sporules ovales. $0^{\mathrm{mm}}, 0028^{\mathrm{mm}}$.

1550. PHYLL ${ }^{\text {ta }}$. TREMULAECOLA. D. C. - Alb. de l'Est. - Sur feuilles de Populus tremula. - AC. - Bois de St-Amand à St-Lumier. Automne-Hiver. - Sporules courbes, cylindriques. - C'est le Depazea Tremulacola Sacc.

15551. PHYLL ${ }^{\text {ta }}$. FRAXINICOLA. Curr. - Alb. de 
l'Est. - Sur feuilles de Fraxinus excelsior. CC. dans les bois de St-Amand. - Fin de l'Été.

1552. PHYLL ${ }^{\text {ta }}$. PODAGRARIE. West. - Alb.de l'Est. - Sur feuilles d'Agopodium podagraria.

- AC. - Bois de St-Amand. - Automne. Phoma selon Sacc.

2e GENRE. - PHOMA. - Eries.

1553. Phoma LABURNi. Sacc. - Alb. de l'Est. - Sur pédoncules des gousses de Cytisus laburnum. - AC. en hiver dans les jardins. St-Amand, etc. - Les sporules longues, fusiformes, courtement pédicellées en font un Macrophoma.

1554. PHOMA PSEUdO. ACACI $\approx$. Sacc. - Alb. de l'Est. - Phoma Oncostoma de Thüm. - Sur rameaux de Robinia pseudo-acacia. - CC. Au printemps. - Jardins. - St-Amand,Ablancourt,etc. - Spermogonie de Diaporthe oncostoma.

1555. PHOMA FUCKELII. Sacc. - Alb. de l'Est.

- Sur rameaux de Carpinus. - Accompagne le Calosphcria Fuckelii dont il est la Spermogonie. - AC. - Haies en charmilles, jardins. St-Amand. - Printemps. - (Dendrophoma).

1556. PHOMA RYCKHOLTII. Sacc. -- Alb. de l'Est. - Sur rameaux de Symphoricarpos. Accompagne le Diaporthe Ryckholtii dont il est la Spermogonie. - AC. - Jardins. - St-Amand. - Été.

155\%. PHOMA LONICERA. Cooke. - Alb. de l'Est. - Sur rameaux de Lonicera Xylosteum. 
- AC. - Jardins. - Printemps. -- Ablancourt. St-Amand. - Spermogonie d'Anthostoma Xylostei. 1558. PHOMA RAMEALIS. Desm. - Alb. de l'Est.

- Sur rameaux morts d'Evonymus Europceus; sur le bois dénudé. - Taches entourées d'une ligne noire, couvertes de phoma. - CC. - Bois d'Aulnay-l'Aître. - Automne, printemps.

1559. PHOMA DetruSA. Sacc. - Alb. de l'Est.

- Sur rameaux dénudés de Berberis vulgaris. CC. dans les jardins. - St-Amand. - Spermogonie de Cucurbitaria berberidis.

1560. PHOMA PITHYOPHILA. Sacc. - Alb. de l'Est. - Sur ajguilles de Pinus Sylvestris. CC. en Automne et en hiver. - Bois de Pins. - St-Amand. - C'est le Spharonema Pithyophilum de Corda.

1561. PHOMA PERSICAE. Sacc. - Alb. de l'Est.

- Sur les rameaux de Persica vulgaris. - AR.

- Jardins. - Printemps. - - St-Amand, etc.

1562. PHOMA COOKEI. Pirotta. - Alb. de l'list.

- Sur jeunes sarments secs de Vitis vinifera. En hiver.-CC. - St-Amand. - Jardins. - Partout.

1563. PHOMA DEPRESSA. Sacc. - Alb. de l'Est. - Cette espéce croît sur les jeunès rameaux desséchés de Syringa vulgaris. - Elle doit être rapportée au Melanostroma fusaroides de Sacc. - CC. - Jardins. - St-Amand.

1561. PHOMA SYRINGINA. Thüm. - Alb. de l'Est. - Sur rameaux de Syringa vulgaris dénilldés. - AC. Jardins. -- Printemps. - St-A mand. - Sporules cylindriques, fusiformes. 
1565. PHOMA RHOIS. Sxcc. - Alb. de l'Est. Sur feuilles et pétioles de Rhus typhinum. - C. - Jardins. -- St-Amand. - Hiver-Printemps. 1566. PHOMA CORNI. Fuckel. - Alb. de l'Est. Sur rameaux de Cornus sanguinea. - C. au printemps. - Dans les bois, parcs, etc. - Partout. - Les basides des sporules sont disposées en rosette et courbées en crochet.

156\%. PHOMA PULLA. Sacc. - Alb. de l'Est. Sur tiges mortes d'Hedera helix. - Aspect d'une hysteriacée. - CC. - Sur le tronc des arbres envahis depuis longtemps par le Lierre. - CC. - Printemps. - St-Amand, etc.

1568. PHOMA OPULI. Thüm. - Alb. de l'Est. Sur rameaux de Viburnum opuli. - AC. dans les bois de St-Amand à St-Lumier. - Printemps. 1569. PHOMA PROTRACTA. Sacc. - Alb. de l'Est. - Sur branches d'Acer campestre, en compagnie de Didymosphreria acerina. - CC. - Dans les bois. - St-Lumier, Lisse, etc. Printemps.

1570. PHOMA RADULA. - $B$. et $B r$. - Alb. de l'Est. - Sur rameaux de Platanus orientalis. - AR. - Printemps. - Vitry-la-Ville, Ablancourt.

15\%. PHOMA JUGLANDINA. Sacc. - Alb. de l'Est. - Sur rameaux de Juglans. - Accompagne souvent le Cytisporina heteracantha. CC. - St-Amand, St-Lumier. - Hiver.

15\%2. PHOMA ACERVALIS. Sacc. - Alb. de l'Est.

- Sur branches dénudées de Salix caprcea. - 
AR. en hiver. - St-Amand. - Spermogonie de Gibberella acervalis.

1573. PHOMA Ligustrina. Sacc. - Alb. de l'Est. - Sur rameaux de Ligustrum vulgare. - AC. - Haies, jardins. - St-Amand. Printemps.

15\%. Phoma oblongum. Desm. - Alb. de l'Est. - Sur branches mortes et desséchées d'Ulmus campestris. - AR. - St-Amand. Printemps. - Spermogonie de Diaporthe Eres.

1575. PHOMA PICEA. Sacc. - Alb. de l'Est. Sur tige morte d'Hypericum perforatum. - C. Bois de St-Amand. - Printemps. - Sporules cylindriques à 4 gouttelettes.

1576. PHOMA TAXI. Sacc. - Alb. de l'Est. - Sur feuilles et tigelles de Taxus baccatus. - CC. - Jardins. - Printemps. - Anlnay-l'Aître, StAmand. - Suivant Saccardo c'est un état jeune de Diplodia taxi.

157\%. PHOMA HYSTERELlA. Sacc. - Alb. de l'Est. - Sur tiges et feuilles de Taxus baccatus. - AR. - St-Amand. - Jardins. - Hiver. Les sporules sont bien différentes de celles de Ph. Taxi. Leur forme est arrondie-ovale, elles ont un court pédicelle et sont granuleuses. - C'est sans doute la Spermogonie de Physaiospora gregaria.

15\%8. Phoma Petiolorum. Desm. - Alb. de l'Est. - Sur pétioles des feuilles de Juglans mortes, après l'hiver. - AC. - St-Amand. -Apparence d'un Hypoderma. 
1579. PHOMA MIRBELII. Sacc. - Alb. de l'Est.

- Sur feuilles de Buxus sempervirens. - AR.

- Jardins. - St-Amand. - Printemps.

1580. PHOMA MERVISEQUA. Sacc. - Alb. de l'Est. - Sur les nervures des feuilles de chêne. - AR. - St-Amand. - Aprés l'hiver. - Apparence d'un Sclerotıum puncturn.

1581. PHOMA LiNGAM. Desm. - Alb. de l'Est. - Sur tiges de Brassica oleracea desséchées et sur les plantes herbacées en général. - CCC. - Jardins. - St-Amand.

1582. PHOMA ALBICANS. Rob. et Desm. - Alb. de l'Est. - Sur tiges de Chicoracées. - Jardins. - Printemps. - C.

1583. PHOMA LONGISSIMA. West. - Alb. de l'Est. - Sur tiges d'Ombellifères et de Chénopodées. - CC. - Jardins, champs. - Partout. - Automne. Printemps.

1584. Phoma ComplanAtA. Desm. - Alb. de l'Est. - Sur tiges d'Urtica dioica, de Chcerophyllum Temulum. - CCC. - Jardins, bois, etc. - Partout. - Printemps.

1595. PHOMA CAULOGRAPHA. Dur. et Mont. Alb. de l'Est. - Sur tiges mortes d'Ombellifères, notamment Chcerophyllum Temulum. -Haies, bois. - St-Amand. - CC. - Printemps.

1586. PHOMA PHLOGIS. Sacc. - Alb. de l'Est. - Sur tiges desséchées de Phlox des Jardins, apres l'hiver. - CC. - St-Amand.

158\%. PHOMA STRIEFORMIS. Dur. et Mont. Alb. de l'Est. - Sur les tiges et tigelles de 
Dipsacus sylvestris. - AC. - Bois, lieux incultes, jardins, elc. - Partout.

1588. PHOMA SPIREE. Desm. - Alb. de l'Est.

- Sur tiges de Spircea Ulmaria. - AR. Bords du Fion. - Printemps.

1589. PHOMA HERBARUM. West. - $\Lambda \mathrm{lb}$. de

l'Est. - Sur les tiges des grandes herbes. CCC. - Partout. - Automne, Printemps.

1590. PHOMA BACCILLARIS. Sacc. - Alb. de I'Est. - Sur tiges d'Aconit napel. - AR. Jardins. - St-Amand. - Printemps. - Sporules cylindriques munies de 4-6 gouttelettes.

1591 PHOMA EPILOBII. Preuss. - Alb. de l'Est.

- Sur tiges d'Epilobium augustifolium. - AC.

- Lieux humides des bois de Lisse, St-Lumier.

- Hiver.

1592. PHOMA ENOTHERE. Sacc. - Alb. de l'Est. - Sur tiges mortes d'Enothera biennis - Au Printemps. - Jardins. - St-Amand. AR. - Sporules avec courtes basides pyriformes.

1593. PHOMA AENOTHERELLA. Sacc. Alb. de l'Est. - Sur tiges d'Enothera biennis. - IIiver. - Jardins. - AR. - St-Amand. - Sporules sans basides apparentes et sans gouttelettes.

159'. PHOMA NEBULOSA. Mont. - Alb. de l'Est. - Snr tiges mortes d'Althcea. Malva. AC. - Jardins. - Printemps. - St-Amand. 1595. PHOMA VINCE. Sacc. - Alb. de l'Est.

- Sur pétioles et feuilles de vinca maior. AR. - Jardins. St-Amand. - Printemps. 
1596. Phoma Leguminum. Sacc. - Alb. de l'Est. - Sur gousses de Phaseolus, de Cytisus, etc. - CC. - Jardins. - St-Amand. - Printemps.

1597. PHOMA LAGENARIÆ. Thüm. - Alb. de l'Est. - Sur les fruits de la Coloquinte en décomposition. - Jardins. - AC. - St-Amand. 1598. PHOMA UVICOLA. $B$. et $C$. - North. ameri. - Alb. de l'Est. - Black-rot. des Américains. - Sur les grains de raisins des treilles. - N'a pas encore été observé dans le vignoble de Champagne. - St-Amand. - RR. - Fin de l'Été.

1599. Phoma STROBIligena. Desm. - Alb. de l'Est. - Sur les cônes du Pin d'Autriche. - Spermogonie de Diatrype occulta. - AC. Printemps. - Soulanges, Ablancourt.

1600. Phoma GLANDiCola. Desm. - Alb. de l'Est. - Sur les glands du chêne, en compagnie du Discosia Glandicola. - AC. - HiverPrintemps. - St-Amand.

1601. PHOMA JUGLANDIS. Sacc. - Alb. de l'Est. - Sur péricarpe de Juglans. - Après l'hiver. - CC. - St-Amand. Lisse.

1602. PHOMA ORNITHOGALI. Thüm. - Alb. de l'Est. - Sur les feuilles d'Ornithogalum umbellatum, en compagnie d'Heterosporium Ornithogali. - AC. à St-Amand, dans les bois, all mois de Mai.

1603. PHOMA CONVALLARIE. West. -- Alb. de l'Est. - Sur feuilles mortes de Convallaric maïalis. - Sporules cylindriques, renflées aux 
extrémitẻs. - Dans les bois de St-Amand à St-Lumier. - En Été. - CC.

160'. PHOMA DAHLIAE. Berk. - Alb. de l'Est.

- Sur tiges pourrissantes de Dahlia Variabilis.

- Après l'hiver. - CC. - St-Amand. Printemps.

1605. PHOMA PODAGRARIA. West. - Alb. de

l'Est. - Sur feuilles d'Egopodium podagraria. - CC. à St-Amand, mais rare ailleurs. - ÉtéAutomne. - Dans les bois aux abords du Fion.

$3^{e}$ GENRE. - APOSPIITERA. - Berk.

1606. APOSPHARIA PINEA. Sacc. - Alb. de l'Est. - Sur les écailles pourrissantes du Pinus sylvestris tombées à terre en Automne. - Printemps. - C. - St-Amand, etc. - (Sphceronema des auteurs.)

$4^{\mathrm{e}}$ GENRE. - DENDROPHIMNA. - Sacc.

160\%. DENDROPHOMA PRUINOSA. Sacc. - Alb. de l'Est. - Sur rameaux de Viburnum Lantana. - Dans les bois de St-Amand à St-Lumier. Printemps. - AR. - Aspect d'un Cytispora.

1608. DENDROPHOMA THERRYANA. Sacc. et Roum. - Alb. de l'Est. - Sur glands de chêne tombès à terre au printemps. - Bois de St-Amand, La Chaussée. - AC.

5e GENRE. - MYCOGALA. - Rost.

1669. MYCOGALA PARIETINUM. Sacc. - Alb. de l'Est. - Sur les chaumes de Graminées et 
sur les vieux murs humides. - AC. - Été. - St-Amand. - Sporule sphérique, tuberculeuse. jaunâtre.

6e GENRE. - SPIIAERONENA. - Fries.

1610. SPHARONAMA DECORTICANS. Lév. Voir le Cytisporina heteracantha. Sacc. - Spermogonie d'Eutypa heteracantha. Sacc. - Sur rameaux secs de Noyer. - Automne. - StAmand. - AR.

1611. SPHARONAMA PILIFERUM. Sacc. Alb. de l'Est. - Commun sur les écailles de Chêne humides à l'air libre. - Dans les bois, chantiers, etc. partoul et toute l'année. - Coniothyrium longirostrum. (h. R.)

1612. SPHARONAEMA PITH YUM. Sacc. - Alb. de l'Est. - Sur écaille de Pinus sylvestris. Spermogonie de Ceratostoma piliferum. Var. pinastri. - C'est l'Apospharia pinea de Sacc. - AC. - St-Amand. - Printemps.

1613. SPHARONAMA CONICUM. Fr. - Alb. de l'Est. - Sur branches dènudées et écailles de bois d'Alnus glutinosa humides. - Périthèces velus, à papilles blanches, couronnés par un globule de sporules ovales. - AR. - StAmand. - Printemps.

\% GENRE. - ASTERTMM. - D. C.

1614. ASTEROMA HYPERICI. Lasch. - Alb. de l'Est. - Sur les tiges sèches d'Hypericum perforatum. - Périthéces situés au centre de fila- 
ments septès, rayonnants; sporules elliptiques. - AR. - Printemps. - St-Amand. - Bords des chemins.

1615. Asteroma Robergir. Desm. - - Alb. de l'Est. - Dans l'intérieur des tiges d'Althea rosea et des Ombellifères. - C'est le Dendrina pulla de Fries. - AC. - Jardins. - St-Amand. - Hiver-Printemps.

1616. ASTEROMA VERNiCosum. Fuckel. - Alb. de l'Est. -- Sur tiges mortes d'Angelica sylvestris. - Bords des rivières. - St-Amand, StLumier, La Chaussée. - CC. - Printemps.

161\%. ASTERoma Reticulatum. Chev. - Alb. de l'Est. - Convallaria polygonatum. - Fibrilles radiées entremêlées de périthéces pezizoïdes. Bois de St-Lumier, Aulnay. - AR. - Été.

1618. AStEROMA. ZEE. Wesi. - Alb. de l'Est,

- Sur tiges pourries de Zea maialis. - Périthéces hémisphériques, filaments rayonnants à la base. - Aulnay-l'Aître. - AR.

8e GENRE. - CIClNvobands. - Cesati.

1618 bis. CICINNOBolUs CESATI. Sacc. - Sur Senecio vulgaris. - Parasite sur Oidium Erysiphoides. - Automne. - Champs d'Aulnay-l'Aître. $-\mathrm{RR}$.

9e GENRE. - verduicularia. - Fries.

1619. VERMICULARIA COMPACTA. $C$. et E. Alb. de l'Est. - Sur jeunes sarments morts de 
vigne. - Dans les Jardins. - St-Amand. AC. - Hiver. - Sporules grandes, un peu courbes.

1620. VERMICULARIA ERYNGII. Corda. - Alb. de l'Est. - Croît abondamment sur les tiges mortes d'Eryngium campestri. - Automne-hiver. - Partout.

1621. VERMICULARIA LILIACEARUM. West. - Alb. de l'Est. - Sur tiges de Lilium album desséchées. - Jardins. - St-Amand. - AC. Sporules elliptiques des Phoma.

1622. VERMICULARIA CULMIFRAGA. Fr. Alb. de l'Est. - Commun sur les chaumes de seigle exposés à l'humide en hiver. - Spermogonie de Pleospora relicina. - CC. - St-Amand. 1623. VERMICULARIA GRAMINICOLA. West. Alb. de l'Est. - A la base des chaumes de Briza media et sur les feuilles radicales. AR. - St-Amand, Aulnay-l'Aître. - Hiverprintemps.

10 GENRF。 - DOTHIORERLA. - Sacc.

162'. DOTHIORELLA POPULEA. Sacc. - Alb. de l'Est. - Sur branches et rameaux morts de Populus Italica. - Voir Dothischiza populea. - CC. - Automne. - St-Amand.

1625. DOTHIORELLA ADVENA. Sacc. - Alb. de l'Est. - Sur branches de Hêtre et de Frêne. - Spermogonie de Botryosphceria advena. - AC.

- Souvent mélangé avec l'état thécasporé. Bois de St-Amand. - Printemps. 
1626. DOTHIORELLA GLANDULOSA. Sacc. -

Alb. de l'Est. - Sur Ailanthus glandulosa. Branches et rameaux morts. - Accompagne le Valsa glandulosa, dont il est la spermogonie. - RR. - Parc d'Ablancourt. - Hiver.

\section{GENRE. - RA BENHOTSTHA. - Fries.}

162\%. RABENHORSTIA TILIE. Fr. - Alb. de l'Est. - Sur branches mortes de Tilia Europaca, qu’il envahit complètement. ainsi que Hercospora Tilice, son état ascophore. - CCC. Jardins. - Parcs. - Hiver, printemps. - Partout.

12e GENRE. - PLA COSPIM AERA. - Sacc.

1628. PLACOSPHARIA ONOBRYCHIDIS. SAcc. - Alb. de l'Est. - Sur feuilles languissantes d'Onobrychis sativa. - CCC. à la fin de l'Eté. - Champs. - St-Amand, Lisse. etc.

$13^{\mathrm{e}}$ GENRE. - TSICOCCUM. - Corda.

1629. FUSICOCCUM KUNZEANUM. Sacc. - Alb. de l'Est. - Sur rameaux secs de Carpinus betulina, en compagnie de Diaporthe carpini. Spermaties fusiformes, grandes; courtes hasides. - AC. - Haies, jardins. - St-Amand. - Printemps. 
1'a GENRE. - PYREMACH RETA. Sacc.

1630. PYREN. BERBERIDIS. Ch. R. - Alb. de l'Est. - Sur rameaux dénudés de Berberis vulgaris. - Périthéces couronnés de poils noirs, spermaties á basides arborescentes. - AC. Jardins. - St-Amand. - Hiver.

1630) bis. PYREN. BROMI Ch. R. - Sur gaines de Bromus asper. - R. - St-Amand.

15e GENRE. - CYTOSPORA. - Erhenb.

1631. CYTOSPORA ROSARUM Grev. - Alb. de l'Est. - Sur tiges mortes de Rosa canina. Suus épidermique. - Sa présence est dévoilée par les Cirrhes jaunes qui paraissent au dehors. - CC. - Bois, haies. - Partout. - Hiver.

16i3\%. CYTOSPORA RUBESCENS Sr. - Alb. de l'Est. - Sur écorce de Cerasus avium. Spermogonies à Cirrhes rouges. - CC. - Bois, Jardins. - Hiver, printemps. - Partout.

1633. CYTOSPORA VITIS Mont. - Alb. de l'Est. - Sur sarments morts de Vitis viniferce. - Spermogonie de Valsa vitis. - CCC. Fagots de vignes exposés à l'humide. - Printemps.

1634. CYTOSPORA CHYSOSPERMA Sr. - Alb. de l'Est. - Sur branches mortes de Peuplier. - Cirrhes jaune-orange trés longs, spermo- 
gonie de Valsa nivea. - CCC. - Hiver, printemps. - St-Amand.

1635. CYTOSPORA MACROBASIS Sacc. - Alb. de l'Est. - Sur branches mortes de Salix caprcea. - Spjermaties portées sur des Basides arborescentes. - Doit être la spermogonie de Cenangium salignum. - AR. - Lisse, Bassu. - Autornne.

1636. CYTOSPORA ATRO-NITENS Chev. - Alb. de l'Est. - Sur écorce de branches de Peuplier et sur tiges de Rosa canina. - Périthéces hémisphériques, luisants. - CC. - Bois de St-Amand. - Hiver, printemps.

1637. CYTOSPORA FUGAX $\mathrm{Sr}$. - Alb. de l'Est.

- Sur rameaux de Salix caprcea. - CCC. Printemps. - Partout - Spermogonie de Valsa salicina.

1638. CYTOSPORA INCARNATA Sr. - Alb. de l'Est. - Sur branches et rameaux de Peuplier. - Cirrhes rose - incarnat; sporules fusiformes. - AC . - Bois de St-Amand. - Hiver. Automne.

1630. CYTOSPORA PLATANI $C h$. $R$. - Alb. de l'Est. - Sur rameaux de Platanus orientalis. - AR. - Bords du Canal à Ablancourt. Printemps.

1610. CYTOSPORA LEUCOSPERMA Sr. - Alb. de l'Est. - Sur branches mortes de Chêne. Spermogonie de Valsa Leiphamia. - CC. Bois de La Chaussée, St-Amand, etc. - IIiver, Printemps. 
SEGT. II. - PH EOSPORÉES. - Sacc.

16e GENRE. - SPHAEROPSIS. - Lév.

1611. SPHAROPSIS VISCI. Sacc. - Alb. de l'Est.

- Sur feuilles et rameaux de Viscum album.

- Jadis Diplodia visci. - Labsence de cloison en fait un Sphceropsis. - CCC. - Sur toutes les feuilles tombées. - Soulanges, La Chaussée, etc. - Hiver-Printemps.

1\% GENRE. - CONHOTIIYRIUM. - Corda.

16ł2. CONIOTHYRIUM OLIVACEUM. Bon. - Alb. de l'Est. - Sur les sarments de Hedera helix - Bois de St-Amand. - AC. - Hiver.

1613. CONIOTHYRIUM INSITIVUM. Sacc. - Alb. de l'Est. - Sur rameaux de Berberis vulgaris et d'Ulmus campestris. - Pycnide de Valsaria insitiva. - CC. - Jardins, bois. - St-Amand. - Printemps.

1614. CONIOTHYRIUM FUCKELII. Sacc. - Alb. de l'Est. - Sur sarments de Rubus truticosus. - AC. - St-Amand. - Printemps.

1645. CONIOTHYRIUM LABURNI. Ch. R. - Alb. de l'Est. - Sur branches dénudées de Cytisus laburnum. - AR. - Jardins. - St-Amand. Hiver. 
1646. CONIOTHYRIUM ULMEUM. Karst. - Alb. de l'Est. - Sur rameaux d'Ulmus campestris, envahis par Ccelosphoria cupularis. - AR. -. Printemps.

1647. CONIOTHYRIUM POPULI ? Ch. $R$. - Alb. de l'Est. - Sur branches de Peuplier. - Souscutané. - Sporules brunes munies d'une gouttelette. - RR. - St-Lumier.

1648. CONIOTHYRIUM CONCENTRICUM. SAcc. - Alb. de l'Est. - Sur feuilles de Jucca gloriosa. - C'roît en zones concentriques. - CCC. -- Jardins. - St-Amand. - Hiver.

1649. CONIOTHYRIUM SYLVATICUM. 'SAcc. Alb. de l'Est. - Sur tige morte d'Euphorbia sylvatica. - Bois de la montagne de Reims. RR. - Automne.

SEGT. III. - PHÆEODIDYMÉES. - Sacc.

18 GENRE. - DIPLODIA. - Fries.

1650. DIPLODIA TILIÆE. Fuck. - Alb. de l'Est.

- Sur branche de Tilia Europcea qu'il envahit complètement. - CC. - Parcs et jardins. Ablancourt, Aulnay-l'Aître. - Printemps.

1651. DIPLODIA SUBTECTA. Fr. - Alb. de l'Est.

- Sur branches d'Acer campestre. - Pycnide de Didymosphieria. - CC. - Bois de St-Amand.

- Automne et Printemps. 
1652. DIPLODIA ESCULI. Lév. - Alb. de l'Est.

- Sur jeunes rameaux d'Esculus. - Périthéces 4-こ réunis. - C. - St-Lumier, Ablancourt, etc.

- Printemps.

1653. Diplodia Viticola. Desm. - Alb. de l'Est. - Sur sarments de Vitis vinifera desséchés. - Périthèces disposés en lignes. - CC. - Pycnide de Valsa Vitis. - Vignes et jardins.

- Partout. - Hiver et Printemps.

165'. DIPLODIA BAMULICOLA. Desm. - Alb. de l'Est. - Sur rameaux d'Evonimus Europeus. - Disposé en ligne. - Bois de St-Amand à St-Lumier. - AR. - Hiver et Printemps.

1655. DIPLODIA FRANGULAE. Fuck. - Alb. de l'Est. - Sur rameaux de Rhamnus frangula. - Pycnide de Cucurbitaria rhamni. - C. - La Chaussée. - Printemps.

1656. DIPLODIA RUDIS. Desm. - Alb. de l'Est. Sur branches de Cytisus laburnum. - C. - Parcs et jardins. - Hiver et Printemps. - Partout. 165\%. DIPLODIA PETIOLORUM. Sacc. - Alb. de l'Est. - Sur les pétioles des feuilles de Juglans. - Après l'hiver. - St-Amand, Soulanges. - CC. 1658. DIPLCDIA ROSA. B. et $C$. - Alb. de l'Est.

- Sur tige de Rosa canina. - Bois et broussailles. - Partout. - Hiver et Printemps.

1659. DIPLODIA CRATAEGI. West. - Alb. de l'Est. - Sur rameaux de Cratcegus Oxyacantha. - CC. au Printemps. - St-Amand, etc.

1660. DIPLODIA HEDERICOLA. Sacc. - Alb. de l'Est. - Sur les feuilles mortes d'Hedera helix. 
- Forme de larges taches gríses. - AC. au Printemps. - St-Amand. - Sur les murs envahis par le lierre.

1661. DIPLODIA LONICERAE. Fuck. - Alb. de l'Est. - Sur tiges de Lonicera Xylostei. - Pycnide d'Amphisphceria Xylostei. - AC. - Haies et jardins. - Ablancourt, La Chaussée. Automne.

1662. DIPLODIA SAMBUCINA. Sacc. - Alb. de l'Est. - Sur le sambucus nigra. - Rameaux dénudés, disposés en lignes. - C. - Printemps, - St-A mand, etc.

1663. DIPLODIA RIBIS. Sacc. - Alb. de l'Est. R. - Sur branches de Ribes rubrum. - Les Périthèces ne contiennent que des stylospores incolores qui ne brunissent qu'après leur sortie. - Jardins. - St-Amand. - Été.

1664. DiplodiA. MiCROSPORA. $B$. et $C$. - Alb. de l'Est. - Sur rameaux de Viburnum opulus. - En compagnie du Phoma opuli. - AR. Bois de St-Amand à St-Lumier. - Printemps. 1665. DIPLODIA INQUINANS. West. - Alb. de l'Est. - Sur branches et troncs du Fraxinus excelsior. - CC. - Dans les bois. - Partout. - Hiver et Printemps.

1666. DIPLODIA LILACIS. West. - Alb. de l'Est.

- Sur les jeunes branches de Syringa vulgaris. mortes avant l'hiver. - C. - Printemps. Jardins. - St-Amand, etc.

1667. Diplodia MELAeNA. Lév. - Alb. de l'Est. - Sur rameaux d'Ulmus campestris. - Pycnide 
de Cucurbitaria naucosa. - CC. au Printemps. - St-Amand, Soulanges.

1668. DIPLODIA MORI. West. - Alb. de l'Est.

- Sur rameaux de Morus alba. - Périthéces très petits. - K. - Automne et Printemps. - Vitry-le-Brûlé.

1669. DIPLODIA POPULINA. Fuck. - Alb. de l'Est. - Sur écailles de Peuplier. - Pycnide d'Otthia populina. -- Lieux humides des bois. après l'hiver. - R. - St-Amand.

16\%. DIPLODIA. HEDERE. Fuckel. - Alb. de l'Est. - Sur feuilles et tiges d'Hedera helix. AR. au Printemps. - St-Amand.

1671. DIPLODIA STAPHYLAE. Sacc. - Alb. de l'Est. - Sur rameaux de Staphylea pinnata. - AR. - Jardins. - St-Amand. - Hiver.

1672. DIPLODIA CARPINI. Sacc. - Alb. de l'Est.

- Sur rameaux de Carpinus. - Périthèces en groupe sur l'épiderme noirci. - Stylospores incolores étant jeunes, bruns et cloisonnés à la maturité. - AC. - St-Amand. - Printemps. 1673. DIPLODIA CORYLI. Fuck. - Alb. de l'Est. - Sur rameaux de Corylus avellana. - Pycnide d'Otthia coryli. - AC. - Printemps. - St-Amand. 1674. DIPLOdIA FAGINEA. Fr. - Alb. de l'Est. - Sur rameaux de Fagus sylvatica. - Pycnide de Cucurbitaria macrospora. - C. - Aulnayl'Aître. - Printemps. - Les périthèces de cette espéce sont réunis en groupe de 15 à 20 .

16\%. DIPLODIA BETULE. West. - Alb. de l'Est. - Sur écorce de Betula alba. - Péri- 
thèces de 5 à 10. - Pycnide de Cucurbitaria conglobata de Fuckel. - AR. - Printemps. - St-Lumier.

16\%6. DIPLODIA JUNIPERI. West. - Alb. de l'Est. -- Sur rameaux de Juniperus communis.

- Dans les plantations de Pins sylvestres. AC. - Printemps. - Soulanges.

167\%. DIPLODIA SAPINE\&. Fuck. - Alb. de l'Est. - Sur les trones de Pins sylvestres. Les stylospores sont ordinairement grosses et privées de cloison. - AR. - Automue. St-A mand.

1678. DIPLODIA TAXI. de Not. - Alb. de l'Est. - Sur tiges et feuilles de Taxus baccata, disposé en ligne. - C. au printemps, dans les jardins. - St-Amand, La Chausseee.

16\%9. DIPLODIA ILICIS. Fr. - Alb. de l'Est. Sur les feuilles et les jeunes branches de Houx, mortes et tombées à terre avant l'hiver - C. - Printemps. - Bois de Sermaize.

1680. DIPLODIA GENIST $Æ$. Fuckel. - Alb. de l'Est. - Sur tiges mortes de Genista prostrata. - Pycnide de Didymosphceria Genista. - R. - Lieux incultes près de Bassu.

1681. DIPLODIA ABROTANI. Fuck. - Alb. de l'Est. - Sur rameaux d'Arthemisia Abrotanum. - R. dans les jardins á St-A mand. - Automne et hiver. 1682. DIPLODIA VINCAE. Ch. R. - Alb. de l'Est. - Sur tiges mortes de Vinca major qui ont èté envahies pendaut l'été par Puccinia Vincce. - R. - St-Amand. - Hiver. 
19e GENRE. - DIPLODIELLA. - harsten.

1683. DIPLODIELLA FIBRICOLA. Sacc. - Alb. de l'Est. - Sur branches dénudées de Populus Pyramidaiis. - Périthéces presque astomes, stylospores fusoïdes, à une ou deux cloisons. R. - Printemps. - St-Amand.

168'. DIPLODIELlA JUNCI. Ch. $R$. - Alb. de l'Est. - Sur les chaumes de Juncus communis. - R. - Fossés humides entre St-Amand et Lisse. - Hiver.

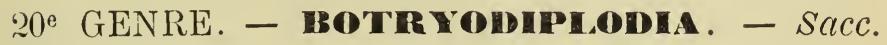

1685. BOTRYODIPLODIA JUGLANDICOLA. Sacc.

-- Alb. de l'Est. - Commun sur les branches mortes du Noyer en compagnie du Cytispora heteracantha. - Bois de St-Amand à St-Lumier. - Automne.

SEct. IV. - HYALODIDYMÉES. - Sacc. 21. GENRE. - ASCOCHYTA. - Lib.

1686. ASCOCHYTA PHILAdELPHI. Sacc. - Alb. de l'Est. - Sur les feuilles encore vertes de Philadelphus coronarius. - AC. en Automne ủans les jardins à St-Amand.

1687. ASCOCHYTA THLASPIDIS. Ch. $R$. - Alb. de l'Est. - Sur tige et feuilles de Thlaspi per- 
foliatum. - CC. dans les lieux incultes. - En Été. - St-Amand, Lisse. - Sporules grandes $0^{\mathrm{m}}, 014$.

1688. ASCOCHYTA AMPELINA. Sacc. - Alb. de l'Est. - Sur les jeunes sarments de vigne coupés nouvellement. - Stylospores incolores munies d'une cloison à la maturité. - C. dans les jardins au Printemps. - Partout.

1689. ASCOCHYTA SORBI. Ch. R. - Alb. de l'Est. - Sur teuilles mourantes de Sorbus acupariı dans les jardins. - St-Amand. - Automne. - CC.

1690. ASCOCHYTA CLEMATIDINA. Thum. Alb. de l'Est. - Sur feuilles de Clcmatis vitalba. - A la surface des taches noires de Euriachora clematidina. - R. - Ablancourt. bords du canal. - Automne.

22 GENRE. - DARECCA. - Cast.

1691. DARLUCA FILUM. Cast. - Alb. de l'Est. - Variété Rumicum. Ch. R. - Sur pustules d'Uredo rumicum. Parasite des tiges de Rumex acetosa. - AR. - Jardins. - En Été. - StAmand. - Variété Clinopodii. Sacc. - Sur Puccinia clinopodii. - Parasite des feuilles de Clinopodium vulgare. - R. - Aulnay-l'Aître. - Automne.

1692. DARLUCA VAGANS. Sacc. - Alb. de l'Est. - Sur feuilles de Salix capreca envahies par Podosporium caprcearum. - RR. - Entre Lisse et Bassu. - Automne. 
23e GENRE. - DMPLOMHNA. - West.

1693. DIPLOdINA. SALICIS. West.-Alb. de l'Est.

- Sur rameaux de Salix babylonica. - AR.

- Jardins à St-Amand. - Printemps.

1694. DIPLODINA ANTIRRHINI. Ch. $R$. - Alb. de l'Est. - Sur les capsules d'Antirrhinum maius. - RR. - Jardins. - St-Amand. Printemps.

1695. Diplodina. Minima. Ch. $R$. - Alb. de l'Est. - Sur rameaux de Ligustrum vulgare.

- Stylospores couleur café au lait. - R. St-Amand. - Printemps.

SEGT. V. - PRAGMOSPOREES. - Sacc. $24^{\mathrm{e}}$ GENRE. - HENDERSOMIA. - Berk. 1696. HENDERSONIA SARMENTORUM. West. Alb. de l'Est. - Sur sarment de vignes jeunes. - CC. - Dans les jardins au Printemps. Partout.

1697. HENDERSONIA FIEDLERI. West. - Alb. de l'Est. - Sur rameaux de Cornus sanguinea. - Sporules longuement pedicellées. - C. dans les bois. - St-Lumier, St-Amand. - Printemps. 1698. HENDERSONIA SYRINGE. Fr. - Alb. de l'Est. - Sur rameaux de Syringa vulgaris. Pycnide de Diaporthe resecans. - AC. dans les jardins. - St-Amand. - Printemps. 
1699. HENDERSONIA LONICERA. Fries. - Alb. de l'Est. - Sur rameaux de Lonicera caprifolium. - AR. - Jardins à St-Amand. Printemps.

1700. HENDERSONIA PHRAGMITIS. Sacc. - Alb. de l'Est. - Sur chaumes d'Arundo phragmites. - Espéce trés petite. - R. - Fossés humides à St-Lumier. - Hiver.

1701. HENDERSONIA RUBI. West. - Alb. de l'Est. - Sur les sarments de Rubus fruticosus. - CC. au Printemps. - St-Lumier, Lisse.

1702. HENDERSONIA SALICINA. Sacc. - Alb. de l'Est. - (Var. Biseptata. Ch. R.) - Sur les rameaux de Salix caprea. - Sporules olive clair 2 cloisons. - AR. - St-Amand. - Printemps. 1703. HENDERSONIA FOLIORUM. Fuck. - Alb. de l'Est. - Sur feuilles de Fagus sylvatica. Périthéces très petits. - R. - Bois de St-Amand. -. Printemps.

170'. HENDERSONIA ACONITI. Ch. $R$. - Alb. de l'Est. - Sur pétiole des feuilles d'Aconit.

- Sporules brunes, à court pédicelle. - R. Jardins à St-Amand. - Hiver.

1705. HENDERSONIA HYPERICI. Ch. $R$. - Alb. de l'Est. - Sur tige d'Hypericum perforatum. - Bois de St-Amand à St-Lumier. - Printemps. 1706. HENDERSONIA JUNIPERI. Ch. $R$. - Alb. de l'Est. - Sur rameaux de Juniperus communis, en compagnie de Diplodia juniperi. - R. Sapinière de St-Amand. - Au Printemps.

170\%. HENDERSONIA LIGUSTRI. Ch. $R$. - Alb. 
de l'Est. - Sur branches de Ligustrum vulgare. - Périthèces sous-cutanés émettant des masses de sporules. - AR. - St-Amand. - $\mathrm{Au}$ Printemps.

1708. HENDERSONIA. PLATANI. Ch. R. -Alb. de l'Est. - Sur branches de Platane. Périthèces disposés en rosette. - R. - Ablancourt. - Printemps.

1709. HENDERSONIA. LABURNI. West. - Alb. de l'Est. - Sur rameaux de Cytisus laburnum.

- Sporules trés petites. - C. - Jardins. Printemps. - Partout.

1710. HENDERSONIA CULMICOLA. Sacc. - Alb. de l'Est. - Sur chaumes de Festuca ovina. Sporules olive-clair cylindriques, 7 cloisons. R. - St-Amand. Printemps.

1711. HENDERSONIA ARUNDINACEA. Sacc. Alb. de l'Est. - Sur Arundo phragmites. Sporules cylindriques, fusiformes: 3 cloisons. R. - Fossès du château d'Aulnay-l'Aître. Automne.

1712. HENDERSONIA ARUNDINIS. Lib. - Alb. de l'Est. - Sur Phragmites communis. - Périthèces sous - cutanés, munis de poils bruns à la base. - AR. - St-Lumier. - Printemps.

1713. HENDERSONIA DEMAZIERI. Mont. - Alb. de l'Est. - Sur rameaux de Platanus orientalis. - Périthèces en rosette, sporules grandès brun-clair. - R. - Ablancourt, le long du canal. - Printemps. 
25 GENRE. - CRYPTOSTRTHS. - Fuckel.

1714. CRYPTOSTICTIS CYNOSBATI. Sacc. Alb. de l'Est. - Sur feuilles mortes du Rosier à cent feuilles. - Périthéces épars sur des taches brunes, sporules terminées par deux cils très longs. - R. - Jardins. - St-Amand. Automne.

1715. CRYPTOSTICTIS HYSTERIOIDES. Fuck.

- Alb. de l'Est. - Sur jeunes sarments de vigne secs. - Disposé en ligne prés des bourgeons. - Sporules à 4 loges. les deux extrêmes hyalines, garnies d'une arête. unique supérieurement. - R. - St-Amand. - Printemps.

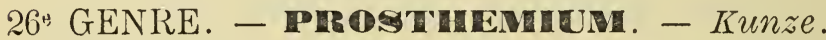

1716. PROSTHEMIUM BETULINUM. Kunze. Alb. de l'Est. - Sur rameaux morts du Bouleau. - Périthéces sous-cutanés, sporules brunes, ovoïdes allongées. disposées en faisceau sur un pédicelle hyalin. -- C. - Bois de St-Lumier. - Printemps.

2\% GENRE. - STA GONOSPORA. - Sacc.

1717. STAGONOSPORA CAESITOSA. Sacc. Alb. de l'Est. - Sur rameaux dénudés de Sambucus nigra. - Sporules fusiformes bi et tri-septées. - C. dans les haies à St-Amand.

- Printemps. 
1718. STAGONOSPORA GRAMINELLA. Sacc. Alb. de l'Est. - Sur chaumes de Phragmites notamment à la base. - Sporules cylindriques un peu courbes: munies de 4-6 gouttelettes. R. - St-Amand. - Eté.

1719. STAGONOSPORA DOLOSA. Sacc. - Alb. de l'Fist. - Sur chaume d'Arundo Phragmetes. Périthéces disposés en lignes. Sporules très grandes, fusiformes pluricloisonnées. - C. - Bords des eaux à St-Amand. - Printemps.

1720. STAGONOSPORA VEXATA. Sacc. - Alb. de l'Est. - Sur Arundo Phragmites. - Même disposition que la précédente espéce, mais sporules cylindriques recourbées. - C. - Bords des eaux á St-Amand. - Printemps.

SECT. V1. - DICTYOSPORÉES. - Sacc. 28 GENRE. - CAMAROSPORHUM. - Schulz.

1721. CAMAROSPORIUM ROBINIEE. Sacc. - Alb. de l'Est. - Sur rameaux de Robinia pseudoacacia. - Pycnide de Cucurbitaria elongata. - C. au printemps, dans les jardins á St-Amand.

1722. CAMAROSPORIUM XYLOSTEI. Sacc. Alb. de l'Est. - Sur jeunes tiges de Lonicera Xylosteon. - Conidies d'Amphishceria Xylostei. - AR. - Jardins et parcs. Ablancourt et Soulanges. - Printemps. 
1723. CAMAROSPORIUM MACROSPORUM. SACC. - Alb. de l'Est. - Sur branches mortes de Philadelphus Coronarius. - R. - Jardins. St-Amand. - Automne. - Pycnide de Diaporthe landeghemice.

1724. CAMAROSPORIUM VISCI. Sacc. - Alb. de l'Est. - Sur tiges et feuilles de Viscum album en compagnie du Diplodia Visci. - R. - Soulanges, Aulnay-l'Aître. - Automne, printemps.

1725. CAMAROSPORIUM CRUCIATUM. Sacc. Alb. de l'Est. - Sur petits rameaux secs d'Ulmus campestris. - AC. au printemps. - Bois à StAmand. - Sporules arrondies, à cloisons disposées en croix.

1726. CAMAROSPORIUM PICASTRUM. Sacc. Alb. de l'Est. - Sur tiges et racines dénudées. de Juniperus communis. - R. - Plantations de Pins sylvestres à Soulanges. - Automne.

172\%. CAMAROSPORIUM CORONILLE. Var. Colutos. Sacc. - Alb. de l'Est. - Sur les pèdoncules des fruits vésiculeux du Colutea arborescens. AC. - Jardins. - Printemps. - St-Amand.

1728. CAMAROSPORIUM BERBERIDIS. Ch. $R$. Alb. de l'Est. - Sur rameaux encore verts de Berberis vulgaris. - AR. - Jardins et parcs à St-Amand et Aulnay-l'Aître. - Automne, Hiver.

29e GENRE. - DICHOMERA. - Cooke.

1729. DiCHOMERA TILIE. Sacc. - Alb. de l'Est.

- Sur branches mortes de Tilleul. - Pustules 
sous-cutanées, rangées en lignes; sporules mures, brunes uni-biseptées. - R. - Jardins á Ablancourt. - Printemps.

SEGT. VII. - SCOLECOSPORÉES. - Sacc.

Sporules allongées. filiformes.

30" GENRE. - SEPTORIA. - Fries.

1730. SEPTORIA CYTISI. Desm. - Alb. de l'Est.

- Sur feuilles languissantes de Cytisus laburnum. - CC. - Automne. - Soulanges, St-A miand. 1731. SEPTORIA RUBI. West. - Alb. de l'Est.

- Sur feuilles de Rubrs Ccesius encore vertes, inséré au milieu de taches blanches, rondes, bordées de brun pourpre. - CC. dans les bois. - Partout. - Automne.

1732. SEPTORIA CRATAEGI. Kickx. - Alb. de l'Est. - Sur feuilles de Cratcegus Oxyacantha. - CC. mais rarement fertile. - Haies, bois à St-Amand. etc. - Automne.

1733. SEPTORIA PIRICOLA. Desm. - Alb. 'de l'Est. -- Sur les feuilles vivantes du Poirier, au centre d'une tache grise, bordée de brun.

- Sporules aculiformes. courbes. - Jardins.

- en Automne. - Partout.

1734. SEPTORIA HEDERE. Desm. - Alb. de l'Est. - C. sur feuilles d'Hedera helix, au centre d'une tache blanche avec bordure brune. - Printemps. - St-Amand. 
1735. SEPTORIA RIBIS. Desm. - Alb. de l'Est.

- Sur feuilles de Ribes rubrum. - Spermogonie de Sphceria ribis (Fuckel). - C. Jardins à St-Amand. - Automne.

1736. SEPTORIA CORNICOLA. Desm. - Alb. de l'Est. - Sur feuilles vertes de Cornus sanguinex; inséré sur taches brunes, à contours violacés - C. Dans les haies et bois à StAmand. - Étè.

173\%. SEPTORIA XYLOSTEI. Sacc. - Alb. de l'Est. - Sur feuilles de Lonicera Xylosteon. inséré sur de petites taches blanches, qui brunissent en détruisant les feuilles. - Sporules aculèiformes, à 4-5 cloisons. - AR. - Jardins à St-Amand. - Automne.

1738. SEPTORIA VIBURNI. West. - Alb. de l'Est. - Sur feuilles de Viburnum opulus. Taches rondes, rouge-vineux, centre gris-brun. - C. dans les bois à St-Lumier, Soulanges. - En Été.

1739. SEPTORIA SALICIS. West. - Alb. de l'Est. - Sur feuilles de Salix viminais, à la face inférieure, peu apparente. - Spermogonie de Sphcerella salicina. AC. - St-Amand, Lisse. - Automne.

1740. SEPTORIA POPULI. Desm. - Alb. de l'Est. - Sur feuilles de Popuius nigra. - CC. en Automne. - Partout.

1711. SEPTORIA CAULINCOLA. Var. Ebuli. $C h$. R. - Alb. de l'Est. - Sur tiges mortes de Samuucus ebulus, formant des taches grises, poin- 
tillées par les périthéces. - Sporules aculéiformes sans cloison apparente. - R. - Aulnay. l'Aître. - Printemps.

1742 SEPTORIA. STELLARIA. Rob. et Desm.Alb. de l'Est. - Sur feuilles de Stellaria media, insérè al bas de la tige. - C. au Printemps, sur le bord des chemins. -- St-Amand. Printemps.

1743. SEPTORIA. FICARIE. Desm. - Alb. de l'Est. - Sur feuilles de Ranunculus ficaria, au centre de taches blanches. entourées d'une zone brune. - C. dans les bois au Printemps. St-Amand, etc.

1\%44. SEPTORIA SEDI. West. - Alb. de l'Est. - Sur feuilles de Sedum téléphium. - Périthèces anguleux, sur taches grises. - C. Bois de St-Amand a St-Lumier. - En Automne. 1745. SEPTORIA. PODAGRARIA. Lasch. - Alb. de l'Est. - Sur feuilles d'Egopodium Podagraria, encore vertes. - C. Bois de St-Amand. - R. ailleurs. - Automne.

1\%46. SEPTORIA. PETROSELINI. Desm. - Alb. de l'Est. - Sur feuilles vivantes du Persil, les parties envahies sont décolorées. - C. au printemps. - Jardins à St-Amand.

174. SEPTORIA HERACLEI. Desm.-Alb. de l'Est. - Sur les deux côtés des feuilles d'Heracleum spondylum. - C. dans les bois, prairies. -- Fin de l'été. - Partout.

1\%8. SEPTORIA CALYSTEGIE. West. - Alb. de l'Est. - Sur feulles de Convolvulus sepium 
languissantes. - Périthèces à peine visibles, sporules relativement grandes. - C. à StAmand. - En Automne.

1749. SEPTORIA. STACHYDIS. Rob. et Desm. Alb. de l'Est. - Sur feuilles de Stachys sylvatica, rarement fertile. - Sporules aculéiformes petites. - C. dans le bois à St-Amand. - En Eté.

1750. SEPTORIA VIRGAUREAE, Desm. - Alb. de de l'Est. - Sur feuilles languissantes de Solidago virgaurece. - C. en Automne, dans les bois. - Partout.

1751. SEPTORIA. POLYGONORUM. Desm. - Alb. de l'Est. - Sur feuilles de Polygonum Persicaria, paraît des deux côtés de la feuille, sur taches pourprées noirâtres. - C. - Bords des eaux. - A l'Automne. - Partout.

1752. SEPTORIA. CANNABIS. Sacc. - Alb. de l'Est. - Sur feuilles vertes de Cannabis sativa. AC. en Automne. - Lisse, St-Amand.

1753. SEPTORIA MERCURIALIS. West. - Alb. de l'Est. - Sur feuilles de Mercurialis annua encore vertes. - C. en Automne. - Champs et jardins. - Partout.

1754. SEPTORIA ARI. Desm. - Alb. de l'Est. Sur feuilles d'Arum maculatum. - Taches jaunâtres, irrégulières, sporules sans cloisons apparentes. - AC. - Bois de St-Amand.-Au Printemps.

1755. SEPTORIA SCILLA. West. - Alb. de l'Est. - Sur feuilles de Muscari Comosum, languissantes. - C. au printemps dans les champs et bois à St-Amand. 
1756. SEPTORIA HUMILI. West. - Alb. de l'Est. - Sur feuilles de Houblon. - Les Périthèces sont très nombreux et décolorent à peine la feuille. - AR. - Bois et jardins. - Au printemps à St-Amand.

1757. SEPTORIA ALLIORUM. West. - Alb. de l'Est. - Sur feuilles d'Allium Porum. - CC. - Printemps, Eté. - Dans les jardins. - Partout.

1758. SEPTORIA. CHÆLIDONII. Sacc. - Alb. de l'Est. - Sur feuilles vertes de Chœlidonium majus. - C. en Eté. - St-Amand. - Le long des haies.

1759. SEPTORIA ANAGALLIS. Ch. $R$. - Alb. de l'Est. - Croît au printemps sur les feuilles inférieures d'Anagallis, au centre d'une tache jaunâtre. - R. -- Champs et jardins à StAmand.

1760. SEPTORIA VERONICA. Sacc. - Alb. de l'Est. - Sur les feuilles inférieures des tiges de Veronica hoederefolia, à peine visible à l'œil nu; souvent stérile. - Sporules aculéiformes. - AC. dans les champs au printemps. - St-Amand.

31e GENRE. - PHLAEOSPORA - Wallr.

1\%61. PHL EOSP. ULMI. Wallr. - Alb. de l'Est. - A la face inférieure des feuilles d'Ulmus campestris. - Périthèces 5-10 réunis, sporules pluriseptées. - CC. - En Automne. - Bois de St-Amand. 
$32^{e}$ GENRE. IRHABDOSPORA . - Mont.

1762. RHABD. HELLEBORINA. Sacc. - Alb. de l'Est. - Sur les tiges mortes d'Helleborus viridis qu'elle noircit par l'abondance de ses périthèces, à nucléus rose. - AR. - Printemps. - Soulanges.

33 GENRE. - SPH REROGRAPHIUM. - Sacc.

1763. SPHER. MICROPORE. Sacc. - Alb. de l'Est. - Sur branches de Prunus spinosa. Spermogonie de Cenangium Prunastri, qu'il entoure sur le même support. - AR. - Ablancourt. - Printemps.

1764. SPHÆER. SERIATUM. Sacc. - Alb. de l'Est. - Sur écorce de Bouleau. - Périthéces en cupules, disposés en lignes. Sporules en crochet - AC. - Hiver et Printemps. - Partout. 1765. SPHAR. COLUTE E. Ch. R. - Alb. de l'Est.

- Sur jeunes rameaux de Colutea arborescens.

- R. dans les jardıns de St-Amand. - Hiver. 1766. SPHÆR. CORYLI. Ch. R. - Alb. de l'Est.

- Sur branches mortes de Corylus avellana. Périthèces à long col, simples ou réunis en faisceau, sporules fusoïdes au sommet de basides arborescentes. - RR. - Automne. - La Chaussée, bords de la Marne.

166\%. SPAER. SYRINGAE. Ch. R. - Alb. de l'Est.

- Sur rameaux de Syringa Vulgaris. - Péri- 
théces sphériques, ressemblant à ceux du Genre Diplodia. - Sporules aculéiformes, sur des basides de même longueur réunies en faisceau. - R. - Jardins à St-Amand. - Hiver.

34'e GENRE. - COBNULARA. - Karsten.

1768. CORN. BOUdIERI. Ch. R. - Alb. de l'Est. - - Sur écorce de Pin. - Périthèces très petits, allongés, noirs, renflés au sommet et couronnés par un globule de sporules aculéiformes. - RR. - Ablancourt. - Au Printemps. - Bull. Soc. des Sc. de Vitry-le-François.

35 GENRE. - DHLOPIIOSPORA. Desm.

1769. DILOPH. GRAMINIS. Desm. - Alb. de l'Est. - Envahit les épis de blé et des autres graminées, sous forme de cruste noire. - Sporules cylindriques garnies aux extrémités de plusieurs cils. - Trés nuisible aux céréales. Heureusement devient rare aujourd'hui. - StAmand, St-Lumier, etc. - Été. - Bull. Soc. des Sc. de Vitry-le-François.

36 GENRE. - CYTOSPORINA. - Sacc.

1770. CYTOS. HETERACANTHA. Sacc. - Alb. de l'Est. - Sur écorce de branches de Noyer mortes. - Périthéces nombreux. plus ou moins allongés en forme de bouteille. remplis de sporules filiformes courbes. - AC. dans de certaines années. - St-Amand, Lisse et Bassu. - Hiver et Printemps. 
3\% GENRE. - MICROPERA. - Lév.

1\%\%1. MICROPERA DRUPACEARUM. Lév. - Alb. de lEst. - Sur branches mortes et pourrissantes de Cerasus avium. - Groupes de Périthèces allongés, jaunâtres ou rosés. Sporules filiformes, - Pycnide de Cenangium cerasi. C. - Hiver, Printemps. - Partout.

$2^{e}$ Fan. - Nectroidées. - Sacc.

Sect. I. - HYALOSPORÉES. - Sacc.

Sporules ovoïdes ou globuleuses, hyalines.

1er GENRE. - POLYSTHGMiNa. - Sacc.

17\%2. POLYST ${ }^{\text {na }}$. RUBRA. Sacc. - Alb. de l'Est.

- Sur feuilles vivantes de Prunus spinosa et Domestica. - Taches d'un beau rouge pourpre. - Sporules filiformes, bifurquées au sommet.

- AC. - Soulanges, St-Amand. - Automne.

- Etat thécasporé Polystigma rubrum. 
$3^{\triangleleft}$ FAM. -- Leptostromacées. - Sacc.

SEGT. I. - HYALOSPORÉES. - Sacc.

Sporules ovoïdes-globuleuses, hyalines.

1er GENRE. - LEPTOTHYRIUM. - Kunze.

1773. LEPT. SCORODONIE. Sacc. - Alb. de l'Est. - Sur tiges de Teucrium scorodonia, situé à la base. - Basides et sporules très visibles. - Récolté à Charmont dans les bois de Bâtis. en Automne. - AR.

17\%4. LEPT. PROTUBERANS. Sacc. - Alb. de l'Est. - Sur les stolons de Potentilla reptans. - Pustules elliptiques, noires luisantes, sporules courbes acuminées. - R. - Bois de St-Lumier. - Printemps.

1775. LEPT. LYCOPI. Ch. R. - Alb. de l'Est. Sur tiges mortes de Lycopus Europeus. - Périthèces noirs, aplatis, irréguliers Sporules semilunaires courbes. - AC. -- Fossés humides, bords du Fion. - Printemps.

1776. LEPT. BERBERIDIS. Ch. $R$. - Alb. de l'Est. - Sur feuilles mortes de Berberis Vulgaris, à la face inférieure. - Périthèces hémisphériques, puis affaissés au centre; noirâtres. R. - A Aulnay-l'Aître, en Automne. 
2e GENRE. - MELASMIA. - Lév.

17\%\%. Melasm. ACerina. Lév. - Alb. de l'kist.

- Sur feuilles d'Acer Pseudo-Platanus. - Taches noires, luisantes, arrondies, fendillées, laissant èchapper les sporules. - Spermogonie de Rhytisma acerinum. - C. dans les bois. - Fin de l'Été.

3e GENRE. - LEPTOS'Troma. - Fries.

17\%8. LEPT. CAPREæ. Lib. - Alb. de l'Est. Sur rameaux morts de Salix Caprea. - Périthèces aplatis, elliptiques, dont la partie supérieure se détache avant la maturité des sporules. - C. - Bois de St.Amand, Lisse, etc. Automne et Printemps.

1779. LEPT. SCIRPINUM. Fr. - Alb. de l'Est. Sur chaumes de Scirpus lacustris. - Périthèces scutiformes, elliptiques, noirs striés, sporules cylindriques. - AR. - Fossés humides. St-Lumier. - Automne.

1780. LEPT. NITIDUM. Wallr. - Alb. de l'Est. Sur feuilles mortes d'Iris Pseudo-acorus. Pèrithèces noirs, elliptiques rangés en ligne.AC. - Bois de St-Lumier. - Automne.

1781. LEPT. CARICINUM. Fr. - Alb. de l'Est.

- Sur feuilles de Carex riparia. - Périthèces noirs, épars; sporules cylindriques un peu cour- 
bes. - C'est la spermogonie de Lophodermium caricinum. - C. Lieux humides des bois. Partout.

1782. LEPT. PTERIDIS. Ehrenb. - Alb. de l'Est.

- Sur tiges de Pteris aquilina. - Périthéces irréguliers, allongés, disposés en lignes longitudinales. Sporules cylindriques courtes. - AC. - Bois de Sermaize. - Automne et Printemps.

1783. LEPT. SPIREA. Fr. - Alb. de l'Est. Sur tiges de Spirea Ulmaria. - Périthèces noirs, épars, de forme irréguliẻre, rarement fertiles. - C. - St-Amand, bords du Fion. Printemps.

178'. LEPT. JUNCACEARUM. Sacc. - Alb. de l'Est. - Sur chaumes de Juncus communis. Périthèces elliptiques, épars. Sporules portées par des basides arborescentes ressemblant à de petites clavaires. - AR. - Fossés humides entre Lisse et St-Amand. Hiver.

1785. LEPT. RUMICIS. Ch. $R$. - Alb. de l'Est.

- Sur tiges mortes de Rumex acetosa. - Périthéces petits, épars. - AR. - Jardins, StAmand. - Fin d'Automne.

$4^{\mathrm{e}}$ GENRE. - PIROSTOMA - Fries.

1786. PIROST. CIRCINANS. Fries. - Alb. de l'Est. - Sur chaumes d'Arundo phragmites. Périthèces très nombreux, groupés en cercle. Sporules incolores assez grandes, piriformes. CC. - Lieux humides. - Partout. - Au Printemps. - Très souvent stérile. 
5e GENRE. - DISCOSIA. - Lib.

178\%. Discosia ARTOCREAS. Fr. - Alb. de l'Est. - Variété Oxyacanthce. - Sur les feuilles de Cratcegus oxyacantha tombées á terre, après l'hiver. - Sporules courbes, munies de 3 cloisons et d'un cil, à chaque extrémité. - Var. - AR. - St-Amand. - Printemps.

1788. DISCOSIA ALNEA. Berk. - Alb. de l'Est.

- Sur la face supérieure des feuilles encore vertes d'Alnus glutinosa. - AC. - En automne, mais rarement fertile. - St- $A$ mand et Lisse.

1789. DISCOSIA CLYPEATA. de Not. - Alb. de l'Est. - Var Glandicola. - Sur glands de Chêne pourrissants. - Groupe peu nombreux, appréciable à la loupe seulement. - Périthèces ronds, noirs: déprimés au centre. Sporules semblables à celles de Disc. Artocreas. - R. - Bois de St-Amand. - Printemps.

6ª GENRE. - ENTOMOSPORIUM. - Lév.

1790. ENTOM. MESPILI. Sacc. - Alb. de l'Est. Sur les feuilles mourantes de Mespilus germanica. disséminé à la surface de taches vertes puis brunes. - Conidie jaune pâle, à 4 ou 5 lobes, disposés en croix, simulant la forme d'un insecte ; chaque lobe est muni de cils droits, incolores. - AC. - Automne à St-Amand. 
ie GENRE. - LEPTOSTROMELLA. - sacc.

1791. LEPT ${ }^{\text {Hla }}$. JUNCINA. Sacc. - Alb. de l'Est. - Croît au printemps à la base des chaumes morts de Juncus acutus et glaucus. - Sporules elliptiques, courbes. - En compagnie de Anthostomella tomicum. - C. - Été. - Lieux humides. - St-Amand, Lisse.

$4^{e}$ FAM. - Excipulacées. - Sacc.

\section{SEGT. - HYALOSPORÉES. - Sacc.}

Sporules globuleuses, ovö̈des, hyalines.

1er GENRE. - GODRoviELLA. - Karsten.

1792. GODRON. JUNCIGENA. - Karst. - Alb. de l'Est. - Sur chaume d'Arundo phragmites. - Périthèces épars, hémisphériques, aplatis, noirs, sillonnés de côtes moins saillantes que dans le type thécasporé Godronia dont il est la spermogonie. - Spermaties aculéiformes, courbes, pluriseplées. - RR. - Printemps.

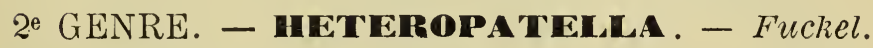

1793 HETER. LACERA. Fuckel. - Alb. de l'Est.

- Sur tiges de Linaria striata. - Périthèces 
sphériques, puis cupuliformes, sporules fusiformes, courbes, munies d'arêtes aiguës aux extrémités. - C. - Bois de St-Amand. - Printemps.

1794. Heter. ChAILletil. Karsten. - Alb. de l'Est. - Sur tiges de Daucus carota. - Périthèces noirs, striés, affaissés au centre ; sporules linéaires. Spermogonie d'Heterosphceria. - C. - Champs, bois. - Partout. - Automne.

\section{$3^{e}$ GENRE. - DoTUICHIZA. - Lib.}

1795. Doth. Populina. Sacc. - Alb. de l'Est.

- Commun sur les rameaux de peuplier. Tubercules noirs verdâtres, pluricellulaires, contenant des sporules rondes, incolores, portées sur des basides droites. - CC. - Automne.

- En temps humides. - Partout.

$4^{e}$ GENRE. - DISCELLA. - Sacc.

1796. DISC la. CARBONACEA. B. et Br. - Alb. de l'Est. - AC. - Sur rameaux de Salix caprea. - Périthéces ronds, aplatis, noirs, luisants, nucléus brun; sporules blanches, uniseptées, elliptiques. - Aulnay-l'Aître. - Automne.

5 e GENRE. - AMEROSPORIUM. - speg.

1797. AMER. CONGREGATUM. Sacc. - Alb. de l'Est. - Sur tiges d'Euphorbia sylvatica. Périthèces hémisphériques, noirs, puis en cupules, munis de quelques poils raides; sporules 
fusiformes, allongèes, portées sur de longues basides. - RR. - Forêt d'Epernay: - Été.

1798. AMER. CARICUM. Sacc. - Alb. de l'Est.

- Sur feuilles de Carex glauca. - Périthèces sphériques puis aplatis, couverts de poils noirs raides; sporules en fuseau, jaunes, puis brunes. - AR. - Printemps. - De St-Amand à Bassu.

6e GENRE. - DinEMASPORIUM. - Lev.

1\%99. DIN. GRAMINUM. Lév. - Alb. de l'Est. Sur tiges de graminées diverses. - Périthèces en cupules, épars, noirs, couverts de poils raides, à disque blanc, dilaté par l'humide ; sporules cylindriques: munies de deux gouttelettes et d'un long cil à chaque extrémité. - C. Automne, printemps. - Partout.

1800. DIN. STRIGOSUM. Sacc. - Alb. de l'Est.

- Sur bois pourri de Pins, vieilles palissades de jardins. - Périthéces noirs, à poils courts, nombreux, disque blanc. - CC. - Automne, printemps. - Partout.

1801. DIN. PURPURASCENS. Ch. $R$. - Alb. de l'Est. - Stir branches mortes de Saule. - De couleur violacée à l'état frais, couvert de poils noirs ; disque rose, très ouvert. Sporules plus courtes que dans les espèces précédentes. AR. - Bois el jardins à St-Amand. - Automne.

ye GENRE. - PILIDIUI. - Kunze.

1802. PILId. FULIGINosum. Auersw. - Alb. de l'Est. - Sur tiges de Salix alba. - Périthèces 
cupulitormes, nombreux, formant des taches arrondies, qui précèdent l'apparition du Rhytisma maximun sa forme thécasporée. - Sporules fusiformes, allongées, à 3 cloisons. - R. - Bords de la Marne. - Soulanges. - Automne.

8 GENRE. - PLEOSPOROPSIS. - Erst.

1803. Pleos. STROBilinA. Auersw. - Alb. de l'Est. - Situé entre les bractées des Cônes de Pinus abies. - Espèce a mbiguë suivant Saccardo Uredinée suivant Max. Cornu. - RR. dans la Marne. - Château d'Ablancourt. - Automne.

\section{MÉLANCONIÉES. - Berk.}

Champignons privés de Périthècẹs et de Thèques ; Conidies portées sur des basides variées, issues d'un substratum proligère.

\section{SEGT. I. - HYALOSPOREES. - Sacc.}

Conidies globuleuses, ovoüdes, ou cylindriques-courtes, hyalines.

\section{1er GENRE. - MAINESIA. - Fll.}

180'. HAIN. RUBI. Sacc. - Alb. de l'Est. - Sur feuilles de Rubus cresius. - Tubercules discoïdes, rouge-orangé, formés de filaments arbo- 
rescents, terminés par des sporules elliptiques courbes. - R. - Bois de St-Lumier. - Fin de l'été.

2e GENRE. - GLAEOSPORIUM. - Desm.

1805. GLæEOS. RIBIS. Mont. - Alb. de l'Est. -

Sur feuilles de Ribes rubrum languissantes. Conidies semilunaires, pédicellées. - AC. Jardins. - Automne. - St-Amand.

1806. GLAEOS. QUERCINUM. Harkn. - Alb. de l'Est. - Sur feuilles de Chêne encore vertes.

- Tubercules roses, épars; conidies incolores. ovales, pédicellées. - R. - Eté. - St-Amand. 180\%. GLæOS. AMPELOPHAGUM. Sacc. - Alb. de l'Est. - Anthracnose. - Sur rameaux, vrilles. feuilles et fruits de la Vigne. - Sa présence occasionne la mort des tissus. - Groupes de conidies. elliptiques, incolores: situées sur des taches brunes à centre blanc. - Jusqu'alors R. dans la Marne. - Fin de l'été. - Près de Reims.

1808. GLAES. GALLARUM. Ch. $R$. - Alb. de l'Est. - Sur la galle des feuilles de Chêne. Amas de conidies, sous l'épiderme noirci. Conidies cylindriques à 6 sporules. - R. - Bois de St.Amand à Aulnay. - Printemps.

1809. GLÆOS. PHASEOLI. - Ch. R. - Alb. de l'Est. - Sur gousses et tiges de Haricot. Amas de conidies, oblongues ovoïdes, pédicellées, recouvert par l'épiderme soulevé ; par- 
ties soulevées, brunes, munies de quelques poils. - AC. - Jardins à St-Amand. - Espéce nuisible.

3e GENRE. - MYXOSPORIUM. - Link.

1810. MyXos. ULMI. Sacc. - Alb. de l'Est. Sur rameaux d'Orme. -- Pustules rougeâtres. sous-cutanées. formées de conidies elliptiques, longuement pédicellées; l'épiderme se fend en lanière pour leur livrer passage. - AR. Automne. - St-Amand.

1811. MYXOS. DEPLANATUM. Sacc. - Alb. de l'Est. - Sur les branches mortes du Charme. - Tubercules verdâtres sous-cutanés. - Conidies incolores cylindriques un peu courbes, pédicellées. - R. - St-Amand. - Printemps.

4e GENRE. - MELANOSTRoma. - Corda.

1812. Melan. Minutum. Corda. - Alb. de l'Est. - Sur sarment de Vigne-Vierge. - Tubercules noires sous-cutanés à disque blanc. Conidies fusoïdes, pédicellées. - R. - St-Amand. — Jardins. - Printemps.

1813. MELAN. FUSARIOIDES. Corda. - Alb. de l'Est. - Sur rameaux de Syringa Vulgaris. Tubercules hémisphériques, aplatis au sommet, sous-cutanés, de substance noirâtre à disque blanc, à point noir central ; conidies incolores, cylindriques, très courtes, disposées en chapelet. - AC. au Printemps. - Jardins à St-Amand. 
5e GENRE. - CYLINDROSPORIUM. - Unger.

1814. CYLIND. RANUNCULI. Sacc. - Alb. de l'Est. - Sur les feuilles de Caltha Palustris encore vertes. - Amas de conidies, cylindriques 1-3 septées. - C. dans les bois au Printemps. St-Amand.

1815. CYLIND. MYOSOTIDIS. Sacc. - Alb. de l'Est. - Sur feuilles de symphytum officinale. - Groupe de conidies, cylindriques, très allongées, disposées en faisceau, de couleur blanche. - C. en Automne à Soulanges.

6e GENRE. - CRYP'TOSPORIUM. - Corda.

1816. CRYPT. NEESII. Corda. - Alb. de l'Est. - Sur branches d'Alnus glutinosa. - Pustules sous-cutanées, conidies fusoïdes, recourbées. AC. - Bois de St-Amand. - Hiver.

ie GENRE. - LHBERTELLA. - Desm.

1817. Libertella FAGineA. Desm. - Alb. de l'Est. - Sur écorce de Hêtre. - Tubercules rouges, entourés d'un duvet blanc, formé par des conidies filiformes. fusoïdes, recourbées, issues de filaments arborescents. - Conidies de Nectria gibbera (Fuckel). - C. - Bois. Partout. 
8 GENRE. - NAMMSPORA. - Pers.

1818. NAMASPORA MICROSPORA. Desm. - Alb. de l'Est. - Sur écorce de Hêtre. - Tubercules orangés. sous-cutanés; conidies cylındriques, arquées, longuement pédicellées. - C. - Bois à Aulnay, etc.

9e GENRE. - MeLANCONiUM. - Link.

1819. MELAN. HEDERE. Pr. F. - Alb. de l'Est. - Sur sarments d'Hedera helix. - Tubercules noirs, formés de conidies brunes, pédicellées, ovales, noircissant le support. - C. dans les bois au printemps.

1820. MELAN. JUGLANDINUM. Kunze. - Alb. de l'Est. - Sur branches et trones morts de Noyer. - Tubercules noirs, formés de conidies ovoïdes, brunes pédicellées. - CC. - Automne, Printemps. - Partout.

1821. MElan. RAMUlorum. Corda. - Alb. de l'Est. - Sur rameaux d'Alnus glutinosa et de Cxrpinus. - Tubercules noirs, saillants, formés ae conidies elliptiques brunes, pédicellées. CC. - Bois. - Automne, Printemps. - Partout.

182\%. MELAN. SPHAROSPERMUM . Link. Alb. de l'Est. - Sur chaumes d'Arundo phragmites. - Amas de Conidies brunes, sphériques. 
sans pédicelle apparent, noircissant le support. - CC. - Printemps. - Bords des eaux. Partout.

\section{GENRE. - THYRSIDIUM. - Mont.}

1823 THYRS. BOTRYOSPORUM. Mont. - Alb. de l'Est. - Sur écorce de Charme en compagnie de Melogramma Bulliardi. - Tubercules noir-mat formés par les faisceaux de filaments rameux du Thyrsidium, surmontés chacun par un glomérule arrondi de conidies, brunes sphé-riques. - R. - Chantiers de Vitry-le-François. - Automne.

11e GENRE. - DIDYMOSPORIUM. - Nees.

1824. DIDYM. CARPINI. Corda. - Alb. de l'Est. - sur rameaux de Carpinus. - Pustules noires sous-cutanées, déchirant l'épiderme, formées par des masses de conidies, brunes elliptiques allongées, uniseptées. - AC. - Haies, charmilles, jardins à St-Amand, etc. - Printemps.

12e GENRE. - MARSONIA. - Fisch.

182.5. MARSONIA JUGLANDIS. Sacc. - Alb. de l'Est. - Sur feuilles de Noyer. - Amas de sporules disséminées sur des taches brunes irrégulières composées de conidies incolores semi-lunaires. - AC. - Été, hiver. - Spermogonie de Gnomonia leptostyla.

1826. MARSONIA POTENTILLE. Fisch. - Alb. de l'Est. - Sur feuilles de Potentilla reptans. 
- Conidies sous-cutanées, allongées, biloculaires, lobe supérieur plus large, et recourbé. Spermogonie de stigmatea potentillarum. - R. - Près de Bassu. - Étè.

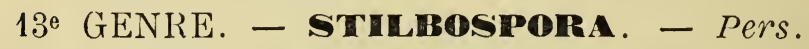

182\%. Stilbos. Angustata. Pers. - Alb. de l'Est. - Sur branches et rameaux d'Acer pseudoplatanus. - Amas de conidies sous-cutanées, oblongues, brunes, à 3 cloisons. Vient aussi sur Carpinus. - Etat conidien de Pseudo valsa macrospora. - C. dans les bois. - Automne. Printemps.

1928. STILBOS. MACROSPERMA. Pers. - Alb. de l'Est. - Sur branches mortes de Chêne. Pustules sous-cutanées, formées par des conidies oblongues, ellipsoïdes, brunes, à 3-4 cloisons longuement pédicellées. - AC. - Printemps. Dans les bois.

1829. STILBOS. CRATAGI. Ch. $R$. - Alb. de l'Est. - Sur branches mortes de Cratcogus oxyacantha. - Conidies oblongues ou piriformes, brun-verdâtre à 3 cloisons. - RR. -Près d'Ablancourt. - Printemps.

\section{GENRE. CORYNEUM. - Nees.}

1830. CORYNEUM UMBONATUM. Nées. - Alb. de l'Est. - Sur branches de Chêne. - Pustules noires, disciformes, placées sur un strome brun. - Conidies fusoïdes 5-8 cloisons, brunolive. - R. - Bois de Sermaize. - Printemps. 
1830 bis. CORYNEUM. KUNZEI. - Var. Castanæ. Sacc. - Sur rameaux de Châtaignier. - Accompagne le Melanconis modonia dont il est la forme conidienne. - Bois de la montagne de Reims. - RR.

1831. CORYNEUM NOTARISTANUM . Sacc. Alb. de l'Est. - Sur branches de Betulus alba; - Amas de conidies, formant des pustules souscutanées, discoïdes ; conidies fusiformes, à 5-6 cloisons, brunes étant mûres. - Etat conidien de Pseudo valsa lanciformis. - AC. - Bois de St-Lumier. - Printemps.

1832. CORYNEUM FUSARIOIDES. Sacc. - Alb. de l'Est. - Sur branches mortes de Rhamnus rrangula. - Conidies brunes, cylindriques, arquées, extrémité hyaline. - RR. - La Chausseee. - Printemps.

1833. CORYNEUM FOLIICOLUM. Fuck. - Alb. de l'Est. - Sur feuilles de Cratagrus oxyacantha. - Amas de conidies, oblongues, fusiformes, 8-10 cloisons de couleur brun-olive, recourbées, formant des taches superficielles. - R. - Bois de Vavray. - Automne.

15e GENRE. - SCOLECOSPORIUM. - Lib.

1834. SCOLECOSP. FAGI. Lib. - Alb. de l'Est. Sur branches mortes de Fagus sylvatica. Amas de conidies fusiformes très allongẻes, hyalines aux extrémités, brunes étant mûres, munies de 8 à 12 cloisons. - Conidies de 
Massaria macrosperma. - CC. - Hiver et printemps. - Partout.

16e GENRE. - ASTEROSPORIUM. - Kunz.

1835. ASTER. HOFFMANNI. Kunze. - Alb. de l'Est. - Commun sur les branches mortes de Hêtre. - Pustules sous-cutanées, formées par des conidies brunes, en forme d'étoile. à 4 rayons coniques, portées par des pédicelles hyalins cloisonnés. - CC. dans les bois au printemps. - Partout.

17e GENRE. - PESTA Lozzia. - De Not.

18:36. PEST. ROSE. West. - Alb. de l'Est. Sur tiges de Rosa Canina. - Conidies fusiformes, 3 cloisons, loge supérieure hyaline, terminée par 3 arêtes divergentes, pédicelle allongé. - CC. - Printemps. - Partout.

1837. PEST. CONIGENA. Lév. - Alb. de l'Est. - Sur bractées de cônes de Pinus abies et Picea. - Conidies fusoïdes, terminées par 4 ou 5 arêtes. - C. - Ablancourt, Soulanges, etc. - Hiver.

1838. PEST. FUNEREA. Desm. - Alb. de l'Est. - Sur teuilles de Thuya. - Conidies fusoïdes allongejes, munies de 4-5 cloisons et 3 ou 4 arêtes. - CC. - Jardins. - St-Amand. - Printemps.

1839. PEST. TRUNCATA. Lev. - Alb. de l'Est. - Sur tiges d'Iyppricum Perforatum. - Conidies brunes, 3 cloisons, loge supérieure hya- 
line à 2 arêtes bifurquées : avec l'âge, les loges extrêmes disparaissent et la conidie reste tronquée. - R. - Bois de St-Lumier. - Printemps. 18'0. PEST. HYPERICINA. Ces. - Alb. de l'Est.

- Sur tiges d'Hypericum Perforatum. - Sporidies cylindriques, courbes, triseptées, munies de 2 ou 3 arêtes, couleur ocre-jaune clair. - R. - Bois de St-Amand à St-Lumier. - Printemps. 18'1. PEST. MONOCHATA. Desm. - Alb. de l'Est. - Sur rameaux de Hêtre. - Conidies réunies en tubercules, brunes, munies de 4 à 5 cloisons, fusiformes allongées ; très long pédicelle ; loge supérieure hyaline, terminée par une seule arête. - AC. - Bois d'Aulnayl'Aître. - Printemps.

1812. Pest. POtentille. Ch. $R$. - Alb. de l'Est. - Sur tiges de Potentilla reptans desséchées à la surface du sol après l'Hiver. - Conidies fusoïdes, 3 cloisons; loge supérieure munie d'aigrette plus longue que la Conidie pédicellée. - RR. - Bois de St-Amand à St-Lumier. - Printemps.

18'3. PEST. PEZIzoIdes. De Not. - Alb. de l'Est. - Sur sarments morts de Vitis vinifera. - Amas de Conidies disciformes pézizoïdes , conidies fusoïdes, à 5 loges et 5 arêtes. AR. - Jardins à St-Amand. - Hiver.

184'. PEST. Liliorum. Ch. R. - Alb. de l'Est. - Sur tiges de Lilium candidum après l'hiver. Groupes de conidies à la base de la tige. - Station exceptionnelle non encore observée. 
18 GENRE. - SEPTOGLAEUM. - Sacc.

1845. SEPTOGL. ACERINUM. Sacc. - Alb.de l'Est.

- Sur les feuilles d'Acer Campestre et Pseudo Platanus. - Tubercules épars sur des taches grisâtres en forme de disque, couleur d'ambre. conidies en fuseau, unicloisonnées portées par de courtes basides. - R. - Bois de St-Amand. - Automne.

19" GENRE. - STEGANOSPORIUM. - Cord.

1846. STEGAN. PIRIFORME. Corda. - Alb. de l'Est. -- Sur Acer Pseudo-Platanus. - Amas de Conidies recouvert par l'épiderme; conidies pirjformes à 4 ou 5 cloisons transversales et 1 ou 2 longitudinales. - C. - Printemps. - Partout. 1847. STEGAN. COMPACTUM. Sacc. - Alb. de l'Est. - Sur jeunes rameaux d'Orme morts. Conidies brunes, oblongues, à 4 ou 6 cloisons transversales et 1 longitudinale, cellule médiane souvent renflée; pédicelle hyalin. - Conidies de Melanconis Berkeleyi. - AC. après l'hiver. - Bois à St-Amand.

1848. STEGAN. CELlulosum. Corda. - Alb. de l'Est - Sur rameaux morts de Tilia. Amas de conidies brunes, piriformes, à 5-7 cloisons murales. - Soulanges. - Printemps. 
20" GENRE. - PHRAgMotrichuM. - Kunze.

18'9. PhRAgM. ChaIlletiI. Kunze. - Alb. de l'Fist. - Sur bractées de cônes de Pinus abies. - Amas de conidies Rhomboïdes, murales, brunes, disposées en chapelet, séparées l'une de l'autre par 2 ou 3 cellules étroites hyalines.

- CC. en Automne et au Printemps. - Parc d'Ablancourt et jardins.

\section{HYPHOMYCÈTES. - Martius.}

Champignons privés de périthèces et de thèques, consistant en filaments plus ou moins développés et en conidies libres.

$1^{\text {re }}$ FAM. - Mucédinées. - Link.

Filaments pâles ou légèrement colorés, dressés puis s'affaissant, non réunis en fascicule; conidies incolores.

SECT. I. - AMÉROSPORÉES. - Sacc. 1er GENRE. - Chromospormum. - Corda.

1850. CHROMOSP. ROSEUM. Corda. - Alb. de l'Est. - Sur chapeau d'agaric en putréfaction. - Petits tubercules roses, formès de conidies 
elliptiques ou globuleux, ayant l'apparence d'un Ascobolus carneus. - AK. - Automne.

\section{2e GENRE. - MICROSTROMA. - Niessl.}

1851. Micr. Album. Sacc. - Alb. de l'Est. A la face inférieure des feuilles de chêne. Conidies ovales, oblongues, pédicellées, réunies en faisceau épars, hyalines. - RR. - Automne. - Aulnay-l'Aître.

3e GENRE. - MOSPORA. - Wallr.

1852. OOSP. MICROCARPA. Schulz. - Alb. de l'Est. - Sur aiguilles de Pin sylvestre, pourrissantes. - Filaments courts, simples, terminés par des conidies rondes-ovales, hyalines, disposées en chapelet. - CC. - Automne. Hiver doux. - Partout.

1853. OOSP. EPIMYCES. Sacc. - Alb. de l'Est. Sur Périthèces de Lasiosphceria crinita. - Flocons blancs de filaments, composés de conidies elliptiques disposées en chapelet. - RR. Automne. - St-Amand.

1854. OOSP. AEQUVOCA. Sacc. - Alb. de l'Est. - Sur racines coupées de Frêne. - Flocons de filaments blancs formés par de longs chapelets de conidies elliptiques apiculées. - R. Bois de St-Amand à St-Lumier. - Automne.

1855. OOSP. CRUSTACEA. Sacc. - Alb. de l'Est.

- Flocons denses crustacés, passant du blanc au rouge minium, formés de filaments terminés 
par des conidies cuboïdes-globuleuses en chapelet. - Sur les vieux fromages, dans les caves. $-\mathrm{CC}$.

1856. OOSP. VIRESCENS. Wallr. - Alb. de l'Est - Sur feuilles de Peuplier et de Saule, pourrissant sur terre. - Flocons denses de filaments verdâtres, allongés tortueux, composés de conidies ovoïdes-oblongues, vertes, en chapelet. - CC. - Automne. - Bois de St-Amand.

185\%. OOSP. SULPHUREA. Sacc. - Alb. de l'Est.

- Sur le pain moisi. - Flocons arachnoïdes couleur de soufre, composés de filaments rampants terminés par des conidies ovales, jaunes, en chapelet. - ('C. - Lieux humides. Partout.

1858. OOSP. SPHARELLE. Ch. R. - Alb. de l'Est. - Sur Périthèces de Nectria Dahlice. (Ch. R.) dont il est l'appareil conidien. Touffes blanches de filaments courts, terminés par des conidies ovoïdes d'abord en chapelet. puis éparses, hyalines. - RR. - Jardins à StAmand. - Automne.

1859. OOSP. CORII. Ch. R. - Alb. de l'Est. Sur lanières de cuir abandonnées dans les lieux humides. - Touffes épaisses et larges de filaments tortueux, rameux, composés en grande partie, par des conidies cuboïdes, en chapelet rouge-clair. - R. - Printemps. - St-Amand . 1860. OOSP. UREDINIS? Sacc. - AJb. de l'Est.

- Sur la face inférieure des feuilles de Rubus.

- Touffes de filaments simples, jaunes-hyalins 


\section{$-414-$}

composés de conidies couries, cuboïdes. - AR.

- Automne. - St-Amand.

4e GENRE. - FUSIDHUM. - Link,

1861. FUSIDIUM PARASITICUM. - Alb. de l'Est.

- Appareil conidien de Xylaria hypoxylon. Conidies blanches, fusiformes, portées sur des basides courtes, le sommet des Xylaria envahis est comprimé, digité et blanc pulvérulent. - CC. - Sur les bois pourris. - Automne, Printemps. - Partout.

$5^{e}$ - GENRE. - Monilia. - Pers.

1862. MON. FRUCTIGENA. Pers. - Alb. del'Est.

- Sur les fruits pourrissants, de poires, de pêches, cerises, etc. - Flocons compacts, blancs, jaunâtres: 'composés de filaments courts en faisceau, terminés par de longs chapelets de conidies ovoïdes, concolores. - CC. - Automne, Hiver. - Partout.

1863. MON. SITOPHILA. Sacc. - Alb. de l'Est.

- Sur mie de pain corrompue, ressemble beaucoup à Oospora Sulphurea, dont il se distingue par sa couleur rose-orangée. - AC. - Lieux humides. - Partout.

6e GENRE. - CYLINDrium. - Bon.

186'. CYLIND. GRISEUM. Bon. - Alb. de l'Est. - Sur feuilles de Chêne humides et pourris- 
santes. - Formant des taches grises, étendues, composées de conidies cylindriques, hyalines. - CC. - Automne. - Partout.

1865. CYLIND. FLAVo-VIRENS. Bon. - Alb. de l'Est. - Taches planes, étendues vert-jaunâtre. composées de conidies cylindriques-fusoïdes, jaunes, hyalines. - Sur feuilles pourrissantes de Chêne et de Hêtre. - CC. - Automne. Partout.

\%e GENRE. - OIDIUM. - Link.

1866. OIDIUM ERYSIPHOIDES. $F r$. - Alb. de l'Est. - Sur les feuilles vivantes d'Humulus, de Papaver Somniferum, etc. - Filaments stériles, formant un tissu arachnoïde, et filaments fertiles dressés, courts, terminés par des conidies ovales, en chapelet 4-8 conidies. - CC. - Jardins. - Automne et Été. - Partout.

186\%. OIDIUM TUCKERI. Berk. - Alb. de l'Est.

- Sur feuilles et fruits vivants de la Vigne. Filaments arachnoïdes, blancs, très nombreux. Filaments fertiles courts, terminés par 2 ou 3 conidies ovoïdes en chapelet. - Parasite très nuisible. - UC. - Fin de l'Été. - Partout.

1868. OIDIUM MONILIOIDES. Link. - Alb. de l'Est. - Sur la face supérieure des feuilles de graminées Hordeum murinum, Triticum repens, etc. - Taches velues d'un blanc-rosé, composées de filaments la plupart fertiles, terminées par des conidies ovoïdes, incolores, en chapelet dressé. - C. - Fin de l’Été. - Partout. 
1869. OIDIUM LEGUMINOSARUM. Ch. $R$, - Alb. de l'Est. - Au sommet des tiges et sur les graines de Sainfoin. - Filaments arachnoïdes et filaments fertiles plus courts, terminés par 2 ou 3 conidies ovales, ponctuées. - La présence de cet Ö̈dium n'influence en rien la germination et la qualité de la graine et cependant en empêche la vente. - Récolté à Reims par le $\mathrm{D}^{\mathrm{r}}$ Thomas et communiqué par le $\mathrm{D}^{\mathrm{r}}$ Jolicœur. - Fin de Juillet.

8e GENRE. - AEDOCEPHALUM. - Preuss.

1870. AEDOCEPH. AGARICINUM. Ch. $R$. - Alb. de l'Est. - Sur chape $u$ d'Agaric. - Groupe fasciculé de filaments hyalins, terminés par un capitule jaune-d'ocre, formé par des conidies ovales. jaunâtres, en chapelet, insérées au sommet du filament un peu renflé et garni de petites verrues. - AR. - Sermaize. - Automne.

$9^{e}$ GENRE. - RMOPALOMYCES. - $B$, et $B r$.

1871. RHOP. CANDIDUS. B.-Br. - Alb. de l'Est. - Sur le crottin de cheval. - Filaments sim. ples, dressés. continus, terminés par un renflement sphérique. aréolé : sur lequel sont insérées les conidies piriformes hyalines formant un capitule sphérique. - AR. - St-Amand. Printemps. 
10 GENRE. - hapLotrichun. - Link.

1872. HAPL. CAPITATUM. Link. - Alb. de l'Est.

- Sur écorce de Pin sylvestre humide. - Groupe de filaments blancs, dressés, fasciculés, septès, renflés au sommet, conidies ovoïdes, insérées sur de petites verrues et formant un capitule blanc sphérique. qui jaunit en vieillissant. AC. - Automne. - St-Amand.

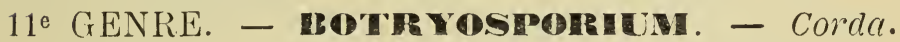

1873. BOtRy. PULCHRUM. Cord. - Alb. de l'Est. - Sur tiges de Dahlia pourrissantes dans les lieux humides. - Touffe de filaments blancs, dressés, simples ou dichotomes. Sporophores insérés en forme d'épi, le long des filaments, terminés par de petites verrues sur lesquelles sont insérées les conidies ovoüdes agglomérées en capitule d'un beau blanc. - AC. - Automne. Hiver. - St-Amand.

12e GENRE. - COEMANSILLLA. - Sacc.

18\% $\%$. Commans. Alabastrina. Sacc. - Alb. de l'Est. - Sur le crottin de cheval. - Filaments simples, dressés terminés par un capitule sphérique d'abord, puis ouvert, à disque central fructifére, composé de 10 à 15 segments radiès: dont l'extrémitè bifide forme une couronne comine les sépales d'une marguerite. Conidies 
ovoïdes insérées sur de petits mamelons basilaires. - AR. -- Automne. - St-Amand.

$13^{e}$ GENRE. - CEPIALOSPORIUM. - Cord.

1875. CEPHAL. ACREMONIUM. Corda. - Alb. de l'Est. - Sur tiges sèches d'Heracleum. Filaments couchés, rameux, émettant des filaments, courts, dressés, aigus, terminés par des capitules sphériques de conidies hyalines, ovales, très nombreuses. - AR. - Automne. - StAmand.

1876. CEPHAL. HERACLEI. Ch. $R$. - Alb. de l'Est. - Sur tiges mortes d'Heracleum spondylum. - Filaments rampants lâches; filaments dressés assez longs, portant au sommet un capitule sphérique de conidies ircolores. - Trés voisin de Cephal. Macrosporum. - AC.

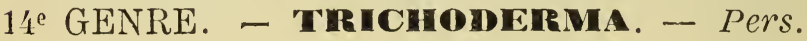

18\%. TRICH. LIGNORUM. Karz. - Alb. de l'Est. - Sur les trones d'arbres exposés à l'humidité. - Petites touffes arrondies de filaments blancs. à l'extérieur, vert-foncé au centre, rameux et septés, les filaments blancs sont stériles: les verts sont fertiles et porteurs de conidies, petites, ovales et vertes à leur maturité. Conidies de IIyprocrea rufa. - CC. -- Automne. Partout. 
$1 \mathrm{o}^{\mathrm{e}}$ GENRE. - ASPERGILICS. - Mirh.

18\%. ASPERGILLUS GLAUCUS. Link. - Alb. de l'Est. - Très commun sur les végétaux et les substances putrescibles - - Filaments stériles rameux, les fertiles dressés simples renflés en vésicules sphériques au sommet. Conidies portées par des sterigmates cylindriques, et disposées en chapelet couleur vert-glauque. Conidies d'Eurotium herbariorum. - CC. - Partout. 1879. ASPERGILLUS CANDIDUS. Link. - All). de l'Est. - Sur les plantes sèches et les champignons. - Caractères semblables á ceux de l'espèce précédente à part l'absence de couleur verdâtre. - CC. - Partout.

16 GENRE. - MHBLIOSPORMU⿴囗十 - Fries.

1880. AMBL. ALBUm. Ch. R. - Alb. de l'Est. - Sur le chapeau d'un Polypore apode, depuis quelque temps à la cave, - Petites touffes blanches composées de filamenis dichotomes, terminés chacun par de nombreuses basides. disposées en ombelles. Chaque baside émet une conidie ovale, qui se détache à la maturité. - RR. Autornne. - St-Amand.

1\% GenRE. - PEMLCLLLUM. - Link.

1881. PENICIL. GLAUCUM. Link. - Alb. de l'Est. - Très commun sur les végétaux et les substances organiques. - Groupes de filaments 
les uns stériles, rampants. les autres fertiles, dressés, divisés au sommet en deux rameaux qui se subdivisent en de nombreux ramuscules. réunis en faisceau. et terminés chacun par un chapelet de conidies ovales, vert-glauque. CC. - Partout.

1882. PENICIL. CANDIDUM. Link. - Alb. de l'Est. - Sur les feuilles, les bulbes des plantes et les champignons. - Caractères de la prècédente espéce, s'en distingue par l'absence de couleur. - CC. - Partout.

18 GENRE. - MAPLARIA. - Link.

1883. HAPL. GRISEA. Link. - Alb. de l'Est. Sur tige de plantes herbacées, et sur fruits en décomposition. - Touffe de filaments dressés. cloisonnés, bifurqués au sommet, grisâtres, garnis dans leur trajet de glomérules sphériques de conidies ovales incolores. - CC. - Automne, Printemps. - Partout.

19e GENRE. - CYLINDROTH CHUM. - Bon.

1884. CYLIND. OLIGOSPERMUM. Bon. - Alb. de l'Est. - Sur les écailles de bois blanc humides. - Taches velues, grisâtres, formées par des fila. ments dressés, rigides, noirâtres, septés. - Les conidies de cette espèce sont incolores, cylindriques, munies de 4 cloisons et 5 sporules; les jeunes filaments sont termınés par des conidies en capitule blanc. - AC. en Automne. - StAmand et St-Lumier. 
20e GENRE. - ACREMONIUM. - Link.

1885. ACREM. Alternatum. Link. - Alb. de

l'Est. - Commun sur les écailles et racines humides et mises en tas. - Filaments arachnoïdes, rampants, munis de rameaux alternes, simples, terminés chacun par une conidie ovale incolore. - CC. - Partout.

1886. ACREM. VERTICILlAtUM. Link. - Alb. de l'Est. - Même station et même caractère que l'espéce précédente; en diffère seulement par ses rameaux disposés en verticilles. - CC. Partout. - Automne.

21 GENRE. - RHINOTRICHUN. - Corda.

188\%. RHIN. SIMPLEX. Cord. - Alb. de l'Est. Sur écailles de Pin sylvestre. - Taches noires. veloutées, composées de filaments rampants, émettant des rameaux dressés, noirs, septés, garnis dans leur longueur de conidies brunes, disposées en verticille. - R. - St-Amand, Soulanges. - Automne.

22. GENRE. - SPOROTRICHUM. - Link.

1888. SPOROTR. DENSUM. Link. - Alb. de l'Est.

- Sur le thorax des coléoptéres. - Touffes blanches, de filaments sinueux, très denses parsemès de conidies sphériques incolores. - AC. - Fin de l’Été. - St-Amand. 
1889. SPOROTR. SCOTOPHILUM. Ehrenb. - Alb. de l'Est. - Sur les crottes de belettes. Touffes de filaments rampants, rameux, les fertiles dressés, simples, entremêlés de conidies rondes-ovales, couleur minium. - AR. - StAmand. - Automne.

\section{3e GENRE. - BO'TRYTTIS. - Mich.}

1890. BOTRYTIS CARNEA. Schum. - Alb. de l'Est. - Sur feuilles de Chêne et de Hêtre tombées. - Groupe de filaments couleur de chair. dressés, dicholomes, rameux, conidies sphériques, roses, verruqueuses, disséminées à l'extrémité un peu renflée des filaments. - AR. dans les forêts. - Sermaize et Charmont. - Automne.

1891. BOTRYTIS DICHOTOMA. Corda. -- Alb. de l'Est. -- Sur écorce de bois humide et sur feuilles pourrissantes. - Touffe jaunâtre, velue, composée de filaments entremêlès, dichotomes, hyalins, parsemés de conidies sphériques, verruqueuses, de couleur jaune-d'ocre. - C. dans les bois. - Partout. - Automie.

1892. BOTRYTIS VIRELLA. Fr. - Alb. de l'Est.

- Sur branches de Saule mortes enfouies sous la mousse. - Touffe de filaments dressés. arborescents, à rameaux verticillés, terminés chacun par une conidie ovale, variant du jaunecitron au vert-pâle. - AR. - Bois de Pins. - St-Amand. - Automne.

1893. BOTRYTIS VULGARIS. $F r$. - Alb. de l'Esl. - Très commun sur les tiges herbacées lan- 
guissantes. - Touffe blanchâtre composée de filaments, bruns á la base, septés, incolores au sommet, qui se divise en plusieurs rameaux terminés chacun par un glomérule sphérique de conidies incolores, ovales. - CC. - Automne et Printemps. - Partout.

189'. BOTRYTIS CINEREA. Pers. - Alb. de l'Est. - Parasite sur Sclerotium durum. - Touffe grisâtre composée de filaments bruns, septés, dressés, dichotome supérieurement, à rameaux terminés par des conidies disposées en capitule; conidies ovales, incolores. - CC. - Partout. - Automne et Printemps.

1895. BOTRYTIS GEMELLA. Sacc. -- Alb. de l'Est. - Sur écorce pourrie de Vigne. - Touffe laineuse, blanche. formée par des filaments dressés, dichotomes, chaque rameau est terminé par un renflement verruqueux, sur lequel s'insẻrent les conidies ovales, réunies en capitule. R. - Récolté à St-Lumier. - Au Printemps.

1896. BOTRYTIS EPIGEA. Link. - Alb. de l'Est.

- Sur la terre argileuse des forêts. - Touffe blanche, tomenteuse, passant au gris en vieillissant; filaments très denses, entremêlès; les fertiles dressés, courts portant au sommet des conidies petites, sphériques, incolores. - C. en Automne. - Dans les bois humides.

2'te GENRE. - OVCLARIA. - Sacc.

1897. OVULARIA LAMII. Sacc. - Alb. de l'Est.

- Sur feuilles languissantes de Lamium amplexi- 
caule. - Groupes épars, composés de faisceaux de filaments courts, terminés par des conidies ovales-fusiformes. - Croît aussi sur Lampsana vulgaris. - C. - Automne. - Partout.

1898. OVULARIA OBLIQUA. Oud. - Alb. de l'Est. - Sur feuilles vivantes de Rumex crispus. - Petits faisceaux blancs insérés sur des taches brunes de la feuille composées de filaments simples, continus, terminés chacun par une conidie ovale piriforme, très développèe. Conidie de spherella rumicis. - AC. - Été. - St-Amand, etc.

1899. OVUlAriA Vitis. Ch. $R$. - Alb. de l'Est. - Sur feuilles de Vigne desséchées. - Taches blanches, assez semblables à celles du Mildiou, dont elles diffèrent par des conidies fusiformes, á courtes basides. - Le Mildiou appartient à un autre genre. - AC. - Automne. - StAmand.

$25 \mathrm{e}$ GENRE. - SEPEDONICM. - Link.

1900. SEPED. CHRYSOSPERMUM. Fr. - Alb. de l'Est. - Sur chapeau des Bolets. - Taches jaune-d'or, velues et trés étendues, formées par les filaments hyalins, septés dressés, dichotomes, terminés chacun par une conidie sphérique. verruqueuse d'un jaune-doré. - CC. Automne. - Jans les bois. - Partout. Conidie d'Hypomyces chrysospermus.

1901. SEPED. CORDE: Sacc. - Alb. de l'Est. Parasite sur le disqne de Peziza macropus.- 
Conidies à 2 lobes sphériques, l'inférieur plus petit; verruqueuses hyalines rosées, passant au jaune. - AC. - Automne. - Dans les bois.

1902. SEPED. BYSSICOLUM. Sacc. - Alb. de l'Est. - Appareil conidien de Rosellinia aquila. - Composé de filaments bruns, bifurqués, dichotomes à rameaux terminaux plus pâles, terminés chacun par des conidies ovales, incolores. C. en Automne. - Sur les vieux bois pourris. - Partout.

26 GENRE. - ASTEROPHORA. - D tm.

1903. Aster. AgAricicola. Corda. - Alb. de l'Est. - Appareil conidien de Nyctalis asterophora. - Prend naissance sous l'épiderme du chapeau. - Il est composé de filaments rameux, incolores, terminés par des conidies tuberculeuses en forme d'étoile, légérement jaunâtres. - AC. en Automne. - Forêts.

$2 \%$ GENRE. - VERTHCHLLUM. - Nees.

1904. VERT. TERRESTRE. Sacc. - Alb. de l'Est. - Sur la terre et sur les écailles de Noyer humides, tournées vers le sol. - Taches blanches, formées par des touffes de filaments, les uns fertiles à rameaux verticillés, munis de conidies incolores sphériques. les autres stériles. très longs, de couleur brun-clair. - AC. Automne.

1905. Vert. AgAricinum. Corda. - Alb. de l'Est. - Commun sur les chapeaux et les 
lamelles des Agaricinées. - Filaments blancs. rameux, à rameaux et ramuscules verticillés; conidies incolores, ovales-oblongues, hyalines. - CC. - Automne. - Partout.

28e GENRE. - AROCYLINDRIUM. - Bon.

1906. ACROCYL. CORDA. Sacc. - Alb. de l'Est. C. sur les Agaricınées. - Composé de filaments dressés, à rameaux simples, verticillés, terminés chacun par une conidie cylindrique.hyaline.

- Automne. - Forêts.

29e GENRE. - ACROSTALAGMUS. - Corda.

190\%. ACROST. CINNABARINUS. Cord. - Alb. de l'Est. - Commun sur les tiges pourries des plantes herbacées, et sur les pommes de terre malades. -- Large plaque velue, rouge brique composée de filaments à rameaux et à ramuscules verticillés, terminés chacun par un globule sphérique de conıdie ovale-rougeâtre agglutinée. - CC. - Automne. - Partout.

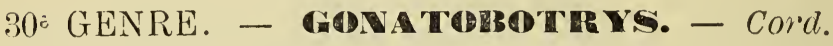

1908. GONAT. SIMPLEX. Corda. - Alb. de l'Est. - Sur grain de Maïs. - Filaments dressés. incolores, septés, fasciculés, munis de distance en distance de renflements globuleux et verruqueux, sur lesquels sont disposées en spirale. les masses de conidies hyalines, ovales. $-A R$. - Automne.

1909. GONAT. FLAVA. Bonord. - Alb. de l'Est. 
- C. sur les vieux trones humides de Noyer

- Filaments rameux, munis dans leur longueur de renflements verruqueux, sur lesquels s'insèrent des glomérules arrondis de conidies ovales jaune-d'ocre. - CC. - Automne, Printemps. Partout.

\section{1e GENRE. - SPICARIA. - Harts.}

i910. SPICAR. VERTICILlATA. Sacc. - Alb. de l'Est. - Sur les tubercules de Solanum tuberosum et sur les tiges pourries de Dahlia mis á la cave pendant l'hiver. - AC. - Cette espéce me paraît être une variété du Verticillium candidum.

\section{SEct. II. - DIDYMOSPORÉES. - Sacc.}

\section{GENRE. - DHPLOCLAIDIUM. - Bon.}

1911. DIPLO. MAJUS. Bon. - Alb. de l'Est. Sur les chapeaux et les lamelles des agaricinées. - Cette espèce est l'état jeune du Dactylum dendrö̈des, les conidies sont uniseptées et deviennent pluriseptées à la maturité. - CC. - Partout.

1912. DIPLO. MiNUS. Bon. - Alb. de l'Est. Sur les tubes du Polyporus versicolor. - Appareil conidien d'Hypomyces aurantius. - Filaments dressés. à rameaux verticillés, terminés 
chacun par une conidie ovale, septée comme celle de Trichothecium roseum. - AR. Automne. - St-Amand.

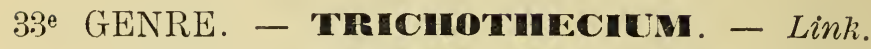

1913. TRICHOT. ROSEUM. Link. - Alb. de l'Est.

- C. sur rameaux de Frêne et de Peuplier.

- Touffe de filaments roses, fasciculés, continus, terminés chacun par une ou deux conidies piriformes, uniseptées, apiculées inférieurement. CC. - Automne, Printemps. - Partout.

1914. TRICHOT. DOMESTICUM. Fr. - Alb. de l'Est. - AC. sur les bois et substances putrescibles, dans l'intérieur des habitations; ressemble à l'espéce précédente. - Conidies de couleur blanche. - CC. - Printemps, Automne. Partout.

34 GENRE. CEPHALO'THECHUM. - Corda.

1915. CePhal. CAPITATUM. Corda. - Alb. de l'Est. - Sur tige de Papaver somniferum en compagnie ủu Brachycladium, sur lequel il croît en parasite. - Filaments rampants stériles. Filaments dressés terminés au sommet par de petites verrues sur lesquelles s'insėrent en forme de capitule, des conidies ovales, uniseptées, incolores. - RR. - Jardins. - St-Amand. Printemps.

1916. CEPHAL. TETRASPERMUM. Ch. $R .-$ Alb. de l'Est. - Sur détritus de balles d'orge en décomposition. - Taches blanches, formées par 
la masse des filaments dressés du Cephalothecium. Chaque filament est simple à 1 ou 2 cloisons, terminé au sommet par 3 ou 4 conidies incolores, ovales uniseptées. - RR. - - St-Lumieren-Champagne. - Automne.

\section{$3 \check{e}^{e}$ - GENRE. - DIDYMOPSIS. - Sacc.}

191\%. DIDYM. SPICATA. Ch. R. Lebr. - Alb. de l'Est. - Sur le disque de la Pezize en ciboire - Duvet blanchâtre, formé par des filaments dressés, rameux munis de conidies allongées, cunèiformes, hyalines uniseptées, disposèes le long du filament, en verticille et en forme d'épis.

- RR. - Communiqué par M. André Lebreton.

- En Été.

36" GENRE. - MYCOGONE. - Ditus.

1918. MYCOG. ROSEA. Link. - Alb. de l'Est. Sur les chapeaux et lamelles des Agaricinées en putréfaction. - Filaments incolores. Conidies obovales, roses, uniseptées. - AC. en Automne dans les bois de Pins.

1919. MICOG. PEZIZA. Sacc. - Alb. de l'Est. Sur le disque de Peziza juniperi. - Espéce se rapprochant beaucoup de Didymaria helvellae (de Corda). Elle en diffère par la disposition de ses rameaux, qui sont tous terminés par 2 conidies allongées. cunéiformes, didymes. -RR. - Aulnay-l'Aître, - Automne. 
3\% GENRE. - HORMIAC'TIS. - Preuss.

1920. HORM. ALBA. Succ. - Alb. de l'Est. Sur tiges mortes d'Orties. - Touffes blanches. velues, composées de longs chapelets de conidies cylindriques uniseptées, insérés sur de courts pédicelles. - RR. - St-Amand. Printemps.

\section{SEGT. III. - PRAGMOSPORÉES. - Sacc.}

$38^{e}$ GENRE. - DACTYLUM. - Nées.

1921. DACTYL. DENDROIDES. Fries. - Alb. de l'Est. - Sur lames et chapeaux d'Agaricinées en décomposition. - Filaments stériles rampants, très nombreux ; filaments dressés, rameux. subverticillès; conidies oblongues, tri-septées, hyalines apiculées à la base. - CC. - Automne. - Forêts.

39e GENRE. - RAMULARIA. - Unger.

1922. RAMULARIA AUGUSTISSIMA. Sacc. Alb. de l'Est. - A la face inférieure des feuilles de Cornus sanguinea. - Petits groupes épars sur des taches brunes composées de filaments rameux munis dans leur longueur et au sommet de conidies étroites fusiformes, incolores. - AR. - Automne. - St-Amand. 
19:3. RAMULARIA SAMBUCINA. Sace. - Alb. de l'Est. - Sur feuilles de Sambucus nigra. - Faisceaux épars de conidies cylindriques, uniseptées : incolores; insérées sur des taches blanchâtres. - AC. - Fin d’été. - St-Amand. 1924. RAMULARIA URTICA. Ces. - Alb. de l'Est. - C. sur les taches noires qui envahissent la face inférieure des feuilles d'Ortie en Automne. - Petits groupes de conidies elliptiques, allongées ; portées sur de courts filaments disposés en faisceau. - Partout.

1925. RAMULARIA GIBBA. Fuck. - Alb. de l'Est. - A la face inférieure des feuilles de Ranunculus repens. - Petits groupes blanc de neige sur une tache jaunâtre, boursouflés, Conidies fusiformes, droites, à 3 gouttelettes, hyalines. - AR. - Été.

1926. RAMULARIA PARIETARIE. Pass. - Alb. de l'Est. - A la face supérieure des feuilles de Parietaria officinalis. - Touffes blanches. composées de filaments fasciculés, porteurs de Conidies cylindriques, la plupart uniseptées. AC. - St-Amand. - Automre.

192\%. RAMULARIA ULMARIZE. Cooke. - Alb. de l'Est. - Sur feuilles de spirea ulmaria. - Taches blanches, composées de conidies cylindriques uniseptées, disposées en chapelets fasciculés. - C. - Automne. - Bords des eaux.

1928. RAMULARIA GERANII. West. Fuck. - Alb. de l'Est. - Sur les feuilles de Geranium molle, à la face inférieure. - Taches brunes, coll- 
vertes de groupes épars de conidies blanches, fusoïdes, fasciculées. - C. - Printemps. - StAmand.

1929. RAMULARIA VARIABILIS. Fuck. - Alb. de l'Est. - A la face inférieure des feuilles du Verbascum thapsus. - Groupes blanchâtres sur des taches brunes, composées de filaments fasciculés trés courts. porteurs de conidies de formes variées, ovales, elliptiques ou cylindriques. - AC. - Printemps. - St-Amand.

19:30. RAMULARIA PLANTIGINEA. Sacc. - Alb. de l'Est. - A la face inférieure des feuilles de Plantago lanceolata. - Groupes épars sur des taches ochracées, composés de filaments simples. continus, porteurs de conidies cylindriques, droites à 1-3 cloisuns. - AC. - Automne. - Dans les champs.

1931. RAMULARIA VIOLA. Fuck. - Alb. de l'Est. - Sur les feuilles de riola canina et odorata. - Groupes épars sur des taches orbiculaires. composés de filaments très courts, fasciculés, porteurs de conidies cylindriques, obtuses. AC. - En Automne à St-Amand.

$19: 31$ bis. RAMULARIA SYMPHYTI. Sacc. - Alb. de l'Est. - Sur feuilles languissantes de Consoude. - Prés, bords de la Marne, - Soulanges. Automne. - AC. - Très longues conidies.

1932. RAMULARIA. LAMII. Fuck. - Alb. de l'Est. - Sur feuilles de Lamium amplexicaule. - Groupes épars, sur des taches orbiculaires. composés de filaments trés courts, simples, por- 
teurs de conidies elliptiques, continues. - AC. - Automne. - Jardins. - Partout.

40 GENRE. - SEPTOCYLINDRIUM. - Sacc.

1933. SEPTOCYL. VIRESCENS. Sacc. - Alb. de l'Est. - Sur feuilles d'Ombellifères. - Taches velues, assez étendues, vert.glauque; apparence de Cladosporium herbarum, composées de filaments courts, septés, rameux; porteurs de conidies cylindriques, pluriseptées, disposėes en chapelet. - R. - Printemps. - Bois de StLumier.

41e GENRE. - HELYCOMYCES. - Link.

1934. HELICOM. ROSEUS. Link. - Alb. de l'Est. - Sur les Pericarpes pourris de l'Esculus hyppocestanum. - Petites taches blanches, rosées formées par la réunion de conidies contournées en spirales, munies de 4 à 6 cloisons, incolores, portées par des filaments courts, septés, terminés par 1 ou 2 pointes sur lesquelles s'insèrent les conidies. - AR. - Automne. - StAmand. 
2 e FAM. - Dematriées. - Fries.

Filaments bruns ou noirs, rigides, non réunis en fascicule, rarement hyalins, dans ce cas, porteurs de Conidies noires.

\section{SEGT. I. - AMÉROSPORĖES. - Sacc.}

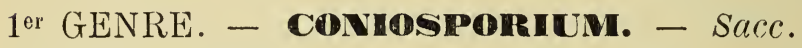

1935. CONIOSP. SOCIUM. Sacc. - Alb. de l'Est. -- Sur la face inférieure des feuilles de Malva sylvestris. - Taches noires intenses, formées par l'agglomération de nombreuses conidies. brunes, ovoïdes, apiculées inférieurement. - R. - Automne. St-Amand.

1936. CONIOSP. RHIZOPHILUM. Sacc. - Alb. de l'Est. - Sur Rhizomes de Triticum repens. - Aspect d'un Torula herbarum. - Groupes très noirs vus en masses, composées de nombreuses conidies, rondes-ovales, de couleur bruneolive sous le microscope. - AR. - Printemps. - St-Amand.

193\%. CONIOSP. CAULINCOLUM. Ch. R. - Alb. de l'Est. - Sur tiges mortes et dénudées de Reseda luteola. - Taches noires, formèes par des masses de conidies, brunes, en forme d'amande. Sous un fort grossissement, on constate 
une couche muqueuse émettant de courts pedicelles, terminés par de jeunes conidies incolores. - RR. - Chemin de St-Amand à Lisse. Hiver.

$2 \sharp$ GENRE. - TORULA. - Pers.

1938. TORULA ANTENNATA. Pers. - Alb. de l'Est. - Sur Prunus cerasus, Vitis vinifera, et Rosa canina. - Taches brunes, plus ou moins larges, velues, composées de chapelets de conidies brunes. elliptiques, allongées, portées sur de courts pédicelles. - AC. - En Automne, Printemps. - Partout.

1939. TORULA HERBARUM. Link. - Alb. de l'Est. - Très C. sur les tiges herbacées. formant des taches noir-olive, velues; composées de conidies globuleuses, 4-6 portées par de courts predicelles. - CC. - Partout.

1940. TORULA COMPNIACENSIS. Ch. R. - Alb. de l'Est. - Inscrit pour mémoire. - Envoi de M. Petit. - Récolté sur les murs des fabriques d'eau-de-vie à Cognac. - Taches noires, composées de conidies, brunes, sphériques, tuberculeuses, 10-20 en chapelets portées par un court pédicelle. - Automne, hiver. - Espéce nouvelle $C h$. $R$.

19'1. TORULA GRAMINIS. Desm. - Allb. de l'Est. - Sur les Rhizomes des graminées déracinėes. - Taches noires, brunes, formées par des chapelets de conidies globuleuses portėes par de courts pédicelles. - CC. - Hiver. - Partout. 
1942. TORULA. CORII. Ch. R. - Alb. de l'Est. - Plaques rougeâtres, étendues, sur lanières de cuir exposées à l'humıdité, composées de chapelets allongés, rameux, de conidies cuboïdes rougeatres, portées par de courts pédicelles. AR. - Printemps. - St-Amand.

1913. TORUla. PUNCTAta. Corda. - Alb. de l'Est. - Sur tiges herbacées, surtout de Malva sylvestris. - Taches noirâtres, ètendues, veloutées, formées par des chapelets très longs de conidies brunes sphériques, tuberculeuses, ponctuées, portées par de courts pédicelles. - AC. - Automne. - St-Amand.

19'1'. TORULA. TUBERCULARIA FORMIS. $C h . R$. - Alb. de l'Est. - Tubercules noirs, discoïdes á surface blanche pulvérulente, épars, sur le bois de Saule pourri, ayant servi de rames dans les jardins. Composés de chapelets de conidies, rondes-cuboïdes, réunies en faisceaux denses portées par des pédicelles de même longueur que les chapelets, septés. - RR. - Automne. ... St-Amand. - Cette espéce pourrait former un genre nouveau de la famille des Tuberculariées.

\section{$3^{e}$ GENRE. - MormischuM. - Kunze.}

1945. HORMiSC. ALTUM. Ehrenb. - Alb. de l'Est. - Sur rameaux d'Alnus Glutinosa. - Groupes de filaments noirs, luisants, placés dans les fissures de l'épiderme, formés par la réunion des 
longs chapelets de conidies cuboïdes, brunes, persistantes. - AC. - Automne, printemps. St-Amand.

1946. HORMISC. VERMICULARE. Sacc. - Alb. de l'Est. - Sur écorce de Châtaignier. Larges plaques d'un noir intense; formées par la réunion des nombreux filaments monilitormes, bruns ; composées de conidies cuboïdes, et réunies en faisceaux de 2 à 5 chapelets. - RR. - Bois de Reims. - Fin de l'été.

194\%. HORMISC. HYSTERIOIDES. Sacc. - Alb. de l'Est. - Sur branches mortes de Rosa canina. - Groupes linéaires, parallảles, incrustrés dans l'écorce ; composés de chapelets jaunâtres. de conidies cuboïdes, transparentes. - RR. -- StAmand. - Automme.

1948. HORMISC. STILBOSPORUM. Sacc. - Alb. de l'Est. - Sur écailles de bois de chêne. Taches velues, très noires, irréguliẻres, formées par des conidies brunes, à 4 cloisons, placées bout-à-bout irrégulièrement; disposées en faisceaux tortueux. - R. - St-Amand. - Printemps.

1949. HORMISC. PITHYOPHILUM. Sacc. - Alb. de l'Est. - Sur jeunes rameaux de Pinus Picea qu'il entoure d'une croûte noire velue, formée par des chapelets de conidies brunes. cuboïdes de longueur très variée. - AC. - Jardins. Partout.

1950. HORMISC. CEREVISCIE. Fuck. - Alb. de l'Est. - A là surface de la biére et des 
liquides acides en fermentation. - Plaques mesentériformes composées de filaments, les uns continus, et les autres composés d'articles ou conidies hyalines sub-ovoïdes disposées en chapelet. - AC. dans les caves et les officines.

\section{GENRE. - G YRochans. - Corda.}

1951. GYROCER. PLANTAGINIS. Sacc. - Alb. de l'Est. - A la face inférieure des feuilles vertes de Plantago media. - Trés longs chapelets de conidies brunes cuboïdes, disposés en faisceaux, contournés, tortueux. - R. - Bords des chemins, entre St-Amand et Bassu. Automne.

$5^{e}$ GENRE. - ECHIMOHB TRYUM. - Corda.

1952. ECHIN. ATRUM. Corla. - Alb. de l'Est.

- Sur bois pourri dénudé, envahi par Campsotrichum, podospermum. - Parasite sur les filaments de cette espèce. — Conidies obovales, brunes, munies d'aspérités saillantes plus claires réunies en glomérules de 5. - AR. - - StAmand. - Automne.

19.ว3. ECHIN. PARASITANS. Cord. - Alb. de l'Est. - Parasite sur les stipes de Slysanus stemonitis. - Glomérules de conidies 8-10. piriformes. jaunâtres, maculées de brun, à col incolore. - AC. - Automne. - Printemps - StA mand. 
6e GENRE. - STACHYBOTRYS. - cord.

195'. STACHYBOTRYS ALTERNANS. Bon. Alb. de l'Est. - Sur les tiges de liliacées et sur le papier humide. - Taches noirâtres, formées par des filaments bruns, lisses rameux, terminés par une couronne de $5-6$ conidies hyalines, sur laquelle s'insèrent des masses de conidies brunes, formant une tête globuleuse noire. Les rameaux de cette espéce sont alternes. - R. - Printemps. - à St-Amand.

1955. STACHYBOTRYS ATRA. Corda. - Alb. de l'Est. - Sur écailles de bois humide et sur tige de Lys. - Groupe de filaments, simples. rugueux. brunâtres, assez courts, terminés par une couronne de 6 conidies hyalines, donnant naissance à des sporidies brunes, elliptiques, uniseptées, disposées en globules noirs, spériques. AC. - Jardins. - Printemps. - St-Amand.

1956. STACHYBOTRYS LOBULATA. Berk. Alb. de l'Est. - Sur tiges d'Arundo phragmites humides. - Ressemble beaucoup à Stachyobotrys alternans, mais en diffère par ses conidies sphériques, échinulées - - AR. Automne. - Aulnay-l'Aître.

Ye GENRE. - PERICONIA. - Bon.

195\%. PERICONIA PYCNOSPORA. Fres. - A!b. de l'Est. - Groupe de petits filaments bruns. septès, simples, dressés, surmontés chacun d'un 
glomérule sphérique composé de conidies brunes; rondes, échinulées. - AC. sur Urtica dioica. Printemps, Automne. - Partout.

1958. PERICONIA ATRA. Corda. -- Alb. de l'Est. - Taches brunes, velues, sur écailles de bois pourrissant, formés de filaments bruns, septés, dressés, terminés par un glomérule sphérique de conidies brunes, rondes. chagrinées. - CC. - Printemps, Automne. - Partout.

1959. PERICONIA FUSCA. Corda. - Alb. de l'Est. - Sur les feuilles putrides des plantes aquatiques. - Caractères semblables à ceux des espèces précédentes mais en diffère par les conidies plus petites, ovales et brunes. - AC. - Bords des ruisseaux. - St-Lumier, Soulanges. Automne.

1960. PERICONIA ELLIPSOSPORA. Sacc. - Alb. de l'Est. - Sur les pédoncules du Pceonia officinalis. - Taches noires, velues, assez étendues, composées de filaments dressés, bruns, septés, surmontés d'un capitule sphérique de conidies vert-olive, puis brunes, voilées et lisses; on remarque à la base du capitule plusieurs conidies sporigéres. - CC. - St-Amand. Printemps.

8e GENRE. - CAMP'TOUM. - Link.

1961. CAMPt. Curvatum. Link. - Alb. de l'Est. - Sur feuilles languissantes de I'oa aquatica. - Petits groupes velus elliptiques, épars, composés de filaments sep̣tés, noduleux, hyalins. 
simples, le long desquels s'insèrent des conidies brunes, courbées, réniformes. - AC. - Bords des eaux. - Printemps.

9e GENRE. - ARTHRINIUM. - Kunze.

1962. ARTHR. CARICICOLUM. Kunze. - Alb. de l'Est. - Sur les fevilles mortes du Carex crespitosa. - Ressemble par ses caractères au Camptoum curvatum, en diftère seulement par la forme de ses conidies elliptiques atténuées aux extrémités. - CC. - Printemps, Automne.

10 GENRE. - GOMROSPORIUM. - Link.

1963. GONIOS. PUCCINIOIDES. Link. - Alb. de l'Est. - C'roît au Printemps sur les feuilles mortes des Poa et des Carex. - Il diffère des deux espéces précédentes par la forme de ses conidies, qui est cuboïde anguleuse. - C. au Printemps dans les lieux humides à St-Amand. etc.

11 GENRE. - STEPTOTRIX. - Cord.

1964. STEPT. FUSCA. Cord. - Alb. de l'Est. Sur les tiges mortes de Pteris aquilina. - Petites touftes velues, brun-verdâtre, composées de filaments brun-clair café au lait, continus noduleux, tordus, le long desquels s'insèrent de petites conidies ovoïdes brun-clair. - RR. - Sermaize. - Automne. 
120 GENRE. - THICHOSPOARIM. -- Fries.

1965. TRICH. FUSCUM. Sacc. - Alb. de l'Est. Sur l'écorce des bois pourris amassés en tas sur la terre humide. - Filaments bruns.rameux entrecroisés, septés, parsemés de conidies ovoïdes, brunes. - Cette espèce précède l'apparition du Rosellinia aquila dont il est l'appareil conidien. - CC. - Partout.

1966. TRICH. ATERRINUM. Sacc. - Alb. de l'Est. - Sur troncs et racines déterrées du Peuplier et du Noyer. - Larges plaques d'un noir intense, composées de filaments simples, entremêlés, très noirs, parsemés de petites conidies elliptiques, brunes et très nombreuses. C. en Automne, Printemps. - St-Amand.

196\%. TRICH. CHARTACEUM. Sacc. - Alb. de l'Est. - Sur papier abandonné dans les lieux humides. - Taches noires, irréguliẻres, composées de filaments, rameux, incolores, et anastomosés, parsemés de conidies ovoïdes, noires, et très petites. - C. -- Automne. - Partout.

13e GENRE. - MONo'TOSPORA. - Corda.

1968. MONOT. SPHAROCEPHALA. $B$. et $B r$. Alb. de l'Est. - Sur la tranche d'une vieille souche pourrie. - Taches noires, formées par les groupes de Monotospora qui examinés à la loupe ressemblent á une forêt de petites épingles à têtes noires; chaque espèce est composée d'un 
filament brun, septé, aminci au sommet et terminé par une conidie noire, Juisante, sphérique et très grosse. - RR. - Automne. - St-Amand.

1969. MONOT. CUNEIFORMIS. (Rich.) Sacc. Alb. de l'Est. - Sur écailles pourries de Pin sylvestre. - Taches velues. placées dans les sinus creusés par les insectes; composée de filaments fasciculés, bruns, simples, septés et terminés au sommet par une conidie unique, brune, cunéiforme; les cloisons des filaments sont quelquetois garnies d'une gaine, résultant d'une spore avortée. - RR. - Automne. Soulanges.

1970. MONOT. OVATA. Sacc. - Alb. de l'Est. (iroupes de conidies ovales ou ovoïdes, noires. luisantes portées par de courts filaments courbes. - Sur bois de Frêne. -- St-Amand. - Automne. - R.

14 GENRE. - HADOTRICHUM. - Fuckel.

1971. HADOTR. PHRAGMITIS. Fuckel. - Alb. de l'Est. - Voir Henderzonia arundinacea.

15e GENRE. - Ca TENULARIA. - Grov.

1972. CATEN. ATrA. Sacc. - Alb. de l'Est Sur écailles de Frêne. - Taches noires, larges, velues, composées de filaments dressés, trés nombreux, septés simples, parsemés d'une masse de conidies très petites, ovoïdes noirâtres. AR. - Printemps. - St-Amand. 
$16^{e}$ GENRE. - HA PLOGRA PHIUM. - $B r$.

1973. HAPLOG. PENICILLATUM. Sacc. et $C h$. $R$. - Alb. de l'Est. - Sur les fruits d'Alnus glutinosa. - Groupes velus, formés par des filaments simples, noirs, dressés. terminés au soinmet par une couronne de rameaux courts, 4-6. surmontés chacun par un très long chapelet flexueux, de conidies cylindriques courtes et hyalines. - RRR. - Entre St-Lumier et StAmand. - Au Printemps.

1974. HAPLOG. TORULOIDES. Sacc. - Alb. de l'Est. - Sur les tiges des plantes herbacées. Groupes noirâtres, formés par des filaments simples, septés, bruns, terminés au sommet par des chapelets de conidies globuleuses, noiresolivacées, assez courts, formant une tête arrondie. - CC. - Automne. - Partout.

$1 \%$ GENRE. - DEMATIUM. - Pers.

1975. DEMAT. HISPIDULUM. Fries. - Alb. de l'Est. - Sur feuilles de Glyceria aquatica. Groupes épars de petites touffes noires-velues, composés de filaments fasciculés. noirs, terminés en pointe, munis à la base de conidies sphériques, brunes, de différentes grosseurs. Lieux humides. - En Automne. - Partout.

18 GENRE. - HORMODENDRUM. - Bon.

19\%6. HORM. OLIVACEUM. Bon. - Alb. de l'Est.

- Sur feuilles de Chêne et sur tiges d'Om- 
bellifëres. - Groupes de filaments velus, vertfoncé ou olive, simples d'abord, et septés, puis rameux au sommet, les rameaux sont terminés par des chapelets de conidies verdâtres, ovoïdes ou petites. - AR. - Bois de St-Lumier. Automne,

19e GENRE. - SARCOPODIUM. - Ehren.

197\%. SARC. FUSCUM. Sacc. - Alb. de l'Est. Sur ramilles de bois tombé à terre, et à la base des tiges des grandes herbes desséchées. - Groupe de filaments velus de couleur ocracée ou brune, composé de filaments trés longs, septés, tortueux, concolores, au milieu desquels gisent des masses de conidies cylindriques, incolores. - AC. - Bois de St-Amand, St-Lumier. - Automne.

1978. SARC. ROSEUM. Fries. - Alb. de l'Est. - Sur tiges pourries des plantes herbacées. Groupes elliptiques, velus, roses, formés de longs filaments tortueux, concolores, qui entourent un globule de conidies elliptiques, allongées et très nombreuses. - AC. - Automne, Printemps. - Partout.

20e GENRE. - CIRCINOTRICHUM. -- Nées.

1979. CIRC. MACULIFORME. Nées. - Alb. de l'Est. - Sur écorce de Hêtre. - Taches noires, éparses, velues, composées de filaments, dressés, noirs, septés. trés rameux au sommet, à 
rameaux plus pâles, dichotomes et recourbés; conidies fusoïdes, incolores, insérées à la base des filaments. - C. - St-Amand. - Printemps,

21e GENRE. - MYXotRICHUM. - Kunze.

1980. MYX. CHARTARUM. Kunze. - Alb. de l'Est. - Sur papier pourrissant. - Taches noirâtres, composées de filaments noirs, enchevêtrés, très rameux ; les rameaux dichotomes plus pâles sont terminés par des conidies sphériques incolores, disposées en glomérules. - Etat conidien de Chatomium chartarum. - AC. Lieux humides. - Automne.

2̧e GENRE. - CHAETOPSHS. - Grév.

1981. CHATOPS. GRISEA. Sacc. - Alb. de l'Est. - Sur le bois pourri, notamment du Corylus avellana. - Groupes velus, de couleur grise due au mélange des filaments noirs et des conidies blanches, filaments dressés, très longs, terminés en pointe, entourés à la base de rameaux incolores, porteurs de conidies cylindriques, hyalines. - C. - Automne. - Partout.

23e GENRE. - MENISPORA. - Pers.

1982. MenisP. GlauCA. Pers. - Alb. de l'Est. - Sur le bois de Chêne pourri. - Ressemble à l'espèce précédente, par ses filaments noirs, terminés en pointe, et par la coloration grise due aux conidies blanches; ces conidies de Menispora sont groupées en capitule sphérique 
au sommet des rameaux d'autres filaments plus courts situés à la base des premiers; conidies incolores, cylindriques, arquées. - C. - Automne. - Partout.

1983. MENisP. Ciliata. Corda. - Alb. de l'Est. -- Sur bois de Chêne pourri. - Taches grises, velues, composées de filaments bruns, septés. rameux, munis dans leur longueur de conidies arquées, blanches, terminés à chaque extrémité par un long cil. - C. - Lieux humides. Partout. - Automne et Printemps.

24' GENRE. - GONYTRICHUM. - Nées.

1984. GON. CÆSIUM. Nées. - Alb. de l'Est. Sur bois pourri humide, dans lieux sombres.Taches velues, grisâtres, composées de filaments, rameux à angle droit, anastomosés, garnis à l'entrecroisement des filaments de courts ramuscules, terminés chacun par un globule de conidies ovoïdes, incolores. - CC. - Lieux humides. - Partout.

$25^{\circ}$ GENRE. - FUCKELINA. - sacc.

1985. FUCK. SOCIA. Sacc. - Alb. de l'Est. Sur écailles de Pin sylvestre. - Filaments dressés, simples, septés, noirs, amincis au sommet, terminés par 6-10 stérigmates, émettant chacun une conidie ovale. incolore. - Etat conidien de Leptospora caudata, Fuck. - AR. - Automne. - St-Amand. 


\section{6" GENRE. - STACHYLIDHOM. - Link.}

1986. STACHYL. BICOLOR. Link. - Alb. de l'Est.

- Commun sur les tiges pourrissantes de Dahlia.

- Filaments dressés, bruns à la base. incolores au sommet, munis dans leur longueur de rameaux simples, verticillés, portant chacun un glomérule de conidies ovales oblongues, hyalines. C. en Automne et Printemps. - St-Amand.

198\%. STACHYL. SAMBUCI. Ch. $R$. - Alh. de l'Est. - Sur branches dénudées du Sambucus nigra. - Filaments dressés, bruns à la base, incolores supérieurement, munis de rameaux disposés en verticilles, chaque ramuscule terminé en pointe porte un glomérule de conidies incolores. ovales. - C. en Automne et au Printemps, à St-Amand.

\section{2\% GENRE. - CHALARA. - Corda.}

1988. ChAlAR. STROBILINA. Sacc. - Alb. de l'Est. - Sur les bractées de cônes de Pinus abies. - Taches grises, formées de filaments courts. renflés en forme de bouteille, bruns, terminés par un trés long chapelet de conidies cylindriques, courtes, munies de 2 sporuies. Appareil conidien d'Helotium strobilinum. - AR. - Ablancourt. - Automne. 
SECT. II. - DIDYMOSPORÉES. - Sacc.

28 GENRE. - DICOCCUM. - Corda.

1989. DICOCCUM UNISEPTATUM. Sacc. - Alb.

de l'Est. - Sur tiges pourrissantes de Clematis Vitalba. - Conidies ovoïdes, brunes, uniseptées inférieurement, munies d'une grosse gouttelette, insérée sur des basides courtes, en mamelon.

- Accompagne le stysanus clematidis. - AC.

- St-Amand. - Printemps.

1990. DICOCCUM SENECIONIS. Ch. R. - Alb. de l'Est. - Sur tiges de Senecio vulgaris. Groupes de conidies elliptiques, munies d'une cloison et resserrées au milieu. de couleur brune.

- AR. - St-Lumier. - Printemps.

\section{9" GENRE. - HBSPORA. - Corda.}

1991. BISP. MONILIOIDES. Corda. - Alb. de l'Est. - Sur écailles de bois de Chêne. Taches noires, formées par de longs chapelets de conidies brunes, fusiformes. obscurément septées. disposées en fascicules. - R. - Bois de Sermaize. - Automne.

30 GENRE. - PASSALORA. - Fr. et Mont.

1992. PASS. BACCILligerA. Mont et Fries. Alb. de l'Est. - Sur feuilles d'Alnus glutinosa. 
envahies à la face inférieure, par des groupes de filaments bruns-grisâtres, fasciculés, septés, toruleux. émettant à leur. sommet de grandes conidies incolores, uniseptées; lobe supérieur renflé fusiforme, l'inférieur cylindrique. $-R$. - Bois de St-Lumier. - Automne.

31e GENRE. - FCSICLADIUM. - Bon.

1993. FUSICL. DENTRITICUM. Fuck. - Alb. de l'Est. - Sur feuilles de Pommier encore vertes. - Taches noires, velues. formées par des filaments bruns, rameux, rampants ou dressés, et alors terminés par des conidies variées, brunes, munies d'une à trois cloisons. - AC. Automne. - Jardins.

1994. FUSICL. PIRINUM. Fuck. - Alb. de l'Est. - A la face inférieure des feuilles de Poirier. - Taches brunes, irrégulières, formées par des conidies brunes-verdâtres, portées par de courts filaments de 1 à 2 cellules. - C. en Automne. - Jardins. - Partout.

1995. FUSICL. DEPRESSUM. Sacc. - Alb. de l'Est. - A la face inférieure des feuilles vivantes d'Angelica sylvestris, à la fin de l'Été. Petits groupes noirs épars, composés de fascicules denses de filaments simples, continus, bruns, entremêlés de conidies de même longueur, uniseptées, renflées supérieurement, amincies à la base, de couleur jaunâtre, ou nankin clair. - C. de St-Amand à St-Lumier. - Automne. 
32 GENRE. - SCOLECOTRICHUM. - Kunze.

1996. SCOLECOT. CLAVARIARUM. Sacc. -- Alb. de l'Est. - Parasite sur Clavaria Cristata, dont il rend le tronc et les rameaux velus-noirâtres. - Composé de filaments dressés. bruns, peu rameux, cloisonriés terminés chacun par une conidie brune, uniseptée, elliptigue, allongée. AC. - Automne. - Bois de Sermaize.

1997. SCOLECOT. VENOSUM. Bon. - Alb. de l'Est. - A la face supérieure des feuilles de Pommier. - Taches grises, rondes, formées par des faisceaux de filaments courts, portant des conidies ovales allongées, uniseptées, brunes, les faisceaux sont disposés en lignes rayonnantes du centre vers la circonférence. - AC. - Automne. - Jardins.

33e GENRE. - POLYTHRHNCIUM. - kunze.

1998. POLYTHR. TRIFOLII. Kunze. - Alb. de l'Est. - C. sur feuilles de Trifolium repens. - Il forme à la face inférieure de petits groupes velus, noirâtres, composés de faisceaux de filaments moniliformes, gris-verdâtres, terminés par une conidie hyaline, ovale, uniseptée. -AC. - Automne. - Partout.

34e GENRE. - CLA DOSPORIUM. - Link.

1999. ClAdOSP. HERBARUM. Link. - Alb. de l'Est. - C. en Automne et au Printemps sur 
les grandes herbes languissantes, et sur le bois humide. - Taches vert-foncé, passant au brun; composées de filaments dressés, rameux. septés, vert-clair , terminés par des conidies de formes diverses, oblongues, ovoïdes, ou cylindriques, 1-3 cloisons. - Partout.

2000. CLADOSP. AMPHITRICUM. Sacc. - Alb. de l'Est. - Sur bois dénudè de Pin sylvestre. - Taches noirâtres ou olives, formées par de petits tubercules, donnant naissance à des fila. ments bruns, dressés, simples, sur lesquels s'insèrent des conidies brunes, ovales-oblongues, continues ou uniseptées. - AR. - Automne. - Soulanges.

:001. CLADOSP. EPIPHYLLUM. Mart. -- Alb. de l'Est. - Groupes velus, olive-noirâtre, disséminés à la surface des feuilles humides; composés de filaments fasciculés septés, concolores, tortueux, terminés par des conidies, ellipsoïdes, verdâtres, unipluri-septées. - CC. - Partout.

200\%. CladosP. TYPHARUM. Desm. - Alb. de l'Est. - Sur Typha et Scirpus lacustris. Groupes épars de filarnents dressés, fasciculés, verdâtres, septés, terminés chacun par une spore elliptique-allongée, concolore, 1-3 cloisons. C. - Bords des eaux. - Partout.

2003. ClAdosP. PILICOLA. Ch. $R$. - Alb. de l'Est. - Sur tiges sèches de Galium mollugo dont les poils sont envahis à leur sommet, par des faisceaux de filaments bruns, rameux, porteurs de conıdies cylindriques, 1-3 septées, con- 
colores; le Cladosporium parasite, donne à chaque poil, l'apparence d'un stysanus ou d'un Coremium. - RR. - Soulanges. - Printemps.

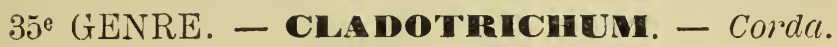

2004. CLADOT. SCYPHOPHORUM. Corda. - Alb. de l'Est. - Sur écorce de Bouleau et de Syringa vulgaris. - Groupes bruns, noirâtres, formés par une masse de filaments bruns, dressés. rameux, septés, noduleux-renflés, ou en forme de coupe. terminés par des conidies elliptiques uniseptées, resserrées à la cloison, brunes, souvent disposées en chapelet. - AC. - St-Amand, Soulanges. - Automne.

2005. CLADOT. POLYSPORUM. Corla. - Alb. de l'Est. - Sur rameaux morts d'Alnus glutinosa. - Taches noires velues, composées de filaments dressés, rameux, fertiles, terminés par des conidies elliptiques, brunes, uniseptées et resserrées à la cloison. mucronées aux deux extrémités. - AC. - Automne. - Soulanges.

36 GENRE. -- DIPLOCOCCIUM. - Grev.

2006. DIPLOCOC. PULCHRUM. Sacc. et $C h$. $R$. - Alb, de l'Est. - Sur le bois pourri. Groupes épars de flocons noirâtres, composés de filaments arborescents, ressemblant à une fougére en miniature; les rameaux principaux sont garnis de ramuscules formés par des chapelets de conidies brunes, elliptiques, didymes.

- AR. - St-Amand, Lisse. - Automne. 
SEGT. III. - PHRAGMOSPOREEES. - Sacc.

3\% GENRE. - Clastertesporhum. - schu.

200\%. CLASTER. SARCOPODIOIDES. Sacc. Alb. de, l'Est. - Sur les siliques de Lunaria annua. - Taches grises, formées par les groupes épars de conidies, disposées en fascicules, dressés. renflés vers le bas, allongés en pointes, munis de 10 à 20 cloisons, de couleur brun-clair; basides très courtes. - AC. - Automne. Jardins.

:008. ClASTER. CURVATUM. Sacc. - Alb. de l'Est. - Sur les feuilles vertes de Cratagus oxyacantha. - Taches noires formées par des fascicules de conidies cylindriques-fusiformes, brunes, trés longues, munies de 10 à 20 cloisons, recourbées. - R. - Bois de Vavray-lePetit. - Automne.

2009. ClASTER. SPARSUM. Sacc. - Alb. de l'Est. - Sur rameaux de Syringa vulgaris dans les fentes de l'épiderme. - Taches velues, noires, composées de conidies, brunes, claviformes, allongées, munies de 7 à 10 cloisons. - AR. - Printemps. - Jardins à St-Amand. 2010. CLASTER. FASCICULARE. Sacc. - Alb. de l'Est. - Sur bois de Peuplier pourri. Taches noires, composées des filaments d' Helminthosporium truncatum, à la base desquels 
croissent les conidies de Clasterosporium, piriformes 4-5 septées, réunies en faisceaux. AC. - Automne. - St-Lumier et St-Amand.

2011. CLASTER. CONGESTUM. Sacc. - Alb. de

l'Est. - Sur bois pourri d'Alnus glutinosa. Taches noires: étendues, formées par des filaments dressés, simples, bruns 2-4 septés porteurs de longues conidies, brunes, cylindriques 5-8 septées. - AC. - Automne. - St-Amand. 2012. ClAster. ABRUPtUM. Sacc. - Alb. de l'Est. - Sur bois pourri d'Alnus glutinosa. Tache brune, formée par les masses de conidies, cylindriques, longues, munies de 5 à 7 cloisons, et de gouttelettes, brunes, portees sur de courts pédicelles. - AC. - Automne. St-Amand et St-Lumier.

2013. CLASTER. CLAV EFORME. Sacc. - Alb. de l'Est. - Sur bois pourri tombé á terre. Taches grises. formées par les masses de conidies, dressées, fusiformes brun-clair, munies de 6-7 cloisons, terminées en pointe par un pédicelle plus clair, à 2-3 cloisons. - AC. Automne, Printemps. - Partout.

2014. CLASLER. EREMITA. Sacc. - Alb. de l'Est.

- Sur brindilles de Chêne en compagnie de Menispora oligosperma. - Conidies très longues, cylindriques, fusiformes, peu colorées, munies de 10 à 20 cloisons, an Clost. vagum? - AR. - St-Amand. - Automne.

2015. CLASTER. OVOIDEUM - Sacc. - Alb. de l'Est. - Sur tiges de Clematis vilalba. - 
Groupes épars, de conidies distinctes, dressées, ovoïdes, brunes, à 2 cloisons, portées sur un court pédicelle, peu coloré. - AR. - Printemps. - St.Amand.

2016. ClASTER. FUSIFORME. Sacc. - Alb. de l'Est. - Sur la tranche horizontale de troncs de Peuplier et d'Aune. - Conidies brunes, ou noires, opaques, fusitormes, munies de 5 à 7 cloisons et portées par des pédicelles de même longueur, cylindriques, septées. - C. - Automne. - Partout.

2017. Claster. CASTANEA. Ch. R. - Alb. de l'Est. - Cette espèce dont les caractères se rapprochent de cenx des Torulacées a été décrite plus haut, sous le nom de Hormiscium vermiculare.

2018. ClASTER. LIGUSTRI. $C h . R$. - Alb. de l'Est. - Sur rameaux de Ligustrum vulgare. - Groupes grisâtres, peu visibles, formés par réunion de nombreuses conidies brunes-claires, cylindriques fusiformes, munies de 7 cloisons, dressées et portées par un très court pédicelle. RR. - St-Amand. - Printemps. - Dans les haies. 2019. CASTER. TRIPARTITUM. Ch. $R$. - Alb. de l'Est. - A la base des tiges mortes de Genista scoparia. - Taches noires, velues, formées par les conidies fasciculées au nombre de 3 , brunpâle : fusoïdes, amincies au sommet, plus ren1lées à la base, munies de 10-15 cloisons, insérées sur un IIyphasma rampant anastomosé. - R. - Sermaize. - Automne. 
38 GENRE. - SEPTONEMA - Corda.

2020. SEPT ${ }^{\mathrm{ma}}$. MULTIPLEX. Sacc. - Alb. de l'Est.

- Nous avons décrit cette espéce sous le nom de Hormiscium stilbosporum; les deux descriptions de Saccardo lui conviennent éga!ement et laissent indécise sa place dans la classification.

39e GENRE. - HELMHNTHOSPOHIUN. - Link.

2021. Helmin. APPENDiCUlatum. Corda. Alb. de l'Est. - C. sur les rameaux d'Alnus glutinosa, d'Acer, etc. - Plaques velues, noires, composées de filaments dressés bruns, septés, terminés en pointes, munis dans leur longueur et au sommet de conidies en massues, courbes. brunes, à base plus pâle amincie, 6-7 cloisons. - CC. - Automne, Printemps. - Partout.

2022. HELMiN. LEUCOSPERMUM. Corda. - Alb. de l'Est. - Sur bois de Saule humide et pourrissant. - Taches noires, velues, formées de filaments fasciculés dressés, bruns, septés, incolores au sommet, et terminés par une conidie fusoïde, bitriseptée, hyaline. - AR. - St-Amand au Printemps.

2023. Helmin. Attenuatum. Feck. - Alb. de l'Est. - Sur les vieux troncs pourris. - Taches noires-velues, composées de conidies droites. multiseptées, la plupart tusiformes ou lancéolées, brunẹs-foncées, excepté la dernière cellule qui est hyaline. Portées par un pédicelle noirâtre. 
- Voisin de Clast. fusiforme. - CC. - Printemps. - Partout.

202'. Helmin. Velatum. Corda. - Alb. de l'Est.

- Sur écailles de bois pourri. - Taches noires, composées de filaments bruns, septés. enveloppés de la base au sommet d'un mucus transparent trés visible, terminés chacun par une conidie fusoïde 5-7 septées-brunes. - AC. -Hiver, Printemps. - Partout.

2025. HELMIN. RHOPALOIDES. Fres. - Alb. de l'Est. - Sur tiges humides et mortes de Buis, daus les jardins. - Taches noirâtres entourant la tige, composées de conidies fusiformes très allongées, brunes. plus pâles au sommet. portées par des pédicelles concolores, un peu plus courtes. -- R. - St-Amand. - Automne. 2026. HELMIN. ClAVARIARUM. Sacc. - Alb. de l'Est. - Cette espèce qui est l'état conidien d'Helmintosphceria clavarice nommé par Fuckel Helminthosporium a été décrite plus haut sous le nom de Scolecotrichum Sacc.

202\%. helmin. TRUNCATUM. Corda. - Alb. de l'Est. - Sur écailles humides de Peuplier mises en tas. - Taches noires-velues, composées de filaments fasciculés, les uns stériles, bruns, septés. amincis, les autres plus épais, tertiles; les deux tiers supérieurs sont bruns, septés et chaque article consiste en une conidie triseptée et tronquée aux deux bouts. - AC. - St-Lumier, St-Amand. - Automne.

2028. HELMIN. MACROCARPUM. Grév. - Alb. 
de l'Est. - Sur les trones et rameaux d'Acer. de Fraxinus, etc. - Taches velues, noiresolivâtres, composées de filaments droits, noirs, subulés, portant au sommet des conidies allongées-claviformes, 6-9 cloisons. - AC. - Automne, Printemps.

2029. HELMIN. MOLLE. $B$. et $C$. - Alb. de l'Est. - Sur rameaux de sambucus ebulus. - Taches brunes peu étendues, composées de filaments, bruns, septés, plus clairs au sommet, porteurs de conidies cylindriques. obtuses, munies de 3 cloisons. - AC. - Aulnay, St-Amand. - Automne.

40 GENRE. - BRACHYSPORHUM. - sacc.

2030. BRACHY. STEMPHYLIOIDES, Sacc. - Alb. de l'Est. - Sur tiges de Spirea Ulmaria. Groupes épars de filamerits distincts ou fasciculés, dressés, brun-clair, septés, terminés par une conidie elliptique, brune, à 5 ou 6 loges, dont las extrêmes sont hyalines, parfois surmontées d'un globule sphérique qui semble être une conidie supplémentaire; des gaines, restes de conıdies avortées existent souvent le long des filaments. - RR. - Aulnay, Printemps.

41 ${ }^{\mathrm{e}}$ GENRE. - CERCOSPORA. - Fres.

2031. CERCOSP. VITICOLA. Sacc. - Alb. de l'Est. - A la face inférieure des feuilles de Vigne. - Taches grises, rondes, formèes par des faisceaux de chapelets de conidies verdâtres. 
cylindriques 1-3 septées. - C. dans les années humides en Automne. - Jardins. - St-Amand. 20:32. Cercosp. Liliicola. Ch. $R$. - Alb. de l'Est. - Sur tige de Lilium Candidum. Ûroupes peu nombreux de filaments blancs, dressés, à 2-3 cloisons terminés chacun par une conidie de même longueur, allongée, fusiforme, concolore, munie de 6-7 cloisons. - RRR. St-Amand. - Jardins. - Printemps.

42 GENRE. HETEROSPORICM. - Klotzsch.

2033. HETER. ORNITHOGALI. Klotzsch. - Alb. de l'Est. Sur feuilles d'Ornithogalum umbellatum. - Etat conidien d'un Phyllachora; composé de filaments simples. dressés, septés, bruns, plus clairs au sommet, terminés par des conidies ovoïdes puis cylindriques, brunes, à $3-5$ cloisons, et á épispore verruqueux. - R. St-Amand. - Printemps.

$43^{e}$ GENRE. - ACERT'THECHUM. - Sacc.

2034. ACROTh. obovatum. Cooke. - Alb. de l'Est. - Sur écailles de bois pourri. - Taches noires velues, peu étendues, composées de filaments bruns, septés clairs au sommet, terminés par une ou deux conidies brunes, ovoïdes, á 2 cloisons inférieures. - R. - St-Amand, StLumier. - Dans les bois. - Été.

4住 GENRE. - SPOROSCHISMA. - Berk.

2035. SPOROS. MIRABILE. Berk. - Alb. de 
l'Est. - Cette espéce selon nous fait double emploi avec Helminthosporium truncatum citèe plus haut.

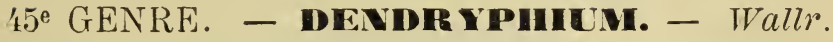

2036. DENDRY. ATRUM. Corda. - Alb. de l'Est.

- Sur écorces de Pin sylvestre. - Touffe de de filaments bruns-noirâtres, assez longs, simples, septés jusqu'au tiers supérieur. présentant à partir de ce point des rameaux nombreux. opposés, formés par des chapelets de conidies. cylindro-elliptiques, brunes à 3 cloisons. - AR. - Soulanges. - Automne.

203\%. DENDRY. FUMOSUM. Fr. - Alb. de l'Est.

- Fréquent sur les tiges pourrissantes de Dahlia et d'Ortie. - Taches très noires, composées de conidies brunes, cylindriques fusiformes, munies de 6 à 10 cloisons, disposées par 3-4 en chapelet, portées par un court filament brun septé, à base rampante. - CC. - Printemps. Partout.

2038. DENDRY. PENICILlatum. Fr. - Alb. de l'Est. - Sur tiges mortes de Papaver somniferum. - Larges taches noirâtres, velues, composées de filaments dressés, bruns. septés, garnis au sommet de quelques rameaux et ramuscules fasciculés, porteurs de conidies elliptiquesallongées à 2-3 cloisons incolores. - C. Printemps. - Jardins. 
SEGT. IV. - DICTYOSPORÉES. - Sacc.

46 e GENRE. - SPORODESMIUM. - Limk.

2039. SPOROD. ULMICOLUM. Sace. - Alb. de l'Est. - Sur rameaux morts d'Ulmus campestris. - Groupes bruns, placés sous l'épiderme déchiré, composés de conidies incolores, puis brunes, fusiformes, renflées, munies de cloisons murales, terminées au sommet par une cellule incolore, courbe et inférieurement par un pédicelle court 1-2 septé. - C'est l'appareil conidien de Massaria pupula. - AC. - Printemps. - St-Amand.

2040. SPOROD. POLYMORPHUM. Corda. - Alb. de l'Est. - Sur rameaux de Pinus sylvestris, formant des amas noirâtres, grumeleux, qui recouvrent d'anciennes cicatrices du conifère. composés d'une masse de conidies de diverses formes, pluri-cloisonnées, brunes, issues d'un Hyphasma constant, mais difficile à apprécier. - AC. - Soulanges, Bassu.

20'1. SPOROD. ALITOSPORII. Ch. R. - Alb. de l'Est. - Sur tiges de Rosa canina. Disséminé parmi les filaments bruns, qui accompagnent le Tapezia rose. - Les conidies sont brunes, elliptiques, ou subtriangulaires, munies de cloisons murales. - RR. Entre St-Amand et Lisse. - Printemps. 
$4 \%$ GENRE. - STHGMELLA. - Lév.

2012. STIGM. DRYINA. Lév. - Alb. de l'Est. A la face inférieure de feuilles de Chêne. Petits groupes noirâtres, épars, sous-cutanés; composés de conidies rondes, irrégulières. brunes, à cloisons murales. - AR. - Bois de Sermaize. - Printemps.

$48^{\circ}$ GENRE. - CONHOTHECIUM. - Corda.

2043. Conioth. EFfusum. Corda. - Alb. de l'Est. - Sur rameaux morts de Pin sylvestre et de Saule. - Taches noirâtres, formées par des amas de conidies subglobuleuses, ou irréguliẻres. brunes, transparentes, réunies en globules difformes. - C. - Bois de pins. Partout.

20'1'. CONIOTh. CONGLUTinatum. Corda. Alb. de l'Est. - Sur branches de Bouleau. Glomérules noirs, confluents, composés de conidies petites, ovoïdes, brunes, conglutinées. AR. - Printemps. - St-Lumier.

20'5. CONICTH. AMENTACEARUM. Corda. Alb. de l'Est. - Sur les rameaux morts des Saules après les gelées. - Groupes noirâtres, pulvinés, épars, composés de conidies oblongues, brunes, agglutinées en glomérules. CC. au Printemps. - Partout.

20'6. Conioth. Betulinum. Desm. - Alb. de l'Esst. - Sur les rameaux morts de Betulus 
alba. - Groupes noirs, épars, rompant l'èpiderme, composés de conidies, concolores subglobuleuses, peu transparentes. - CC. - Printemps. - Partout.

204\%. CONIOTH. QUESTIERI. Desm. - Alb. de l'Eist. - Sur teuilles languissantes de Cornus sanguinea. - Taches noires irréguliéres, composées de glomérules noirs, luisants, entremêlés de filaments très ténus; chaque glomérule est formé de la réunion de 8-10 conidies incolores. puis brunes, ovoïdes. - AR. - St-Amand, St-Lumier. - Automne.

2048. CONIOTH. FASCICULATUM. Ch. $R$. Alb. de l'Est. - Sur tiges desséchées de Ballota nigra. - Petits groupes noirâtres, hémisphèriques, disposés régulièrement à la surface de l'épiderme, composés de conidies conglutinées, superposées, monilitormes, et fasciculées. - AC. - St-Amand, Ablancourt, etc.

49" GENRE. - DIC'TYOSPOLIUM. - Corda.

2049. DiCTY. ELEGANS. Corda. - Alb. de l'Est.

- Sur rameaux de Corylus avellana. - Taches grisâtres, formées par la réunion des Dictyosporium; visibles seulement à la loupe et au microscope. - Les conidies sont simples, dressées, cloisonnées rnurales, linguiformes, munies d'un très court pédicelle. - AR. - Printemps. - St-Amand, St-Lumier. 
50 GENRE. - SPEIRA. - Corda.

2050. SPEIRA TORULOIDES. Corda. - Alb. de l'Est. - Sur feuilles de Carex. - Conidies ovoïdes, ou en fer à cheval, brun-clair, composées de cellules, moniliformes disposées sur trois rangs, munies d'un très court pédicelle. - AR. - Printemps. - Lieux humides.

j̃e GENRE. - S'TEMPHYLIUM. - Waltz.

2051. STEMP. PARAdOXUM. Fuck. - Alb. de l'Est. - Sur écorce de Pommier. - Taches noires, composées de groupes épars, de conidies noires-luisantes, de formes ovales ou cylindriques, dressées. munies d'un court pédicelle et formées de nombreuses cellules murales. - R. - St-Amand. - Printemps.

2052. STEMP. PIRIFORME. Bon. - Alb. de l'Est.

- Sur le papier humide. -- Taches grisâtres. composées de conidies piriformes, brunes, murales, insérées sur un hyphama à peine visible. rameux. -. AR. - Hiver. - St-Amand.

\section{ت̃2 GENRE. - MaCROSPORIUM. - Fries.}

2053. MACROS. BRASSICAE. Berk. - Alb. de l'Est. - Sur les tiges mortes de Brassica oleracea et d'Hesperis matronalis. - Taches grises. formées par les groupes épars de Macrosporium. Composées de filaments courts, dressés, bruns, 
surmontés chacun d'une conidie grosse. piriforme-allongée, brune, cloisonnée murale. - AC. - Printemps. - St-Amand.

2054. MACROS. MALVAE. Thüm. - Alb. de l'Est.

- Sur tiges de Malva sylvestris. - Taches grisâtres, composées de filaments dressés, brunclair, munis de nombreuses cloisons, terminés chacun par une conidie grande, piriforme ou fusoïde à cloisons murales,á peine teintées presque hyalines. - AC. - Printemps. - St-Amand.

2055. MACROS. LAGENARIA. Thüm. - Alb. de l'Est. - Sur le fruit de Lagenaria vulgaris. Taches grises, à zones concentriques, formées par les groupes mamelonnés des fascicules de Macrosporium, composées de filaments brunclair, septés, terminés chacun par une conidie irrégulièrement ovoïde, brune à cloisons murales. - AC. - En hiver.

53e GENRE. MYSTROSPORIUM. Cordl.

2056. MYSTR. STEMPHYLIUM. Corda. - Alb. de l'Est. - Sur tige pourrissante de Chou. Taches grisâtres formées par les groupes de conidies brunes-olivacées, à cloisons murales. et de formes très diverses, portées par des pédicelles courts concolores. - C. - Automne, printemps. - Jardins. - Partout.

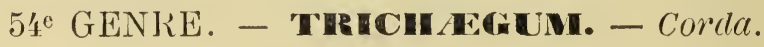

205\%. TRICH $A$ G. CLADOSPORIOIDES. Corda. Alb. de l'Est. - Sur branches de Saule. mortes. 
- Taches grises, formées par les faisceaux épars, de Trichcegum, composées de filaments courts, noduleux, bruns, portant à leur sommet une conidie ovale irrégulière, cloisonnée. murale et verruqueuse. - AC. - Printemps. Partout.

55̃ GENRE. - SEP'TOSPOAICM. - Corda.

2058. SEPT. BULBOTRICHUM. Corda. - Alb. de l'Est. - Sur bois pourri et sur grains de Maïs. - Taches grisâtres, formées par les filaments de deux natures, les uns simples. septès, brunâtres, stériles, renflés-bulbeux à la base. les autres courts concolores portant une conidie piriforme, brune, pluri-cloisonnée, murale. - C. - Printemps. - Partout.

2059. SEPT. VELUTiNUM. C. et $E l$. - Alb. de l'Est. - Sur branches d'Acer. - Taches grisbrun, composées de faisceaux de filaments stériles, bruns, septés, et de filaments fertiles, courts, portant des conidıes ovoïdes piriformes. brunes, murales, pas de bulbes à la base. - Automne, printemps.

56" GENRE. - Acterditia. - Nées.

2060. Altern. Tenius. Nées. - Alb. de l'Est. - Sur tiges de Lis mortes et humides. Taches noires, intenses, composées de filaments courts: terminés chacun par un long chapelet de conidies brunes, murales, lageniformes. AR. - Jardins. - Automne. - St-Amand. 
$5 \%$ GENRE. - FUMAGO. - Pers.

2061. Fumago. Vagans. Pers. - Alb. de l'Est.

- Sur feuilles vivantes de Peuplier. de Ronce. de Vigne, etc. - Taches noires-opaques; formées de filaments divers, olivacés-noirâtres, rameux, entremêlés, confus, imitant le noir de fumée, munis de conidies la plupart didymes, pluri-septées. - CC. - Automne. - Partoul.

2062. FUMAGo. LATERITIORUM. Sacc. - Alb. de l'Est. - Sur les briques des toits. - Taches noires, formées de filaments rayonnants, la plupart stériles, les fertiles plus courts, portant des conidies agglomérées ou en chapelet. - AR. - Automne. Printemps.

SEGT. V. - STAUROSPOREES. - Sacc.

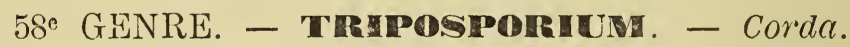

2063. TRIPosp. Elegans. Corda. - Alb. de l'Est. - Sur rameanx pourris de Coudrier et de Charme. - Taches brunes, velues, composées de filaments bruns, septés, un peu rameux. à rameaux à angle droit; les stériles sont allongés terminés en pointes; les fertiles plus courts portent à leur sommet, des conidies, à 3-ð segments, disposés en étoile, bruns, septes. plus pâles au sommet. - AR. - Bois de Sl. A mand. St-Lumier. - Automne. 
SEGT. VI. - HELICOSPOREES. - Sacc.

59ª GENE. - HELICOSPOIRUM. - Nées.

2064. Helicos. PUlvinatum. Fr. - Alb. de l'Est. - Sur écaille de Peuplier. - Taches grisâtres composées de filaments brun-pâle, septés, très rameux, le long desquels s'insèrent des conidies cylindriques, septées et munies de gouttelettes, contournées en spirales, incolores. - AC. au Printemps en temps humide. - St$\Lambda$ mand et St-Lumier.

2065. HELICOS. MULLERI. - Sacc. - Alb. de l'Est. - Sur petits rameaux de Chêne ou d'Acer. ayant séjourné dans les lieux inondés l'hiver. - Taches couleur gris de fer, composées d'une masse de conidies, brunes-verdâtres. contournées en spirales ou en vrilles, cloisonnées el munies de gouttelettes, issues de filaments, dressés, un peu rameux. d'une teinte plus pâle. AR. dans les bois entre St-Amand et St-Lumier.

- Printemps. 


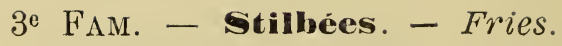

Filaments pâles ou bruns, réunis en faisceau allongé en forme de stipe.

SEGT. I. - AMÉROSPORÉES. - Sacc.

$$
\text { 60 GENRE. - STHLPUM. - Tode. }
$$

2066. STILBUM. ERYTHROCEPHALUM. Ditm.Alb. de l'Est. - Sur les excréments du chien. - Petites clavules, blanches, ou rosées, terminées par un capitule rougeâtre. - Cette espéce est formée par la réunion dense de longs filaments, terminès chacun par une conidie, ovale; l'épaisse couche de conidies constitue !e capitule rougeâtre. - AC. - Automne. - Partout.

2067. STILBUM. Villosum. Mérat. - Alb. de l'Est. - Sur les crottes de rat, dans un tas de vieille paille exposée à l'air humide. - Il se distingue du précédent par son port plus svelte, sa couleur blanche, et par ses filaments fertiles bi-quadrifurqués au sommet. - R. - St-Amand. - Automne.

2068. STILBUM. VULGARE. Tod. - Alb. de l'Est. - C. sur les écailles humides de Chêne, de Bouleau, de Peuplier. - Petits groupes de clavules blanchâtres, glabres, munies d'un capi- 
tule blanc-jaunissant, presque sphérique, conidies ovales, hyalines. - CC. - Automne-Printemps. - Partout.

2069. STILBUM. AURANTIACUM. Bab. - Alb. de l'Est. - Sur rameaux morts de l'Ulmus campestris tombés à terre. - Petits groupes de clavules simples ou fasciculées; pédicelle assez long, rouge-pourpre; capitules globuleux d'une teinte plus pâle; conidies oblongues, et tronquées-incolores. - AC. - Automne-Printemps. - St-Amand.

20\%0. STILBUM. PILACRIFORME. Ch. $R$. - Alb. de l'Est. -- Sur bois pourri, humide, d'Ulmus campestris et d'Alnus glutinosa. - Etat conidien de Pilacre Friesii. - Petites clavules, blanchâtres, courtes-velues. munies d'un capitule recouvert de conidies vert-glauque, insérées sur une couche noire formée par le sommet bifurqué et de couleur brune des filaments du pédicelle; conidie hyaline piriforme. - R. - StAmand, Soulanges.

\section{GENRE. - ChMLIOPODIUM. - Corda.}

2071. Cilic. SANGUineum. Corda. - Alb. de l'Est. - Croît en groupes sur les tiges herbacèes pourries en tas, notamment sur Dianthus plumarius. - Clavules allongées, fibrilleuses, blanches, amincies à la base, terminées au sommet, par un capitule rouge-sanguin, formé par l'agglomération des nombreuses conidies ovoïdes 
qui terminent les filaments. - AR. - Jardins. - St-Amand. - En Automine.

62 GENRE. - ECCHYMA. - Fries. (Pilacre Sacc). 2072. ECCHINA FAGINEA. $B$. et $B r$. - Alb. de l'Est. - Sur le bois de Hêtre mis en tas. Groupes épars, de petites clavules, composées d'un pédicule jaunâtre, strié, cylindrique terminé par un capitule hémisphérique, recouvert par une masse de conidies sphériques brun-clair. - Récolté à Cheminon en Automne. - AR.

2073. ECCHYNA. WEINMANNI. Fr. - Alb. de l'Est. - L'espèce décrite sous ce nom par Fries est à n'en pas douter la forme conidienne de Pilacre ou Vibrissea Friesii qui attaque fréquemment les racines de la vigne malade; nous en avons donné la description parmi les Discomycètes, page 201. - AC. - Automne.

$20 \%$ ' ECCHYNA. PORICOLA. Ch. $R$. - Alb. de l'Est. - S Sur les tubes de Polyporus salicinus en décomposition. - Cette espèce présente tous les caractères d'Ecchyna faginea, à part sa station et ses dimensions plus grandes. - RRR. - Bois de St-Amand, à la Cense des Près. Automne.

\section{3e GENRE. - COREMIUMT. - Link.}

2075. COREm. GlauCUm. Fries. - Alb. de l'Est. - C. sur toutes les substances sucrées et putrescibles, telles que les fruits. les confitures, etc. - Petites clavules. munies d'un pédicule blanc 
composé de filaments nombreux réunis, et qui en s'étalant au sommet forment un capitule gìauque, composé de conidies disposées en chapelet. - C'est évidemment une des formes nombreuses du Penicillium glaucum. - CC. Partout.

2076. COREM. NIVEUM. Corda. - Alb. de l'Est. - AC. - Partout, notamment sur écorce et aiguille de Pin sylvestre. - Ressemble au Coremium glaucum dont il diffère 'seulement par sa blancheur de neige et ses formes plus délicates. - Automne et printemps.

\section{GENRE. - TsATIA. - Pers.}

2077. ISARIA FARINOSA. Fr. - Alb. de l'Est.

- Sur les larves et chrysalides des insectes, recouvertes de terre. - Elle paraît au dehol's sous forme d'une petite clavaire arborescente au sommet, et saupoudrée d'une masse de conidies blanches; la tige est composée de filaments très serrés, terminés supérieurement en plusieurs rarneaux courts; conidies ovales allongées hyalines; c'est la forme conidienne de Cordiceps militaris. - AR. - Bois à St-Amand.

2078. ISARIA. ARACHNOPHILA. Ditm. - Alb. de l'Est. - Sur les corps des araignées mortes. - Se présente sous forme de rameaux allongés. simples, disposés en faisceaux; de la tige des rameaux, formée par la réunion des filaments partent des ramuscules nombreux, en épis, terminés chacun par un capitule composé de 
basides piriformes et de petites conidies ovales hyalines. - C'est probablement le Sterigmalocystis candida $(S a c c)$. AR. - Printemps. - St-Amand.

2079. ISARIA ELEUTHERATORUM. Nées. - Alb. de l'Est. - Sur larves d'insectes. - Rameaux filamenteux blancs, très longs, sinueux terminés par 3 ou 4 ramuscules, couverts de nombreuses conidies, hyalines, très petites, ovales-allongẻes - AR. dans les bois á St-Lumier.

2080. ISARIA BRACHIATA. Schum. - Alb. de l'Est. - Sur les chapeaux des Agaricinés pourris et desséchés. - Se présente sous forme de petits rameaux, simples, amincis au sommet, composés de filaments réunis, et garnis de sporophores nombreux, terminés par un capitule de conidies, petites hyalines. - AC. Bois de pins. - Bassu, Soulanges.

2081. ISARIA UMBRINA. Pers. - Alb. de l'Est. Sur Fraxinus et Prunus spinosa. - Petites clavules velues, simples ou rameuses, couleur café au lait, entourant ordinairement l'Irypoxylon fragiforme, dont il est l'appareil conidien, les filaments fertiles semblent appartenir au genre Verticillium, - AC. - Automne. - St-Amand.

65e GENRE. - CERATHAI. - A. S.

2082. CerATIUM HYDNOIDES. Alb. et Schw. Alb. de l'Est. - Sur le bois pourri de Chêne et de Pin - Groupes de petits troncs, rameux, à rameaux contournés, de consistance molle; 
fermos avec l'âge, de couleur blanc de neige puis jaunâtres, couverts sur toute la surface de spicules terminées chacune par une conidie elliptique, grosse, hyaline. - AC. - Automne, Printemps.

2083. Ceratium. PyXidatum. Alb. et Schw. Alb. de l'Est. - Sur rameaux de Chêne pourris et sur tige sarmenteuse de Houblon. - Se présentant sous forme de petites clavules blanches, fasciculées, réunies à la base, 2-4; la surface des clavules est hérissée de basides acuminées, surmontées chacune d'une conidie elliptique munie d'une cloison médiane. -- Cette espéce me semble appelée à faire partie d'un genre particulier. - C. - Automne. - Printemps. Partout.

\section{SÉR. - PHÆOSTILBÉES. - Sacc.}

\section{SeGt. I. - AMEROSPORÉES. - Sacc.}

66e GENRE. - SHORdoCYBE. - Fries.

2084. SPOROCYBE BYSSOIDES. Bon. - Alb. de l'Est. - C. sur les tiges des grandes herbes. - Petites taches velues, formées par les groupes nombreux de sporocybe, composés chacun d'un pédicule noirâtre, rigide et d'un capitule olivacé produit par l'écartement de l'extrémité des filaments munis de nombreuses conidies 
otovales, olives. - AC. - Automne-Printemps. - Partout.

2085. SPOROCYBE CUNEIFERA. Sacc. - Alb. de l'Est. - Sur tiges de Saponaria et de Dianthus. - Taches noirâtres, formées par les groupes de sporocybe, à pédicules, filamenteux, noirs, écartés au sommet, en gerbe, presqu'incolores. et surmontés de conidies hyalines cunéiformes. - R. - St-Amand. - Jardins. - Au Printemps. 2086. SPOROCYBE FASCICULATA. Sacc. - Alb. de l'Est. - Cette espèce ressemble au Sporocybe Atra et n'en diffère que par sa disposition en faisceaux et par ses conidies ovoïdes plus petites. - Croît en Automne sur le bois pourri. - AR. - St-Amand.

2087. SPOROCYBE ATRA. Succ. - Alb. de l'Est. - Sur feuilles et chaumes de Carex et de Festuca. - Epars et en petit nombre. à pédicules rigides, noirs, formés de filaments qui s’écartent brusquement au sommet, pour former un capitule hémisphérique, couvert de conidies oblongues-fusoïdes-olivacées. - AC. - Automne-Printemps. - St-A mand.

2088. SPOROCYBE CARNEA. Ch. $R$. - Alb. de l'Est. - Sur lattes de Chêne depuis longtemps dañs une cave. - Cette espèce a l'aspect et la couleur d'un Adocephalum agaricinum, c'est une stilbinée à stipe formé de filaments réunis, septés, couleur de chair, capitule sphérique, compact, couvert de conidies roses, elliptiques.

- R. - St-Amand. - Été. 
6\% GENRE, - GRAPHIUM. - Corda.

2089. GRAPH. RIGIDUM. Sacc. - Alb. de l'Est.

- Sur écailles de bois humide, notamment du Pin sylvestre. - Groupes de Graphium épars, munis chacun d'un pédicelle noir, rigide. subulé, portant au sommet un capitule sphérique, blanc, aqueux puis compact et gris: conidies petites, fusoïdes. -- C. - Automne, Printemps. - St-Amand.

2090. GRAPH. FISSUM. Preuss. - Alb. de l'Est.

- Sur tiges mortes de Solanum dulcamara.

- Formant des groupes très nombreux de Graphium à pédicule noir, fibrilleux, les filaments du sommet sont dilatés en gerbes; et parsemés çà et lá. même le lıng de la tige, de conidies hyalines, fusoïdes. - AR. - St-Amand. Automne.

2091. GRAPH. STRICTUM. Preuss. - Alb. de l'Est. - Sur le bois putride. - Petits groupes noirâtres de Graphium, munis d'un pédicule noir, plus pâle au sommet, disposé en pinceau formant un capitule muqueux, blanc, de conidies ovales. hyalines. - Printemps. - StAmand.

68 GENRE. - HARPOGEAPHIUM. - Sacc.

2092. HARP. FASCICULATUM. Sacc. - Alb. de l'Est. - Sur Prunus domestica et sur Acer. - Etat conidien d'Eutypa heteracantha. qu'il 
accompagne sur le support, formant de petites massues brunes, filamenteuses, couvertes presqu'entièrement de conidies. - AR. - St-Amand. - Automne.

\section{9e GENRE. - S'TYSANUS. - Corda.}

2093. STYSANUS. STEMONITES. Corda. - Alb. de l'Est. - Sur racines pourries et sur plantes herbacées mises en tas. Groupes très denses, formant des taches grises, composées de pédicules rigides, noirâtres, filamenteux. terminés par un capitule cylindrique, blanchâtre, formé par l'extrémité des filaments, muni de conidies ovales, incolores. - CC. - Automne, Printemps. - Partout.

2094. STYSANUS CLEMATIDES. Fuck. - Alb. de l'Est. - Sur les sarments pourris de Clématis Vitalba. - Cette espéce forme. une petite forêt de capitules ovoïdes, gris-argentés, portés par des pédicules bruns-noirâtres, filamenteux, les conidies insérées au sommet des filaments, sur de petites basides verruqueuses, sont ovales, allongées, incolores. - C. - Jardins. - Automne, Printemps.

\% 0 e GENRE. - ANTHOMYCES. - Fres. -

?095. ANTROMYC. COPRIDIS. Fres. - Alb. de l'Est. - Espèce communiquée par M. Boudier de Montmorency. - Inscrite ici pour mémoire. - Très voisine du genre Arthrobotryum. Naissant dans la coque d'une chrysalide de 
Copris, ses conidies sont uniseptées, disposées en chapelet. - RRR.

SEGT. II. - PHRAGMOSPORÉES. - Sacc. $71^{\text {e }}$ GENRE. - ARTHROBOTRYUM. - Ces. 2096. ARThr. STILboideum. Ces. - Alb. de l'Est. - Sur les troncs pourris de Peuplier, coupés prés du sol. - Petites clavules noires, dressées, un peu renflées au milieu, dont les filaments s'écartent en gerbe au sommet et portent chacun une conidie elliptique fusnïde. à 2-3 cloisons. - C. Bois de St-Amand à StLumier. - Printemps.

\section{$4^{\theta}$ FAM. - Tuberculariées.}

Filaments pâles ou bruns, agglutinés et réunis en forme de tubercule ayant souvent pour base un strôme un peu épais.

SEGT. I. - AMÉROSPORÉES. - Sacc.

1er GENRE. - TUBERCULARIA. - Tode.

209\%. TUBERCULARIA VULGARIS. Tode. - Alb. de l'Est. - CC. sur l'écorce d'Erable. -- C'est l'appareil conidien de Nectric cinnabarina. Tubercules nombreux, hémisphériques rouge- 
vermillon, à strome plus pâle, composé d'une masse de filaments rameux, portant sur leur trajet et au sommet des conidies elliptiquesallongées. incolores; toute l'année -- Partout.

2098. TUBERCULARIA PEZIZOIDEA. Schw. Alb. de l'Est. - Sur jeunes rameaux de Peuplier. - Tubercules en torme de disques, jaunesorangés, formés par la réunion compacte de filaments dressés, terminés chacun par une conidie incolore sphérique. - AR. - Bois de St-Amand et St-Lumier. - Automne.

2099. TUBERCULARIA SARMENTORUM. Fr. Alb. de l'Est. - Sur les sarments de Vigne, et de Clematis Vitalba. - Tubercules petits, couleur pourpre. sortant de dessous l'épiderme, disposés en lignes longitudinales; conidies cylindriques courbes, petites, hyalines. - AC. Partout.

2100. TUBERCULARIA HYDNOIDEA. Ch. $R$. Alb. de l'Est. - Sur écailles de Peuplier humides; affectant deux formes, l'une sphérique ou lobée. l'autre divisée en rameaux claviformes couleur. rouge-pâle ou rose, couverte à la surface par des conidies incolores, elliptiques relativement trés grandes. - $R$. en hiver. - Bois de StLumier.

2101. TUBERCULARIA VERSICOLOR. SAcc. Alb. de l'Est. - Sur rameaux de Buxus sempervirens. - Tubercules rouge-pâle, aplatis ou un peu convexes, formés par des filaments continus trés longs. portant chacun à leur sommet. 
une conidie incolore, elliptique, très grande. Etat conidien de Nectria Demazieri. - AC. Jardins. - St-Amand. - Printemps.

2e GENRE. - DENDHODOCHIUM. - Bon.

2102. DENDR. CITRINUM. Grave. - Alb. de l'Est.

- Sur cône de Pinus Sylvestris. - Tubercules très petits. jaune-citron, ovoïdes ou pezizoïdes, composés de filaments droits, septés, fasciculés, donnant naissance dans leur longueur et au sommet à de petites conidies incolores, elliptiques avec deux gouttelettes. - AC. - Printemps-Automne. - Bois de Pins. - Partout.

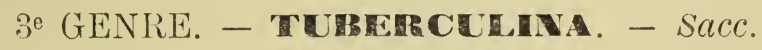

2103. TUBER. PERSICINA. Sacc. - Alb. de l'Est. - Sur feuilles d'Euphorbiacées attaquées par diffèrents Ecidium, dont il remplit la cupule avec ses tubercules, arrondis, saillants, et violacés; ces tubercules sont formés des basides irrégulières qui portent à leur sommet des ccnidies ovales, incolores. - AR. -. Été.

$4^{2}$ GENRE. - HLLOSTPHETM. - Mart.

2101. IllosP. ROSEUM. Mart. - Alb. de l'Est. - Croît sur les thalles de plusieurs lichens, sous forme de tubercules roses, arrondis ou lobés, composés de filaments tortueux, donnant naissance à des conidies ovoïdes. - AR. Automne. - St-Amand. 
2105. ILLOSP. COCCINEUM. Fr. - Alb. de l'Est.

- Sur rameaux d'Orme, envahis par un Parmelia. - Tubercules rouge-carmin, sphériques granuleux, placés sur une membrane blanche, étalée sur le lichen; conidies ovales, ou globuleuses, recouvrant la surface. $-\Lambda R$. en Automne. - Aulnay-l'Aître.

\section{GENRE. - REGRITA. - Pers.}

2106. EGERITA ALBA. Sacc. - Alb. de l'Est. - Sur les Péricarpes humides de l'Asculus hypocastanum. - Groupes de tubercules sphériques, blancs. formés par l'agglomération des conidies ovales, hyalines. - AR. - Bois de St-Lumier. - Automne.

2107. AEGERITA TORULOSA. Sacc. - Alb. de l'Est

- Récolté sur la terre des fosses à Pulpes.

- Tubercules de 2 à 4 millimètres, sphériques, blancs, composés de cellules basilaires, fusoïdes. surmontées de conidies ovales-cylindriques, en chapelet,incolores. - R. - St-Amand. - Automne.

2108. EGERITA CANDIDA. Sacc. - Alb. de l'Est.

- Sur branches de Chêne pourries. - Tubercules nombreux, très petits, sphériques, blancs, groupés, composés de sporophores sinueux, terminés à la Périphérie de l'Egerita, par des conidies incolores, rondes ou ovales. - AC.

- Dans les forêts. - Automne.

6e GENRE. - FUSHCOLLA. - Bon.

2109. FUSICOLLA CORTICALIS. - Karst. - Alb. 
de l'Est. - Sur les rameaux de Saule Niarceau humides. - Petits tubercules, blancs, ronds, aplatis, confluents, se désagrégeant dans l'eau. et répandant des masses de conidies en batonnets incolores. - AR. - St-Amand, St-Lumier. - Hiver.

2:10. FUSICOLLA PHRAGMITIS. Karst. - Alb. de l'Est. - Sur chaumes et rhizomes d'Arundo Phragmites. - Tubercules jaune-orangé, de diverses formes, sphériques. coniques ou en cupules, composés de filaments rameux, dressés. porteurs de conidies elliptiques, incolores. munies de deux gouttelettes. $\Lambda$ R. - Automne. - Fossés du château d’Aulnay.

ye GENRE. - SPHACELIA. - Lév.

2111. SPHACELIA SEGETUM. Lév. - Alb. de l'Est. - Membrane enveloppant l'Ergot de Seigle, blanche ridée, munie de conidies petites: hyalines, elliptiques, portées par des basides fasciculées. - Etat conidien du Claviceps Purpurea. - CC. - Été. - Partout.

2112. SPHACELIA TYPHINA. Sacc. - Alb. de l'Est. - Sur les gaines du Dactylis Glomerata. - Précéde l'apparition des pẻrithèces d'Epichloe typhina. - Sous forme d'un duvet blanchâtre, composé de basides simples, terminées par des conidies elliptiques, hyalines. - C. en Automne. - Bois, prairie. - Partout.

8 GENRE. - hymenela. - Fries.

2113. HYMEN. RUBELla. Fries. - Alb, de l'Est. 
- Sur tiges mortes d'Arundo Phragmites. Tubercules disciformes, épars, jaune-citron, céracés, gélatineux, composés de filaments allongés. continus. terminés chacun par une conidie hyaline, cylindrique. - AR. - Bords des ruisseaux. - St-Amand, Lisse. - Automne.

9e GENRE. - CYLINDIRdCOLLA. - Bon.

2114. CYLINDR. URTICAE. Bon. - Alb. de l'Est. - Sur tiges mortes d'Ortie. - Tubercules rouge-orangé, céracés, composés de filaments rameux porteurs de conidies cylindriques, un peu courbes, ou fusoïdes. - Etat conidien de Calloria fusarioides. - CC. - Automne-Printemps.

2115. CYLINDR. DUBIA. Sacc. - Alb. de l'Est. Sur écailles de bois pourri. - Tubercules blanc de neige, petits, sphériques, formés de filaments, porteurs de conidies cylindriques, granuleuses, placées bout à bout comme dans Chalara. AR. -- Bois de St-Amand. - Hiver.

$10^{\circ}$ GENRE、 - SRHAERHDIUM. - Fres.

2116. SPHAR. CITRINUM. Sacc. et Ch. R. - Alb. de l'Est. -- Sur écailles humides de Peuplier. - Très petits flocons jaunes. épars, sessiles ou pédicellés, composés de filaments, formés par des conidies cylindriques, en chapelets trés longs, et rameux. jaunes, hyalins. - R. St-Amand. - Automne. 


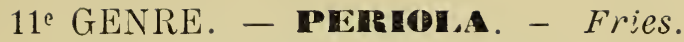

211\%. PERIOla hirsuta. Fr. - Alb. de l'Est. - Sur bois pourri. - Tubercules blancs, subsphériques, couverts de longs cils, concolores, terminés en crochet; ces tubercules sont composés d'un amas de conidies, elliptiques, incolores, granulées. - AR. - St-Amand. Printemps.

2113. PERIOLA TOMENTOSA. Fr. - Alb. de l'Est.

- Sur tiges pourries de Pommes de terre. Petits tubercules blancs, velus, épars, composés de cellules centrales, qui émettent à leur sommet des conidies elliptiques, blanches, disposées en chapelets. - AC. - Lieux humides. - Automne. - St-Amand.

12e GENRE. - volutella. - Tode.

2119. Volutella SetosA. Berk. - Alb. de l'Est. - C. sur les tiges herbacées, et sur le bois pourri. - Tubercules sphériques, blancs, couverts de longs poils, dressés, concolores, composés de conidies globuleuses, hyalines, très petites. - CC. - Automne, Printemps. St-Amand.

2120. Volutella BuXi. Berk. - Alb. de l'Est. - Sur feuilles et tiges de Buis. - Tubercules, sub-sphériques, roses, couverts de longs poils, concolores, septés; composés de filaments tortueux, rameux, hyalins, terminés par des conidies elliptiques, fusoïdes, munies de deux gout- 
telettes. - CC. - Automne, Printemps.- Partout. 2121. VOLUTELlA ARUNDINIS. Desm. - Alb. de l'Est. - Sur Arundo Phragmites. - Plaques velues, elliptiques, blanc-rosé, composées de longs filaments simples, à la base desquels sont amassées des conidies, elliptiques, fusoïdes, hyalines, très nombreuses. - C. - Lieux marécageux. en Hiver. - A St-Amand.

212\%. VOLUTELLA CYPERACEARUM. Sacc. Alb. de l'Est. - Sur feuilles de Poa aquativa. - Petits tubercules hémisphériques, rose-rouge, entourés de longs poils noirs, septés, composés de conidies cylindriques, courbes, amincies à la base, munies de gouttelettes incolores, et portées par un strome granuleux. - AR. - Lieux humides. - St-Amand. - Automne.

\section{Segt. II. - PHRAGMOSPORÉES. - Sacc.}

\section{3e GENRE. - HACTRHDIUM. - Kunze.}

2123. BACTR. FLAVUM. $K$. et $S .-A l b$. de l'Est. - Sur troncs pourris et humides d'Orme et d'Aulne. - Tubercules globuleux, jrune-orangé, tuberculeux épars. composés de filaments qui émettent des pédicelles courts, terminés chacun par de longues conidies claviformes, à ' cloisons, les 3 cellules médianes sont jaunes, et les deux extrêmes hyalines. - C. - IIiverPrintemps. -- St-A mand. 
14. GENRE. - NUSARIUM. -- Link.

2124. FUSARIUM PYROCHROUM. Sacc. -- dlb. de l'Est. - Sur rameaux morts d'Acer et de Sambucus nigra. - Tubercules en groupes, arrondis, de couleur d'ocre; composés de courts pédicelles, porteurs de trés nombreuses conidies. fusoïdes arquées, munies de 3-5 cloisons. AC. - Automne-Printemps. - St-Amand.

2125. FUSARIUM PALlENS. Nées. - Alb. de l'Est. - Sur tiges mortes d'Angelica sylvestris. - Tubercules blanc-rosé, couverts de conidies cylindriques, continues ou uniseptées, portées par de longues basides, simples ou bifurquées, insèrées sur un strome brunâtre. - AC. - Bois. - St-Amand. - Automne.

2126. FUSARIUM SAMBUCINUM. Fuck. - Alb. de l'Esst. - Sur troncs et rameaux de Sambucus nigra. - Tubercules hémisphériques, rougepâle. pruineux; composés de conidies, fusiformes, courbes. apiculées, portées par des sporophores concolores. septés. - C. - Printemps. - Partout.

212\%. FUSARIUM ROSEUM. Link. - Alb. de l'Est - Sur les feuilles de Buis. -- Tubercules roseorangé, petits, épars, formés par un strome, portant à sa surface des conidies fusiformes, triseptées, recourbées aux extrémités. - C. au Printemps. - Partout.

2128. FUSARIUM SOLANI. Sacc. - Alb. de l'Est. 
- Sur Pommes de terre pourries. - Tubercules globuleux, irréguliers, tomenteux, composés de filaments rameux, portant des conidies hyalines, fusiformes, arquées, triseptées. CC. - Partout en Automne et Printemps.

2129. FUSARIUM HETEROSPORUM. Nées. - Alb. de l'Est. - Sur les épis de Seigle et de Bromus Pinnatus. - Attaque les grains sous forme de petites plaques roses, rougeâtres au centre, composées de courtes basides, surmontées de conidies, fusoïdes, arquées. - C. - St-Amand. - Soulanges. - Bords des chemins au Printemps.

2130. FUSARIUM ZEEE. Sacc. - Alb. de l'Est. Sur les chaumes et feuilles de Maïs. - Tubercules petits, rosés, composés de filaments rameux, terminés par des conidies fusoïdes, arquées, triseptées. - AC. - Aulnay-l'Aître. Printemps, Hiver.

2131. FUSARIUM. GALlinACEUM. Cooke. - Alb. de l'Est. - Sur les pattes et sur les ailes de poules mortes. - Tubercules aplatis, rougevermillon. ciliés sur les bords blanchâtres, composès de filaments rameux, porteurs de conidies, fusiformes, irrégulières, munies de gouttelettes. - Récolté à Soulanges plusieurs fois au Printemps. - R.

2132. FUSARIUM AURANTIACUM. Sacc. -- Alb. de l'Est. - Sur les Cucurbitacées. - Tubercules hémisphériques, aplatis, confluents, entourés de filaments blancs, stériles, de couleur rouge-orangée, composés de filaments tẹnus, 
très rameux, portant des conidies fusiformes, arquées, triseptées. - CC. - Automne. - Jardins. - Partout.

2133. FUSARIUM MINUTULUM. Corda. - Alb. de l'Est. - Sur rameaux morts de Corylus avellana.-Tubercules blancs, très petits, groupés, confluents,composés de filamerits simples,ou bifurqués, portant chacun une conidie elliptique.fusoïde incolore. - AR. - St-Lumier. -- Printemps.

213'. FUSARIUM OXYSPORUM. Schlecht. - Alb. de l'Est. - Sur l'écorce des melons. - Tubercules, convexes, verruqueux, confluents, composés de conidies courbes, aiguës, de couleur rose. - Semble ne différer du Fusarium aurantiacum que par la teinte des tubercules. - CC. - Jardins. - Automne.

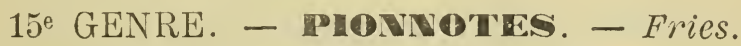

2135. PIONN. BET 2 E. Sacc. - Alb. de l'Est. Sur racines de Betteraves en décomposition. Tubercules gélatineux, irréguliers, aplatis, composés de filaments fasciculés ou rameux, portant des conidies hyalines, fusiformes, arquées. - CC. - Automne, Hiver. - Partout.

\section{TUBERCULARIÉES DEMATIÉES. - Sacc.}

SEGT. I. - AMEROSPOREES. - Sacc. 16 e GENRE. - EPICOCCUM. - Link.

2136. EPICOCCUM VULGARE. Corda. - Alb. de 
l'Est. - Sur les tiges et feuilles des herbes pourrissantes. - Tubercules convexes, de couleur pourpre-noircissant, placés sur des taches concolores, plus pâles, composées de conidies brunes. aréolées, pointillées, portées par un court pédicelle hyalin. - CC. - Automne, Printemps. - Partout.

2137. EPICOCCUM NEGLECTUM. Desm. - Alb. de l'Est. - Sur les feuilles languissartes et sur les chaumes de Maïs. - Tubercules ponctiformes, très noirs, petits, groupés, mais non insérés sur une tache, composés de conidies brunes, réticulées, munies d'un court pédicelle hyalin. - AC. - En Automne. - Partout.

1\% GENRE. - HYMEMOPSIS. - Sacc.

2138. HYMENOPSIS ELLIPSOSPORA. Sacc. Alb. de l'Est. - Sur gaines d'Arundo Phragmites. - Tubercules noirs, elliptiques ou hémisphériques, formés par des filaments denses, simples, portant à leur sommet des conidies brunes, elliptiques-fusiformes. - AR. - StLumier, St-Amand. -- Automne, Printemps.

2139. HYMENOPSIS TYPHAE. Sacc. - Alb. de l'Est. - Sur tigas de Typha et de Juncus communis. - Tubercules en disques noirs, marginés, épars, composés de basides simples, dressées. denses, verdâtres,portant des conidies fusiformeselliptiques, concolores, munies de 2 gouttelettes. - AC. - Lieux marécageux - St-Amand, Lisse. 
18 GENRE. - CHAETOSTHOMA - Corda.

2110. CHATOS. CARICIS. Sacc. -- Alb. de l'Est.

- Sur les tiges desséchées de Carex. -- Cette espéce indiquée par Saccardo, avec un point de doute me paraít être la même que Hymenopsis Typhce citée plus haut.

2141. Chetos. ATrum. Sacc. - Alb. de l'Est. - Sur chaumes de Scirpus lacustris. - Tubercules noirs, trémelloïdes, entourés de poils concolores très longs, peu nombreux, formés de basides simples, courtes, terminés par des conidies fusoïdes, verdâtres, munies de deux gouttelettes. -- AC. - Lieux marécageux. Automne: Printemps.

\section{9e GENRE. - MYRO'THECRCM. - Tode.}

2142. MYROTH. CINCTUM. Sacc. - Alb. de l'Est.

- Sur tiges de Poa, desséchées. - Tubercules noirs, hémisphériques, entourês à la base d'une collerette de filaments tortueux, blanchâtres, formés par des basides simples, denses. terminés par des conidies fusoïdes, brunes-verdâtres. sans gouttelettes. - AR. - Bois de St-Amand, St-Lumier. - Printemps.

2143. MYROTH. GRAMINUM. Libert. - Alb. de REst. - Sur tiges d'Agrostis vulgaris. - Tubercules noirs, ronds, un peu convexes, entourés à la base, de longs poils, blanchâtres; composés de filaments ou basides, simples, terminés par 
des conidies cylindriques, fusiformes; hyalines. - AR. - Automne. - St-Amand.

SEGT. II. - ASTÉROSPORF́ES. - Sacc.

20" GENRE. - S'TEPHIINOMA. - Wallr.

2144. STEPH. STRIgOSUM. Wallr. - Alb. de

l'Est. - Parasite sur le disque de Peziza hemispherica. - Forme conidienne d'Hyponyces pezizce. - Composé de filaments rampants, qui émettent sur leur trajet des pédicelles dont le sommet renflé est entouré de conidies à lobes arrondis, disposés en étoiles hyalines. - $R R$. Bois de Sermaize. -- En Été.

SEGT. III. - PHRAGMOSPORÉES. - Sacc. 21e GENRE. - EXISPOMUMM. - Link.

2145. EXOSP. TILIE. Link. - Alb. de l'Est. Tubercules noirs, convexes, pulvinés, portant à leur surface des conidies, claviformes, ou fusoïdes, pluriseptées, brunes-olivâtres. - CC. Sur les rameaux morts du Tilleul. - Automna, Printemps. - Partout. 
MYCELIA STERILIA. - Fries.

Tissus fongiques prives de Fructification.

\section{SCLEROTIUII. Mycelium Quiescens.}

2146. SCLEROT. SPHARIEFORME. Lib. - Alb. de l'Est. - Sur tiges mortes, notamment d'Allium capa, parmi les détritus des Jardins. CC. - St-Amand, etc. - Automne. - Mycelium de Pleospora allii.

214\%. SCLEROT. CORNUTUM. Fr. - Alb. de l'Est. - Sur le chapeau et les lamelles de Russula nigricans. - CC. dans les bois de TroisFontaines, Charmont. - Automne. - Mycelium d'une Agaricinèe.

2148. SCLEROT. PUSTULA. D. C. - Alb. de l'Est. - Sur feuilles de Chêne mortes et encore adhérentes aux rameaux. - AC. - St-Lumier. Automne, Hiver. - Selon Léveillé, Mycelium de Peziza candollebana.

2149. SCLEROT. FUNGORUM. Pers.-Alb. de l'Est. - Sur chapeau et lamelles d'Hebeloma crustuliniforme. - AC. - Plantations de Pins. St-Amand. - Été, Automne. - Mycelium de Collybia Tuberosa.

2150. SCLEROT. LACUNOSUM. Fr. - Alb. de l'Est. - Sur chapeau et lamelles d'Agaricinées en décomposition, dans les bois de Pins. -- Se distingue de la précédente espéce par ses tuber- 
cules irréguliers et creusés de lacunes. - AC. - St-Amand, Soulanges. - Été-Automne.

2151. SCLEROT. SCOLYMI. West. - Alb. de l'Est.

- Sur les feuilles anciennes d'Artichaut. - Placé en petits groupes brunâtres près de l'insertion de la feuille sur le talon. - CC. - Jardins. Autoinne. - St-Amand, etc.

2152. SCLEROT. ELONGATUM. Fuck. - Alb. de l'Est. - Sur la nervure principale des feuilles de Chêne tombées à terre. - Elliptique, rougeâtre, rugueux, strié, superficiel. - AC. - Bois de St-Amand. - Printemps.

2153. SCLEROT. LILIACEARUM. Kickx. - Alb. de l'Est. - Sur les tiges de Muscari comosum et sur Ornithogalum pyreneaicum. - CC. Automne. - St-Amand.

2154. SCLEROT. COMPLANATUM. Tode. - Alb. de l'Est. - Sur feuilles humides et pourries de Peuplier et d'Aulne. - Tubercules blanes, aplatis, cunéiformes. - CC. - St-Amand. - Automne.

2155. SCLEROT. PIRINUM. Fr. - Alb. de l'Est.

- Sur l'épiderme des poires pourries à la cave.

-- Tubercules noirs, ronds, irréguliers. - CC.

- Hiver. - Partout.

2156 SCLEROT. SARMENTICOLUM. West. Alb. de l'Est. - Sur jeunes rameaux de Vigne morts, encore adhérents au ceps. - Elliptique. gris-noirâtre. - C'est sans doute une variété de scler. Durum - Il donne naissance au Botrytis cinerea. - CC. - Jardins.

215\%. SCLEROT. SEMEN. Tode. - Alb. de l'Est. 
- Sur les tiges mortes et pourrissantes des légumes et des grandes herbes. - Tubercules sphériques, petits, passant du blanc au noir. chagrinés. - CC. - Hiver-Printemps. - Partout. 2158. SCLEROT. JUGLANDINUM. West. - Alb. de l'Est. - A l'intérieur des écailles de Noix pourries, dans les lieux humides. - AC. Jardins. - St-Amand, Aulnay, etc. - Partout. - Automne, Printemps.

2159. SCLEROT. SCUTELLATUM. Alb. et Schu. - Alb. de l'Est. - Sur feuilles d'Acer et leurs pétioles. - Tubercules ronds, aplatis en scutelle, bruns. - AR. - Bois de St-Amand à St-Lumier. - Mycelium de Typhula Erythropus.

\section{RHIZOCTONIA. - D. C.}

2160. RHIZOCT. MEDICAGINIS. D. C. - Alb. de l'Est. - Sur racines de Medicago Sativa qu'il entoure d'un Mycelium filamenteux, violacé, très abondant et parfois parsemé de périthèces sphériques, noirâtres, stériles. - AC. - Champs. - Automne-Hiver. - St-A mand, etc. - Nuisible. 2161. RHIZOCT. DAUCI. Rebent. - Alb. de l'Est. - Sur racines ues Carottes pourrissantes dans les caves. - Composé d'un Mycelium filamenteux, rougeâtre, moins dense que le précédent et plus rare. - St-Amand. - Hiver.

2162. RHIZOCT. SOLANI. Kühn. - Alb. de l'Est. - Sur les tubercules de Solanum tuberosutn á la cave, dans l'Hiver. - Filaments rameux, anastomosés, bruns-rougeâtres. - Peu développè 
par conséquent moins nuisible que les précèdents. - St-Amand.

\section{RHIZOMORPHA. - Roth.}

2163. RHIZOM. CORTICALIS. - Alb. de l'Ést. Stromes noirâtres, luisants, trẻs allongés, de formes diverses, en filets arrondis, ou comprimés élargis, à rameaux anastomosés; croissant sous l'écorce des vieux troncs cariés. - C'est le Mycelium d'une Agaricinée nuisible Armillaria mellea. - CC. - Bois. - Partout.

2164. RHIzOM. SAMBUCI. Chev. - Alb. de l'Est. - Sur la moelle des rameaux de Sambucus nigra pourris. - Longs filaments brunsnoirâtres, parallẻles et longitudinaux. - CC. dans les jardıns, vieilles haies. - St-Amand. - Automne.

2165. RHIZOM. FRAGILIS. Schmitz. - Alb. de l'Est. - Stromes très allongés, cylindriques, d'un brun-rougeâtre, luisants ; rameaux étalés, alternes, les supérieurs opposés ou verticillés. - C. sur les troncs pourris. - Je pense que c'est une variété moins robuste de Rhiz. corticalis.

2166. RHIZOM. SETIFORMis. - Alb. de l'Est.

- Sur tiges de scirpus lacustris pourrissant.

- Long filament brun-clair, partant de la base du chaume au sommet, émettant des rameaux latéraux parsemés de globules ovales, jaunâtres, tuberculeux. - (conidies?). - AR. - St-Lumier. 
himlantia. - Pers.

216\%. Himantia CANDIDA. Pers. - Alb. de l'Est. - Sur les feuilles et sur le bois pourris mis en tas. - Expansion filamenteuse, blanche. à filaments radiés, plus ou moins membraneuse. - AC. en Automme. - Partout.

2168. HimANTIA CELlaRIS. Pers. - Alb. de l'Est. - Sur les murs et sur la terre des celliers et caves humides. - Tissu membraneux. arachnoïde, brun-noirâtre, composé de filaments partant d'un centre commun. - AC. - Hiver. printemps. - Partout.

\section{HYPHА. - Rabh.}

2169. HYPHA GIGANTEA. Chev. -- Alb. de l'Est. - Sur les vieilles charpentes et dans les plafonds de masures abandonnées. - Plaques très larges, blanches puis jaunâtres, ressemblant à de l'amadou. - $\Lambda$ C. - St-Amand, etc.

21\%. HYPHA ALUTA. Link. - Alb. de l'Est.

- Croît dans les mêmes localités que la précédente espéce, sous forme de plaques très minces, veloutées, jaune.cannelle. - AC.

\section{OZONIUII. - Link.}

21\%1. OZONIUM AURICOMUM. Link. - Alb. de l'Est. - Sur le bois de chêne travaillé, vieux et pourri, charpentes, seaux domestiques, etc. - Houppes fasciculées de filaments orangés, 
raides. - Mycelium d'un Coprin. - AC. Automne. - Partout.

21\%2. OZONIUM AUREUM. - Alb. de l'Est. -

Sur les racines languissantes des arbres fruitiers et autres. - Coussinets velus, de couleur d'or puis rougeâtre. - C'est sans douie le Chroolepus aureus. - AC. - Automne. - StLurnier, St-Amand.

\section{EXANTHEMATA. - Kickx.}

Pseudo-Mycétes.

Aitérations pathologiques dues à des piqures d'Insectes.

\section{PHYLLERIUM. - Fries.}

21\%3. PHYLL. PIRINUM. - Alb. de l'Est. Taches velues', roux-ferrugineux,' assez larges. envahissant la face inférıeure des feuilles du Poirier. - C. - Automne.

21\%. PHYLL. Vitis. - Alb. de l'Est. - Taches velues, blanches, puis rosées, sous les feuilles de Vigne qu'elles déforment sans nuire à la végétation. - CC. - Partout. - Eté.

2175. PHYLL. JUGLANDIS. - Alb. de l'Est. Taches velues, oblongues-rectangulaires, jauneroussâtre, sous les feuilles du Noyer. - CC. - Eté, automne.

2176. PHYLl. TiliaceUm. - Alb. de l'Est. Taches velues, jaunes puis pourpres, sous les feuilles de Tilleul. - CC. - Automne. 
21\%. PHYLL. PADI. - Alb. de l'Est. - Taches velues d'une belle couleur rose, puis orangée; sous les feuilles de Prunus Padus. - AR. Automne.

\section{ERINEUM. - Pers.}

2178. ERINEUM. FAGINEUM. - Alb. de l'Est. Taches velues, blanc-rosé, sous les feuilles de Fagus sylvatica. - C. - Automne.

2179. ERINEUM ALNEUM. - Alb. de l'Est. -

Taches velues, blanches, puis rouge-cinabre sous les feuilles d'Alnus glutinosa. - C. - Eté.

2180. ERINEUM PLATANOIDEUM. - Alb. de l'Est. -. Taches velues, blanches puis ferrugineuses. - Sous les feuilles d'Acer pseudo-platanus. - C. - Eté, automne.

\section{NOSOPHLEEES. - Fries.}

Renflements bulleux de l'épiderme des troncs et rameaux vivants, s'ouvrant par fentes et laissant échapper sous forme de corpuscules pulvérulents, les cellules désagrégées de l'écorce.

2181. NOSOPHLAEA ALNEA. - Sur les trones vivants d'Alnus glutinosa. - Epiderme soulevé en phlyctènes rougeâtres, participant de la coloration des cellules sous-jacentes. - Il n'est pas rare de voir les troncs des pommiers atteints de la même altération pathologique. 



\section{LISTE ALPHABÉtIQUE DES GENRES.}

Chaque nom de Genre est suivi d'un nom d'Espèce-Type dont la Spore est représentée avec l'indication de sa dimension réelle.

PAGES GENRES HPÈCES SPORES ET MESURE

187. ACETABULA. Fuckel. Acet. Acetabulum.

Q 0

121. ACREMONIUM. Sacc. A. Alternatum.

O $0^{m m} \cdot 003$

426. ACROCYLINDRIUM. Sacc. A. Gordæ.

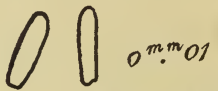

351. ACROSPERMUM. Tode. A. Compressum.

$0 m \cdot 16$

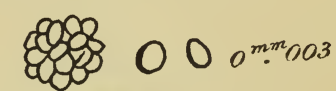

426. ACROStalagmus. Cord. A. Cinnabarinus

460. ACROTHECIUM. Sacc. A. Obovatum

2:31. ACTINOSPIRA. Cord. A. Chartarum.

166. ACIDIUM. Pers. AE. Tragopogonis.

482. ÆGERITA. Pers. $\mathbb{E}$. Candida.

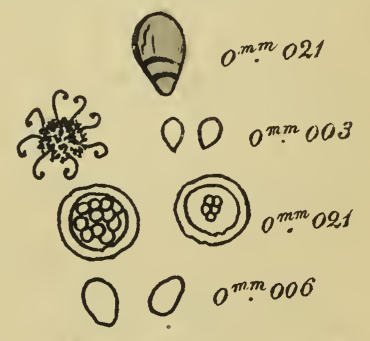

131. ETHALIUM. Link. A. Septicum.

303. AGLAOSPORA. de Not. A. Profusa.

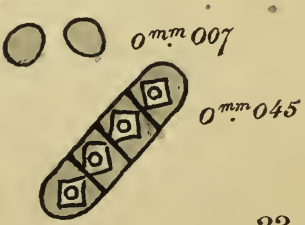


PAGES GENRES ESPECES SPORES ET MESURE

187. ALEURIA. Fries. A. Vesiculosa.

467. ALTERNARIA. Nees. A. Tenuis

25. AMANITA. Quel. A. Muscaria.

419. AMBLIOSPORIUM. Fres. A. Album.

398. AMEROSPORIUM. Speg. A. Caricinum.

289. AMPHISPHARIA. Ces. A. Umbrina.

135. ANGIRIODORUM. Grev. A. Sinuosum.

236. ANIX1A Fries. A. Perichœnoides

259. ANTHOSTOMA. Sacc. A. Gastrina

258. ANTHOSTOMELLA. Sacc. A. Limitata.

194. ANTHRACOBIA Boud. A. Melaloma

478. ANTHROMYCES. Fres. A. Copridis

364. APOSPH ARIA. Berk. A. Pinea.

132. ARCYRIA. Hill. A. Nutans.

30. ARMILLARIA. Q. A. Mellea.
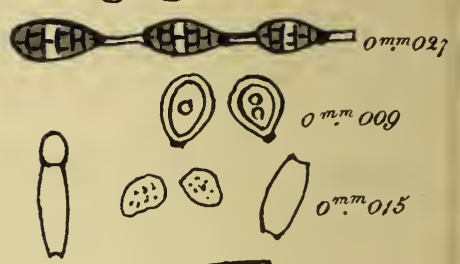

H
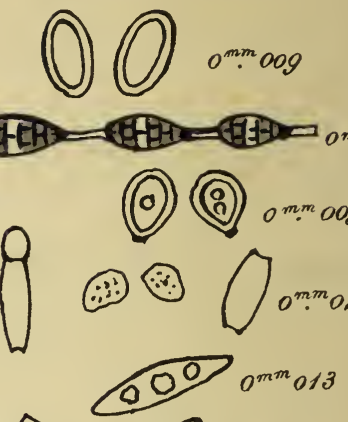

8)

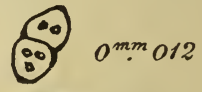

(O) (O) ommol

(0) (0) 0 mmo05

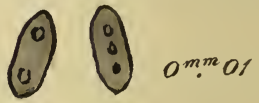

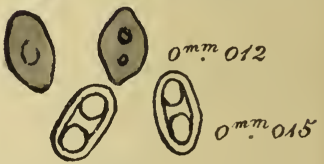

$\square$ 의 $0^{m m} \cdot 013$

(1) $0^{m} \cdot 005^{5}$

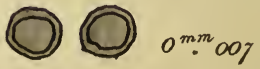

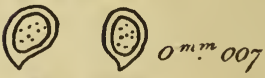

441. ARTHRINIUM. $K \approx e$. A. Caricicolum

479. ARTHROBOTRYUM. Cés. A. Stilboideum

196. ASCOBOLUS. Pers. A. Furfuraceus.

377 ASCOCHYTA Lib. A Philadelphi

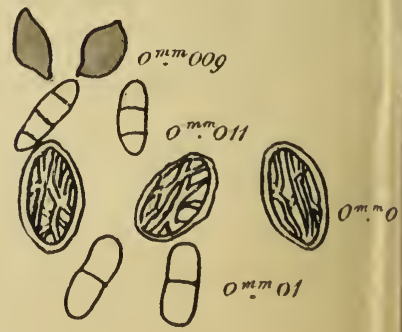


PAGES GENRES ESPĖCS

197. ASCOPHANUS. Boud. A Garneus.

235. ASCOTRICHA. Berk. A. Chartarum.

198. ASCOZONUS. Boud. A. Cunicularis

419. ASPERGILLUS. Mich. A. Glaucus.

235. ASTERINA Lév. A, Scabiosæ.

365. ASteroma. D. C. A. Hyperici.

425. ASTEROPHORA. Ditm. A. Agaricicola,

408. ASTEROSPORIUM. Kze. A. Hoffmanni

348. AULOGRAPHUM. Lib. A. Juncinum.

109. AURICUlaria. Fries. A. Mesenterica.

486. BACTRIDIUM. Kze. B. Flavum.

135 BADHAMIA. Berk. B. Pallida

277. BERTIA. De Not. B. Moriformis

449. BISPORA. Cord. B. Monilioides

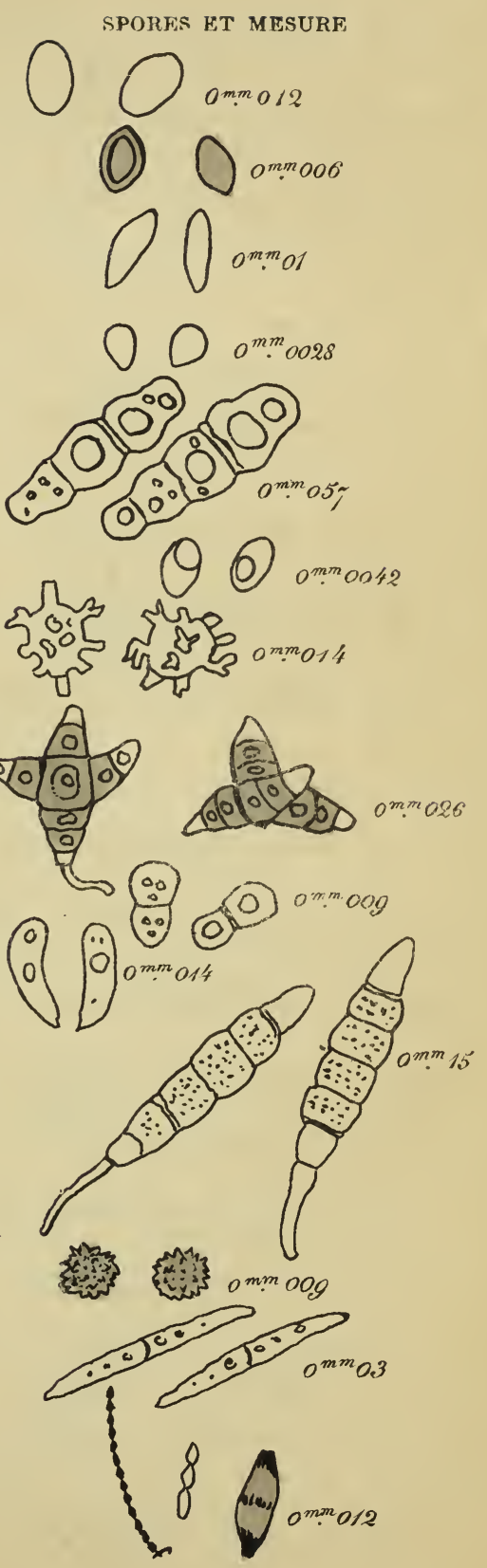


88. BOLETUS. Dill. B. Scaber.

257. BOMBARDIA. Fries. B. Fasciculata

377. BOTRYODIPLODIA. Sacc. B. Juglandicola.
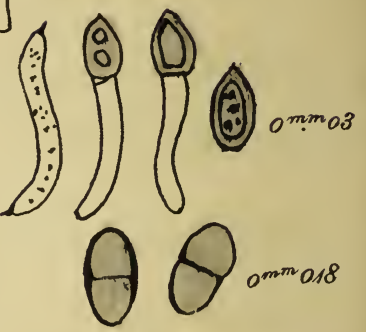

268. BOTRYOSPHARIA. Ces. B. Advena.

417. Botryosporium. Cord. B. Pulchrum.

422. BOTRYTIS. Mich. B. Cinerea

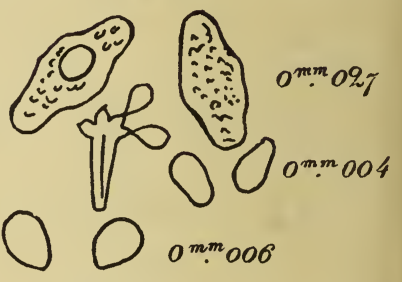

459. BRACHYSPORIUM. Cord. B. Stemphylioides

203. BULGARIA. Fries.-B. Inquinans.

211. CALOSPHARIA. Sacc. C. Cupularis.
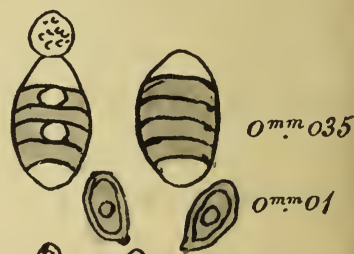

117. CALOCERA. Fries. C. Viscosa.

339. CALONECTRIA. De Not. C. Richoni.

203. CALLORIA. Fries. C. Fusaroides.

190. CALOSCYPHA. Boud. C. Fulgens.

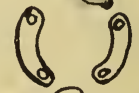
ommor

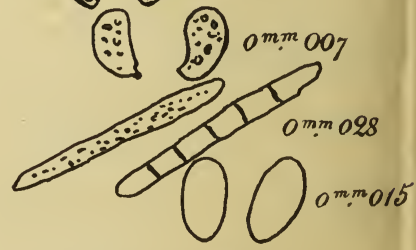

2(0). CALOSPHARIA. Tul. C. Vibratilis.
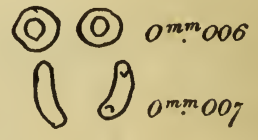

202. CALYCELLA. Boud. C. Citrina.

383. CAMAROSPORIUM. Schlz. C Berberidis.

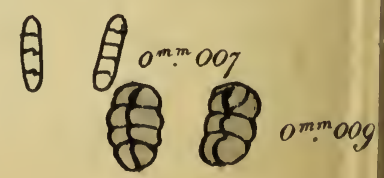


PAGES GENRES ESPÈCES

440. CAMPTOUM. Link. C. Curvatum.

82. CANTHARELLUS. Fries. C. Cibarius.

238. CAPNODIUM. Mont: C. Salicinum

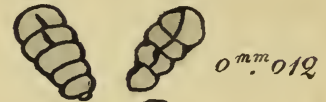

316. CAPRONIA. Sacc. C. Juniperi.

135. CARCERINA. Fries. C. Spumaroides

448. CATENULARIA. Grev. C. Atra.

219. CENANGIUM. Fck . C. Prunastri.

234. CEPHALOTHECA. $F c k^{3}$. C. Sulfurea.
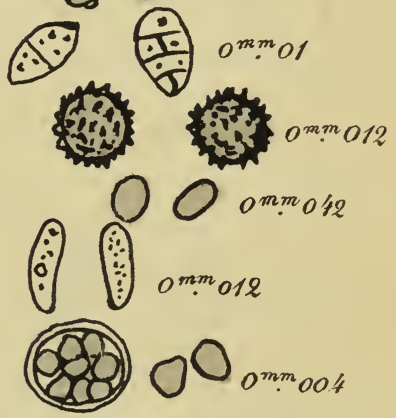

418. CEPHALOSPORIUM. Cord. C. Acremonium.

(0) (0) $0^{m m} \cdot 004$

428. CEPHALOTHECIUM. Cord. C. Tetrasporum

474. CERATIUM. Alb. et Schw. C. Hydnoides

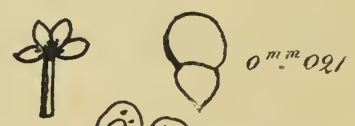

(0.) (4.) $0^{m m} n_{1}$

312. CERATOSPH ERIA. Niessl. C. Lampadophora

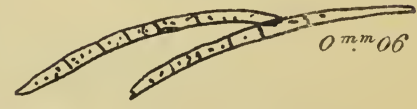

251. CERATOSTOMA. Ces. C. Rosœ.

264. CERATOSTOMELLA. Sacc. C. Rostrata.

459. CERCOSPORA. Eres. C. Viticola.

308. CERIOSPORA. Niessl. C. Xantha.

179. CHATOCLADIUM. Fres. C. Jonesii.

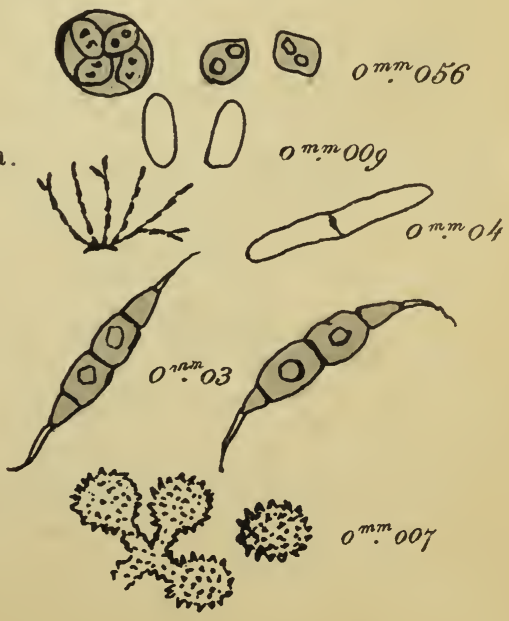


251. СН任OMIUM. Kze. C Chartarum

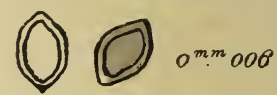

Sup ${ }^{1}$. CHАTOPHOMA. Cooke. C. Antirrhini.

O0 $0^{m m} \cdot 004$

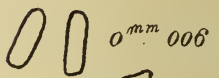

446. CHATOPSIS Grev. C. Grisea.

301. CH ETOSPHAERIA. Tul. C. Callimorpha CHATOSTROMA. Cord. C. Atrum.

448. CHALARA. Cord. C. Strobilina.

193. CHEILYMENIA. Boud. C. Stercorea

206. CHLOROSPLENIUM . Fr. C. Aruginosum

411. CHROMOSPORIUM. Cord. C. Roseum.

205. CIBORIA. Fck C. Amentacea.

366. CICINNOBOIUS Ehr C. Cesatii

193. CILIARIA. Quél. C. Scutellata.

471. CILICIOPODIUM. Cord. C. Sanguineum.

445. CIRCINOTRICHUM. Nees. C. Maculiforme.

451. CLADOSPORIUM. Link. C. Herbarum

453. CLADOTRICHUM. Cord C. Scyphophorum

451 CLASTEROSPORIUM. Schwo C. Fasciculare
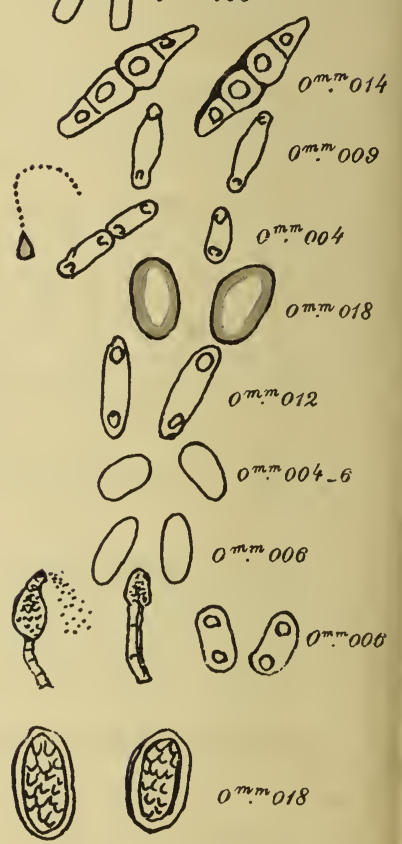

$0000^{m \cdot m} 0042$

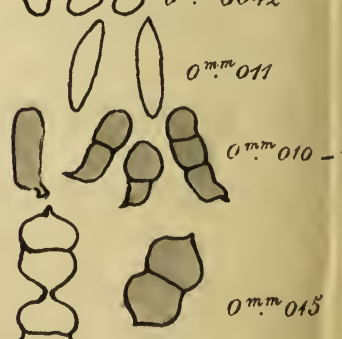




\section{PAGES GENRES ESPÈCES}

51. CLAUDOPUS, Quel. C. Variabilis.

114. ClaVARIA. Linn. C. Botrytis.

342. CLAVICEPS. Tul. C. Purpurea

34. CLITOCYBE. Q. C Suaveolens

49. CLITOPILUS. Q. C. Orcella.

301. CLYPEOSPHARIA. Fck . C. Notarisii.

417. CERANSIELLA. Sacc. C. Alabastrina.

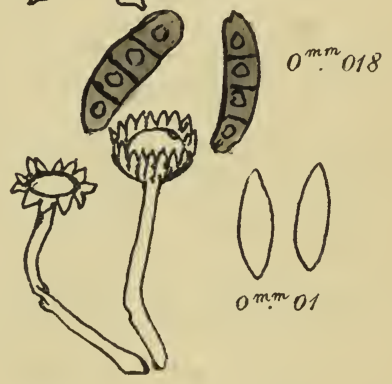
SPORES ET MESURE

Q $0_{0}^{m m} 006$

$\therefore 001$

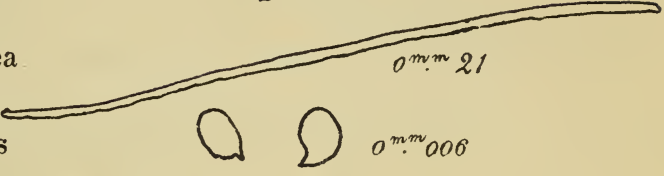

144. COLEOSPORIUM. Lév. C. Miniatum

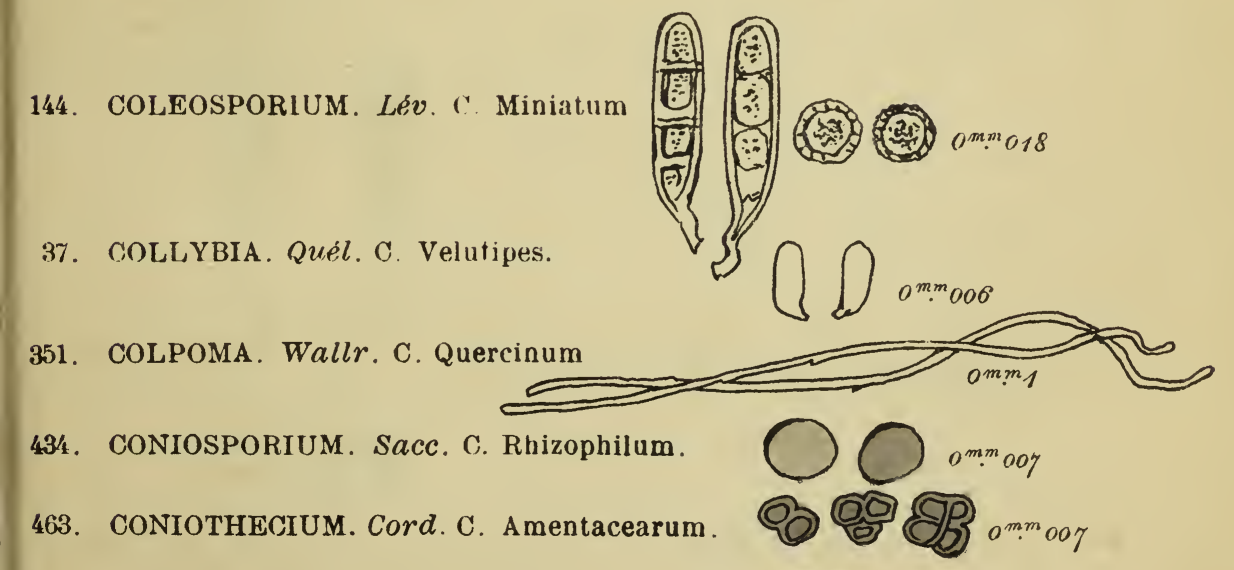

351. COLPOMA. Wallr. C. Quercinum

37. Collybia. Quél. C. Velutipes.

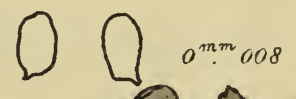

371. ConiothyriUm. Cord. C. Fuckelü.

$0000^{m ! m} m_{003}$

67. COPRINUS. Pers. C. Lagopus. 
PAGESS GENRES ESPLECES

SPORES HT MESURE

195. COPROBIA. Boud. C. Granulata

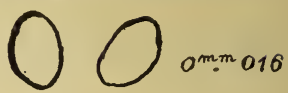

255. COPROLEPA. Fries. C. Merdaria.

342. CORDICEPS. Link. C Ophinglossoides.

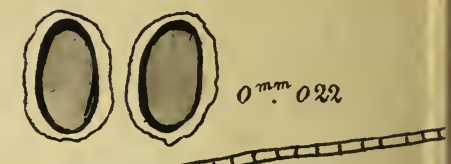

472. COREMIUM. Link. C. Glaucum.

391. CORNILARIA. Karsten. C. Boudieri

109. CORTICIUM. Fries. C. Quercinum.

39. CORTINARIUS. Fries. C. Violaceo Cinereus.

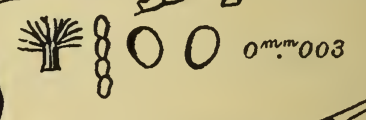

240. CORONOPHORA. F' $k^{\prime}$. C. Annexa.

202. CORYNE. Tul. C Sarcoides

303. CORYNELIAA. Boud. C. Atro-Virens.

406. GORYNEUM. Nees. C. Notarisianum

107. CRATERELlus. Hries. C. Cornucopioides.

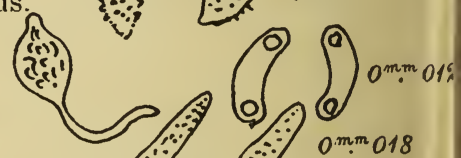

138. CRATERIUM. Fries. (. Leucophalum.

61. CREPIDOTUS. Q. C. Mollis.

133 CRIBARIA Schr. C. Vulgaris.

171. CRONARTIUM. Tul. C. Pæonix

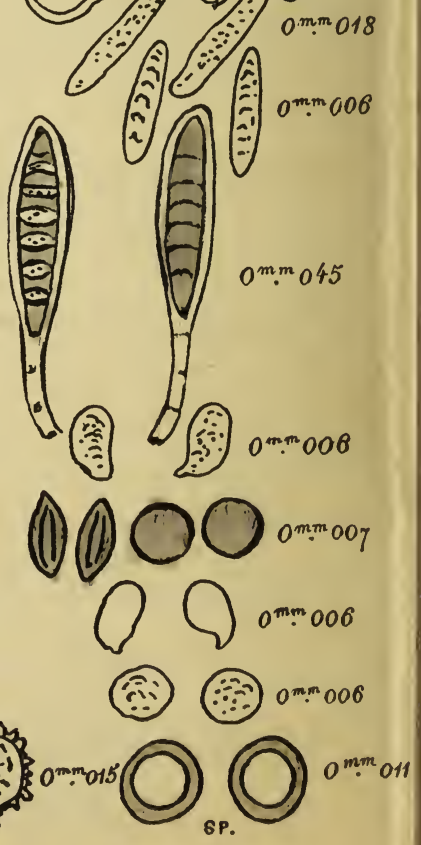


47. CRYPTOSPHÆRIA. Grev. C. Populina.

28. CRYPTOSPORA. Tul, C. Suffusa.

39. CRYPTOSPORELlA. Sacc. C. Hypodermia.

3. CRYPTOSPORIUM. Cord. C. Neesii.

i2. CRYPTOSTICTIS. Fick! C. Cynosbati.

8. CRYPTOVAlsa. Ces. C. Pruni.

7. CUCURBITARIA. Gray's. C. Elongata

9. CYATHICUla. De Nopt. C. Coronata.

4. CYATHUS. Hall. C. Striatus.

4. CYLINDRIUM. Bon. C. Flavo.Virens.

4. CYLINDROCELla. Bon. C. Dubia.

3. CYLINDROSPORIUM. Unger. C. Ranunculi.
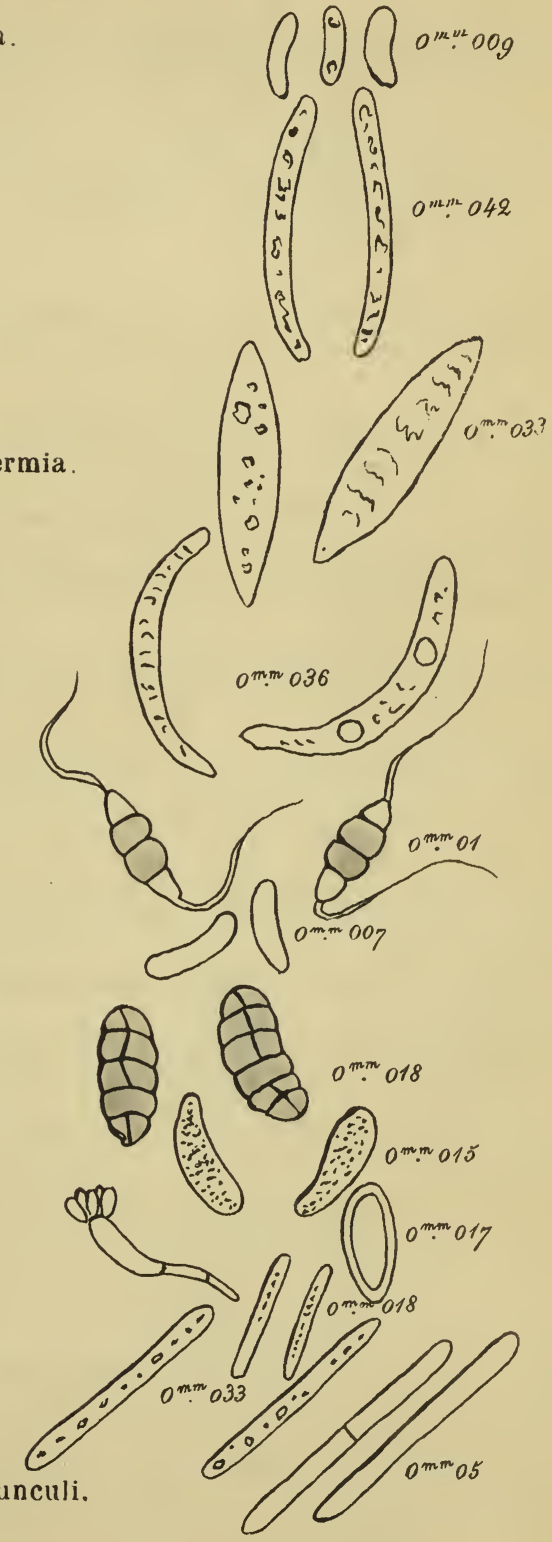
PAGES

GENRES

ESPE⿱CECES

SPORES ET MESURE

420. CYLINDROTRICHUM. Bon Oligospermum

125. CYNOPHALLUS. Fries. C. Caninus.

111. CYPHELlA. Fries. C. Muscigena.

175. CYSTOPUS. De By. C. Candidus.

369. CYTOSPORA. Ehr. C. Leucosperma.

Sup'. CYTOSPORELLA. Sacc. C. Conspersa.

391. CYTOSPORINA. Sacc. C. Heteracantha.

Sup'. CYSTOTRICHA. B. et Br. C. Striola.

122. DACRYMYCES. Nees. D Fragiformis.

100. DEDALAA. Fries. D. Unicolor.

430. DACTYLIUM Nees. D. Dendroides.

264. DALDINIA. De Not. D. Concentrica.

378. DARLUCA. Cast. D. Vagans.

209. DASYSCYPHA. Boud D. Palearum.

290. DELITSCHIA. Auersw. D. Auerswaldii.

244. DEMATIUM. Pers. D. Hispidulum.

481. DENDRODOCHIUM. Bon. J). Citrinum

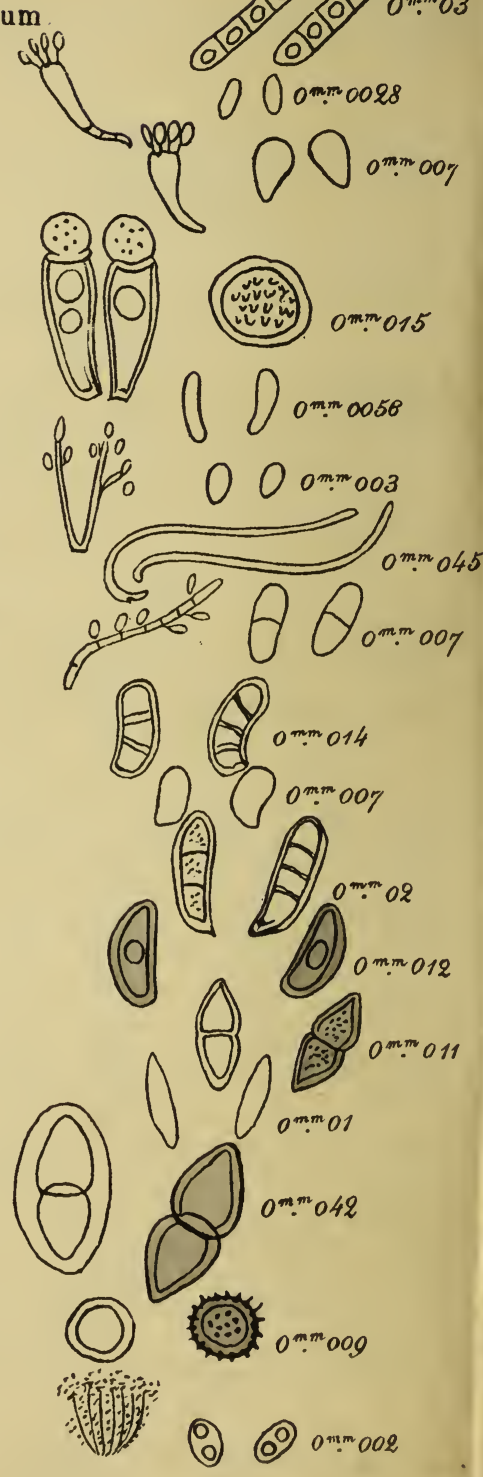




\section{PAGES \\ GENRES \\ ESPÈCES}

364. DENDTOPHOMA. Sacc. D. Pruinosa.

461. DENDRYPHIUM. Wallr. D. Atrum.

Sup'. DEPAZEA. Fries. D. Tremulæcola.

218. DERMATEA. Fries. D. Furfuracea.

280. DIAPORTHE. Nits. D. Quercina. 218. IJATRYPE. Fries. D. Acericola. 249. DIATRYPELLA. Ces. D. Minuta. 349. DICHENA. Fries. D. Strobilina

384. DICHOMERA. Cooke. D. Tiliæ.

449. DICOCCUM. Cord. D. Uniseptatum

133. DICTYDIUM. Schr. D. Umbilicatum .

46\%. DICTYOSPORIUM. Cord. D. Elegans.

136. DIDERMia. Pers. D. Gilobosum.

129. DIDYMOPSTS. Sacc. D. Spicata.

274. DIDYMFILA.A. sacc. औ) Bryonia.

137. DIDVMIUM. Schrad. D. IJemisphæricum. 288. DIDYMOSPILARTA. Sacr. n. Acerina.

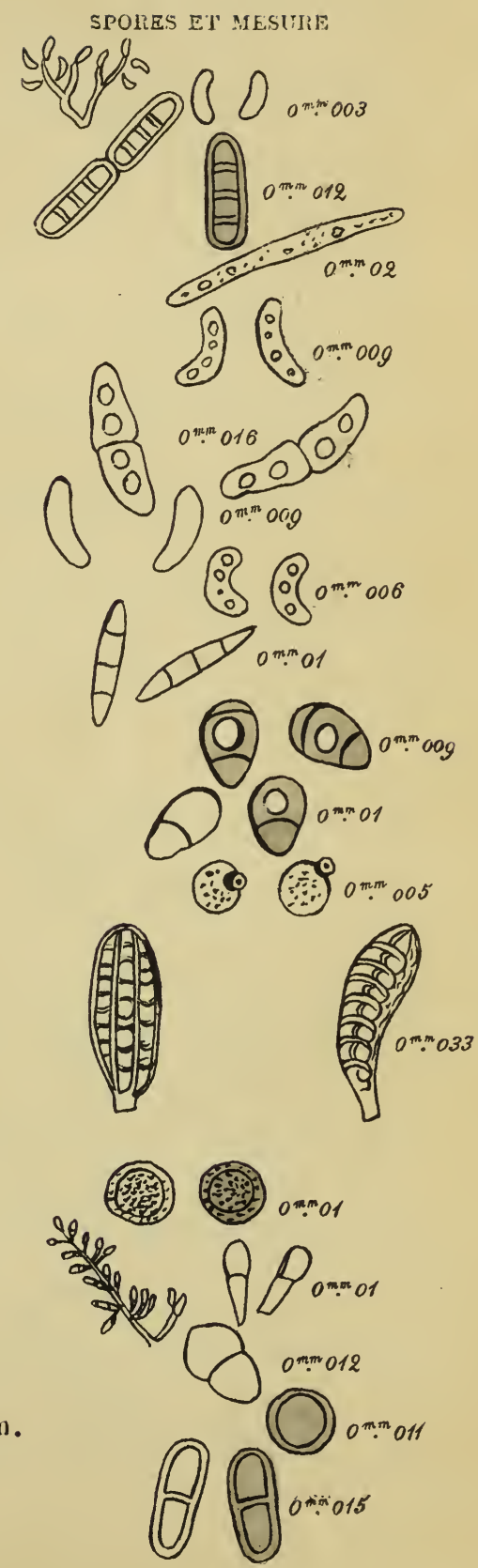


PAGES GENRES ESPÈCES

405. DIDYMOSPORIUM. Sacc. D. Carpini.

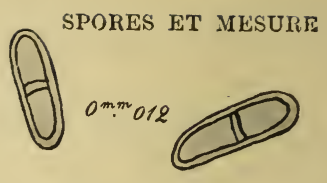

327. DILOPHIA. Sacc.

391. DILOPHOSPORA. Desm. D. Graminis.

405. DINEMASPORIUM. Sacc. D. Graminis.

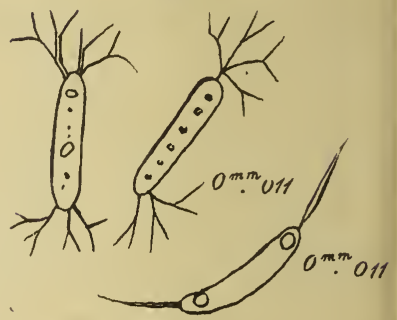

238. DIMEROSPORIUM. Sacc.

127. DIPLOCLADIUM. Bon. D. Minus.

453. DIPLOCOCCIUM. Grev. D. Pulchrum.

372. DIPLODIA. Fries. D. Populina.

377. DIPLODIELLA. Karst. D. Fibricola.

379. DIPLODINA. West. D. Salicis.

398. DISCELLA. Sacc. D. Carbonacea.
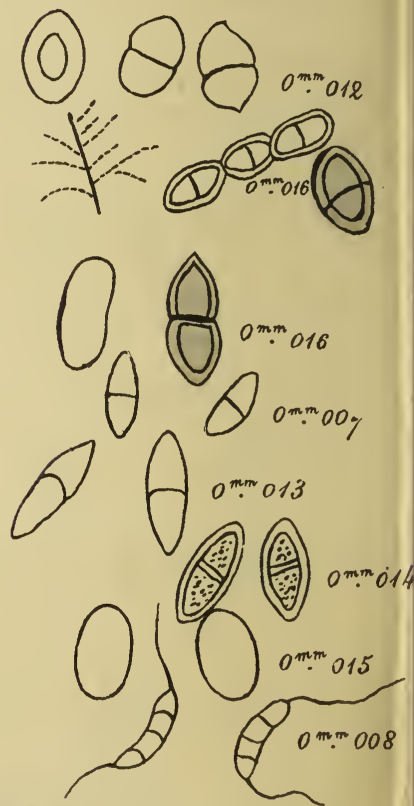

267. DITOPELLA. De Not. (Voir Cryptospora Suffusa. p. 328).

186. DIscIotis. Boud. D. Venosa.

396. DISCOSIA. Lib. D. Artocreas.

398. DOTHICHIZA. Lib. D. Populea.

344. DOTHIDEA. Sacc. D. Puccinioides.

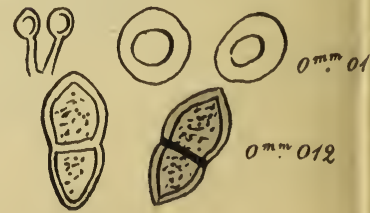


PAGES GENRES ESPËCES

367. DOTHIORELLA. Sacc. D. Advena.

$\left\{\begin{array}{l}\text { 126. ECCHYNA. Fries. E. Faginea. } \\ \text { 472. }\end{array}\right.$

438. ECHYNOBOTRYUM. Cord. E. Parasitans.

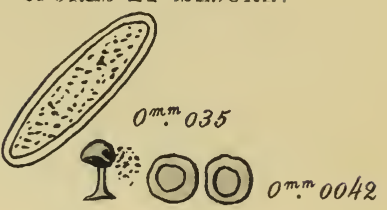
Nob $\therefore$ EN $\because \because 0$ ommo1

51. ECCILIA. Quél. E. Polita.

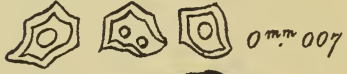

226. ELAPHOMYCES. Tul. E. Aculeatus.

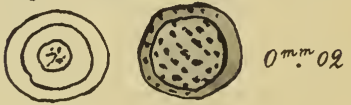

331. ELEUTHEROMYCFS. Fuck. (Voir Sphæronema Subulatum Suppl.)

239. ENCHNOA. Eries. E. Friesii.

$\left(\begin{array}{l}0 \\ 0 \\ 0\end{array}\right) \quad \begin{aligned} & 0 \\ & 0 \\ & 0 \\ & 0\end{aligned} 0^{m \cdot n} 012$

311. ENCHNOSPH ÆRIA. Fuck'. E. Pinetorum.

144. ENDOPHYLLUM. Lév. E. Persoonii.

19. ENTOLOMA. Quél. E. Rhodopolium.

๕96. ENTOMOSPORIUii. Lév. E. Mespili.

342 EPICHLOE. Tul. E. Typhina.

489. EPICOCCUM. Link. E. Neglectum

121. EPIDOCHIUM. Fries. E. Atro.Virens.

499. ERINEUM. Pers. E. Alneum.

278. ERIOSPHARIA. Sacc. E. Alligata.

231. ERYSIPHE. Lév. E. Umbelliferarum.

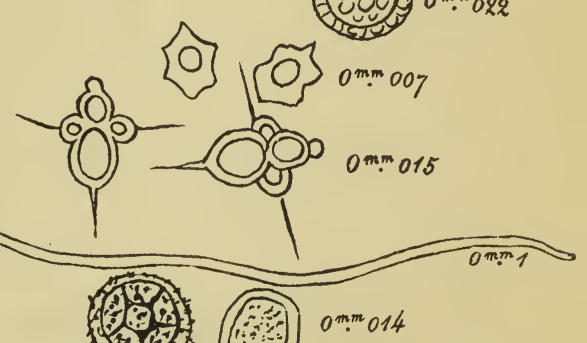


PAGES GENRES ESPECES

233. EUROTILM. Link. E. Lipixylon.

245. EUTYPA, Tul. E. Aspera.

SPURES ET MESURE

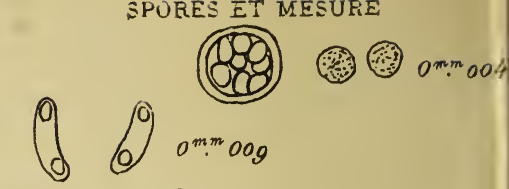

245. EutyPELLA. Nits. E. Prunastri

123. EXIDIA Fries. E. Recisa.

180. EXOAsCUS. De By. E. Alni.

492. EXOSPORIUM. Link. E. Tiliæ.
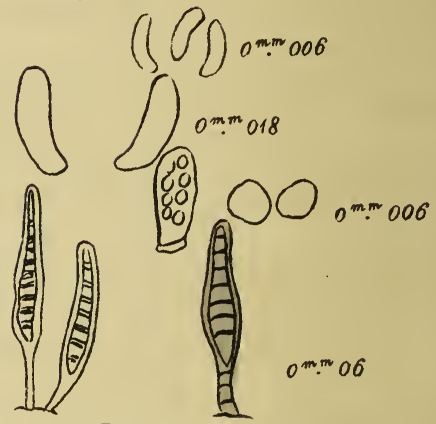

321. FENESTELLA. Tul. F. Princeps.

93. FISTULINA. Bull. F. Hepatica.

57. FI AMMLLA. Quél. F. Conissans.

447. FUCKELINA. Sacc. F. Microspora.
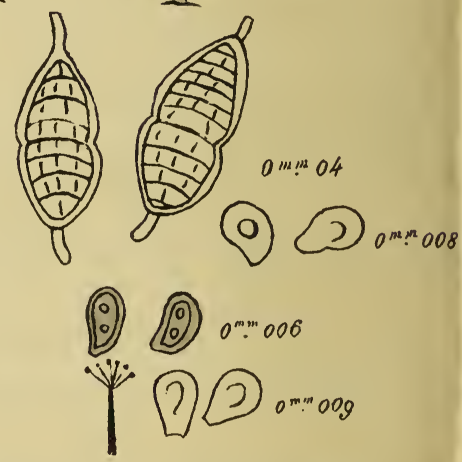

468. étimago. Pers. F. Vagans.

48\%. FUSARIUM. Link. F. Roseum.

450. FUSICLAl)IUM. Bon. F. Pirinum.

363. PUSICOCCUM. Cord. F. Kunzeanum.

482. FUSICOLIh, Bon, F. Phraguitis.

414. FUSIDIUM. Link. F. Parasiticum.

188. GaLACTINIA. Corke. G. Succosa.
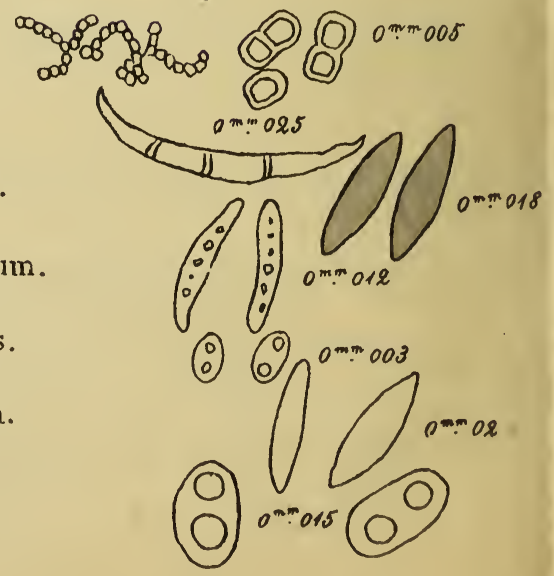


\section{PAGES GENRES ESPECES}

60. GALERA. Ouél. G. Tenes.

128. GEASTER. Micil. G. Hygrometricus.

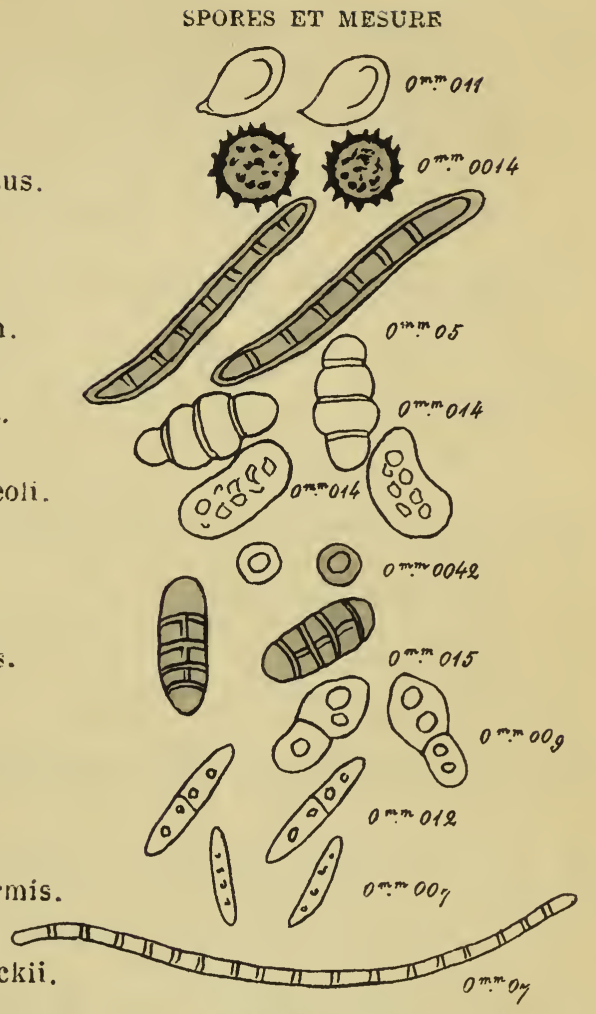

199. GEOGLOSSUM. Pers G. Glabrum.

339. GIEBERELLA. Sacc. G. Acervalis.

401. GLAOSPORIUM. Desm. G. Phaseoli.

127. GLO3ARIA. Rers. G. Gigantea

34.9. GLONIOPSIS. De Not. G. Biformis.

318. GLONIU.M. Mich. G. Pygmæum.

275. GNCNONIA. Ces. G. Ischnuslyla.

264. GNOMONIELLA. Sacc. G. Tubiformis.

352. GODRONIA. C\%. K. G. Mühlenbeckii.

397. GODRONIELLA. Karst G. Juncina

73. GOMPIIIDIUS. Fries. G. Viscidus.

426 GONATOBOTRYS Cord. G. Flava.

111. GONIOSPORIUM. Link G. Pucccinioides.

44i GONYTRICHUM. Nees. G. Cæsiurn

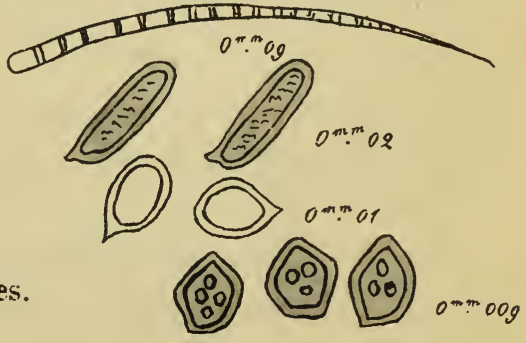

106. GRANDINIA. Fries. G. Crustosa

() (2) om: oors

Q $000 \% \cdot 0028$

171. GRAPHiOLA, Poit. G Phænicis.

$0 \bigcirc 00003$ 
PAGES GENRES ESPÈCES

4i7. GRAPHIUM. Cord. G. Rigidum.

488. GYROCERAS. Cord. G. Plantaginis.

184 GYROMITRA. G. Esculenta.

224. $\left\{\begin{array}{l}\text { HABROSTICTIS. Fuck. } \\ \text { OCELLARIA. Tul. }\end{array}\{\right.$ Aurea.

443. HADOTRICHUM. Fuck'. H. Phragmitis.

400. HAINESIA. Ell. H. Rubi.

420. HAPLARIA. Link. H. Grisea.

444. HAPLOGRAPHIUM. Br. H. Toruloides.

417. HAPLOTRICHUM. Link. H. Capitatum.

477. HARPOGRAPHIUM. Sacc. H. Fasciculatum.

56. HEBHLOMA. Quél. H. Crustuliniformis.

469. HELICOSPORIUM. Nees. H. Mulleri.

433. HELICOMYCES. Link. H. Roseus.

40̄7. HELMINTHOSPORIUM. Link. Appendiculatum
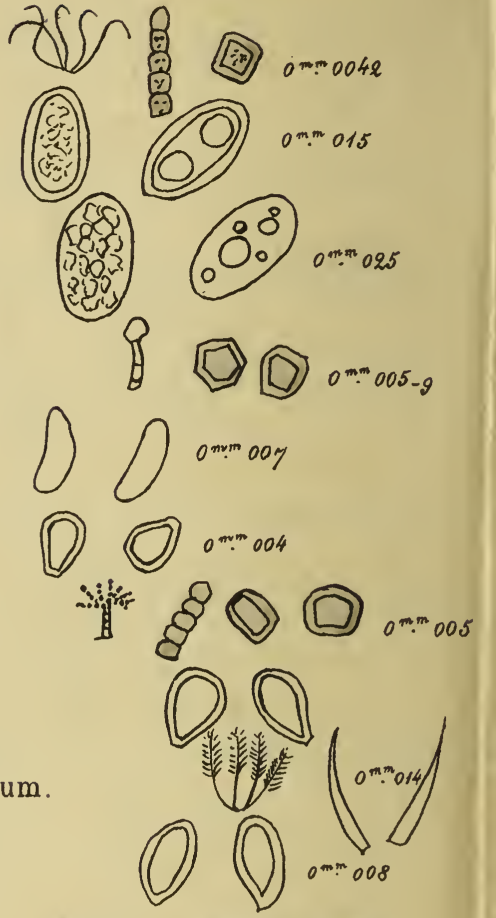

\& $000.005-9$

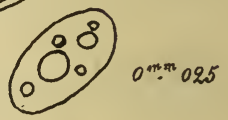

(O) 0 m.m 015 $0 m: 0042$

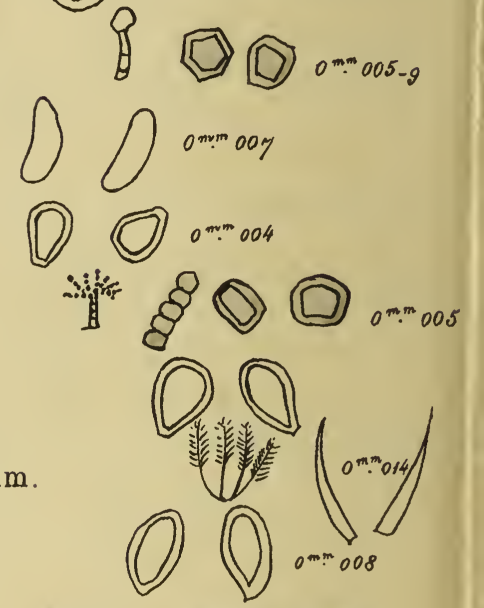

SPORES ET MESURE
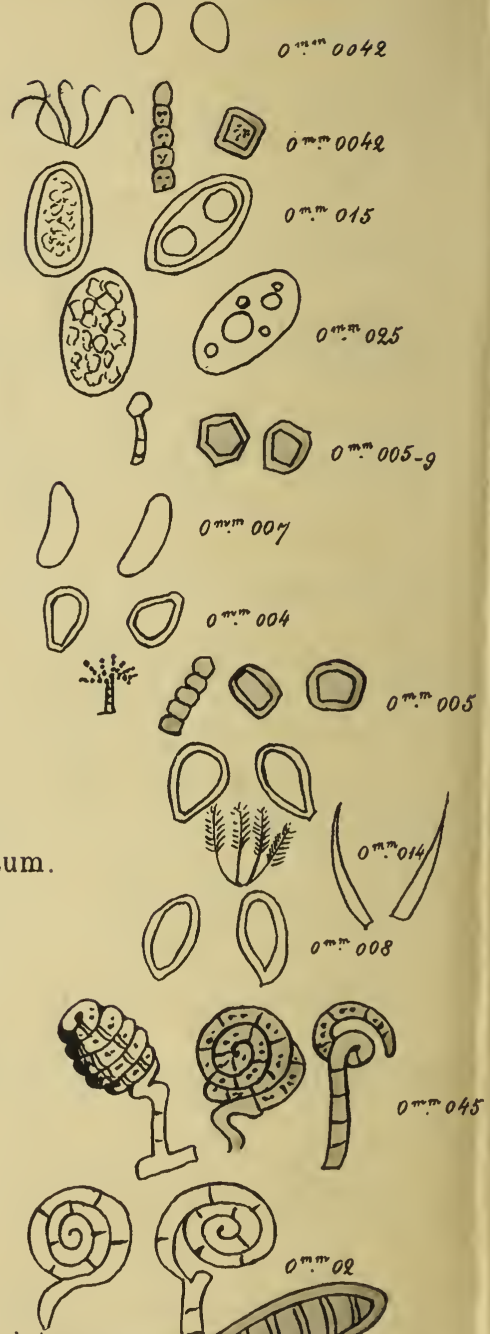

IIIIIII $0=011$

252. HELMINTHOSPHARIA. Fuck, H.Clavariarum.

207. HELOTIUM. Fries. H. Alniellum. 
PAGES GENRKS SSPËCES SPORES ET MESURE.

185. HELVELLA. Linn. H. Crispa.

379. HENDERZONIA. Berk. H. Syringæ.

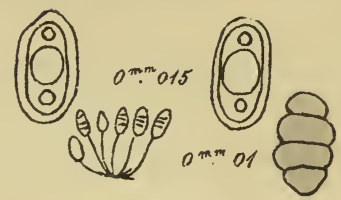

397. HETEROPATELLA. Fuck. H. Lacera

217. Heterospharia. Grev. H, Patella.

280. HERCOSPORA. Tul. H. Tiliæ.

460. HETEROSPORIUM. Klots. H. Ornithogali.

200. HEYDERIA. Fries. H. Cucullata.

497. HyMantia. Pers. Mycelium stérile.

103. HYDNUM. Linn. H. Ernaceum

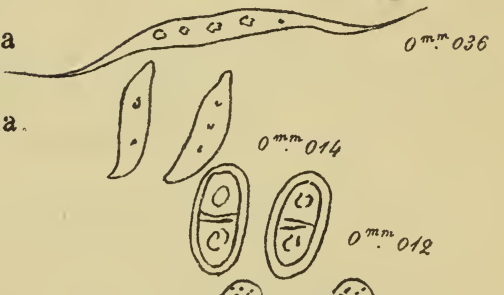

i4. HYGROPHORUS. Fries. H. Virgineus

123. HIRNEOLA Fries. H. Auricula-Judoe

430. HORMIACTIS, Preuss. H. Alba

436. HORMISCIUM. Kunz. H. Vermiculare.
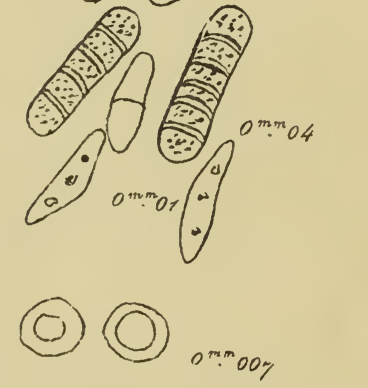

444. HORMODENDRON. Bun. H. Olivaceum.
194: HUMARIA. Fries. H. Leucoloma.
201. HYALINA. Boud. H. Crystallina. 
PAGES GENRES ESPL̀CES

213. HYALOSCYPHA. Boud. H. Dentata.

129. HYMENOGASTER. Witt. H. Leptoniæsporus.

490. HYMENOPSIS. Sacc. G. Ellipsospora.

483. HYMENULA. Eries. G. Rubella.
SPORES ET MESURE.

$\bigcap \Omega$

497. HYPHA. Rabh. H. Gigantea. Mycelium stérile.

63. HYPHOLOMA. Quel. H. Fascicularis.

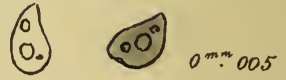

111. HYPOCHNUS. Froies. H. Ferrugineus.

254. HYPOCOPRA. Fuck. H. Maxima.

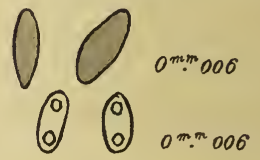


PAGES

GENRES

55. INOCYBE. Quel. I. Dulcamara.

106. IRPEX. Fries. I. Fusco-Violaceus.

473. ISARIA. Pers. I. Farinosa.

Sup'. KALMUSIA. Niessl. K, Hypotephra.

192. LACHNEA. Fries. I. Mollissima.

211. LACHNELLA. Fries. L. Prasina.

76. LACTARIUS. Fries. I. Volemus.

265. LAESTADIA. Auersio. L. Carpinea.

195. I.AMPROSPORA. De Not. I. Wrightii.

197. LASIOBOLUS. Boud. L. Pilosus.

309. I.ASIOSPHARIȦ. C'es. L. Crinita.

217. IECANIDIUM. Rabh. L. Atrum.

85 I.ENTINUS. Fries. L. Tigrinus.

277. LENTOMITA. Niessl. L. Auerswaldii.

87. LENZITES. Fries. L.

137. LEOCARPUS. Pers. L. Vernicosus.

æ01. LEOTIA. Bull. L. Lubrica.

28. LEPIOTA. Quél. i. Procera.

50. LEPTONIA. Quél. L. Euchlorus.
SPORES ET MESURE.

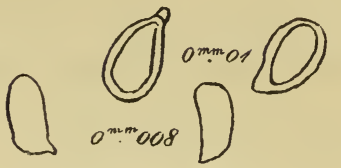

$000^{m ! n} 002$
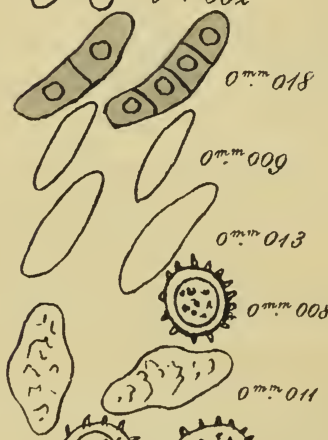
6. $\cdots$ ?
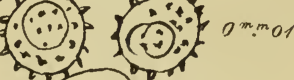

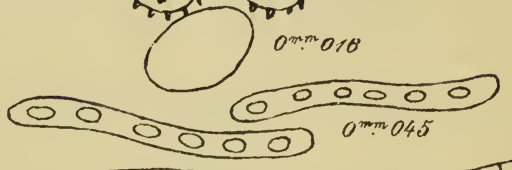

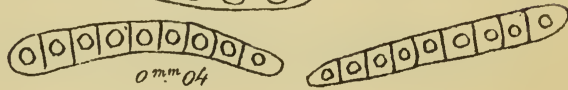
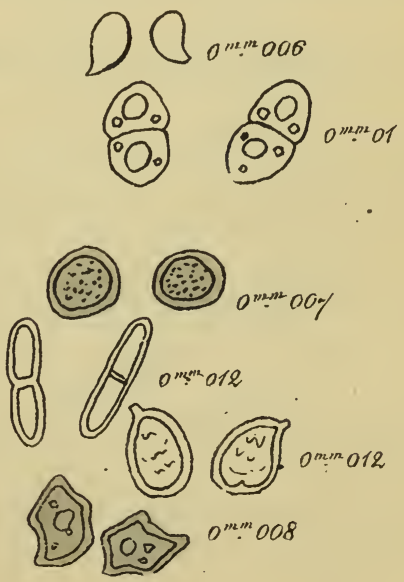
PAGES GENRE

SPORES ET MESURE

185. I.EPTOPODIA. Boud. L. Elastica.

20c. LEPTOGLOSSUM. Cooke. L. Olivaceum.

295. IAPTOSPH ERIA. C'es.' L. Culmifraga

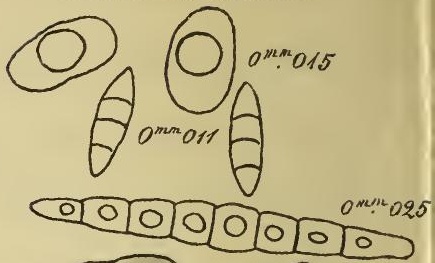

300. LEPTOSPH ERITES. Ch. R. I. Lemoinii

39\%. LEPTOSTROMA. Fries. I. Scirpinum

397. I.EPTOSTROMELLA. Sacc. L. Junci

393. LEPTOTHYRIUM. Kunz. L. Protuberans.

338. LETENDRFA. Sacc. L. Eurotioides.

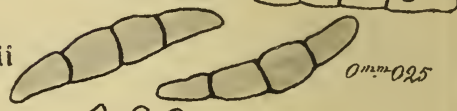

$00 \sqrt{0,212003}$

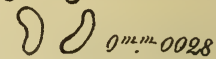

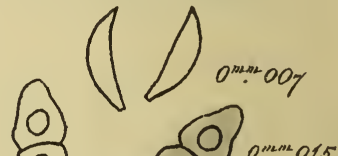

191 I.EUCOSCYPItA. Boud, I. I.encotrichi

403. LIBERTELLA. Desm. I. Fagınea.

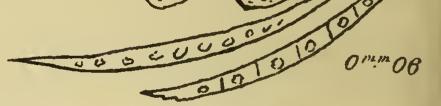

336 LINOSPORA. Fuck. I. Capres:

$0^{m ! 12} 135$

347. LOPHIDIIIM. Sace. I.. Compressum.

348. LOPHIOSPHERA. Trev. L. Fuckelii.

347. LOPHIOSTOMA. Ces. L. Quadrinucleatum

346. LOPHIOTREMA. Sacc. L. Crenatum.
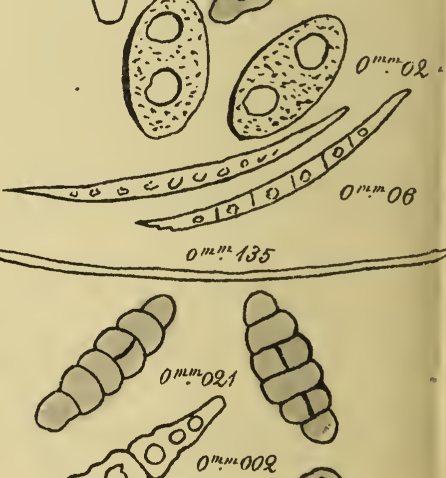
PAGES GENRES ESPECES SPORES ET MESURE.

35\%. LOPHODERMIUM. Chev. L. Arurdinaceum

130. LYCOGALA. Mich. L. Epidendron.

187. MACROPODIA. Fuck. M. Macropus.

465. MACROSPORIUM. Fries. M. Legenarix.

83. MARASMIUS. Fries. M. Urens.

405. MARSONIA. Eisch. M. Potentillæ.

293. MASSARIA. De Not. M. Pupula

306. MASSARINA. Sacc. M Eburnea.

343. MAZZANTIA. Mont. M. Galii

14i). MELAMPSORA. Tul. M. Lini.

291. MELANCONIELLA. Sacc. M. Spodiæa

979. MELANCONIS, Tul. M. Alni.

404. MELANCONIUM. Link. M. Ramulorum.

30?. MELANOMMa. Nits. M. Vile.
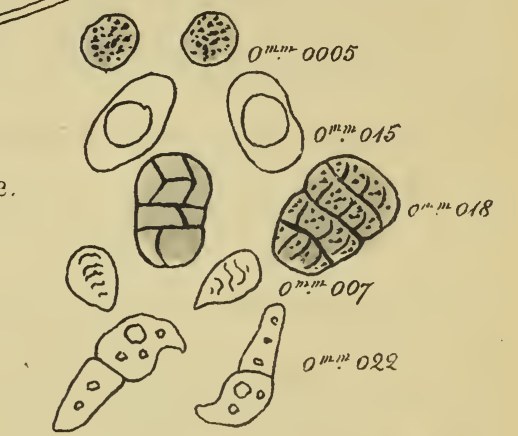

276. MELANOPSAMMA. Niessl. M. Pomiformis.

331. MELANOSPORA. Cord. M. Vervecina.

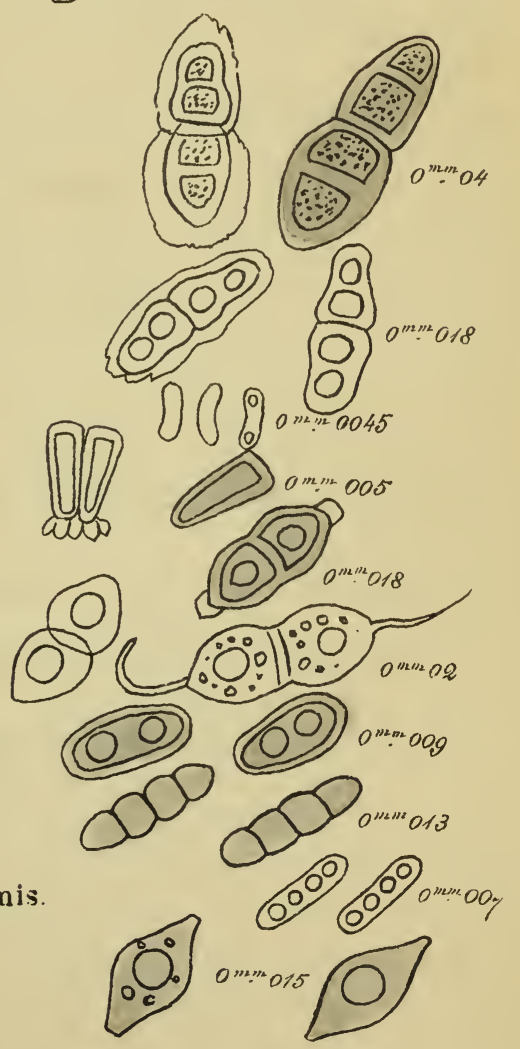


PAGES GENRES FSPL̀CES

402. MELANOSTRoma. Cord. M. Minutum.

394. MELASMIA. Lév. M. Acerina.

194. MELASTIZA. Boud. M. Miniata.

305. MELOGRAMMA. Tul. M. Vagans.

311. MELOMASTIA. Nits. M. Friesii.

446. MENISPORA. Per's. M. Ciliata.

101. MERULIUS. Eries. M. Lacrymans.

306. METASPHERIA. Sacc. M. Sæpincola.

200. MICROGLOSSUM. Sacc. M. Viride.

392. MICROPERA. Lév. M. Drupacearum.

230. MICROSPH ERA. Lév. M. Grossulariæ.

412. MICROSTROMA. Niessl. M. Album.

345. MiCROThyriUm. Desmi. M. Visci. Ch.

184. MiTROPHORA. Lév. M. Semilibera.

215. MOLLISIA. Fries. M. Cinerea.

414. MONILIA. Pers. M. Fructigena.

442. MONOTOSPORA. Cord. M. Sphærocephala

183. MORCHILLA. Dill. M. Esculenta.

179. MORTIERELLA. Coem. M. Polycephala.
SPORES ET MLSURE
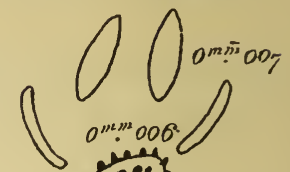

0"'?":006
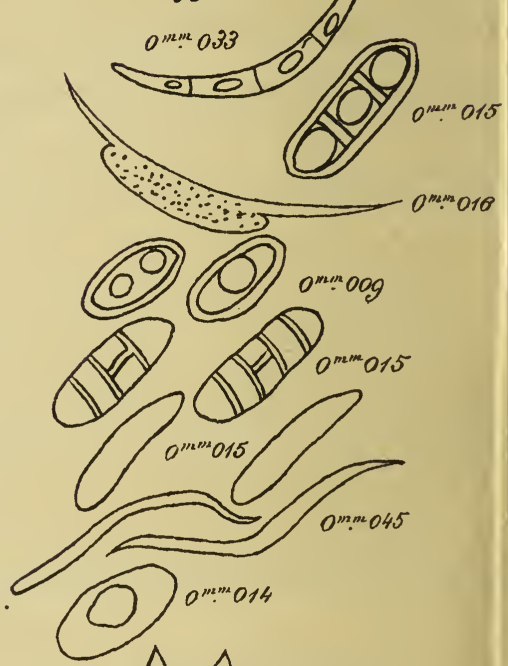

(0) (0) $0^{m 2 m} 005$
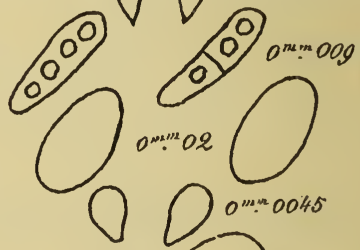

on:m 02

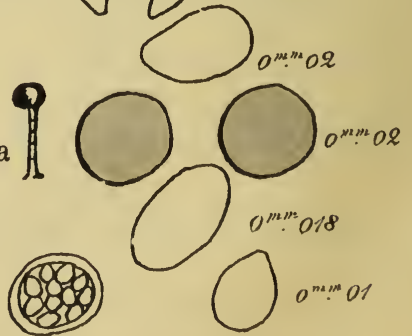

0000045 
ESPÈCES

SPORES ET MESURE.

175. MUCOR De By. M. Mucedo.

40. MYCENA. Quél. M. Galericulata.

364. MYCOGALA. Rost. M. Parietinum.

129. MYCOGONE. Ditm. M. Rosea.

491. MYROTHECIUM. Tode. M. Cinctum.
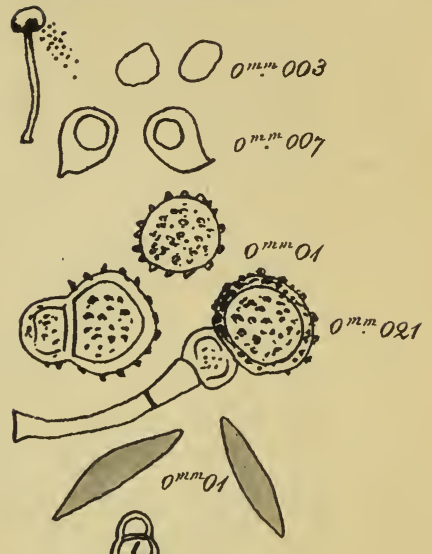

466. MYSTROSPORIUM. Cord. M. Stemphylium.

402. MYXOSPORIUM. Link. M. Deplanatum

440. MYXOTRICHUM. Knnz. M. Chartarum.

58. NAUCORIA. Quél. N. Melinoides.

334. NECTRIA. Fries. N. Sinopica.

330. NECTRIELLA. Sacc. N. Rousseliana.

404. N\&MASPORA. Pers. N. Microspora.

50. NOLANEA. Quél. N. Pascua.
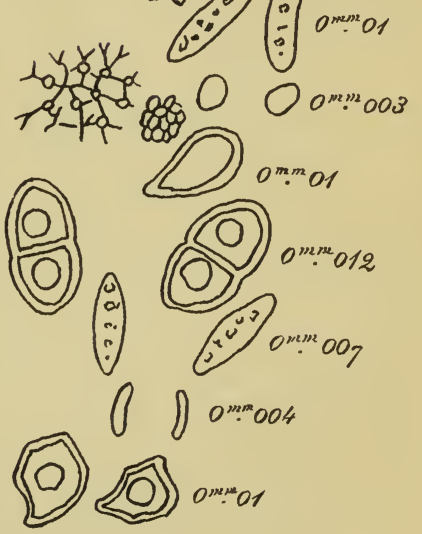

499. NOSOPHLAA. Fries. N. Alnea. altération du tissu cellulaire.

83. NYCTALIS. Fries. N. Asterophora.

$\bigcirc 00.0^{m, 2} 003$

224. OCELLARIA. Tul. O. Aurea. (Voir Habrostict.is.)

416. AEDOCEPH ALUM. Preuss. CE. Agaricinum.

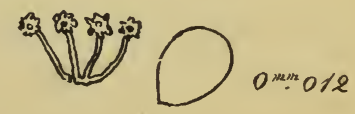


PAGES GENRES RSPECES

415. OIDIUM. Link. O. Tuckeri.

202. OMBROPHILA. Fries. O. Purpurascens.

181. ONIGENA. Pers. O. Corvina.

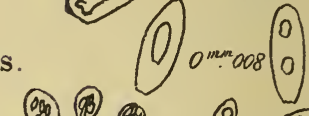

SPORES ET MESURE
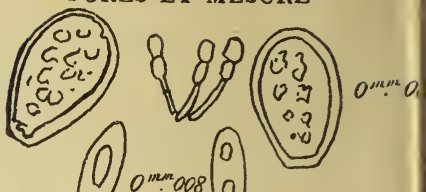

43. OMPHALIA. Quél. O. Fibula.

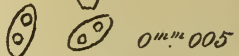

227. OOGASTER. Cord. O. Cibarium.

341. OOMYCES. $B$, et $B r$. O Carneo-Albus

412. OOSPORA. Wallr. O. Epimyces.

322. OPHIOBOLUS. Riess. O. Hyperici.

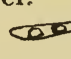

327. OPHIOCERAS. Sacc. O. Gorni. Ch. R.
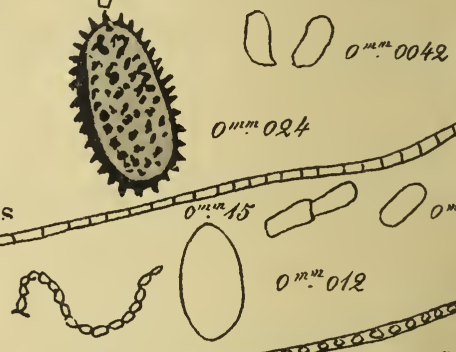

204. ORBILIA. Fries. O. Rozei.

$\bigcirc \bigcirc 0$

351. OSTROPA. Fries. (). Cinerea.

181. OTIDEA. Fuck. O. Onotica.

291. OTTHIA. Nits. O. Brunaudiana.

432. OVULARIA. Sacc. O. Lamii.

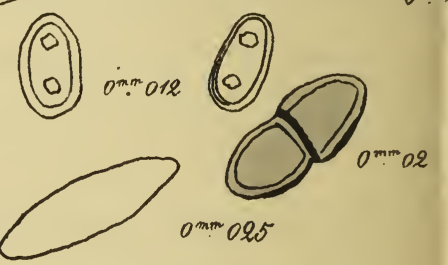

498. OZONIUM. Link. O. Aurantiacum. Mycelium de Coprin.

66. PANAEOLUS. Quél. P. Papillonaceus.

86. PANUS. Fries. P. Rudis.

449. PASSALORA. Mont. P. Baccilligera.

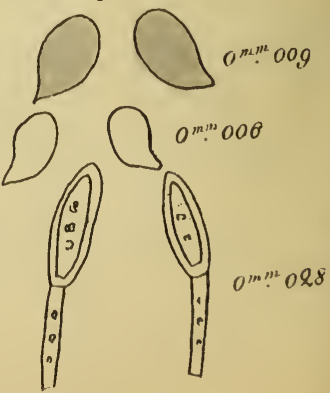


1GES GENRES ESPÈCES

SPORES ET MESURE

73. PAXILLUS. Fries. P. Atro-Tomentosus.

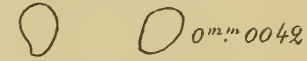

419. PENICILLIUM. Link. P. Glaucum.

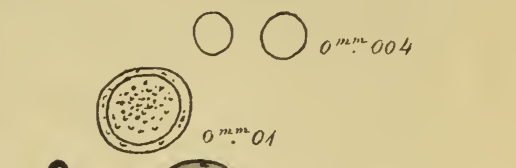

183. PERICHÆNA. Fries. P. Populina.

439. PERICONIA. Bon. P. Atra,

143. PERIDERMIUM. Tul. P. Pini.

185. PERIOLA. Fries. P. Tomentosa.

237. PERISPORIUM. Fries. P. Fimeti.

173. PERONOSPORA. De By. P. Viticola.

108. PeStalozZia. De Not. P. Funerea.

319. PEZICULA. Fuck. P. Cratægi.

223. PHACIDIUM. Fries. P. Repandum.

125. PHALLUS. Linn. P. Impudicus.

206. PHIALEA. Fries. P. Firma.

189. PHLÆOSPORA. Wallr. P. Ulmi.

.08. PHLEBIA. Fries. P. Radiata.

51. PHOLIOTA. Quél. P. Cylindracea

157. PHOMA. Fries. P. Herbarum.
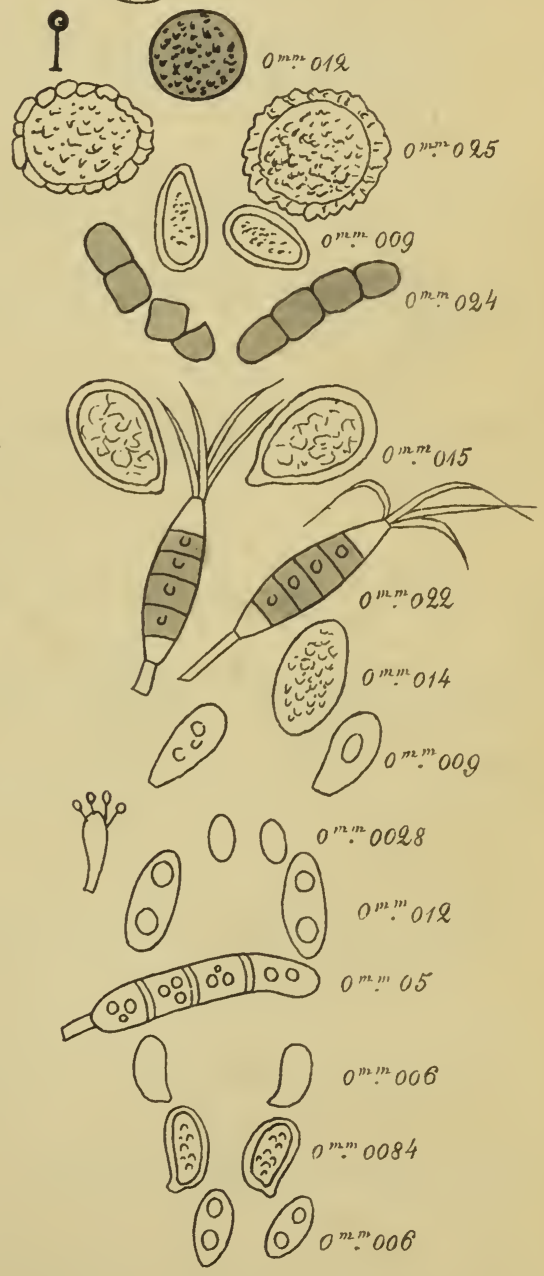
PAGES GENRES ESPF'CES

SPORES ET MESURE.

266. PHOMATOSPORA. Sacc. P. Molluginis

146. PHRAGMIDIUM. Tul. P. Incrassatui.

411. PHRAGMOTRICHUM. Kze. P. Chailleiil.

343. PHYLILACHORA. Nits. P. Graminis.

230. PHYLtaACTINIA. Lév. P. Suffulta.
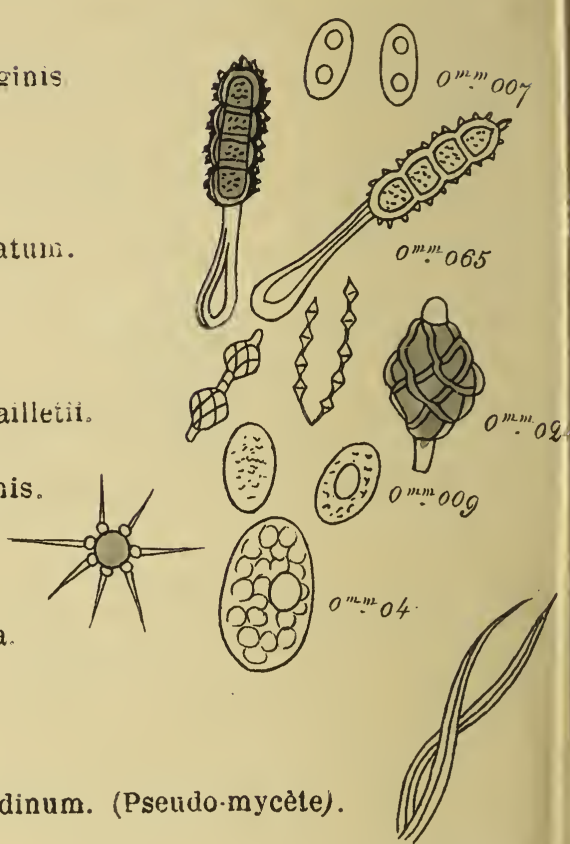

499. PHYLLERIUM. Fries. P. Juglandinum. (Psetido-mycète).

353. PHYLlosticta. Pers. P. Syringæ.

256. PHYLOCOPRA. Speg. P. Setosa.

266. PHYSALOSPORA. Niessl. P. Gregaria.

138. PHYSARUM. Pers. P. Columbinum.

1\%2. PHYSODERMA. Wallr. P. Maculare.

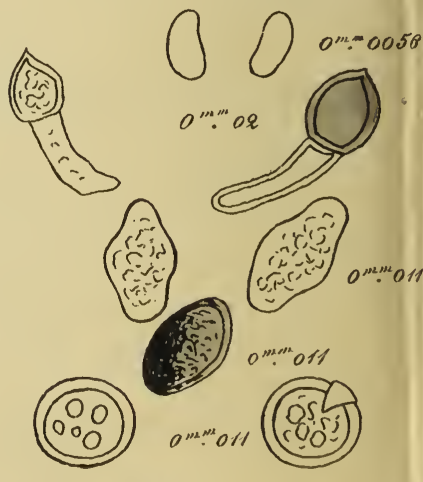

201. PILACRE. Fries. P. Friesii.

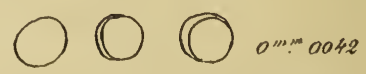

399. PILIDIUM. Kunz. P. Fuliginosum.

176. FILOzOr US. Coem. P. Crystallinus.

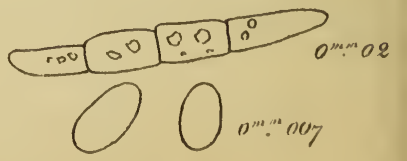


489. PIONNOTES, Fries. P. Hetæ.

395. PIROSTOMA. Fries. P. Circinans.

119. PISTILLARIA. Fries P. Micans.

312. PLEOMASSARIA. Speg. P. Siparia.

340. PLEONECTRIA. Sacc. P. Lamii.

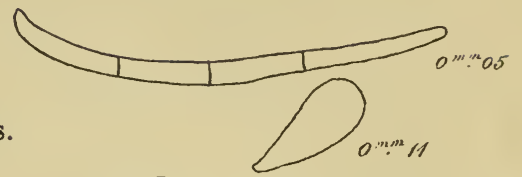

368. PLACOSPH FRIA. Sacc. P. Onobrychidis.

317. PLEOSPHARIA. Speg. P. Piłosella.

312. PLEOSPORA, Rab. P. Herbarum.

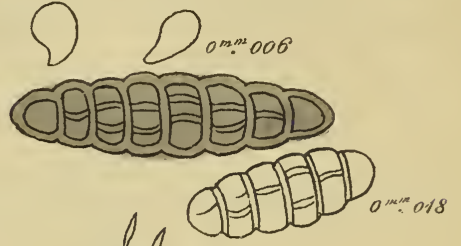

400. PLEOSPOROPSIS. Erst. P. Strobilina.

44. PIEUROTUS. Quél. P. Ostreatus.

344. PLOWRIGH'TIA. Sacc. P. Ribesia.

47. PLUTEUs. Quél. P. Cervinus.

170. PODISOMA. Rst. P. Juniperi.

229. PODOSPIIERA. Kunz. P. Tridactyla.

93. POLYPORUS. Fries. P. Brumalis.
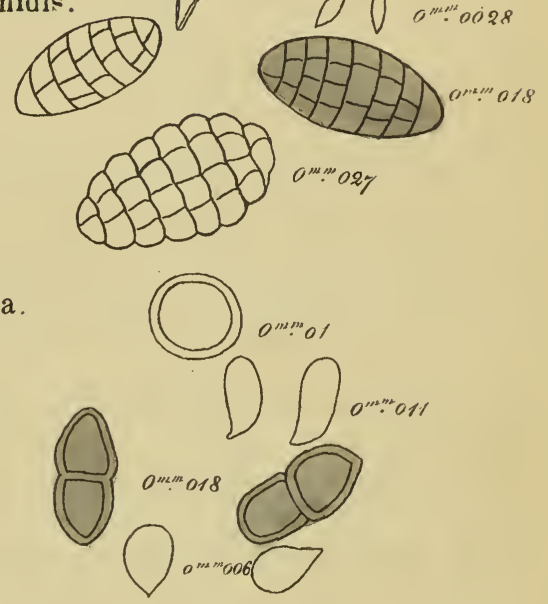

$100^{m 1 / 0} \cdot 0028$
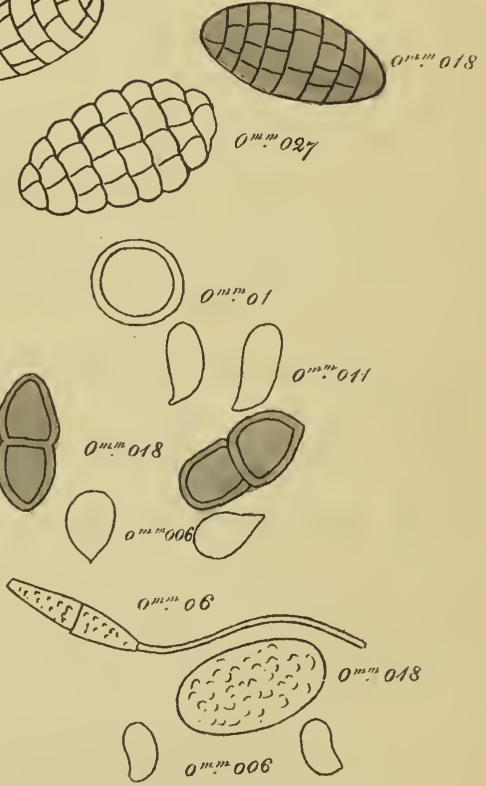

331. POLYSTIGMA. Fuck. P. Rubrum.

392. POLYSTIGMINA. Sacc. P. Rubra.

457. POI,YTHRINCIUM. Kunze. P. Trifolii.

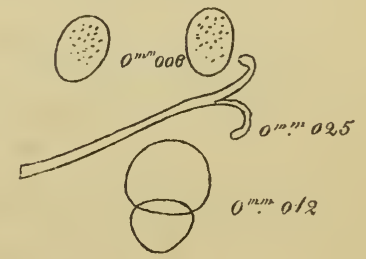


SPORES ET MESURE.

262. PORONIA. Wild. P. Punctata.

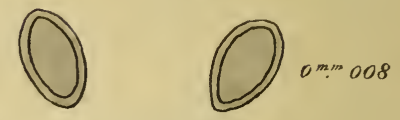

224. PROPOLIS. Eries. P. Versicolor.

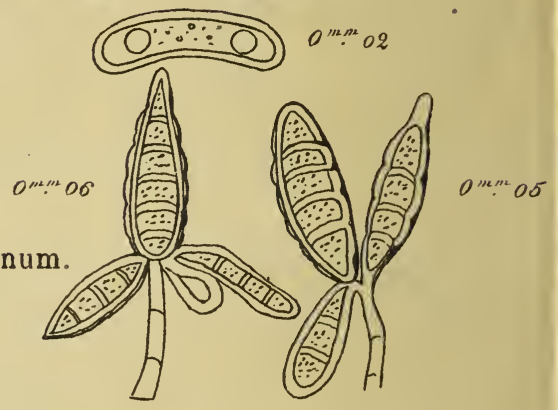

61. PSAlliota. Quél. P. Campestris.

65. PSATHYRA. Quél. P. Corrugis.

67. PSATHYRELLA. Quél. P. Gracilis.

216. PSEUDO-PEZIZA. Fuck. P. Trifolii,

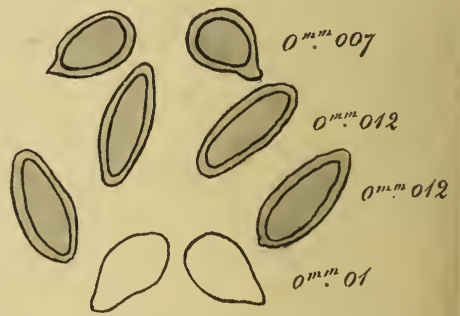

191. PSEUDO-PLECTANIA. Fuck. P. Nigella.

303. PSEUDO-VALSA. Ces. P. Berkeleyi.

65. PSILOCYBE. Quél. P. Spadicea.

125. PTYChOGASTER. Cord. P. Albus.

148. PUCCINIA. De By. P. Graminis.

190. PUSTULARIA. Fuck. P. Cupularis.
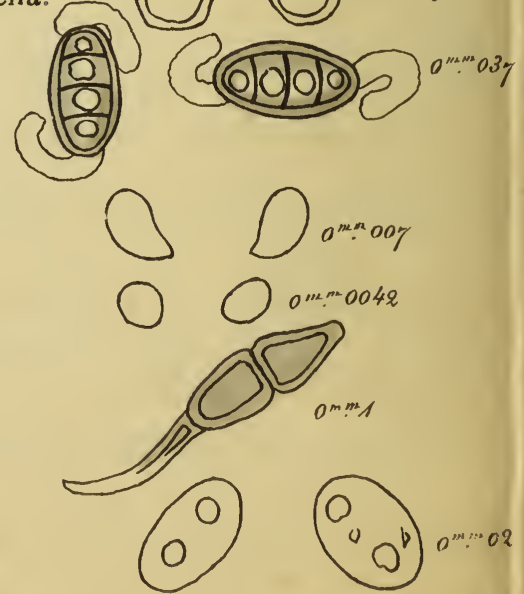


\section{PAGES GENRES ESPECES}

369. PYRENOCHETA. SAcc. P. Berberidis.

215. PYRENOPEZIZA. Fuck. P. Eryngii.

315. PYRENOPHORA. Fries. P. Relicina.

196. PYRONEMA. Carus. P. Marianum.

242. QUATERnARIA. Tul. Q. Personii. Sup'. QUELETIA. Quel. Q. Mirabilis.

430. RAMUlaria. Ung. R. Ulmaria.

368. RABENHORTZIA. Fries. R. Tiliæ.

130. RETICULARIA. Bull. R. Umbrina.

390. RHABDOSPORA. Mont. R. Helleborina.

317. RHAMPHORIA. Niessl. R. Buxi.

421. RHINOTRICHUM. Cord. R. Simplex.

186. RHIZINA. Fries. R. Undulata.

495. RHIZOCTONIA. D. C. R. Medicaginis. Pycnides stériles.

SPORES ET MESURE

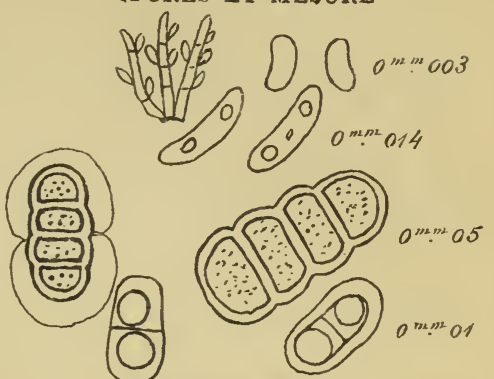

496. RHIZOMORPHA, Roth. R. Adna. Sp. ?

191. RHIZOPODELLA. Cooke. R. Melastoma.
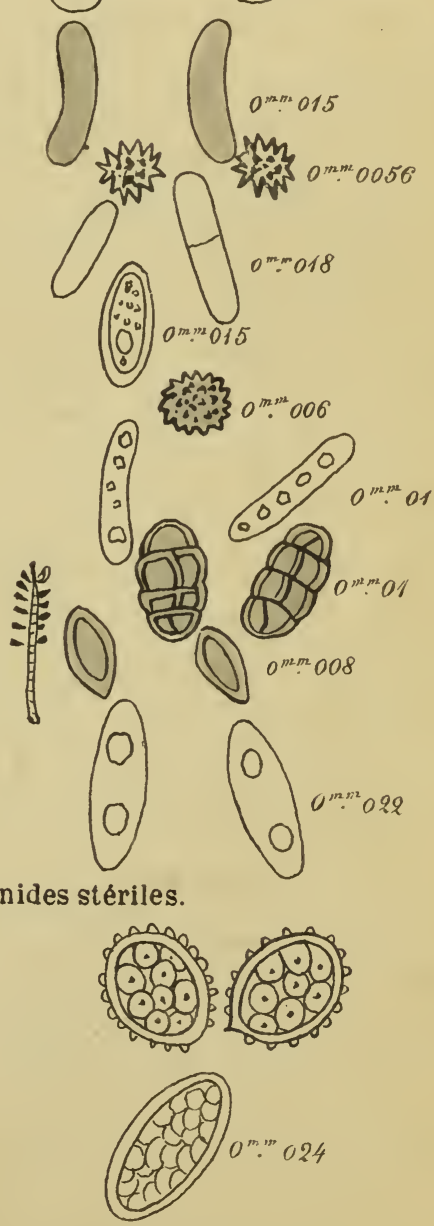
129. RHIZOPOGON. Fries. R. Tuteolus

178. RHIZOPUS. E'hr. R. Nigricans.

116. RHOPAI OMYCES. $B$. et $B r$. R. Gandidus

345. ROPOGRAPHUS. Fuck. R. Filicinus.

221. RHYTISMA. Fries. R. Salicinum.

296. RICHONIA. Boud. R. Variospora.
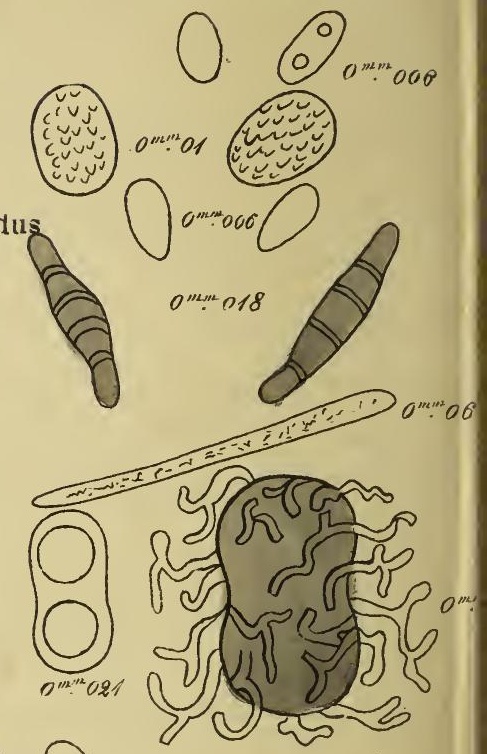

190. RIPARobius. Boud. R. Cookei

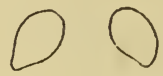

jäl. ROBhrgeA. Desm. R. Unica.

170. R(ESTELia. Rel. R. Cancellata. Spermatie (0) 0 on... 004

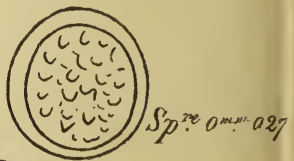

250. ROSELIINIA, De Not. R, Mammæformis.

79. Russula. firies. R. Cyanoxantha. 197. SACCOBOLLis, Boud. S. Neglectus. 415. SARCOPODILY. Nhr. S. Roseum. 191. SARCOSCYPIIA Fries. S. Coccinea. 18y. SAROOSPIIARA A $A$. S. Macrocalix.

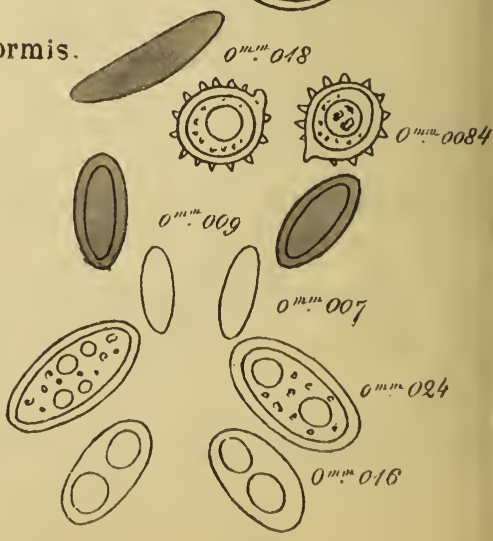


AGLS GENRES LSWF'WS SPORES FT MESUPE

37. SCHizopityulitm. Frit's. S. Commune.

そ心. SCHI7,OXYLUM. Pers. S. Berkeleyana.

344. SCTRRHIA. Nits. S. Rimosa.

126. SCLERODERMA. Pers. S. Verrucosum.

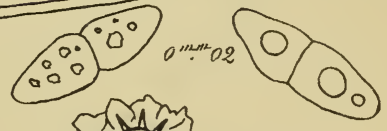

40\%. SCOLECOSPORIUM. S. Macrosporum.

493. SCLEROTIUM. Pers. S. Semen. Mycelium dur. Stérile

451. SCOLECOTRICHUM. Kunz.S. Clavariarum.

424. SEPEDONIUM. Link. S. Chrysospermum.

410. SEP'TOGLAUM. Sacc. S. Acerinum

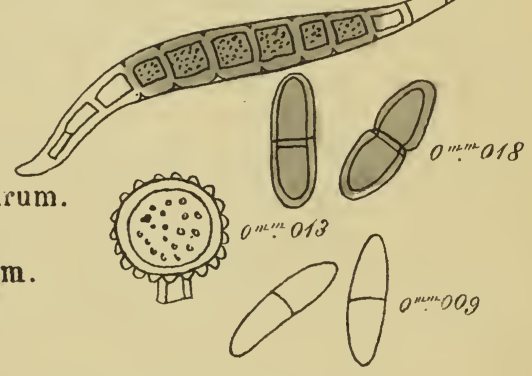

4:3. SEPTOCYLINDRIUM. SACC. S. Viride

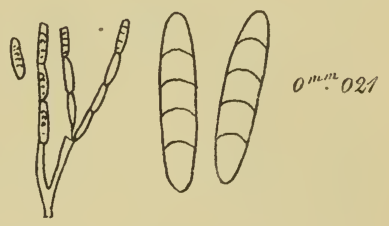

385. SEPTORIA. Fries. S. Calystegiæ.

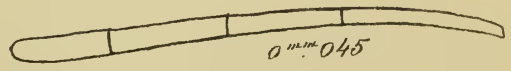

457. SEPTONFMA. Cord. S. Multiplex. Voir Hormiscium Stilbosporum.

167. SEPTOSPORTum. Cord. S. Velutinum.

192. SEPUL'TARTA. Cooke. S. Arenaria.

327. SILlia. K'urst. S. Ferruginea.

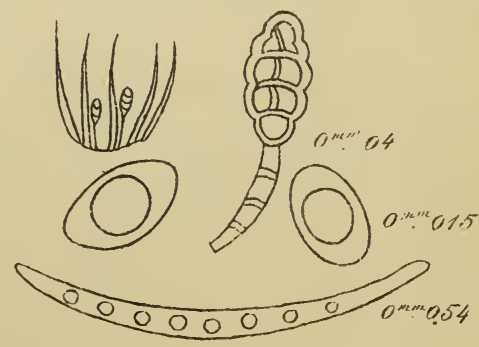


PAGES

GENRES

ESPÈCES

SPORES ET MESURE

Supl. SIRODESMIUM. De Not. Sub Fumago. p. 468.

105. SISTOTREMA. Pers. S. Confluens.

$\bigcirc 00$ ment.004

102. SOLENIA. Fuck. S. Anomala.

253. SORDARIA. Ces. S. Caudata.

423. SPHACELIA. Lév. S. Segetum.

270. SPHÆRELLA. Ces. S. Crassa.

329. SPI ERIA. Auct. S. Fissa? Sacc.

484. SPHARIDIUM. Fres. S. Citrinum.

390. SPHAROGRAPHIUM. Sacc. S. Micropera.

124. SPHEROBOLUS. Tode. S. Stellatus.

365. SPHARONEMA. Fries. S. Conicum.

371. SPHÆROPSIS. Lév. S. Longissima.

229. SPHÆROTHECA. Livo. S. Castagnei.

308. SPH ERULINA. Sacc. S. Intermixta.

127. SPICARIA. Harts. S. Verticillata.
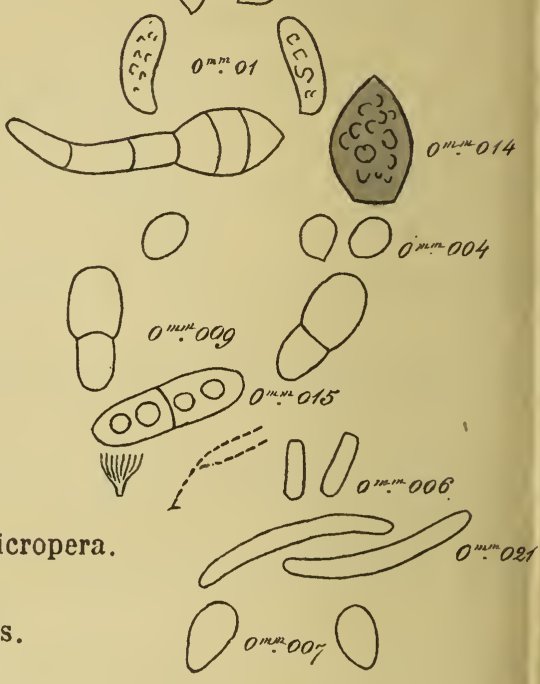

000 on:002

000 1"1.12005

.


PAGES GENRES FIPECES

SPORES ET MESURE .

462. SPORODESMIUM. Link. S. Ulmicolum.

46). SPOROSchisma. Berk. S. Mirabile.

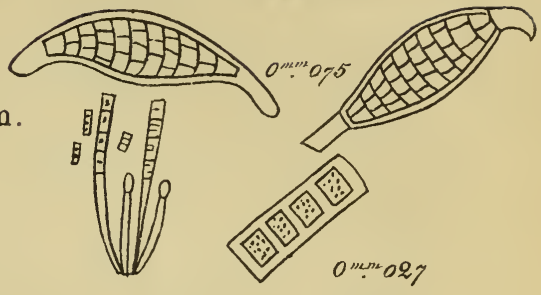

421. SPOROTRICHUM. Link. S. Scotophilum.

134. SPUMARIA. Pers. S. Alba.

448. STACHYLIDIUM. Link. Bicolor.

439. STACHYOBOTRYS. Cord. S. Alternans. 382. STAGONOSPORA. Sacc. S. Graminella. 410. STEganOSPORIUM. Cord. Compactum 222. STEGIA. Fries. S. Ilicis.

134. STEMONITIS. Fries. S. Ovata

465. STEMPhylium. Wallr. S. Paradoxum.

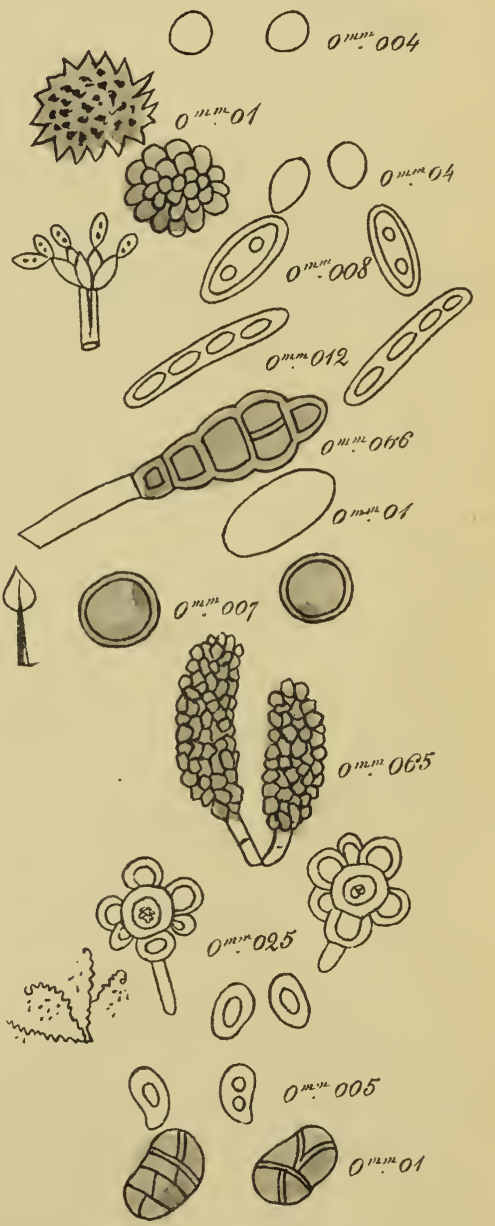

492. STEPHANOMA. Wrillr. S. Strigosum.

441. StREptotrix. Cord. S. Fusca.

108. STEREUM. Pers. S. Hirsutum.

463. STIGMELLA. Lév. S Dryina. 
PAGES GENRES RSPLCES

273. STIGMATEA.Fries. S. Hobertiani

225. STICTIS. Per's. S. Radiata

4u6. STILBOSPORA. Pers. S. Macrosperma.

470. STILBUM. Tode. S. Villosum

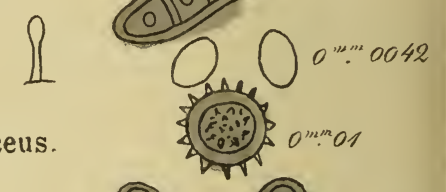

62. STROPHARIA. Quel. s. Airuginosa.

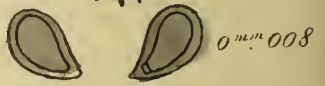

478. STYSANUS. Cord. S. Stemonites.

214. 'TAPESIA. Fries. 'T. Rosæ.

181. TAPHRINA. Tul. T'. Populina.

316. TEICHOSPORA. Fuck. T. Obducens.

178. THAMNIDIUM. Link. T. Elegans.

316. THECAPHORA. Tul. T. Delastrina.

107. THELEPHORA. Elis. 'T. T'errestris.

305. THYRIDARIA. Sacc. T. Incrustans.
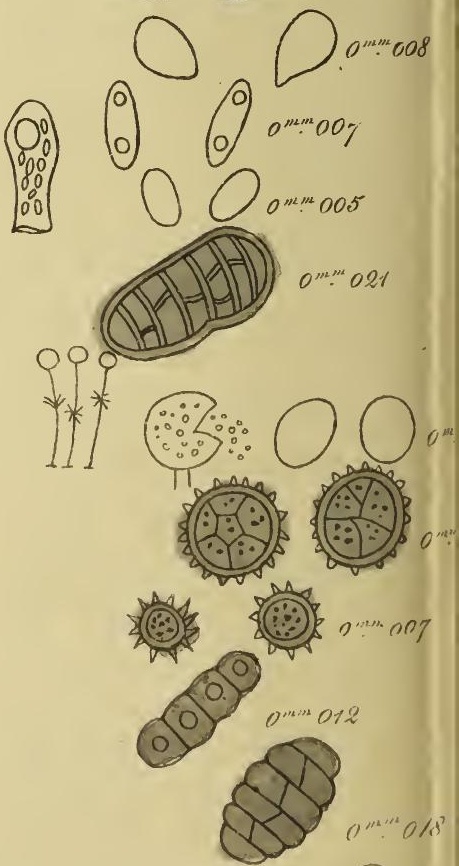

321. THYRIDIUM. Sacc. T. Philadelphi.

341. THYRONECTRIA. Sacc. T. Pyrrhochlora.

405. THYSIDIUM.Mont. T. Botryosporum.

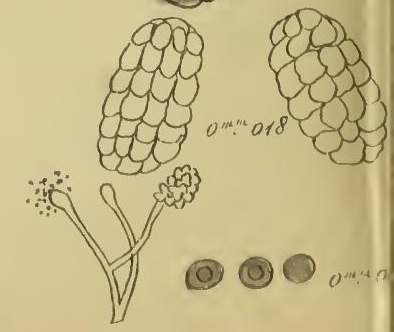


AGES TENRES ESPÈCES

141. TILLETIA. Tul. L. Caries.

43j. TORUIAA. Pers. T. Herbarum.

100. TRAMETES. Fries. T. Hexagonoides.

302. TREMA TOSPHÆRTA. Fuck. T. Fraxini.

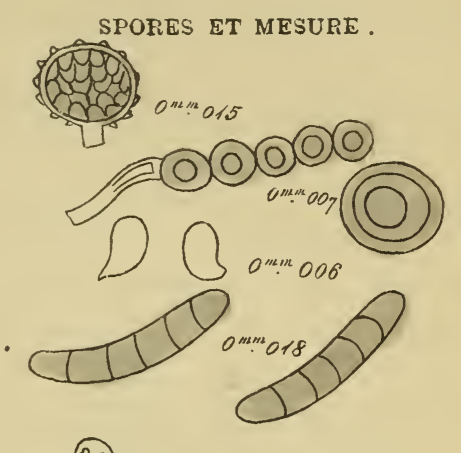

120. TREMELLA. Dill. T. Fimbriata.

466. TRICHEGUM. Cord. T. Cladosporioides.

131. TRICHIA. Hallv. T. Chrysosperma.

418. TRICHODERMA. Pers. T. Lignorum.

199. TRICHOGLOSSUM. Boud. T. Hirsutum.

30. TRICHOI,OMA. Quél. T. Nudum.

193. TRICHOPHÆA. Boud. T. Hispidula.

212. TRICHOSCY̌PHA. Boud. T. Pygmæa.
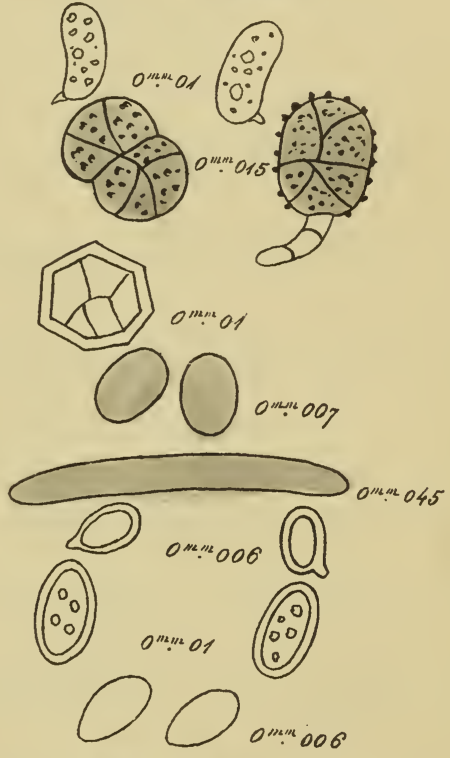

442. TRICHOSPORIUM. Fries. T. Chartaceum.

428. TRICHOTHECIUM. Link. T. Roseum.

148. TRIPHRAGMIUM. Tul. T. Ulmariæ.

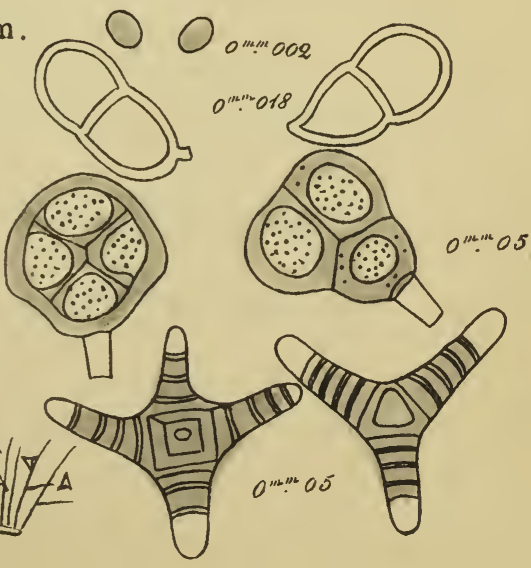

468. TRIPOSPORIUM. Cord. T. Elegans. 
PAGES GENRES ESPÈCES

221. TROCHILA. Fries. T. Craterium.

86. TROGIA. Fries. T. Crispa.

60. TUBARIA. Quél. T. Furfuracea,

227. TUBER. Mich, T. Mesentericum.

479. TUBERCULARIA. Tode. T. Vulgaris.

481. TUBERCUIARINA. Sacc. T. Persicina.

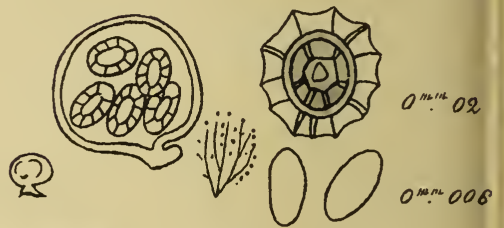

SPORES ET MESURE.

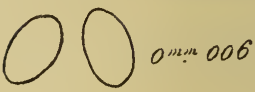

Sporis non visis. Sec. Sacc. om.mo04
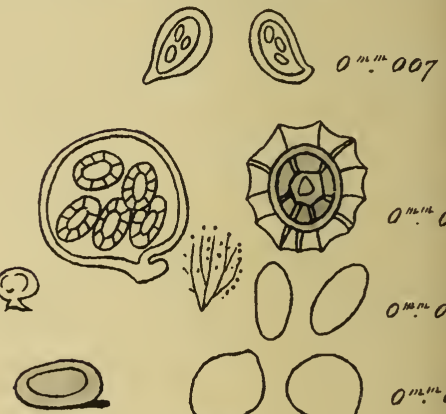

134. TUBULINA. Pers. T. Cylindrica. (Fragiformis).

128. TULASNODFA. Fries. T. Mammosa.

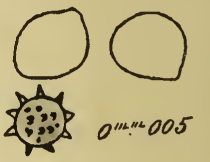

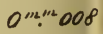

118. TYPHULA. Fries. T. Erythropus.

230. UNCINUILA. Iév. U. Adunca.

213. URCEOLEILA. Boud. U. Hyalina.

158. UREDO. Pers. IJ. Potentillarum.

142. UROCYSTIS. Lèv. U. Colchici.

156. UROMYCES. De By. U. Alliorum.

139. USTILAGO. Tul U. Segetum.
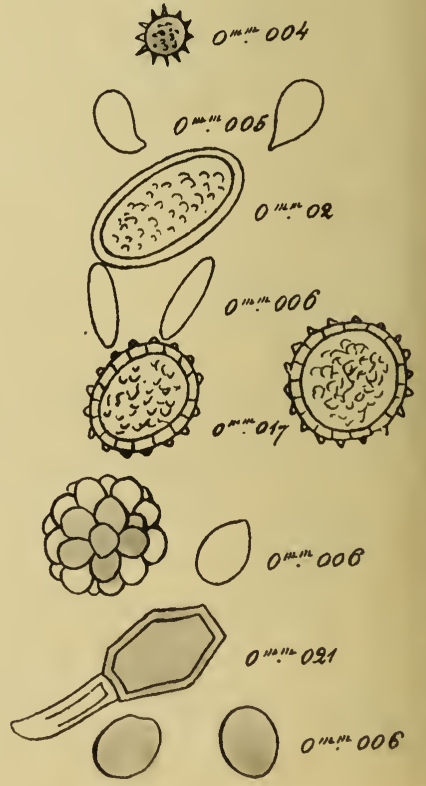
$-537-$

AGES

GENRES

ESPÈCES

SPORES ET MESURE.

262. USTULINA. Tul. U. Vulgaris.

137. UTRARIA. Tourn. U. Excipuliformis.

2'2. VALSA. Fries. V. Pini.

292. VAI.SARIA. De Not. V. Cincta.

Sup'. VELUTARIA. Fuck. V. Rufo-Olivacea.

278. VENTURIA. De Not. V. Circinans.

300\%. VERMICULARIA. Fries. V. Graminicola

125. VERPA. Schwartz. V. Digitaliformis.

125. VERTiCillium. Nées. V. Agaricinum.

485. VOLUTELLA. Tode. V. Buri.

47. VOLVARIA. Quél. V. Speciosa.
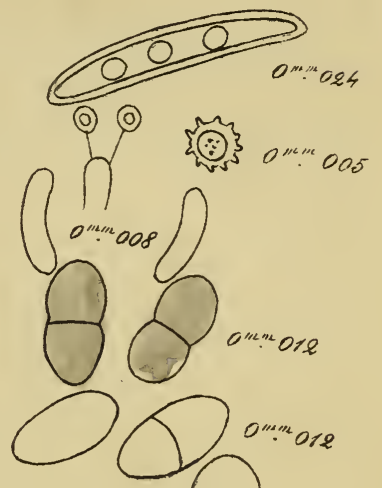

267. WALLROTIELLA. Sace. V. Minima.

00 an:-ooke

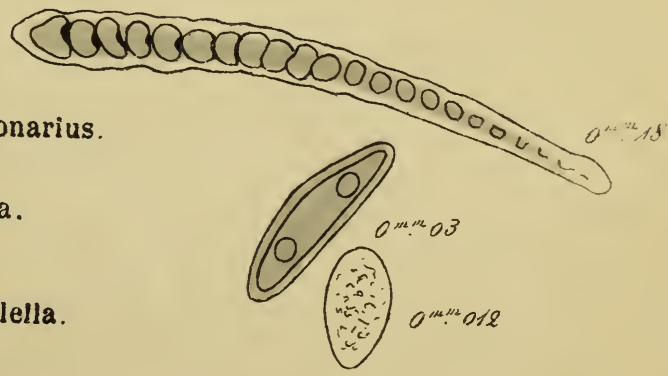

48. XENODOCHUS. Fuck. X. Carbonarius.

60. XYLARIA. Hill. X. Polymorpha.

25. XYLOGRAPHA. Fries. X. Paralella.
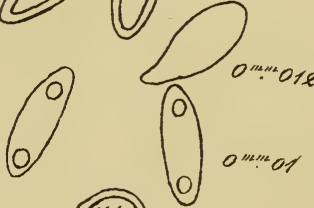

0,013 
PAGES GENRES

234. ZASMIDIUM. Fries, Z. Cellare.

311. ZIGNOELLA. Sacc, Z. Ovoidea.

338. ZOPFIA. Raしh. Z Rhizophila.
SPORES ET MESURE.
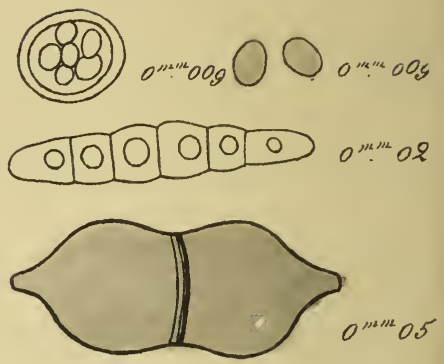
SUPPLÉMENT 



\section{SUPPLÉMENT.}

APIOSPORIUM MELAMPYRI. Sacc. - Sphæria Nervisequa. D. C. - Alb. de l'Est. - Sur Mclampyrum pratense. - Hypophylle; périthèces petits, sphériques, noirs, luisants, situés le long des nervures de la feuille; nucleus blanchâtre, stérile. - AC. - Automne. Sermaize. Charmont.

ASCOCHYTA CLEMATIDIS. Ch. $R$. - Alb. de l'Est. - (non Asc. Clematidina. Sacc.) - Croît sur les feuilles de Clematis vitalba encore vertes, à la surface de larges taches noires, irrégulières. - Périthèces hémisphériques, jaunâtres, entourés à la base de quelques filaments blanchâtres; ostiole assez large; sporules incolores, elliptiques, uniseptées, $0^{\mathrm{mm}}, 012$. - AR. Soulanges. - Automne.

ASCOPHANUS SPADICEO-NIGER. $C h . R$. - Rec. 8. p. 22. - Réceptacles sphériques, épars, superficiels, rugueux, roux étant frais, noirs et ridés étant secs, ressemblant à Peziza nidulus et à 
Riparobius Cookei. - Thèques larges, octospores; spores bisériées. elliptiques, incolores, $0^{\mathrm{mm}}, 015$; paraphyses cylindriques, continues ou septées, non renflées au sommet. - Espéce voisine d'Ascoph Ochraceus de Boud. - Récolté sur le fumier et les crottes de lapin. - StAmand. - Printemps.

CAMAROSPORIUIM MACROSPORUM. SACC. Alb. de l'Est. - Sur rameaux morts de Philadelphus coronarius. - Pẻrithèces sphériques, sous-cutanés, paraissant à travers les fentes de l'épiderme; sporules lancéolées, brunes à 4-5 cloisons. - AR. - Jardins. - Hiver, Printemps. - St-Amand.

CHAETOPHOMA ANTIRRHINI. Ch. $R$. - Alb. de l'Est. - Sur capsules d'Antirrhinum majus. - Périthèces bruns, subsphériques, puis aftaissés, glabres; munis à la base de fibrilles radiées; ostiole central; sporules ovales-oblongues, incolores, très nombreuses, 0 mm,004. - Précéde l'apparition de Pleospora herbarum. - Var. Antirrhini. - AC. - St-Amand. - Jardins. Automne.

CLASTEROSPORIUM SPARSUM. Sacc. -- Alb. de l'Est. - Sur rameaux de Syringa vulgaris. Taches velues, noirâtres, formées par les conidies claviformes, brunes, munies de la base au sommet de 8 à 10 cloisons. - AR. - Jardins.

- St-Amand. - Printemps, Hiver.

CONIOTHYRIUM AILANTHI. Ch. R. - Alb. de l'Est. - Pycnide de Thyridaria ailunthi. - 
Périthéces 4, réunis en cercle, entourés d'un duvet rouge à la base, renfermant des sporules incolores puis brunes, courtement pédicellées. $0^{\mathrm{mm}} .0045$. - Croît en compagnie du Thyridaria sur rameaux d'Ailanthus glandulosa. - AR. Jardins. - Soulanges, Ablancourt. - Printemps.

CORTICIUM AMORPHUM. Conidies. Ch. $R$. Alb. de l'Est. - J'ai découvert l'appareil conidien du Corticium dans l'épaisseur du tissu soushyménial ; il consiste en filaments arborescents, hyalins, porteurs sur chaque ramuscule, de conidies simples ou en chapelet remplies de sporidioles. - St-Amand. - 18\%7. - Bull. Soc. Bot. de France.

\section{Genre CV'TOSTPGERLLA. - Sace.}

CYTOSPORELLA CONSPERSA. Ch. R. - Alb. de l'Est. - Pycnide de Tympanis conspersa. Conceptacles ovoïdes, noirs, entourant la base des cupules de Tympanis et issus du même strôme, laissant échapper de leur sommet des cirrhes de sporules incolores, elliptiques, $0^{\mathrm{mm}}, 003$; basides continues, simples ou un peu rameuses. - Sur branches mortes de Pommier. - Jardins, vergers. - St-Amand, St-Lumier. - AR. - Printemps.

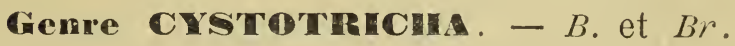

CYSTOTRICHA STRIOLA. $B$. et $B r$. - Rec. 11. - Sur bois dénudé d'Alnus glutinosa servant 
de barrière à un jardin de St-Amand. - Périthèces incrustés dans le bois, bruns, noircissant, hystẻriiformes; nucleus blanchâtre composé de basides longues, rameuses, septées, garnies latéralement de sporules incolores. elliptiques, uniseptées: $0^{\mathrm{mm}}, 00 \%$ - Hiver-Printemps. - AR.

CYTOSPORA CORNI. Fuchl. - Alb. de l'Est. Spermogonie de Valsa fallax sur rameaux de Cornus sanguinea. - Petites pustules corticoles à peine proéminentes; périthèces 8-10 disposés en cercle, émettant des cirrhes blanchâtres de sporules botuliformes, hyalines $0^{\mathrm{mm}}, 0045$. - AC. - Ablancourt. St-Amand. - Automne.

DENDROPHOMA PINI. Ch. $R$. - Alb. de l'Est. - Sur branches et rameaux de Pin sylvestre. - Spermogonie de Phacidium pini. - Périthèces hémisphériques, s'ouvrant en fentes comme l'êtat ascophore; spermaties petites, cylindriques, courbes, portées sur des basides rameuses, - St-Amand, Soulanges. - AC.

DEPAZEA TREMULACOLA. D. C. et Sacc. Alb. de l'Est. - Sur feuilles de PeuplierTremóle. - Taches rondes, grises, bordées par une zône plus foncée, occupées au centre par des groupes de périthéces noirs, ronds, aplatis, disposés la plupart en cercles concentriques; sporules cylindriques, $0^{\mathrm{mm}}, 02$. - AR. - Bois de St-Lumier. - Automne.

DIPLODIA SALICINA. Lév. Sacc. - Alb. de l'Est. - Sur rameaux de Saule. ... Groupes de périthèces sous-cutanés, brunissant l'èpiderme soll- 
levẻ en forme de petites pustules hémisphériques; du sommet déchiré sortent des cirrhes blanchâtres de sporules incolores; ces sporules d'abord continues brunissent en vieillissant et prennent la cloison médiane. - AR. - StAmand. - Automne. - C'est la pycnide de Cucurbitaria salicis.

HENDERZONIA CARPINI. Sacc. - Alb. de l'Est.

- Sur branches mortes de Carpinus. - Tubercules noirs, saillants, aplatis au sommet, composés de sporules brunes; oblongues-ovales. á 4-5 cloisons, très longuement stipitées. - Pycnide de Massaria Carpini. - AC. - St-Amand. Printemps.

HENDERZONIA POLYCYSTIS. $B$. et $B r^{r}$. Alb. de l'Est. - Sur branches mortes de Bouleau. - Périthèces aplatis, sous-cutanés, réunis en groupes; sporules brunes, oblongues-fusiformes, larges, à 5-7 cloisons. - AR. - St-Amand. Stylospore de Pseudo-valsa lenciformis. - Printemps, automne.

HYDNUM ERINACEUM. Conjdies. Ch. $R$. Alb. de l'Est. - Conidies intracellulaires situées dans l'épaisseur du parenchyme près du revêtement du chapeau ; conidies de deux sortes, les unes ovales-elliptiques munies de deux gouttelettes. $0^{\mathrm{mm}}, 007$; les autres baculoïdes-fusiformes, 0mm.011. - St-Amand, Bassu. - RR. - Bull. Soc. Bot. de France, 1881. 
Genre rámulasia. - Niessl.

KALMUSIA HYPOTEPHRA. Sacc. - Alb. de l'Est. - Sur branche de Chêne dénudée. Strômes elliptiques, noirâtres, minces, épars, percés par les ostioles des périthéces logés dans le bois ; thèques cylindriques, renfermant 8 sporidies brunes, allongées, un peu courbes, munies de 3 cloisons et de 4 gouttelettes, $0 \mathrm{~mm}, 018$. - AR. - Bois de St-Lumier. - Automne.

PHOMA AQUILEGIE. Ch. R. - Rec. 11. - Cette espèce croît en été sur les tiges mortes d'Aquilegia vulgaris dont l'épiderme est détaché, exposées à l'humidité. - Elle se présente en groupes de petits points disposés en ligne, de couleur brune; les sporules, assez grosses $0^{\mathrm{mm}}, 006$, terminent des basides un peu arquées et deux fois plus longues. - Jardins. - StAmand.

PHOMA BACCACOLA. Ch. R. - Rec. 11. - Sur baies de Symphoricarpon noircies par la vétusté. - La surface de la tache est parsemée de périthèces hémisphériques, à large ostiole laissant échapper un globule de sporules elliptiques à 2 gouttelettes; les sporules sont portées par des basides droites, assez longues. - AC. - Jardins. - St-Amand. - Aulomne.

PHOMA CISSI. $C h . R$. - Rec. 11.-J’ai récolté cetle espèce dans mon jardin, sur la Vignevierge. - Les sarments envahis présentent à leur surface des pustules éparses, formées par 
les périthèces sous-jacents, recouverts par l'épıderme soulevé et fendu ; les périthèces noirs, hémisphériques contiennent des sporules allongées, $0^{\mathrm{mm}}, 006$, renflées aux extrémités, munies de 2 gouttelettes, insérées sur de longues basides droites. - St-Amand. - Printemps.

PHOMA COLPOMATIS. Ch. $R$. - Rec. $8 .-\mathrm{Ce}$ phoma est la spermogonie du Colpoma quercinum. - Il croît au centre du Colpoma en vétusté ou dans son voisinage; ses périthèces sont noirs, sphériques, superficiels ; ils renferment des sporules ovales ou cylindriques très courtes, $0^{\mathrm{mm}}, 0028$. - Bois de St-Amand. - Automne.

PHOMA RHAMNI. Ch. $R$. - Alb. de l'Est. Sur rameaux de Rhammus frangula en compagnie du Diplodia frangulce. - Spermogonie de Cucurbitaria Rhamni. - Périthèces noirs, ronds, aplatis, renfermant des sporules de phoma grosses, $0^{\mathrm{mm}}, 008$, elliptiques, à 2 gouttelettes ; basides longues. - AR. dans les haies des jardins. - La Chaussée. - Printemps.

PHOMA THLASPIDIS. Ch. $R$. - Rec. 8. - Sur tiges mortes de Thlaspi perfoliatum. - Périthèces hémisphèriques, noirâtres, épars ; sporules elliptiques à 2 gouttelettes, $0^{\mathrm{mm}}, 006$; basides droites, assez longues. - C. - St-Amand. Juillet. - Le long des chemins arides.

PHOMA. VISCI. Sacc. - Alb. de l'Est. - Sur les feuilles mortes et tombées du Viscum album. - Périthèces disséminés sur des taches rondes, blanches, entourées d'une ligne rougeâtre : 
apparence d'un Phyllosticta. - AC. - Soulanges, Ablancourt. - Hiver.

PHOMA ULMICOLA. Ch. $R$. - Rec. 9. - Spermogonie de Cryptosporella hypodermia. - Cette espéce croît sur les mêmes rameaux que le Cryptosporella ; ses périthèces sont épars, sousépidermiques, plus petits que ceux de la forme thécasporée ; sporules grandes, $0^{\mathrm{mm}}, 012$, ovales, remplies de gouttelettes, portées par des basides longues, rappellant celles de Melanconium juglandis. - Saccardo en fait un Fusicoccum avec un point de donte. Je n'ai pas vu les loges, cellulaires qui caractérisent ce dernier Genre. - Bois de St-Amand. - Hiver. - Sur les rameaux d'Ulmus campestris.

PHYLLACHORA POPULI. (Botulispora). Ch. $R$. - Alb. de l'Est et Rec. 12. - Cette espéce remarquable croît au printemps sur les feuilles de Populus cordifolia. - Je l'ai récoltée entre St-Lumier et St-Quentin-les-Marais, en février 1882. - Comme nous l'avons dit page 3ł3, elle est ambiguë et ne peut trouver une place fixe dans la Classification. Elle se rapproche d'un côté du Genre Cryptosphceria par ses sporidies botuliformes, brunissantes et ses théques longuement pédicellées, de l'autre du Genre Phylla(hora (Phyll. ulmi) par sa station foliicole et ses périthéces aplatis, confluents, réunis en strôme.

RHABDOSPORA TRAGOPOGONIS. Ch. $R$. - Rec. 12. - Cette espéce se rapproche de Rhabd. 
solidaginis, cependant elle en diftère par sa station, par ses périthéces plus saillants et disposés en ligne, enfin par la taille plus petite des sporules, $0^{\mathrm{mm}}, 018$, exemptes de gouttelettes. - Récoltẻe en décembre 188\%, sur le chemin de Lisse à Bassu.

SCHIZOTHYRIUM JUGLANDIS. Ch. $R$. - Alb. de l'Est. - Croît sur les pétioles des feuilles mortes du Noyer. - Elle forme, sur l'épiderme décoloré. des points noirs, très petits, ressemblant à des hysterium. - Plongés dans l'eau les réceptacles s'ouvrent en cupules dont les bords sont un peu laciniés. Théques en massue; sporidies 4-6, incolores, elliptiques-fusoïdes : $0^{\mathrm{mm}}, 008$; paraphyses continues, renflées au sommet. - AC. - Automne, hiver. - St-Amand.

SCLEROTIUM DURUM. Kickx. - Alb. de l'Est.

- Trés commun sur les grandes herbes sèches.

- Pustules noires, de formes variées, rugueuses, allongées ; substance interne blanche. Très souvent envahi par Botrytis cinerea. - Partout. - Hiver, printemps.

SCLEROTIUM SCOLYMI. West. - Alb. de l'Est. A. la base des feuilles d'Artichaut (involucre). - Pustules saillantes, coniques, pâles puis brunes. - Substance interne blanche. - AC. - Jardins.

SIRODESMIUM FUMAGO. Sacc. - Rec. 12. Fumago vagans, var. Armeniaca. West. Cette espéce rampe en forme de rosace sur les feuilles de Pêcher. - Ses filaments sont nodu- 
leux, torulacés, bruns, entremêlés de sporules elliptiques presqu'incolores. - Jardins. - Automne. - St-Amand.

SPHARONEMA. SUBULATUM des auteurs. Eleutheromyces de Fuckel. - Cette espèce croît en groupes très denses sur les shapeaux des agarics desséchès après l'hiver. - Les périthéces sont en forme de bouteille allongée, d'un brun-rougeâtre plus pâle au sommet, terminés par une couronne de filaments aigus qui s'écartent pour le passage d'un globule de conidies ; à l'intérieur ces conidies incolores, fusiformes, $0^{\mathrm{mm}} .01$, munies d'un cil à chaque extrémité sont portées par des filaments simples ou peu rameux, fasciculés, dressés, septés, terminés en pointe. - Les conidies prennent naissance au dessous de chaque cloison. - J'ai vainement cherché les théques indiquées par Fuckel et la description exacte que je donne ici permet, sans nier l'existence possible de la forme thécasporée, de conserver à cette spermogonie le nom de Sphieronema que Fuckel et Saccardo ont écarté de leur classification.

TREMATOSPHERIA FRAXINI. Ch. $R$. - Rec. 2. - Sur les rameaux morts de Fraxinus excelsior. - Périthèces épars, sphẻriques, à ostiole conique émergeant á peine de l'épiderme noirci par la sortie des sporidies: théques fusiformes : longuement atténuées en pédicelle. octospores, entourées de paraphyses continues ; sporidies brunes, cylindrigaes, botuliformes, dis- 
tiques, munies de 5 cloisons. - R. - Bois de St-Amand. - Automre, printemps.

TRIBLIDIUM ABIETIS. Ch. $R$. - Rec. 12. Sur bois dénudé de Pinus abies. - Périthèces petits (à peine $1 \mathrm{~mm}$.) hystériiformes, noirs ; thèques obovales, $0^{\mathrm{mm}}, 051$, sessiles, contenant de 4 à 8 spores hrunes, biloculaires, $0^{\mathrm{mm}}, 014$; paraphyses peu distinctes rameuses au sommet. -R. - St-Amand. - Hiver.

TRICHOSTROMA. QUERCUS. Ch. R. - Rec. 12. - Cette espéce vient en groupes sur le bois dénudẻ et humide des branches de Chêne, sous forme de tubercules très petits, sphériques, d'un jaune sale un peu verdâtre, glabres d'abord puis hérissés de poils noirs, excepté au sommet qui se dilate comme le disque d'une pézize pour le passage de la masse des sporules ; la substance des tubercules est formée d'une matière mucilagineuse, entremêlée de filaments incolores, rameux et parsemés de sporules ovoïdes, $0^{\mathrm{mm}}, 003 .-\mathrm{R}$. - Récolté à St-Amand, le 22 décembre $188 \%$.

URCEOLELLA RICHONIS. Boudier. - Alb. de l'Est. - Mon savant ami M. Boudier, Président de la Societé Mycologique, m’a fait la gracieuseté de me dédier cette espéce que j'ai trouvée en Décembre 1885, entre Lisse et St-Amand. Elle a été décrite dans le Bulletin de la Société mycologique, tom. IV, sous le nom de Urceolella Richonis.

UROCYSTIS OROBANCHES. Sacc. - Alb. de 
l'Est. - Sub Tuburcinia. - Croît à la base des tiges de Phelipcea ramosa parasite des racines du Chanvre. - Tubercules saillants formés par l'épiderme soulevé par la masse de sporules sous-jacentes, noires, rondes-irrégulières, assez grosses. - AR. mais commun à St-Lumier-enChampagne, dans les chenevières. - En Automne.

\section{Genre VELUTARIA. - Fuckel.}

VELUTARIA RUFO-OLIVACEA. Fuck. - Alb. de l'Est. - Sur rameaux desséchés de Rosu canina, encore adhérents à la souche. - Pustules velues, brun-clair, pulvérulentes; dabord fermées puis ouvertes en cupules; chair épaisse; hymenium couvert de thèques cylindriques, $0^{\mathrm{mm}}, 065 ;$; paraphyses simples, plus longues ; 8 spores elliptiques-ovales, incolores, $0^{\mathrm{mm}}, 007$. Espèce souvent stérile. - R. - St-Amand. Automne.

ZASMIDIUM CELLARE. Fries. - Cephalotheca Cellaris. Ch. R. - Nous avons dit, page 234, que le Zasmidium était un Eurotium pour la fructification; des échantillons de périthèces, fraîchement récoltés m'ont permis de découvrir certains caractères essentiels qui m'avaient échappé dans les analyses antérieures et m'autorisent à fixer définitivement l'espéce en question dans le Genre Spharotheca trẻs voisin en effet du Genre Eurotium ; ces caracteres con- 
sistent dans le mode de formation des théques au sommet des filaments hyméniens et dans la couleur brune ou noire des périthèces et des spores.

Selon moi, le Genre Zasmidium disparaît et rentre dans le Genre Sphcerotheca; notre espèce devient le Sphcerotheca cellaris et les noms de Racodium de Pers. Antennaria et Zasmidium de Fries, sont réservés pour désigner les larges plaques velues qui envahissent les tonneaux que je considère comme le Mycelium, ordinairement stérile, de Cephalotheca cellaris. 



\title{
CONTRIBUTION
}

\author{
A LA
}

\section{Flore Mycologique Générale.}

Découvertes faites par Ch. RICHON, INSCRITES DANS LE CATALOGUE.

\section{DANS LE CaTALOGUE.}


No d'ordre.

10\%8. Cephalotheca Hispida.

1728. Camarosporium Berberidis.

1133. Ceratostoma Rosa.

20:32. Cercospora Liliicola.

Suppl. Chætophoma Antirrhini.

2003. Cladosporium Pilicola.

2018. Clasterosporium Ligustri.

2019. Clasterosporium Tripartitum.

1937. Coniosporium Caulincolum.

1615. Coniothyrium Fasciculatum.

164\%. Coniothyrium? Populi.

1645. Coniothyrium Laburni.

1768. Cornularia Boudieri.

Suppl. Corticium Amorphum. Conidies.

1430. Cryptospora Quercus.

Suppl. Cryptosporella Conspersa.

1399. Cucurbitaria Mahoniæ.

Suppl. Cystotricha Striola. Sacc.

985. Dasyscypha Hypocastani.

191\%. Didymopsis spicata.

Suppl. Dendrophoma Pini.

1769. Dilophospora Graminis.

1801. Dinemasporium Purpurascens.

2006. Diplococcium Pulchrum. 
No d'ordre.

1682. Diplodia Vincæ.

168'. Diplodiella Junci.

169'. Diplodina Antirrhini.

1685. Diplodina Minima.

20\%. Ecchyna Poricola.

1116. Eutypa Ligustri.

1808. Glæosporium Gallarum.

1809. Glæosporium Phasæoli.

1231. Gnomonia Rhoïs.

1524. Godronia Mühlenbeckii.

1973. Haplographium Penicillatum.

1\%04. Henderzonia Aconiti.

1705. Henderzonia Hyperici.

1706. Henderzonia Juniperi.

170\%. Henderzonia Ligustri.

1708. Henderzonia Platani.

Suppl. Hydnum Erinaceum. Conidies.

632. Hymenogaster Leptoniæsporus.

1330. Leptosphæria Berberidis.

1329. Leptosphæria Stellariæ.

1331. Leptosphærites Lemoinii.

1775. Leptothyrium Lycopi.

17\%6. Leptothyrium Berberidis.

1500. Lophiostoma Genistæ. Var. 
No d'ordre.

1'96. Lophiotrema Epilobii.

1192. Lophiotricha Viburni.

988. Lachnella Juniperi .

403. Marasmius Festucæ.

135\%. Metasphæria Gei.

1491. Microthyrium Platani.

1490. Microthyrium Visci.

1969. Monotospora Cuneiformis.

1454. Nectria Dahliæ.

1870. CEdocephalum Agaricinum.

1859. Oospora Corii.

1858 Oospora Sphærellæ.

1418. Ophiobolus Galii.

1419. Ophiobolus Meliolæoides.

1819. Ovularia Vitis.

1085. Perisporium Matricariæ. Conidie.

1084. Perisporium Secale.

1841. Pestalozzia Liliorum.

18'2. Pestalozzia Potentillæ.

10'1. Phacidium Viride.

Suppl. Phoma Aquilegix.

Suppl. Phoma Baccæcoia.

Suppl. Phoma Cissi.

Suppl. Phoma Colpomatis . 
No d'ordre.

Suppl. Phoma Ulmicola.

1191. Phomatospora Berberidis.

709. Phragmidium Sparsum.

Puccinia Malvacearum, en 1872.

93\%. Pilacre, en $18 \%$.

$\{1480$.

Suppl.

Phyllachora Populi.

1549. Phyllosticta Trifolii.

119\%. Physalospora Ononidis.

1630. Pyrenochæta Berberidis.

1011. Pyrenochœta Jaceæ.

Suppl. Rhabdospora Tragopogonis.

1389. Rhamphoria Buxi.

Suppl. Schizothyrium Juglandis .

1759. Septoria Anagallis.

1221. Sphærella Ebuli.

1431. Sphæria Reniformis.

1432. Sphæria Dichænoides.

2016. Sphæridium Citrinum .

1765. Sphærographium Coluteæ.

1766. Sphærographium Coryli.

2041. Sporidesmium ? Alitosporii.

20S8. Sporocybe Carnea.

198\%. Stachylidium Sambuci. 
No d'ordre.

1829. Stilbospora Cratægi.

20\%(). stilbum Pilacriforme.

1403. Thyridium Philadelphi.

1940. Torula Compniacensis.

1942. Torula Corii.

1944. Torula Tuberculariæformis.

Suppl. Triblidium Abietis.

$\left\{\begin{array}{l}133 \% . \\ \text { Suppl. }\end{array}\right.$ Trematosphæria Fraxini.

Suppl. Trichostroma Quercus.

2100. Tubercularia Hydnoidea.

$\left\{\begin{array}{c}998 . \\ \text { Suppl. }\end{array}\right.$ Urceolella Richonis.

10)\%. Zasmidium Cellare. 


\section{ISTE}

PAR ORDRE DE CLASSIFICATION,

des Champignons récoltós dans les pays circonvoisins, dont nous recommandons la recherche aux botanistes de la Marne.

I. Amanita Strangulata. Fries.

II. Amanita Nitida. Fries.

III. Tricholoma Album. Fries.

IV Tricholoma Splendens. Fries.

V. Tricholoma Arnaricans. Fries.

VI. Clitocybe Gilva. Fries.

VII. Clitocybe Amara. Fries.

VIII. Mycena Pelianthina. Fries.

IX. Mycena Rosella. Fries.

X. Mycena Frumentacea. Roze.

XI. Mycena Seynii. Quélet.

XII. Enteloma Clypeatum. Fries. 
XIII. Entoloma Sericellum. Boud.

XIV. Entoloma Queletii. Boud.

XV. Nolanea Pascua. Fries.

XVI. Leptonia Serrulata. Fries.

XVII. Pleurotus Cornucopioides. Fries.

XVIII. Inocybe Incarnata. Gillet.

XIX. Inocybe Bongardi. Weinn.

XX. Inocybe Strigiceps. Fries.

XXI. (Psalliota Sylvatica. Quélet.

XXII. Psalliota Peronata. Rose.

XXIII. Psalliota Duriuscula.

XXIV. Psalliota Flavescens.

XXV. Psalliota Tenuipes.

Ces cinq dernières espèces sont décrites dans l'Album des Ch. Vénéneux et Comestibles de Richon et Roze.

XXVI. Stropharia Cotonea. Quélet.

XXVII. Cortinarius Orellanus, Fries.

XXVIII. Cortinarius Cotoneus. Fries.

XXIX. Cortinarius Purpurascens. Quélet.

XXXI. Cortinarius Claricolor. Fries.

XXXII. Cortinarius Dibaphus. Fries.

XXXIII. Cortinarius Torvus. Fries.

XXXIV. Cortinarius Sanguineus. Fries.

XXXV. Cortinarius Cinnabarinus. Fries. 
XXXVI. Cortinarius Saturninus. Fries.

XXXVII. Hygrophorus Penarius. Fries.

XXXVIII. Hygrophorus Pudorinus. Quélel.

XXXIX. Hygrophorus Unguinosus, Fries.

XL Hygrophorus Chrysodon. Fries.

XLI. Hygrophorus Streptopus. Fries.

XLII. Lactarius Turpis. Fries.

XLIII. Marasmius Gilobularis. Quélet.

XLIV. Boletus Boudieri. Quélet.

XLV. Boletus Estivalis. Fries.

XLVI. Polyporus Leucomelas. Pers.

XLVII. Polyporus Schweinitzii. Fries.

XLVIII. Polyporus Betulinus. Bull.

XLIX. Polyporus Evonymi. Bern.

L. Polyporus Aureolus. Fries.

LI. Trametes Pini. Fries.

LII. Hydnum Acre. Quélet.

LIII. Hydnum Imbricatum. Fries.

LIV. Hydnum Fuligineo-Album. Fries.

LV. Hydnum Velutinum. Fries.

LVI. Tremellodon Gelatinosum. Fries.

LVII. Odontia Fimhriata. Pers.

LXIII. Craterellus Pusillus. Fries.

LIX. Stereum Disciforme. Pers.

LX. Corticum Mougeoti. Fries. 
LXI. Cyphella Digitalis. Alb. et Schw.

LXII. Clavaria Crispula. Fries.

LXIII. Tremella Viscosa. Berk.

LXIV. Polysaccum Crassipes. D. C.

LXV. Utraria Cælata. Bull.

LXVI. Geaster Fimbriatus. F'ies.

LXVII. Battarea Giucciardiana. (pour mémoire. Espèce étrangère).

LXVIII. Morchella Deliciosa. Fries.

LXIX. Gyromitra Esculenta. Pers.

LXX. Helvella Monachella. Pers.

LXXI. Sparassis Laminosa. Fries.

LXXII. Peziza Wrightii. Cooke.

LXXII. Geoglossum Peckianum. Boud.

LXXIV. Trichoglossum Hirsutum. Pers.

LXXV. Microglossum Olivaceum. Pers.

LXXVI. Mitrula Paludosa. Fries.

LXXVII. Cudonia Circinans. Pers.

LXXVIII. Ombrophila Sigilliformis. Boudier.

LXXIX. Mniæcia Jungermanniæ. Boudier.

LXXX. Sclerotinia Tuberosa. Hedw.

LXXXI. Sclerotinia Curreyana. $B$.

LXXXII. Phialea Squamicola. Quél.

LXXXIII. Phialea Echinophila. Boud.

LXXXIV. Dasyscypha Pygmea. I' Fires. 
LXXXV. Dasyscypha Godroniana. Mont.

LXXXVI. Dasyscypha Schumacheri. Boud.

Lxxxvir. Tapesia Aurelia. Pers.

LXXXVIII. Tapesia Sanguinea. Pers.

LXXXIX. Mollisia Collematis. Boud.

LC. Rhytisma Andromedæ. D. C.

LCI. Tuber Estivum. Witt.

LCII. Tuber Magnatum. Picol.

LCIII. Tuber Brumale. Witt.

LCIV. Tuber Uncinatum. Chatin.

LCV. Tuber (Oogaster) Cibarium. Witt.

LCVI. Elaphomyces Leveillei. Tul.

LCVII. Elaphomyces Aculeatus. Witt.

LCVIII. Queletia Mirabilis. Fries.

LCIX. Lepiota Carneifolia. Gillet.

C. Pleurotus Olearius. Fries.

CI. Mollisia Retincola. Fuckel.

Les dessins de toutes les espéces indiquées sur cette liste font suite á mon album et portent le même $\mathrm{N}^{\circ}$ en chiffres romains. 



$$
\text { L I S T E }
$$

\section{DES CHAMPIGNONS ALIMENTAIRES}

DU DÉPARTEMENT DE LA MARNE. 


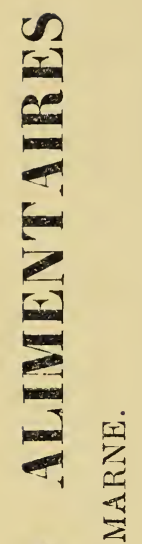

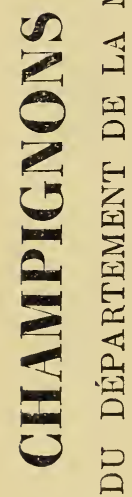

os

$+$

2

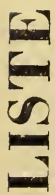

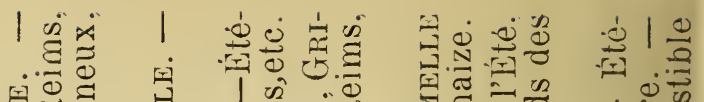
舟过界 1

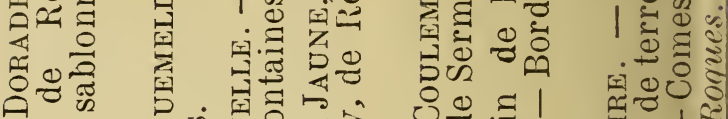

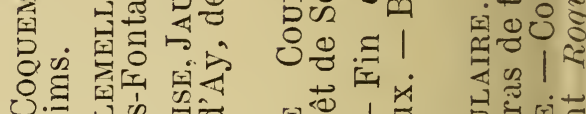

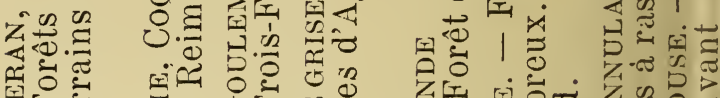

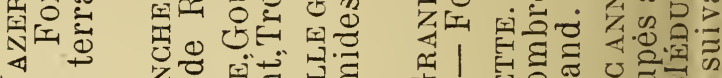

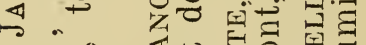

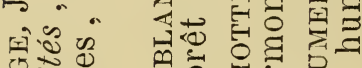
of

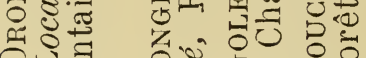
过过 궁

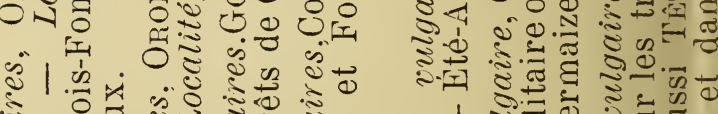

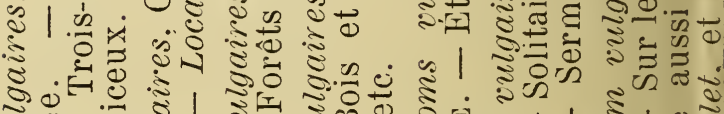

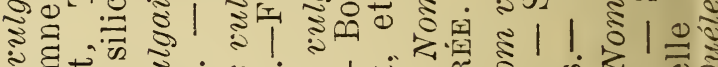

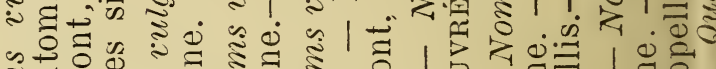

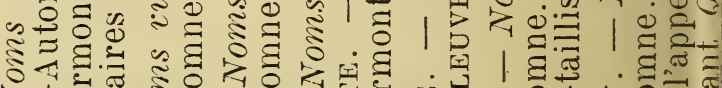
궁

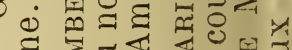

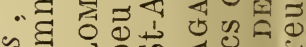
अ $\therefore \equiv 0=0$ व o7 क0

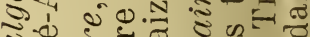
政

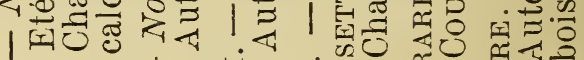

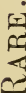

$\dot{8}$

ช

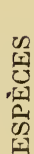

!

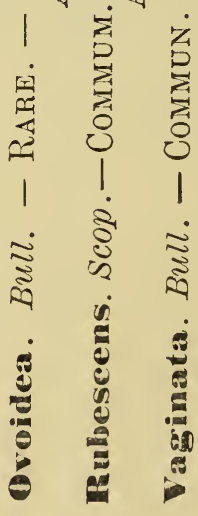
赵

悹

岕点点

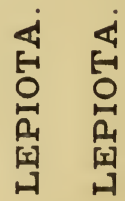

运 


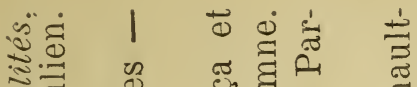

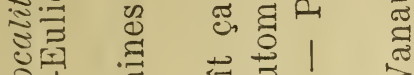

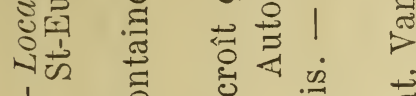

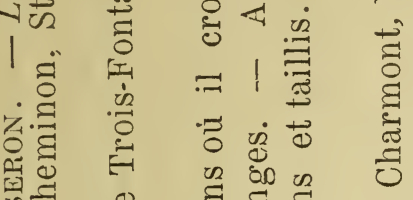

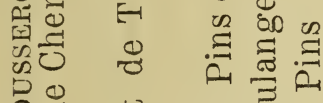

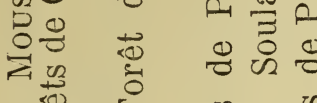

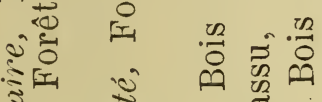

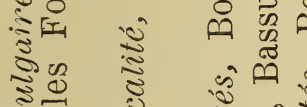
政 ํํㄹ.

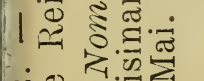

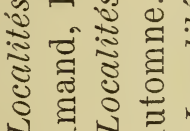
(1) 年 㱐

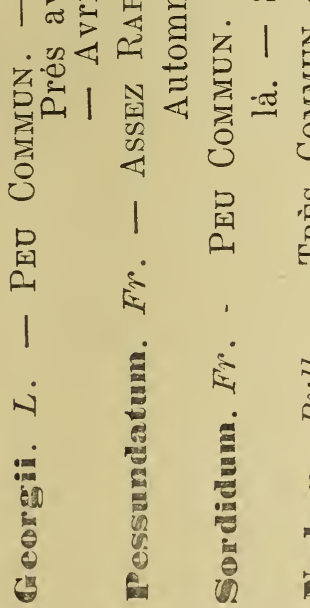

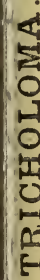

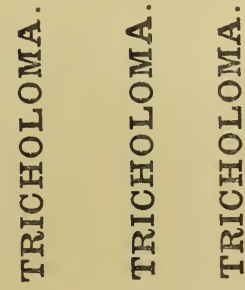

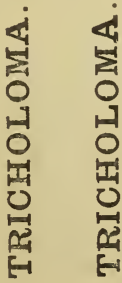

遥

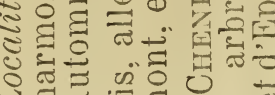

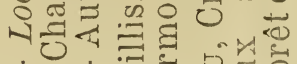

111100

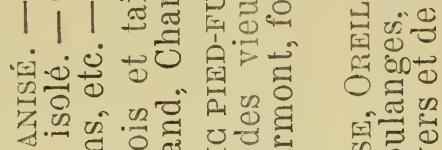

\& 7 .

Un

.

诸

\%

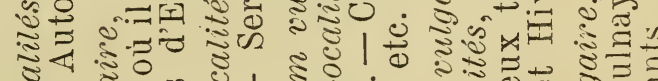
ช.

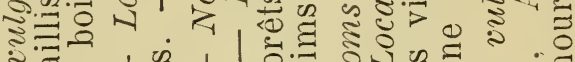
|

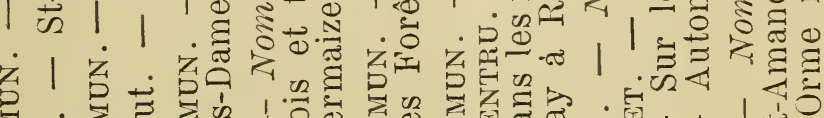

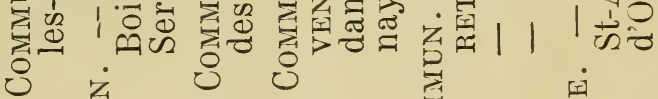

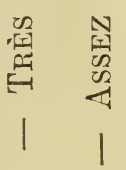

$\int_{2} \stackrel{i}{N}$

赔
8

¿্ّ

象
लि

芯

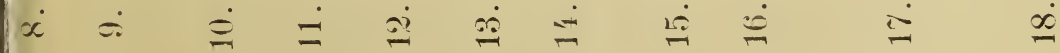




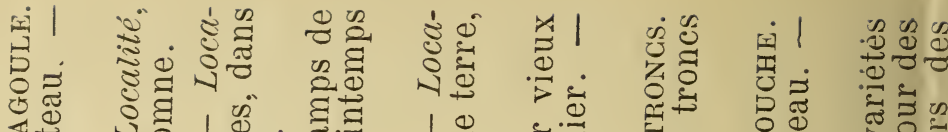
乐更

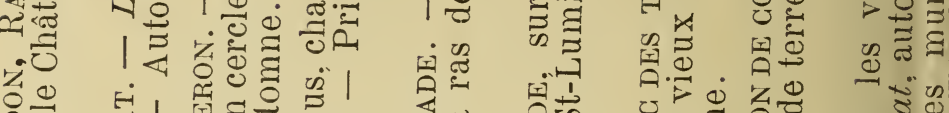

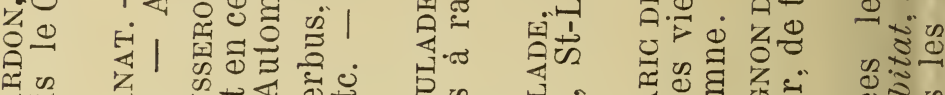
级 过

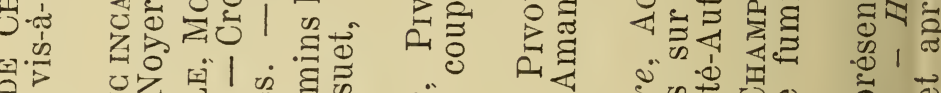
$A$ एँ

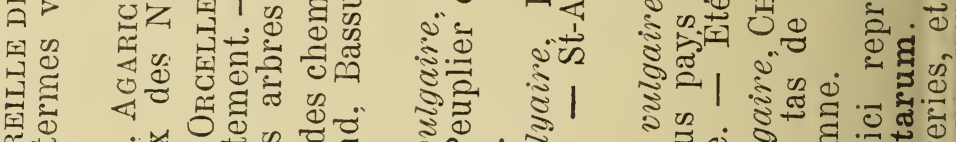

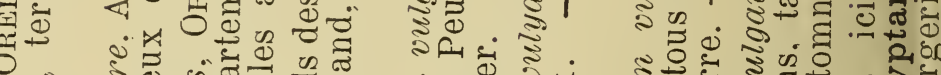

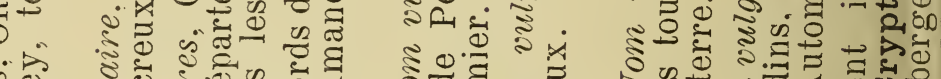

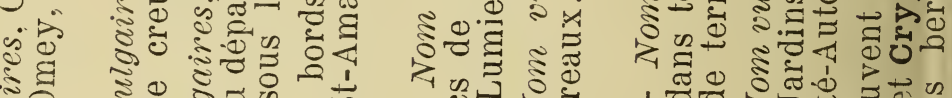

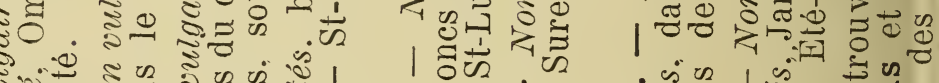

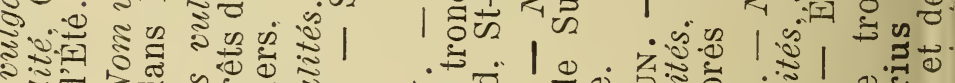
语 ङ 0 द

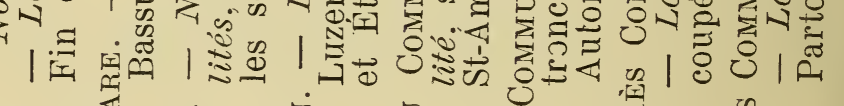

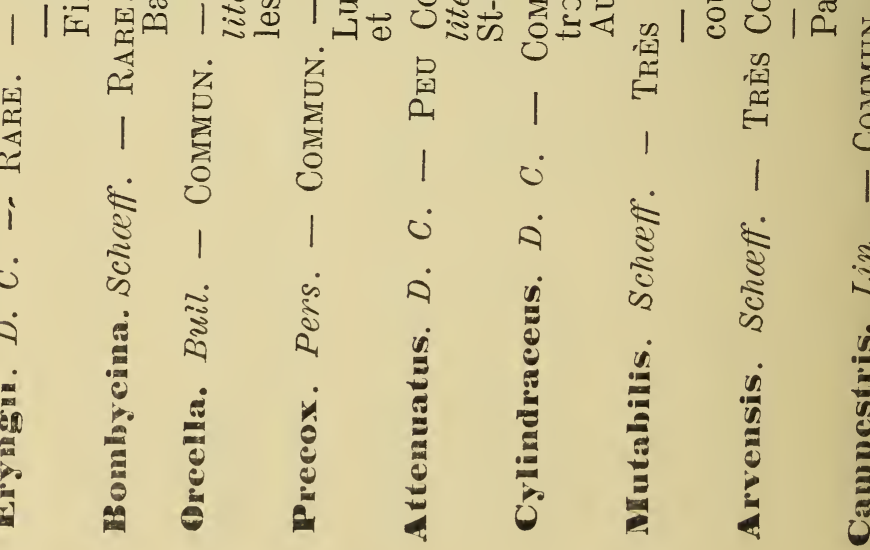

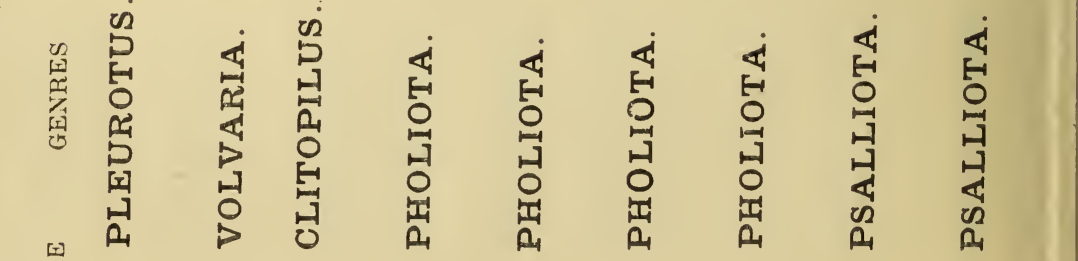

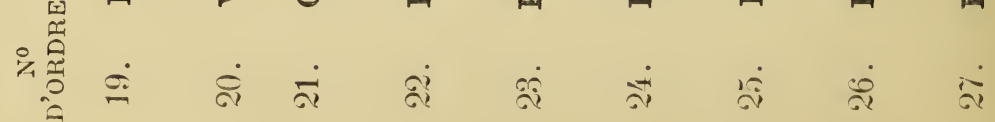




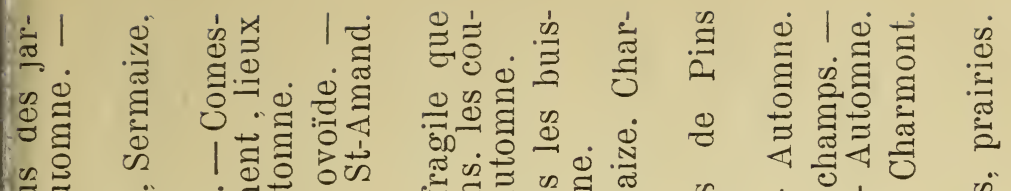

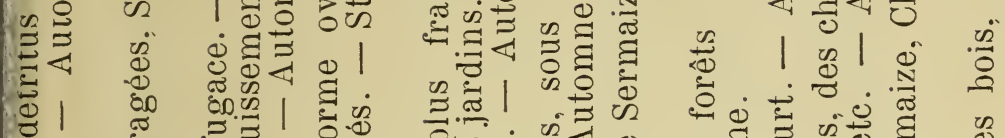

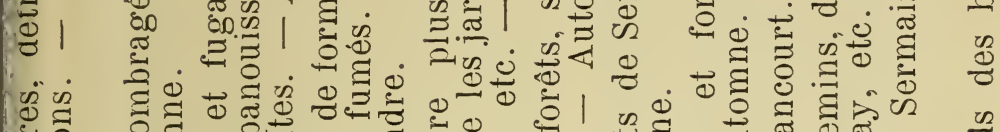
.

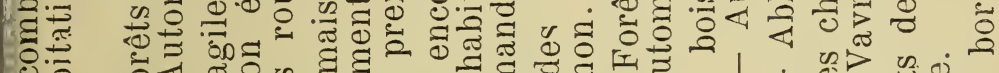

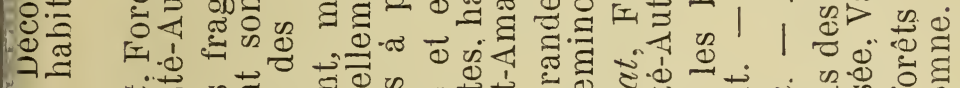

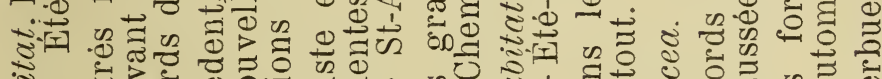

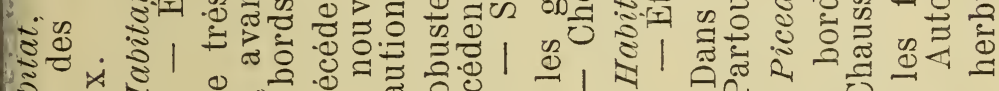
今.

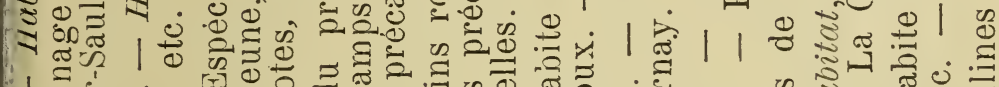

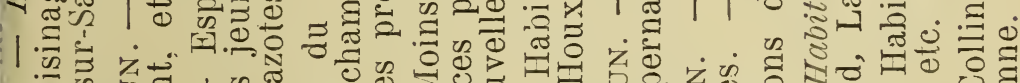
.

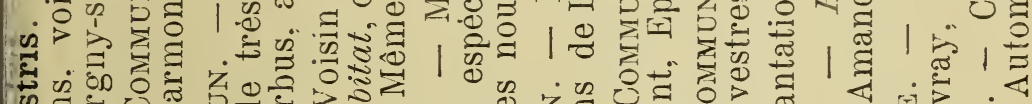

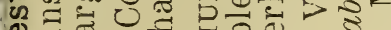
次地

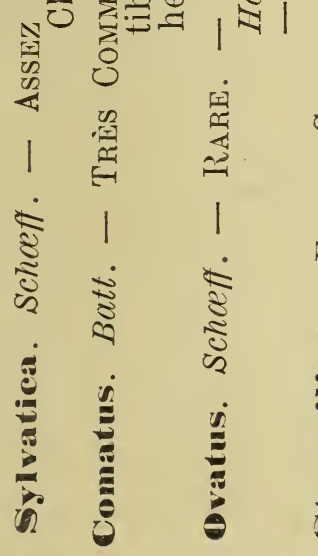

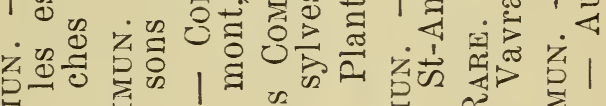

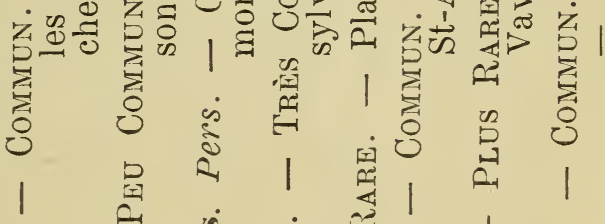

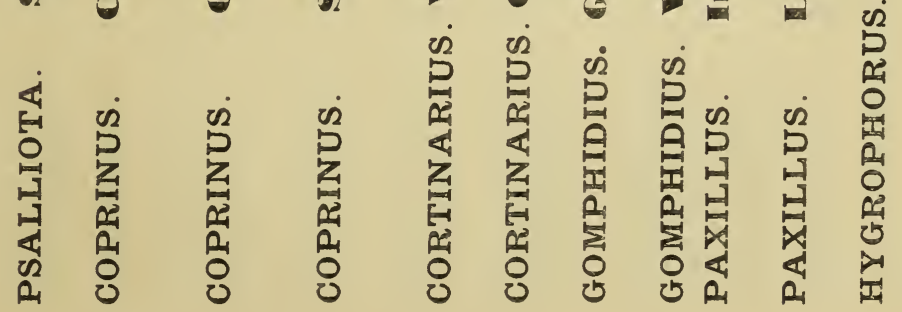

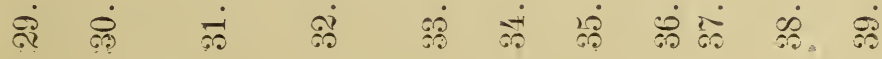




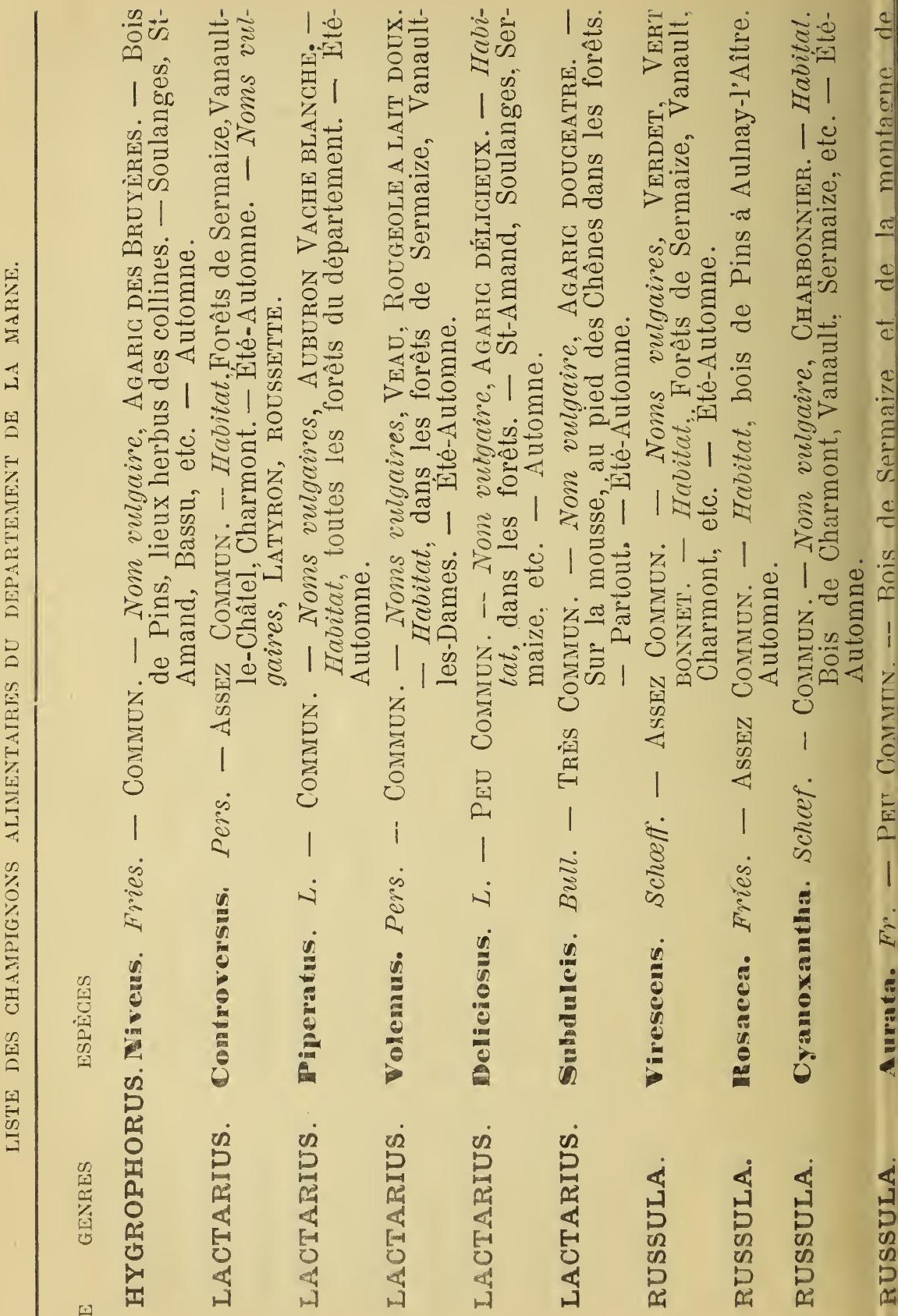
$\longrightarrow 8$ 光

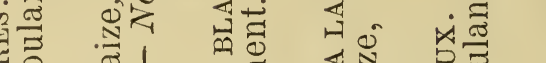

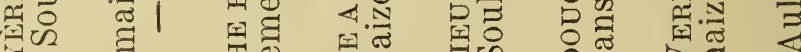

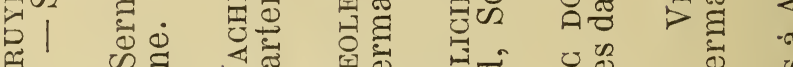

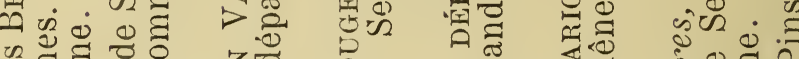

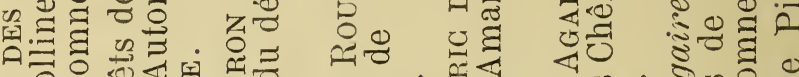
00 용 थय 에

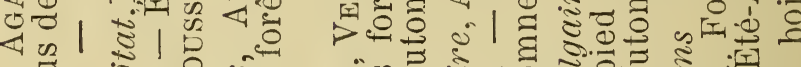

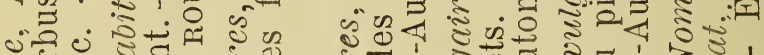

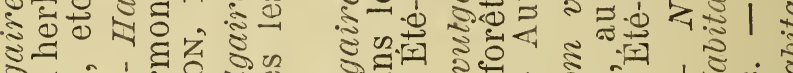

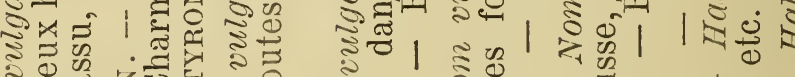
ईच

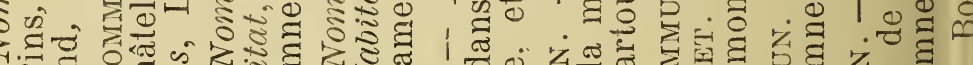

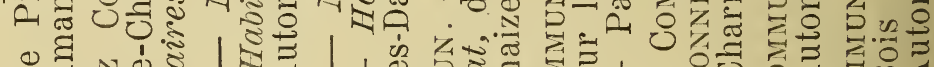
.

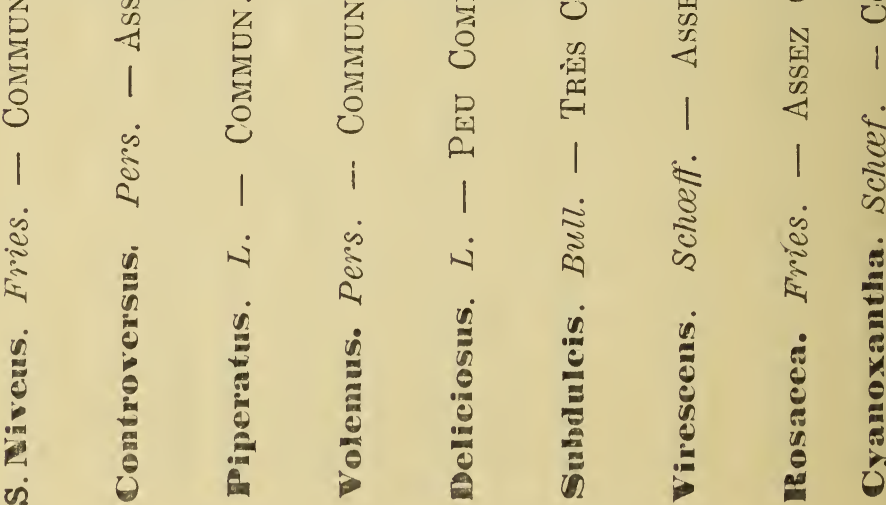




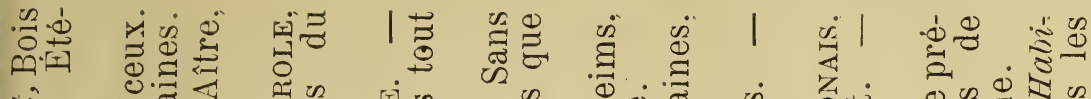

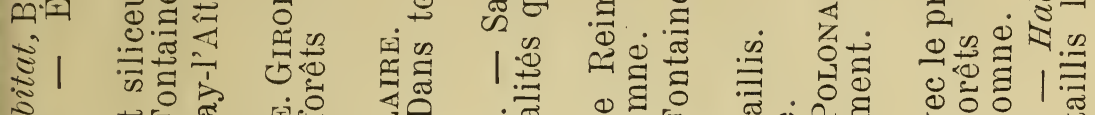
过 ।

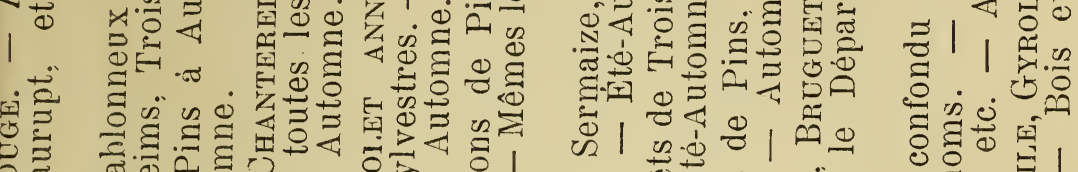

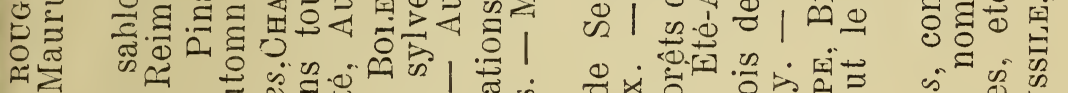

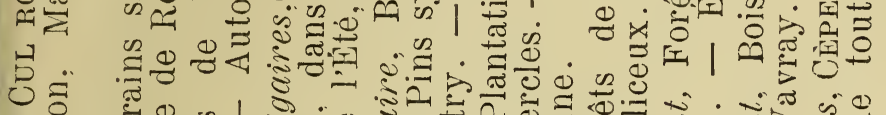
ن.

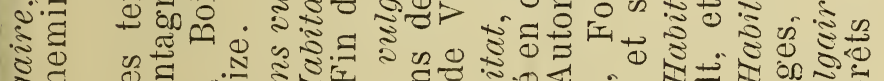
उٓ की डे ڤ

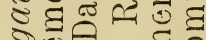
정 is

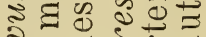

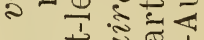
क n

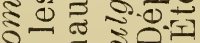

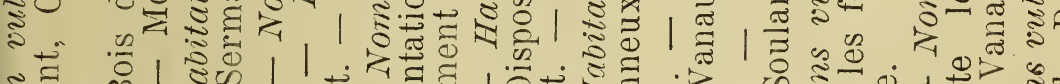

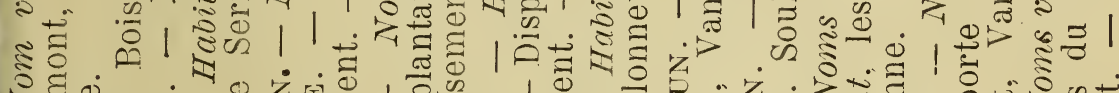

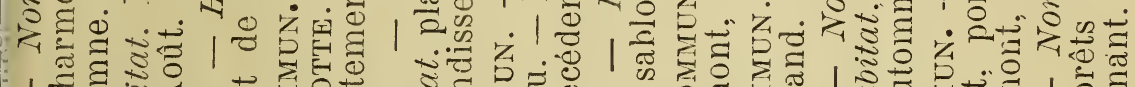

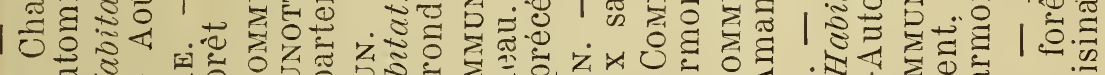
. 范更

尊

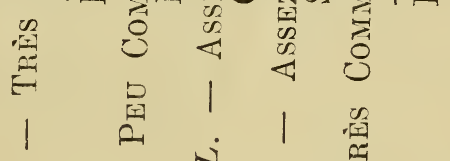

ฐ

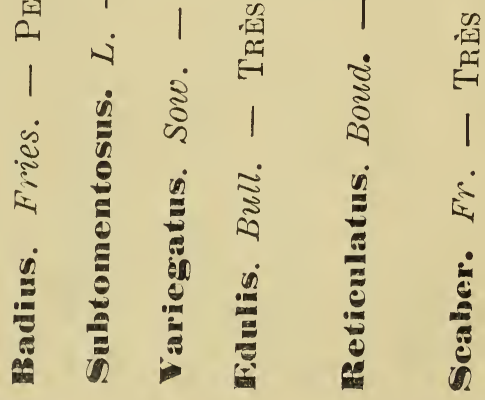

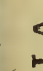

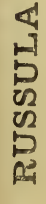

(2)

(1) 8

룰

ช2

A

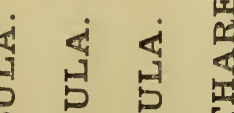

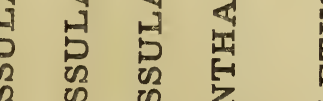

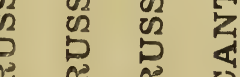

$\therefore$

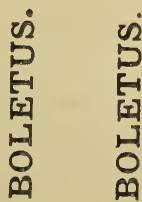

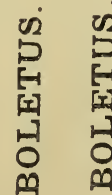

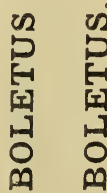

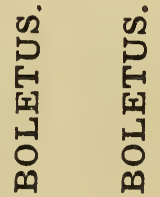

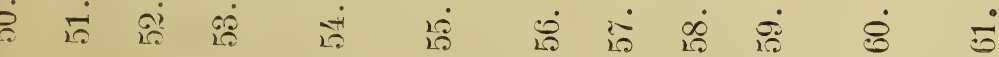




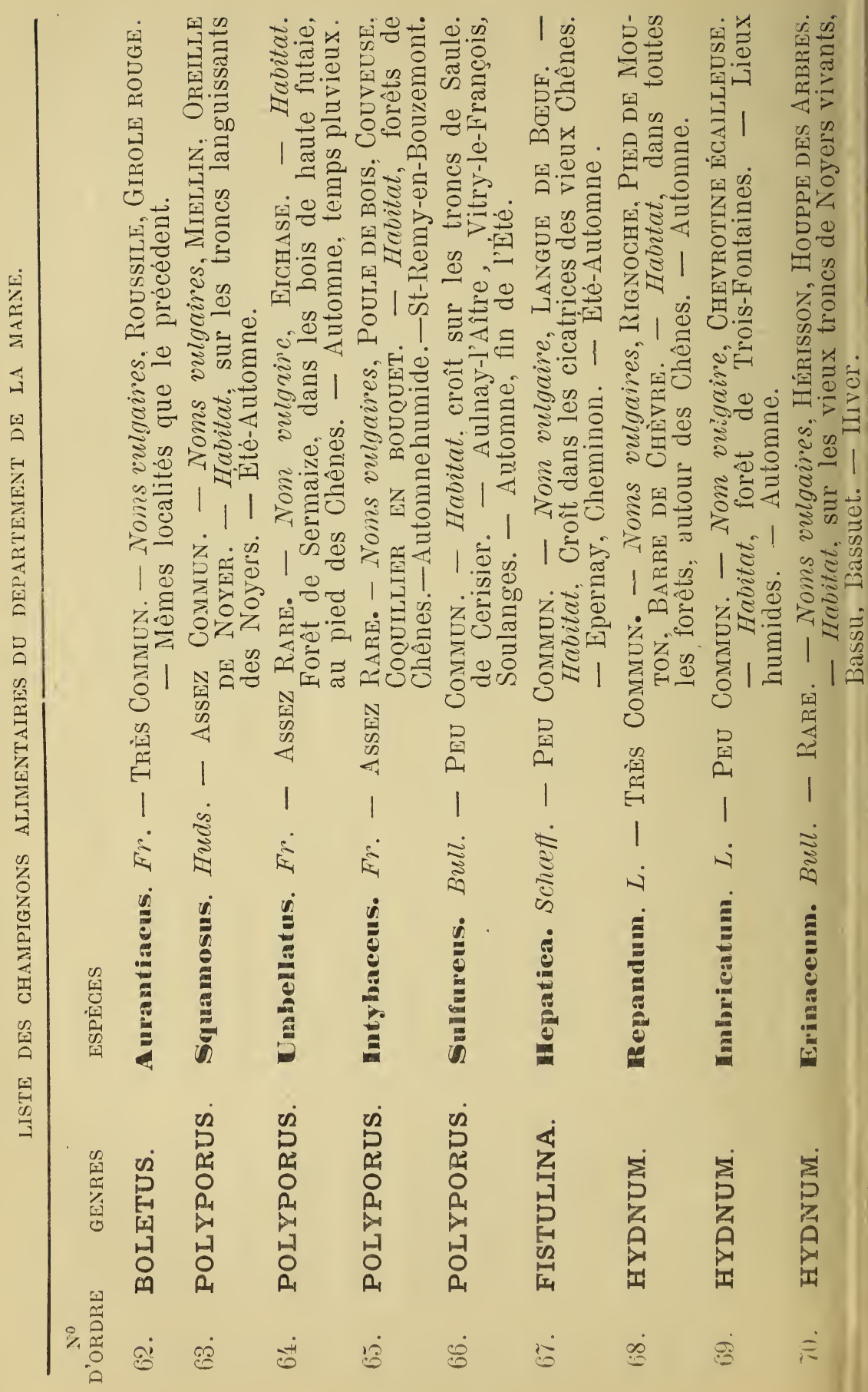




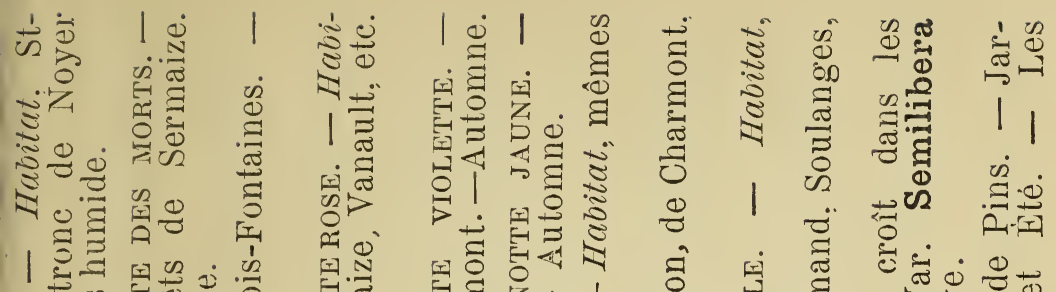

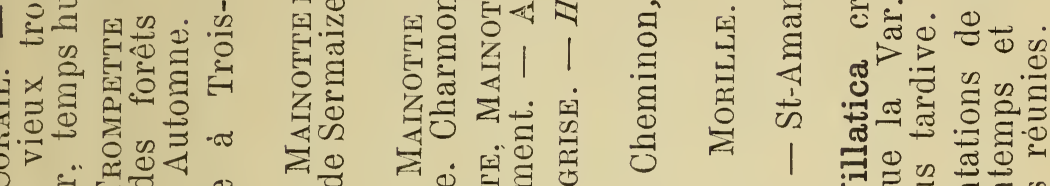
$\exists$ Jै

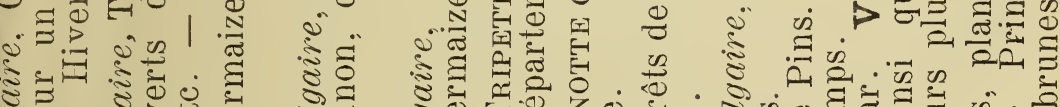

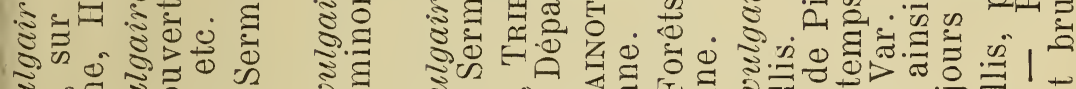
สँ

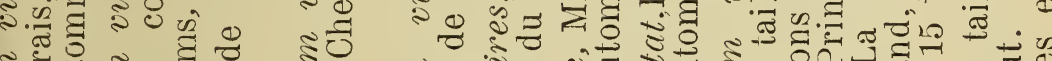

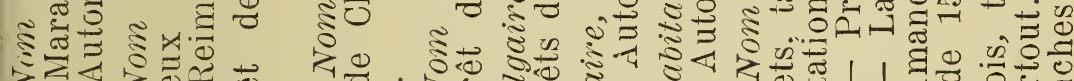

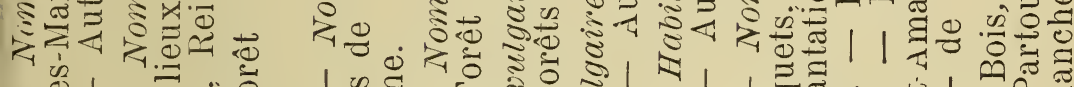

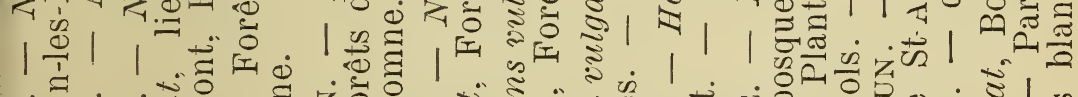

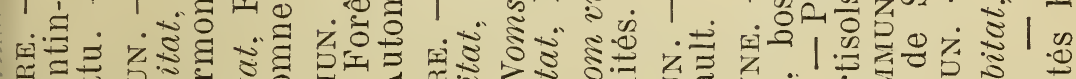

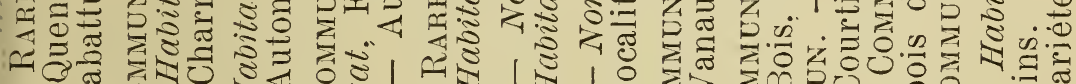

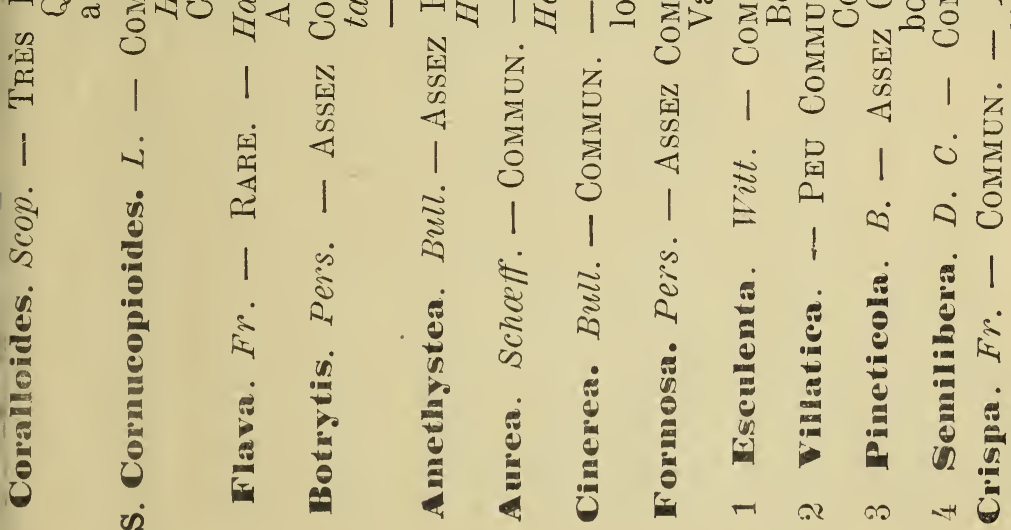

水

$\stackrel{i}{i} \quad \stackrel{i}{i}$

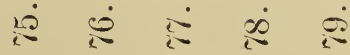




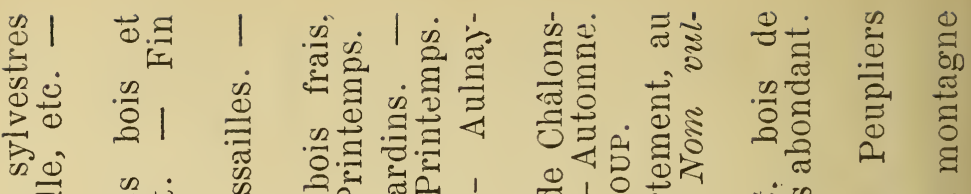

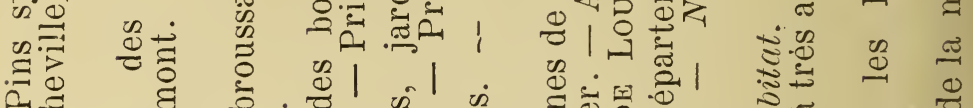

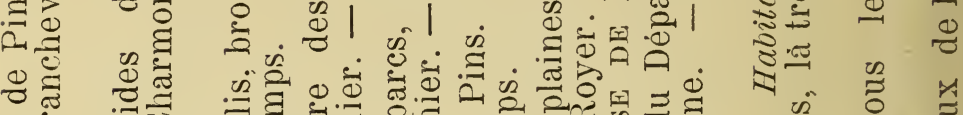

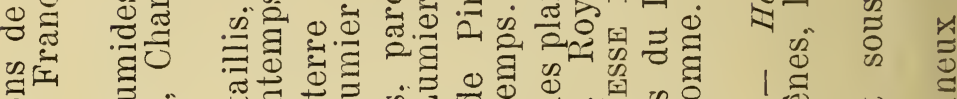
.

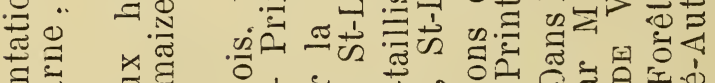

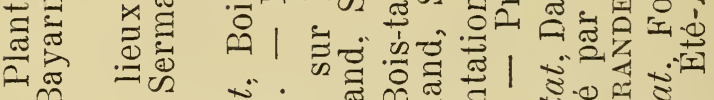

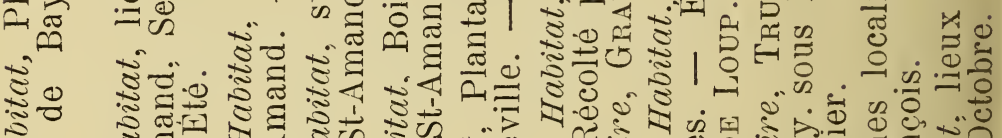

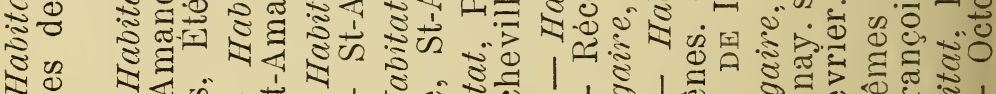

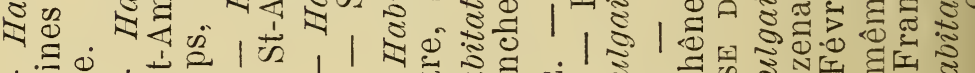

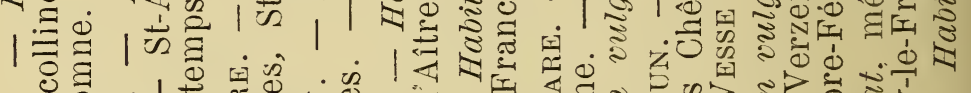

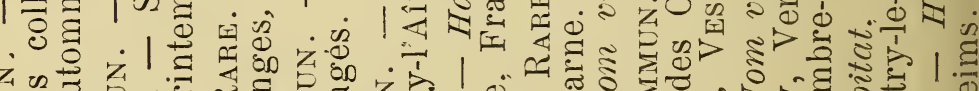

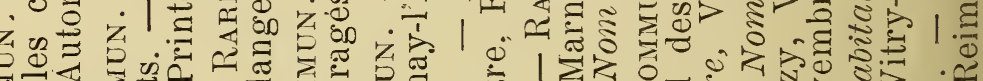

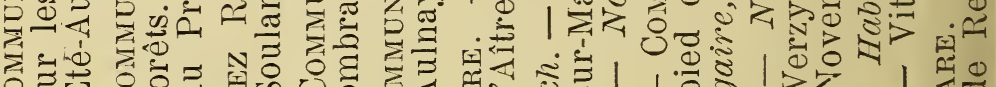

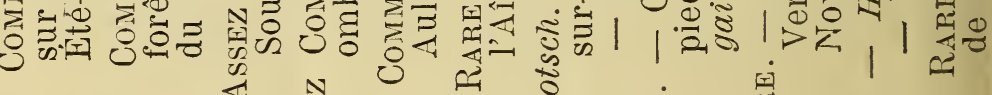
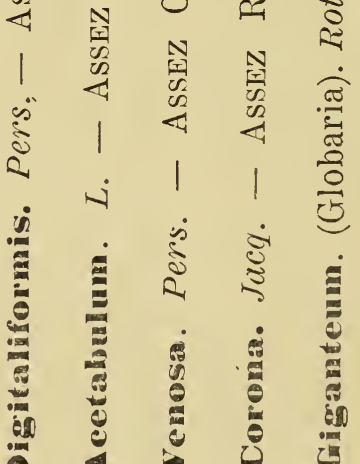

$\therefore \quad \stackrel{\sim}{\stackrel{\sim}{\pi}}$

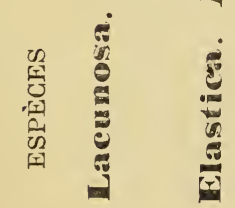

룰
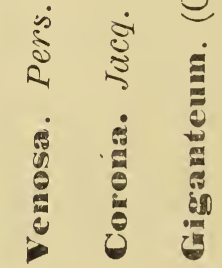

苍

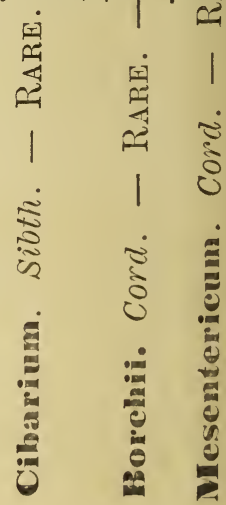

红

7

r

\%

- $\quad$ अं

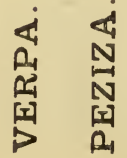

S

\section{4 व}

$N$

N

0

ज्ञा 뙤 मे

ค

คै

되

A

0 ज्ञा

0

ต

ल 9

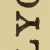

p

E

בֶ.




\section{I S T E}

\section{DES CHAMPIGNONS VÉNÉNEUX ET SUSPECTS}

$$
\text { DU DÉPARTEMENT DE LA MARNE. }
$$




\section{is

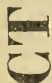 \\ 5 \\ 2. \\ a

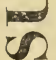

5

$\sum_{-1}^{\infty}$

$>$ 동

盗

齐

$\sum_{103}$

공

음

$a$

$\sum$

in

둥

$=$

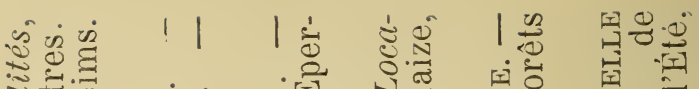

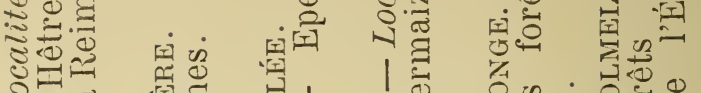

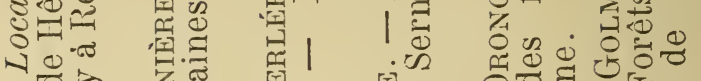
的管

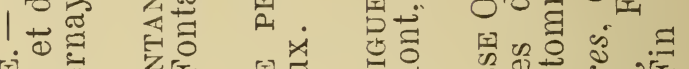

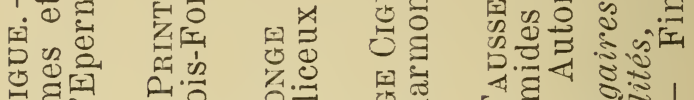

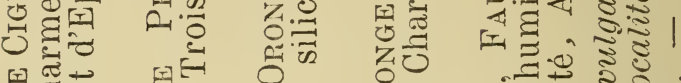

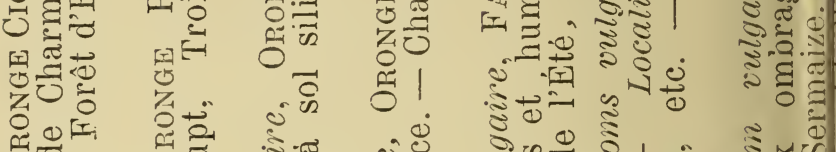

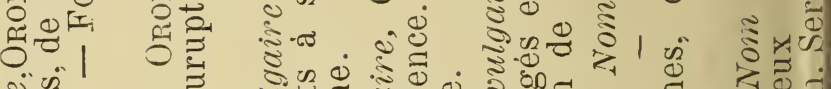

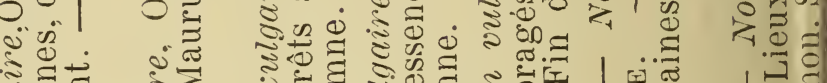

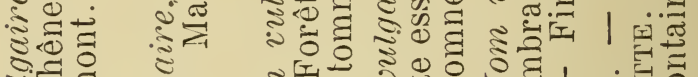

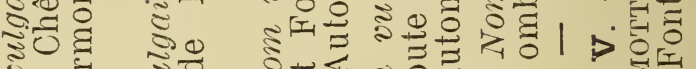

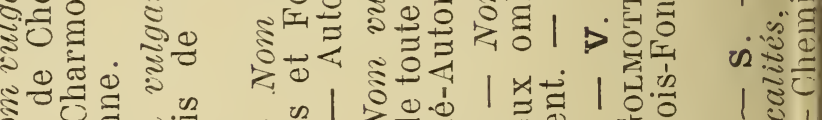

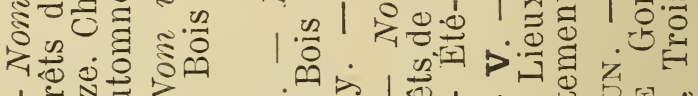
।

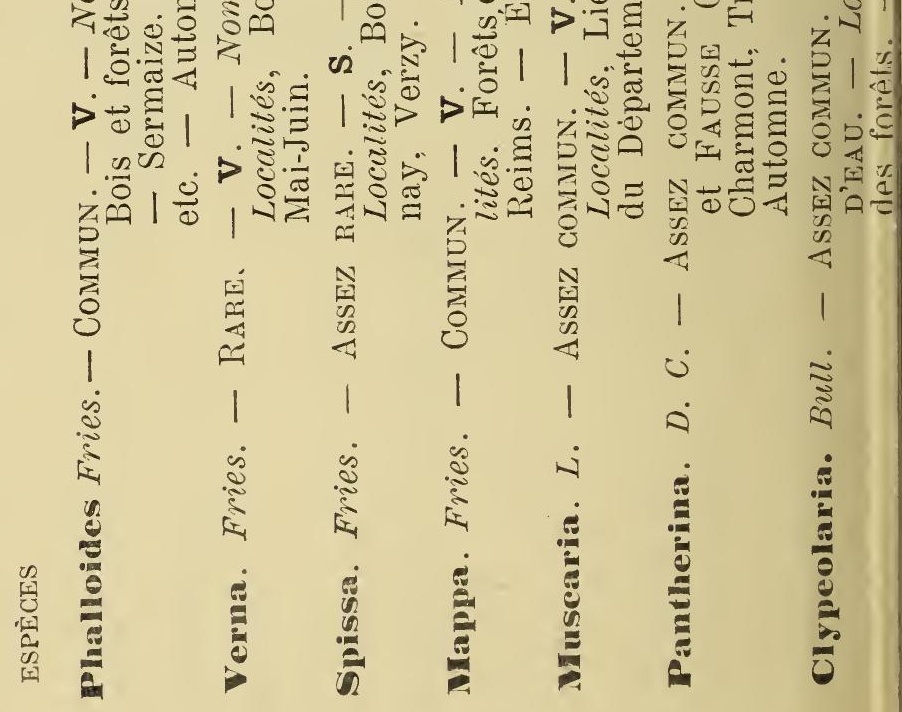

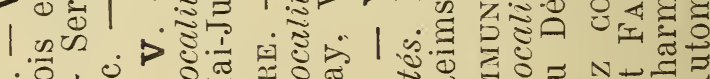
动 $1 \frac{0}{0}$ 


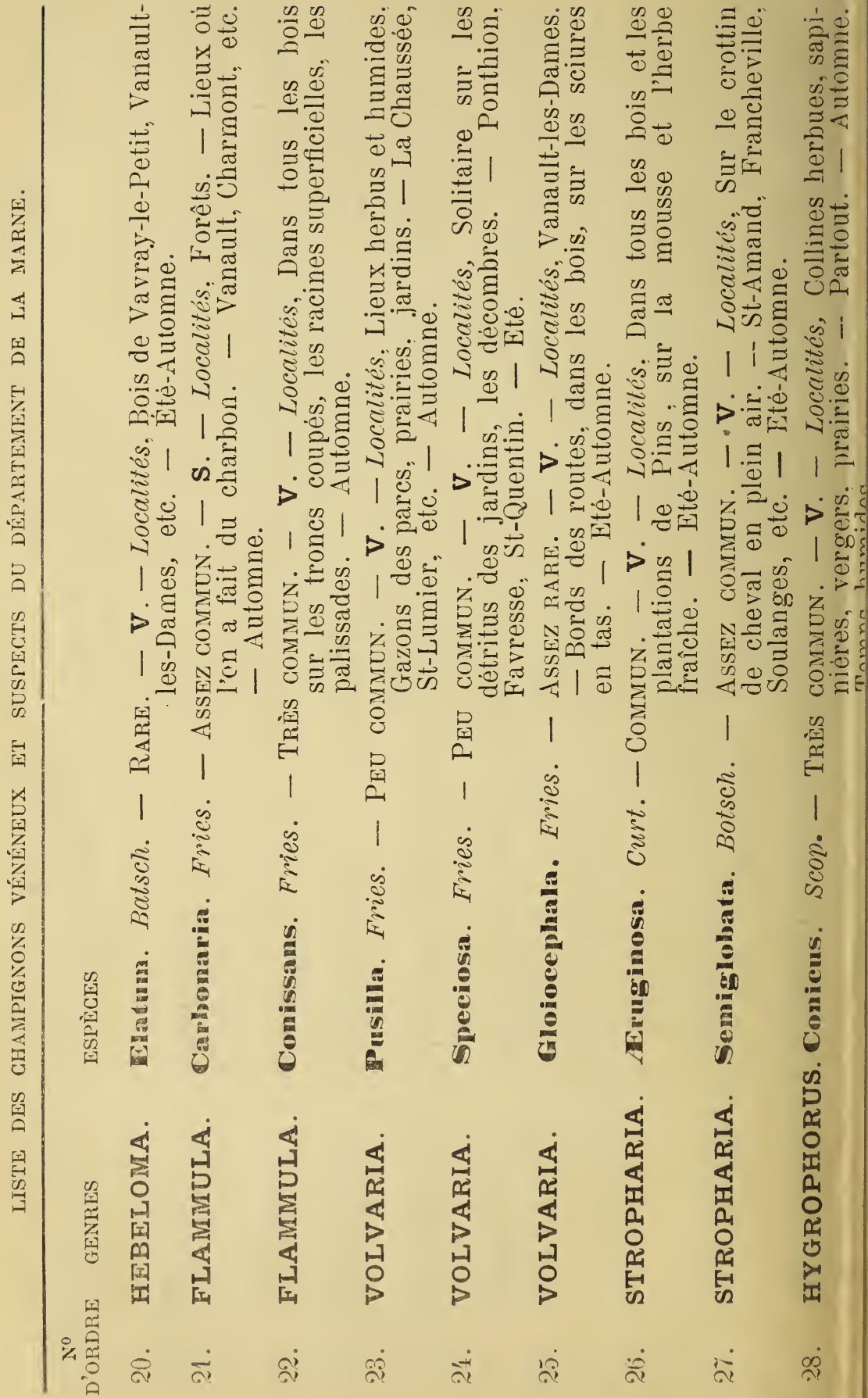




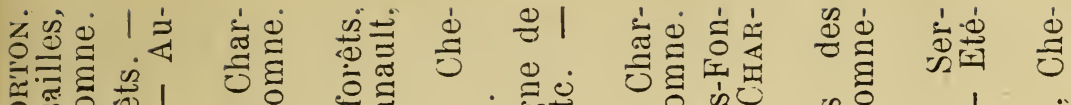

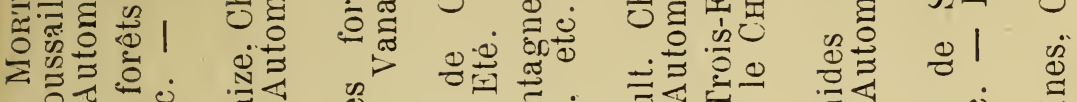
政

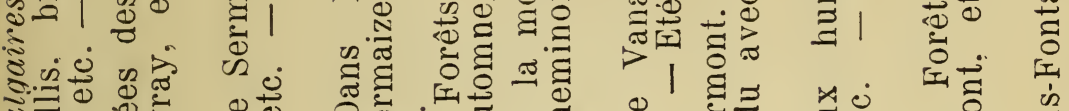

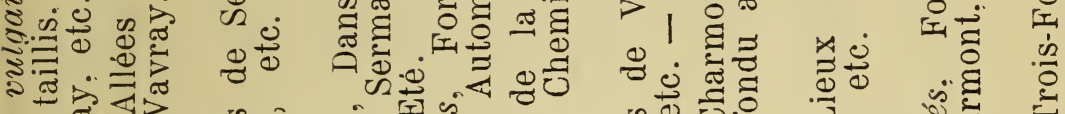

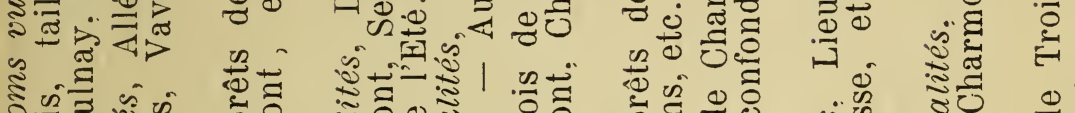

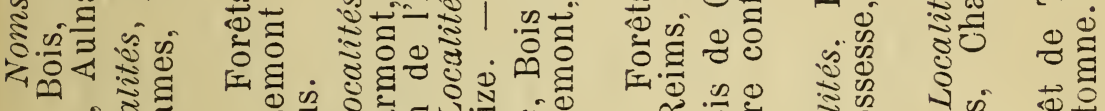

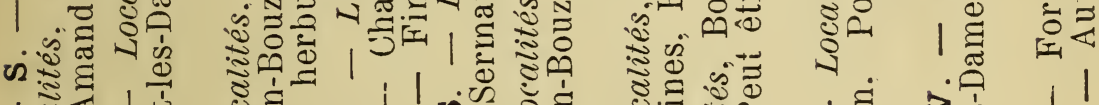

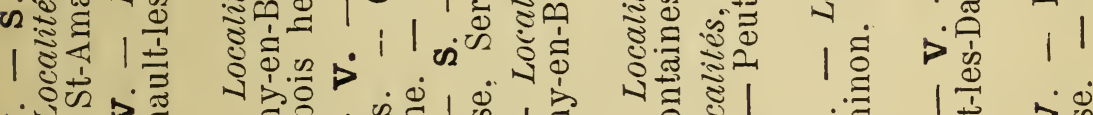
虫

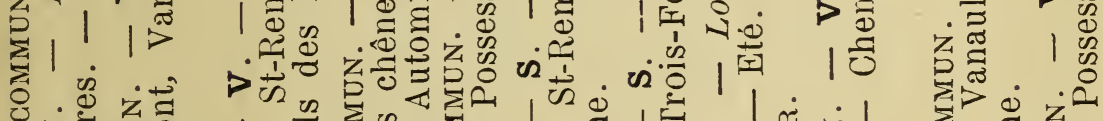

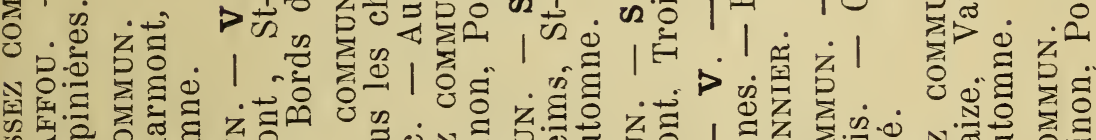

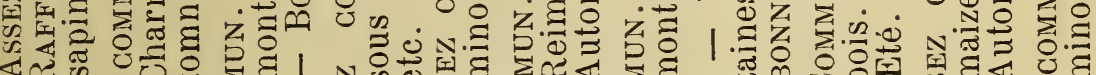

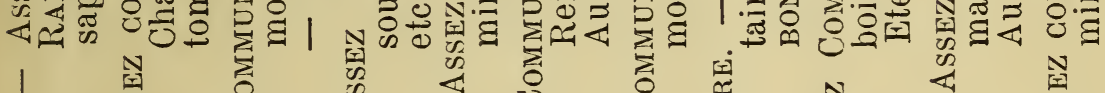

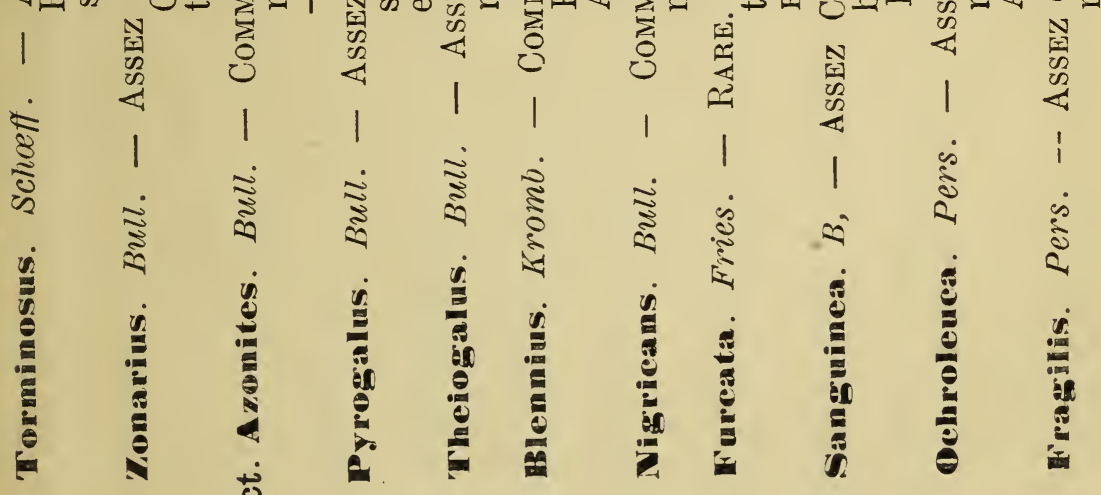
D.

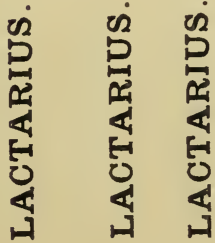

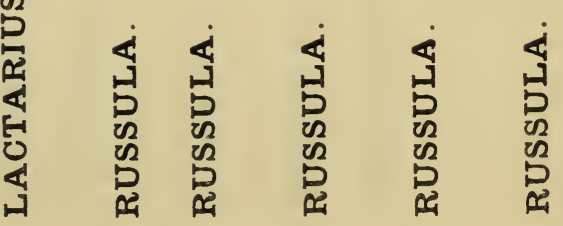

म

จั

总

ชं क्ष

:

$x$

$\infty$

के 


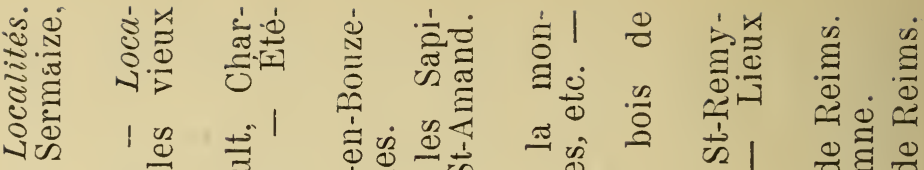

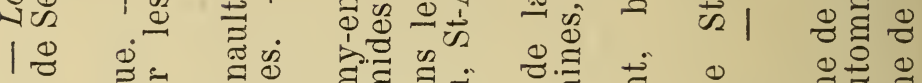
هi

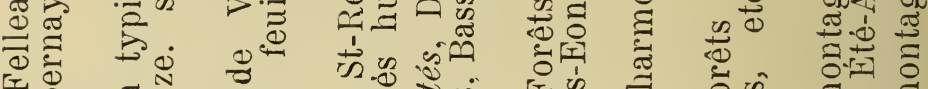

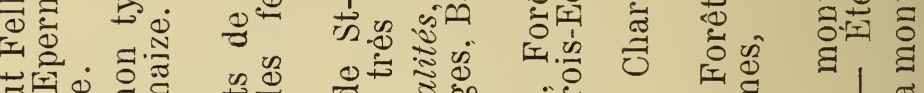

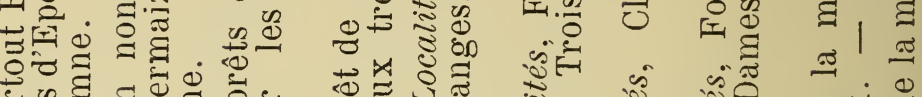

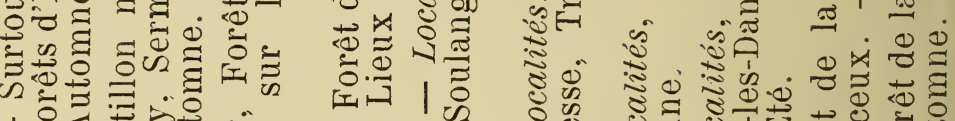
0 寻䔽 I

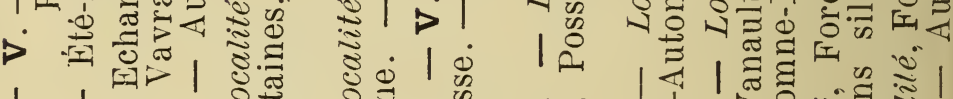

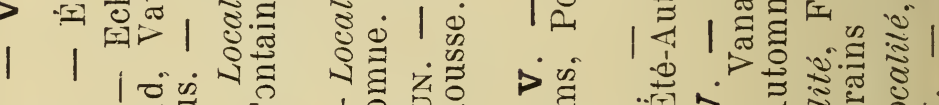

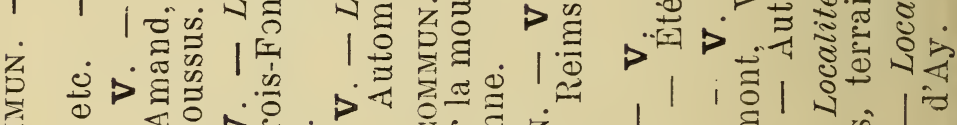

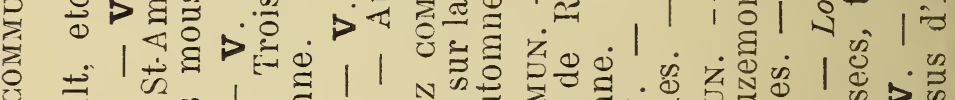
○

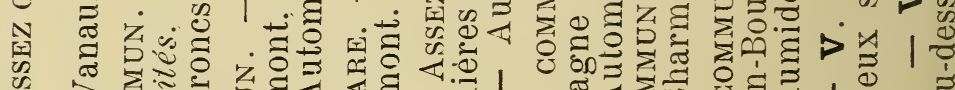

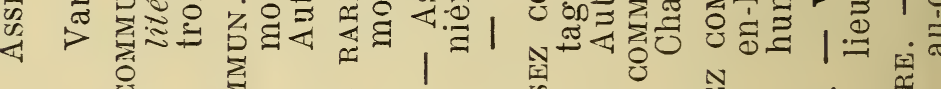<smiles>[C-]#[C-]</smiles>

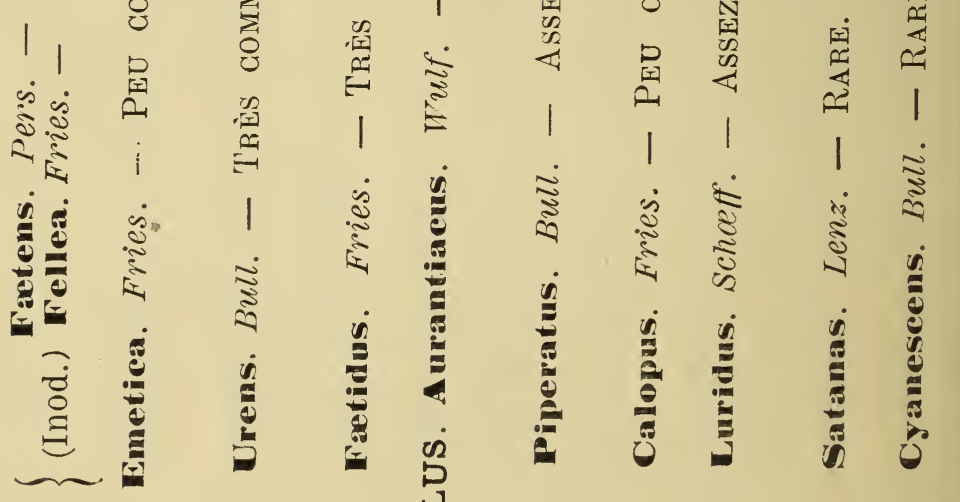
닝

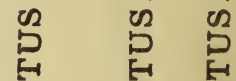

디 되 디 띠

싱 00 잉 


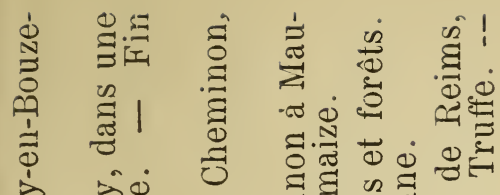

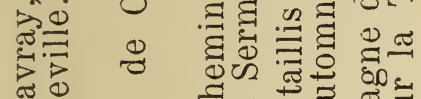

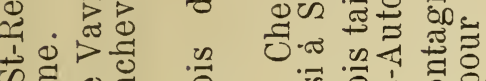

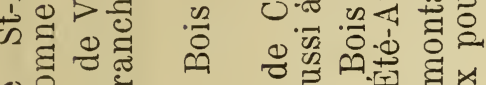
을 证

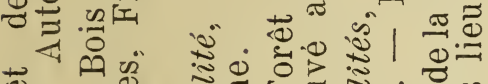
卷। . 马ुँ

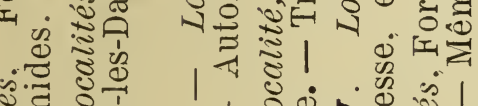

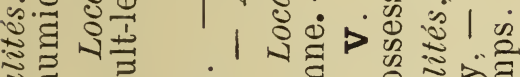

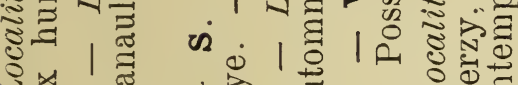

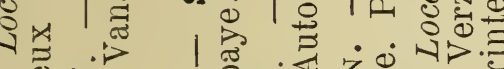

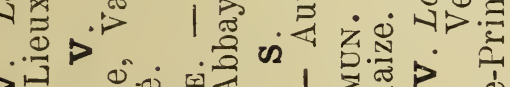

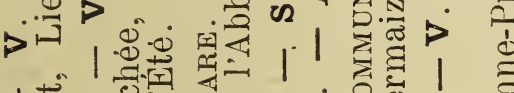
।

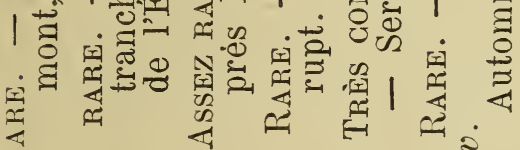

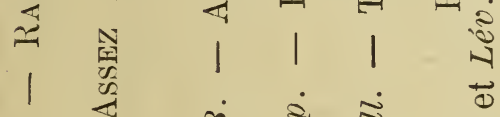

।

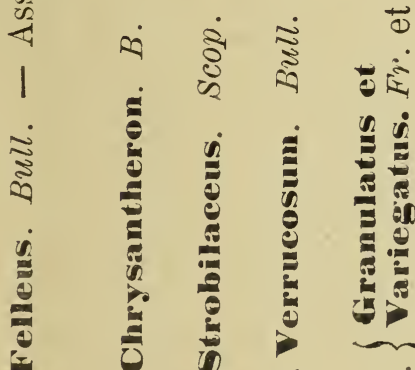

(1)

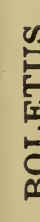

要

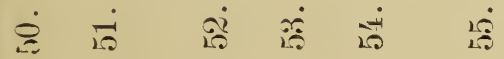



TAB LE.

Pages.

INTRODUGTION..................... I

Classification des Champignons. - Disposition générale des familles suivie de planches représentant les types principaux............... Essai d’une Classification des Agaricinées, E.Roze, tableaux et dessins à l'appui......... 19 Champignons : Hymêniés.................... 25 Péridiés.................. 124

Plasmodiophores.............. 130

- Hypodermés................. 139

- Phycomycètes............... 178

- Asconémés................. 180

- Onige̊nés.................. 181

- Gupulés................... 183

- Nucléés.................... 229

- Spheropsidés................ 853

- Mélanconiés................. 400

- Hyphomycètes............... 411

- Mycelia Sterilia.............. 498

- Exanthemata................ 498 
$-586-$

Liste Alphabétique des Genres a vec les dessins DES SPORES........................ 501

Supplément au Gatalogue................ $5 / 1$

Contribution a la Flore mycologiQue générale. $5.5 \bar{J}$

Liste des Ghampignons RÉCOltés dANS LES PAYS CIRGONVOISINS DU DÉPARTEMENT DE LA MARNE. $\overline{6} 61$ Champignons almentatres............... 568 Champignons véNÉNEUX E'T SUSPEGTS........... 578 


\section{E R R A T A.}

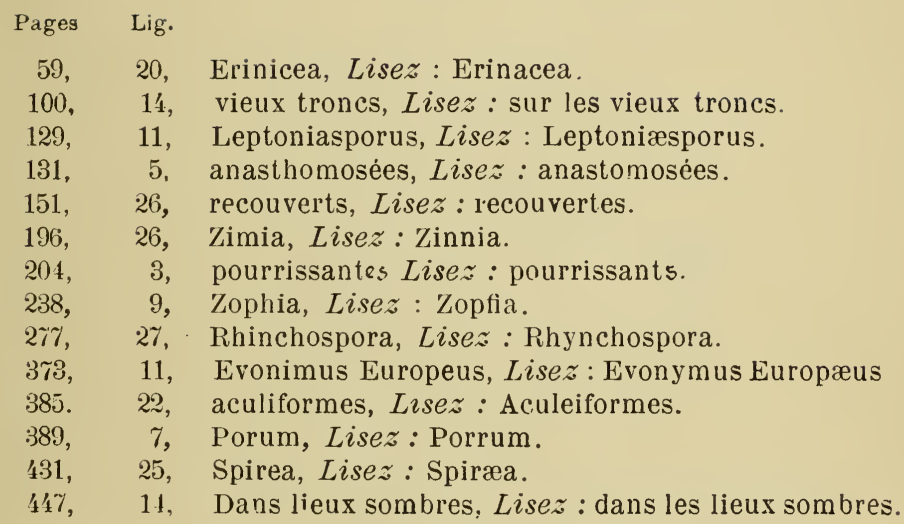






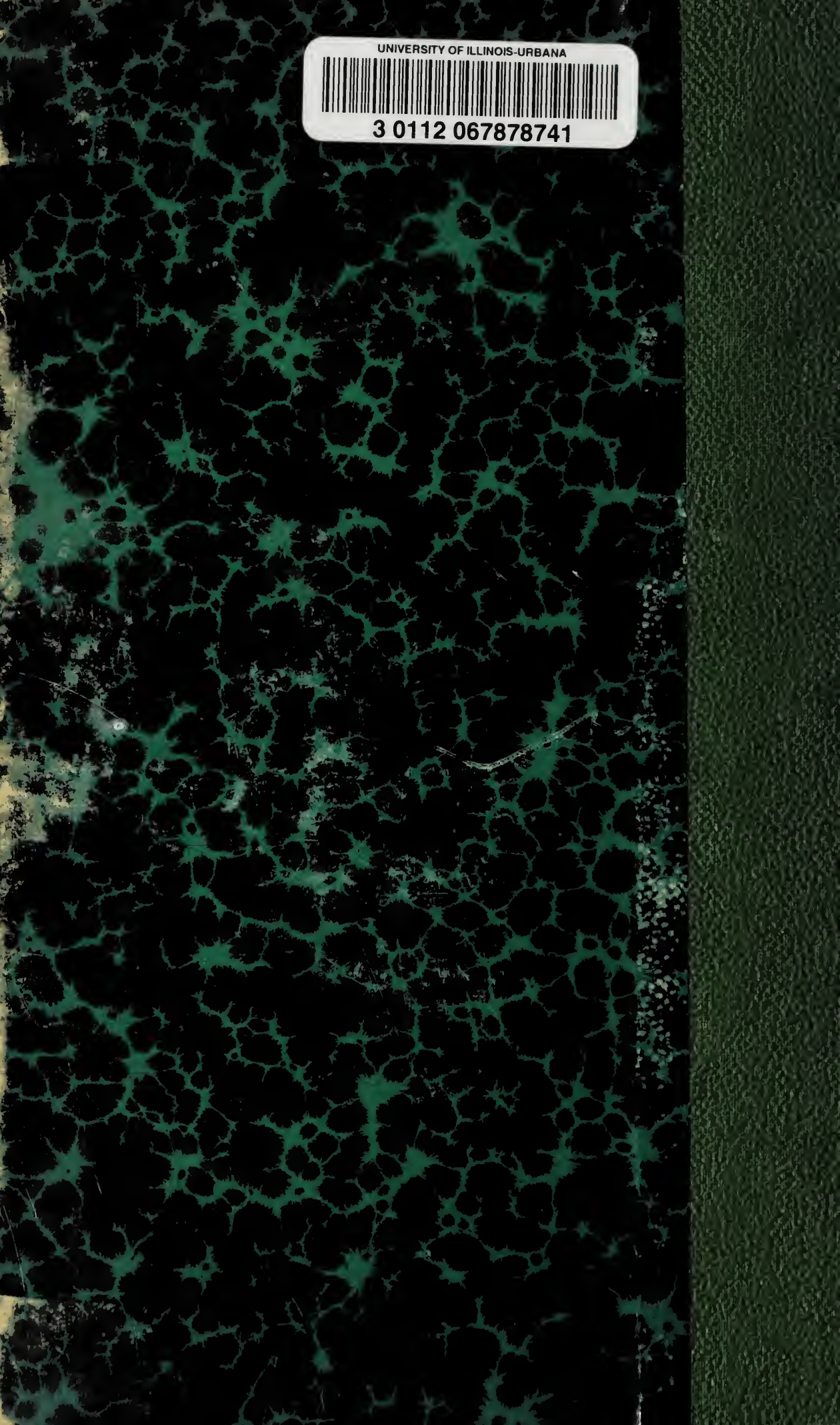

